\title{
THE EFFECTS OF URBAN SETTINGS ON AIRFLOW CHARACTERISTICS FOR URBAN AIR MOBILITY APPLICATIONS
}

\author{
Maryam Al Labbad
}

A Thesis submitted to the Faculty of Graduate Studies and Research in partial fulfilment of the requirements for the degree of

\section{Master of Applied Science}

in

Aerospace Engineering

\author{
Ottawa-Carleton Institute for \\ Mechanical \& Aerospace Engineering \\ Department of Mechanical and Aerospace Engineering \\ Carleton University \\ Ottawa, Ontario, Canada
}

(C) 2021

Maryam Al Labbad 
The undersigned recommend to

the Faculty of Graduate Studies and Research

acceptance of the thesis entitled

\title{
The Effects of Urban Settings on Airflow Characteristics for Urban Air Mobility Applications
}

\author{
Submitted by \\ Maryam Al Labbad \\ in partial fulfilment of the requirements for the degree of \\ Master of Applied Science in Aerospace Engineering
}

Dr. Alanna Wall, Supervisor

Dr. Guy L. Larose, Supervisor

Dr. Fidel Khouli, Supervisor

Dr. Ron Miller, Department Chair

Carleton University

2021 


\section{Abstract}

The urban wind environment is characterized by complex flow structures and high levels of turbulence due to the densely built up regions and the presence of tall buildings. This renders the flight operations in urban areas challenging since the turbulent airflow characteristics may impair the control system of the flying vehicles, leading to loss of control, which could be catastrophic considering that the areas are densely populated. This thesis investigates the urban airflow characteristics that are expected to pose significant challenges to the flight of aircraft systems and impair their aerodynamic stability.

Complex airflow structures were analyzed through wind tunnel experiments in two different facilities on scaled models that represent a realistic urban setting in Toronto, Canada. Flow measurements were conducted at various locations around and above buildings for five different wind incident angles to capture the full range of flow features that are suspected to have influence on flight including wind speed acceleration and deceleration, wind direction changes, wind speed and directional shear, turbulence intensity and vortex shedding phenomenon. The location of the worst-case flow features were identified with respect to buildings geometry and arrangements. The overall findings of the experiment revealed the severity of the flow conditions in urban environments, particularly, within the height of the buildings.

The effect of testing facility, model scale, approach flow exposure profile and near field modelling on the accuracy of data measurements were investigated by conducting 
additional wind tunnel experiments at a different facility on a different model of the same urban site to identify the sensitivity of the flow features studied to the experimental approach deployed. 
I dedicate this work to my brother Mohammed Al Labbad for his endless sacrifices and continuous support in life and throughout my academic journey. 


\section{Acknowledgments}

"It always seems impossible until it is done"- Nelson Mandela

These eight words explain it all, and now it is done. This academic journey was as wonderful as challenging, thanks to those who helped in making the impossible so possible.

First and foremost, my profound gratitude goes to my team of thesis supervisors. I am very fortunate to have had the opportunity to work under your supervision, from which I learned a lot. Thank you for sharing your knowledge, for your support, for your patience and for making this academic journey so fun and memorable.

I would like to thank Dr. Fidel Khouli for offering me this research opportunity at the first place. Thanks for believing in my ability and for your continuous support.

I would like to thank Dr. Alanna Wall for supporting me through every step in this research work. I really appreciate your trust in my ability, guidance and the time you dedicated for me even in your vacations. I truly believe that many of the challenges I faced during this research work went smoothly due to your kind approach. You have been a role model for me, and I learned a lot from you. Your support was beyond the call of duty and will never be forgotten.

I would like to thank Dr. Guy L. Larose for inspiring me, motivating me and helping me throughout this research work. I am really honoured to have you as one of my supervisors. I really appreciate your kind personality and sense of humour. I learned a lot from your expertise and I loved the wind engineering more because of 
you. Thank you for making my visit to Rowan Williams Davies \& Irwin (RWDI) Inc. happened despite the COVID-19 situation. I know that you wanted to give me the maximum of a learning opportunity during this academic journey, which you did.

I would like to thank Hali L. Barber, who had been an unofficial supervisor for me during this research work. I really appreciate all the help and support. You always encouraged me and your efforts in this research are highly acknowledged. Thank you for feeding me with ideas and visions, which made my work even better.

I would like to thank Dr. Ron Miller and Dr. John Hayes for postponing the wind tunnel decommissioning date in order for me to finish my wind tunnel experiment.

I would like to thank my M.A.Sc. colleague Richard McKercher, who worked on the second half of this research project. Thank you for the sharing the thoughts during our research meetings, and for the interesting discussions we had at the RWDI trip. I wish you the best of luck.

I gratefully acknowledge the financial support from the National Research Council Canada (NRC) which allowed me to focus on my research work. Thank you for providing with all what I needed to work from home comfortably during the COVID19 situation. It is much appreciated.

Thanks are due to the aerodynamics lab team at the NRC M2: Sean McTavish, Brian McAuliffe, Annick D’Auteuil, Fenella De Souza, Fa Ghorbanishohrat, Richard Lee, Arash Raeesi and Bernard Tanguay, for the inspiring discussions and sharing the knowledge. A special thanks to Sean McTavish for helping me out during my wind tunnel experiment through answering my questions and lending me the books I needed, all of which had improved the quality of my work. I would like to thank Joshua Raeside from the NRC technical department for the continues help in relation to technical matters. Also, I would like to thank the NRC technical department for providing me with the experimental equipment, which made my wind tunnel experiment possible. 
I would like to acknowledge the effort and support from the technical staff at Carleton University. Alex Proctor, Kevin Sangster and Aric Adcock from the machine shop, thank you so much for your advice, patience and help during my wind tunnel experiment. I really appreciate the time you spent preparing my test set-up and model components. Stephan Biljan, thanks for 3D printing my test components. David Raude, thank you very much for the training sessions before my first use of the wind tunnel, for your quick acts in getting the wind tunnel fixed so I can presume my test before the wind tunnel decommissioning, and for re-printing the 3D parts when they got damaged. James Cann, thanks for helping me out finding the necessary test equipment, and spending the time in teaching me how to use them. Steve Truttmann, thanks for the lovely discussions and giving me extra time to complete my experiment. Neil McFadyen, thank you for all the technical assistance that made working from home possible.

I would like to thank the technical team at RWDI Inc. for the positive environment and the lovely talks. I really enjoyed my visit and it will be one to remember !

My gratitude, appreciation and love to my parents Hameedah Al-Ghawas and Abdullah Al Labbad for their endless love and support. Without you mom and dad, I would not be here writing this thesis. You encouraged me to pursue my dream and you always believed in my ability. I appreciate the financial support from you along the way, and I never forget your continues prayers.

I would like to thank my wonderful sister Kawthar Al Labbad for her support and encouragement during this research work. Thank you for the continuous calls and messages from overseas to make sure that I am right on track on writing this thesis. Thank you for the lovely talks which reduced the intensity of the most stressful moments. I love you more than I can say.

I also would like to thank my brother Abbas Al Labbad for his love and sense of humour that always helped in keeping the energy level up. 
I would like to thank my sister in law Forat Alismail for her love and kindness, and for the moments we shared together here in Canada during my academic life.

Lohay Al-Bess, you have been an amazing partner both in life and in academia. I really appreciate your support during this academic journey, and I really enjoyed sharing the engineering love with you. Thanks for helping me out during my wind tunnel experiment whenever I needed a second hand, I owe you a lot ! 


\section{Table of Contents}

Abstract

\begin{tabular}{|ll} 
Acknowledgments & vi
\end{tabular}

$\begin{array}{ll}\text { List of Tables } & \text { Xv }\end{array}$

List of Figures xvii

List of Symbols $\quad$ Xxx

$\begin{array}{ll}\text { List of Acronyms } & \text { xxxv }\end{array}$

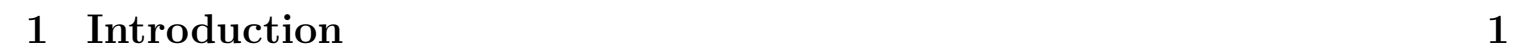

1.1 CU-NRC-RWDI collaboration . . . . . . . . . . . . . . . 1

1.2 Motivation . . . . . . . . . . . . . . . . . . . . . . . . . . . 1

1.3 Objectives . . . . . . . . . . . . . . . . . . 2

1.4 Thesis structure . . . . . . . . . . . . . . . . . . . 3

\begin{tabular}{|ll|}
\hline 2 & Background and Literature Review
\end{tabular}

2.1 Characteristics of urban wind environment . . . . . . . . . . . . . . 5

2.2 Flow structure around buildings . . . . . . . . . . . . . . . . . . . . 9

2.3 Operations of UAS in urban environment . . . . . . . . . . . . . 13

2.3.1 Urban flow features of significance to UAS operations . . . . . 14 
2.3 .2 Canadian regulations for UAS operations . . . . . . . . . . 15

2.4 Laboratory requirements for model testing . . . . . . . . . . . . . 16

2.4 .1 Wind modelling . . . . . . . . . . . . . . . . . 16

2.4 .2 Similarity and scaling . . . . . . . . . . . . . . . . 17

2.4 .3 Blockage effect . . . . . . . . . . . . . . . . . . 17

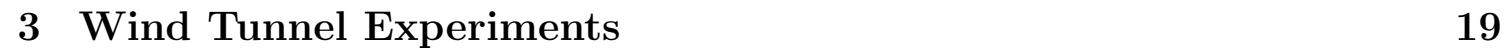

3.1 CU wind tunnel experiment: $\frac{1}{400}$ city model . . . . . . . . . . . . 20

3.1.1 ABL wind tunnel . . . . . . . . . . . . . . . . . . . . . . . . 20

3.1 .2 Simulation of the small-scale natural wind . . . . . . . . . . 23

3.1 .3 Model characteristics and details . . . . . . . . . . . . . . . . 29

3.1.3.1 Model description, domain, and geometry . . . . . . 29

3.1.3.2 Model construction . . . . . . . . . . . . . 34

3.1.3.3 Model scale and solid blockage . . . . . . . . . 36

3.1.3.4 Velocity scale and Reynolds number scaling . . . . . 38

3.1.4 Selection of wind angles and measurement locations . . . . . . 38

3.1 .5 Instrumentation and data acquisition system . . . . . . . . . . 41

3.1.5.1 Hardware setup . . . . . . . . . . . . . . . . . 41

3.1.5.2 $\quad$ Software configuration . . . . . . . . . . . . . 43

3.1.5.3 Instruments specifications and mounting arrangements 44

The Cobra probes . . . . . . . . . . . . . . . 44

The Pitot-static tube system . . . . . . . . . . . . . . 48

3.1.5.4 Sensitivity analysis . . . . . . . . . . . . . 50

Sampling time selection . . . . . . . . . . . 50

Effects of temperature and pressure variations on Cobra

probe readings . . . . . . . . . . . 53

3.1.5.5 Instruments Calibration . . . . . . . . . . . . 54 
Electronic manometer calibration . . . . . . . . . 55

Cobra probes calibration . . . . . . . . . . 55

$3.1 .6 \quad$ Experimental procedures $\ldots \ldots \ldots \ldots$. . . . . . . . . 58

$3.1 .6 .1 \quad$ Empty wind tunnel test $\ldots \ldots \ldots$. . . . . . . 58

Test section flow uniformity $\ldots \ldots \ldots$. . . . . . . 59

Reference velocity calibration curve development . . . . 61

3.1.6.2 Scaled model test . . . . . . . . . . . . . . . . . 62

Flow visualization experiment . . . . . . . . . . . 62

Cobra probe measurements . . . . . . . . . . . 66

$3.1 .7 \quad$ Experimental uncertainties . . . . . . . . . . . . . . . . 73

$3.2 \quad$ RWDI wind tunnel experiment: $\frac{1}{300}$ city model $\ldots \ldots \ldots \ldots \ldots$

3.2 .1 The B12 wind tunnel . . . . . . . . . . . . . . . . . 74

3.2 .2 Model characteristics and details $\ldots \ldots \ldots \ldots . \ldots 75$

$3.2 .3 \quad$ Instrumentation and DAQ setup. . . . . . . . . . . . 77

3.2 .4 Experimental procedures $\ldots \ldots \ldots \ldots \ldots$

$3.2 .4 .1 \quad$ Empty wind tunnel measurements . . . . . . . . 80

$3.2 .4 .2 \quad$ Scaled model vertical profiles measurements . . . . . 80

$3.2 .4 .3 \quad$ Scaled model horizontal profiles measurements. . . . 85

\begin{tabular}{|ll|l|l|l|l}
4 & Wind Tunnel Experimental Results and Discussions & 87
\end{tabular}

4.1 CU wind tunnel experiment: empty tunnel . . . . . . . . . . . . 90

4.1 .1 Approach-flow boundary layer . . . . . . . . . . . . . . 91

4.1 .2 Assessment of lateral flow uniformity . . . . . . . . . . . 92

4.2 CU wind tunnel experiment: data corrections $\ldots \ldots \ldots \ldots$

4.2 .1 Corrections for blockage effect $\ldots \ldots \ldots \ldots$

4.2 .2 Corrections for flow angles $\ldots \ldots \ldots \ldots \ldots$

$4.3 \quad$ CU wind tunnel experiment: $\frac{1}{400}$ city model $\ldots \ldots \ldots \ldots$ 
4.3 .1 Extreme values of flow features . . . . . . . . . . . . . 101

4.3 .2 Worst-case urban flows in relation to model geometry . . . . . 115

$4.3 .2 .1 \quad$ Wind speed . . . . . . . . . . . . . . . . . . 115

Flow speed increase . . . . . . . . . . . . . . . . 115

Flow speed reduction . . . . . . . . . . . . . . . 122

4.3 .2 .2 Wind direction . . . . . . . . . . . . . . 131

Vertical airflow . . . . . . . . . . . . . . . . 131

Horizontal airflow . . . . . . . . . . . . . . . . 140

4.3 .2 .3 Wind shear . . . . . . . . . . . . . . . . . 145

Vertical speed shear . . . . . . . . . . . . . . . 145

Vertical directional shear . . . . . . . . . . . . . . 151

Horizontal directional shear . . . . . . . . . . . . 155

4.3 .2 .4 Turbulence intensity . . . . . . . . . . . . . . . 161

Along-wind turbulence intensity . . . . . . . . . . . . 164

Across-wind turbulence intensity . . . . . . . . . . . . 169

Vertical turbulence intensity . . . . . . . . . . . . 171

Effect of surface roughness on turbulence intensity . . . 179

4.3 .2 .5 Vortex shedding . . . . . . . . . . . . . . . 183

4.3 .3 Regions with complex flow conditions . . . . . . . . . . . 196

$4.3 .4 \quad$ Flow conditions above the Hockey Hall of Fame . . . . . . . . 199

4.3 .5 Effect of adding the future buildings to the model . . . . . . 208

$4.4 \quad$ RWDI wind tunnel experiment. . . . . . . . . . . . . . . . 255

$4.4 .1 \quad$ Empty wind tunnel test . . . . . . . . . . . . . . 255

4.4 .2 Effect of model scale and testing facility . . . . . . . . . 256

4.4 .3 Effect of approach flow boundary layer . . . . . . . . . . 259

4.4 .4 Effect of near field modelling . . . . . . . . . . . . . . 264

4.4 .5 Lateral wake characteristics . . . . . . . . . . . . . . . 269 
$4.4 .5 .1 \quad$ Transect $12 \ldots \ldots \ldots$

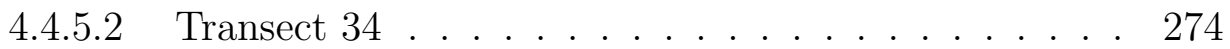

4.4 .5 .3 Transect $56 \ldots \ldots \ldots \ldots \ldots$

5 Conclusions, Contributions and Recommendations for Future Work281

5.1 Contributions . . . . . . . . . . . . . . . . . . . . . . . . . . 281

5.2 Conclusions . . . . . . . . . . . . . . . . . . . . . 282

5.3 Future work . . . . . . . . . . . . . . . . . . . . . 286

\begin{tabular}{ll}
\hline List of References & 288
\end{tabular} 


\section{List of Tables}

3.1 Model blockage at various wind directions . . . . . . . . . . . . . . . 38

3.2 Wind direction designations for the model city experiment . . . . . . 39

$3.3 \quad$ Sampling time and the corresponding number of samples . . . . . . . 52

3.4 Electronic manometer calibration results . . . . . . . . . . . . 56

3.5 Cobra probe $_{1}$ calibration results . . . . . . . . . . . . . . . . 57

3.6 Cobra probe ${ }_{2}$ calibration results . . . . . . . . . . . . . . . . . 58

3.7 Cobra probes readings check in a side-by-side arrangement . . . . . . 58

3.8 Vertical traverse points for CU empty wind tunnel measurements . . 60

$3.9 \quad$ Vertical traverse points for the 1:400 model city experiment. . . . . . 72

3.10 Horizontal traverse points for RWDI empty wind tunnel measurements 80

3.11 Vertical traverse points for the 1:300 model city experiment. . . . . . 82

3.12 Vertical measurements details for the 1:300 model city experiment . . 84

3.13 Horizontal measurements details for the 1:300 model city experiment 86

4.1 Correction factors for blockage effects . . . . . . . . . . . . . . . . . . 99

4.2 Extreme values of speed, direction, shear and turbulence flow features of the 1:400 city model experiment . . . . . . . . . . . . . . . . . . . 103

$4.3 \quad$ Locations of the extreme values of speed, direction, shear and turbulence flow features of the 1:400 city model experiment . . . . . . . . . 104

4.4 Severe turbulence intensity values (in \%) at each of the vertical zones of the city model . . . . . . . . . . . . . . . . . . . 163 
4.5 Locations of severe turbulence intensity values at each of the vertical zones of the city model . . . . . . . . . . . . . . . . . . . . 163 


\section{List of Figures}

2.1 The structure of the lower atmosphere . . . . . . . . . . . . 6

2.2 Mean wind speed profiles over terrains of different roughness . . . . . 8

2.3 Changes in the mean wind speed profile within the UCL . . . . . . . 9

2.4 Airflow structure around rectangular building . . . . . . . . . . . 10

2.5 Effect of wind incident angle on the wake size of square building . . . 11

2.6 Regions of common flow accelerations . . . . . . . . . . . . . . 15

3.1 Drawing of the ABL wind tunnel at Carleton University (SI units) . . 22

3.2 Downstream test section of the ABL wind tunnel . . . . . . . . . . . 23

3.3 Irwin's methodology for boundary layer generation . . . . . . . . . . 24

3.4 Illustration of boundary layer growth at a distance of $6 h+\Delta x \ldots 27$

3.5 Spires and roughness elements in the ABL wind tunnel . . . . . . . . 28

3.6 Spires and roughness elements dimensions . . . . . . . . . . . . . . 29

3.7 2D and 3D model domain in real settings . . . . . . . . . . . . . . . 30

3.8 Initial full-scale (top), and final sub-scale (bottom) CAD models for the city model . . . . . . . . . . . . . . . . . . . . . . . . . . . 32

3.9 The height of the buildings of the 1:400 city model measured from

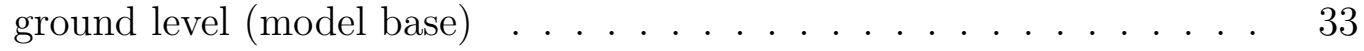

3.10 Foam disc with buildings layout . . . . . . . . . . . . . . . 34

3.11 1:400 scale physical model . . . . . . . . . . . . . . . . . 35

3.12 Bottom view of the 1:400 city model turntable . . . . . . . . . . . . 36 
3.13 Wind directions and measurement locations for the 1:400 model city experiment. . . . . . . . . . . . . . . . . . 40

3.14 Dwyer inclined liquid manometer . . . . . . . . . . . . . . . 42

3.15 Equipment setup details . . . . . . . . . . . . . . . . . . 42

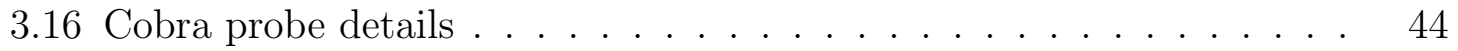

3.17 Cobra probe flow axis system . . . . . . . . . . . . . . 45

3.18 Cobra probes stainless-steel tubes . . . . . . . . . . . . . 46

3.19 CAD model for Cobra probe holder and indexing system . . . . . . . 47

3.20 Pitot-static tube configuration . . . . . . . . . . . . . . . 48

3.21 Pitot-static tube mounting arrangements . . . . . . . . . . . 50

3.22 Cobra probe mounting arrangement for the sensitivity checks . . . . . 51

3.23 Mean wind speed versus sampling time (top) and velocity rms versus sampling time (bottom) . . . . . . . . . . . . . . 52

3.24 Mean wind speed versus temperature (top) and mean wind speed versus pressure (bottom) . . . . . . . . . . . . . . . . 54

3.25 Instruments mounting arrangements for the calibration procedure . . 57

3.26 Side and top views of measurement locations for empty wind tunnel test vertical profiles . . . . . . . . . . . . . . . . . . . . . . . . . . 59

3.27 Reference velocity calibration curve . . . . . . . . . . . . . . 62

3.28 Tuft rod used for the flow visualization experiment . . . . . . . . 63

3.29 Smooth and turbulent flows observation from the flow visualization experiment. . . . . . . . . . . . . . . . . . 64

3.30 Observation of certain flow features from the flow visualization experiment........................ 65

3.31 Measurement locations for WSW, $\mathrm{WSW}_{+15}$, and $\mathrm{E}_{17.1}$ wind directions 67

3.32 Measurement locations for $\mathrm{N}_{16.7}$ and $\mathrm{S}_{15}$ wind directions. . . . . . . . 68

3.33 Cobra probes setup for the scaled model experiment . . . . . . . . . . 70 
3.34 Locations of vertical traverse points for the 1:400 model city experiment 71

3.35 The B12 wind tunnel facility . . . . . . . . . . . . . . . . 75

3.36 CAD model of the scaled physical model tested at RWDI . . . . . . . 76

3.37 Model domain in real settings for the 1:300 city model . . . . . . . . 76

3.38 Instruments mounting arrangements at B12 wind tunnel . . . . . . . 77

3.39 The 1:300 scale physical model along with instrument arrangements in the B12 wind tunnel . . . . . . . . . . . . . . . . . . . . . . . . . . . 79

3.40 Locations of vertical traverse points for the 1:300 model city experiment 81

3.41 Measurement locations for the 1:300 city model vertical profiles . . . 83

3.42 Designations for cross-wind transects . . . . . . . . . . . . . . 85

4.1 Wind speed and directional shear . . . . . . . . . . . . . . . . 89

4.2 Comparisons between the wind speed profile measured at the ABL wind tunnel and the power-law velocity profiles with different exponents 92

$4.3 \quad$ Empty wind tunnel mean wind speed and turbulence profiles measured at CU ABL wind tunnel . . . . . . . . . . . . . . . . . . . . . . . . . 93

$4.4 \quad$ Interpolated mean wind speed profiles between the Middle and Right locations . . . . . . . . . . . . . . . . . . . . 95

4.5 The correction factors for the reference velocities measured by the Pitot-static tube . . . . . . . . . . . . . . . . 96

$4.6 \quad$ Locations of the interpolated profiles with respect to the disc center . 97

4.7 The colour scheme of the \%GD for the Cobra probes measurements . 100

4.8 Example of the minimal percent change in the mean wind speed above buildings level . . . . . . . . . . . . . . . . . . . . . . . . 105

4.9 The location of spot 20 with a model orientation representing $\mathrm{S}_{15}$ wind direction . . . . . . . . . . . . . . . . . 106

4.10 Isometric views of the $1: 400$ model part 1 . . . . . . . . . . . . 107

4.11 Isometric views of the $1: 400$ model part 2 . . . . . . . . . . . 108 
4.12 Example of the minimal flow yaw angle above buildings level . . . . . 109

4.13 The location of Spot 18 with a model orientation representing $\mathrm{N}_{16.7}$ wind direction . . . . . . . . . . . . . . . . . . . . . . . 110

4.14 The location of Spot 30 with a model orientation representing $\mathrm{WSW}_{+15}$ wind direction . . . . . . . . . . . . . . . . . . . . . . . . . 111

4.15 Example of the minimal speed shear within the height of the buildings 112

4.16 The location of Spot 11 with a model orientation representing WSW wind direction . . . . . . . . . . . . . . . . . . . . . . . . . . . 114

4.17 Mean wind speed profiles at Spots 25 (a) and 26 (b) in $\mathrm{S}_{15}$ wind direction 116

4.18 The location of Spots 25 and 26 with a model orientation representing $\mathrm{S}_{15}$ wind direction $\ldots \ldots \ldots \ldots \ldots \ldots \ldots \ldots$

4.19 The location of Spots 4 and 6 with a model orientation representing $\mathrm{S}_{15}$ wind direction $\ldots \ldots \ldots \ldots \ldots$

4.20 Mean wind speed profiles at Spots 4 (a) and 6 (b) in $\mathrm{S}_{15}$ wind direction 120

4.21 Mean wind speed profile at Spot 21 for wind direction $\mathrm{WSW}_{+15}$. . . 120

4.22 The location of Spot 21 with a model orientation representing WSW +15 wind direction . . . . . . . . . . . . . . . . . . . . . 121

4.23 The location of Spot 9 with a model orientation representing WSW wind direction . . . . . . . . . . . . . . . . . . . . . . . . 123

4.24 Mean wind speed profiles (a) and Mean wind speed against overall turbulence intensity (b) at Spot 9 in WSW wind direction . . . . . . 124

4.25 The location of Spot 5 with a model orientation representing $\mathrm{N}_{16.7}$ wind direction . . . . . . . . . . . . . . . . . . 127

4.26 Example of the flow speed reduction at increments above upstream building level . . . . . . . . . . . . . . . . . . . . . . . . . . 128

4.27 Example of the flow speed reduction at measuring spot located upstream of a tall building . . . . . . . . . . . . . . . . 128 
4.28 The location of Spot 24 with a model orientation representing $\mathrm{E}_{17.1}$ wind direction . . . . . . . . . . . . . . . . . . . . . . . . . . . . . . . 129

4.29 Pitch angle profile at Spot 24 for wind direction $\mathrm{E}_{17.1}$. . . . . . . . . 130

4.30 The location of Spot 19 with a model orientation representing WSW wind direction . . . . . . . . . . . . . . . . . . . . . . . . . . . . . . . 134

4.31 Pitch angle profile at Spot 19 for wind direction WSW . . . . . . . . 135

4.32 The location of Spot 17 with a model orientation representing $\mathrm{N}_{16.7}$ wind direction . . . . . . . . . . . . . . . . . . . . . . . . . . 136

4.33 The location of Spot 28 with a model orientation representing $\mathrm{E}_{17.1}$ wind direction . . . . . . . . . . . . . . . . . . . . . . . . . . . . 137

4.34 Pitch angle profile at Spot 28 for wind direction $\mathrm{E}_{17.1}$. . . . . . . . . 138

4.35 The location of Spot 5 with a model orientation representing $\mathrm{S}_{15}$ wind direction . . . . . . . . . . . . . . . . . . . . . . . . . . 139

4.36 The location of Spots 18, 23 and 26 with a model orientation repre-

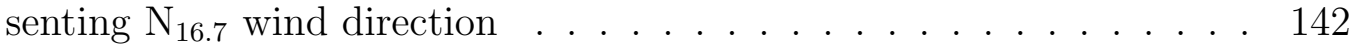

4.37 Yaw angle profile at Spots: (a) 18, (b) 23 and (c) 26 for wind direction $\mathrm{N}_{16.7} \ldots \ldots \ldots \ldots \ldots \ldots \ldots$

4.38 The location of Spot 11 with a model orientation representing WSW wind direction . . . . . . . . . . . . . . . . . . . . . . . . . . . . . . . 144

4.39 Normalized mean wind speed and overall turbulence intensity profiles at Spots: (a) 23 in $\mathrm{N}_{16.7}$, (b) 26 in $\mathrm{S}_{15}$ and (c) 9 in WSW wind directions 146

4.40 The location of Increments 12-13 at Spot 23 with a model orientation representing $\mathrm{N}_{16.7}$ wind direction $\ldots . . . . . . . .148$

4.41 The location of Increments $13-14$ at Spot 26 with a model orientation representing $\mathrm{S}_{15}$ wind direction $\ldots$. . . . . . . . . . . . . . . . . . . 149

4.42 The location of Increments $11-12$ at Spot 9 with a model orientation representing WSW wind direction . . . . . . . . . . . . . . . . . . . . 150 
4.43 Pitch angle profile at Spot 17 for wind direction $\mathrm{N}_{16.7}$. . . . . . . . . 151

4.44 The location of Increments 1-2 at Spot 6 with a model orientation

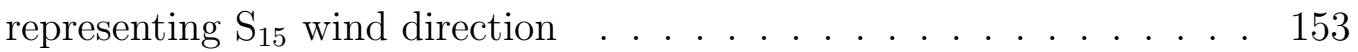

4.45 Pitch angle profile at Spot 6 for wind direction $\mathrm{S}_{15}$. . . . . . . . . . . 154

4.46 The location of Increments 11-12 at Spot 23 with a model orientation representing $\mathrm{N}_{16.7}$ wind direction . . . . . . . . . . . . . . . . . 157

4.47 The location of Increments $2-3$ at Spot 9 with a model orientation representing WSW wind direction . . . . . . . . . . . . . . . . . . . . 158

4.48 Yaw angle profile at Spot 9 for wind direction WSW . . . . . . . . . 159

4.49 The location of Increments 11-12 at Spot 31 with a model orientation

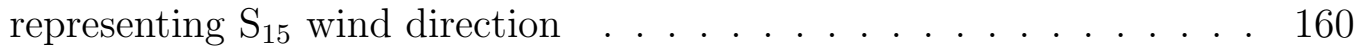

4.50 Vertical zones for the assessment of turbulence intensity within and above the city model . . . . . . . . . . . . . . . . . . . . . . . . . . . 162

4.51 The location of Spot 30 with a model orientation representing WSW wind direction . . . . . . . . . . . . . . . . . . . . . . . . . . 166

4.52 Longitudinal velocity and turbulence intensity profiles at Spots: (a) 5 in $\mathrm{N}_{16.7}$ and (b) 30 in WSW wind directions . . . . . . . . . . . . . . 167

4.53 The location of Spots 31 and 35 with a model orientation representing

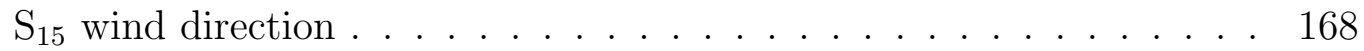

4.54 The location of Spots 32 and 35 with a model orientation representing

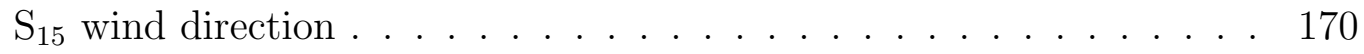

4.55 Lateral velocity and lateral turbulence intensity profiles (a) and yaw angle profile (b) at Spot 35 for wind direction $\mathrm{S}_{15}$. . . . . . . . . . . 171

4.56 The location of Spot 12 with a model orientation representing $\mathrm{S}_{15}$ wind direction . . . . . . . . . . . . . . . . . . . . . . 173

4.57 Vertical velocity and vertical turbulence intensity profiles without (a) and with the future buildings (b) at Spot 12 for wind direction $\mathrm{S}_{15} . .174$ 
4.58 The location of Spot 18 , increment 5 with a model orientation repre-

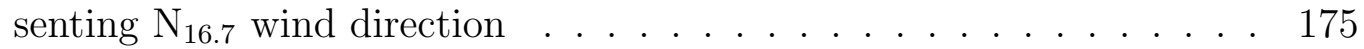

4.59 Vertical velocity and vertical turbulence intensity profiles at Spot 18 for wind direction $\mathrm{N}_{16.7}$. . . . . . . . . . . . . . . . . . . . . . . . . . 176

4.60 The location of Spot 5 and 35 with a model orientation representing

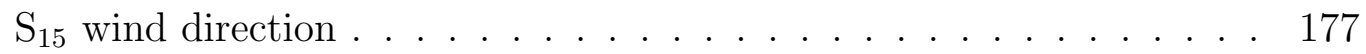

4.61 Vertical velocity and vertical turbulence intensity profiles at Spots: (a) 5 and (b) 35 for wind direction $\mathrm{S}_{15} \ldots \ldots$. . . . . . . . . . . . . . 178

4.62 Longitudinal (top), lateral (middle), and vertical (bottom) turbulence

intensities for measurements taken at Increment 20 in $\mathrm{N}_{16.7}$ wind direction 180

4.63 Longitudinal (top), lateral (middle), and vertical (bottom) turbulence intensities for measurements taken at Increment 20 in $\mathrm{S}_{15}$ wind direction 181

4.64 Measurement locations for: (a) $\mathrm{N}_{16.7}$ and (b) $\mathrm{S}_{15}$ wind directions . . . 182

4.65 The location of Increments 7,8 and 9 of Spot 9 with a model orientation representing WSW wind direction . . . . . . . . . . . . . . . . . . . 185

4.66 PSD plots for longitudinal (top) and lateral (bottom) velocity signals at Increment 7 of Spot 9 for wind direction WSW . . . . . . . . . . . 186

4.67 PSD plots for longitudinal (top) and lateral (bottom) velocity signals

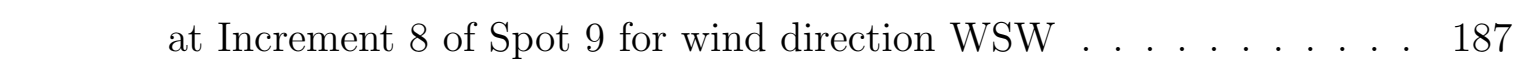

4.68 PSD plots for longitudinal (top) and lateral (bottom) velocity signals at Increment 9 of Spot 9 for wind direction WSW . . . . . . . . . . . 188

4.69 The location of Increments 9 and 11 of Spot 11 with a model orientation representing WSW wind direction . . . . . . . . . . . . . . . . . . . . 190

4.70 PSD plots for longitudinal (top) and lateral (bottom) velocity signals at Increment 9 of Spot 11 for wind direction WSW . . . . . . . . . . 191

4.71 PSD plots for longitudinal (top) and lateral (bottom) velocity signals at Increments 11 of Spot 11 for wind direction WSW . . . . . . . . . 192 
4.72 The location of Increments 8 and 9 of Spot 1 with a model orientation

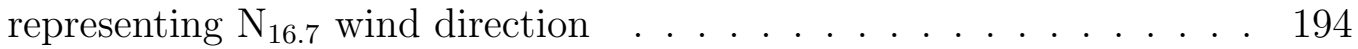

4.73 PSD plots for lateral velocity signal at Increments 8 (top) and 9 (bottom) of Spot 1 for wind direction $\mathrm{N}_{16.7}$. . . . . . . . . . . . . . . . . 195

4.74 Locations of the more complex flow regions for wind direction $\mathrm{E}_{17.1} \quad$ • 197

4.75 Locations of the more complex flow regions for wind direction $\mathrm{N}_{16.7}$. 198

4.76 Velocities, turbulence intensities and flow angles profiles at Spot 33 for WSW wind direction . . . . . . . . . . . . . . . . . . . . 201

4.77 Velocities, turbulence intensities and flow angles profiles at Spot 33 for

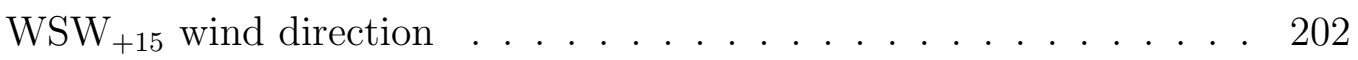

4.78 Velocities, turbulence intensities and flow angles profiles at Spot 33 for $\mathrm{E}_{17.1}$ wind direction . . . . . . . . . . . . . . . . . . . . . . . . . . . . 203

4.79 Velocities, turbulence intensities and flow angles profiles at Spot 33 for $\mathrm{N}_{16.7}$ wind direction . . . . . . . . . . . . . . . . . . . . . . . . 204

4.80 Velocities, turbulence intensities and flow angles profiles at Spot 33 for

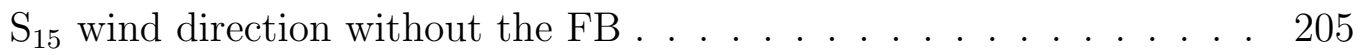

4.81 Velocities, turbulence intensities and flow angles profiles at Spot 33 for $\mathrm{S}_{15}$ wind direction with the $\mathrm{FB}$. . . . . . . . . . . . . 206

4.82 Location of Spot 33 in WSW (left) and $\mathrm{WSW}_{+15}$ (right) wind directions 207

4.83 Location of Spot 33 in $\mathrm{N}_{16.7}$ wind direction . . . . . . . . . . . . . . . 207

4.84 Overall turbulence intensity at Increment 1 (0.063 $\mathrm{m}$ above wind tunnel floor) without (top) and with (bottom) the future buildings. . . . . . 210

4.85 Overall turbulence intensity at Increment 1 (0.073 $\mathrm{m}$ above wind tunnel floor) without (top) and with (bottom) the future buildings . . . . . . 211

4.86 Overall turbulence intensity at Increment 2 (0.103 $\mathrm{m}$ above wind tunnel floor) without (top) and with (bottom) the future buildings. . . . . . 212 
4.87 Overall turbulence intensity at Increment $3(0.143 \mathrm{~m}$ above wind tunnel floor) without (top) and with (bottom) the future buildings . . . . . . 213

4.88 Overall turbulence intensity at Increment 4 (0.183 $\mathrm{m}$ above wind tunnel floor) without (top) and with (bottom) the future buildings. . . . . . 214

4.89 Overall turbulence intensity at Increment 5 (0.223 $\mathrm{m}$ above wind tunnel floor) without (top) and with (bottom) the future buildings. . . . . . 215

4.90 Overall turbulence intensity at Increment $6(0.263 \mathrm{~m}$ above wind tunnel floor) without (top) and with (bottom) the future buildings . . . . . . 216

4.91 Overall turbulence intensity at Increment 7 (0.303 $\mathrm{m}$ above wind tunnel

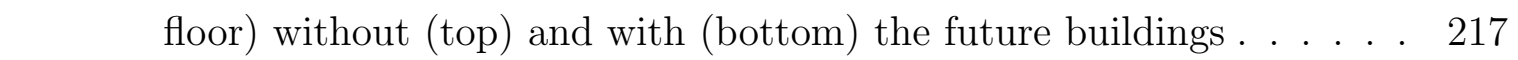

4.92 Overall turbulence intensity at Increment 8 (0.343 $\mathrm{m}$ above wind tunnel floor) without (top) and with (bottom) the future buildings. . . . . . 218

4.93 Overall turbulence intensity at Increment 9 ( $0.383 \mathrm{~m}$ above wind tunnel floor) without (top) and with (bottom) the future buildings. . . . . . 219

4.94 Overall turbulence intensity at Increment $10(0.4230 \mathrm{~m}$ above wind tunnel floor) without (top) and with (bottom) the future buildings. . 220

4.95 Overall turbulence intensity at Increment 11 (0.483 $\mathrm{m}$ above wind tunnel floor) without (top) and with (bottom) the future buildings . . . 221

4.96 Overall turbulence intensity at Increment 12 (0.543 $\mathrm{m}$ above wind tunnel floor) without (top) and with (bottom) the future buildings . . . 222

4.97 Overall turbulence intensity at Increment 13 (0.603 $\mathrm{m}$ above wind tun-

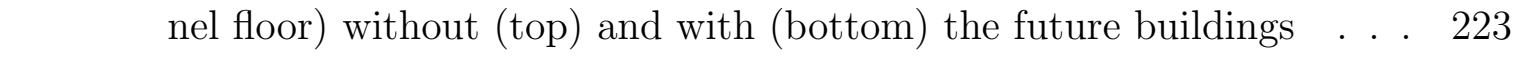

4.98 Overall turbulence intensity at Increment 14 (0.663 $\mathrm{m}$ above wind tunnel floor) without (top) and with (bottom) the future buildings . . . 224

4.99 Overall turbulence intensity at Increment 15 (0.723 $\mathrm{m}$ above wind tunnel floor) without (top) and with (bottom) the future buildings . . . 225 
4.100Overall turbulence intensity at Increment 16 (0.783 $\mathrm{m}$ above wind tunnel floor) without (top) and with (bottom) the future buildings . . . 226

4.101Overall turbulence intensity at Increment 17 (0.843 m above wind tunnel floor) without (top) and with (bottom) the future buildings . . . 227

4.102Overall turbulence intensity at Increment 18 (0.903 $\mathrm{m}$ above wind tunnel floor) without (top) and with (bottom) the future buildings . . . 228

4.103Overall turbulence intensity at Increment 19 (0.963 m above wind tunnel floor) without (top) and with (bottom) the future buildings . . . 229

4.104Overall turbulence intensity at Increment 20 (1.023 $\mathrm{m}$ above wind tunnel floor) without (top) and with (bottom) the future buildings . . . 230

4.105Locations of Spots 2, 10, 28 and 35 in $\mathrm{S}_{15}$ wind direction . . . . . . . 231

4.106Mean wind speed profiles for Spots 28, 35, 2 and 10 with and without the future buildings . . . . . . . . . . . . . . . . . . . . . . . 232

4.107Flow angles at Increment $1(0.063 \mathrm{~m}$ above wind tunnel floor) without (top) and with (bottom) the future buildings . . . . . . . . . . . . . . 234

4.108Flow angles at Increment 1 (0.073 $\mathrm{m}$ above wind tunnel floor) without (top) and with (bottom) the future buildings . . . . . . . . . . . . . . 235

4.109Flow angles at Increment $2(0.103 \mathrm{~m}$ above wind tunnel floor) without (top) and with (bottom) the future buildings . . . . . . . . . . . . . . 236

4.110Flow angles at Increment $3(0.143 \mathrm{~m}$ above wind tunnel floor) without (top) and with (bottom) the future buildings . . . . . . . . . . . . . 237

4.111Flow angles at Increment $4(0.183 \mathrm{~m}$ above wind tunnel floor) without (top) and with (bottom) the future buildings . . . . . . . . . . . . . . 238

4.112Flow angles at Increment $5(0.223 \mathrm{~m}$ above wind tunnel floor) without (top) and with (bottom) the future buildings . . . . . . . . . . . . . 239

4.113Flow angles at Increment 6 (0.263 $\mathrm{m}$ above wind tunnel floor) without (top) and with (bottom) the future buildings . . . . . . . . . . . . . . 240 
4.114Flow angles at Increment $7(0.303 \mathrm{~m}$ above wind tunnel floor) without (top) and with (bottom) the future buildings . . . . . . . . . . . . . . 241

4.115Flow angles at Increment $8(0.343 \mathrm{~m}$ above wind tunnel floor) without (top) and with (bottom) the future buildings . . . . . . . . . . . . . . 242

4.116Flow angles at Increment 9 (0.383 $\mathrm{m}$ above wind tunnel floor) without (top) and with (bottom) the future buildings . . . . . . . . . . . . . . 243

4.117Flow angles at Increment 10 (0.4230 $\mathrm{m}$ above wind tunnel floor) without (top) and with (bottom) the future buildings . . . . . . . . . . . 244

4.118Flow angles at Increment 11 (0.483 $\mathrm{m}$ above wind tunnel floor) without (top) and with (bottom) the future buildings . . . . . . . . . . . . . . 245

4.119Flow angles at Increment 12 (0.543 $\mathrm{m}$ above wind tunnel floor) without (top) and with (bottom) the future buildings . . . . . . . . . . . . . . 246

4.120Flow angles at Increment 13 (0.603 $\mathrm{m}$ above wind tunnel floor) without (top) and with (bottom) the future buildings . . . . . . . . . . . . . . 247

4.121Flow angles at Increment 14 (0.663 $\mathrm{m}$ above wind tunnel floor) without (top) and with (bottom) the future buildings . . . . . . . . . . . . . . 248

4.122Flow angles at Increment 15 (0.723 $\mathrm{m}$ above wind tunnel floor) without (top) and with (bottom) the future buildings . . . . . . . . . . . . . . 249

4.123Flow angles at Increment 16 (0.783 $\mathrm{m}$ above wind tunnel floor) without

(top) and with (bottom) the future buildings . . . . . . . . . . . . . . 250

4.124Flow angles at Increment 17 (0.843 $\mathrm{m}$ above wind tunnel floor) without

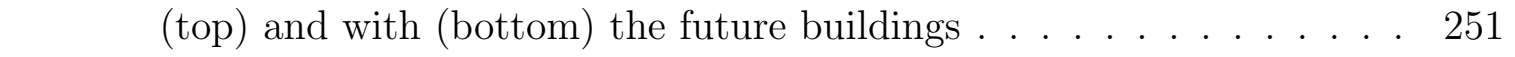

4.125Flow angles at Increment 18 (0.903 $\mathrm{m}$ above wind tunnel floor) without

(top) and with (bottom) the future buildings . . . . . . . . . . . . . . 252

4.126Flow angles at Increment 19 (0.963 $\mathrm{m}$ above wind tunnel floor) without

(top) and with (bottom) the future buildings . . . . . . . . . . . . . 253 
4.127Flow angles at Increment 20 (1.023 $\mathrm{m}$ above wind tunnel floor) without (top) and with (bottom) the future buildings . . . . . . . . . . . . 254

4.128Comparisons between RWDI exposure profiles and CU urban profiles 256

4.129Comparisons between RWDI and CU profiles at Spot 7 in WSW wind direction for urban exposure . . . . . . . . . . . . . . . . . . . . . . . 257

4.130Comparisons between RWDI and CU profiles at Spot 9 in WSW wind direction for urban exposure . . . . . . . . . . . . . . . . . . . . . . 258

4.131Location of Spot 28 for wind direction $\mathrm{E}_{17.1}$. . . . . . . . . . . . . . 260

4.132Location of Spot 33 for wind direction $\mathrm{N}_{16.7}$. . . . . . . . . . . . . . 260

4.133Location of Spot 7 for wind direction WSW . . . . . . . . . . . . . . 261

4.134Comparisons between the profiles measured at Spot 28 in $\mathrm{E}_{17.1}$ wind direction for all three exposures . . . . . . . . . . . . . . . . . . . . . 262

4.135Comparisons between the profiles measured at Spot 33 in $\mathrm{N}_{16.7}$ wind direction for all three exposures . . . . . . . . . . . . . . . . 263

4.136Comparisons between the profiles measured at Spot 7 in WSW wind direction for all three exposures . . . . . . . . . . . . . . . . . 263

4.137Measurement locations for the investigation of the near field modelling effect on data measurements . . . . . . . . . . . . . . . . . . . . . . . 266

4.138Comparisons between RWDI and CU profiles at Spot 10 in $\mathrm{N}_{16.7}$ wind direction for urban exposure . . . . . . . . . . . . . . . . . . . . 267

4.139Comparisons between RWDI and CU profiles at Spot 16 in $\mathrm{N}_{16.7}$ wind direction for urban exposure . . . . . . . . . . . . . . . . . . 267

4.140Comparisons between RWDI and CU profiles at Spot 18 in $\mathrm{N}_{16.7}$ wind direction for urban exposure . . . . . . . . . . . . . . . . . . . 268

4.141Comparisons between RWDI and CU profiles at Spot 33 in $\mathrm{N}_{16.7}$ wind direction for urban exposure . . . . . . . . . . . . . . . . . . . . . 268

4.142The upstream building layers of Spot 33 for both scaled models . . . 269 
4.143Location of $\mathrm{T}_{12} \ldots \ldots \ldots \ldots \ldots \ldots \ldots \ldots \ldots \ldots \ldots \ldots$

4.144Lateral wake velocity, turbulence and flow angles data for $\mathrm{T}_{12}$ at 0.3507 m from the wind tunnel floor . . . . . . . . . . . . . . . . 272

4.145Lateral wake velocity, turbulence and flow angles data for $\mathrm{T}_{12}$ at 0.5640 $\mathrm{m}$ from the wind tunnel floor . . . . . . . . . . . . . . . . . . . . 273

4.146 Location of $\mathrm{T}_{34} \ldots \ldots \ldots \ldots \ldots \ldots \ldots \ldots \ldots \ldots$

4.147Lateral wake velocity, turbulence and flow angles data for $\mathrm{T}_{34}$ at 0.5640 $\mathrm{m}$ from the wind tunnel floor . . . . . . . . . . . . . . . . . . 277

4.148Location of $\mathrm{T}_{56} \ldots \ldots \ldots \ldots \ldots \ldots \ldots \ldots \ldots \ldots$

4.149Mean wind speed and turbulence intensity profiles along $\mathrm{T}_{56}$ at 0.4573 $\mathrm{m}$ from the wind tunnel floor for three different wind directions . . . 280 


\section{List of Symbols}

\section{Latin Characters}

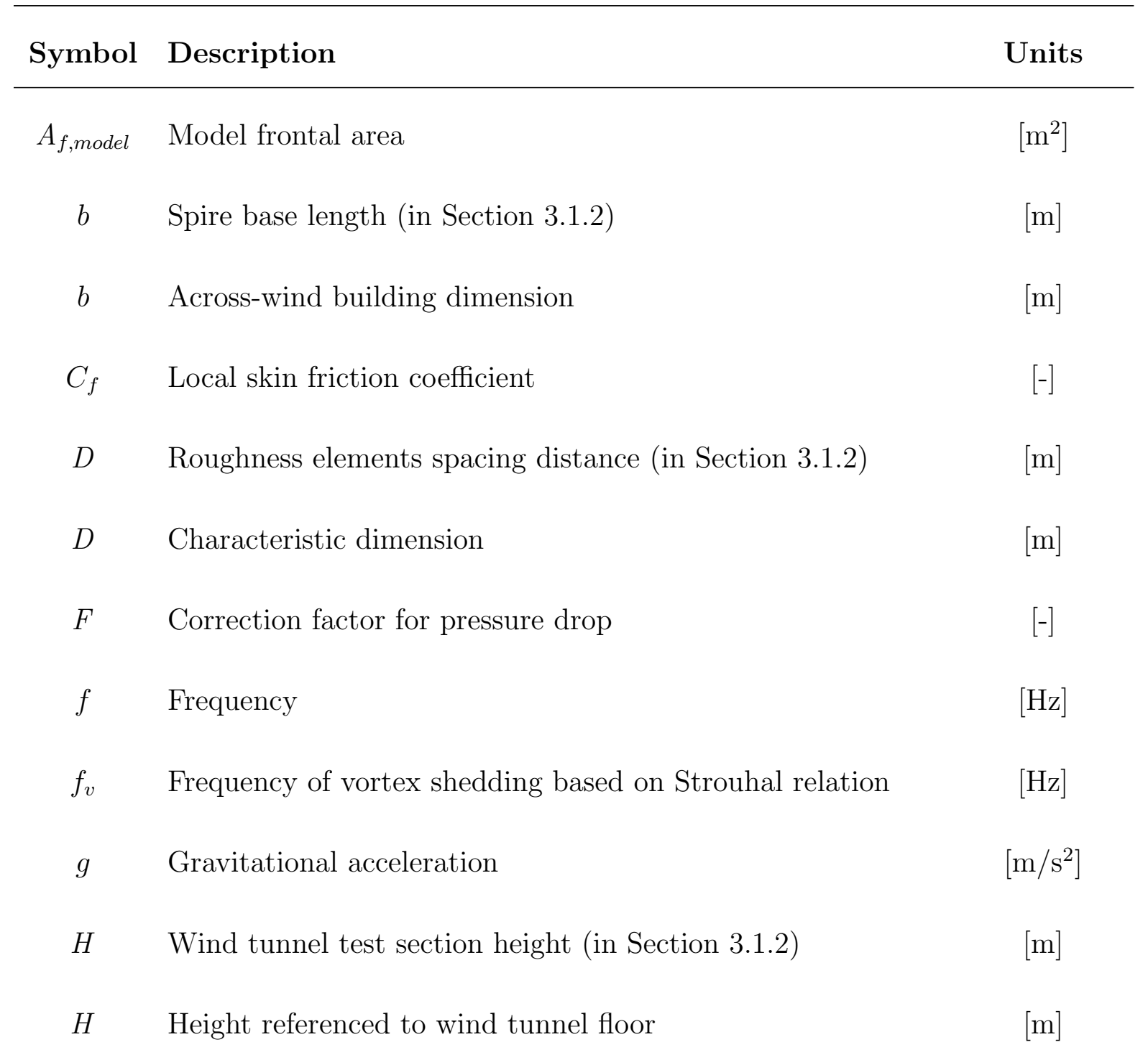


$h \quad$ Spire height

$I_{u u} \quad$ Along-wind turbulence intensity

$I_{v v} \quad$ Across-wind turbulence intensity

$I_{w w} \quad$ Vertical wind turbulence intensity

$I_{\text {uvw }} \quad$ Overall turbulence intensity

$k \quad$ Roughness element height

$P_{0} \quad$ Local total pressure

$P_{a} \quad$ Barometric pressure

$P_{s} \quad$ Local static pressure

$R \quad$ Gas constant

$R e_{b} \quad$ Building Reynolds number

$S_{t} \quad$ Strouhal number

$S_{u}(f) \quad$ Spectrum of longitudinal velocity component at frequency $f \quad\left[(\mathrm{~m} / \mathrm{s})^{2} / \mathrm{Hz}\right]$

$S_{v}(f) \quad$ Spectrum of lateral velocity component at frequency $f \quad\left[(\mathrm{~m} / \mathrm{s})^{2} / \mathrm{Hz}\right]$

T Air temperature

U Wind speed

$U_{G} \quad$ Wind speed at gradient height $\mathrm{Z}_{G}$

$U_{\text {ref }} \quad$ Reference mean wind speed

$U_{\text {refc }} \quad$ Corrected reference mean wind speed 
$U_{r e f_{M}} \quad$ Reference mean wind speed at the middle of the turntable $\quad[\mathrm{m} / \mathrm{s}]$

$U_{r m s} \quad$ Root-mean-square of mean wind speed $\quad[\mathrm{m} / \mathrm{s}]$

$U_{\delta} \quad$ Wind speed at boundary layer thickness $\delta \quad[\mathrm{m} / \mathrm{s}]$

$u \quad$ Along-wind velocity component $\quad[\mathrm{m} / \mathrm{s}]$

$\bar{u} \quad$ Time-averaged longitudinal velocity component $\quad[\mathrm{m} / \mathrm{s}]$

$V \quad$ Velocity vector $\quad[\mathrm{m} / \mathrm{s}]$

$\bar{V} \quad$ Time-averaged velocity vector $\quad[\mathrm{m} / \mathrm{s}]$

$v \quad$ Across-wind velocity component $\quad[\mathrm{m} / \mathrm{s}]$

$\bar{v} \quad$ Time-averaged lateral velocity component $\quad[\mathrm{m} / \mathrm{s}]$

$w \quad$ vertical velocity component $\quad[\mathrm{m} / \mathrm{s}]$

$\bar{w} \quad$ Time-averaged vertical velocity component $\quad[\mathrm{m} / \mathrm{s}]$

$x \quad$ Along-wind axis $\quad[-]$

$y \quad$ Across-wind axis

Z Arbitrary height [m]

z Perpendicular-to-wind axis $\quad[-]$

$Z_{G} \quad$ Gradient height $\quad[\mathrm{m}]$ 


\section{Greek Characters}

\begin{tabular}{|c|c|c|}
\hline Symbol & Description & Units \\
\hline$\alpha$ & Power law exponent & {$[-]$} \\
\hline$\beta$ & Constant for Irwin's empirical formulations & {$[-]$} \\
\hline$\delta$ & Boundary layer thickness (in Section 3.1.2) & {$[-]$} \\
\hline$\delta$ & Reference height & {$[-]$} \\
\hline$\Delta \delta$ & Increase in boundary layer thickness & {$[\mathrm{m}]$} \\
\hline$\Delta h$ & Height of water column & {$\left[\right.$ in $\left.\mathrm{H}_{2} \mathrm{O}\right]$} \\
\hline$\Delta P$ & Pressure difference & {$[\mathrm{Pa}]$} \\
\hline$\Delta U$ & Velocity difference & {$[\mathrm{m} / \mathrm{s}]$} \\
\hline$\Delta x$ & Increase in distance & {$[\mathrm{m}]$} \\
\hline$\varepsilon_{T}$ & Total blockage correction factor & {$[-]$} \\
\hline$\eta$ & Constant for Irwin's empirical formulations & {$[-]$} \\
\hline$\theta$ & Flow pitch angle & {$[\mathrm{deg}]$} \\
\hline$\nu$ & Kinematic viscosity & {$\left[\mathrm{m}^{2} / \mathrm{s}\right]$} \\
\hline$\rho_{a}$ & Air density & {$\left[\mathrm{kg} / \mathrm{m}^{3}\right]$} \\
\hline$\rho_{w}$ & Water density & {$\left[\mathrm{kg} / \mathrm{m}^{3}\right]$} \\
\hline$\sigma^{2}$ & Variance & {$\left[(\mathrm{m} / \mathrm{s})^{2}\right]$} \\
\hline$\sigma_{u}$ & Standard deviation of longitudinal velocity component & {$[\mathrm{m} / \mathrm{s}]$} \\
\hline
\end{tabular}


$\sigma_{v} \quad$ Standard deviation of lateral velocity component

$[\mathrm{m} / \mathrm{s}]$

$\sigma_{w} \quad$ Standard deviation of vertical velocity component

$[\mathrm{m} / \mathrm{s}]$

$\psi \quad$ Flow yaw angle

[deg] 


\section{List of Acronyms}

2D Two-Dimensional

3D Three-Dimensional

ABL Atmospheric Boundary Layer

ABS Acrylonitrile Butadiene Styrene

ADC Analogue-to-Digital Converter

ASR Acquisition Scan Rate

ASCE American Society for Civil Engineers

CAD Computer-Aided Design

CFD Computational Fluid Dynamics

CNC Computer Numerically Controlled

CU Carleton University

CW Clock-Wise

DAQ Data Acquisition System

DOR Data Output Rate

dP Pressure drop 


\begin{tabular}{ll} 
EV & Empty wind tunnel Value \\
GD & Good Data \\
FB & Future Buildings \\
FS & Full Scale \\
HHF & Hockey Hall of Fame \\
Incr. & Increment \\
in $\mathbf{H}_{2} \mathbf{O}$ & Inches of Water Column \\
L & Left \\
M & Middle \\
MV & Model Value \\
NI & National Instruments \\
RRC & National Research Council Canada \\
Ref. & Reference \\
P-s & Pitot-static \\
PSD & Power Spectral Density \\
\hline root-mean-square
\end{tabular}

RPAS Remotely Piloted Aircraft Systems 
RSL Roughness Sub-Layer

RWDI Rowan Williams Davies \& Irwin

SI International System

SS Sub Scale

SW Side Wall

TFI Turbulent Flow Instrumentation Ltd

TI Turbulence Intensity

UAM Urban Air Mobility

UAS Unmanned Aerial Systems

UBL Urban Boundary Layer

UCL Urban Canopy Layer 


\section{Chapter 1}

\section{Introduction}

\subsection{CU-NRC-RWDI collaboration}

The research presented in this thesis presents one aspect of a larger research program, which is funded by the National Research Council Canada (NRC), on the topic of Unmanned Aerial Systems (UAS) flight in urban environments. The research program is currently underway through joint partnership between Carleton University (CU), NRC and Rowan Williams Davies \& Irwin (RWDI) Inc.

\subsection{Motivation}

Over the past few decades, an extensive amount of research has been carried out on the topic of urban airflow. This is due to the rapid growth of cities and urbanization that took place, in many different parts of the globe, during the 19th century [1. As a result of urbanization, major cities have become densely populated and urban planners are continuously evolving their standards and designs to accommodate those changes. The rapid increase in global populations pose the need for additional space and cities are now expanding vertically to avoid further encroachment on natural habitats and green space by taking advantage of the sky, which is to some degree 
unlimited. Although the idea of developing vertical cities brought many advantages, the patterns of airflow, which are influenced by the geometry, height, orientation and density of buildings, have brought many challenges, most of which have been related to the aspect of safety. The majority of studies concerning airflow in urban environments are related to the topics of natural ventilation $[2] 4]$, pedestrian-level comfort [5, 6], wind loads on structures [7,8] and pollutant dispersion [9 12], where the urban environment, in many instances, is modeled by the use of idealized blocks in various arrangements.

With the wide range of envisioned applications and growing technology advancement of UAS, multiple studies have been devoted to examining UAS behaviour in urban environments. However, most of the work done has focused on the control aspect of UAS, where flight operations were analyzed in specific flow fields 13 17. Since the major component that poses challenges to safe flight in urban environments is related to the flow structures themselves, there is a need for characterizing the complex flow environment by considering the flow features that are most significant for vehicle stability when flying between and above buildings. A more comprehensive look at challenging flow structures can enable design criteria and safety regulations to be developed.

\subsection{Objectives}

This research aims to analyze the complex airflow structures from a broader perspective, that is by analyzing the urban flow characteristics of an entire region of a real urban city. The urban airflow characterization involves disintegrating the complex flow structure into separate flow features that are expected to influence the safe operation of air vehicles. Such flow features include, changes in flow speed and direction, speed and directional shear, turbulence intensity and vortex shedding. With the 
growing power of Computational Fluid Dynamics (CFD) tools, their use in modelling urban wind conditions become more common [18, 19]. In this research, however, it was decided to investigate the urban airflow features through an experimental method using wind tunnels since localized turbulence data are sensitive to small details of the buildings envelope [20], and CFD tools have some limitations in resolving the complex flow features in the wake of high-rise buildings and the regions of flow accelerations (strong wind conditions) [21]. Thus, the primary overall objective of this research is to analyze worst-case urban flow characteristics of relevance to flying vehicles stability through:

1. defining extremes, and consequently ranges, of the flow features based on measurements at various locations in a physical city model; and

2. determining locations of extreme flow features in relation to the model geometry.

A secondary overall objective is to assess the impact of certain testing parameters including testing facility, model scale, approach flow boundary layer profile and near field modelling on the measurements quality and accuracy in wind tunnel experiments.

\subsection{Thesis structure}

This thesis is divided into six chapters. Chapter 2 presents background information and subject review on urban wind environment, flow patterns around buildings and structures, Urban Air Mobility (UAM) and its regulations, as well as laboratory requirements for wind tunnel testing. The experimental procedures, nomenclatures for measurement locations and wind angles, along with instrumentation setup for two wind tunnel experiments are described in Chapter 3 . Chapter 4 presents the results of the wind tunnel experiments of the scaled city models along with the applied methods for data corrections. Chapter 5 outlines the major findings of the research work, 
identifies the major contributions made throughout the research work and provides recommendations for possible future work. 


\section{Chapter 2}

\section{Background and Literature Review}

The operation of UAS takes place in the lower atmosphere; therefore, background information about the urban wind environment will be provided in Section 2.1. Since flying vehicles may operate between buildings, the general airflow structures around buildings will be described in Section 2.2. Literature survey related to UAS operations in urban areas along with the Canadian laws for UAS operations will be presented in Section 2.3. The investigation of urban flow characteristics was done through an experimental approach using wind tunnels; thus, laboratory requirements and considerations for testing buildings and structures will be presented in Section 2.4.

\subsection{Characteristics of urban wind environment}

The term 'urban environment' is usually defined based on the field of study. In general, an area can be classified as 'urban' based on minimum population density, the determination of which differs widely among different countries 22 . From the perspective of wind engineering, the decision of whether or not an area is to be classified as 'urban' depends mainly on the terrain characteristics. Therefore, for wind related studies, urban environment refers to heavily built-up areas where highrise buildings do exist 23. 


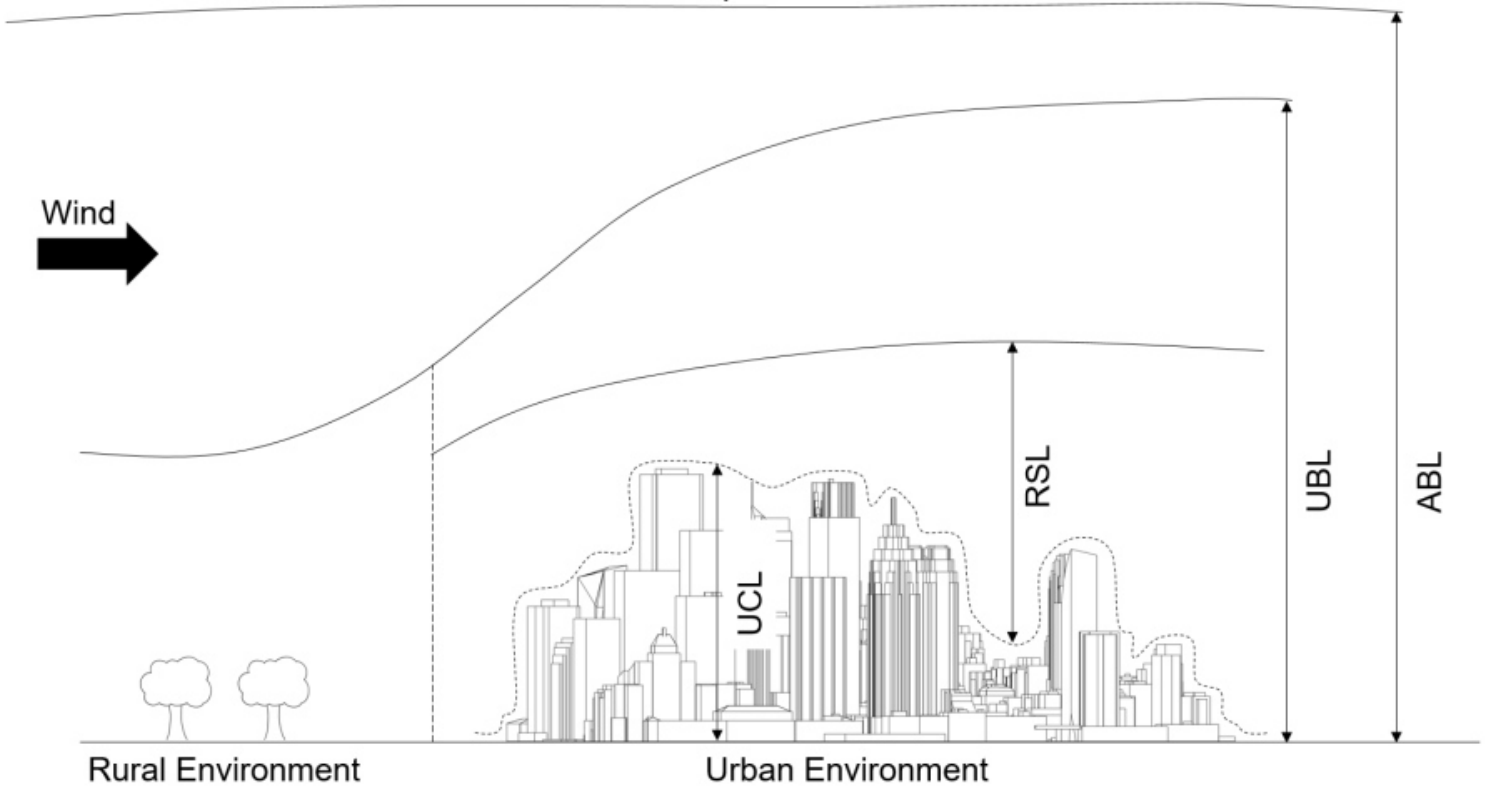

Figure 2.1: The structure of the lower atmosphere.

The wind characteristics are influenced by the surface roughness within the Atmospheric Boundary Layer (ABL), above which is the free atmosphere where wind is mainly driven by pressure gradients, inertial and coriolis forces [24]. The depth of the ABL is referred to as the gradient height, and its typical range or value is not consistent within the literature. Based on publication in 1970, Teunissen [24] reported that ABL depth could range from approximately 305 to $610 \mathrm{~m}$ (1000 to $2000 \mathrm{ft}$ ) depending on the surface roughness of the earth. A handbook for applied fluid dynamics by Blevins in 1984 25] provided an approximate value of the ABL depth, which is about $1000 \mathrm{~m}$ (3000 ft). The American Society for Civil Engineers (ASCE) reported an ABL depth that ranges from 1000 - $2000 \mathrm{~m}$ (3281 - $6562 \mathrm{ft})$ in their journal for wind tunnel testing of buildings and structures published in 1996 [26]. Clearly, the estimated depth increases over years given the increased level of man-made structures, which added an additional roughness to the earth's surface. 
Over the depth of the ABL, wind speed generally decreases from a maximum value at the top of the boundary to a minimum value at the surface of the earth due to drag effect from the earth surface and the drag from buildings and structures. Thus, the decrease in wind speed near the ground is accompanied by an increase in turbulence level 27]. As illustrated in Figure 2.1. the Urban Boundary Layer (UBL) is a sub-layer of the ABL, and its depth is influenced by the detailed roughness of the urban environment, and therefore, UBL is generally turbulent along its entire depth [28]. The UBL is commonly divided into dynamical vertical zones as shown in Figure 2.1, where the airflow characteristics are distinct in each zone. The Urban Canopy Layer (UCL) extends from the ground up to the buildings rooftops, where airflow is extremely affected by topography's local effects, geometry and dimensions of buildings [29]. Above the UCL is the Roughness Sub-Layer (RSL), which extends from buildings rooftops up to a vertical distance equals to 2-5 times the average height of buildings [30]. The airflow in the RSL is also under the influence of urban structures arrangements; however, the production and dissipation of turbulent kinetic energy is almost in equilibrium state 29].

The urban wind properties of high significance are the mean velocity profile and turbulence characteristics [31]. The mean wind speed profile represents the changes in wind velocity from the earth's surface to the top of the ABL. The mean wind speed profile over various types of terrain can be adequately represented by the power law expression:

$$
\frac{U}{U_{G}}=\left(\frac{Z}{Z_{G}}\right)^{\alpha}
$$

where $U$ is the wind speed at height $Z, U_{G}$ is the wind speed at gradient height $Z_{G}$ and $\alpha$ is the power law exponent.

Typical mean wind speed profiles over terrains of different roughness under neutrally-stable conditions are shown in Figure 2.2 provided that the wind has blown 


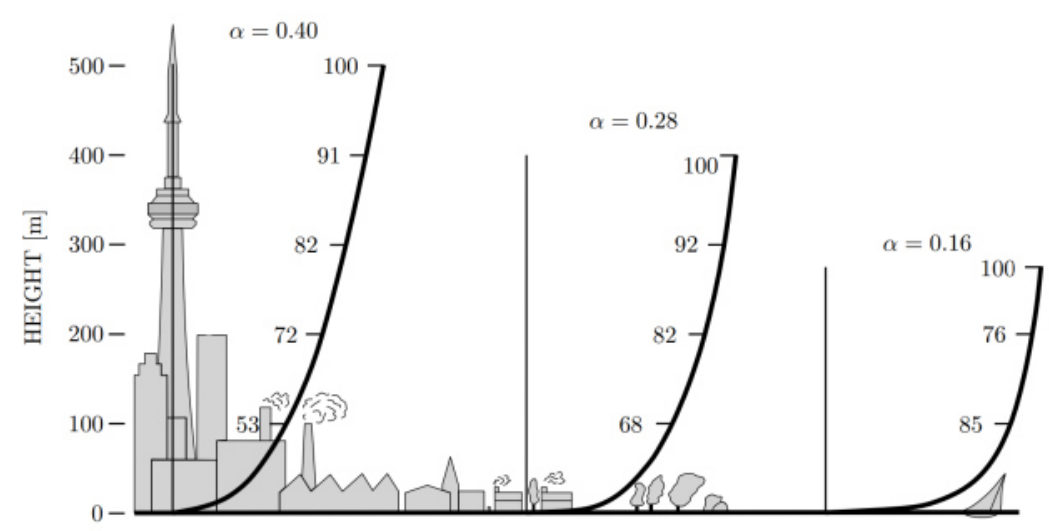

Figure 2.2: Mean wind speed profiles over terrains of different roughness. Adapted from: [32].

over a distance of uniform roughness. The terrain roughness is what governs the power law exponent. For urban, sub-urban, and flat open country terrain roughness, typical values for $\alpha$ are $0.40,0.28$, and 0.16 respectively. The profiles shown in Figure 2.2 demonstrate smooth wind speed increase with height since the power law profiles are developed with the assumption of consistent turbulence mixing over the terrain [33]. However, this is not the case when considering the wind profiles within the height of buildings (within the UCL), especially in the wake of buildings. When wind is being blocked by an urban building, decrease in the wind speed profile is dominant within the wake of the building as illustrated schematically in Figure 2.3. This decrease in wind speed is accompanied by an increase in turbulence level in the wake of buildings 33. 


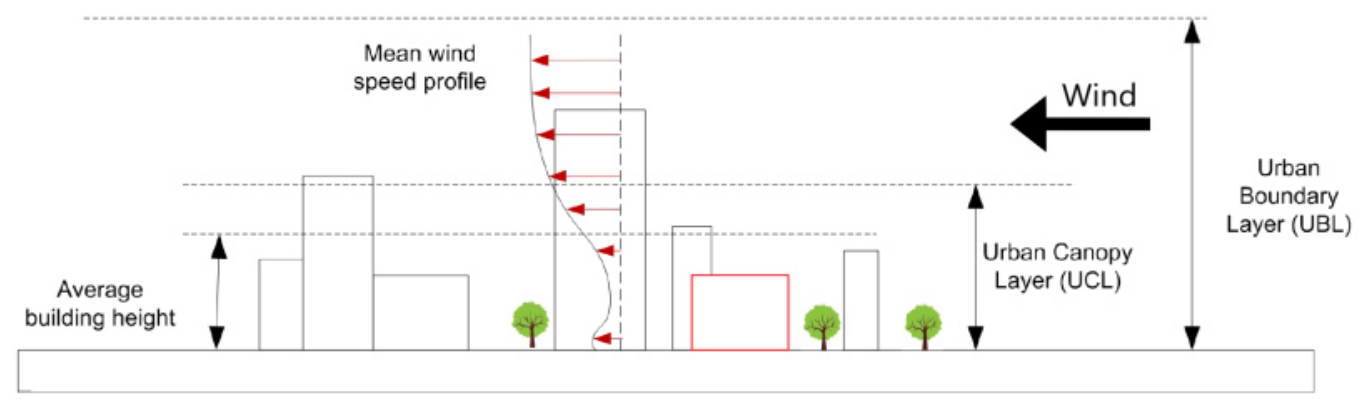

Figure 2.3: Changes in the mean wind speed profile within the UCL. Adapted from: [34].

\subsection{Flow structure around buildings}

Before going into details about the flow structure around buildings, the definition of 'urban buildings' will be explained. Urban buildings are bluff bodies that are clustered together in different configurations, whose influence on the airflow characteristics is significantly notable in the RSL, and even more notably, in the UCL [35]. Most urban buildings are sharp-edged bluff bodies where flow separation is initiated from the structure edges, resulting in curved shear layers as well as unsteady velocity field, high turbulence level, and unsteady vortex shedding in the buildings wake region 36, 37.

In the urban environment, the wind speed and the turbulence characteristics are influenced by the flow interactions between the various buildings; nonetheless, the airflow characteristics at a specific point within the built-up environment is mainly influenced by the characteristics of the nearby building [38]. The flow structures around buildings may vary depending on the buildings geometry, dimension and orientation to the incoming wind [35].

Considering an idealized block of a building with a rectangular cross-section (see Figure 2.4 , the flow structure based on a perpendicular incident wind angle on the wide building facet (Figure 2.4(a)] is explained as follows [39]:

- From the building windward face, airflow diverges at approximately $70 \%$ of the height of the building from a zone of a maximum pressure, called the stagnation 


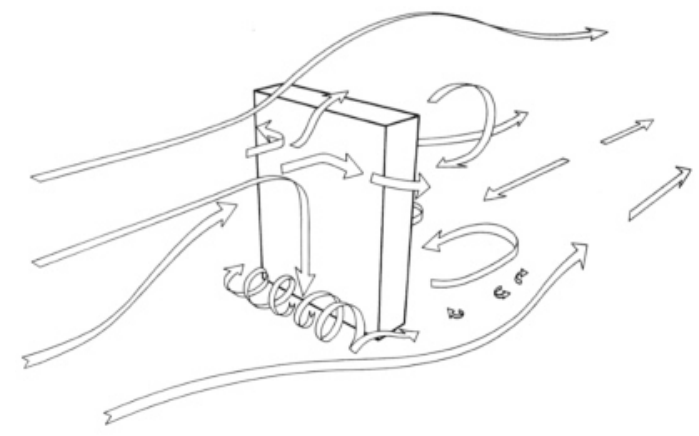

(a)

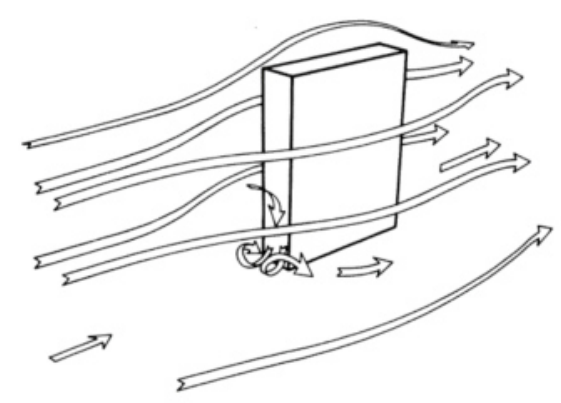

(b)

Figure 2.4: Airflow structure around rectangular building. Reproduced from: 38]. zone. From the stagnation zone, airflow deviates to regions of low pressure by moving upward, downward, and along the side walls of the building.

- Due to the stagnation zone location, airflow moves strongly downward, which cause the formation of a vortex at the ground, commonly known as the horseshoe vortex. The vortex extends to the building side walls and flow separation occurs from the buildings sharp edges.

- As the flow separates from the building edges, zones with high velocity gradients forms, known as the shear layers, which are characterized by small and fast rotating vortices.

- Immediately downstream the building, low pressure zone is created, and flow re-circulation occurs. Flow reversals define the near-wake region, which is also known as the cavity zone 12 .

- Beyond the near-wake region, re-circulation phenomenon ceases, but flow speed remains low for considerable distance downstream the buildings.

For the same building geometry, but with a perpendicular wind angle of incidence to the narrow face of the building (Figure 2.4(b)), the airflow structure changes significantly. For this building configuration, the airflow is to a great extent transported 
along the building side and top walls whereas flow reversals occur over small areas on the windward building facet 39 .

The buildings wake is generally sub-divided into two main regions, the near-wake and the far-wake. As explained earlier, near-wake is where re-circulation takes place downwind of buildings. In the near-wake, the decrease in velocity and increase in turbulence is approximately constant; however, the disturbances in the flow decreases with distance in the far-wake region [40].

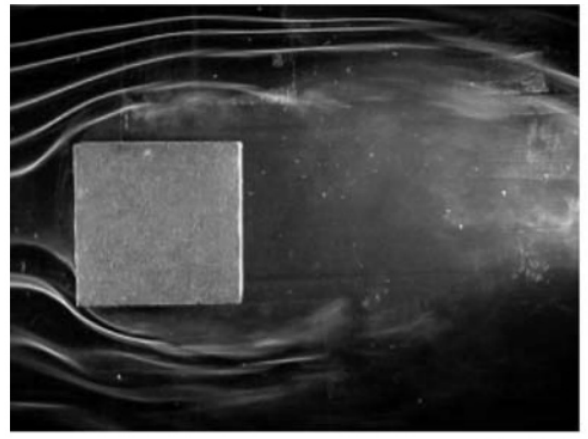

$\theta=0^{\circ}$

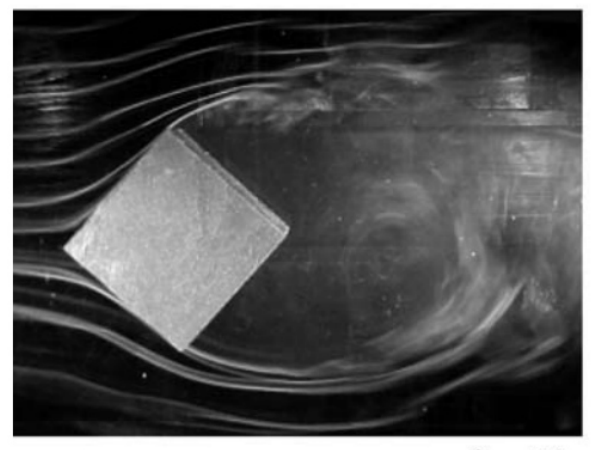

$\theta=45^{\circ}$

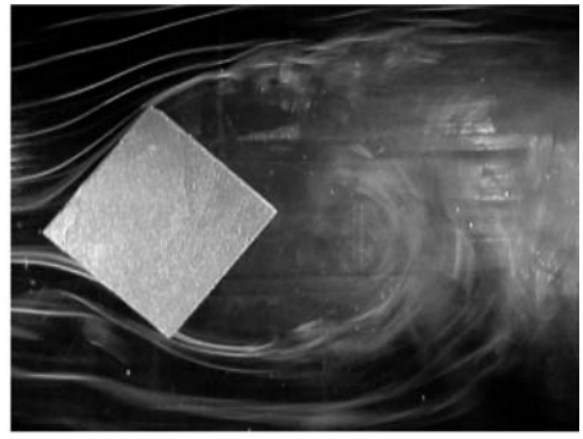

$\theta=30^{\circ}$

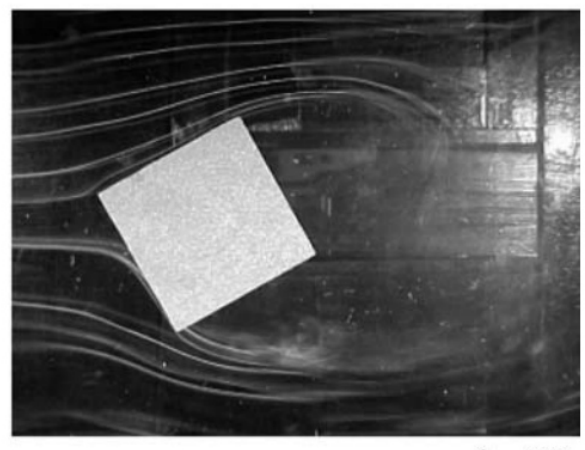

$\theta=60^{\circ}$

Figure 2.5: Effect of wind incident angle on the wake size of square building. Reproduced from: [41.

The wake size is significantly influenced by the building width as well as the building orientation to the incoming wind. Dutta et al. [41] investigated the flow pattern around a square cylinder with considerations of the cylinder orientation to the incoming flow. Utilizing a smoke wind tunnel facility, flow visualizations around a square cylinder were performed for angles of inclination from $0^{\circ}$ to $60^{\circ}$ (see Figure 
2.5). For the case of $0^{\circ}$ flow incident angle, the flow separation from the cylinder occurred at the leading edges, resulting in a wake region of similar width to the cylinder. However, as the angle of inclination increases, the points of separation are shifted downstream, leading to a larger wake size.

The wake length downstream of buildings is influenced by the relative building width and the wind incident angle. The width-to-height aspect ratio of a building plays a major role in the wake characteristics. Buildings with small aspect ratio have small velocity and turbulence defect in their wake. Additionally, the flow normal conditions are restored quickly downstream the buildings, leading to a reduction, up to eight times, in the wake length for width-to-height aspect ratios in the range of 1 to 10 . As for the wind angle of incidence, the wake length is larger for the case of perpendicular incident wind. For oblique winds, reduction in the wake length is expected, particularly, for large width-to-height aspect ratios. Other parameters like building relative depth (along-wind dimension) is known to have minimal impact on the wake characteristics [40].

The airflow patterns around buildings within a city cannot be generalized since they are highly influenced by the wake interactions between the buildings, which can vary significantly depending on the buildings arrangement. Therefore, studies concerning airflow around multiple buildings are usually performed on specific idealized blocks arrangements, in which the flow pattern is mostly case-specific. Example of such studies are listed here.

Reference [39]: presents smoke visualization of the airflow patterns over two adjacent blocks of different height.

Reference [42]: presents the wake patterns of two square cylinders on a side-by-side arrangement with various gap spacings between the cylinders.

Reference [43]: presents the airflow patterns over urban canyons with different 
height-to-width aspect ratios.

Reference [19]: presents the airflow patterns around urban canyons of different geometrical shapes.

Reference [44: presents oil-film visualization of the airflow patterns over multiple blocks of different shapes in certain arrangement.

Reference [45]: presents numerical simulation on the effect of adding high-rise future building to a cluster of low-rise existing buildings.

Reference [10]: presents schematic illustration of the flow field at street intersection with the existence of tall building.

\subsection{Operations of UAS in urban environment}

The recent development in UAS has generated a lot of interest amongst both the general public and industries regarding their use within the urban environment. This is due to the wide range of applications and benefits a flying vehicle may offer, including inter-city transport, emergency response, package delivery, surveillance and reconnaissance, as well as infrastructure inspection. Despite the benefits of operating UAS in urban environments, many restrictions are currently in place worldwide, in which the operations of UAS are prohibited over populated areas. Multiple factors influence the safe operations of UAS which include changes in temperature, pressure, and humidity leading to poor vehicle performance. To the author's knowledge, there are currently no specific weather forecast system or measurement regulations used for urban UAS pre-flight planning, and thus, UAS operators use the available information from weather station reports along with personal judgments on the general weather conditions. The smaller size of UAS, as compared to their traditional manned aircraft 
counterparts, make them more susceptible to atmospheric wind and turbulence, and consequently compromising their stability to the various wind conditions. Since UAS are designed to operate within the ABL, they are usually designed to operate at low wind speeds; therefore, strong wind conditions that occur with increasing altitude may strongly impact the desired navigation. The detailed terrain roughness near the ground increases the turbulence level, where turbulence intensity can be greater than $50 \%$ within city centers comprising high-rise buildings. Such high turbulence is generally mechanical in nature, which implies that the turbulence source is related to surface friction, buildings wake, and directional shear. The turbulence generated from buildings wake spans various length scales with the largest eddies corresponding in size to the characteristic dimensions of buildings. Eddies that are of similar size to the flying vehicle are the eddies the most responsible for vehicle instabilities 46.

\subsubsection{Urban flow features of significance to UAS operations}

The operation of UAS within the UBL is predominantly influenced by the surface conditions, which in city centers, buildings envelope is what shape the airflow structure and characteristics. Turbulence intensity is one of the flow features that challenges the stable flight operation of UAS. High turbulence intensities are found within the UCL as a result of combined effect of buildings wake and intensive shear forming by buildings tops. Flow separation from the buildings sharp edges causes flow disturbances that may persist vertically up to a distance of three times the height of the tallest building within a certain area. Updrafts and downdrafts are commonly formed upstream as a result of flow separation from the buildings windward face. Downdrafts results in reversed flow region with lower wind speed near the buildings base. Flow accelerations are usually found on top and lateral zones of buildings as a result of flow separation and transition from areas of high pressure to areas of lower pressure downwind the buildings [46]. Additionally, airflow may accelerate as it passes 
between two side-by-side buildings [34]. The common regions of flow accelerations are illustrated schematically in Figure 2.6. Flow accelerations may exceed the UAS capabilities leading to catastrophic events in populated area. Flow decelerations are found commonly downwind of buildings, particularly, in the wake region due to the presence of turbulent eddies. Flow re-circulations are significantly found in the buildings near-wake [46]. Vortex shedding, which refers to swirling flows that form at, and detach from, the buildings sides [47, could be of greatest concern if the vortex size is comparable to the vehicle characteristic dimension.

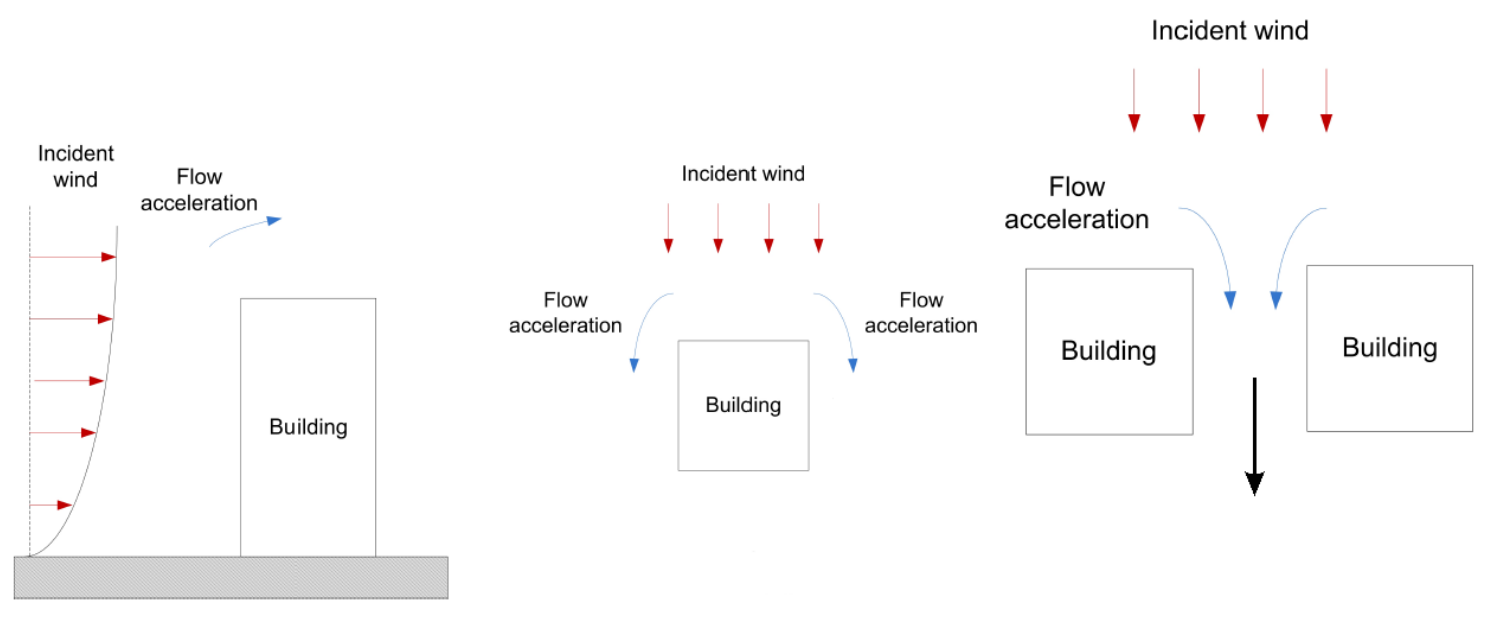

Figure 2.6: Regions of common flow accelerations. Adapted from: [34].

\subsubsection{Canadian regulations for UAS operations}

The Canadian regulations and laws for drone operations are fairly strict due to safety reasons. The current regulations, provided by Transport Canada [48, prohibit the flying operations over populated areas unless specific permission is obtained. Additionally, the current regulations apply only to Remotely Piloted Aircraft Systems (RPAS) whose weight do not exceed $25 \mathrm{~kg}$. The RPAS operation requirements include: 
- flying below $122 \mathrm{~m}$ (400 ft) in the air;

- flying away from people, horizontally, by at least $30 \mathrm{~m}$ (100 ft); and

- maintaining visual line of sight of the drone at all times.

\subsection{Laboratory requirements for model testing}

This section provides background information on the important aspects related to performing wind tunnel experiments on buildings and structures.

\subsubsection{Wind modelling}

For studies involving wind effect on buildings and structures, appropriate modelling of the natural wind at a site is needed for accurate match between model measurements and Full-Scale (FS) data. This involves modelling both the approach flow boundary layer profile, and including the significant nearby buildings to the site of the measurements [26]. This is important since in real settings, wind blows over the entire upstream fetch before reaching to the site of interest. However, in wind tunnel testing, partial city models are utilized.

The approach flow boundary layer profile can be modelled for different types of terrain through utilizing boundary layer wind tunnel facilities, which are characterized by having long working sections. Commonly, flow conditioning devices like spires, vortex generators and fences are used to increase the boundary layer depth in long working section wind tunnels, and to generate the boundary layer characteristics in short working section wind tunnels. The proper modelling of approach flow boundary layer requires the modelling of the mean wind speed and longitudinal turbulence intensity variations with height along with the reproduction of the significant characteristics of atmospheric turbulence. Such requirements can satisfied by modelling 
the entire depth of the ABL.

\subsubsection{Similarity and scaling}

For accurate correspondence between buildings model measurements and the fullscale data, both geometric and dynamic similarities need to be preserved [49]. The geometric similarity is usually preserved by appropriate scaling through maintaining the ratios of overall buildings dimensions between physical models and full-scale structures 26. The typical range of geometric scales of models of buildings and structures is between 1:300 to 1:600, whereby these typical geometric scales, equality between the approach wind inherent length scales and model geometric scale can be maintained [49].

The dynamic similarity is achieved by matching certain dimensionless parameters between the model-scale and full-scale. For incompressible flows of homogenous fluids, it is Reynolds number that needs to be duplicated in the wind tunnel experiment [49]. However, matching Reynolds number requires much higher wind speeds in the wind tunnel, which may introduce compressibility effects. For studies of sharp-edged bluff bodies, matching Reynolds number can be relaxed since the flow separation from such structures is generally insensitive to Reynolds number. Nonetheless, minimum value of Reynolds number, which is about 11,000, should be achieved to minimize viscous effect when studying external flows around buildings [26]. Above 11,000, the flow is considered to be in 'Reynolds independence regime', in which normalized flow field data are equal between model-scale and full-scale.

\subsubsection{Blockage effect}

The placement of the physical model in the test section of the wind tunnel blocks part of the flow, resulting in the flow divergence around the model. By continuity 
and Bernoulli equation, the flow confinement by the wind tunnel solid walls cause an increase in the flow speed near the model, an effect known as solid blockage. Additionally, the confinement of the flow by the wind tunnel solid walls causes an increase in the velocity of the freestream downwind the model, outside the wake region, an effect referred to as wake blockage. This increase in wind speed near and downstream the model depends on the model size relative to the wind tunnel crosssectional area. Ideally, the blockage of the wind tunnel cross-sectional area by the model should not exceed 5\%, above which corrections are needed to account for the effect of freestream velocity increase on the measured parameters [49,50]. 


\section{Chapter 3}

\section{Wind Tunnel Experiments}

This chapter includes detailed descriptions of two wind tunnel experiments performed at two different facilities. The first experiment was conducted in the atmospheric boundary layer wind tunnel at Carleton University, whereas the second experiment was conducted in the Davies Boundary Layer Wind Tunnel (B12) at RWDI Inc.

The wind tunnel experiment at Carleton University is discussed in Section 3.1 as follows. Section 3.1.1 presents the ABL wind tunnel geometry, configuration, and specifications. The design procedure used for the development of the scaled ABL in the wind tunnel is outlined in Section 3.1.2. Section 3.1.3 describes the details of the

physical model and the construction processes. Section 3.1.4. outlines the procedure of selecting the flow angles and the measurement locations. The instrumentation and equipment setup, as well as the probe holder design description, are included in Section 3.1.5. The experimental procedures and related considerations are discussed in Section 3.1.6. Section 3.1.7 subsequently presents the overall experimental uncertainties.

The wind tunnel experiment at RWDI is discussed in Section 3.2 as follows. Section 3.2.1 describes the wind tunnel facility and specifications. Section 3.2.2 presents 
the details of the scaled city model. The instrumentation details and mounting setup are described in Section 3.2.3. Finally, Section 3.2.4 discusses the details of the wind tunnel experiments.

\subsection{CU wind tunnel experiment: $\frac{1}{400}$ city model}

\subsubsection{ABL wind tunnel}

The scaled city model test was conducted at the ABL wind tunnel located in room $2140 \mathrm{ME}$ at Carleton University. The wind tunnel is a suction-type, low-speed, openloop facility with a 7.1:1 contraction ratio. The wind tunnel is driven by a $30 \mathrm{~kW}$ $\mathrm{AC}$ motor coupled to a fan with a diameter of $1.67 \mathrm{~m}$. The wind tunnel has two test sections, upstream (close to the inlet) and downstream (close to the outlet/fan), each with a length of $2.44 \mathrm{~m}$ and a cross sectional width of $1.68 \mathrm{~m}$. The height of each section is different due to the slight divergence in the test section ceiling. Thus, the cross section height of both upstream and downstream test sections are $1.12 \mathrm{~m}$ and $1.23 \mathrm{~m}$, respectively. Both test sections are accessed through plexiglass doors located at the side walls of the wind tunnel. The floor of the downstream test section is constructed with a turntable with a diameter of $1.21 \mathrm{~m}$, which allows the investigation of different angles of incidence on a model. The turntable can be rotated manually from an opening beneath the test section. The ABL wind tunnel can operate with a maximum flow speed of $17 \mathrm{~m} / \mathrm{s}(60 \mathrm{~km} / \mathrm{h})$. The wind tunnel speed is determined from the static pressure difference obtained by a pressure transducer that is connected to two static pressure taps on the wind tunnel contraction. The pressure transducer is connected to a computer through an Analogue-to-Digital Converter (ADC), and the readings are displayed on the computer screen in inches of $\mathrm{H}_{2} \mathrm{O}$. A drawing of the wind tunnel is adapted from S. McTavish Ph.D. thesis [51], and is depicted in Figure 
3.1 .

The use of either one of the test sections depends on the purpose of the test. For the presented work, the downstream test section, shown in Figure 3.2, was utilized since rotating the model was necessary to carryout the test. Also, using the downstream test section gave the advantage of having a longer fetch, and hence a better development of ABL. The fetch distance is determined to be $10.69 \mathrm{~m}$ based on measurement from the wind tunnel inlet to the leading edge of the turntable. 

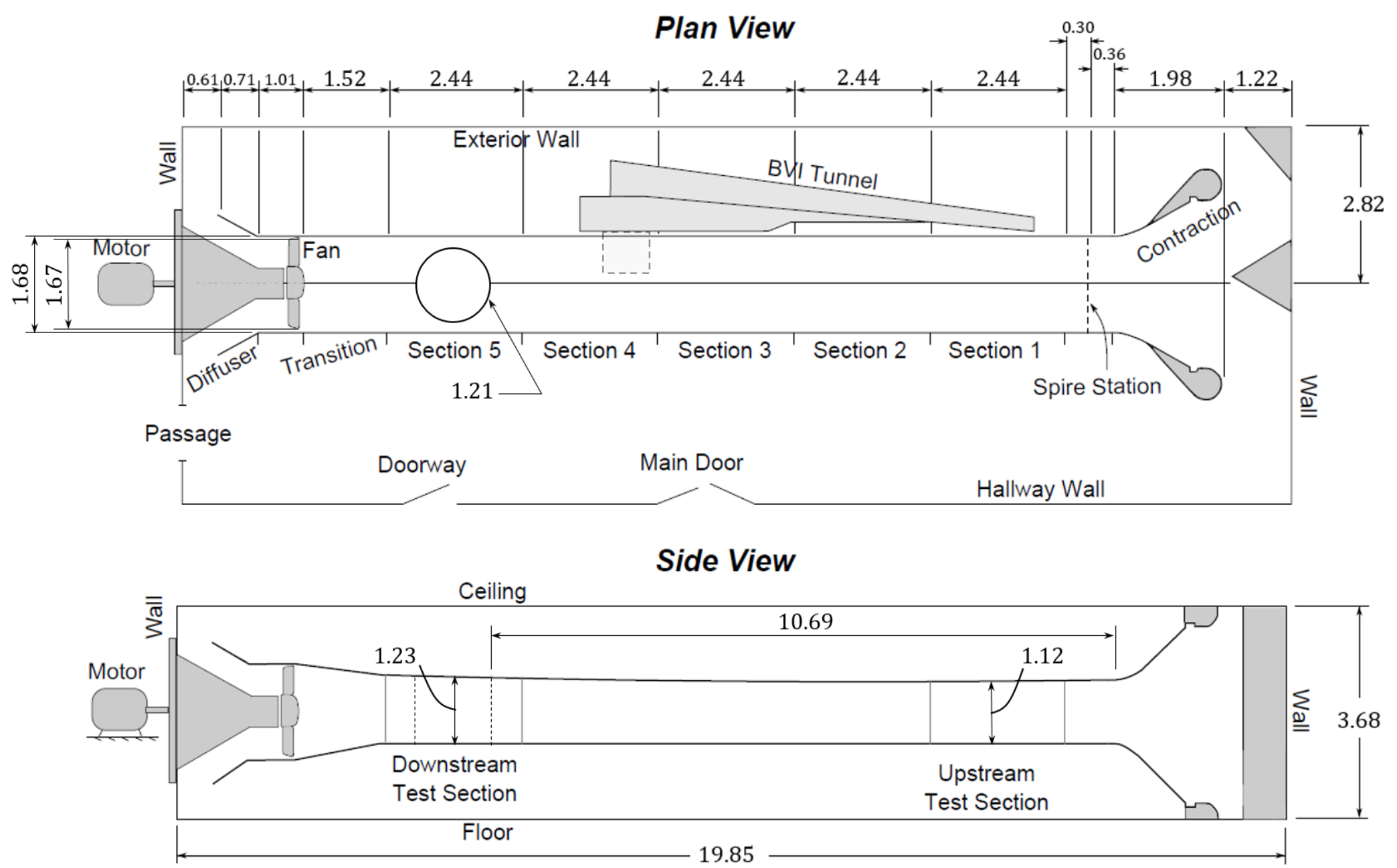

Figure 3.1: Drawing of the ABL wind tunnel at Carleton University with SI units dimensions (meters). Adapted from: [51]. 


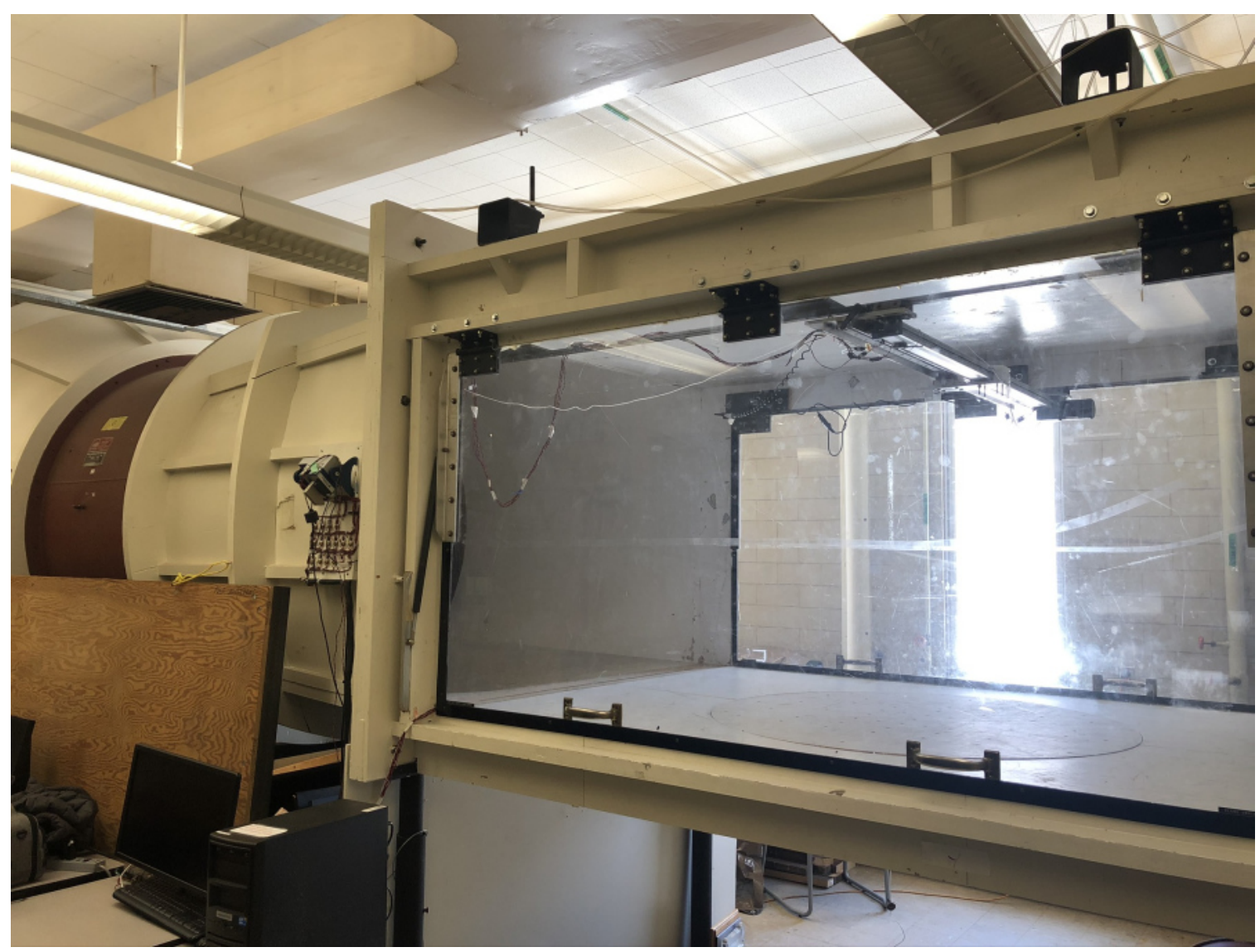

Figure 3.2: Downstream test section of the ABL wind tunnel.

\subsubsection{Simulation of the small-scale natural wind}

Urban air flows characteristics are highly influenced by the properties of the atmospheric boundary layer. Therefore, scaled models of buildings and structures are usually tested in boundary layer wind tunnels which are characterized by having a long upwind fetch, typically 15-30 m. Such wind tunnels should be capable of generating air flows that mimic the basic properties of natural wind at a specific site of interest. Flow conditioning devices such as spires are widely used to generate a thick ABL in wind tunnels having a fetch distance of less than $15 \mathrm{~m}[52$. 
Considering the working section length of the ABL wind tunnel at Carleton University, a spire-roughness technique was implemented to generate a scaled atmospheric boundary layer [53,54]. The setup of this technique is shown in Figure 3.3 .

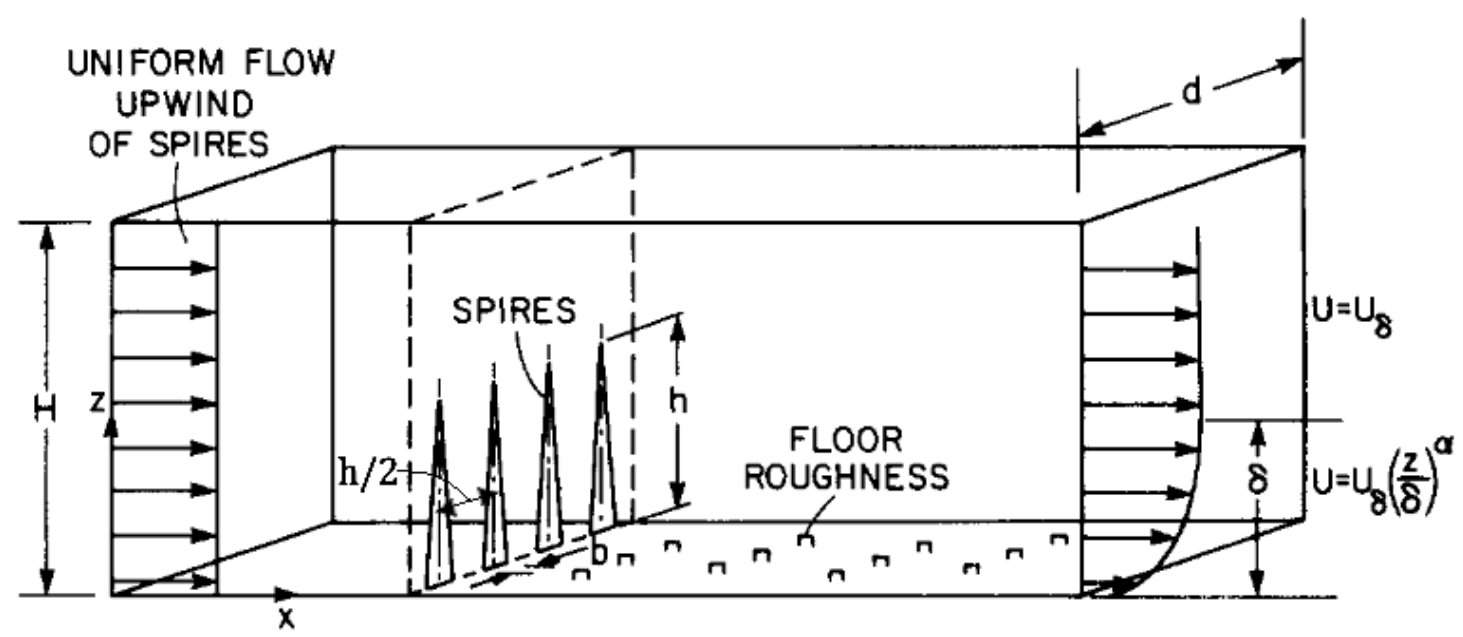

Figure 3.3: Irwin's methodology for boundary layer generation. Adapted from: 53 .

The main function of the spires is to generate the large-scale eddies, whereas the growth of the atmospheric boundary layer and the generation of the correct velocity profile are accomplished by the use of the roughness elements. The spires are placed at the upstream test section downwind of the contraction cone, followed by the roughness elements, which are distributed up until the downstream test section, where the model is located.

The designing formulas of the spires and roughness elements are based on Irwin's work [53,54] which considers a triangular shape of spires with triangular splitter-plate. The methodology builds upon the assumption of having a uniform air flow upstream of the spire array and considering a wind tunnel with a rectangular working section. The spire array should be positioned symmetrically in the working section with the spires center-lines being spaced laterally by a distance of $h / 2$, where $h$ is spire height. At a distance of $6 h$ downstream the spire array, a boundary layer with a height of $\delta$ 
is to be generated with a velocity profile that is represented by the power law:

$$
\frac{U}{U_{\delta}}=\left(\frac{Z}{\delta}\right)^{\alpha}
$$

where $U$ is the velocity at certain height $Z, U_{\delta}$ is the velocity at a reference height (boundary layer thickness) $\delta$, and $\alpha$ is the exponent of the power law, which varies depending on the atmospheric stability and surface roughness.

The generation of the boundary layer is influenced by the spires dimensions, namely base length $(b)$ and height $(h)$, and thus, suitable values of both $\alpha$ and $\delta$ should be chosen first in order to determine the spires size needed to generate the desired boundary layer. Equation $(3.2)$ is used to determine the spires height based on the desired boundary layer thickness. As for the spires base length, Equation (3.3) can be utilized after solving for coefficients $\eta$ and $\beta$ in Equations (3.4) and (3.5), respectively,

$$
\begin{gathered}
h=\frac{1.39 \delta}{1+(\delta / 2)} \\
\frac{b}{h}=\frac{\delta}{h}\left[\frac{\eta(H / \delta)}{1.41(1+\eta)}\right]
\end{gathered}
$$

where $H$ in Equation (3.3) represents the test section height.

$$
\begin{gathered}
\eta=\frac{\beta\left[\left(\frac{2}{1+2 \alpha}\right)+\beta-6\left[\frac{0.188 \alpha}{(1+\alpha)\left(1+\frac{\alpha}{2}\right)}\right]\right]}{(1-\beta)^{2}} \\
\beta=\frac{\delta}{H} \frac{\alpha}{(1+\alpha)}
\end{gathered}
$$

It is worth mentioning that Equation (3.3) holds true only if the test section width is a multiple of $h / 2$.

The boundary layer characteristics can be obtained with an appropriate selection 
of the floor roughness. The aerodynamic drag effect of the roughened floor is already accounted for in Equation (3.4). A correlation between the local skin friction coefficient $\left(C_{f}\right)$ and the power law exponent $(\alpha)$ is represented by Equation 3.6 .

$$
C_{f}=0.136\left[\frac{\alpha}{1+\alpha}\right]^{2}
$$

The required value of $C_{f}$ can be produced in wind tunnels by the use of cubic roughness elements whose height $(k)$ is approximated in relation to the desired boundary layer thickness using Equation 3.7

$$
\frac{k}{\delta}=\exp \left[(2 / 3) \ln (D / \delta)-0.1161\left(\left(2 / C_{f}\right)+2.05\right)^{1 / 2}\right]
$$

where $D$ is the spacing between the roughness elements which must be selected first in order to determine the $k$ value.

The empirical expressions developed by Irwin [53,54] are expected to produce the desired boundary layer thickness and profile at a distance of $6 h$ downstream of the spire array. At this distance, lateral uniformity of the flow should be attained if the spire array is positioned symmetrically in the working section with the center-lines of the two end spires being away from the wind tunnel wall by a distance of $h / 4$.

In the case of a working section with a length of more than $6 h$ (see Figure 3.4), the increase in the boundary layer thickness $(\Delta \delta)$ at a distance $\Delta x$ can be calculated using Equation (3.8),

$$
\Delta \delta=0.068 \alpha[(1+2 \alpha) /(1+\alpha)] \Delta x \cdot F
$$

where $F$ is a correction factor used to account for the pressure drop resulting from the growth of the boundary layer in the wind tunnel working section, and it can be 


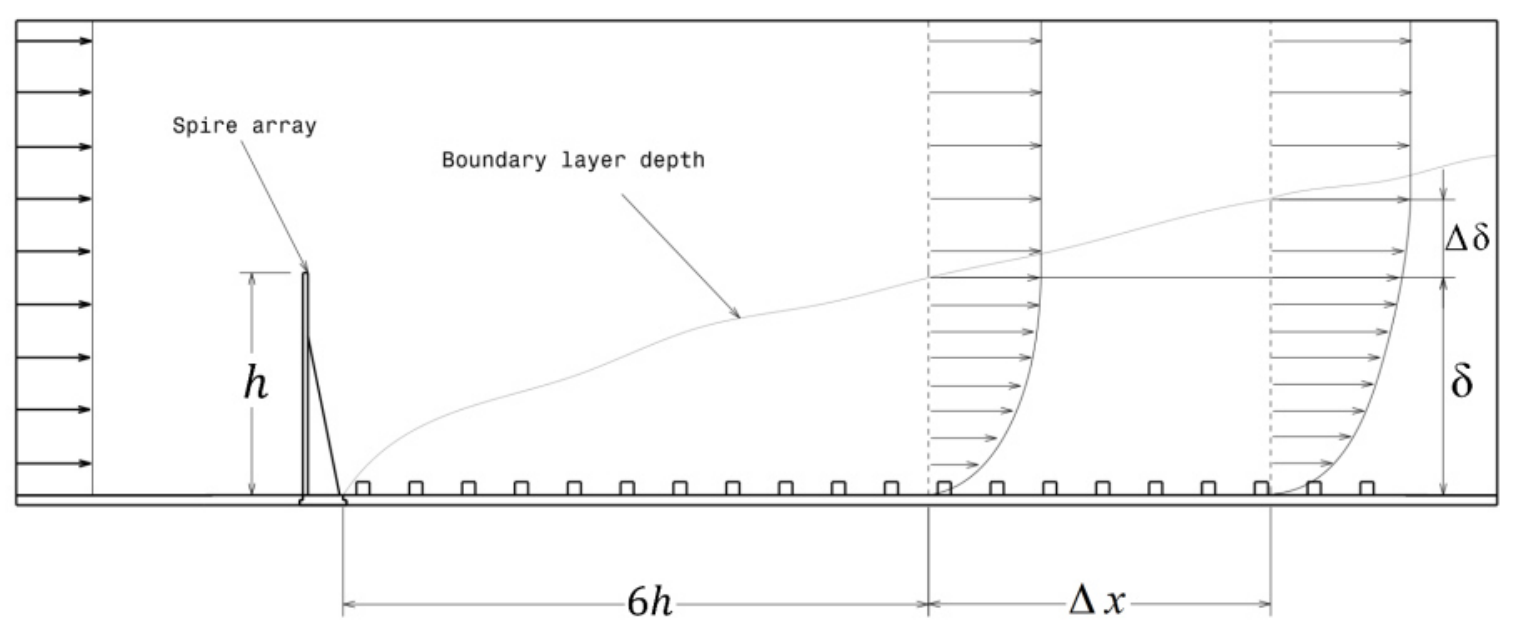

Figure 3.4: Illustration of boundary layer growth at a distance of $6 h+\Delta x$.

(Dimensions are not to scale)

determined as described in Equation 3.9 .

$$
F=\left[1+(\delta / H)\left[\alpha(3+2 \alpha) /\left(1+\alpha\left(1-\frac{\delta}{H}\right)\right)\right]\right]^{-1}
$$

The scaled boundary layer for the presented work was generated according to the empirical relationships discussed above. An array of four spires with a height of $84 \mathrm{~cm}$ and base length of $17.69 \mathrm{~cm}$ was placed immediately downstream of the contraction cone as seen in Figure 3.5 .

The spires blocks $15.74 \%$ of the incoming flow, so the effect on the maximum wind tunnel speed was insignificant considering the desired wind speed for the study, which was $10 \mathrm{~m} / \mathrm{s}$. The spires were constructed from $19 \mathrm{~mm}(3 / 4 \mathrm{inch})$ thick plywood. The calculated spire size was obtained considering a power-law exponent $\alpha=0.40$, which represents the velocity profile for heavily built up urban centers [31. Downstream the spires, the flow developed further by passing over cubic roughness elements which were distributed along the wind tunnel working section up until $0.5 \mathrm{~m}$ upstream of the turntable leading edge. The size of the elements was also selected based on 


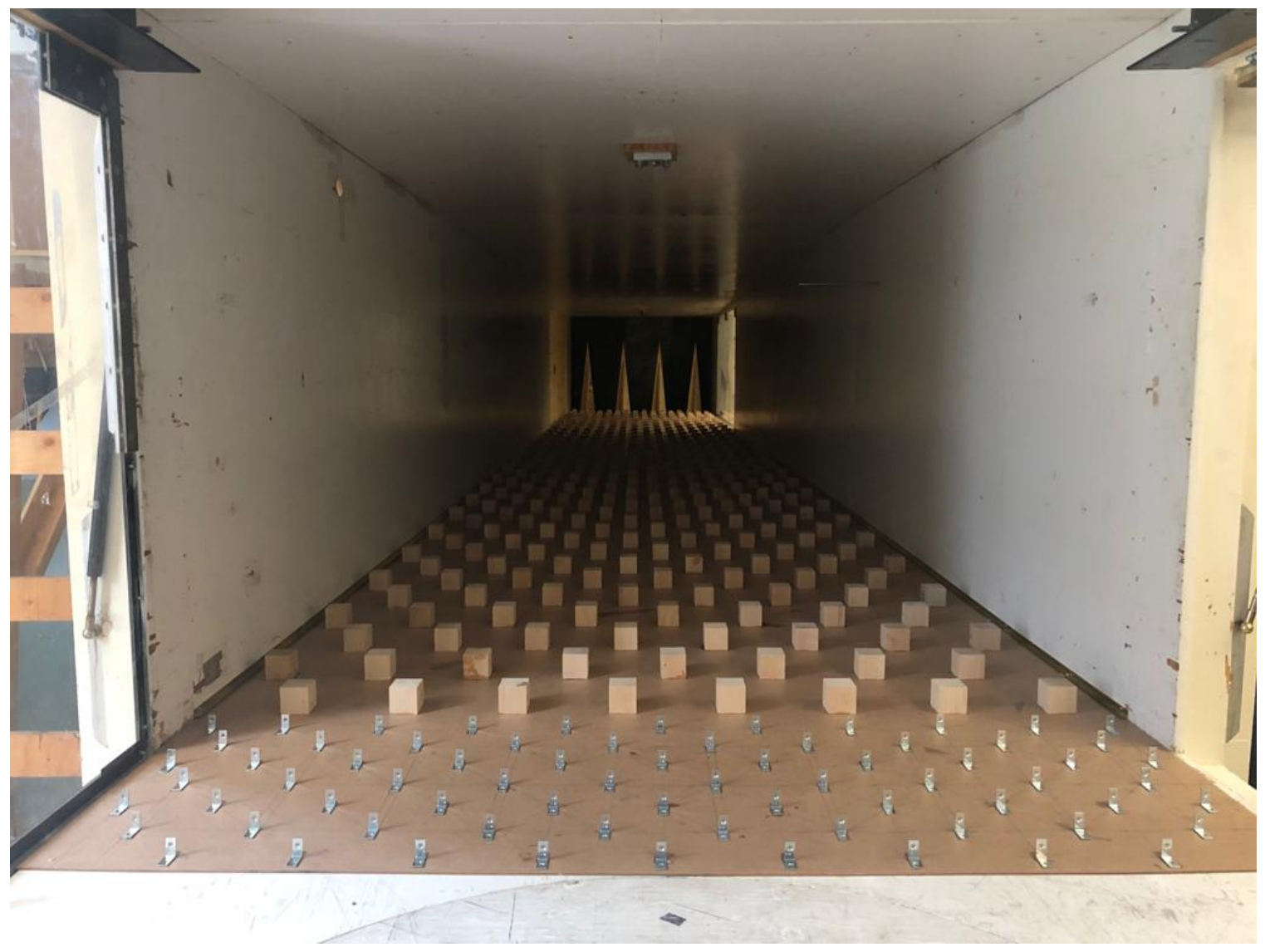

Figure 3.5: Spires and roughness elements in the ABL wind tunnel.

Irwin formulas presented in $[53]$. The formulas suggested a cube height of $5 \mathrm{~cm}$ with spacing distance of $15 \mathrm{~cm}$ in both $x$ and $y$ directions. Thus, $5 \mathrm{~cm}$ wooden cubes were glued to $3.2 \mathrm{~mm}$ (1/8 inch) thick hardboard panels which serve as a replacement for the wind tunnel floor. The wooden cubes were followed by $25.4 \mathrm{~mm}$ (1 inch) angle brackets spaced apart by a distance of $10 \mathrm{~cm}$. The decision to use smaller size roughness elements was made to eliminate local effects on the model measurements while keeping the boundary layer energized. The spires and roughness elements dimensions are illustrated in Figure 3.6 . 


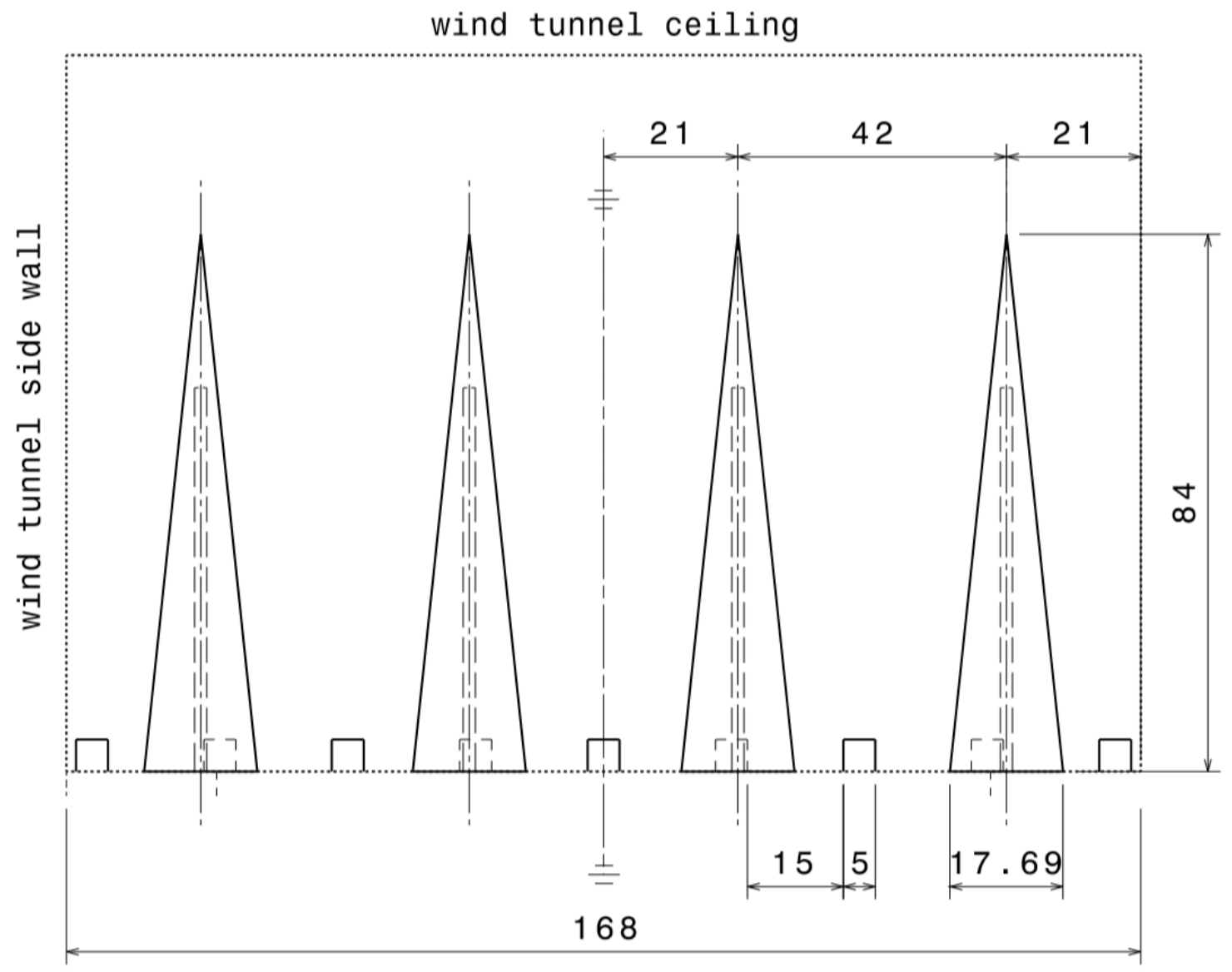

Figure 3.6: Spires and roughness elements dimensions in $\mathrm{cm}$.

\subsubsection{Model characteristics and details}

\subsubsection{Model description, domain, and geometry}

The urban flows characterization was accomplished by testing a scaled-down partial city model in the ABL wind tunnel. The 1:400 scale city model is a representation of a realistic urban setting in downtown Toronto, Canada. The selection of Toronto city was influenced by the research objective of characterizing 'the worst' case urban flows; hence, choosing the most urban city in Canada was the optimal option. The selected part of Toronto city, shown in Figure 3.7, represents a circular region with a diameter of $484 \mathrm{~m}$ and an area of $183,984 \mathrm{~m}^{2}$ (FS). The selected region was centered 

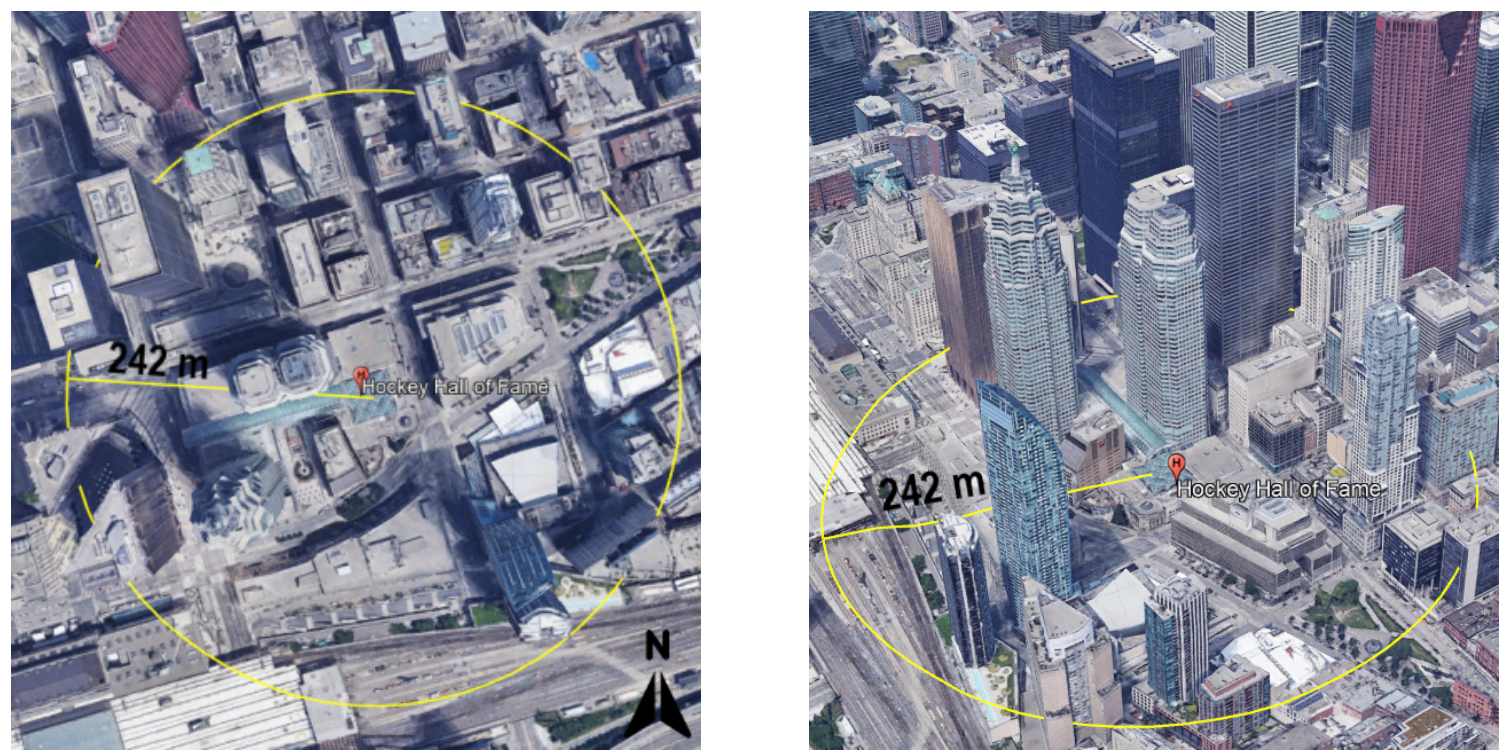

Figure 3.7: 2D and 3D model domain in real settings.

at the Hockey Hall of Fame (HHF), whose rooftop was a potential landing zone in this research for the Bell Nexus flying taxi [55]. The landing zone was chosen to be on the top of the HHF for the following reasons: it is surrounded by buildings with heterogeneous heights, of which are multiple high-rise buildings; it is close to the ground level, making it an ideal landing zone; and its footprint can fit the Bell Nexus flying taxi.

To construct the Sub-Scale (SS) partial city model, a FS Computer-Aided Design (CAD) model of Toronto, consisting of simplified buildings geometry, was provided by RWDI to be used as a basis for building the physical model. Features such as vegetation, bus shelters, and other small-scale architectural details were not part of the model. The initial CAD model was scaled down, and all buildings surrounding the HHF that would fit in the $1.21 \mathrm{~m}$ turntable were extracted. Since the model represents a circular region, some of the buildings at the edge of the circle were either entirely removed or partially included based on the geometrical shape. The CAD models of the FS and SS city models are shown in Figure 3.8. On the selected 
region, there are 28 buildings, two of which are Future Buildings (FB) and coloured in green as shown in Figure 3.8. The remaining 26 buildings are combination of low-, mid- and high-rise buildings. There are seven buildings of interest due to their FS height, which is greater than $150 \mathrm{~m}$. Nonetheless, the flow features of all buildings whose height is greater than the rooftop of the HHF were within the scope of the research. Details of the height of the major buildings are illustrated in Figure 3.9 . The height is measured from the model base to the rooftop of the buildings. In the same figure, the seven high rise buildings mentioned above are coloured in red, and the future buildings are coloured in blue (shown previously in green in Figure 3.8). Furthermore, multiple holes appears on the model base and buildings top surfaces, which represent possible measurement locations for the model test. Details about the selection of the measurement locations will be discussed in Section 3.1.4 

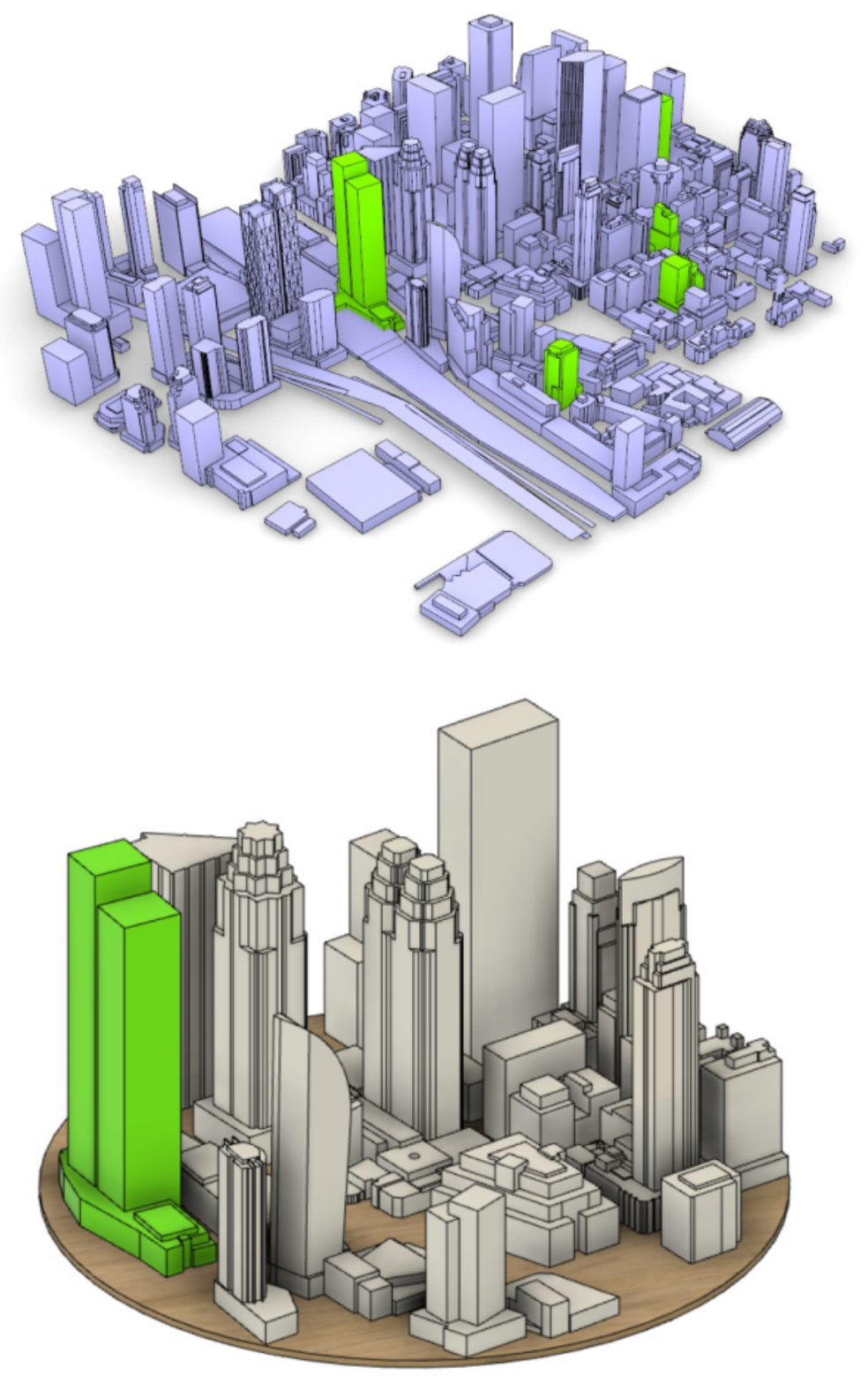

Figure 3.8: Initial full-scale (top) [56, and final sub-scale (bottom) CAD models for the city model. 


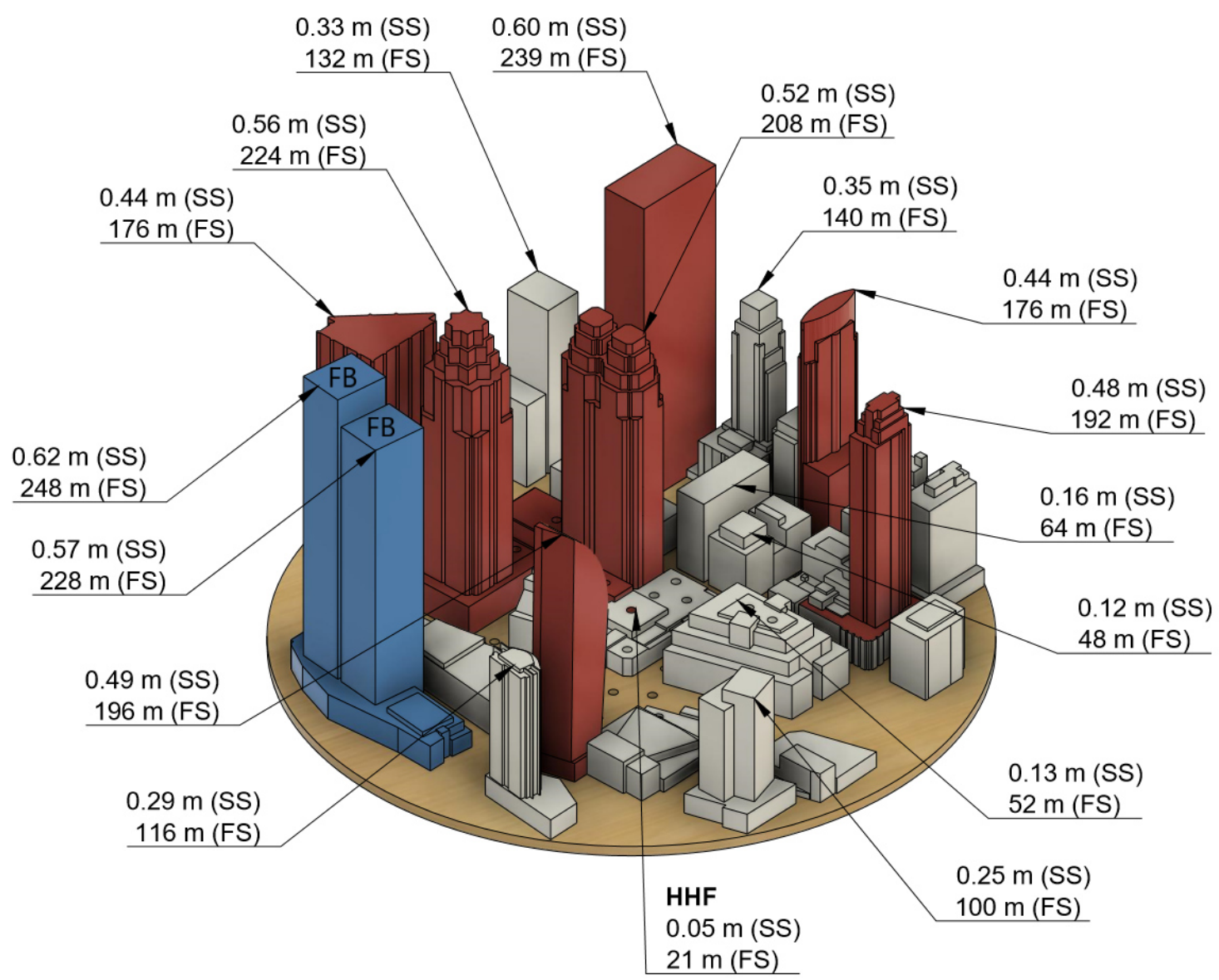

Figure 3.9: The height of the buildings of the 1:400 city model measured from ground level (model base). 


\subsubsection{Model construction}

The physical model was constructed by the use of a Computer Numerically Controlled (CNC) router machine, available in the machine shop at Carleton University. The router table dimensions are $2.44 \mathrm{~m}(8 \mathrm{ft})$ by $1.22 \mathrm{~m}(4 \mathrm{ft})$ in $x$ and $y$ directions, respectively. The machine allows $20.32 \mathrm{~cm}$ ( 8 inch) of travel in the $z$ direction; however, the tool length influences the part thickness. Considering the height of the scaled buildings, it was decided to construct the partial city model in vertical sections using polyurethane foam boards, which were provided by the NRC. To ensure appropriate placement of the buildings, a $6.3 \mathrm{~mm}(1 / 4 \mathrm{inch})$ foam disc was constructed with a layer of each building as shown in Figure 3.10. The remaining sections of the
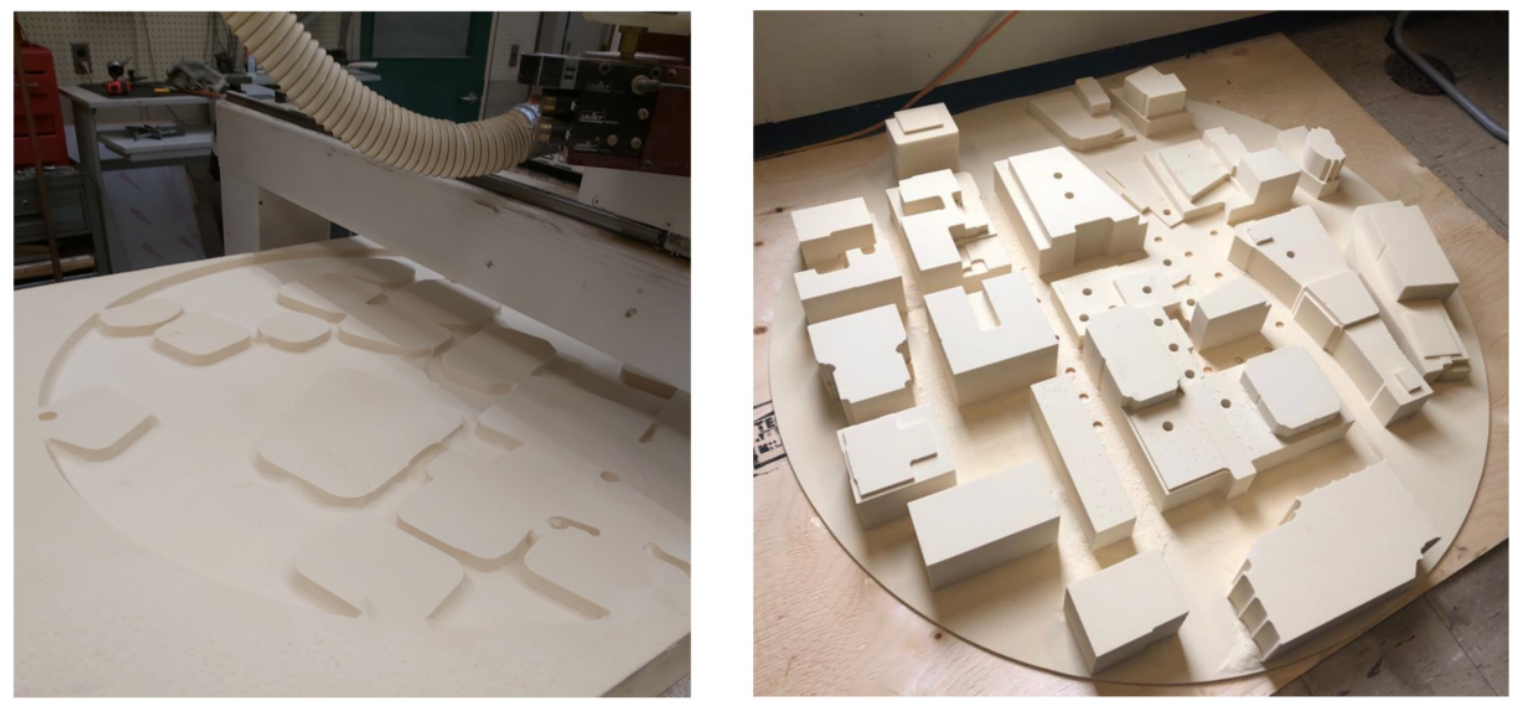

Figure 3.10: Foam disc with buildings layout.

buildings were glued together at first, and subsequently glued to the foam disc, making up the complete physical model shown in Figure 3.11. The future buildings, however, were not glued to the base since the model was to be tested with and without them.

The city model foam disc was then glued to the top face of a newly built 12.7 mm $(1 / 2$ inch $)$ thick turntable that was constructed to accommodate for the foam 

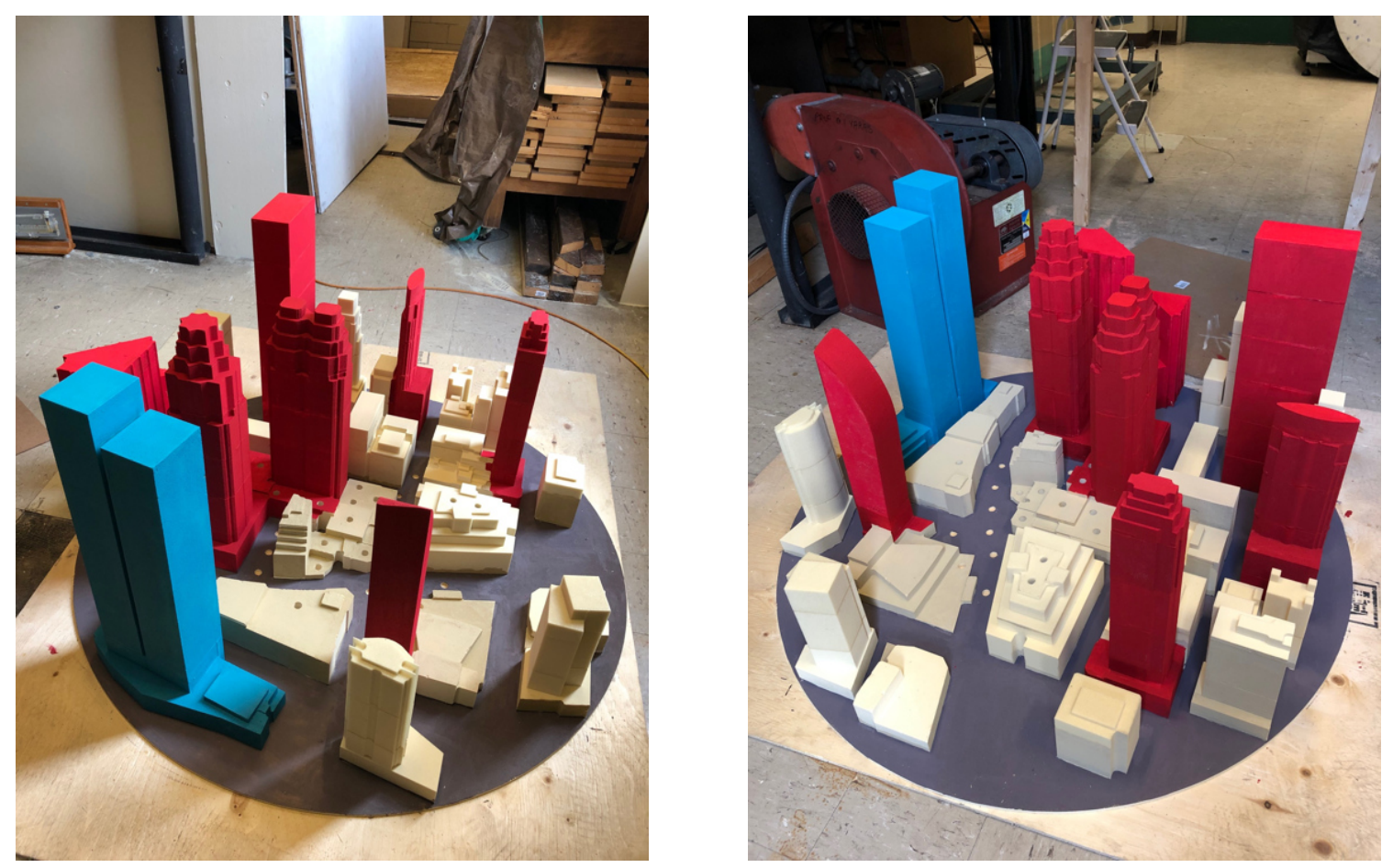

Figure 3.11: 1:400 scale physical model.

disc thickness of $6.3 \mathrm{~mm}(1 / 4 \mathrm{inch})$. This was done to ensure that the model base would be flush with the wind tunnel floor since the original thickness of the wind tunnel turntable is $19 \mathrm{~mm}(3 / 4 \mathrm{inch})$. Additionally, the turntable bottom surface was machined with grooves, representing multiple wind directions, to ensure appropriate model orientation to the incoming flow. In the turntable, small and large holes were also drilled for both mounting the measuring instrument holder and indexing system as well as inserting the measuring instrument into the flow field, externally, through the opening beneath the test section. Thus, in order to fasten the measuring instrument holder system to the turntable, multiple T-nuts were installed in the predrilled small holes. T-nuts refer to the threaded inserts that can be inserted into a wood or composite to make a threaded path for fastening parts together. Commonly, each T-nut has three to four prongs on a thin flange at one end of the nut, which can be screwed or hammered into the wood to secure the nut in position. Figure 
3.12 shows the bottom surface of the constructed turntable with the inserted T-nuts. The holes in the model base and buildings top surfaces, as seen in Figures 3.10 and 3.11, are aligned with the large holes in the turntable, and were made for the same aforementioned purpose. Details about the measuring instrument and its holder and indexing system will be discussed further in Section 3.1.5.3.

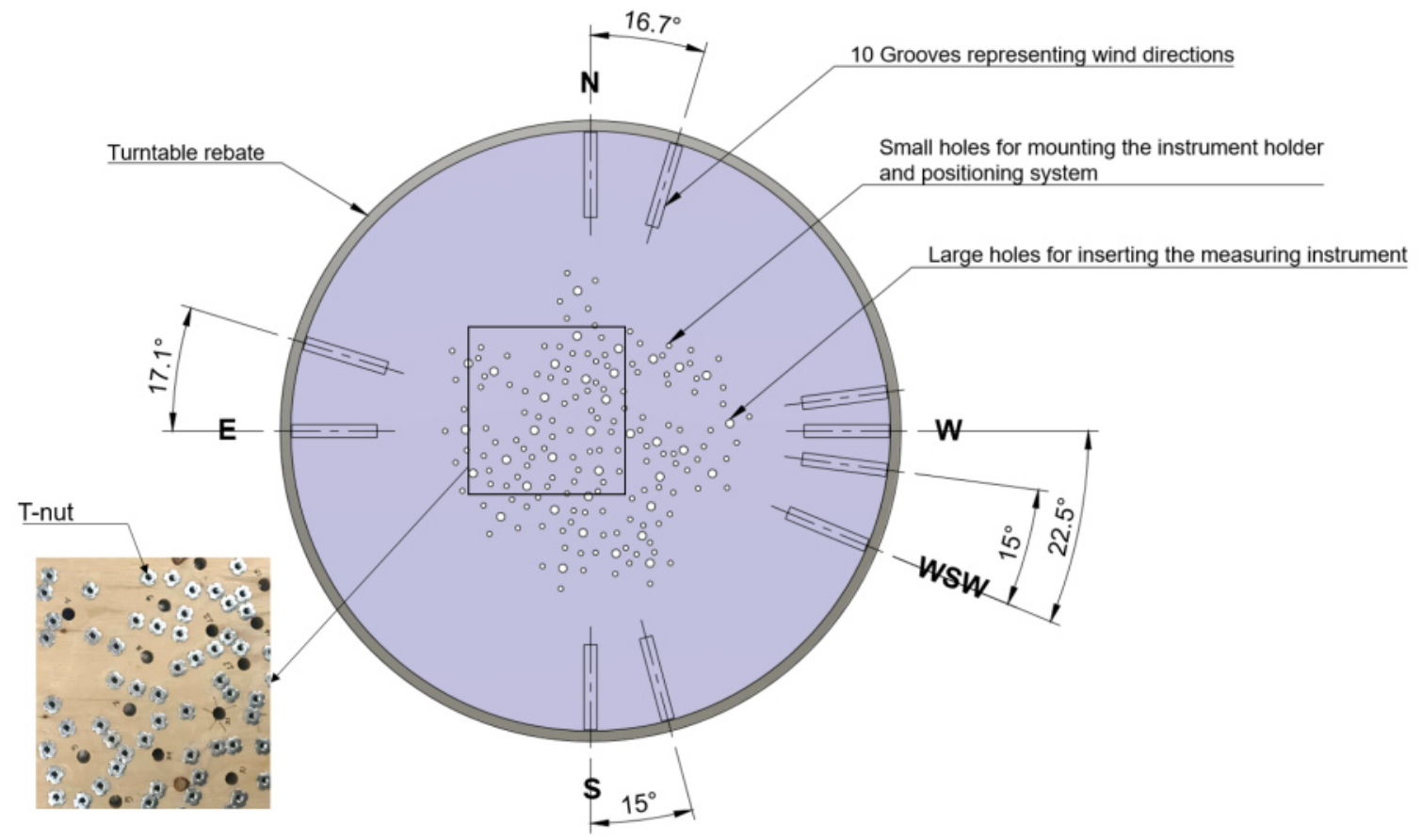

Figure 3.12: Bottom view of the 1:400 city model turntable.

\subsubsection{Model scale and solid blockage}

The model geometric scale of 1:400 was chosen with considerations of: i) the typical scales for model testing of large buildings and structures; and ii) the wind tunnel test section dimensions.

Respecting the typical scales, which range between 1:300 and 1:600 [26], is necessary since, according to Jensen's model law [57], representative wind speed and atmospheric turbulence profiles of the full scale natural wind can be modelled adequately 
in wind tunnels at the aforementioned geometric scales; plus, both the buildings geometric scale and the length scale of the modeled natural wind has to be consistent. In other words, appropriate model testing should be carried out in turbulent boundary layer with a velocity profile that is in scale with geometric size of the model [58].

As for the wind tunnel test section size, choosing a larger scale was impractical since the city model would have included fewer buildings, and at the same time, the model would be closer to the wind tunnel ceiling. Similarly, going with a smaller scale was not preferable since the building Reynolds number, $R e_{b}$, which is dependent on the characteristic dimension (D) of the building, would be correspondingly lower. Additionally, if the model was on a smaller scale, the measurement process would have been more challenging due to the instrument size with respect to the model geometry.

The blockage for the 1:400 model was estimated by considering the ratio between the model frontal area and the test section cross-sectional area. Consequently, model rotation for simulating different angles of incidence resulted in different blockage by the model. The solid blockage for the model was estimated for five wind directions, and the results are presented in the Table 3.1. $\mathrm{S}_{15 \text {,without }}$ and $\mathrm{S}_{15 \text {,with }}$ are referring to the same wind direction, $\mathrm{S}_{15}$. However, $\mathrm{S}_{15 \text {,without }}$ refers to the solid blockage without the future buildings while $\mathrm{S}_{15 \text {, with }}$ refers to the solid blockage when the future buildings were included. More information about the wind directions are included in Section 3.1 .4 
Table 3.1: Model blockage at various wind directions.

\begin{tabular}{cc}
\hline \hline Wind direction & Solid Blockage [\%] \\
\hline WSW & 20.4 \\
WSW $_{+15}$ & 21.0 \\
$\mathrm{E}_{17.1}$ & 19.6 \\
$\mathrm{~N}_{16.7}$ & 18.0 \\
$\mathrm{~S}_{15, \text { without }}$ & 22.1 \\
$\mathrm{~S}_{15, \text { with }}$ & 25.1 \\
\hline
\end{tabular}

\subsubsection{Velocity scale and Reynolds number scaling}

For the model city experiment, the selection of the wind tunnel speed was solely based on achieving an aerodynamic similarity between the model and full-scale flows; that is, both the model and full-scale flows are to be independent of the Reynolds number. Provided that the city model consists of buildings with sharp edges, it was necessary to achieve a minimum of $R e_{b}>11,000$ in order to satisfy the aerodynamic similarity [26]. Thus, $R e_{b}=U D / \nu=11,000$ was used as a limiting case, with $\mathrm{U}=10$ $\mathrm{m} / \mathrm{s}$ and $\nu$ value at $22{ }^{\circ} \mathrm{C}$. The limiting case criteria suggested that any building with $\mathrm{D}>0.015 \mathrm{~m}$, which is a very small value compared with the characteristic dimension of the smallest representative building in the model, would satisfy the aerodynamic similarity. Thus, the model was tested at $10 \mathrm{~m} / \mathrm{s}$, which ensured that the flow features over and around the buildings would be fully turbulent.

\subsubsection{Selection of wind angles and measurement locations}

The model city experiment was conducted at five different wind directions. The angles of the wind directions are measured from North, which is $0^{\circ}$, in a clockwise $(\mathrm{CW})$ direction, and are listed in Table 3.2 . 
Table 3.2: Wind direction designations for the model city experiment.

\begin{tabular}{cc}
\hline \hline Wind direction & Angle [deg] \\
\hline WSW & 247.5 \\
WSW $_{+15}$ & 262.5 \\
$\mathrm{E}_{17.1}$ & 72.9 \\
$\mathrm{~N}_{16.7}$ & 343.3 \\
$\mathrm{~S}_{15}$ & 195 \\
\hline
\end{tabular}

The selection of various wind directions was done to widen the scope of characterizing the urban airflows, that is, by examining the effects of the incidence angle on the flow structures together with investigating the impact of the approach flow, due to the nearby buildings, on specific sites. Therefore, the logic employed for selecting the wind directions, mentioned in Table 3.2, was as follows: to include wind directions that represent a smooth and a complex approach flows $\left(\mathrm{E}_{17.1}\right.$ and $\mathrm{WSW}_{+15}$ respectively); to include the prevailing wind direction in Toronto city (WSW); to include a wind direction that is aligned with the model streets or perpendicular to the buildings $\left(\mathrm{N}_{16.7}\right)$; and to include a wind direction from the South to examine the effects of adding future buildings on the flow field $\left(\mathrm{S}_{15}\right)$.

Following the selection of the wind directions, a total of 36 measurement locations were picked, taking into account, the flow features of interest. The selection of the locations was based on a general knowledge of the flow patterns around building blocks of different shapes, heights, and densities with respect to the flow angle, reviewed in References (Refs.) 25, 35, 39, 41, 43, 59,64. Additionally, all measurement locations were selected to be above flat surfaces to avoid destroying the buildings geometry. The measurement locations were contained within a circular region that is centered at the HHF, and has a diameter of $0.58 \mathrm{~m}$. The wind directions and the measurement locations along with their designations are illustrated in Figure 3.13. 


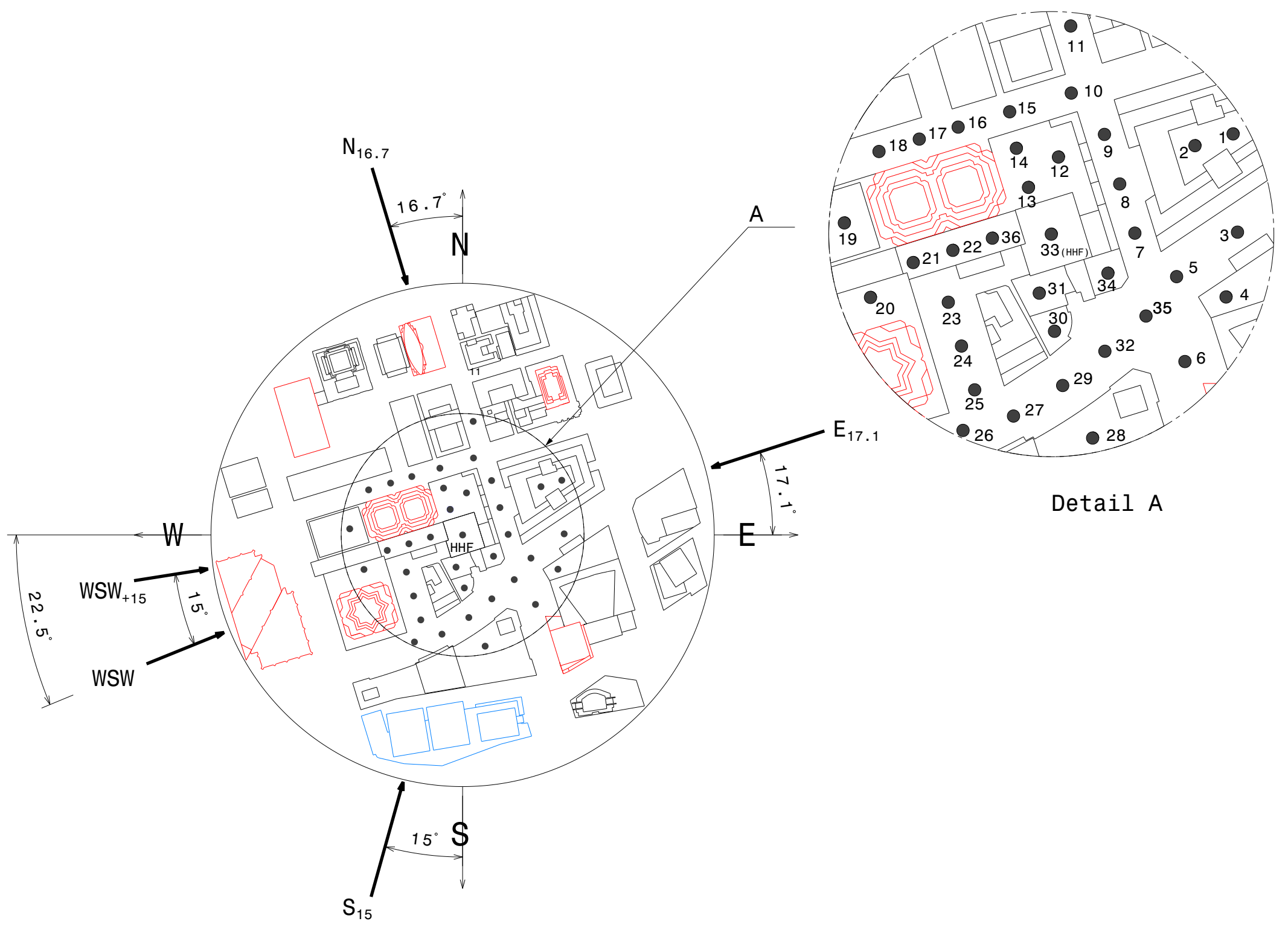

Figure 3.13: Wind directions and measurement locations for the 1:400 model city experiment. 


\subsubsection{Instrumentation and data acquisition system}

This section describes the instrumentation and equipment used to carry out the wind tunnel experiments, as well as the calibration processes and instrumentation sensitivity checks.

\subsubsection{Hardware setup}

The model city experiment was conducted by the use of multiple instrumentation and equipment. The primary measuring instruments were the Cobra probes (refer to Section 3.1.5.3 for the instrument description), which were used for collecting all the data from the city model. Additionally, a Pitot-static (P-s) tube was used in combination with Datametrics 1400-Electronic Manometer with pressure range up to 10 in $\mathrm{H}_{2} \mathrm{O}$. The Pitot-static tube system was utilized to generate a calibration curve for determining the free-stream velocity in absence of the model and to calibrate the Cobra probes. To check the functionality and accuracy of the electronic manometer, multiple pressure readings from the electronic system were cross-checked with readings from Dwyer inclined liquid manometer, which is shown in Figure 3.14.

The Cobra probes and the Pitot-static tube system were connected to an interface unit from Turbulent Flow Instrumentation Ltd (TFI), that was connected to a computer through a National Instruments, NI 6251, data acquisition system (DAQ) with $\pm 10 \mathrm{~V}$ input range and 16-bit ADC resolution. The equipment setup is shown in Figure 3.15, and the instrumentation details will be discussed further in the following subsections. 


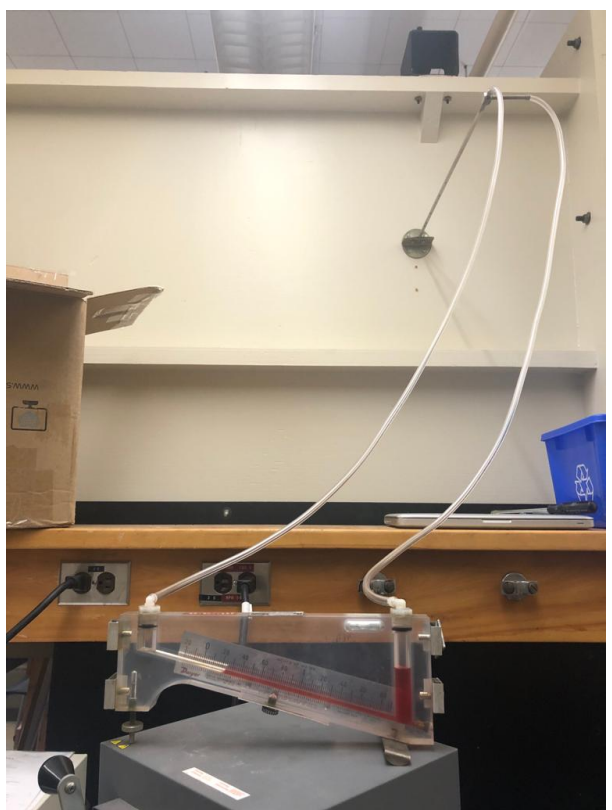

Figure 3.14: Dwyer inclined liquid manometer.

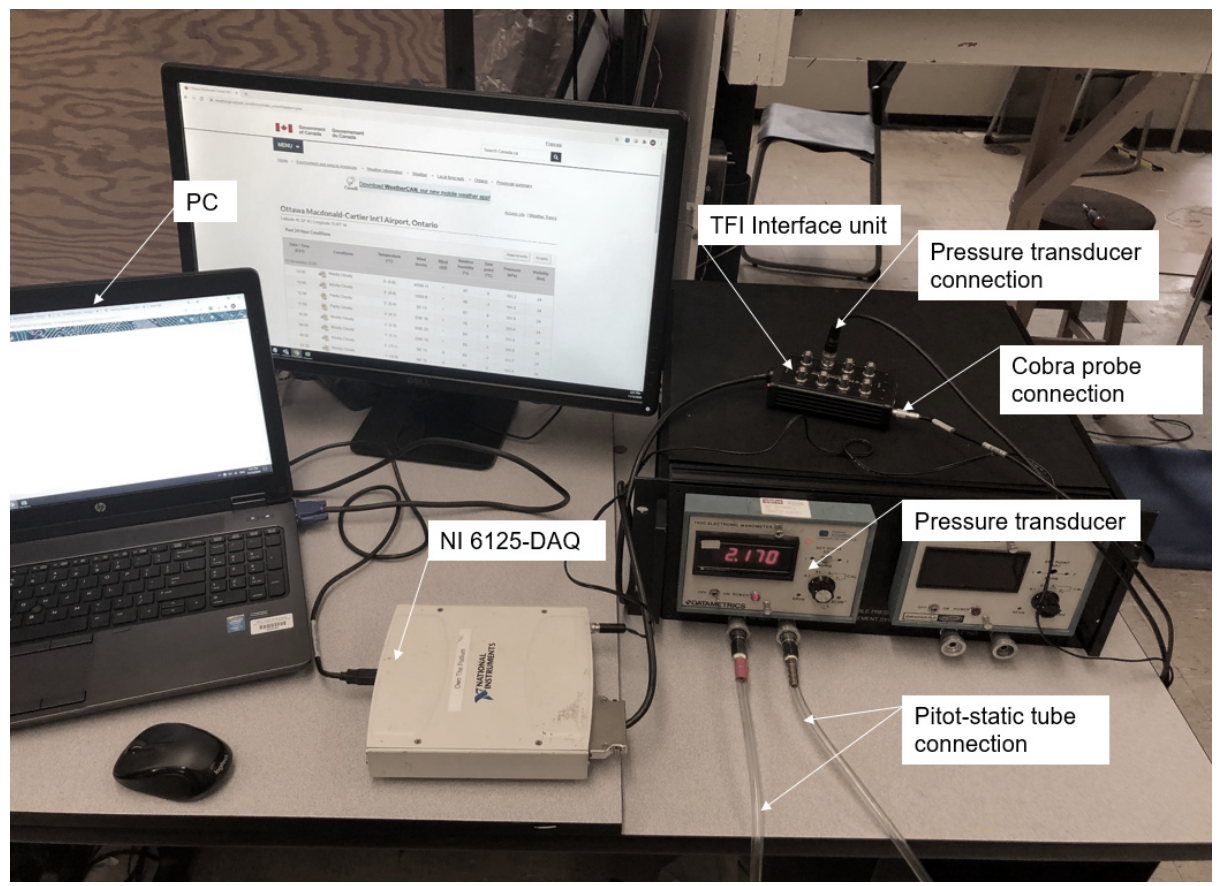

Figure 3.15: Equipment setup details. 


\subsubsection{Software configuration}

The data collection of both the Cobra probes and the Pitot-static tube was done through the use of TFI Device Control software, which is supplied with the Cobra probe package. The software allows the user to set all the sampling information as well as the working fluid pressure and temperature. The temperature and pressure readings were entered manually before each run. Since the wind tunnel is a suction type with an open circuit arrangement, it was decided to take the temperature readings from the room temperature sensor located nearby the downstream test section, and hence, near to the wind tunnel fan. On the other hand, the pressure readings were collected from hourly local weather forecast, specifically, the Ottawa MacdonaldCartier Int'l Airport. Sensitivity analysis to determine the effects of temperature and pressure variations on the probe readings are discussed in Section 3.1.5.4.

On the TFI software, there are two terms to configure the sampling and data rates. The first is the Acquisition Scan Rate (ASR), and it represents the frequency at which the analogue signals are sampled per channel. The ASR should be set between 8000 and 10,000 $\mathrm{Hz}$ according to the manufacturer recommendations to avoid aliasing and reduce measurement noise. The TFI software uses low-pass filtering and down-sampling on the data acquired at the ASR based on the desired Data Output Rate (DOR), which is the second term needed to configure the sampling rates. Thus, the DOR is basically the frequency at which the data is output to file. The output data comes in two different types, summary files and binary time-history files. The summary files for the Cobra probes contain the sampling information, working fluid temperature and pressure, and the mean values of multiple parameters including flow speed, pitch and yaw flow angles. The summary files for the Pitot-static tube contain the sampling information and the mean value of the height of water column, in inches, that is measured by the electronic manometer. 
For the presented work, the acquisition scan rate was set to $8000 \mathrm{~Hz}$, the data output rate was set to $2000 \mathrm{~Hz}$, and the sampling time was set to 30 seconds (s). Sensitivity analysis was also done to ensure appropriate selection of the sampling time, and it is discussed in Section 3.1.5.4.

\subsubsection{Instruments specifications and mounting arrangements}

\section{The Cobra probes}

Due to the nature of urban airflows, which is characterized by high levels of turbulence, it was necessary to use a fast-response measuring instrument in order to capture the rapidly changing flow conditions. Thus, two Cobra probes, manufactured by Turbulent Flow Instrumentation Ltd (TFI) of Australia, were utilized to collect all the measurement data of the city model experiment.

The Cobra probe, depicted in Figure 3.16, is a four-hole pressure probe that provides instantaneous measurements of flow speed magnitude, flow pitch $(\theta)$ and yaw $(\psi)$ angles, as well as local static pressure for flow that resides within $\pm 45^{\circ}$ of the probe x-axis. The probe has an overall length of $155 \mathrm{~mm}$, a body diameter of 14 $\mathrm{mm}$, and a head width of $2.6 \mathrm{~mm}$. On the probe faceted head, there are four $0.5 \mathrm{~mm}$ taps that are connected, by means of tubing, to pressure transducers located inside the body of the probe.

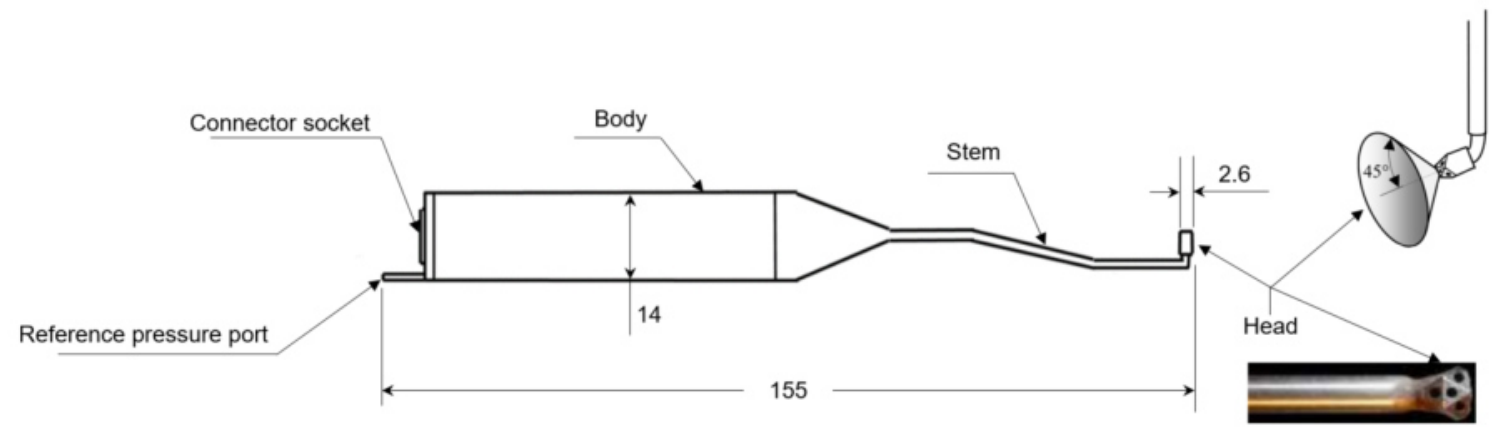

Figure 3.16: Cobra probe details (dimensions in mm). Adapted from: 65], 66]. 
Owing to the close proximity of the pressure transducers to the pressure taps, and hence the short tubing connection, the Cobra probe is capable of measuring time varying data, accurately, up to a frequency of $2000 \mathrm{~Hz}$. The probes module, used in the experiment, can operate effectively in a flow speed ranging from $2 \mathrm{~m} / \mathrm{s}$ to 55 $\mathrm{m} / \mathrm{s}$. The measured flow speed and flow angles are accurate within $\pm 0.5 \mathrm{~m} / \mathrm{s}$ and $\pm 1.0^{\circ}$, respectively. The manufacturer reported accuracies of both flow speed and flow angles were cross-checked by Mallipudi et al. [67], and the results found to be in agreement with the manufacturer specifications. The probe flow axis system and the flow pitch and yaw angles definitions are illustrated in Figure 3.17 based on the descriptions provided in the TFI Cobra probe manual in Reference (Ref.) [65].
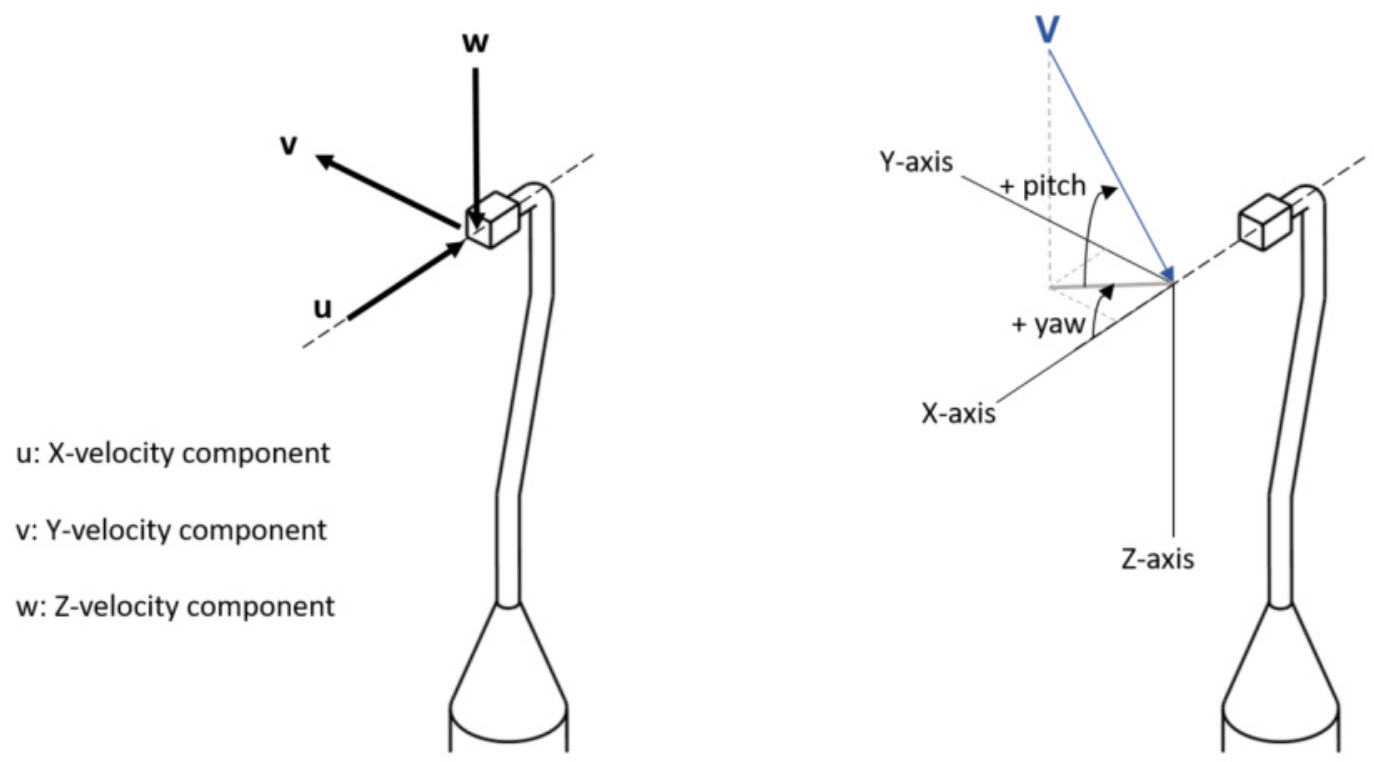

Figure 3.17: Cobra probe flow axis system.

The Cobra probes were inserted into the wind tunnel test section externally from the opening under the test section, and through the drilled holes in the turntable and the model. Due to the short length of the probes, it was decided to use $123.19 \mathrm{~cm}$ (48.5 in) stainless-steel tubes in order to traverse the probes along the test section 
height. For each of the tubes, one end was machined by widening the inner diameter and making a T-shape cut along $2.54 \mathrm{~cm}$ (1 in) of the tube length. The concept of the machined end was developed by Sylvain Bisson, a senior wind tunnel operator for $\mathrm{NRC}$, and is made to create an interference fit between the probe and the tube. Thus, when the probe is inserted in the tube, it would be fixed, and any change in the probe position thereafter is attributed to traversing and/or rotating the stainless-steel tube. The probe reference pressure port was inside the tube, and thus, it was not connected to any tubing as it was already isolated from the flow field. The details of the tubes are illustrated in Figure 3.18 .

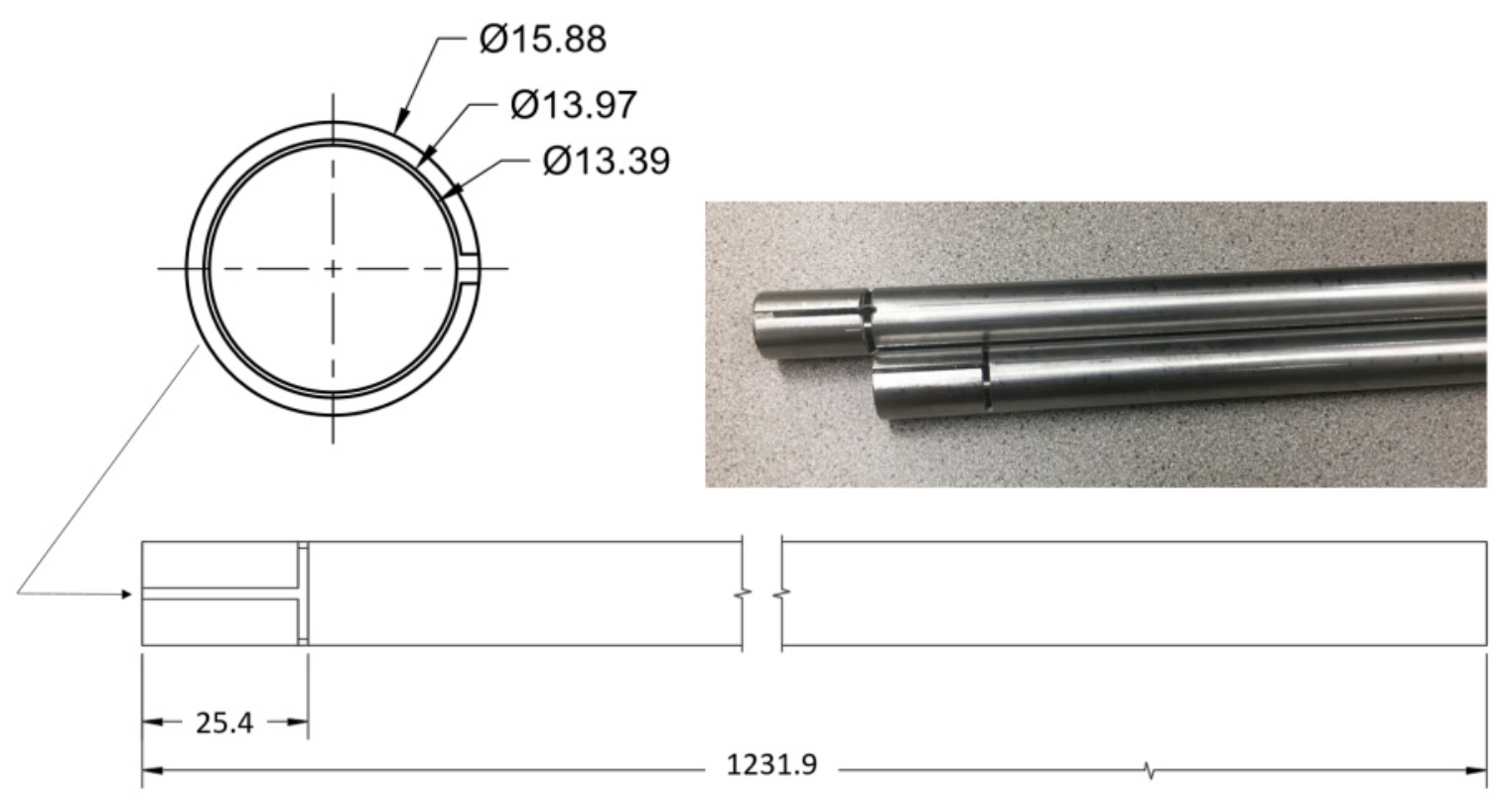

Figure 3.18: Cobra probes stainless-steel tubes (dimensions in $\mathrm{mm}$ ).

Each of the Cobra probes, used in the experiment, was mounted to the bottom surface of the turntable by the use of Acrylonitrile Butadiene Styrene (ABS) 3D printed probe holder and indexing system. The system, depicted in Figure 3.19, consists of two parts: a holder which has three Hex-head screws to secure the probe tube in position, and a flange to mount the entire system to the turntable by fastening 
four bolts to their mating T-nuts, which were shown earlier in Figure 3.12 . On the flange and holder base, there are 36 tapped holes, spaced by 10 degrees, and aligned between the two parts. Additionally, there is a groove that extends along the holder length such that it would be eventually aligned with one of the 36 grooves located on the flange.
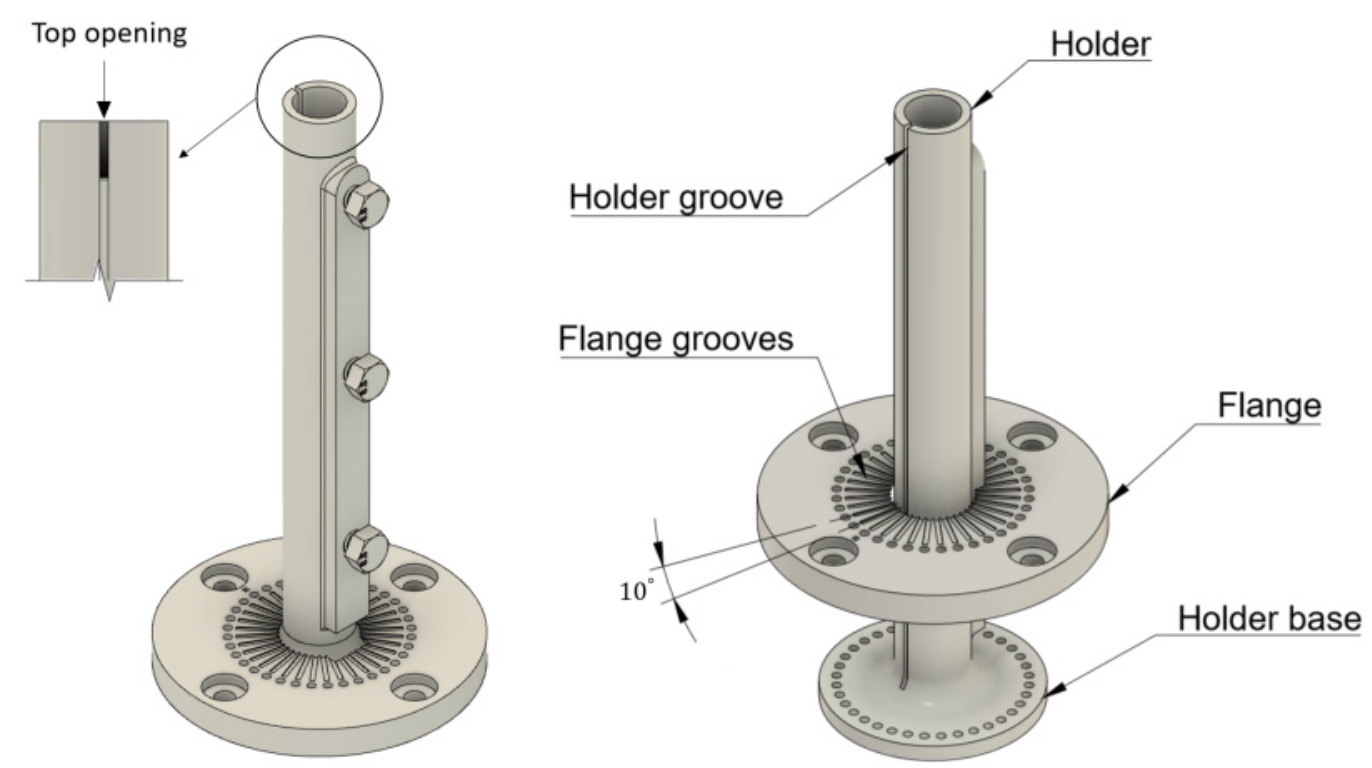

Figure 3.19: CAD model for Cobra probe holder and indexing system.

The reasons of including the grooves and the tapped holes is to create an angular indexing system that would allow for an accurate rotation of the Cobra probe when the flow direction is outside the 45 degrees acceptance cone. Thus, the rotation of the probe tube is controlled by the holder while the flange is mounted to the turntable, and when the desired position is achieved, the holder base is affixed to the flange through the use of screws in some of their aligned tapped holes. Likewise, to ensure that the probe is not rotated during the manual traversing of the tube, the stainlesssteel tubes were marked with a thin, straight line that was aligned with and always kept within the holder groove top opening, shown in Figure 3.19, from the start till the end of each run. 


\section{The Pitot-static tube system}

The Dwyer 160-48 stainless-steel Pitot-static tube is a hollow, open-ended, cylindrical tube with an insertion length of $121.92 \mathrm{~cm}$ (48 in). The Pitot-static tube provides the local dynamic pressure, that is the difference between the local total pressure, $P_{0}$, and the local static pressure, $P_{s}$. As can be seen in Figure 3.20, the tube has an orifice centered on its tip, and in-line with the incoming flow, through which the local total pressure is measured. The local static pressure, on the other hand, is measured from the static holes located on the tube circumference, and perpendicular to the incoming flow. The total pressure hole is isolated from the static pressure holes, and each has its own connection at the end of the tube.

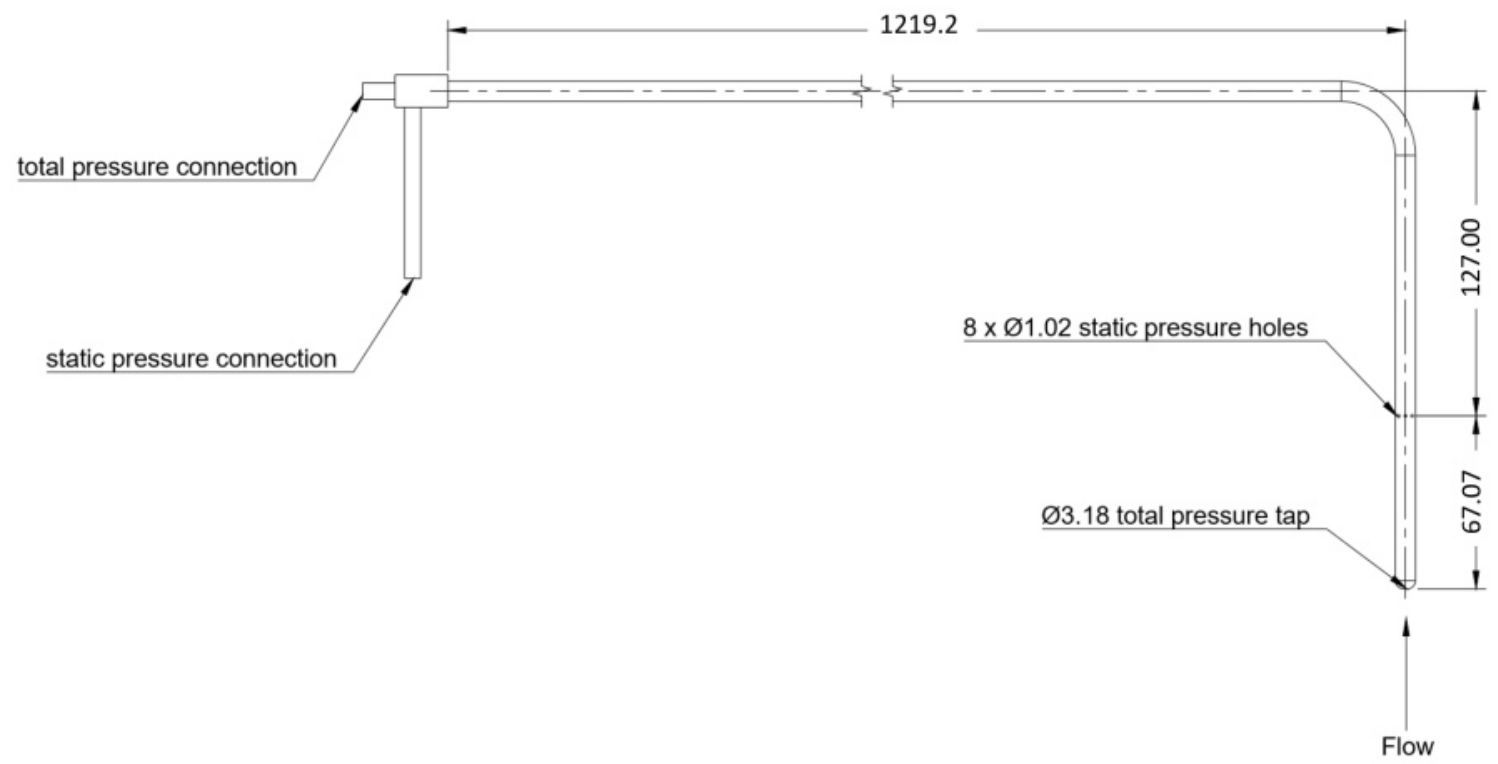

Figure 3.20: Pitot-static tube configuration (dimensions in $\mathrm{mm}$ ).

The total and static pressure connections at the end of the Pitot-static tube were connected to the high and low pressure ports, respectively, of the electronic manometer via Tygon tubing. The electronic manometer measures the difference in pressure in inches of $\mathrm{H}_{2} \mathrm{O}$.

Thus, by the use of the hydrostatic law and Bernoulli's equation, the local flow 
velocity can be determined as follows:

$$
\begin{aligned}
P_{0}-P_{s} & =\Delta P=\rho_{w} g \Delta h \\
U & =\sqrt{\frac{2 \Delta P}{\rho_{a}}}
\end{aligned}
$$

where $g$ is the gravitational acceleration, $\Delta h$ is the reading from the electronic manometer in inches of water, $\rho_{w}$ is the water density, and $\rho_{a}$ is the air density which was determined from the ideal gas law as indicated in Equation (3.12):

$$
\rho_{a}=\frac{P_{a}}{R T}
$$

where $P_{a}$ is the barometric pressure obtained from Ottawa Macdonald-Cartier Int'l Airport, $R$ is the gas constant, and $T$ is the air temperature taken from the room temperature sensor as indicated in Section 3.1.5.2.

For calibrating the electronic manometer, the Pitot-static tube was mounted to the side wall of the wind tunnel by the use of split flange as shown in Figure 3.21 (a) and (b). However, for generating the reference velocity calibration curve and calibrating the Cobra probes, the Pitot-static tube was mounted to the turntable in the downstream test section as seen in Figure 3.21 (c). 


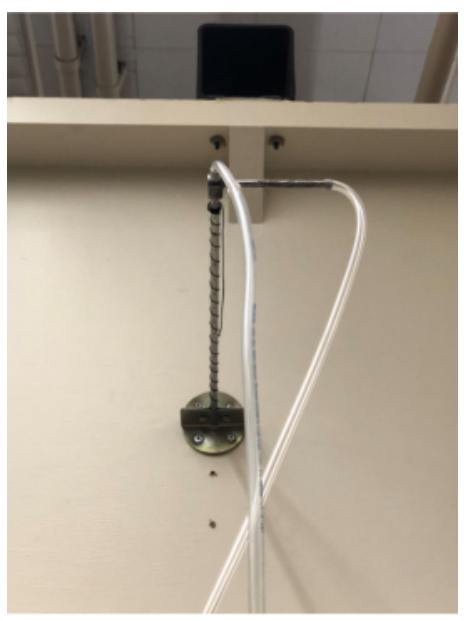

(a)

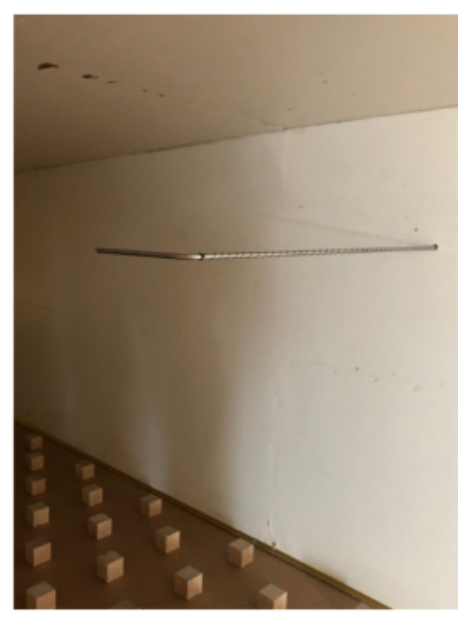

(b)

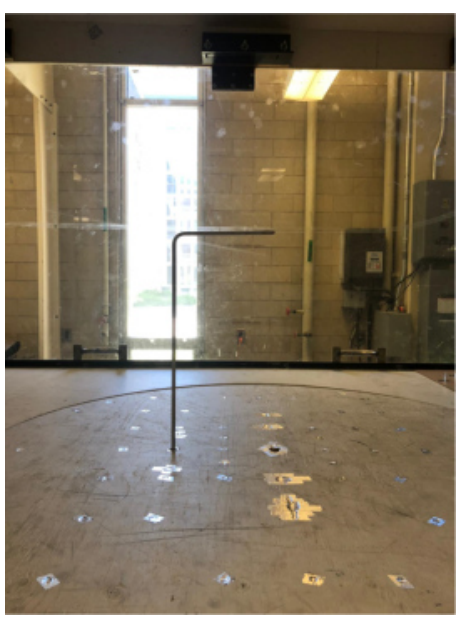

(c)

Figure 3.21: Pitot-static tube mounting arrangements.

\subsubsection{Sensitivity analysis}

All of the sensitivity analyses were done at $10 \mathrm{~m} / \mathrm{s}$, without the model of the city of Toronto, and by the use of one Cobra probe, mounted at the center of the turntable as shown in Figure 3.22 . It is worth noting that both spires and roughness elements were installed in the wind tunnel for all sensitivity check tests.

\section{Sampling time selection}

The sampling time was selected after performing a sensitivity analysis to determine the minimum required sampling time for stable results from the Cobra probe, considering a sampling frequency of $2000 \mathrm{~Hz}$. The analysis was performed at $25.7 \mathrm{~cm}$ above the wind tunnel floor, where measurements were taken from the Cobra probe at 11 different sampling time as indicated in Table 3.3 .

From the Cobra probe measurements, the mean wind speed as well as the rootmean-square (rms) of the velocity were plotted with respect to their corresponding sampling time, as shown in Figure 3.23. From the plot, it can be observed that both the mean and rms values are relatively stable starting from 30.720 seconds. Since the 


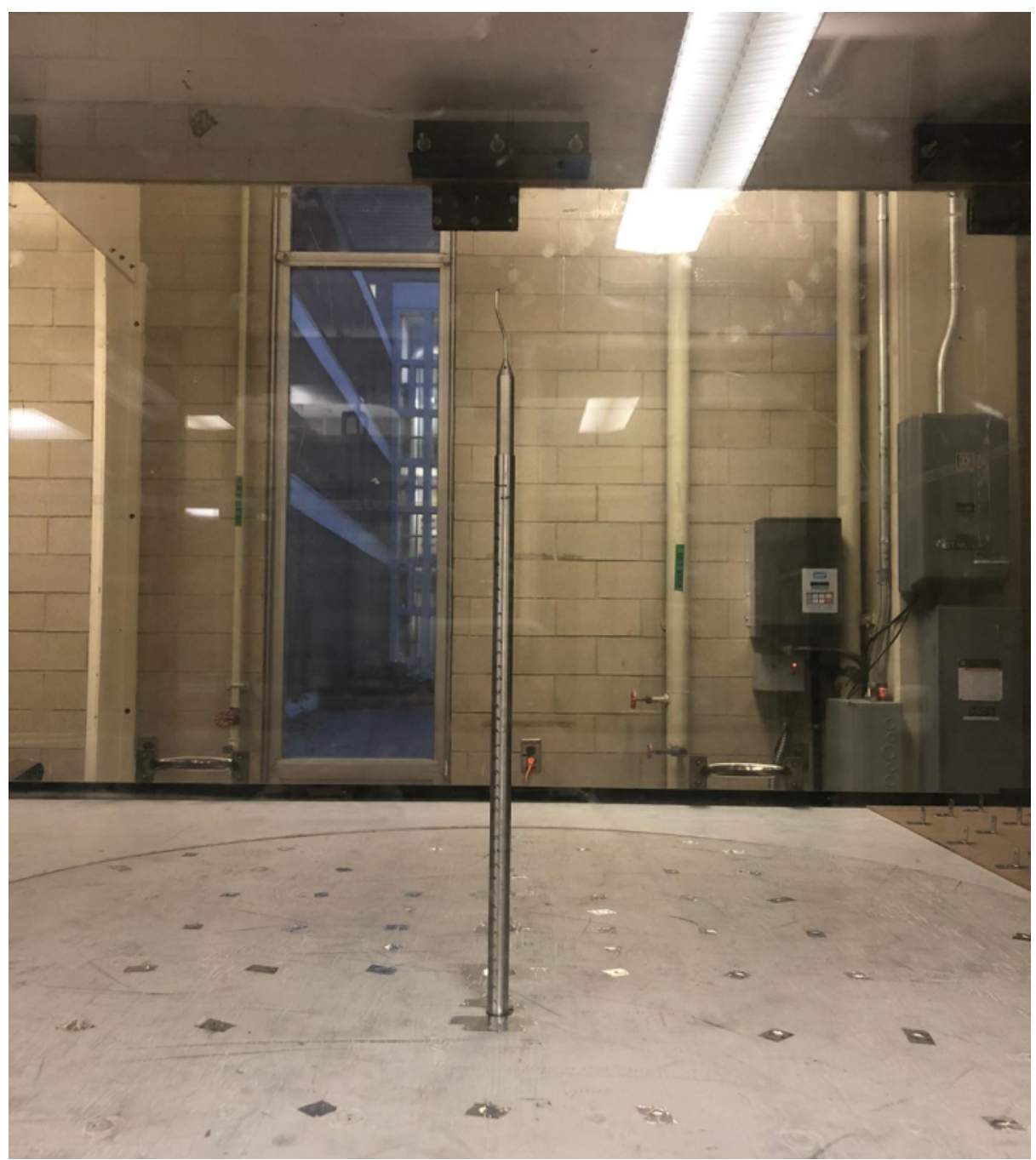

Figure 3.22: Cobra probe mounting arrangement for the sensitivity checks.

amount of data collection is influenced by the sampling duration, $30.720 \mathrm{~s}$ was used for sampling all the experimental data. The selected sampling time deviates less than $0.085 \mathrm{~m} / \mathrm{s}$ from the mean speed values, and less than $0.025 \mathrm{~m} / \mathrm{s}$ from the rms values, measured at higher sampling time. Thus, it was decided to tolerate the relatively small uncertainty to optimize the test time. 
Table 3.3: Sampling time and the corresponding number of samples.

\begin{tabular}{ccc}
\hline \hline Run & Sampling time $[\mathrm{s}]$ & Number of samples \\
\hline 01 & 5.120 & 10,240 \\
02 & 10.240 & 20,480 \\
03 & 15.360 & 30,720 \\
04 & 20.480 & 40,960 \\
05 & 25.600 & 51,200 \\
06 & 30.720 & 61,440 \\
07 & 35.840 & 71,680 \\
08 & 40.960 & 81,920 \\
09 & 45.056 & 90,112 \\
10 & 50.176 & 100,352 \\
11 & 55.296 & 110,592 \\
\hline
\end{tabular}
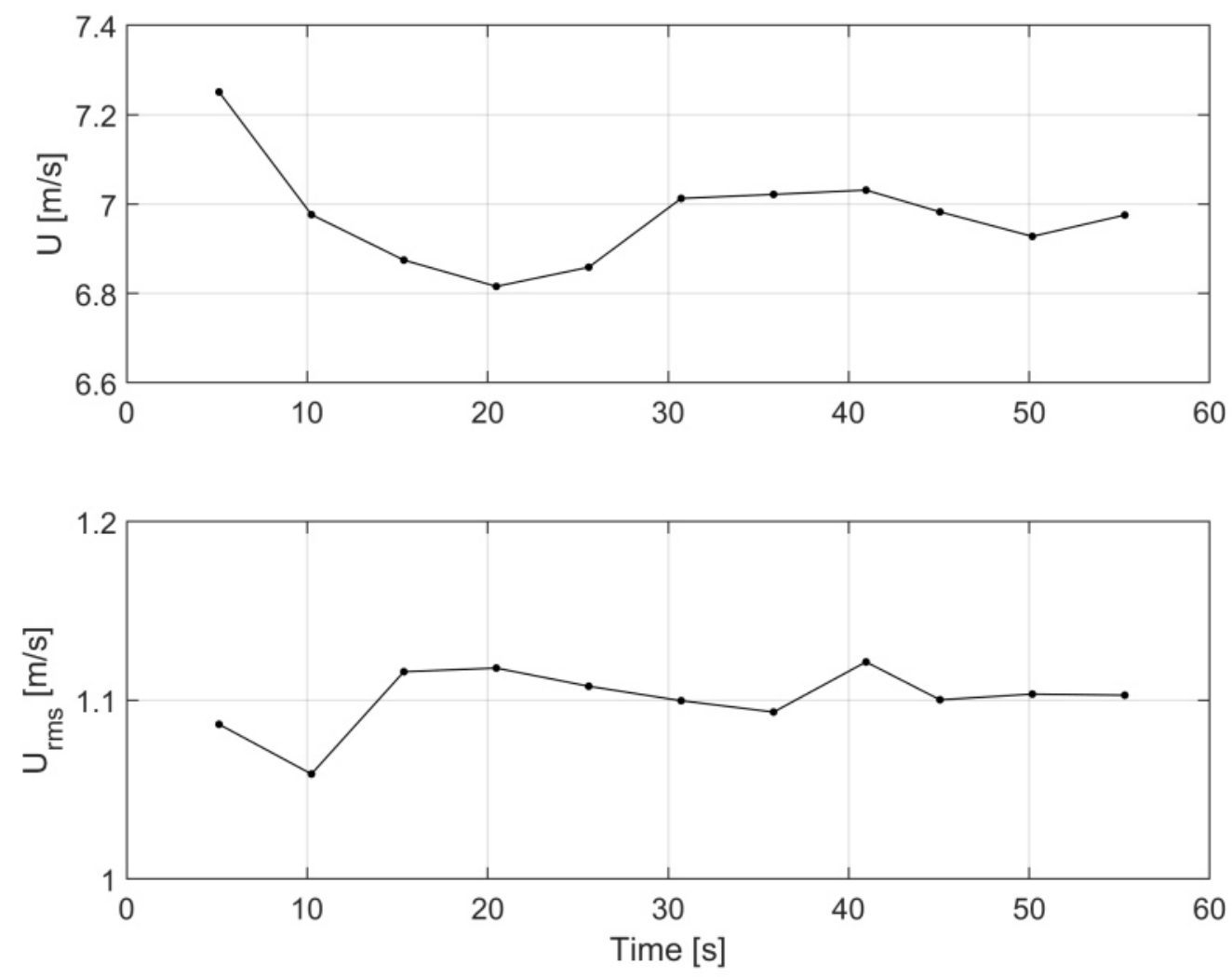

Figure 3.23: Mean wind speed versus sampling time (top) and velocity rms versus sampling time (bottom). 


\section{Effects of temperature and pressure variations on Cobra probe readings}

The TFI software requires a manual input of the temperature and pressure before each sampling. During the experiment, it was observed that the temperature sensor reading could change by $0.1^{\circ} \mathrm{C}$ while the software is sampling the data. Also, since the temperature readings were obtained from a sensor located outside the wind tunnel, it was decided to assess the temperature variations on the probe readings. The pressure readings were taken from Ottawa Macdonald-Cartier Int'l Airport since the elevation difference between the airport location and the testing site is approximately $41 \mathrm{~m}$, which would cause a variation in the wind speed measurements by a maximum of $0.06 \mathrm{~m} / \mathrm{s}$, which is within the uncertainty of the probes. The pressure reading at the airport is posted 15 minutes past the indicated hour, and it could be $\pm 100 \mathrm{~Pa}$ from the preceding value. Therefore, it was also desired to examine the effect of pressure change on the probe readings, and whether or not a correction is needed for the data collected at slightly different pressure value.

Thus, multiple runs were performed with the Cobra probe, which was mounted $64.5 \mathrm{~cm}$ above the wind tunnel floor.

As can be seen in the top plot of Figure 3.24 , the mean wind speed was plotted versus the temperature with an increment of $0.1{ }^{\circ} \mathrm{C}$ between 22 and $22.6{ }^{\circ} \mathrm{C}$, from which the maximum difference between two consecutive points was found to be 0.008 $\mathrm{m} / \mathrm{s}$. This reflects the minimal influence of the $0.1{ }^{\circ} \mathrm{C}$ change on the probe readings. Additionally, the difference in wind speed between 22 and $25^{\circ} \mathrm{C}$ is only 0.047 $\mathrm{m} / \mathrm{s}$, which is within the uncertainty of the probes. Thus, even if the test section temperature was slightly higher or lower than what the nearby sensor is measuring, this should not impact the accuracy of the probe readings. The same applies for the pressure readings, which were plotted with an increment of $100 \mathrm{~Pa}$ against the mean wind speed as shown in the bottom plot of Figure 3.24. The maximum difference 
between two consecutive readings found to be $0.012 \mathrm{~m} / \mathrm{s}$, which is also minimal, and no corrections needed to be made.
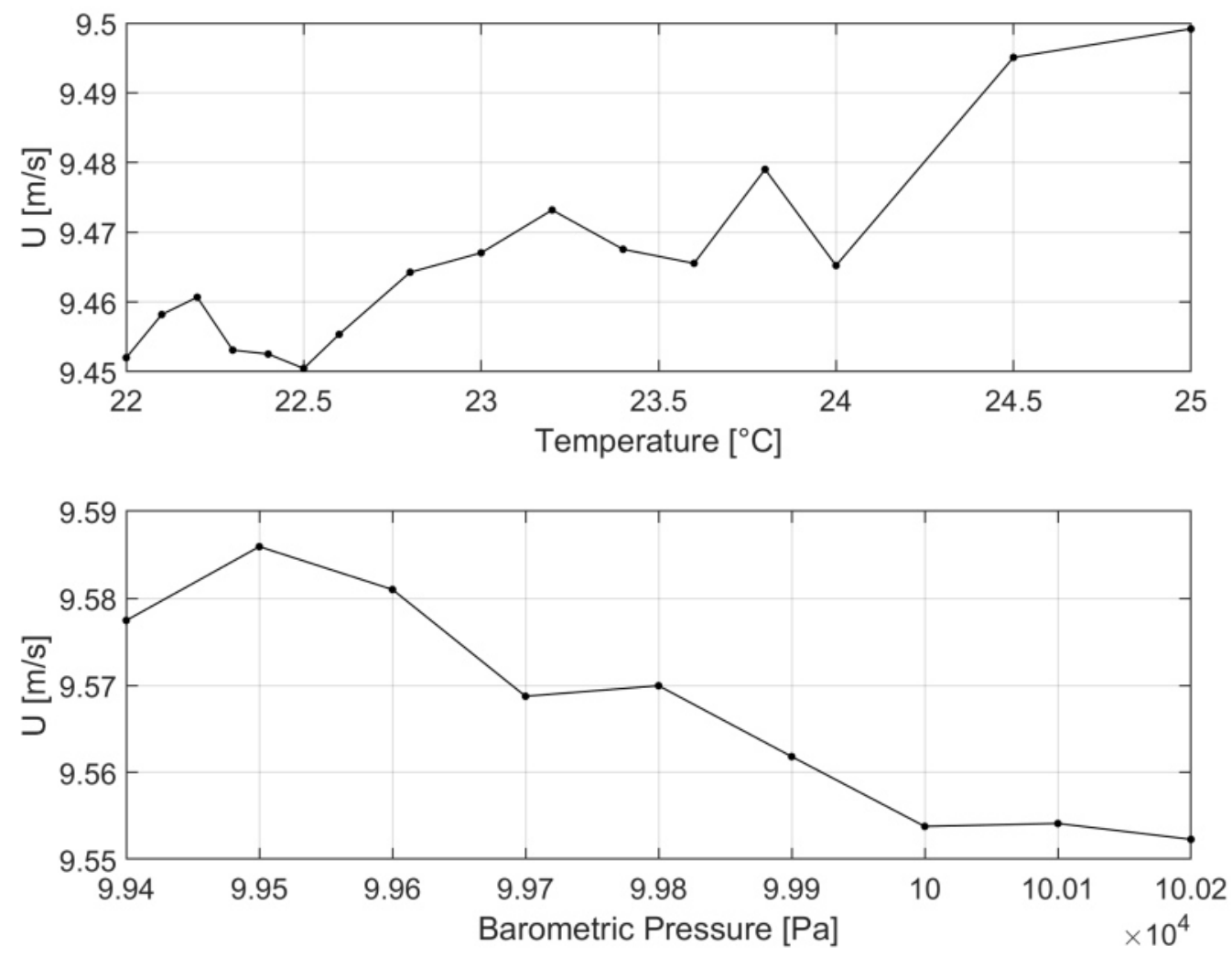

Figure 3.24: Mean wind speed versus temperature (top) and mean wind speed versus pressure (bottom).

\subsubsection{Instruments Calibration}

The instruments calibration was done in partially smooth flow. The spire array was removed from the wind tunnel inlet, whereas the roughness elements were kept in position. The calibration procedure of the electronic manometer and the Cobra probes will be thoroughly discussed in this section, and the instruments height indicated thereafter is always referenced to the wind tunnel floor. 


\section{Electronic manometer calibration}

The Datametrics electronic manometer is calibrated by Alpha Controls \& Instrumentation Inc. on a yearly basis. However, it was found that the equipment calibration is one year past due at the time of the experiment, and thus, it was necessary to ensure that the manometer is operating accurately before starting the data collection. Therefore, an inclined liquid manometer was utilized to verify the differential pressure measurements indicated by the electronic manometer.

The calibration process involved a series of tests, performed at various wind speeds, in which both the liquid and electronic manometers were connected to a Pitot-static tube in an alternating manner while the wind tunnel fan was running continuously. The Pitot-static tube was mounted $68 \mathrm{~cm}$ away from wind tunnel inner side wall, $90.5 \mathrm{~cm}$ above the wind tunnel floor, and approximately $150 \mathrm{~cm}$ from the turntable leading edge.

Thus, readings from both instruments were collected for a total of seven runs, in which the wind speed was increased by approximately $0.3 \mathrm{~m} / \mathrm{s}$ from run 2 to run 4 , and decreased by the same amount at each run thereafter. The velocity was determined from the pressure difference in inches of water measured from both instruments, and the results are presented in Table 3.4 .

The velocities obtained from the measurements of both instruments indicated a maximum deviation of less than $0.2 \mathrm{~m} / \mathrm{s}$, which suggests that the readings from the electronic manometer were accurate despite the past due calibration.

\section{Cobra probes calibration}

Each Cobra probe comes fully calibrated by the manufacturer. The calibration is done for the probe static response, dynamic response, and head geometry. According to the manufacturer, occasional static calibration check is recommend, and therefore, it was decided to compare the mean wind speeds measured by each of the Cobra 
Table 3.4: Electronic manometer calibration results.

\begin{tabular}{cccc}
\hline \hline \multirow{2}{*}{ Run } & Electronic manometer & Liquid manometer & Difference \\
\cline { 2 - 4 } & $\mathrm{U}[\mathrm{m} / \mathrm{s}]$ & $\mathrm{U}[\mathrm{m} / \mathrm{s}]$ & $|\Delta \mathrm{U}|[\mathrm{m} / \mathrm{s}]$ \\
\hline 1 & 8.810 & 8.648 & 0.162 \\
2 & 9.145 & 9.117 & 0.027 \\
3 & 9.437 & 9.562 & 0.125 \\
4 & 9.898 & 9.987 & 0.090 \\
5 & 9.412 & 9.560 & 0.148 \\
6 & 9.216 & 9.229 & 0.013 \\
7 & 8.802 & 8.838 & 0.035 \\
\hline
\end{tabular}

probes with readings from the Pitot-static tube system, which served as a standard. The calibration process, described below, was applied separately for each of the Cobra probes.

The Cobra probe and the Pitot-static tube were both mounted to the turntable at the downstream test section. The instruments were mounted such that their tips will measure the flow at the same location by only changing their elevations and/or rotating the Pitot-static tube. This was possible owing to the Pitot-static tube geometry, with the tube total pressure tap being located $19.41 \mathrm{~cm}$ away from its stem. The mounting of the instruments is illustrated schematically in Figure 3.25

The calibration of each of the Cobra probes, namely probe ${ }_{1}$ and probe $_{2}$, was done at two different heights while the fan was continuously running. The calibration results are presented in Tables 3.5 and 3.6

The Calibration results of both probes shows a deviation of less than $0.3 \mathrm{~m} / \mathrm{s}$, which is within the manufacturer specified tolerance of $\pm 0.5 \mathrm{~m} / \mathrm{s}$.

The test plan of the scaled model experiment involved the use of both Cobra probes, at the same time, for the data collection. Therefore, another check was done to examine the difference between the Cobra probes velocity measurements, 


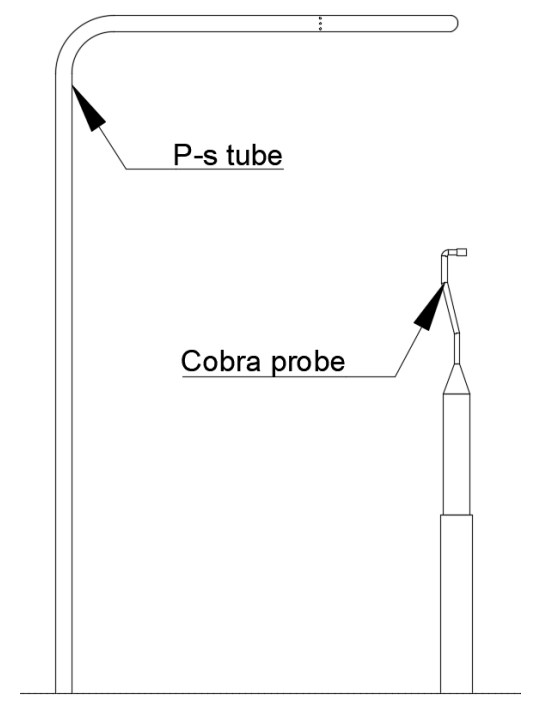

(a)

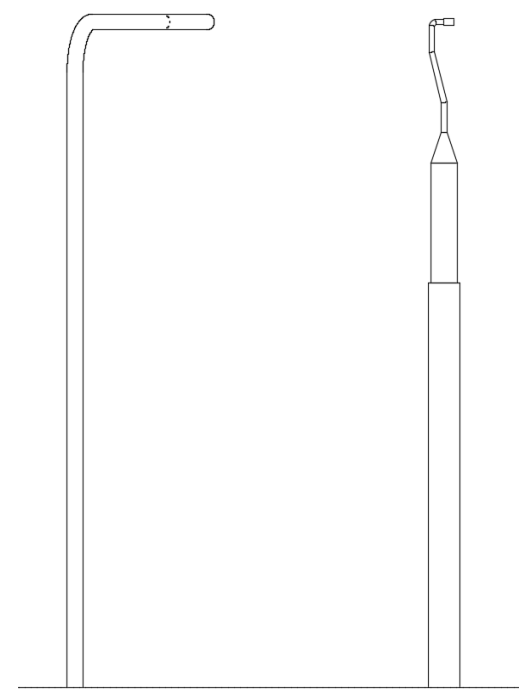

(b)

Figure 3.25: Instruments mounting arrangements for the calibration procedure.

Table 3.5: Cobra probe $_{1}$ calibration results.

\begin{tabular}{cccc}
\hline \hline \multirow{2}{*}{ Height $[\mathrm{m}]$} & Probe $_{1}$ & P-s tube system & Difference \\
\cline { 2 - 4 } & $\mathrm{U}[\mathrm{m} / \mathrm{s}]$ & $\mathrm{U}[\mathrm{m} / \mathrm{s}]$ & $|\Delta \mathrm{U}|[\mathrm{m} / \mathrm{s}]$ \\
\hline 0.343 & 9.542 & 9.423 & 0.289 \\
0.903 & 9.926 & 9.637 & 0.119 \\
\hline
\end{tabular}

in which both probes were mounted side-by-side with a lateral distance of $0.10 \mathrm{~m}$. Measurements from both probes were collected at two elevations, and the run was repeated by swapping the location of the probes to ensure that the difference in the probes readings is not attributed to the mounting location of each instrument.

The results from Table 3.7 show that probe $_{1}$ always recorded higher values of mean wind speed as compared to probe $_{2}$. However, the difference in the measured velocities was smaller in the second run, which suggests that the probe location must have an influence on the results. Nonetheless, the difference between the readings from the probes at both runs was within the manufacturer tolerance of $\pm 0.5 \mathrm{~m} / \mathrm{s}$, and thus, the instruments readings were trusted. 
Table 3.6: Cobra probe 2 calibration results.

\begin{tabular}{cccc}
\hline \hline \multirow{2}{*}{ Height $[\mathrm{m}]$} & Probe $_{2}$ & P-s tube system & Difference \\
\cline { 2 - 4 } & $\mathrm{U}[\mathrm{m} / \mathrm{s}]$ & $\mathrm{U}[\mathrm{m} / \mathrm{s}]$ & $|\Delta \mathrm{U}|[\mathrm{m} / \mathrm{s}]$ \\
\hline 0.343 & 9.323 & 9.466 & 0.143 \\
0.903 & 9.709 & 9.666 & 0.043 \\
\hline
\end{tabular}

Table 3.7: Cobra probes readings check in a side-by-side arrangement.

\begin{tabular}{ccccc}
\hline \hline \multirow{2}{*}{ Run } & Height $[\mathrm{m}]$ & Probe $_{1}$ & Probe $_{2}$ & Difference \\
\cline { 3 - 5 } & & $\mathrm{U}[\mathrm{m} / \mathrm{s}]$ & $\mathrm{U}[\mathrm{m} / \mathrm{s}]$ & $|\Delta \mathrm{U}|[\mathrm{m} / \mathrm{s}]$ \\
\hline \multirow{2}{*}{1} & 0.343 & 9.579 & 9.341 & 0.238 \\
& 0.903 & 9.957 & 9.657 & 0.3 \\
\hline \multirow{2}{*}{2} & 0.343 & 9.516 & 9.410 & 0.106 \\
& 0.903 & 9.871 & 9.805 & 0.066 \\
\hline
\end{tabular}

\subsubsection{Experimental procedures}

This section discusses the processes of the data collection from the wind tunnel experiment before and after the model installation.

\subsubsection{Empty wind tunnel test}

Prior to model installation in the wind tunnel test section, multiple measurements were taken at the empty wind tunnel to examine the lateral uniformity of the flow and to develop a calibration curve from which the reference wind speed can be determined. The details of both procedures will be explained herein below. 


\section{Test section flow uniformity}

Checking the lateral uniformity across the test section was an essential step before taking any measurements from the physical model, which covers the entire turntable of the wind tunnel. This was necessary since the collected data from various spots at the model need to be compared with each other as part of the data analysis. Therefore, vertical velocity and turbulence intensity profiles were measured by the Cobra probe, at three different spots, namely, middle (M), left (L), and right (R) as seen in Figure 3.26. The left and right were separated laterally from the turntable center $(\mathrm{M})$ by $0.25 \mathrm{~m}$, and the height of the vertical points, referenced to the wind tunnel floor, is identified in Table 3.8. The test section flow uniformity results will be discussed in Section 4.1 .
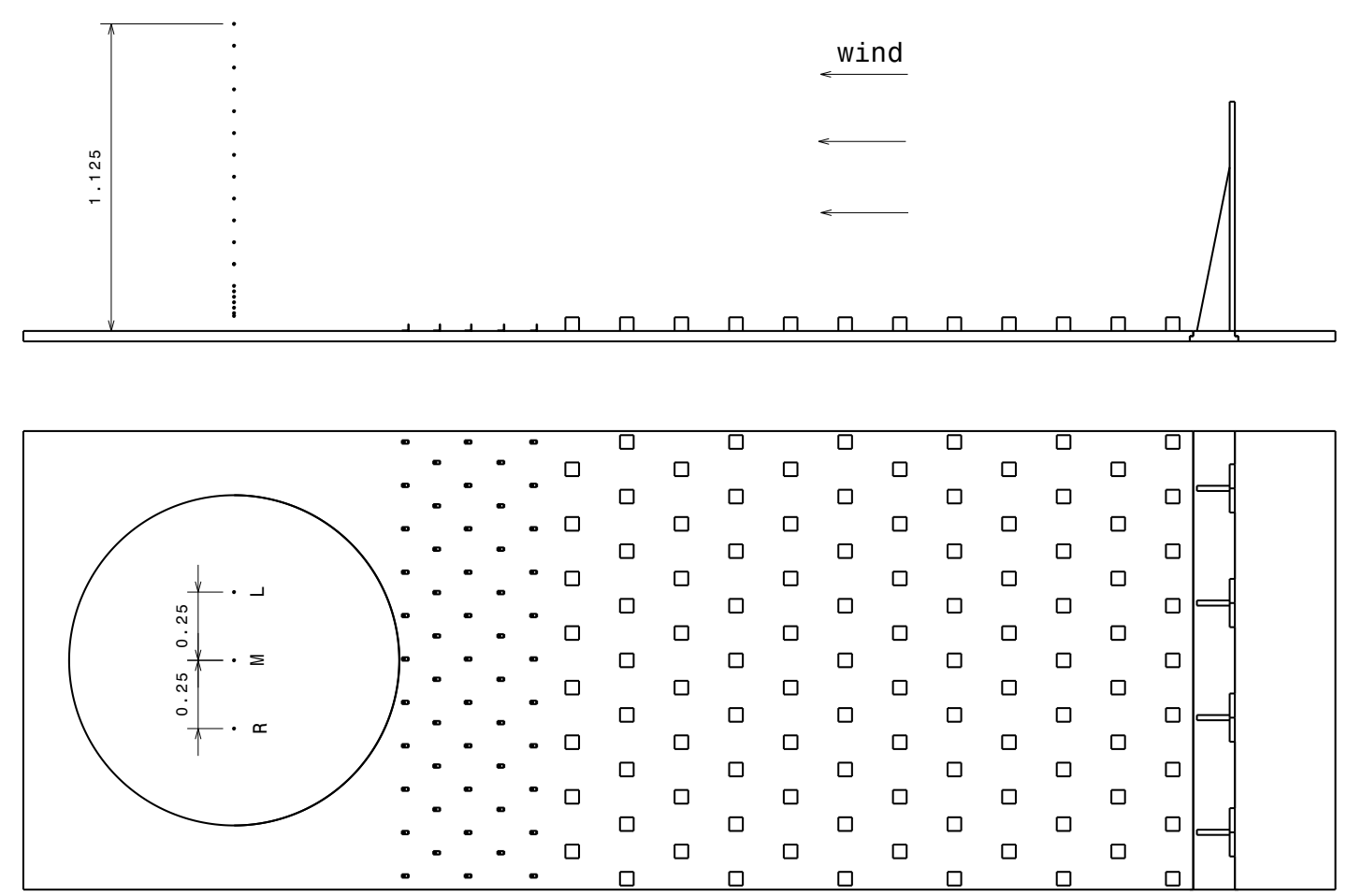

Figure 3.26: Side and top views of measurement locations for empty wind tunnel test vertical profiles (dimensions in $\mathrm{m}$ ). 
Table 3.8: Vertical traverse points for CU empty wind tunnel measurements.

\begin{tabular}{cc}
\hline \hline Increment & Height $[\mathrm{m}]$ \\
\hline 01 & 0.055 \\
02 & 0.065 \\
03 & 0.085 \\
04 & 0.105 \\
05 & 0.125 \\
06 & 0.145 \\
07 & 0.165 \\
08 & 0.245 \\
09 & 0.325 \\
10 & 0.405 \\
11 & 0.485 \\
12 & 0.565 \\
13 & 0.645 \\
14 & 0.725 \\
15 & 0.805 \\
16 & 0.885 \\
17 & 0.965 \\
18 & 1.045 \\
19 & 1.125 \\
\hline
\end{tabular}




\section{Reference velocity calibration curve development}

The speed of the wind tunnel is set by the use of toggle switch that accepts user input of either increasing or decreasing the wind tunnel fan speed. Since the wind tunnel experiment was done at several runs and phases, slight variations on the speed of the wind tunnel was expected. Therefore, to ensure an appropriate comparison between the collected data from different runs, it was decided to normalize the data by a reference wind speed that would be determined from a relationship to the pressure drop $(\mathrm{dP})$ across the contraction of the wind tunnel. The pressure drop was recorded from the wind tunnel computer, which is connected to a pressure transducer and sensitive manometer via ADC.

To generate the calibration curve, the Pitot-static tube was mounted to the center of the turntable at a height of $0.903 \mathrm{~m}$ above the floor of the test section. The Pitot-static tube was connected to the electronic manometer, and data were collected at range of wind speeds. The pressure drop was manually recorded while the TFI software was sampling the data from the P-s system at each wind speed. The reference velocities $\left(\mathrm{U}_{r e f}\right)$ were calculated from the pressure difference indicated by the manometer, and were plotted afterwards against the pressure drop which was measured in inches of $\mathrm{H}_{2} \mathrm{O}$. The developed calibration curve is shown in Figure 3.27 . 


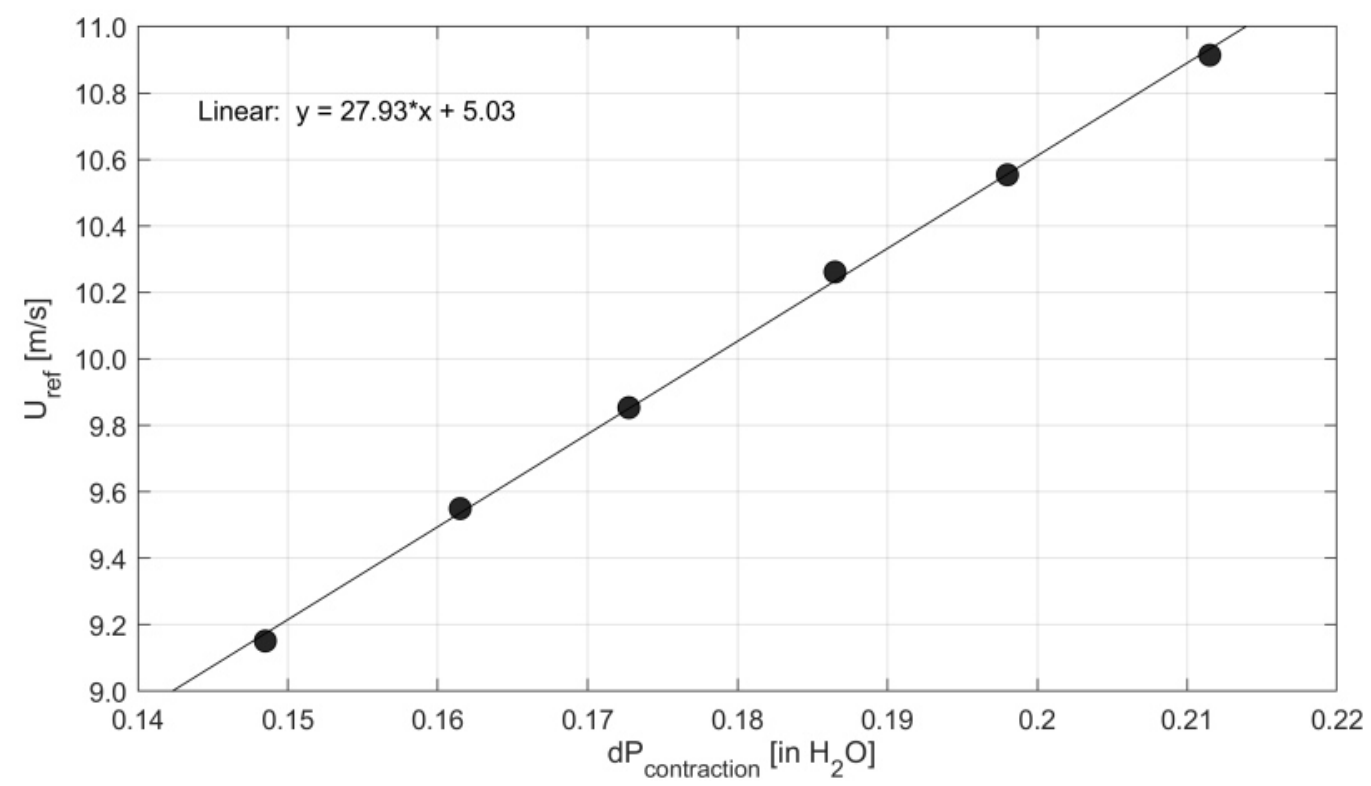

Figure 3.27: Reference velocity calibration curve.

\subsubsection{Scaled model test}

The scaled model experiment was done in two phases, the first involved a visualization of the flow at various wind directions and the second involved the data collection with the Cobra probes. More details will be provided in the reminder of this section.

\section{Flow visualization experiment}

The flow visualization experiment was conducted with the use of tufts, prior to the data collection from the model. Tufts are short pieces of string fastened at one end to an aerodynamic surface, and are used to identify regions of flow separation, high level of turbulence, up-draft, down-draft, as well as severe flow yaw angles 68. Since the measurement locations, identified in Section 3.1.4, are generic for all of the wind directions planned for the test, the flow visualization experiment was intended to select certain spots for each of the wind directions identified previously in Table 3.2. Therefore, short pieces of strings were attached to two streamlined tubes, each with a chord length of $\frac{3}{8}$ in and a total length of 35 in as shown in Figure 3.28 . One 
end of the streamlined tube was connected to a circular rod so that the same probe holder system can be utilized for mounting the tuft tubes to the turntable.

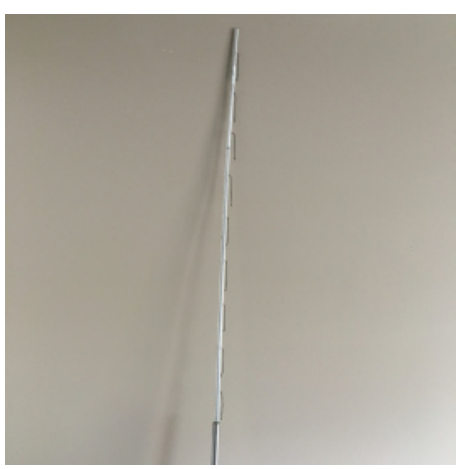

(a)

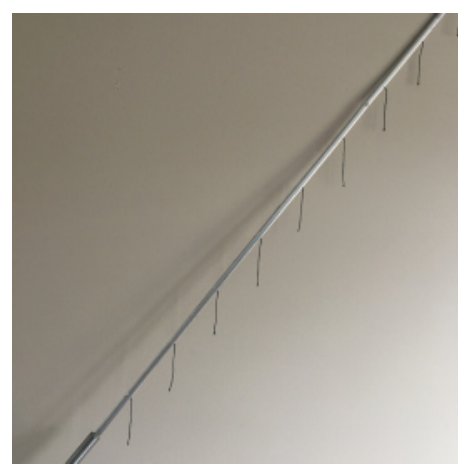

(b)

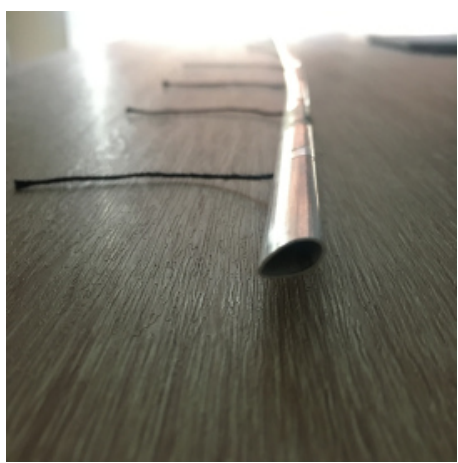

(c)

Figure 3.28: Tuft rod used for the flow visualization experiment.

The model was installed at the test section according to the five selected wind directions. For each of the model orientations to the incoming flow, the tuft rods were inserted at all measurement locations to examine the behaviour of the flow, visually, and consequently decide if a certain spot is a good candidate for the Cobra probe measurements.

Figure 3.29 shows the tufts behaviour at two different flow conditions. The tufts location in case (a) was open to the approach flow with no upstream buildings obstruction. In that case, the tufts were moving in a relatively smooth manner since their movement was solely derived from the approach flow. The tufts behaviour in cases (b) and (c) was indicating heavily turbulent flow conditions since the tufts location was downstream of tall buildings, and such spots were of great interest for further investigation with the Cobra probes. 


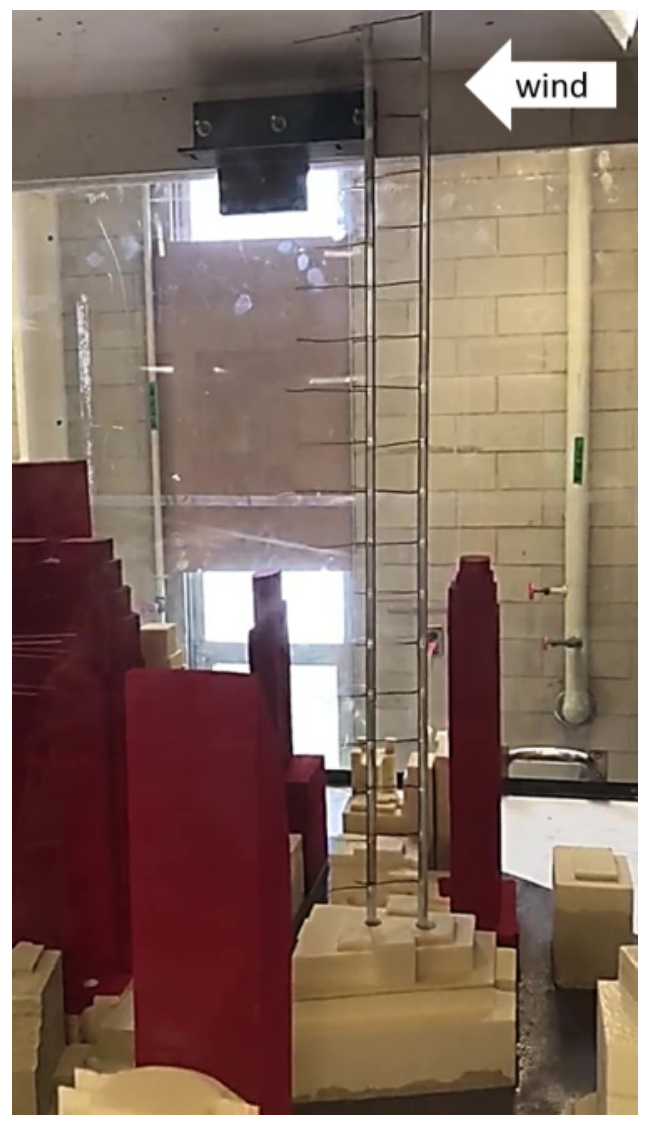

(a) Smooth flow

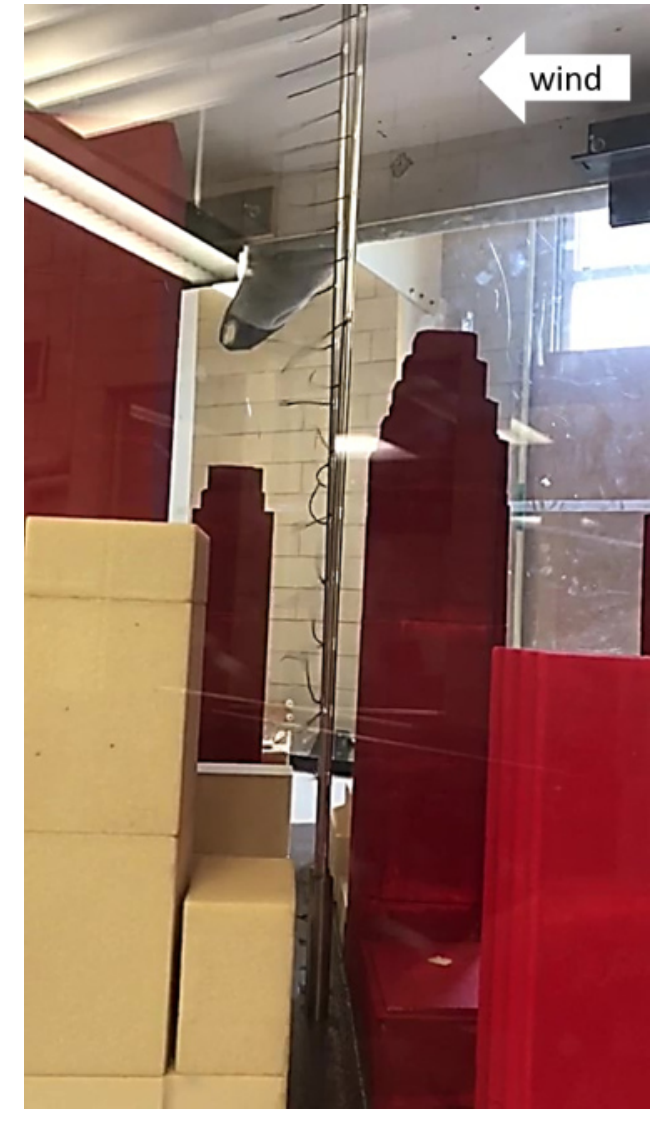

(b) Turbulent flow

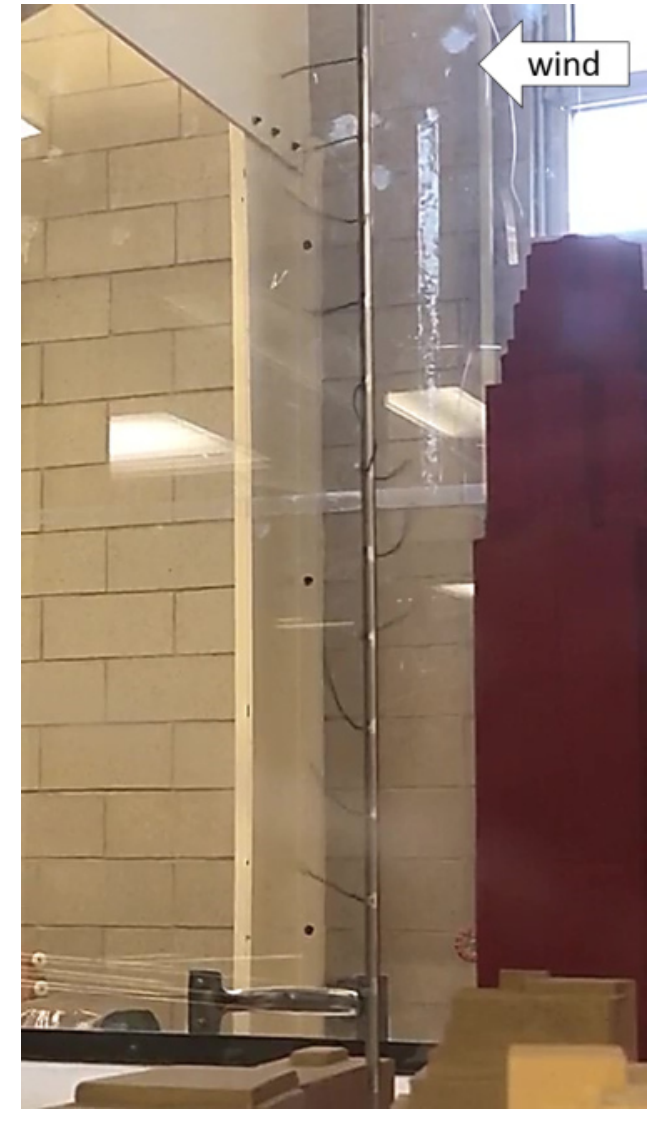

(c) Turbulent flow

Figure 3.29: Smooth and turbulent flows observation from the flow visualization experiment. 
Some of the flow features were also observed from the tufts behaviour as shown in Figure 3.30, where up- and down-drafts along with a change in the flow lateral direction were found upstream of a tall building as shown in case (a) and some flow reversals were observed immediately downstream of a tall building as shown in case (b).

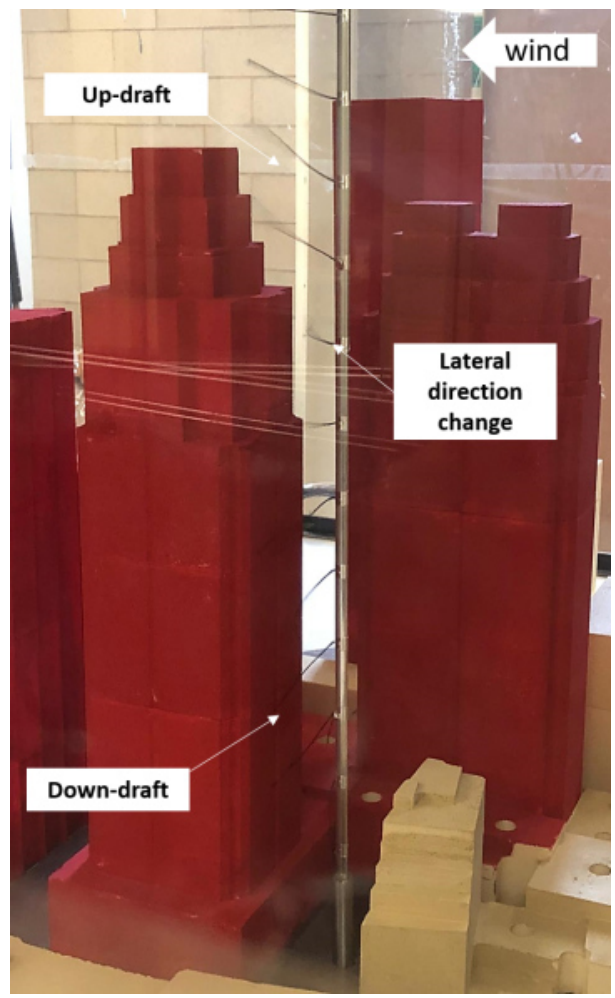

(a)

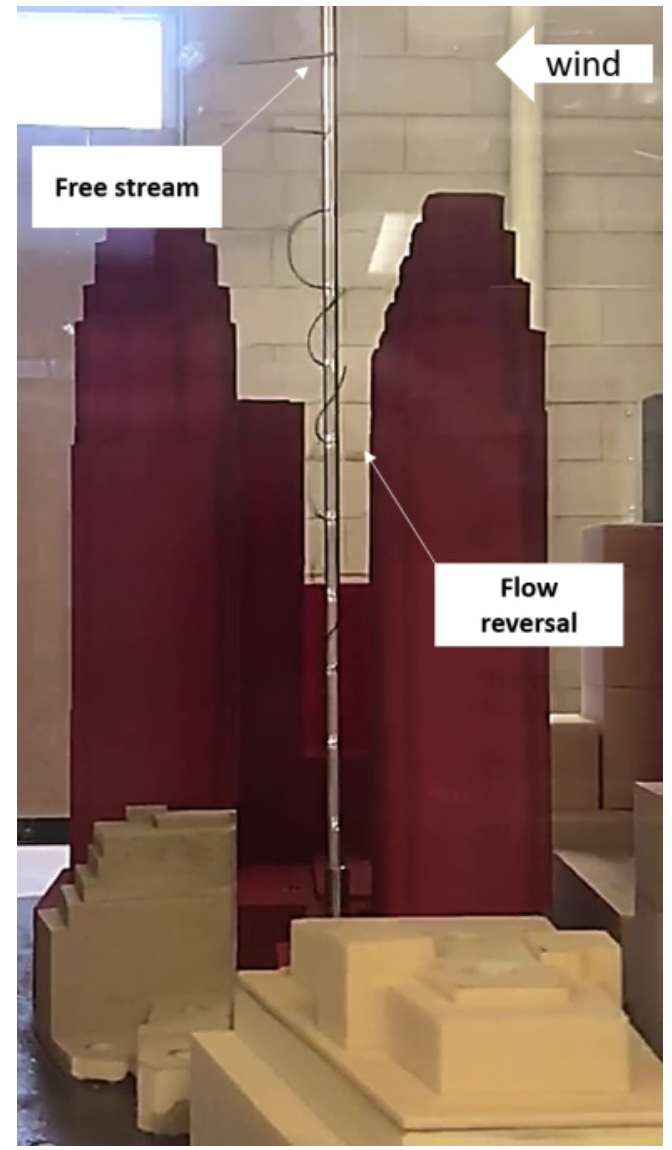

(b)

Figure 3.30: Observation of certain flow features from the flow visualization experiment. 


\section{Cobra probe measurements}

The scaled city model was tested at five different wind directions, in which measurements were taken by the Cobra probes at the selected locations from the flow visualization experiment. The physical model contains multiple holes for mounting the probes, two of which were utilized at each run, and the reminder were covered with an aluminum tape to avoid flow leakage from the test section. Additionally, the future buildings were only included in the model for the $\mathrm{S}_{15}$ wind direction.

The data collection was done in two phases. The first phase included measurements from the WSW, WSW +15 , and $\mathrm{E}_{17.1}$ wind directions at the red spots indicated in Figure 3.31. The second phase included measurements from the $\mathrm{N}_{16.7}$ and $\mathrm{S}_{15}$ wind directions. The future buildings were glued to the base of the model after collecting the data from all the five wind directions including the $\mathrm{S}_{15}$. The measurement locations chosen for the second phase are coloured also in red and shown in Figure 3.32 . Nomenclature for measuring spots was previously indicated in Figure 3.13. 


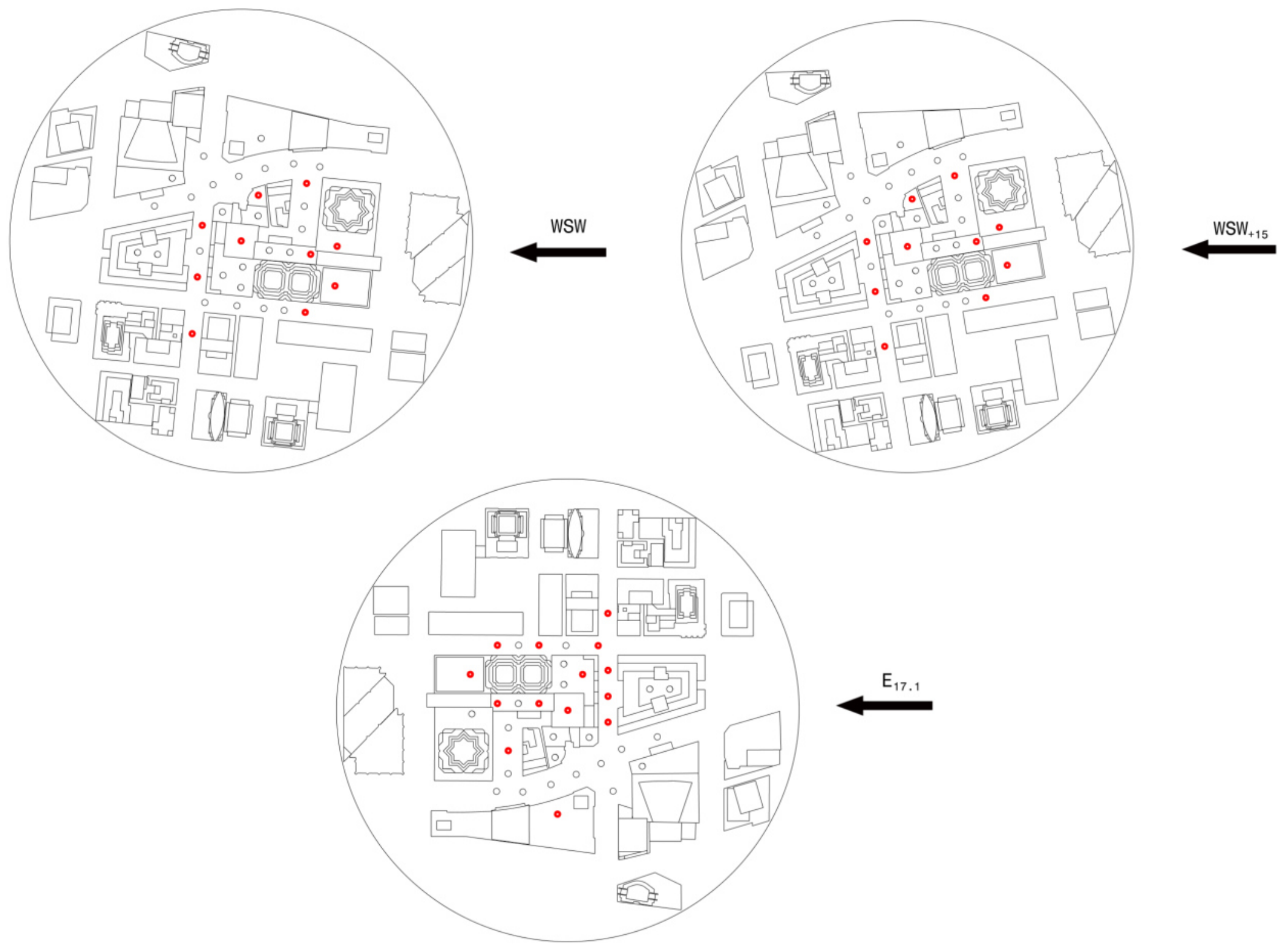

Figure 3.31: Measurement locations for $\mathrm{WSW} \mathrm{WSW}_{+15}$, and $\mathrm{E}_{17.1}$ wind directions. 

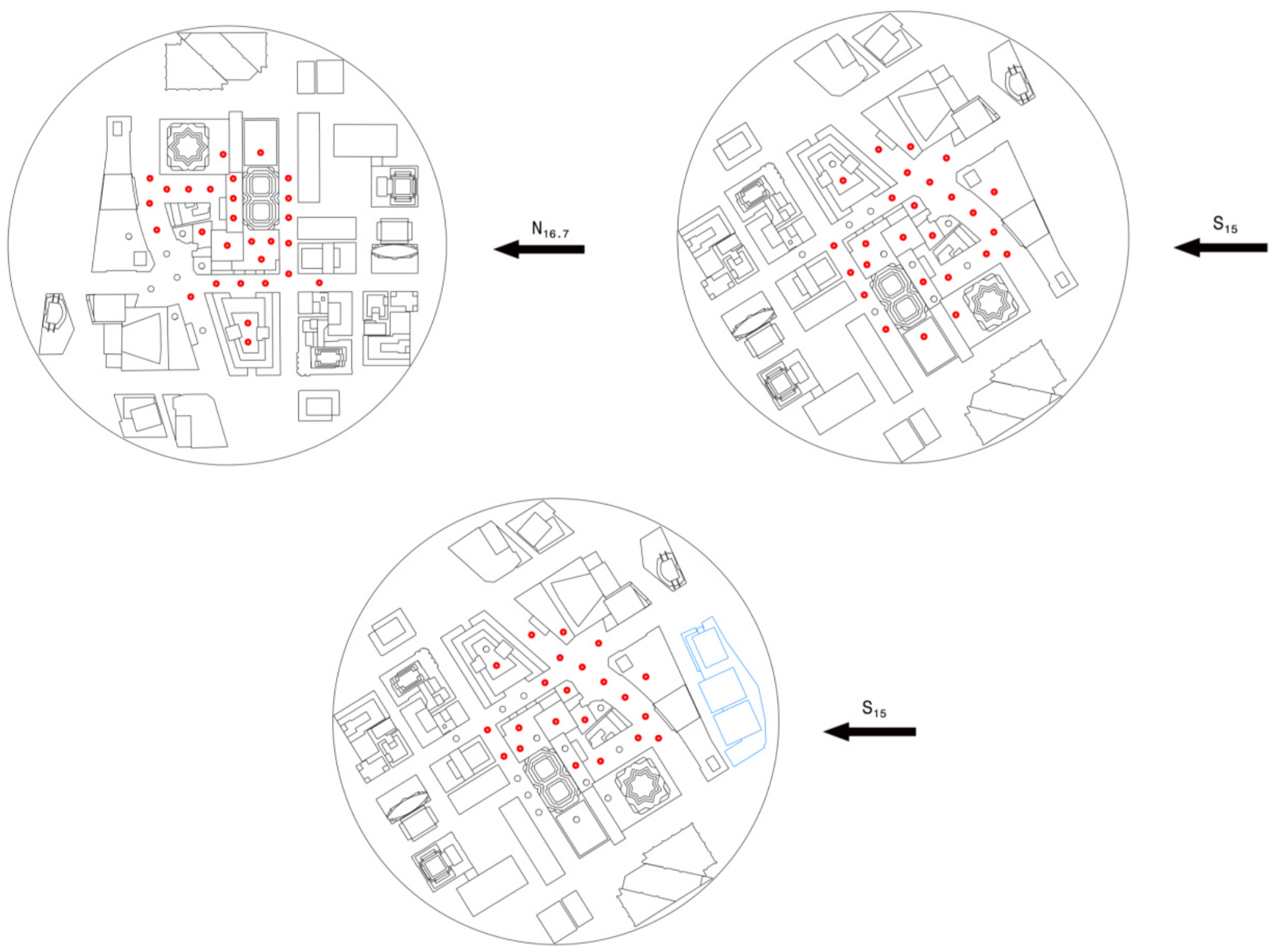

Figure 3.32: Measurement locations for $\mathrm{N}_{16.7}$ and $\mathrm{S}_{15}$ wind directions. 
At each wind direction, the data was collected at several runs, each involved measurements of vertical profiles at two different spots by manually traversing two Cobra probes along the height of the test section. At each elevation of the probes, the pressure drop across the contraction of the wind tunnel was also recorded while the TFI software was sampling the data. The Cobra probes arrangement for data collection from the physical model is shown in Figure 3.33 .

The TFI software can only resolve flow features for flow direction that resides within the probes acceptance cone of $\pm 45^{\circ}$, and thus, when the flow direction exceeds the probes limit, the flow information are registered as zero in the time history files. The ratio between the number of zero values and the number of samples is utilized by the software to calculate the percentage of captured data, which is referred to as \% Good Data (\% GD). During the experiment, the TFI software live screen was monitored before each data sampling to ensure that at least $95 \%$ of the data were captured by the probes. The 95\% GD was selected as the limiting case, below which the probes had to be rotated to maximize the captured flow information. The amount of rotation was accurately known, and recorded, from the indexing system of the probes holders.

The vertical traverse for the data collected at the first phase included 15 increments, the highest of which was $0.723 \mathrm{~m}$ above the wind tunnel floor. However, the vertical traverse for data collected at the second phase included 20 increments, the highest of which was $1.023 \mathrm{~m}$ above the floor of the wind tunnel. The general vertical traverse points are shown with respect to the height of the buildings in Figure 3.34 . and the exact values of all measuring locations are indicated in Table 3.9. It is worth noting that some of the measuring locations were located above buildings, and that caused some variations on the starting point of the traverse depending on the height of the building. The designations of the measuring spots were previously defined in Figure 3.13 . 

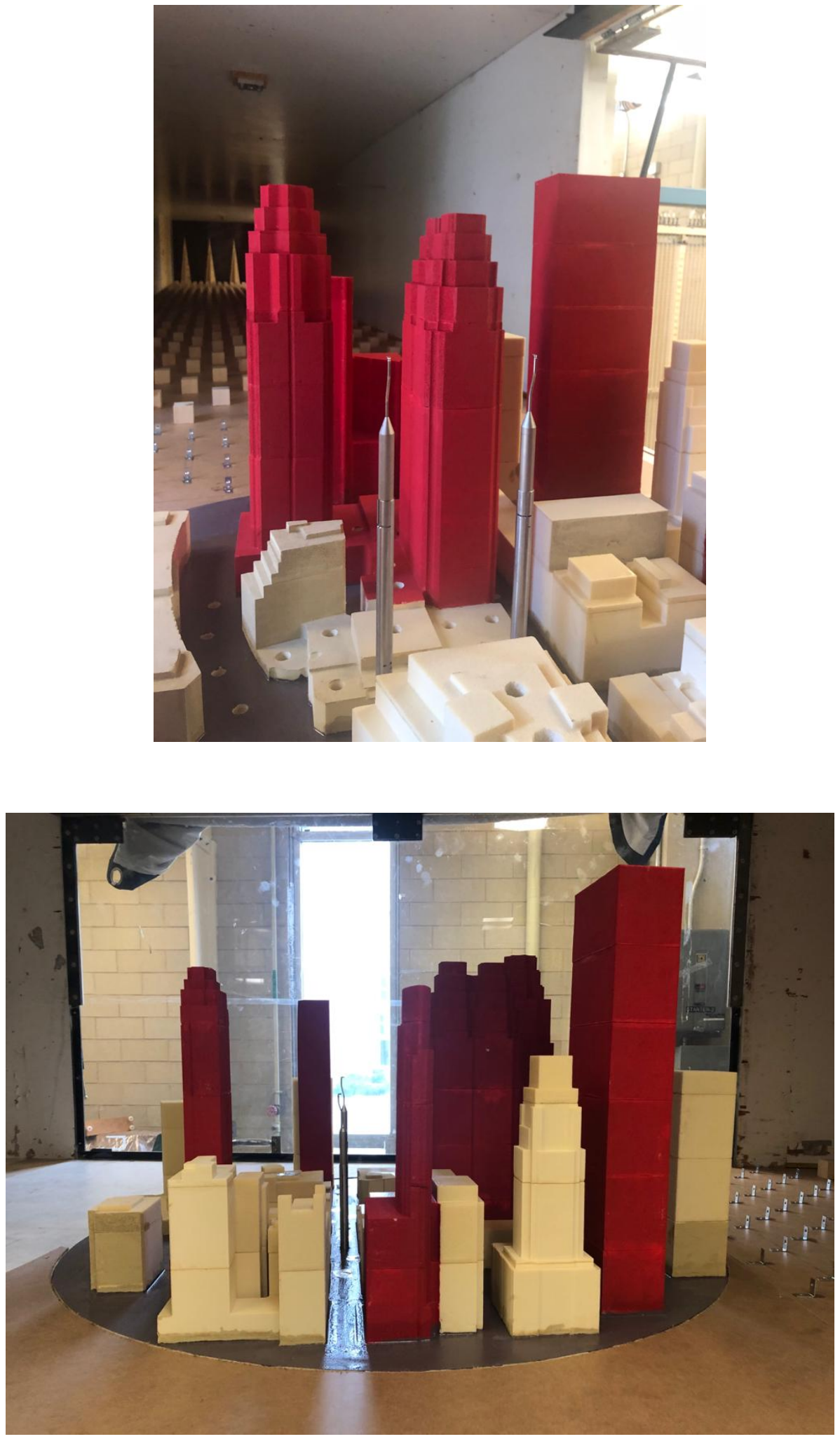

Figure 3.33: Cobra probes setup for the scaled model experiment. 


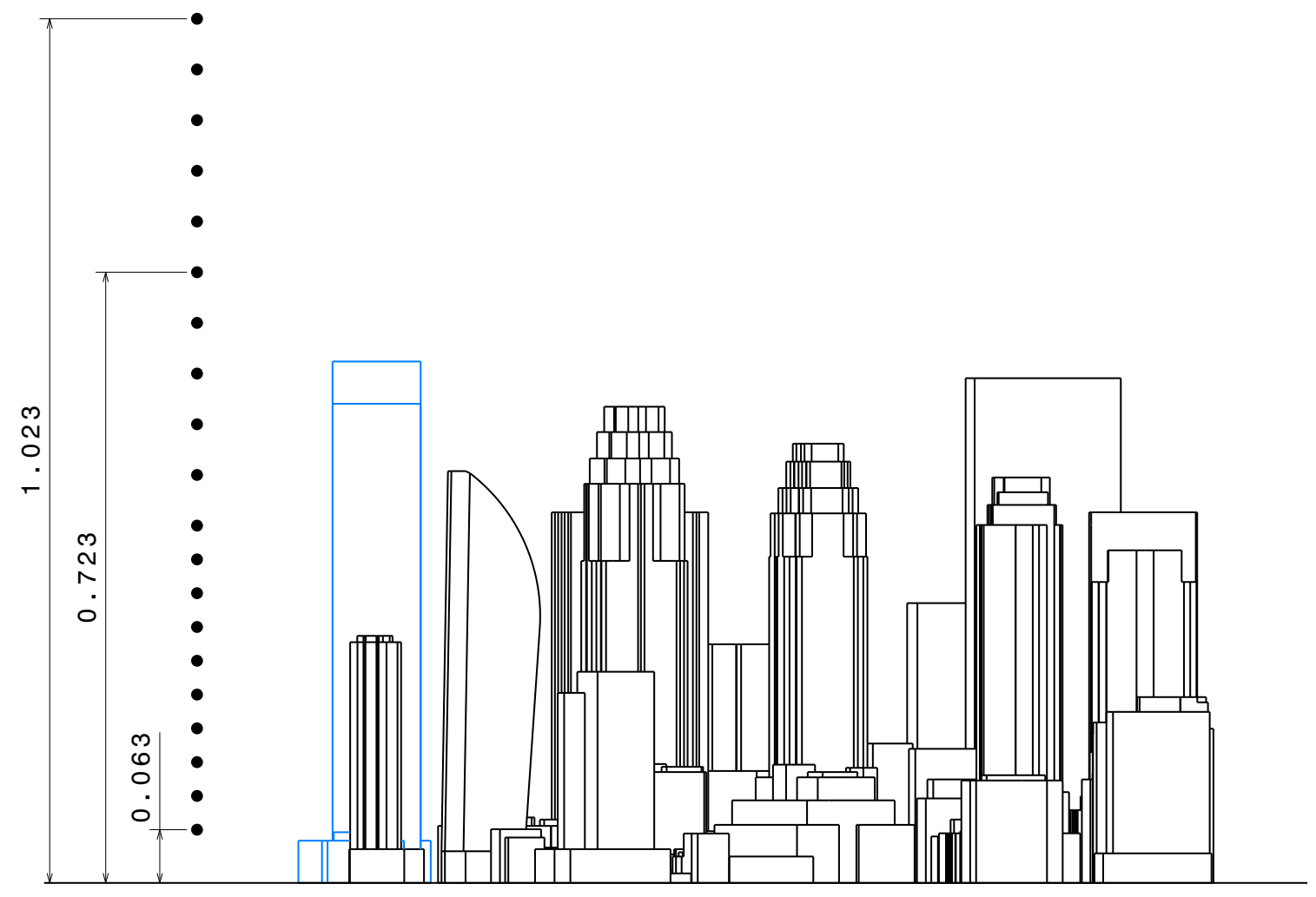

Figure 3.34: Locations of vertical traverse points for the 1:400 model city experiment (dimensions in $\mathrm{m}$ ). 
Table 3.9: Vertical traverse points for the 1:400 model city experiment.

\begin{tabular}{|c|c|c|c|}
\hline \multirow{3}{*}{ Increment } & \multicolumn{3}{|c|}{ Spots } \\
\hline & $(19,20,21,22,36,28)$ & $(1 \& 2)$ & Others \\
\hline & \multicolumn{3}{|c|}{ Height $[\mathrm{m}]$} \\
\hline 01 & 0.073 & - & 0.063 \\
\hline 02 & 0.103 & - & 0.103 \\
\hline 03 & 0.143 & 0.143 & 0.143 \\
\hline 04 & 0.183 & 0.183 & 0.183 \\
\hline 05 & 0.223 & 0.223 & 0.223 \\
\hline 06 & 0.263 & 0.263 & 0.263 \\
\hline 07 & 0.303 & 0.303 & 0.303 \\
\hline 08 & 0.343 & 0.343 & 0.343 \\
\hline 09 & 0.383 & 0.383 & 0.383 \\
\hline 10 & 0.423 & 0.423 & 0.423 \\
\hline 11 & 0.483 & 0.483 & 0.483 \\
\hline 12 & 0.543 & 0.543 & 0.543 \\
\hline 13 & 0.603 & 0.603 & 0.603 \\
\hline 14 & 0.663 & 0.663 & 0.663 \\
\hline 15 & 0.723 & 0.723 & 0.723 \\
\hline 16 & 0.783 & 0.783 & 0.783 \\
\hline 17 & 0.843 & 0.843 & 0.843 \\
\hline 18 & 0.903 & 0.903 & 0.903 \\
\hline 19 & 0.963 & 0.963 & 0.963 \\
\hline 20 & 1.023 & 1.023 & 1.023 \\
\hline
\end{tabular}




\subsubsection{Experimental uncertainties}

This section provides the accuracies of certain components of the experiment, that include accuracies in model construction, instruments and model mounting, wind speed measurements, as well as the accuracies of working fluid input data into the software. The details of each component will be discussed in the following statements.

Model construction: The accuracy of model construction was within $\pm 0.1 \mathrm{~mm}$, which corresponds to the accuracy of the CNC machine.

Instrument mounting: Each of the Cobra probes was aligned with the incoming flow $\left( \pm 1^{\circ}\right.$ in yaw and $\pm 3^{\circ}$ in pitch angles) before each run. The accuracy of vertically traversing the probe was within $\pm 1 \mathrm{~mm}$, and the possible rotation of the tube while traversing was within $\pm 0.3^{\circ}$.

Model orientation: The orientation accuracy of the turntable when simulating different wind angles was within $\pm 0.03^{\circ}$ with respect to the wind tunnel longitudinal (along-wind) axis.

Software input data: The accuracy of the temperature and pressure values, used in the TFI software for data sampling, was $\pm 0.1^{\circ} \mathrm{C}$ and $\pm 100 \mathrm{~Pa}$, respectively.

Wind speed: The variations on the Cobra probes wind speed measurements were evaluated by repeating one of the experimental runs, and cross-checking the readings of 10 different elevations. The results showed variations within $\pm 3 \%$.

\subsection{RWDI wind tunnel experiment: $\frac{1}{300}$ city model}

The wind tunnel experiment at RWDI was the second major component of the body of work presented in this thesis. The objectives of the second experiment are indicated in the following statements. 
- The first objective was to compare the results of two different experiments that were conducted in different facilities using two models constructed at different scales.

- The second objective was to examine the effect of near field modelling on the measured data. The near field refers to the nearby significant buildings which are included in the physical model to appropriately simulate the approach flow in a specific site. For urban settings, the near field should include all major buildings located within 300-800 m (FS) from the site of measurement [26], where this was not possible at Carleton ABL wind tunnel due to limitation on the size of the facility. Thus, the influence of adding more buildings for one of the wind directions was to be investigated through comparison to the results obtained from the first experiment.

- The third objective was to investigate the effect of the modeled atmospheric boundary layer on the flow measurements of urban cities.

- The last objective was to measure horizontal profiles, which was not also possible at Carleton $\mathrm{ABL}$ wind tunnel due to limitations on the instruments mounting arrangements.

\subsubsection{The B12 wind tunnel}

The scaled physical model was tested at RWDI B12 wind tunnel. The wind tunnel is a closed-circuit boundary layer wind tunnel that can operate with a maximum flow speed of $18 \mathrm{~m} / \mathrm{s}$. The wind tunnel has an upstream contraction cone with a ratio of 1:2.15 followed by a fetch distance of $20 \mathrm{~m}$. The wind tunnel test section height and width are $2.13 \mathrm{~m}$ and $3.6 \mathrm{~m}$, respectively. The test section has a $3.35 \mathrm{~m}$ diameter automated turntable, that can both rotate and traverse vertically downward 
to allow for an external mounting of the model. The boundary layer and turbulence generation are achieved by the use of four $2.08 \mathrm{~m}$ high adjustable spires and roughness elements. The spire width and roughness elements height are controlled by a computer to generate the desired boundary layer characteristics. Rendering of the facility is presented in Figure 3.35 .

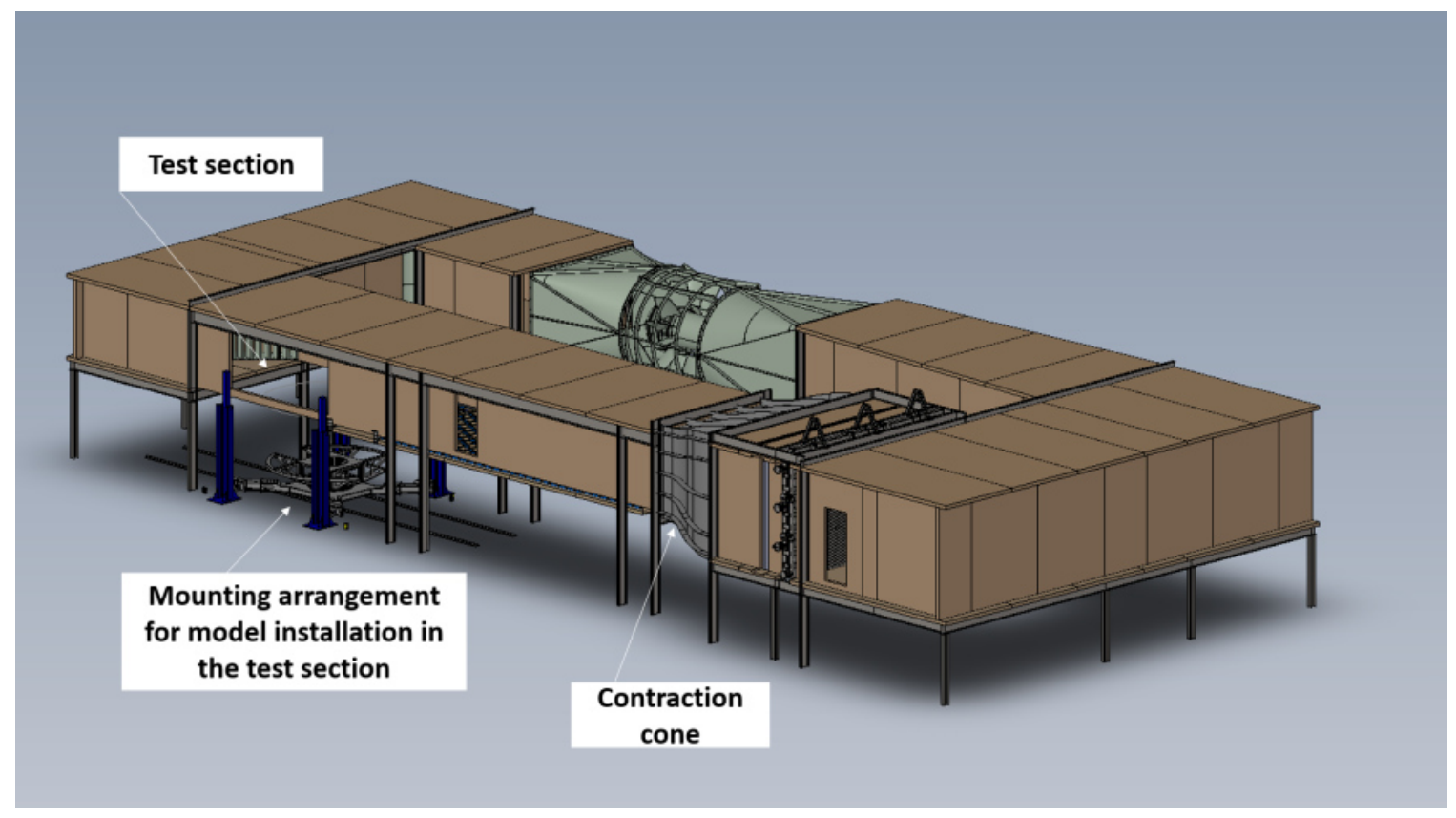

Figure 3.35: The B12 wind tunnel facility [69].

\subsubsection{Model characteristics and details}

The scaled physical model, shown as a CAD model in Figure 3.36, includes all buildings from the 1:400 city model in addition to more structures from certain flow directions, the majority of which are from North. The model was built with a scale of 1:300 from foam boards by the use of hot-wire foam cutter. The use of hot-wire ensured the creation of a model with sharp edges, which is important for aerodynamic studies. Additionally, the model includes four future buildings coloured in green, two of which were part of the 1:400 city model. Figure 3.37 shows the model domain in 
real settings, which is represented by a circular region with a diameter of $870 \mathrm{~m}$ in FS.

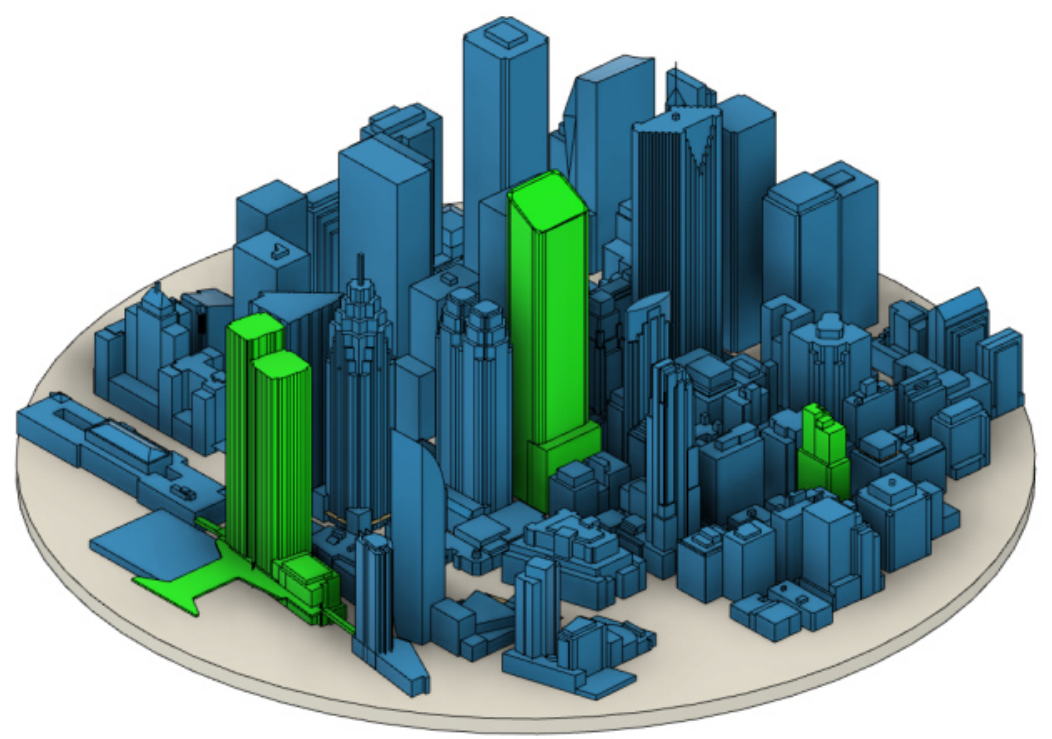

Figure 3.36: CAD model of the scaled physical model tested at RWDI [69].
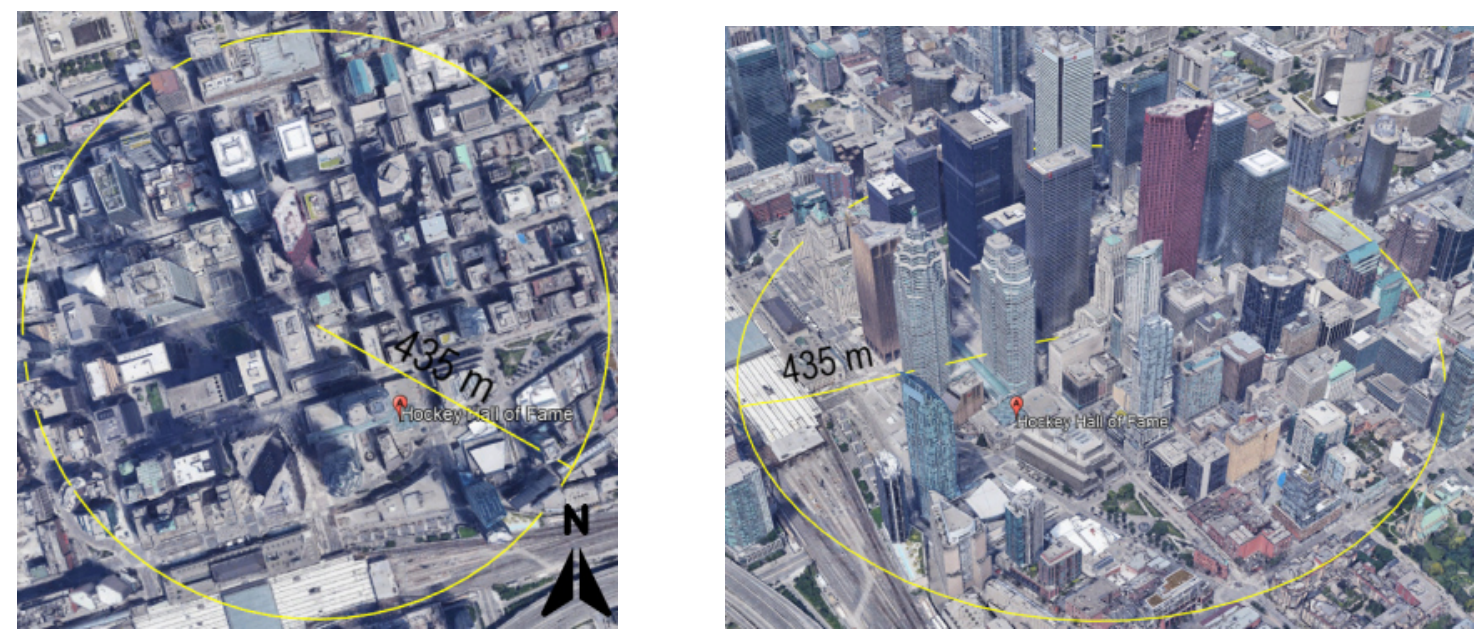

Figure 3.37: Model domain in real settings for the 1:300 city model. 


\subsubsection{Instrumentation and DAQ setup}

The instrumentation setup for the 1:300 model city experiment include one Cobra probe and a Pitot-static tube system. The Cobra probe was used for data collection from the model, and it was mounted from the wind tunnel ceiling on an automated traversing mechanism. The P-s tube was used to collect free stream velocities, and it was also mounted from the wind tunnel ceiling, 60 in upstream of the model leading edge. The setup of both instruments is shown in Figure 3.38 . For sampling the data, both instruments were connected to the TFI software, whose configuration was already discussed in Section 3.1.5.2. The flow measurements were sampled at 1,201.9 $\mathrm{Hz}$ (DOR) for a total time of 90 seconds.
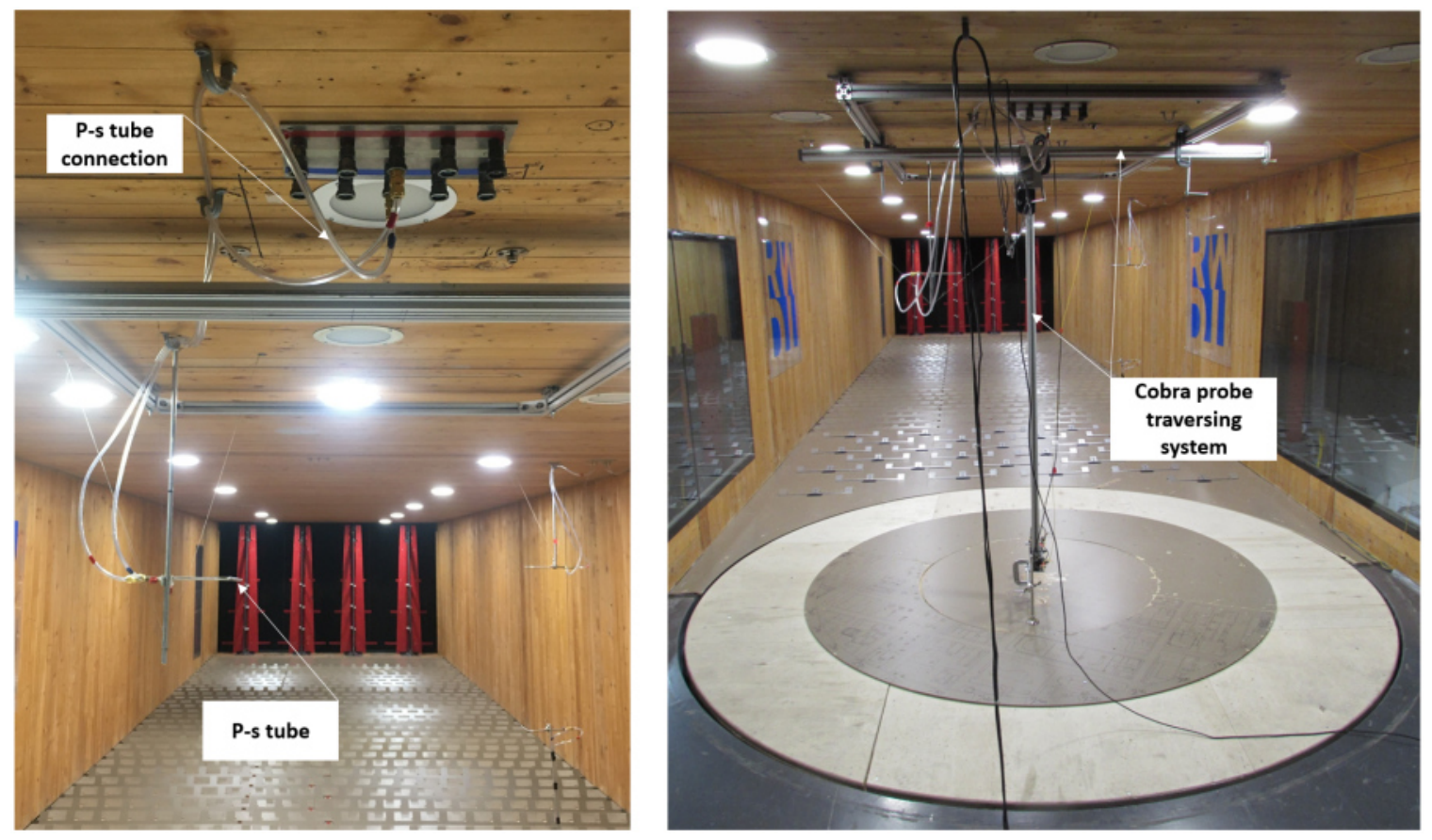

Figure 3.38: Instruments mounting arrangements at B12 wind tunnel. 


\subsubsection{Experimental procedures}

The wind tunnel experiment involved measurements at different approach flow exposure profiles, each was representing the flow characteristics over terrain with a specific roughness. For the model experiment, the three exposures used were flow over city centres (urban), low-rise buildings and wooded country (sub-urban), and open level country (open-sub). These exposure profiles are some of the pre-determined set points for approach flow characteristics that are used by RWDI for their wind engineering studies, and are achieved through the computer controlled adjustable spires and roughness elements.

Prior to model installation in the test section, measurements were taken at the empty wind tunnel at a wind speed of $40 \mathrm{ft} / \mathrm{s}(12.2 \mathrm{~m} / \mathrm{s})$, after which the model was installed. The scaled model experiment, on the other hand, was conducted at two different wind speeds according to the flow exposures. For urban approach flow profile, the model was tested at a wind speed of $35 \mathrm{ft} / \mathrm{s}(10.7 \mathrm{~m} / \mathrm{s})$. For the sub-urban

and open-sub approach flow profiles, the model was tested at wind speed of $40 \mathrm{ft} / \mathrm{s}$ $(12.2 \mathrm{~m} / \mathrm{s})$. Additionally, the experiment was conducted in two phases. The first phase included vertical profiles measurements, whereas the second phase involved measurements of horizontal profiles. For all experiments, the Cobra probe pitch and yaw angles were aligned within $\pm 1^{\circ}$ of the flow prior to any data collection. The model and instrument arrangements in the test section are shown in Figures 3.39, and the details of the wind tunnel experiments are presented in the following subsections. 

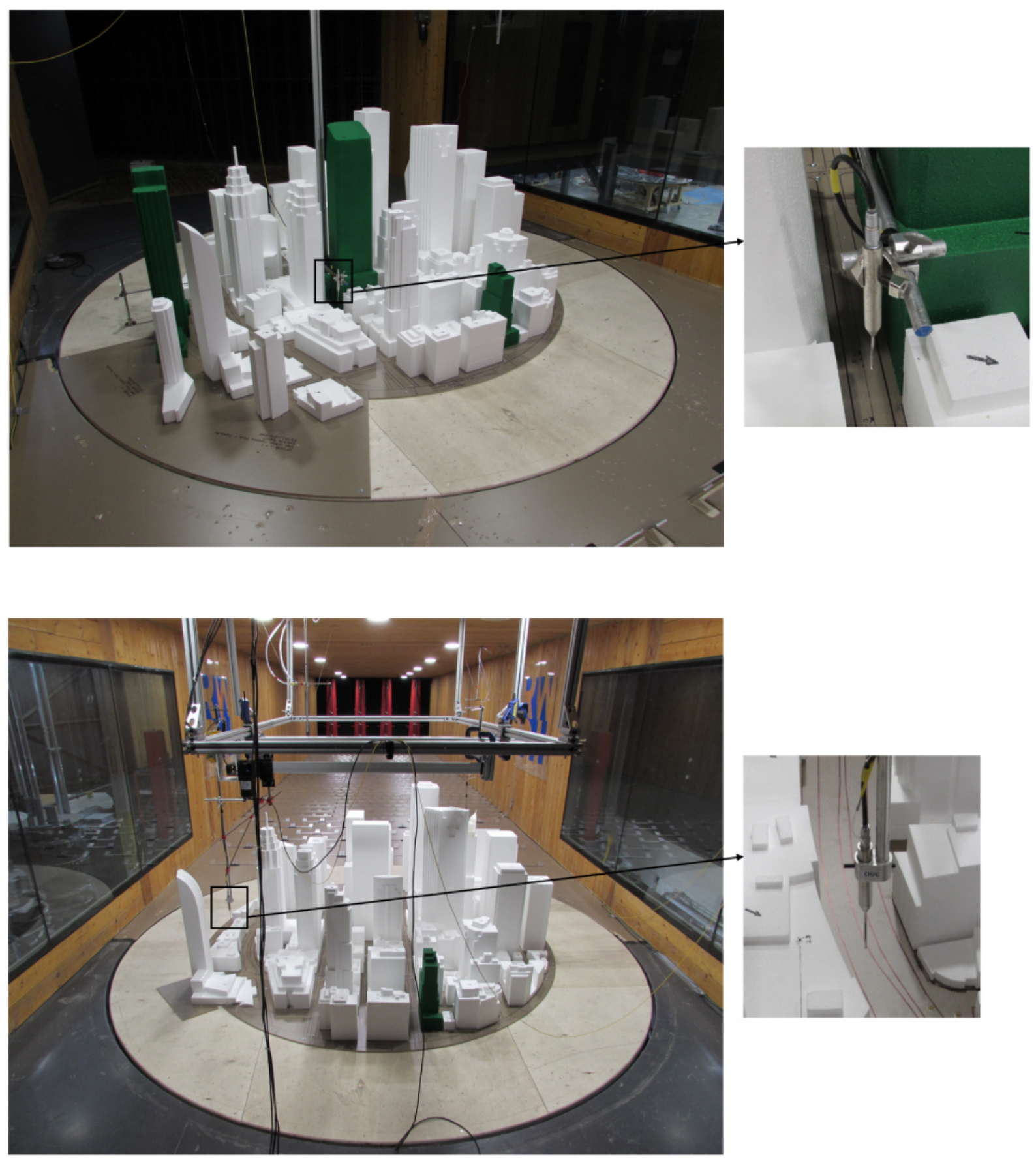

Figure 3.39: The 1:300 scale physical model along with instrument arrangements in the B12 wind tunnel. 


\subsubsection{Empty wind tunnel measurements}

The empty wind tunnel measurements involved data collection of both vertical and horizonal profiles at three exposures, namely, urban, sub-urban, and open-sub.

The vertical profiles were measured at the center of the turntable according to the vertical traverse points planned for the model test, which will be presented in Section 3.2.4.2, Table 3.11.

The measurement of the horizontal profiles was done at an elevation of $0.6 \mathrm{~m}$ above the floor of the test section, the traverse points of which are presented in Table 3.10 .

Table 3.10: Horizontal traverse points for RWDI empty wind tunnel measurements.

\begin{tabular}{cc}
\hline \hline Increment & Lateral distance $[\mathrm{m}]$ \\
\hline 1 & 0.75 \\
2 & 0.50 \\
3 & 0.25 \\
4 & 0 (center) \\
5 & -0.25 \\
6 & -0.50 \\
7 & -0.75 \\
\hline
\end{tabular}

\subsubsection{Scaled model vertical profiles measurements}

For the vertical traverse measurements, the same nomenclatures identified in Figure 3.13 were used for the measurement locations and wind directions. Additionally, the vertical traverse points correspond to the same locations with respect to the buildings geometry of the first experiment; however, the locations of the traverse points, measured from the wind tunnel floor, is different due to the different scale. Thus, the traverse points corresponding to a scale of 1:300 are shown in Figure 3.40 . 
and the increment values are listed in Table 3.11 .

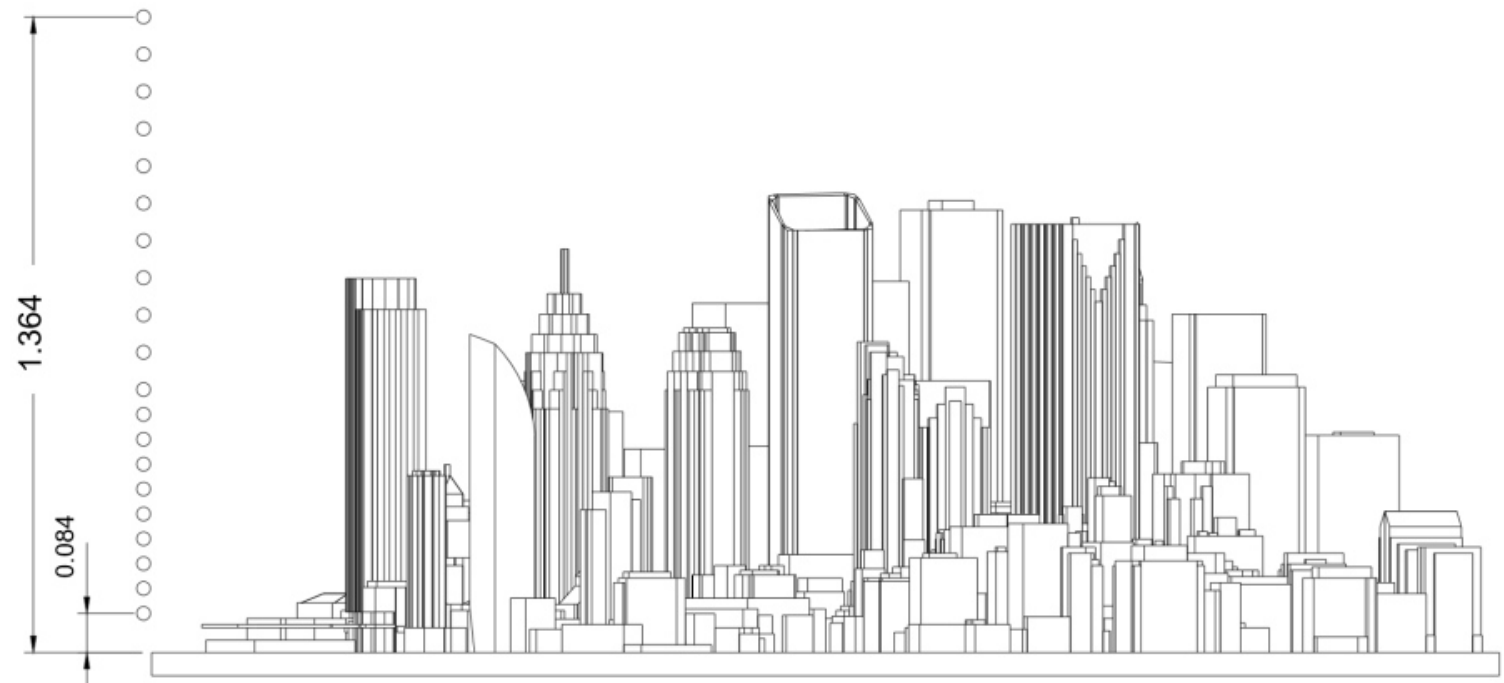

Figure 3.40: Locations of vertical traverse points for the 1:300 model city experiment (dimensions in $\mathrm{m}$ ).

The vertical profiles were measured at three different wind directions, which are the WSW, $\mathrm{E}_{17.1}$, and $\mathrm{N}_{16.7}$ at the red spots shown in Figure 3.41. The flow exposure was urban for all of the spots; however, some spots were tested at the other two exposures (sub-urban and open-sub). The future constructions, A, B, and C shown in Figure 3.41 were included for some of the wind directions. Table 3.12 summarizes the following information:

- the measurement locations corresponding to each wind direction;

- whether or not the future buildings were included; and

- the flow exposure profile used for the data collection. 
Table 3.11: Vertical traverse points for the 1:300 model city experiment.

\begin{tabular}{|c|c|c|}
\hline \multirow{3}{*}{ Increment } & \multicolumn{2}{|r|}{ Spots } \\
\hline & $(28)$ & (Others + empty wind tunnel) \\
\hline & \multicolumn{2}{|r|}{ Height $[\mathrm{m}]$} \\
\hline 01 & 0.0973 & 0.0840 \\
\hline 02 & 0.1373 & 0.1373 \\
\hline 03 & 0.1907 & 0.1907 \\
\hline 04 & 0.2440 & 0.2440 \\
\hline 05 & 0.2973 & 0.2973 \\
\hline 06 & 0.3507 & 0.3507 \\
\hline 07 & 0.4040 & 0.4040 \\
\hline 08 & 0.4573 & 0.4573 \\
\hline 09 & 0.5107 & 0.5107 \\
\hline 10 & 0.5640 & 0.5640 \\
\hline 11 & 0.6440 & 0.6440 \\
\hline 12 & 0.7240 & 0.7240 \\
\hline 13 & 0.8040 & 0.8040 \\
\hline 14 & 0.8840 & 0.8840 \\
\hline 15 & 0.9640 & 0.9640 \\
\hline 16 & 1.0440 & 1.0440 \\
\hline 17 & 1.1240 & 1.1240 \\
\hline 18 & 1.2040 & 1.2040 \\
\hline 19 & 1.2840 & 1.2840 \\
\hline 20 & 1.3640 & 1.3640 \\
\hline
\end{tabular}




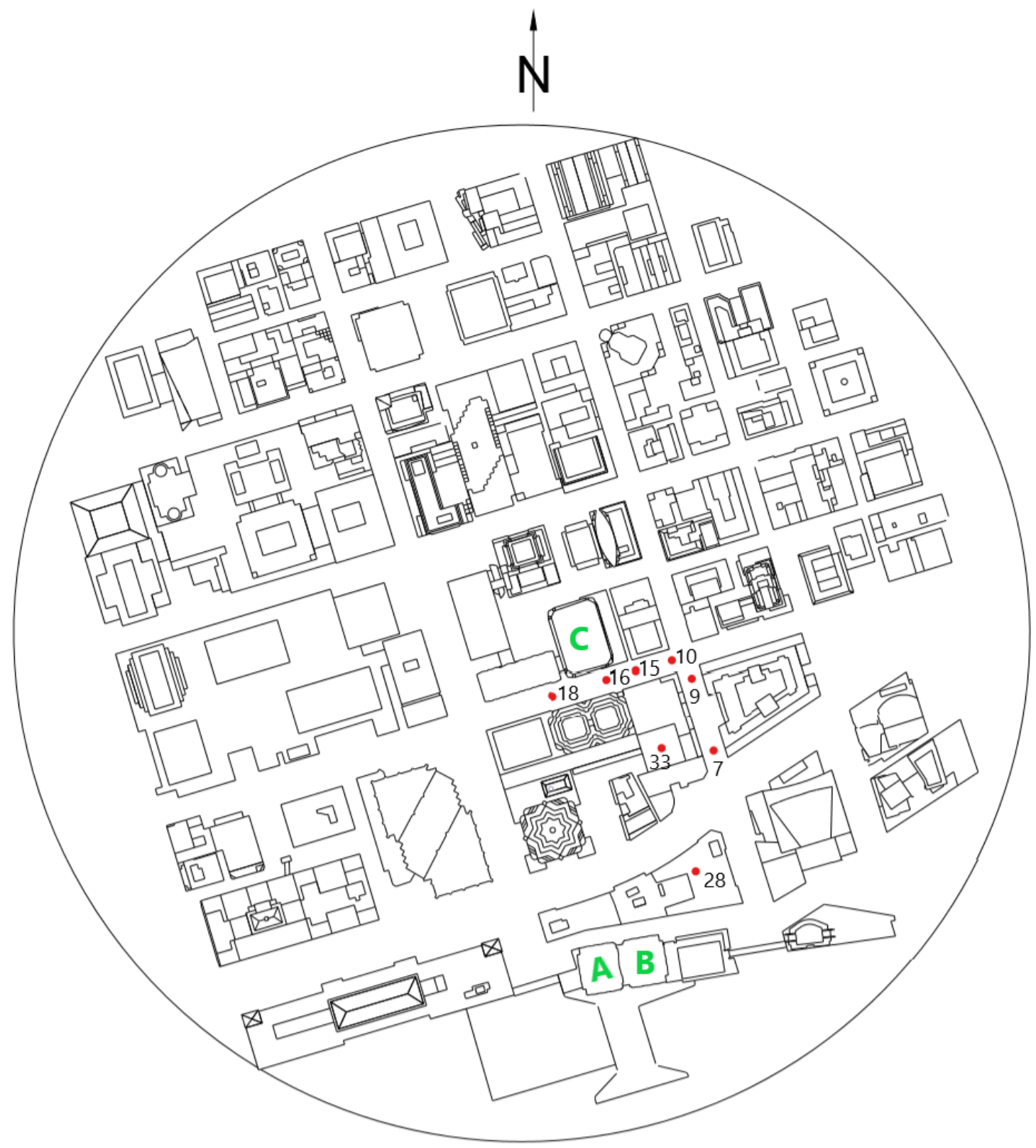

Figure 3.41: Measurement locations for the 1:300 city model vertical profiles. 
Table 3.12: Vertical measurements details for the 1:300 model city experiment.

\begin{tabular}{c|c|c|c}
\hline \hline Wind direction & Measurement location & Exposure profile & Future buildings \\
\hline \multirow{2}{*}{ WSW } & 7 & Urban; sub-urban; open-sub & None \\
& 9 & Urban & None \\
\hline \multirow{2}{*}{$\mathrm{E}_{17.1}$} & 15 & Urban & A; B; C \\
& 18 & Urban; sub-urban; open-sub & A; B; C \\
& 28 & Urban; sub-urban; open-sub & None \\
\hline \multirow{3}{*}{$\mathrm{N}_{16.7}$} & 10 & Urban & None \\
& 16 & Urban & None \\
& 18 & Urban & None \\
& 33 & Urban; sub-urban; open-sub & None \\
\hline
\end{tabular}




\subsubsection{Scaled model horizontal profiles measurements}

The measurement of the horizontal profiles was performed by traversing the Cobra probe with an increment of $0.025 \mathrm{~m}$ along the lateral (cross-wind) transects: $\mathrm{T}_{12}, \mathrm{~T}_{34}$, and $\mathrm{T}_{56}$ which are shown in Figure 3.42, The transects designations are based on the starting and ending points of the traverse.

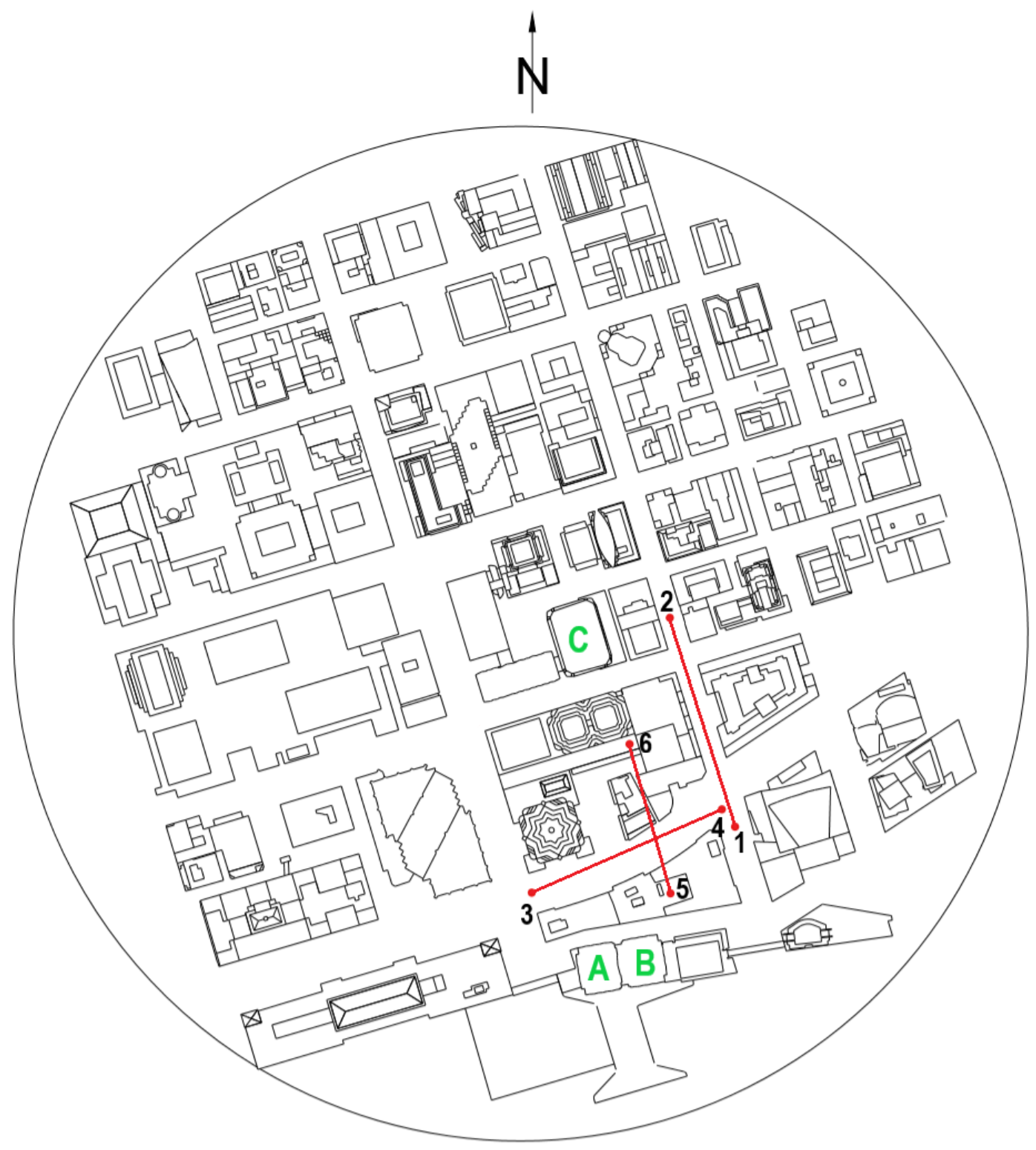

Figure 3.42: Designations for cross-wind transects. 
The horizontal profiles were measured at four wind directions, two of which were the WSW and WSW +15 which corresponds to the azimuth angles of $247.5^{\circ}$ and $262.5^{\circ}$, respectively as indicated in Table 3.2 in the preceding sections. The other two wind angles were $253.2^{\circ}$ and $335^{\circ}$, with the corresponding designations, $\mathrm{N}_{253.2}$ and $\mathrm{N}_{335}$, both measured from North in a CW direction. It is worth mentioning that the future buildings $\mathrm{A}, \mathrm{B}$, and $\mathrm{C}$ were not included in the model for all horizontal traverses. Table 3.13 summarizes the following information:

- the lateral transects for each wind direction;

- the elevation from the wind tunnel floor for measurements along each transect;

- the number of horizontal traverse points of each transect; and

- the flow exposure profile used for the data collection.

Table 3.13: Horizontal measurements details for the 1:300 model city experiment.

\begin{tabular}{|c|c|c|c|c|}
\hline Wind direction & Lateral transect & Height $[\mathrm{m}]$ & Traverse total points & Exposure profile \\
\hline WSW & $\mathrm{T}_{56}$ & 0.4573 & 19 & Urban \\
\hline $\mathrm{WSW}_{+15}$ & $\mathrm{~T}_{56}$ & 0.4573 & 21 & Urban \\
\hline \multirow{3}{*}{$\mathrm{N}_{253.2}$} & $\mathrm{~T}_{12}$ & 0.3507 & 26 & Urban; sub-urban; open-sub \\
\hline & $\mathrm{T}_{12}$ & 0.5640 & 26 & Urban \\
\hline & $\mathrm{T}_{56}$ & 0.4573 & 19 & Urban \\
\hline $\mathrm{N}_{335}$ & $\mathrm{~T}_{34}$ & 0.5640 & 26 & Urban \\
\hline
\end{tabular}




\section{Chapter 4}

\section{Wind Tunnel Experimental Results and Discussions}

This chapter details and discusses the major results obtained from both Carleton University and RWDI wind tunnel experiments. Additionally, the applied methods for data corrections are explained where appropriate.

The wind tunnel experimental results for the 1:400 scale city model are focused on certain airflow features that are expected to pose challenges to the safe operations of UAM. The flow features presented herein were inspired by the acronym SDSTV, described by Barber and Wall in Ref. [33], which represents the Speed, Direction, Shear, Turbulence and Vorticity flow features. However, the angle from which some of the flow features had been looked at in this thesis may vary from the definitions considered in Ref. [33, and thus a description of each of the flow features will be explained in the following statements.

Speed: The wind speed was examined by comparing the flow speed magnitudes with and without the model at a particular location, that is by calculating the percent increase or decrease from the Empty wind tunnel Value (EV) to the Model Value (MV) as illustrated in Equation (4.1). This type of analysis demonstrates the amount of flow acceleration (speed increase) or deceleration 
(speed decrease) that an air taxi may encounter when flying between buildings and urban structures.

$$
\% \text { increase } / \text { decrease }=\frac{\mathrm{MV}-\mathrm{EV}}{\mathrm{EV}} \times 100
$$

Direction: The wind direction was analyzed by examining the yaw and pitch flow angles measured by the Cobra probe at a certain location. The pitch angle however was categorized into up-draft and down-draft, which represent the upward and down-ward flow motions, respectively.

Shear: The wind shear analysis includes an evaluation of the vertical speed shear and directional shear, in which the latter involves a separate analysis for the horizontal directional shear and the vertical directional shear. For each of the wind shear components, the shear was determined from the vertical profile measurements, and by calculating the difference between the flow values measured at two consecutive points, normalized by the distance between the two points as illustrated in the following equations,

$$
\begin{aligned}
& \text { speed shear }=\frac{U_{2}-U_{1}}{H_{2}-H_{1}} \\
& \text { yaw shear }=\frac{\left|\psi_{2}-\psi_{1}\right|}{H_{2}-H_{1}} \\
& \text { pitch shear }=\frac{\left|\theta_{2}-\theta_{1}\right|}{H_{2}-H_{1}}
\end{aligned}
$$

where $U, \psi, \theta$, and $H$ are the mean wind speed, wind yaw angle, wind pitch angle and measurement height respectively. The subscripts 1 and 2 refer to the lower and higher measuring points, respectively. The shear in wind is illustrated 
schematically in Figure 4.1 .

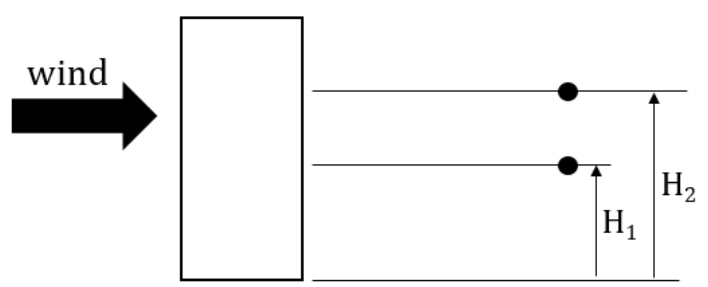

Building block

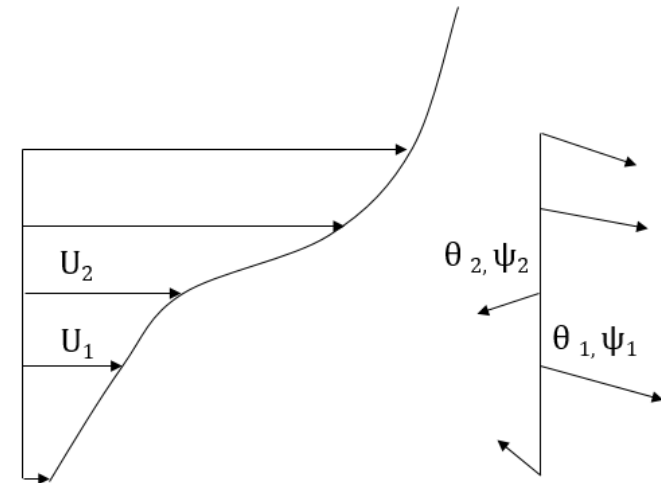

Speed shear

Directional shear

Figure 4.1: Wind speed and directional shear.

Turbulence: The atmospheric Turbulence Intensity (TI) was investigated by examining its three components, namely, along-wind turbulence $\left(\mathrm{I}_{u u}\right)$, across-wind turbulence $\left(\mathrm{I}_{v v}\right)$, and vertical turbulence $\left(\mathrm{I}_{w w}\right)$ intensities. Each of the turbulence components was determined from the ratio between the standard deviation $(\sigma)$ of the corresponding time-varying velocity component and the magnitude of the time-averaged velocity vector, $\bar{V}$, which are described mathematically as follows:

$$
\begin{aligned}
& I_{u u}=\frac{\sigma_{u}}{\bar{V}}=\frac{\sigma_{u}}{\sqrt{\bar{u}^{2}+\bar{v}^{2}+\bar{w}^{2}}} \\
& I_{v v}=\frac{\sigma_{v}}{\bar{V}}=\frac{\sigma_{v}}{\sqrt{\bar{u}^{2}+\bar{v}^{2}+\bar{w}^{2}}} \\
& I_{w w}=\frac{\sigma_{w}}{\bar{V}}=\frac{\sigma_{w}}{\sqrt{\bar{u}^{2}+\bar{v}^{2}+\bar{w}^{2}}}
\end{aligned}
$$

where $\bar{u}, \bar{v}$, and $\bar{w}$ are the longitudinal (along-wind), lateral (across-wind), and 
vertical time-averaged velocity components, respectively.

Vorticity: For the analysis of wind vortices, the emphasis was on the type of vortices that form on and detach from a building sides, leading to the side-to-side vortex shedding phenomenon. The onset of vortex shedding was determined from comparisons between the frequency of vortex shedding given by Strouhal rela-

tionship, $f_{v}=\frac{S_{t} U}{b}$, and the frequencies obtained from the power spectral density (PSD) of the corresponding velocity signal. The parameters of the Strouhal relationship are the frequency of vortex shedding, $f_{v}$, the across-wind building dimension, $b$, the wind velocity, $U$, and the Strouhal number [70], $S_{t}$. The calculation of $f_{v}$ from Strouhal relationship was based on a Strouhal number in the range $0.1-0.2$ 71].

For RWDI experimental results, comparative analysis was performed to examine the effect of model scale, testing facility, near field modelling, and approach flow exposure profile on the flow measurements. Additionally, the capabilities available at RWDI wind tunnel testing facility allowed for the investigation of the lateral wake characteristics downwind of buildings.

\subsection{CU wind tunnel experiment: empty tunnel}

Prior to the 1:400 model city experiment, measurements were taken in the empty test section of the wind tunnel to check the approach flow boundary layer profile and depth as well as the flow uniformity across the wind tunnel test section. The test involved measurements of three vertical profiles across the width of the turntable as indicated previously in Chapter 3, Figure 3.26. Further details are provided in the subsequent sections. 


\subsubsection{Approach-flow boundary layer}

The approach flow boundary layer profile and depth were developed in the wind tunnel by the use of spires and roughness elements, which were designed according to Irwin empirical formulations $[53]$ as described earlier in Chapter 3 , Section 3.1.2.

The vertical distribution of the mean wind speed was measured at the center of the turntable to assess the agreement between the measured velocity profile and the power law velocity profile with $\alpha=0.40$, which was the target profile for the 1:400 scale model city experiment. The measured mean wind speed were normalized by the mean wind speed value at a reference height $\delta=0.885 \mathrm{~m}$. As can be seen in Figure 4.2, the measured profile appears to be more consistent with the power law profiles with $\alpha$ in the range from 0.30 to 0.35 .

The deviation between the measured profile and the 0.40 -power law profile was not unexpected since the profile was measured at the center of the disc as opposed to the disc leading edge. Also, Irwin methodology assumes the formation of the desired boundary layer height and profile downstream of the roughness elements whose size is determined from specific equations. However, as explained earlier in Chapter 3 , it was decided to use smaller roughness elements in the region immediately upstream of the model, which explains the agreement between the measured profile and the profiles with the lower values of $\alpha$.

For the boundary layer depth, the formulation indicated an approximate value of $0.97 \mathrm{~m}$ at the leading edge of the turntable and $1.04 \mathrm{~m}$ at the center of the turntable. However, the test section height influences the boundary layer thickness due to the wall effects, which was not an issue since the main purpose of checking the boundary layer depth was to ensure that the model is fully immersed into the generated boundary layer. 


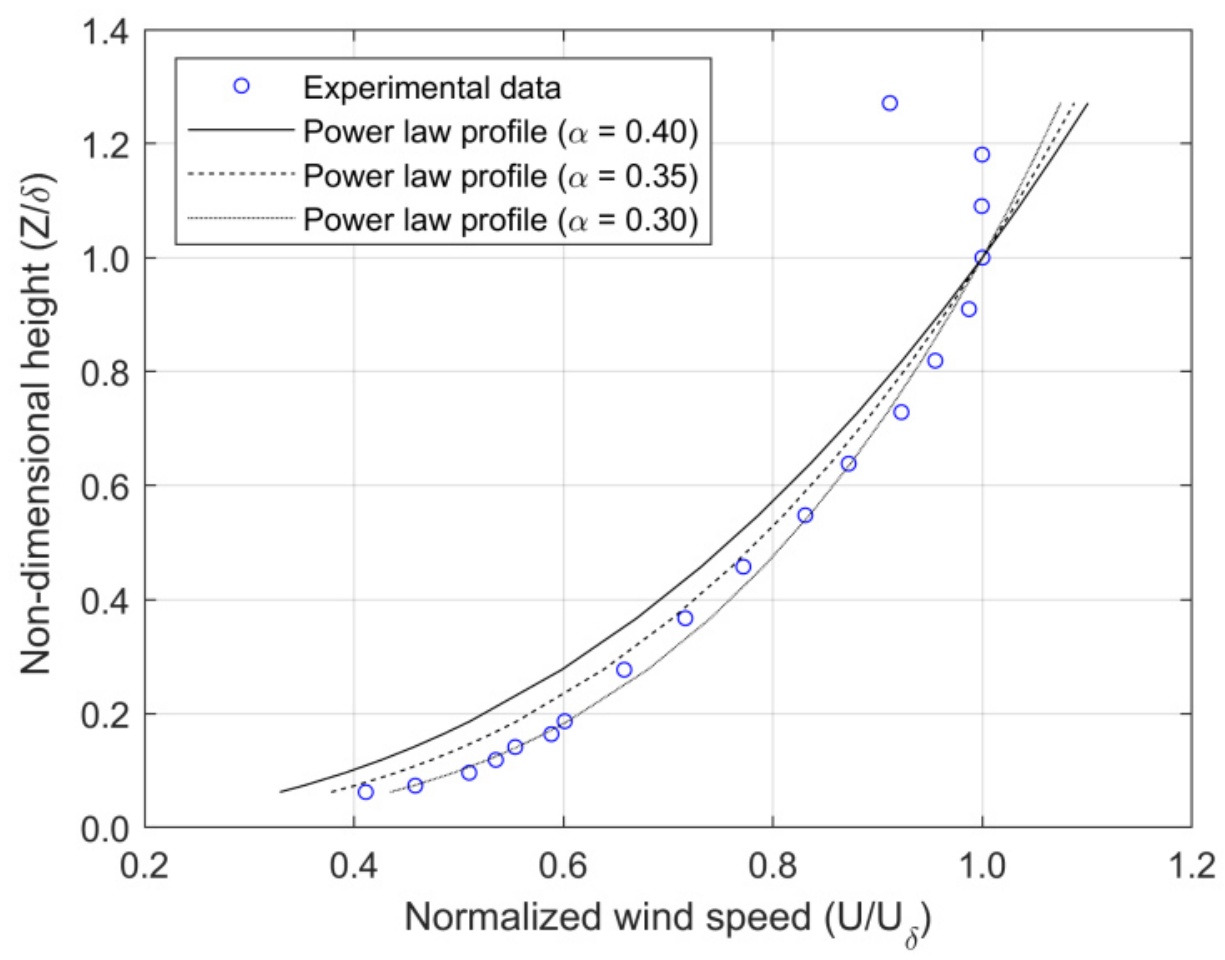

Figure 4.2: Comparisons between the wind speed profile measured at the ABL wind tunnel and the power-law velocity profiles with different exponents. X-axis: mean wind speed normalized by the speed value at a reference height $(\delta)$. Y-axis: measurement height normalized by the reference height $(\delta)$.

\subsubsection{Assessment of lateral flow uniformity}

The test section lateral flow uniformity was verified by measuring three profiles across the width of the turntable. The profiles locations were illustrated schematically in Figure 3.26, in which measurements were taken at the center of the disc, and at a lateral distance of $0.25 \mathrm{~m}$ away from the disc center in both directions.

The profiles shown in Figure 4.3 are representing the vertical distributions of the mean wind speed and the longitudinal turbulence intensity component of the three measurement locations M, L and R. To ensure an appropriate comparison between the data, the mean wind speed profiles were normalized by the corresponding reference 
wind speeds, $\mathrm{U}_{r e f}$, which were determined from the calibration curve based on Pitotstatic tube measurements at the center of the turntable, without the model, and at a height of $0.903 \mathrm{~m}$ above the wind tunnel floor.
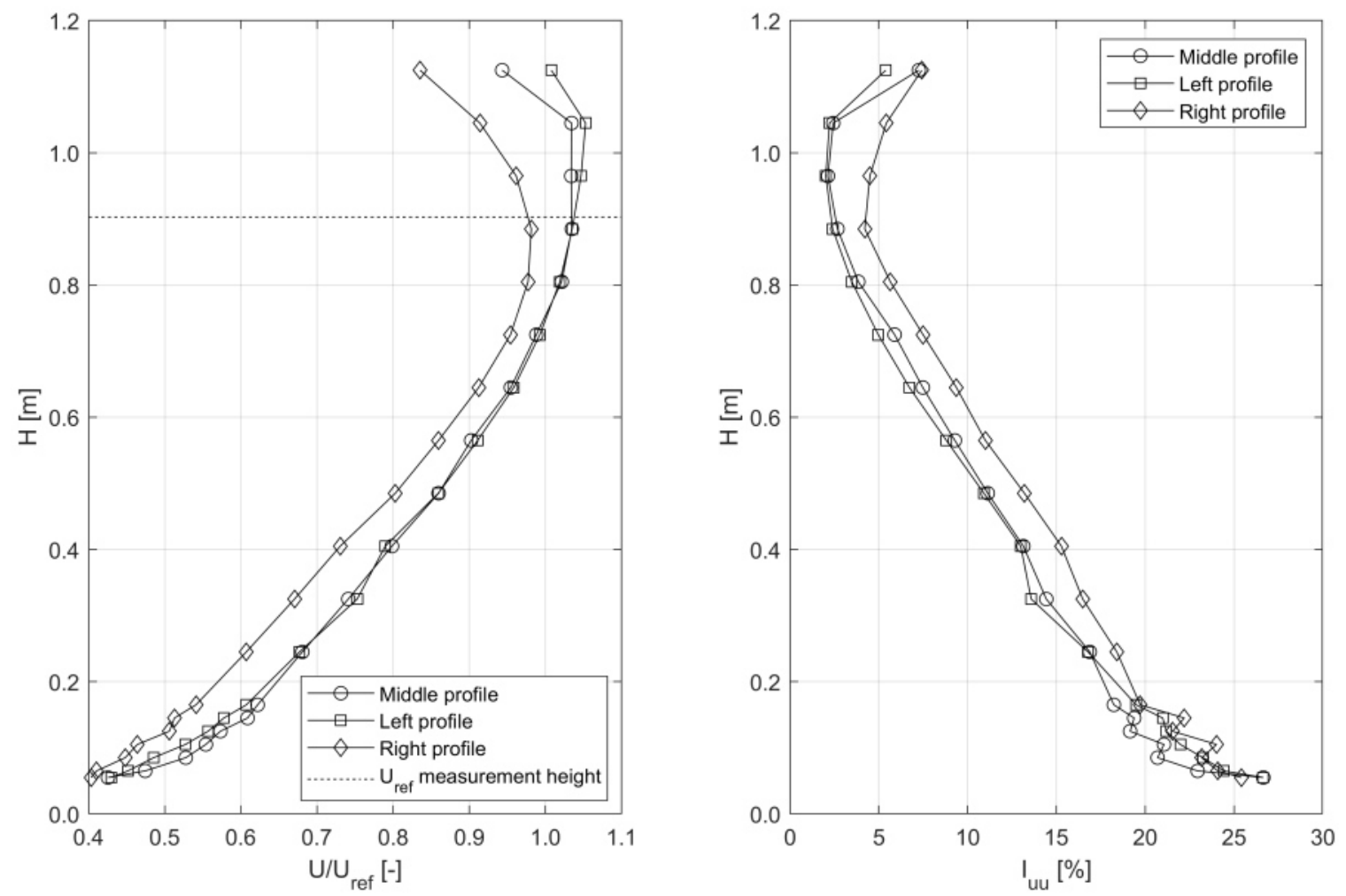

Figure 4.3: Empty wind tunnel profiles measured at CU ABL wind tunnel. Left: mean wind speed vertical profiles versus height. Right: longitudinal turbulence intensity profiles versus height.

By examining the profiles, it was discovered that the flow is not symmetric across the width of the wind tunnel since the $R$ profile clearly deviates from the $M$ and L profiles. This issue of flow non-uniformity was believed to affect the comparison between the measured data since normalization would be based on measurements by the P-s tube located at the center of the disc, which means that for the same conditions, the data measured between the $\mathrm{M}$ and $\mathrm{R}$ locations would always be lower 
in speed as compared to data measured between the $\mathrm{M}$ and $\mathrm{L}$ locations, in spite of normalization.

Considering the height of the $\mathrm{P}_{-\mathrm{s}}$ tube, variations in the measured wind speeds between the $\mathrm{M}$ and $\mathrm{L}$ locations was found to be within $0.3 \%$, and thus, $\mathrm{U}_{\text {ref }}$ measured at the center were used for normalizing the data measured between the $\mathrm{M}$ and L locations. However, the variations in the recorded wind speeds between the $\mathrm{M}$ and $\mathrm{R}$ locations were within $5.6 \%$, which necessitated corrections for the $\mathrm{U}_{\text {ref }}$ values used for normalizing the model measurements between the $\mathrm{M}$ and $\mathrm{R}$ locations. For the longitudinal turbulence intensity, which is the ratio between the standard deviation of the $u$ velocity component to the local mean wind speed, the maximum difference between the $\mathrm{M}$ and $\mathrm{R}$ was found to be less than $3 \%$, which supports the assumption that the flow characteristics of the simulated ABL are insensitive to Reynolds number greater than $\sim 4000[72]$. The reference velocities, $\mathrm{U}_{r e f}$, used for normalizing the measured data between the $\mathrm{M}$ and $\mathrm{R}$ locations were corrected according to the following procedure so that all the data can be compared.

- The variations between the $\mathrm{M}$ and the $\mathrm{R}$ profiles were assumed to be linear, and thus, five profiles were linearly interpolated between the $\mathrm{M}$ and $\mathrm{R}$ profiles as shown in Figure 4.4 .

- The normalized mean wind speed values, coloured in red in Figure 4.4, were normalized by the M mean wind speed value to determine the correction factors for $\mathrm{U}_{\text {ref }}$ as illustrated in Figure 4.5 .

- Based on the measurement location on the model, the $\mathrm{U}_{\text {ref }}$ value was corrected by the correction factor of the nearby profile. For instance, the measurements in Spots 9 and 11 shown in Figure 4.6 were normalized by $0.94 \mathrm{U}_{r e f_{M}}$ and $0.98 \mathrm{U}_{r e f_{M}}$, respectively. 


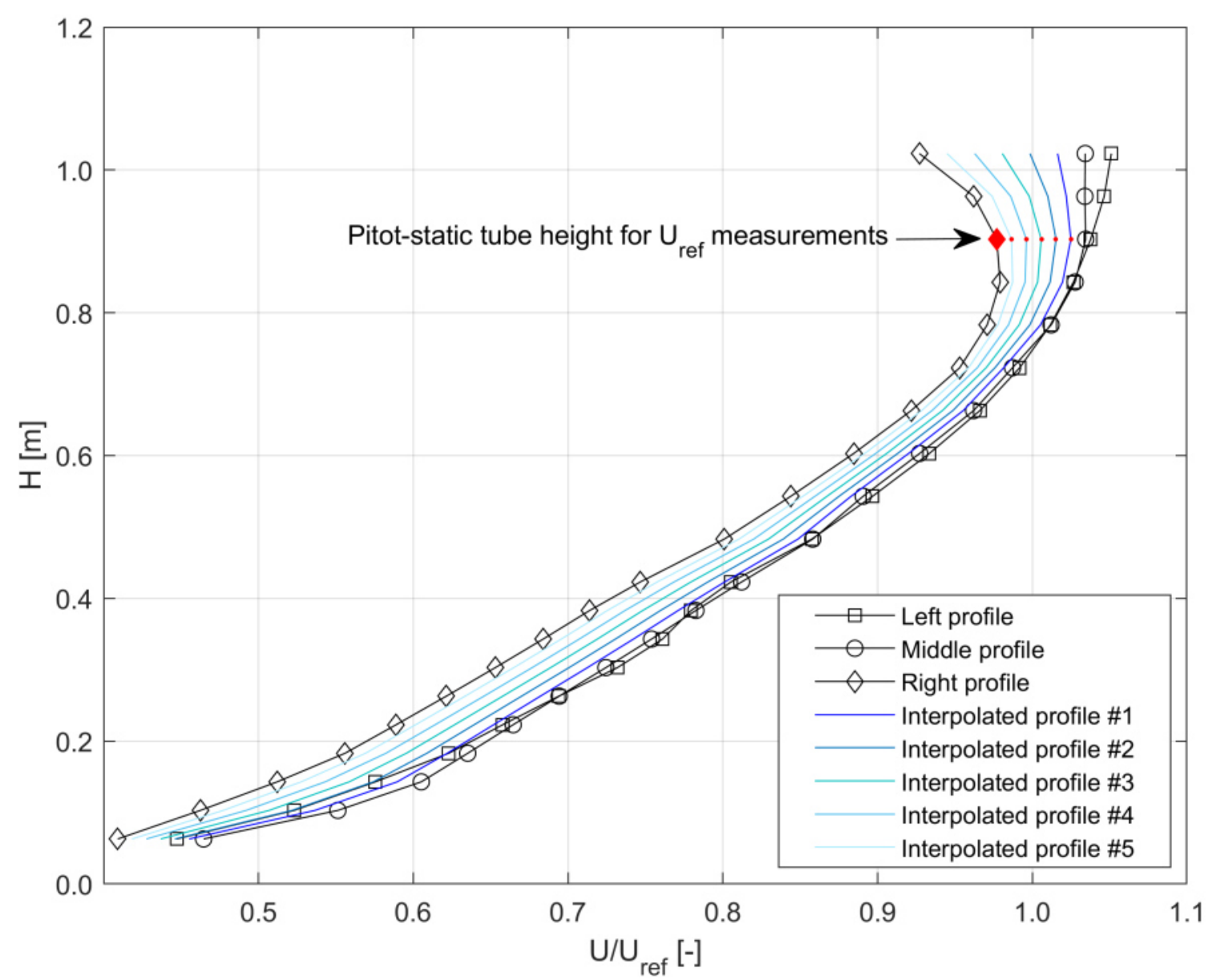

Figure 4.4: Interpolated mean wind speed profiles between the Middle and Right locations.

By applying the aforementioned corrections, the maximum uncertainty in the measured wind speed between the M and R locations was estimated to be within $0.5 \%$. 


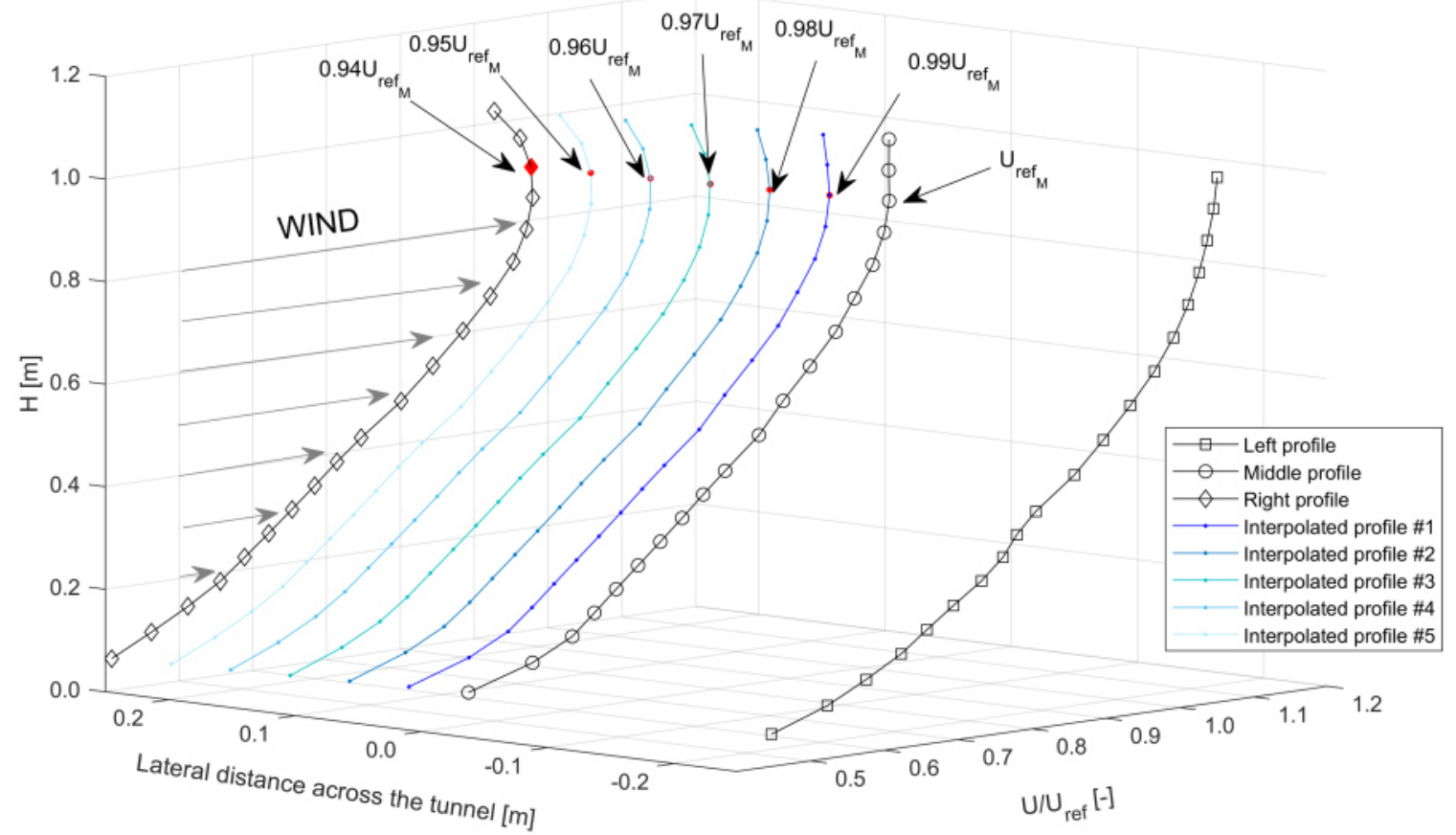

Figure 4.5: The correction factors for the reference velocities measured by the Pitotstatic tube. 


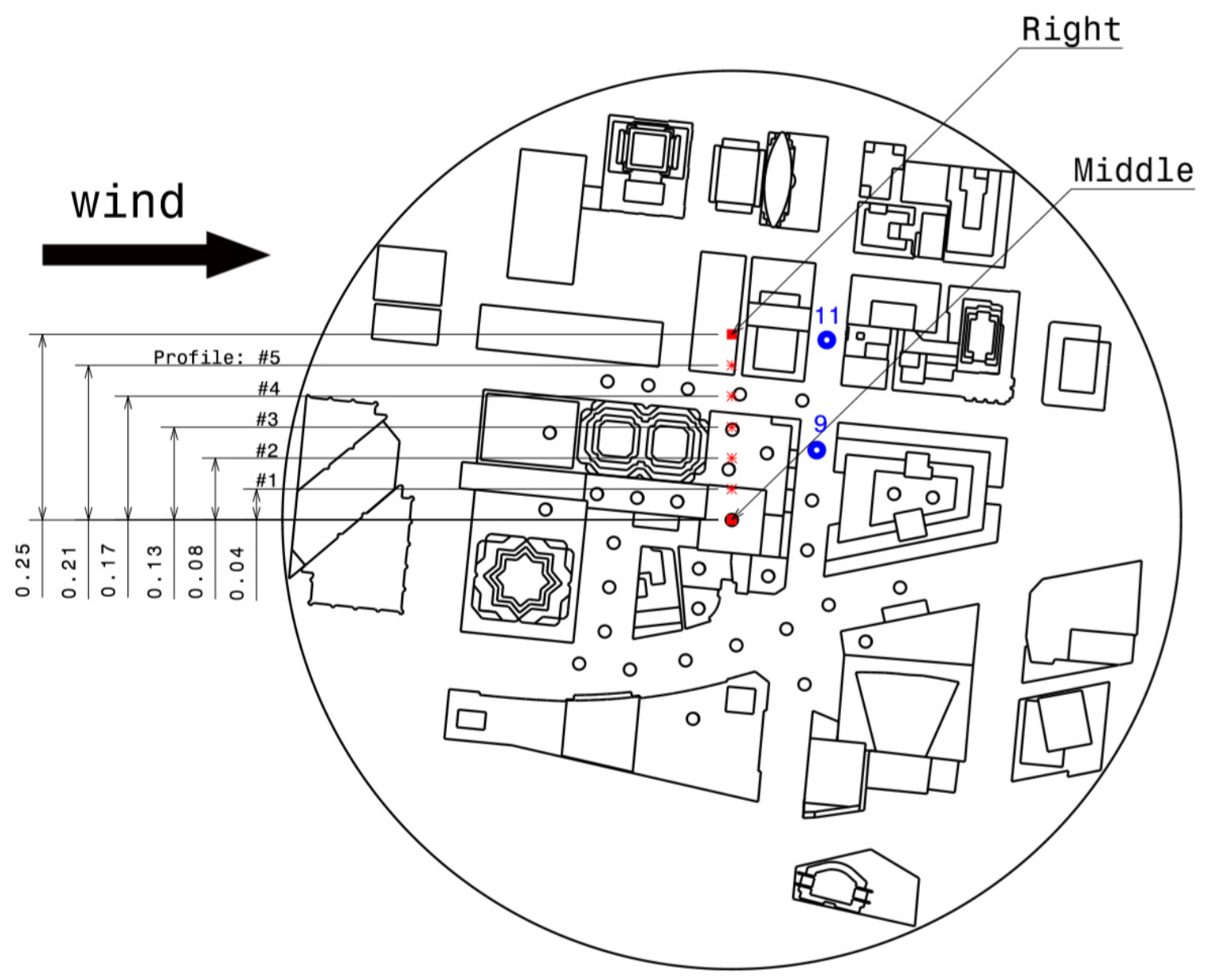

Figure 4.6: Locations of the interpolated profiles with respect to the disc center (dimensions in $\mathrm{m}$ ). 


\subsection{CU wind tunnel experiment: data corrections}

\subsubsection{Corrections for blockage effect}

The 1:400 physical city model size relative to the cross-sectional area of the ABL wind tunnel test section resulted in solid blockage in the range of $18 \%-25 \%$ as indicated previously in Table 3.1. Since the solid blockage was more than 10\%, corrections for blockage effects were required [71]. There are multiple blockage correction techniques, which range in simplicity from normalizing the data by reference velocities measured in the accelerated flow, to the use of analytical relationships for identifying the necessary correction factors.

The method employed for correcting the city model data was based on the formulations explained in Ref. [50], which accounts for solid and wake blockages of models with unusual shapes. The reference velocities measured by the P-s tube, $\mathrm{U}_{r e f}$, were corrected according to the following relations:

$$
\begin{gathered}
U_{r e f_{c}}=U_{\text {ref }}\left(1+\varepsilon_{T}\right) \\
\varepsilon_{T}=\frac{1}{4} \frac{\text { model frontal area }}{\text { test section area }}
\end{gathered}
$$

where $\mathrm{U}_{r e f_{c}}$ is the corrected reference velocity and $\varepsilon_{T}$ is the total blockage correction factor. It is important to note that the term $\mathrm{U}_{r e f_{c}}$ will not be used in this thesis, and the general term $\mathrm{U}_{r e f}$ will be used instead for the corrected reference velocities for model measurements.

For each of the model orientations with respect to the incoming flow, the model frontal area, $\mathrm{A}_{f, \text { model }}$, was calculated utilizing CATIA V5 software. The model frontal area along with the total blockage correction factor for each of the model orientations are summarized in Table 4.1. 
Table 4.1: Correction factors for blockage effects.

\begin{tabular}{|c|c|c|c|}
\hline Wind direction & Projected view & $\mathrm{A}_{f, \text { model }}\left[\mathrm{m}^{2}\right]$ & $\varepsilon_{T}$ \\
\hline WSW & & 0.422 & 0.051 \\
\hline $\mathrm{WSW}_{+15}$ & & 0.434 & 0.053 \\
\hline $\mathrm{E}_{17.1}$ & & 0.406 & 0.049 \\
\hline $\mathrm{N}_{16.7}$ & & 0.372 & 0.045 \\
\hline $\mathrm{S}_{15, \text { without }}$ & & 0.456 & 0.055 \\
\hline $\mathrm{S}_{15, \text { with }}$ & & 0.518 & 0.063 \\
\hline
\end{tabular}




\subsubsection{Corrections for flow angles}

To optimize the flow measurements, the Cobra probes were rotated whenever the $\%$ GD was below $95 \%$. The amount of rotation was initially identified by the indexing mechanism, which was shown previously in Figure 3.19, and subtracted thereafter from the measured yaw angles. Thus, the flow yaw angles to be presented later in this chapter correspond to the actual angles of the flow with respect to the wind tunnel longitudinal axis (along-wind). However, the indexing mechanism was only applicable for rotation about the probe z-axis (consult Figure 3.17 for the probe axis system), and thus, the measured flow pitch angles were restricted by the $\pm 45^{\circ}$ acceptance cone.

\subsection{CU wind tunnel experiment: $\frac{1}{400}$ city model}

The experimental results and findings included in this section are based on the data measured by the Cobra probes with a minimum of $80 \%$ GD. However, in some instances, plots of vertical profiles will be presented, in which data points may correspond to different \%GD. Thus, the data points of model measurements are coloured based on the \%GD according to the colour scheme provided in Figure 4.7.

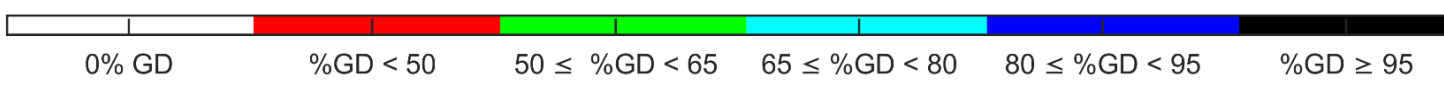

Figure 4.7: The colour scheme of the \%GD for the Cobra probes measurements.

In the following sections, the terms 'Spot' and 'Increment' will be used frequently. The term Spot refers to the measurement location, whereas Increment refers to the vertical traverse points above the measurement location. The reader is encouraged to

consult Figure 3.13 for nomenclature of measurement locations and wind directions, 
Figure 3.9 for the height of major buildings and Figure 3.34 along with Table 3.9 for vertical traverse increments and height. The experimental results are focused on the extreme maximum flow features (worst-case urban flow characteristics), the details of which will be discussed thoroughly in Section 4.3.2. Nonetheless, brief discussion about the extreme minimum will be provided in Section 4.3.1, which discusses the overall extremes that were found from the model measurements.

\subsubsection{Extreme values of flow features}

One of the main goals of the city model wind tunnel experiment was to assess the extent of severity of each flow feature, and subsequently investigate where the extremes exist in relation to the model geometry. Therefore, the maximum and minimum values of the speed, direction, shear, and turbulence flow features were identified from the vertical traverse measurements at each of the wind directions (see Figures 3.31 and 3.32, and the results are given in Table 4.2. The corresponding locations of the presented extremes in Table 4.2 are given in Table 4.3 in the form: (spot,increment) for wind speed, direction and turbulence, and in the form: (spot,lower incrementhigher increment) for wind shear. The vorticity flow feature was not part of the extremum analysis since the investigation of vortex shedding was not exhaustive, and only selected cases of possible vortex shedding phenomena were examined, the results of which are discussed in Section 4.3.2

It is emphasized that the wind shear was examined based on the definitions provided at the beginning of this chapter to ensure proper comparison between all the data given that the vertical traverse points were not equally spaced (0.04 m spacing for Increments 1-10 and 0.06 m spacing thereafter). However, in order to provide an illustrative interpretation of the data, the extreme values of speed shear are presented in terms of the actual change in wind speed, both non-dimensional and in $\mathrm{m} / \mathrm{s}$ (considering $\mathrm{U}_{\text {ref }}=10 \mathrm{~m} / \mathrm{s}$ ) instead of $1 / \mathrm{m}$ and $1 / \mathrm{s}$, respectively. Similarly, directional 
shear extreme values are presented in terms of the actual change in flow angles, in degrees instead of $\mathrm{deg} / \mathrm{m}$. Additionally, the assessment of flow speed increase and decrease was based on comparisons to the empty wind tunnel profiles. Therefore, due to the test section asymmetry, the vertical profiles measured between the $\mathrm{M}$ and $\mathrm{R}$ locations were compared to the most nearby empty wind tunnel profile ( $\mathrm{M}$, R, or one of the interpolated profiles). It was also decided to interpolate five profiles between the $\mathrm{M}$ and $\mathrm{L}$ locations following the same method provided in Section 4.1.2 to account for the slight variations found in the top part between the M and L profiles (see Figure 4.3). 
Table 4.2: Extreme values of speed, direction, shear and turbulence flow features of the 1:400 city model experiment in Carleton University-ABL wind tunnel.

\begin{tabular}{|c|c|c|c|c|c|c|c|c|c|c|c|c|c|c|c|}
\hline \multirow{4}{*}{ Flow features } & & \multicolumn{12}{|c|}{ Wind directions } & \multirow{2}{*}{\multicolumn{2}{|c|}{ Overall }} \\
\hline & & \multicolumn{2}{|c|}{ WSW } & \multicolumn{2}{|c|}{$\mathrm{WSW}_{+15}$} & \multicolumn{2}{|c|}{$\mathrm{E}_{17.1}$} & \multicolumn{2}{|c|}{$\mathrm{N}_{16.7}$} & \multicolumn{2}{|c|}{$\mathrm{S}_{15, \text { without }}$} & \multicolumn{2}{|c|}{$\mathrm{S}_{15, w i t h}$} & & \\
\hline & & & & & & & & Extr & mes & & & & & & \\
\hline & & $\max$ & $\min$ & $\max$ & $\min$ & $\max$ & $\min$ & $\max$ & $\min$ & $\max$ & $\min$ & $\max$ & $\min$ & $\max$ & $\min$ \\
\hline \multirow{2}{*}{ Speed: } & acceleration [\%] & 35.88 & 0.10 & 56.13 & 0.41 & 24.87 & 0.22 & 20.51 & 0.01 & 42.74 & 0.00 & 73.69 & 0.18 & 73.69 & 0.00 \\
\hline & deceleration [\%] & 61.11 & 0.02 & 30.49 & 0.60 & 34.34 & 0.15 & 53.32 & 0.02 & 46.40 & 0.06 & 44.05 & 0.00 & 61.11 & 0.00 \\
\hline \multirow{3}{*}{ Direction: } & up-draft [deg] & 10.42 & 0.08 & 12.32 & 0.03 & 17.60 & 0.00 & 18.82 & 0.01 & 20.47 & 0.00 & 19.40 & 0.02 & 20.47 & 0.00 \\
\hline & down-draft [deg] & 15.42 & 0.08 & 17.73 & 0.00 & 26.26 & 0.01 & 23.29 & 0.01 & 22.29 & 0.01 & 20.51 & 0.05 & 26.26 & 0.00 \\
\hline & yaw angle [deg] & 62.85 & 0.09 & 35.40 & 0.04 & 45.97 & 0.01 & 76.80 & 0.00 & 53.00 & 0.03 & 48.85 & 0.02 & 76.80 & 0.01 \\
\hline \multirow{4}{*}{ Shear: } & speed shear [-] & 0.34 & 0.00 & 0.25 & 0.00 & 0.17 & 0.00 & 0.42 & 0.00 & 0.20 & 0.00 & 0.35 & 0.00 & 0.42 & 0.00 \\
\hline & speed shear $[\mathrm{m} / \mathrm{s}]$ & 3.53 & 0.00 & 2.64 & 0.02 & 1.84 & 0.00 & 4.45 & 0.00 & 2.18 & 0.00 & 3.75 & 0.00 & 4.45 & 0.00 \\
\hline & pitch shear [deg] & 7.14 & 0.00 & 7.02 & 0.04 & 19.33 & 0.01 & 22.4 & 0.00 & 15.21 & 0.00 & 14.88 & 0.01 & 22.4 & 0.00 \\
\hline & yaw shear $[\mathrm{deg}]$ & 25.42 & 0.11 & 14.25 & 0.00 & 17.31 & 0.00 & 49.99 & 0.01 & 17.66 & 0.00 & 25.13 & 0.01 & 49.99 & 0.00 \\
\hline \multirow{3}{*}{ Turbulence: } & longitudinal TI [\%] & 40.22 & 5.75 & 37.68 & 5.90 & 34.33 & 5.29 & 42.07 & 1.58 & 36.56 & 1.55 & 46.48 & 1.52 & 46.48 & 1.52 \\
\hline & lateral TI [\%] & 44.67 & 4.77 & 41.54 & 4.71 & 27.16 & 4.52 & 38.65 & 1.19 & 38.50 & 1.25 & 47.32 & 1.22 & 47.32 & 1.19 \\
\hline & vertical TI [\%] & 36.48 & 4.28 & 33.94 & 4.25 & 30.89 & 4.27 & 35.90 & 1.00 & 34.93 & 0.96 & 37.73 & 0.99 & 37.73 & 0.99 \\
\hline
\end{tabular}


Table 4.3: Locations of the extreme values of speed, direction, shear and turbulence flow features of the 1:400 city model experiment in Carleton University-ABL wind tunnel.

\begin{tabular}{|c|c|c|c|c|c|c|c|c|c|c|c|c|c|}
\hline \multirow{4}{*}{ Flow features } & & \multicolumn{12}{|c|}{ Wind directions } \\
\hline & & \multicolumn{2}{|c|}{ WSW } & \multicolumn{2}{|c|}{$\mathrm{WSW}_{+15}$} & \multicolumn{2}{|c|}{$\mathrm{E}_{17.1}$} & \multicolumn{2}{|c|}{$\mathrm{N}_{16.7}$} & \multicolumn{2}{|c|}{$\mathrm{S}_{15, \text { without }}$} & \multicolumn{2}{|c|}{$\mathrm{S}_{15, w i t h}$} \\
\hline & & \multicolumn{12}{|c|}{ Locations of extremes (Spot, Increment/s) } \\
\hline & & $\max$ & $\min$ & $\max$ & $\min$ & $\max$ & $\min$ & $\max$ & $\min$ & $\max$ & $\min$ & $\max$ & $\min$ \\
\hline \multirow{2}{*}{ Speed: } & acceleration & 18,4 & 7,14 & 21,2 & 20,14 & 36,5 & 16,2 & 13,6 & 14,14 & 25,1 & 33,19 & 25,1 & 6,14 \\
\hline & deceleration & 9,3 & 30,13 & 9,10 & 7,15 & 10,2 & 28,13 & 17,10 & 27,13 & 16,12 & 20,18 & 29,13 & 34,9 \\
\hline \multirow[t]{2}{*}{ Direction: } & down-draft & 19,5 & 30,14 & 20,5 & 30,9 & 24,3 & 7,4 & 17,8 & 9,7 & 26,1 & 29,3 & 25,1 & 5,17 \\
\hline & yaw angle & 9,1 & 9,13 & 25,9 & 19,12 & 28,1 & 10,12 & 18,5 & 24,20 & 23,1 & 26,20 & 26,6 & 26,20 \\
\hline \multirow{3}{*}{ Shear: } & speed shear & $9,11-12$ & $11,10-11$ & $30,12-13$ & $19,3-4$ & $36,2-3$ & $36,11-12$ & $23,12-13$ & $19,10-11$ & $6,1-2$ & $20,17-18$ & $26,13-14$ & $27,18-19$ \\
\hline & pitch shear & $30,6-7$ & $9,14-15$ & $33,2-3$ & $18,6-7$ & $28,1-2$ & $10,12-13$ & $17,9-10$ & $12,11-12$ & $6,1-2$ & $12,18-19$ & $35,1-2$ & $6,14-15$ \\
\hline & yaw shear & $9,2-3$ & $9,14-15$ & $19,2-3$ & $21,2-3$ & $36,1-2$ & $10,13-14$ & $23,11-12$ & $15,14-15$ & $23,1-2$ & $27,7-8$ & $31,11-12$ & $7,14-15$ \\
\hline \multirow{2}{*}{ Turbulence: } & longitudinal TI & 30,3 & 25,15 & 30,9 & 30,15 & 10,2 & 18,15 & 26,11 & 36,20 & 10,1 & 5,20 & 31,6 & 28,20 \\
\hline & vertical TI & 9,4 & 30,15 & 33,4 & 25,15 & 10,2 & 18,15 & 18,5 & 16,20 & 18,6 & 30,20 & 12,2 & 28,20 \\
\hline
\end{tabular}


As can be seen in Table 4.2, the presence of buildings caused an increase in the mean wind speed value by a maximum of $74 \%$. Similarly, some locations were subjected to reduction in the mean wind speed, the maximum of which was found to be $61 \%$. However, there were some cases of $0 \%$ change or minimal change in the mean wind speed despite the presence of the model, the majority of which were found to be well above buildings level. Figure 4.8 illustrates the case of the minimal change in the mean wind speed well above buildings level (Increments 15-20), in which 0\% acceleration/deceleration from the empty wind tunnel data was found in Increment 18. The profile was measured at Spot 20, without the future buildings, and at $\mathrm{S}_{15}$ wind direction. The vertical traverse points with respect to the model geometry are shown in Figure 4.9 with Increments 15-20 represented by filled markers. Isometric views of the model are provided in Figures 4.10 and 4.11 to aid in visualizing the location of the indicated spots throughout this chapter.

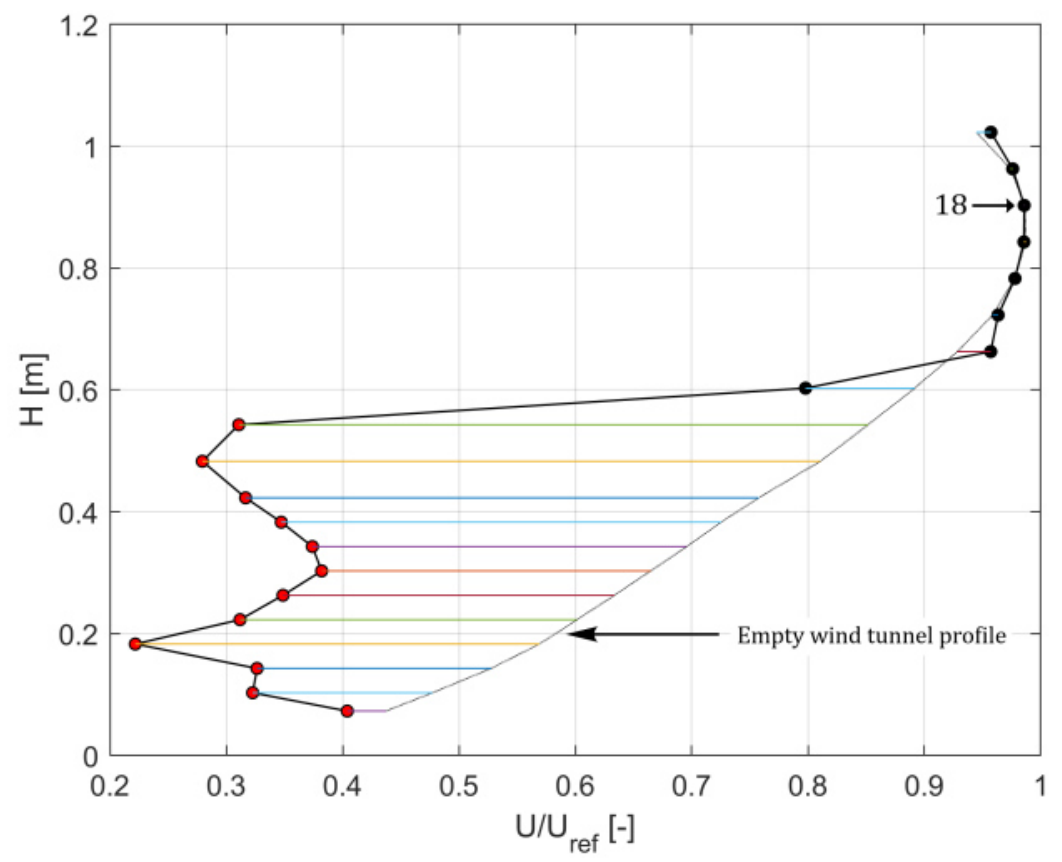

Figure 4.8: Example of the minimal percent change in the mean wind speed above buildings level. Measurement at Spot 20 for $\mathrm{S}_{15}$ wind direction. Total increments $=20$. 


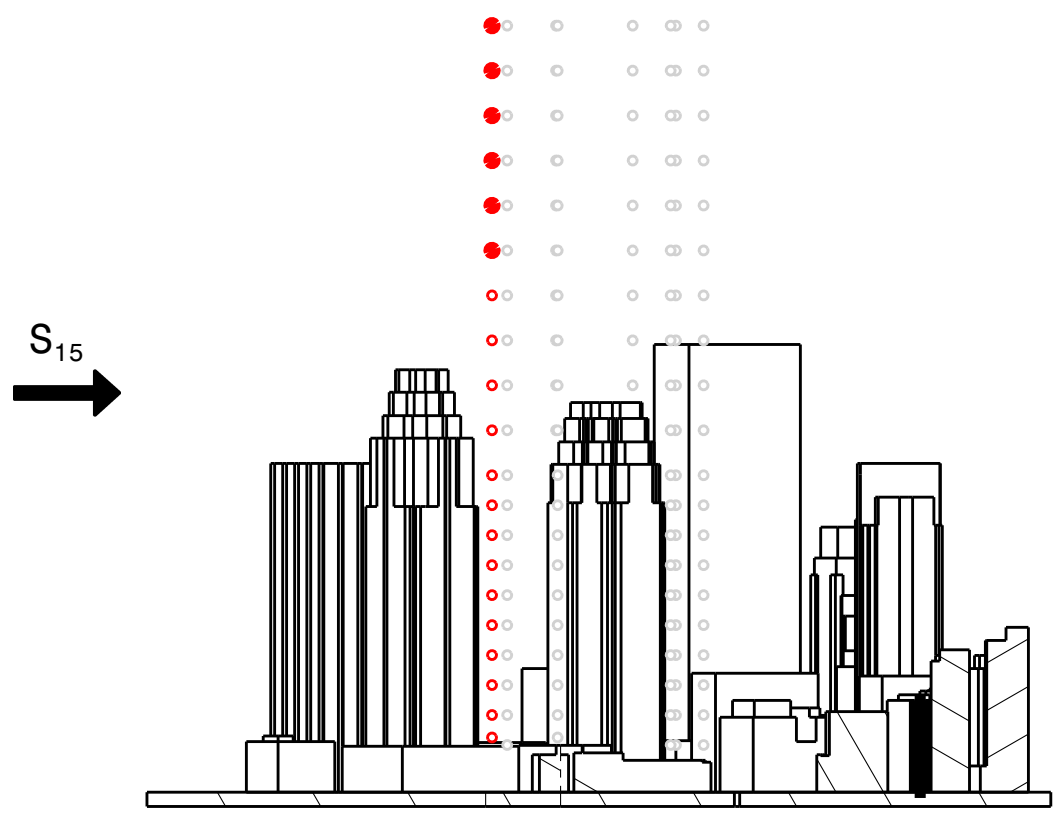

Section view A-A

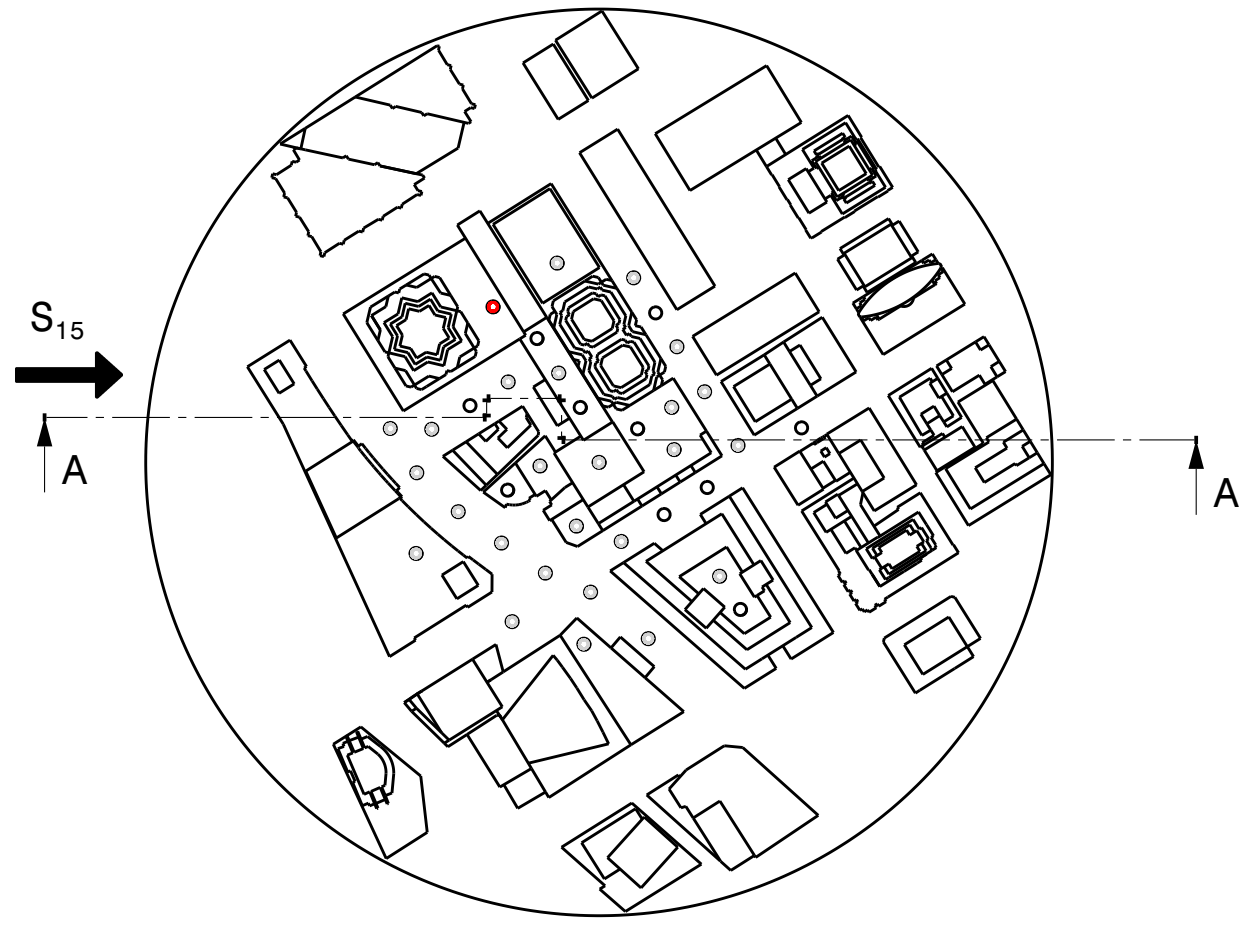

Figure 4.9: The location of spot 20 (red markers) with a model orientation representing $\mathrm{S}_{15}$ wind direction.

(Smooth case of speed percent change) 

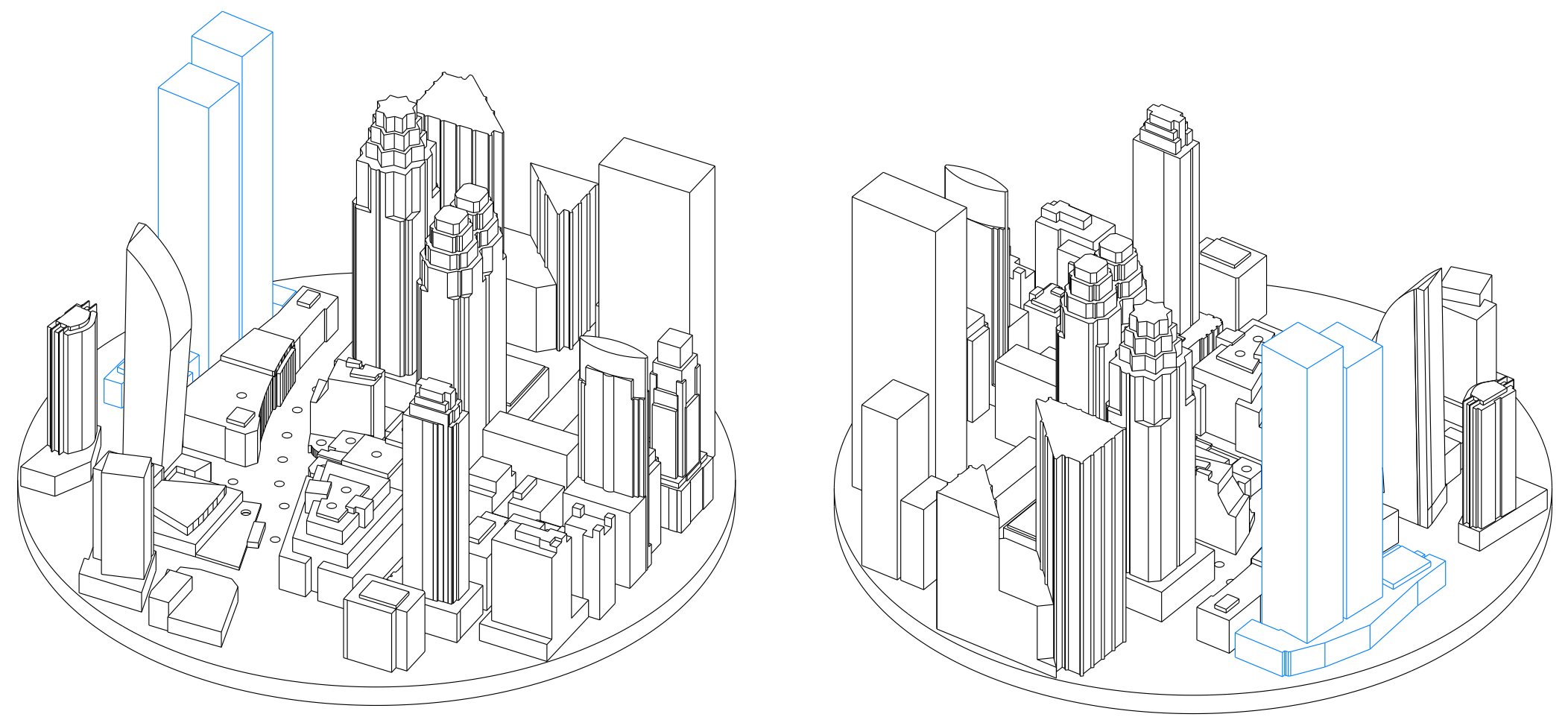

Figure 4.10: Isometric views of the 1:400 model part 1 . 

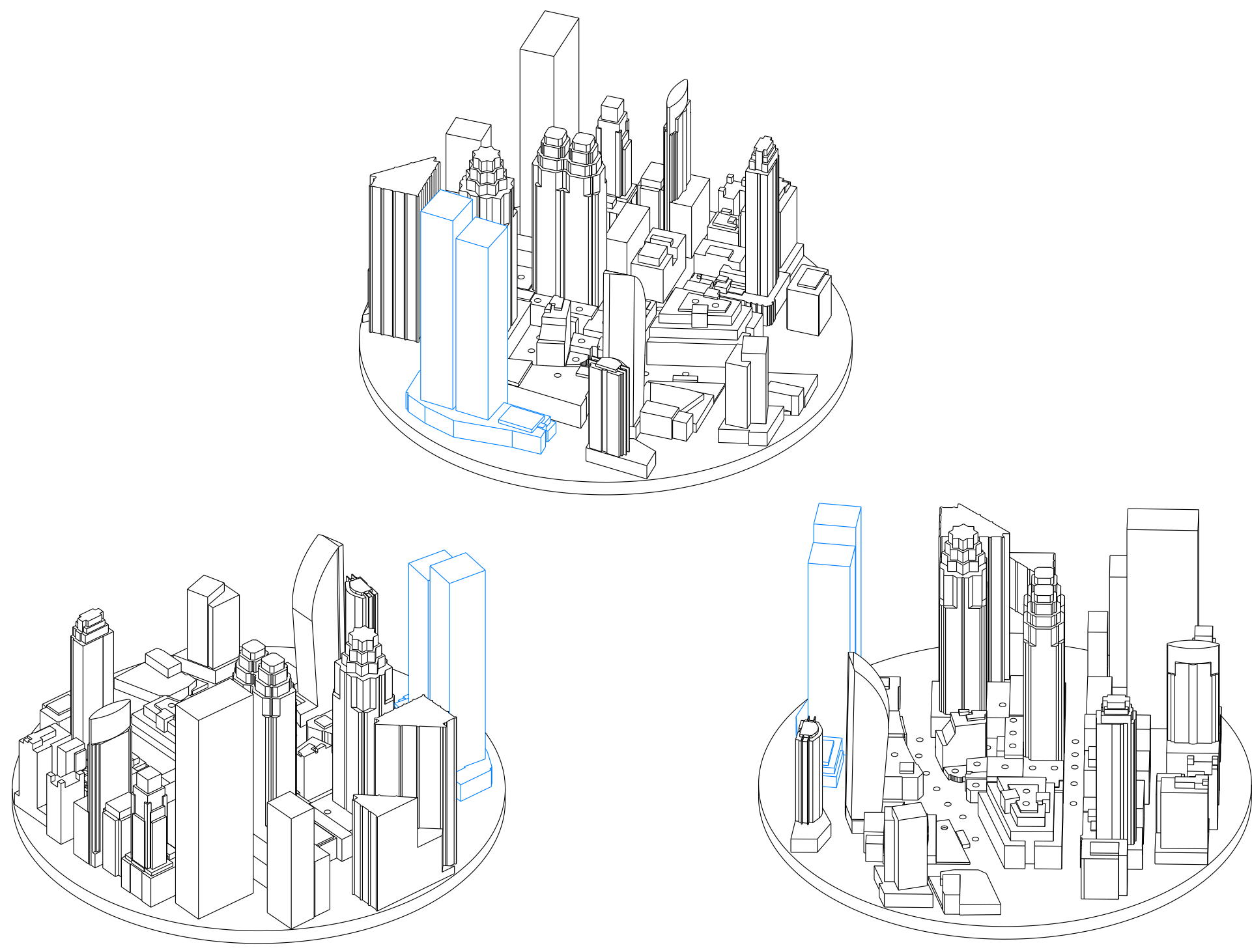

Figure 4.11: Isometric views of the 1:400 model part 2. 
For wind flow angles, maximum up-draft, down-draft, and yaw angle were found to be 20, 26, and 77 degrees, respectively. However, small flow angles less than 0.1 degrees were found at certain locations in the model. Figure 4.12 shows the flow yaw angle versus height of Spot 18 for wind coming from $\mathrm{N}_{16.7}$. From the figure, it can be seen that the variations on flow yaw angles are minimal and around $0^{\circ}$ at higher altitudes, especially above buildings level (above $0.60 \mathrm{~m}$ ). The minimum value of $0^{\circ}$ was found at the highest point of the traverse, which corresponds to Increment 20, the location of which is shown with respect to the model geometry in Figure 4.13 .

Although flow yaw angle was generally low at higher altitudes, cases of minimal flow pitch angle (up-draft and down-draft) were found at various height in the model. The upward and downward motion of the flow was strongly influenced by the proximity to buildings and whether or not the measurement height is close to the building top surface; hence, in the region of flow separation in the vertical direction. Figure 4.14 shows Spot 30 for wind coming from $\mathrm{WSW}_{+15}$, in which flow pitch angle was $0^{\circ}$ at Increment 9 (filled markers in Figure 4.14) despite being located below buildings level.

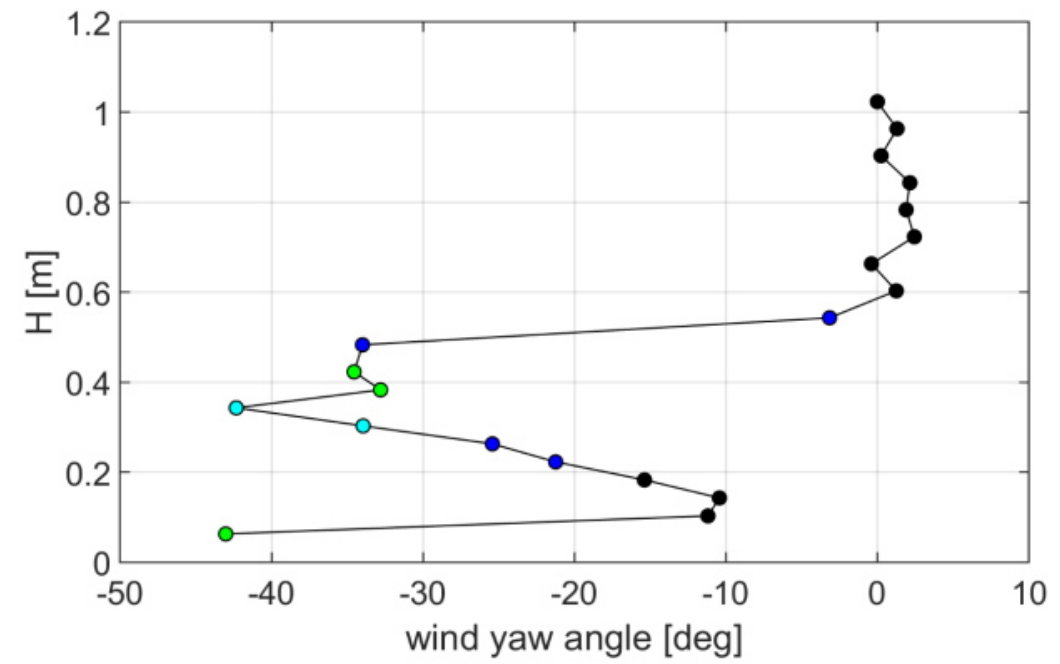

Figure 4.12: Example of the minimal flow yaw angle above buildings level. Measurement at Spot 18 for $\mathrm{N}_{16.7}$ wind direction. Total increments $=20$. 

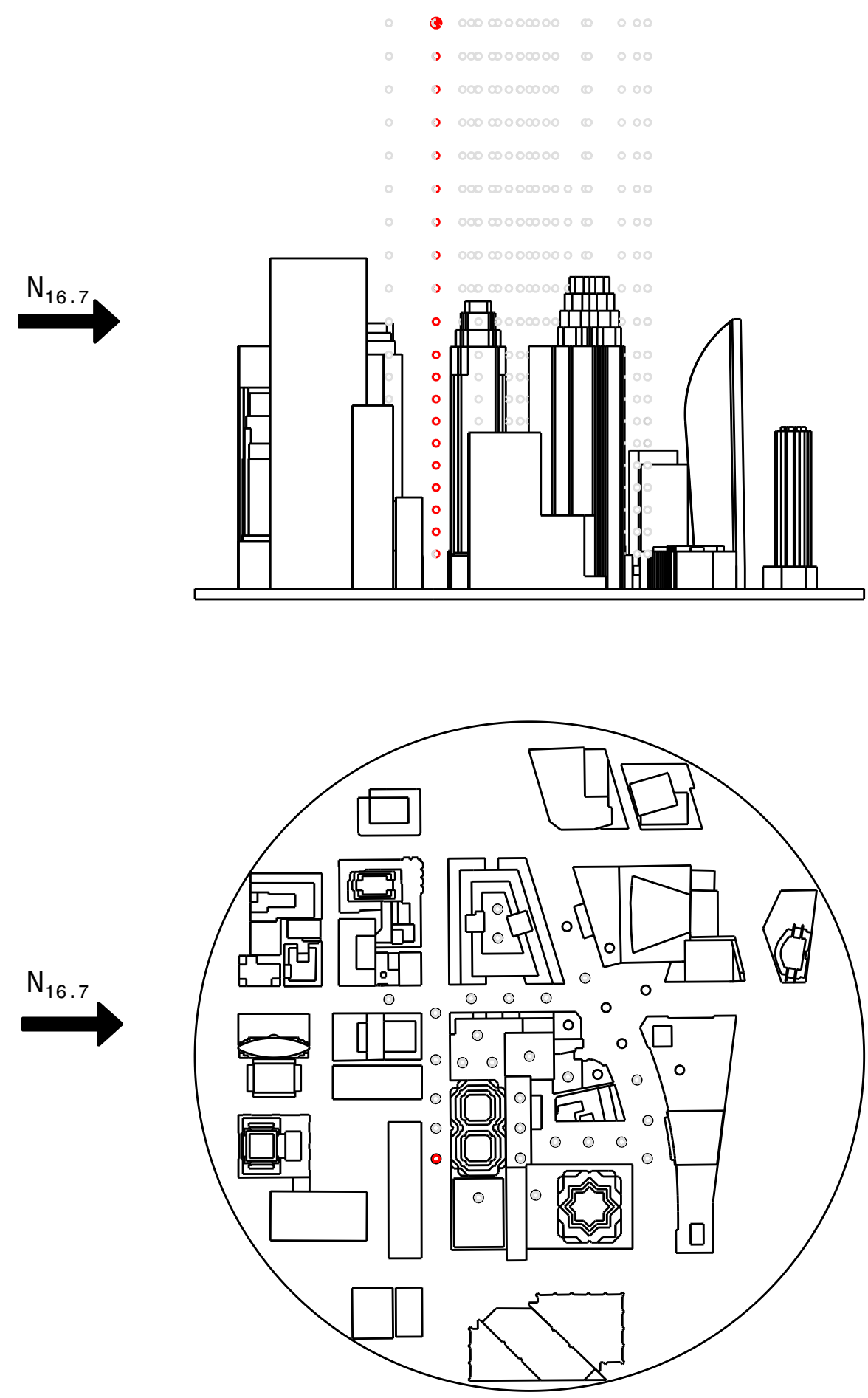

Figure 4.13: The location of Spot 18 (red markers) with a model orientation representing $\mathrm{N}_{16.7}$ wind direction.

(Smooth case of flow yaw angle) 

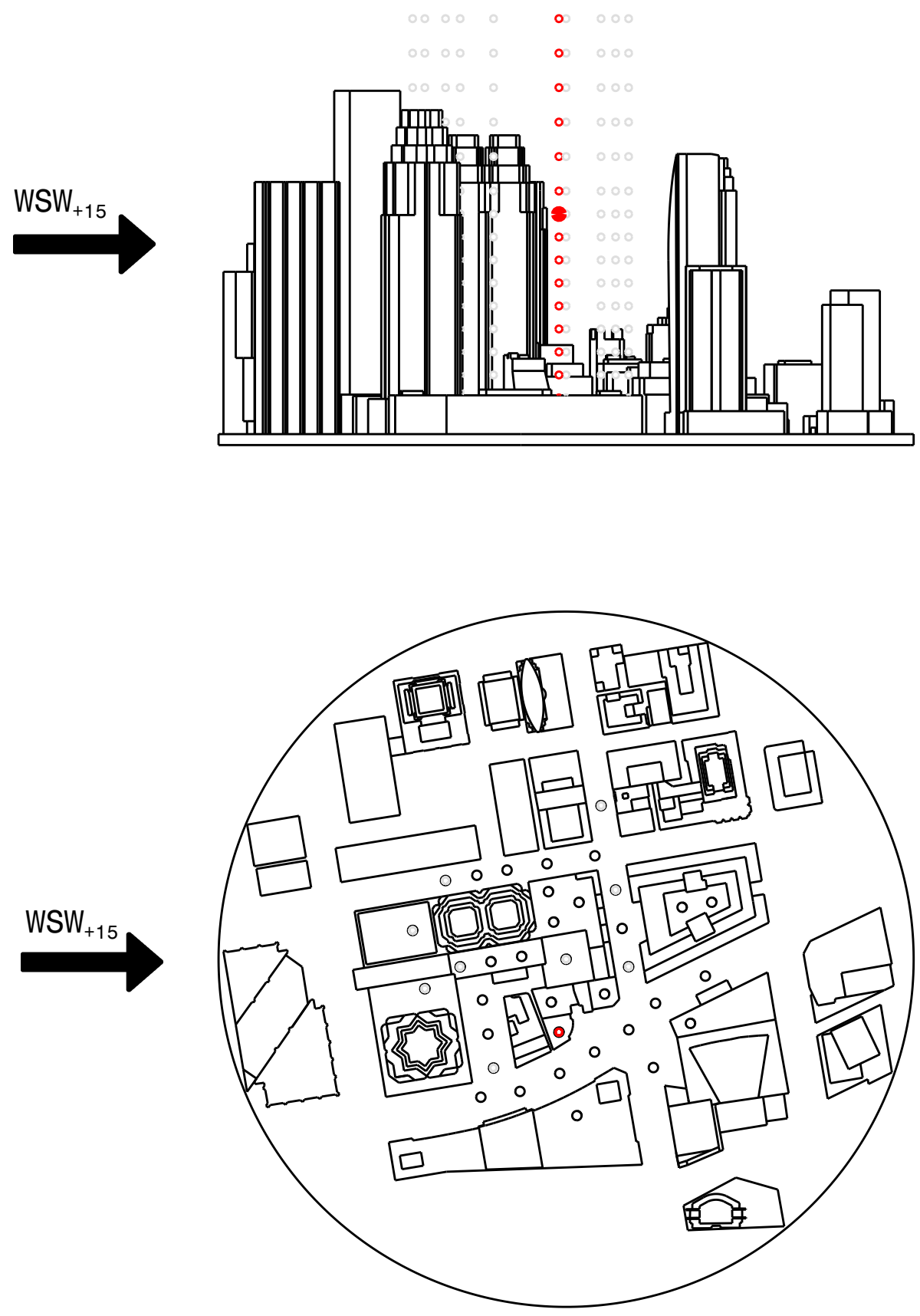

Figure 4.14: The location of Spot 30 (red markers) with a model orientation representing $\mathrm{WSW}_{+15}$ wind direction.

(Smooth case of flow pitch angle) 
The maximum shear in mean wind speed between two consecutive vertical increments was found to be $4.45 \mathrm{~m} / \mathrm{s}$. Nonetheless, there were some cases of minimal change in the mean wind speed that were found within the height of the buildings such as the case demonstrated in Figure 4.15. The plot in Figure 4.15 shows the normalized mean wind speed $\left(U / U_{r e f}\right)$ in the bottom axis, and the overall turbulence intensity $\left(\mathrm{I}_{u v w}\right)$ in the top axis.

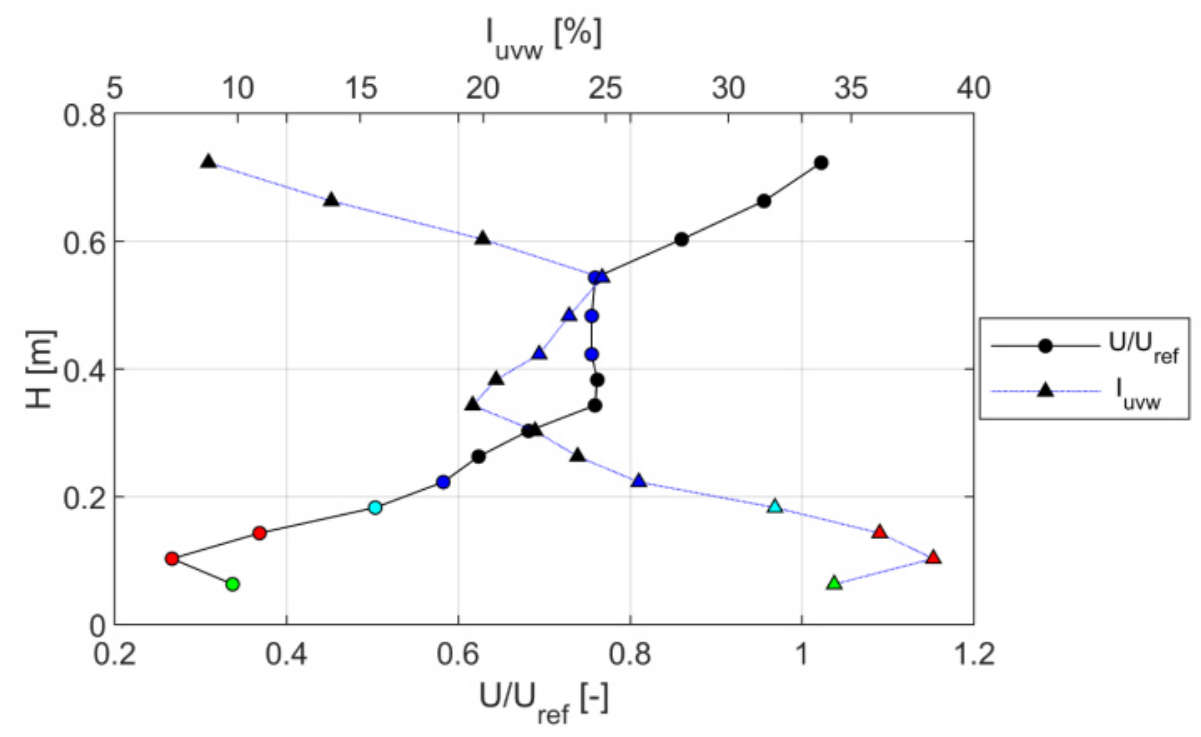

Figure 4.15: Example of the minimal speed shear within the height of the buildings. Measurement at Spot 11 for WSW wind direction. Total increments $=15$.

In the plot, the normalized mean wind speed was almost the same from Increment 8 to 12 while the overall turbulence intensity was gradually increasing with height for the same increments range. This behaviour can be explained by the effect of turbulent wake of an upstream building as a result of flow separation from the building top surface as illustrated in Figure 4.16. Thus, a minimal speed shear found within the height of the buildings could be the result of interacting flow features and not an evidence of smooth flow conditions. In the case of directional shear, the maximums were found to be $22^{\circ}$ and $50^{\circ}$ for vertical and horizontal directional shear, respectively. However, there were cases of minimal or no directional shear, which were generally 
found at locations above the height of the buildings. Few exceptions were found within the height of the buildings; however, the measurement location was in a street canyon where the flow passes through with no upstream buildings obstruction.

The turbulence intensity maximum values were found to be $46 \%$ along-wind, $47 \%$ across-wind, and 38\% perpendicular-to-wind. The minimum turbulence intensities indicated in Table 4.2 were always found at the highest points in the traverse (Increment 15 for phase 1 measurements and Increment 20 for phase 2 measurements). Some cases of low turbulence were found within the height of the buildings; however, the measurement locations were either in urban canyons or in spots that are open to the incoming flow with no upstream blockage. 


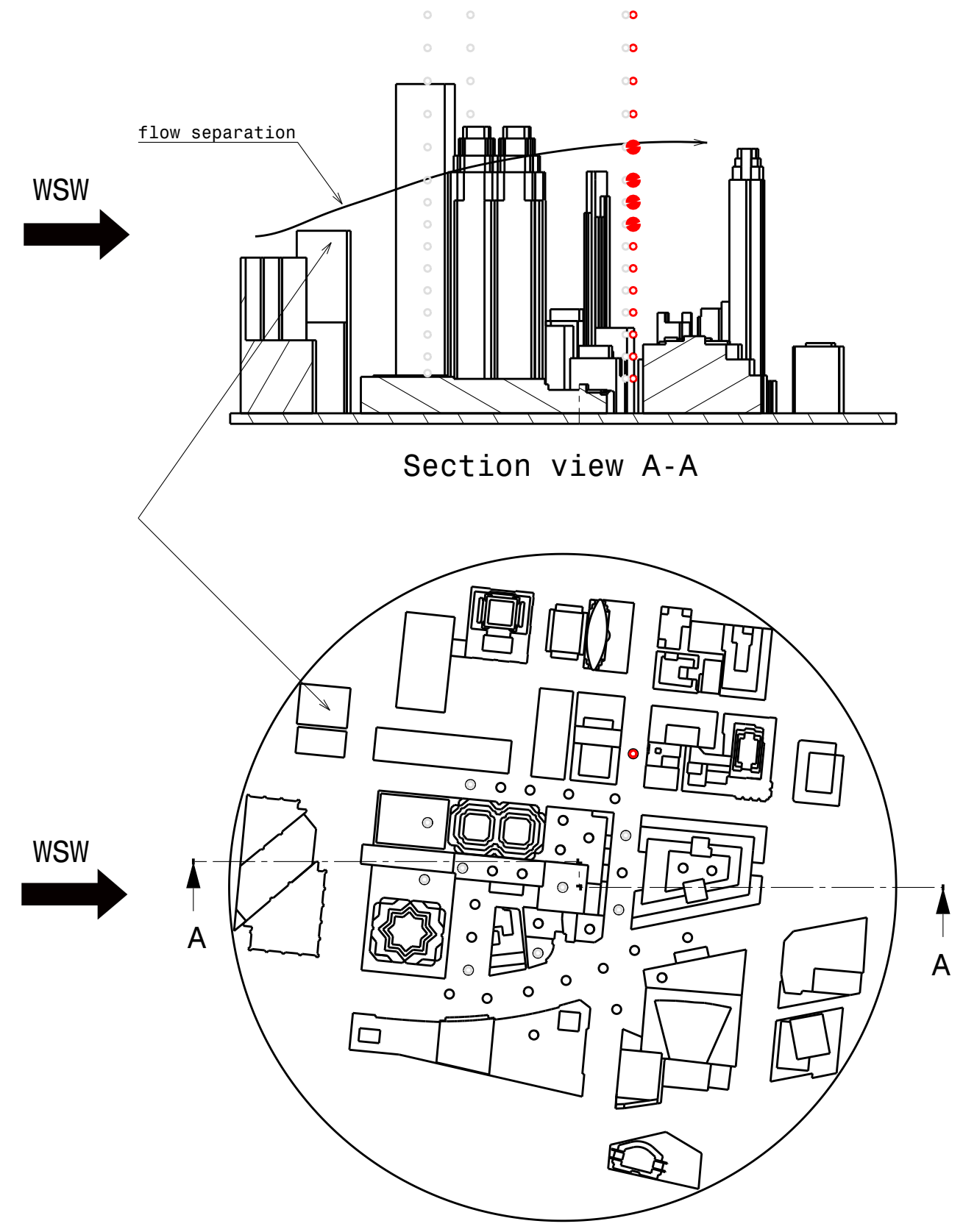

Figure 4.16: The location of Spot 11 (red markers) with a model orientation representing WSW wind direction.

(Smooth case of speed shear) 


\subsubsection{Worst-case urban flows in relation to model geometry}

From the results presented in Table 4.2 , it is clear how the model orientation relative to the incoming flow influences the severity of flow conditions; therefore, what is considered severe in one wind direction could be considered moderate in the others. Hence, in the analyses, the maximum value of a certain flow property found across all of the considered wind directions is what is categorized as severe. Less severe cases are categorized as either adverse or moderate according to their severity relative to the aforementioned maximum. The discussions of worst cases might include multiple spots from the same wind direction, the values of which were not listed in Table 4.2 , and thus, they will be mentioned where appropriate. Additionally, certain flow patterns like flow separations from buildings are described schematically in certain figures to illustrate the concept; however, it is emphasized that those flow patterns are only shown to qualitatively illustrate the general features of the flow and not to provide exact flow patterns.

\subsubsection{Wind speed}

For urban air mobility applications, wind speed is an essential parameter to consider before flight. The weather station reports include information about the mean wind speed in a specific region; however, local wind conditions within urban areas are not readily available. Therefore, when it comes to flying within the urban canopy, following weather report data may not yield safe flight operations. This is due to the complex flow nature around buildings and structure, which was alluded to, above, in terms of local increase or decrease in mean wind speed that may exceed the performance limit of the flying vehicle.

\section{Flow speed increase}

The severe cases of percent increase in the mean wind speed were found in Spots 25 
and 26 for a wind coming from $\mathrm{S}_{15}$ with the inclusion of the future buildings. As can be seen in Figure 4.17, both spots were subjected to similar amount of increase in the mean wind speed at Increment 1, where the flow accelerated by $74 \%$ at Spot 25 and $73 \%$ at Spot 26.

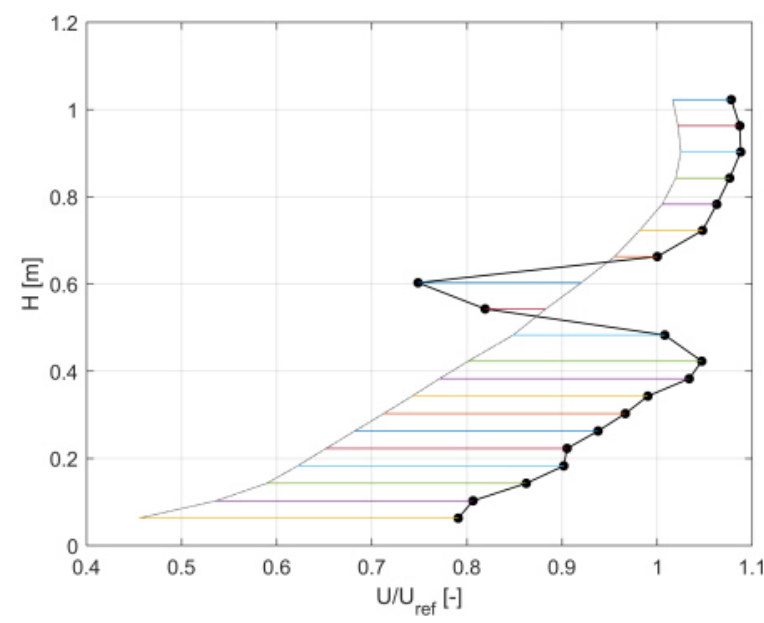

(a)

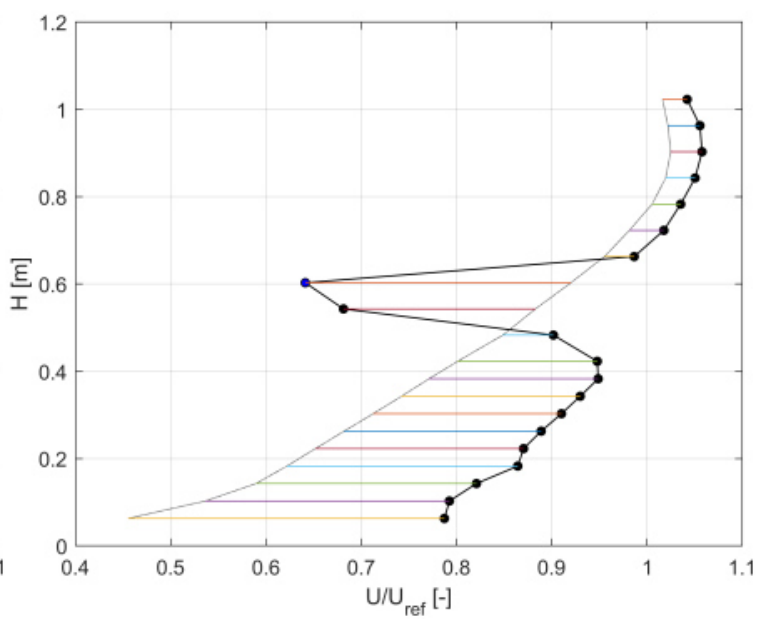

(b)

Figure 4.17: Mean wind speed profiles with and without the model at Spots 25 (a) and 26 (b) in $\mathrm{S}_{15}$ wind direction. Total increments $=20$.

By examining the spots locations in relation to the surrounding buildings (see Figure 4.18, it was reasoned that the spots were subjected to the influence of flow separations from one side of the future buildings highlighted with blue, one side of Building II, the top surface of Building I, and the top surface of the base of Building II. The location of measurements was $4.50 \mathrm{~mm}$ below the top surface of Building I and $1.75 \mathrm{~mm}$ above the base of Building II; therefore, in addition to being located at the edge of the wake of the future buildings and Building II, they are almost located at the same height as Building I and base of Building II, where flow acceleration is expected as flow moves over the buildings surfaces. Although the two spots were subjected to almost the same amount of flow acceleration at Increment 1, the increase in the mean wind speed profile at Spot 25 was always higher than that of Spot 26 (consult Figure 4.17). 


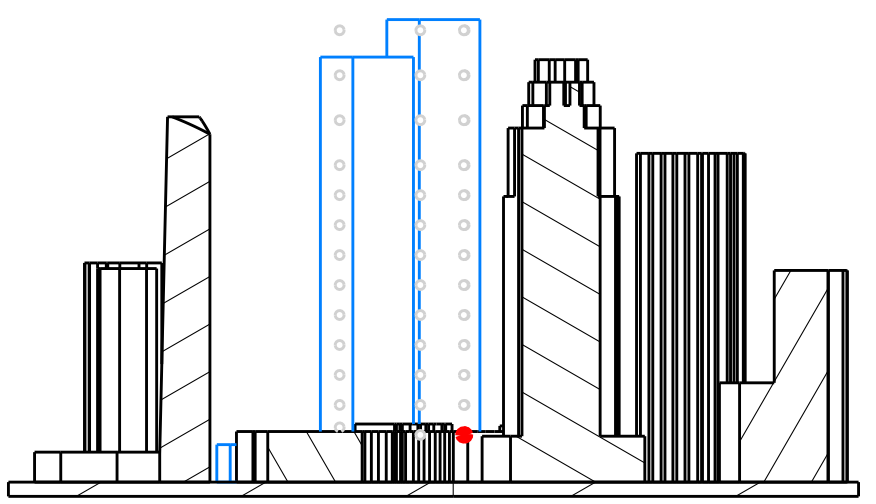

Section view A-A

$S_{15}$ is out of the page

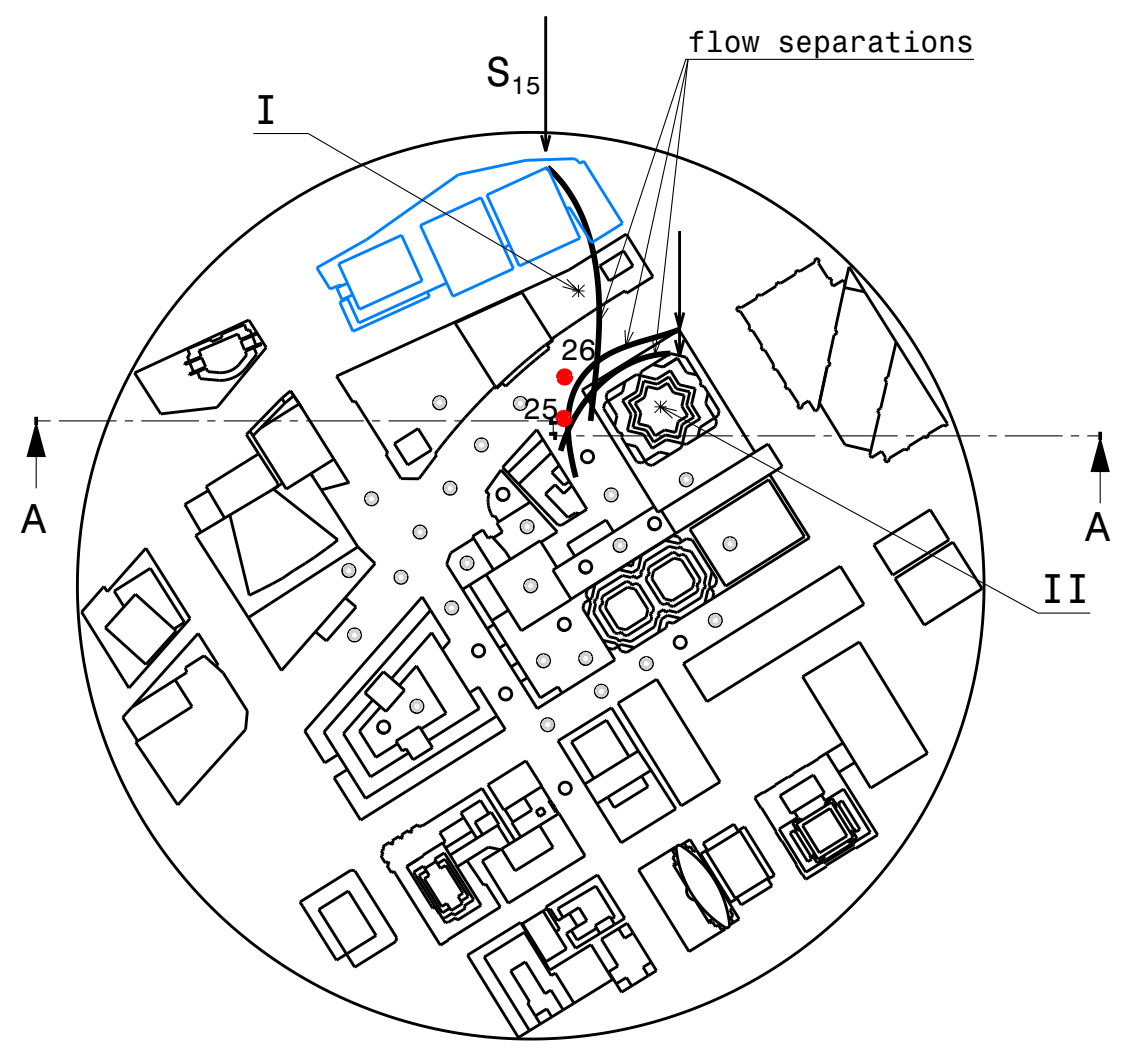

Figure 4.18: The location of Spots 25 and 26 (red markers) with a model orientation representing $\mathrm{S}_{15}$ wind direction.

(Severe cases of flow acceleration) 
This can be explained by the location of the spots in relation to the future buildings and Building II, where Spot 26 was primarily influenced by the flow separation from the future buildings. On the other hand, Spot 25 was primarily influenced by the flow separation from both the future buildings and Building II. This can explain the higher flow accelerations at Spot 25.

Similar observation, but with less severity, was found at Increment 1 of Spots 4 and 6 for the same wind direction, $\mathrm{S}_{15}$. Spot 6 was subjected to flow acceleration of $57 \%$, whereas the acceleration in the flow at Spot 4 was found to be $54 \%$. Figure 4.19 shows the location of both spots, where Spot 6 was likely influenced by the flow separation from both the future buildings and Building I, where it is likely located at the edge of the wake of both buildings. Spot 4, on the other hand, was probably located at the edge of the wake of the future buildings and more in the centre of the wake of Building I main wake. Spot 6 mean wind speed profile was also showing higher flow accelerations as compared to Spot 4 (see Figure 4.20). This could be the result of it being located at the edge of the wake of two buildings plus being in close-proximity to the future buildings.

Some cases of adverse flow accelerations were also observed in spots located close to the separation point from the leeward side of certain buildings. Figure 4.21 shows the mean wind speed profile of Spot 21 with a model orientation representing the $\mathrm{WSW}_{+15}$ wind direction, where a flow acceleration of $56 \%$ was found at Increment 2 , the location of which is indicated in Figure 4.22 . 


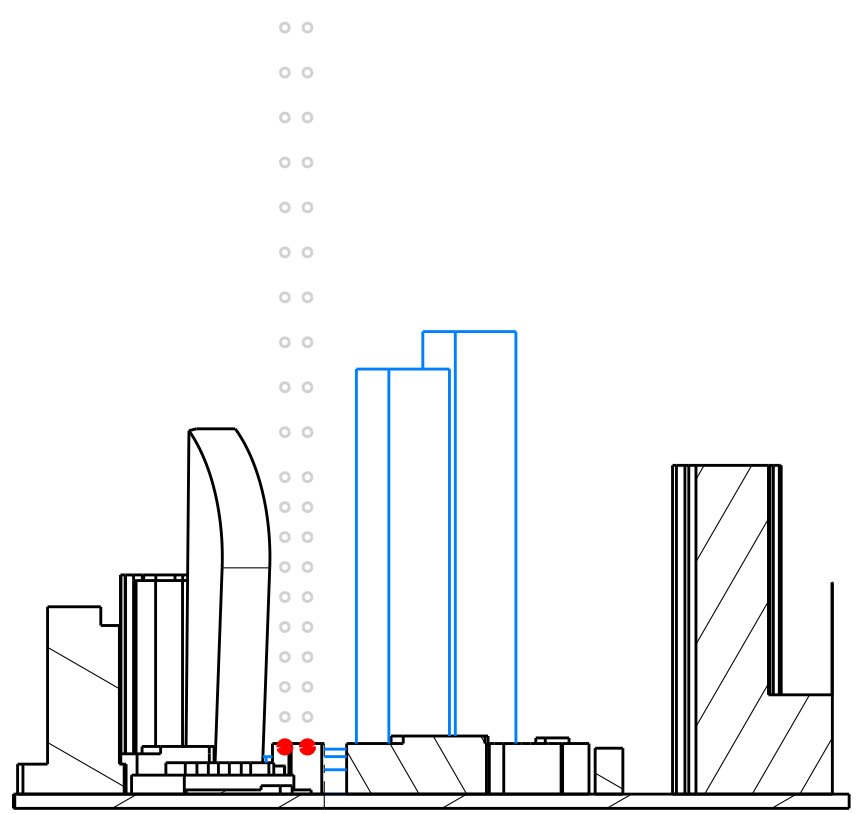

Section view A-A

$S_{15}$ is out of the page

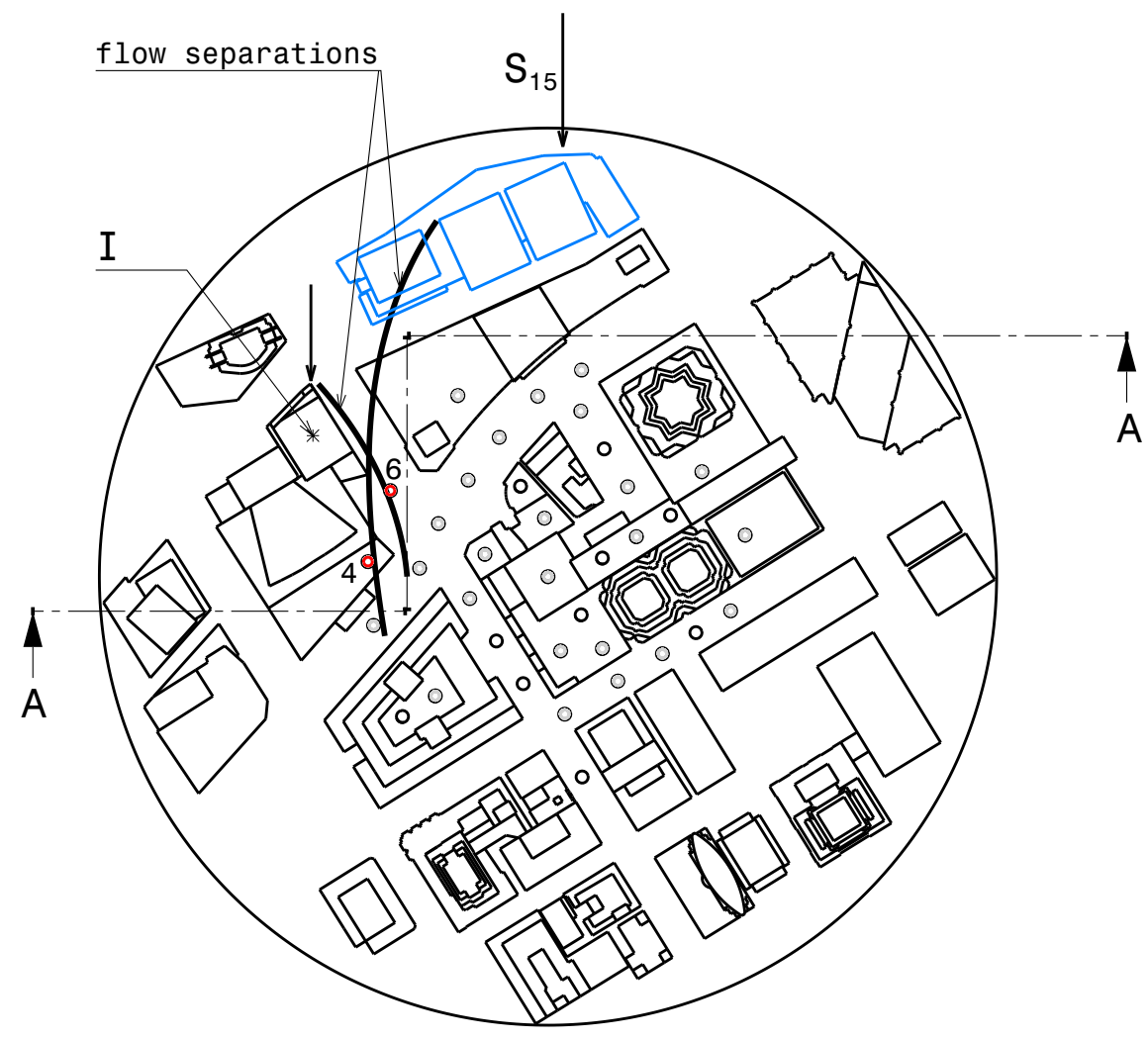

Figure 4.19: The location of Spots 4 and 6 (red markers) with a model orientation representing $\mathrm{S}_{15}$ wind direction.

(Adverse cases of flow acceleration) 


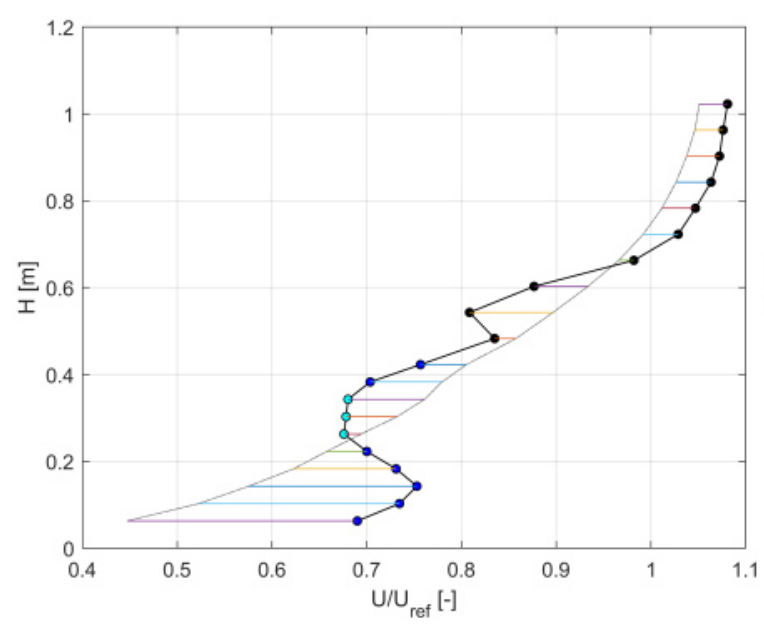

(a)

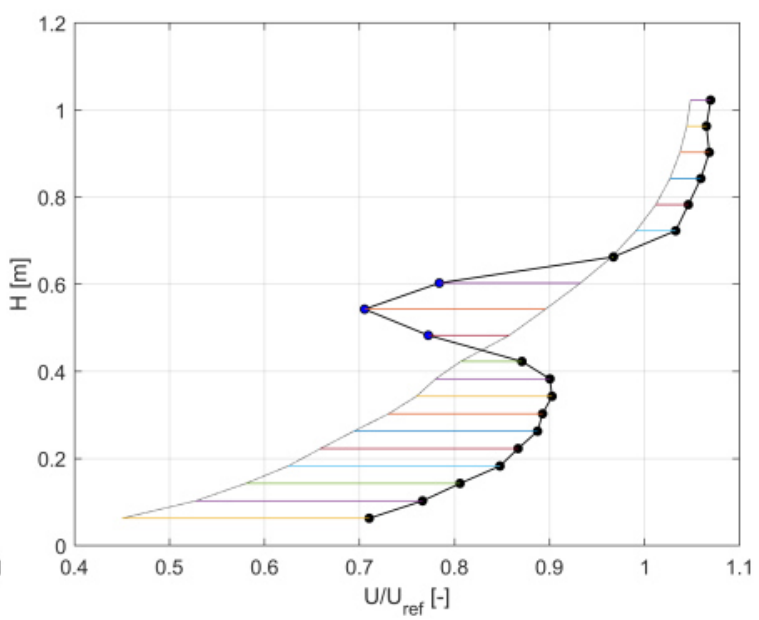

(b)

Figure 4.20: Mean wind speed profiles with and without the model at Spots 4 (a) and 6 (b) in $\mathrm{S}_{15}$ wind direction. Total increments $=20$.

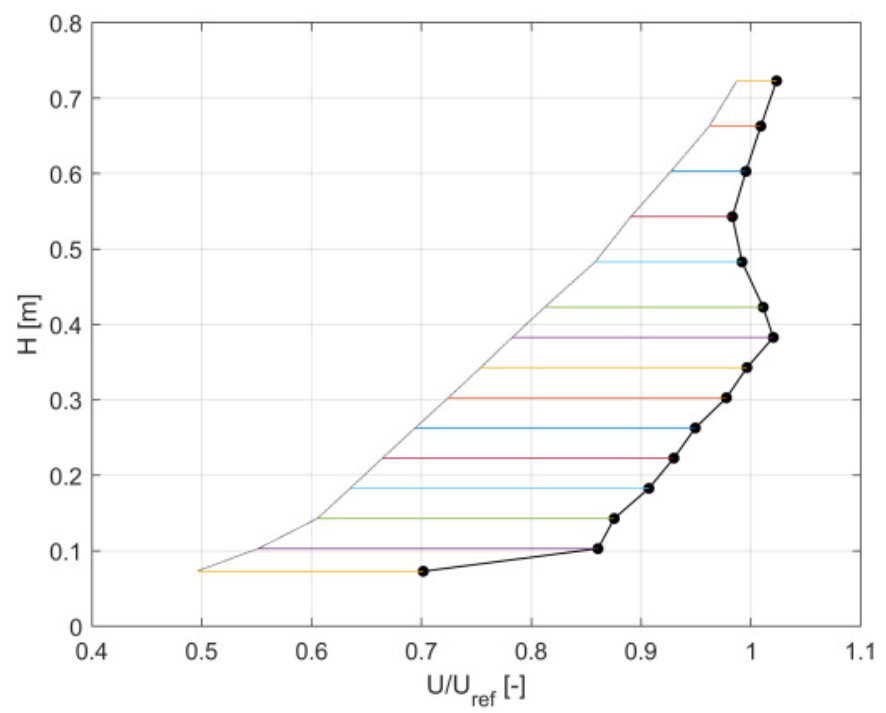

Figure 4.21: Mean wind speed profile at Spot 21 for wind direction $W_{S W} W_{+15}$. Total increments $=15$. 


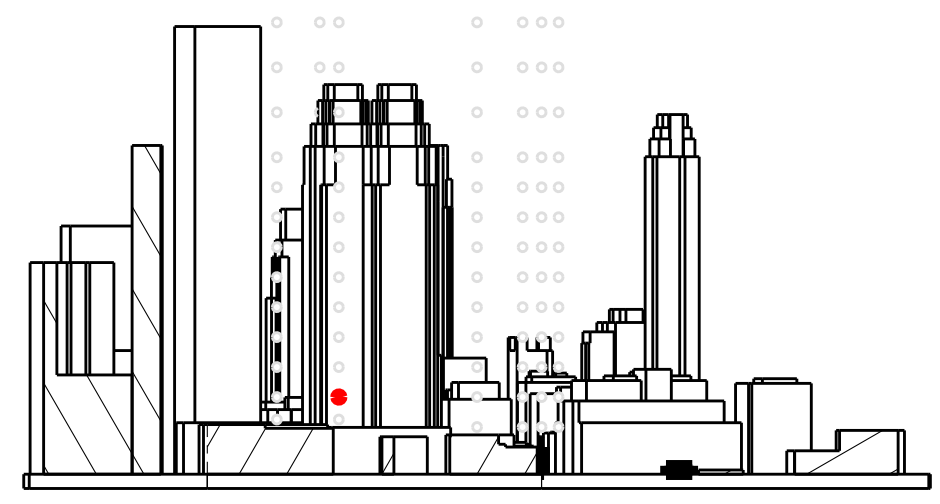

Section view A-A

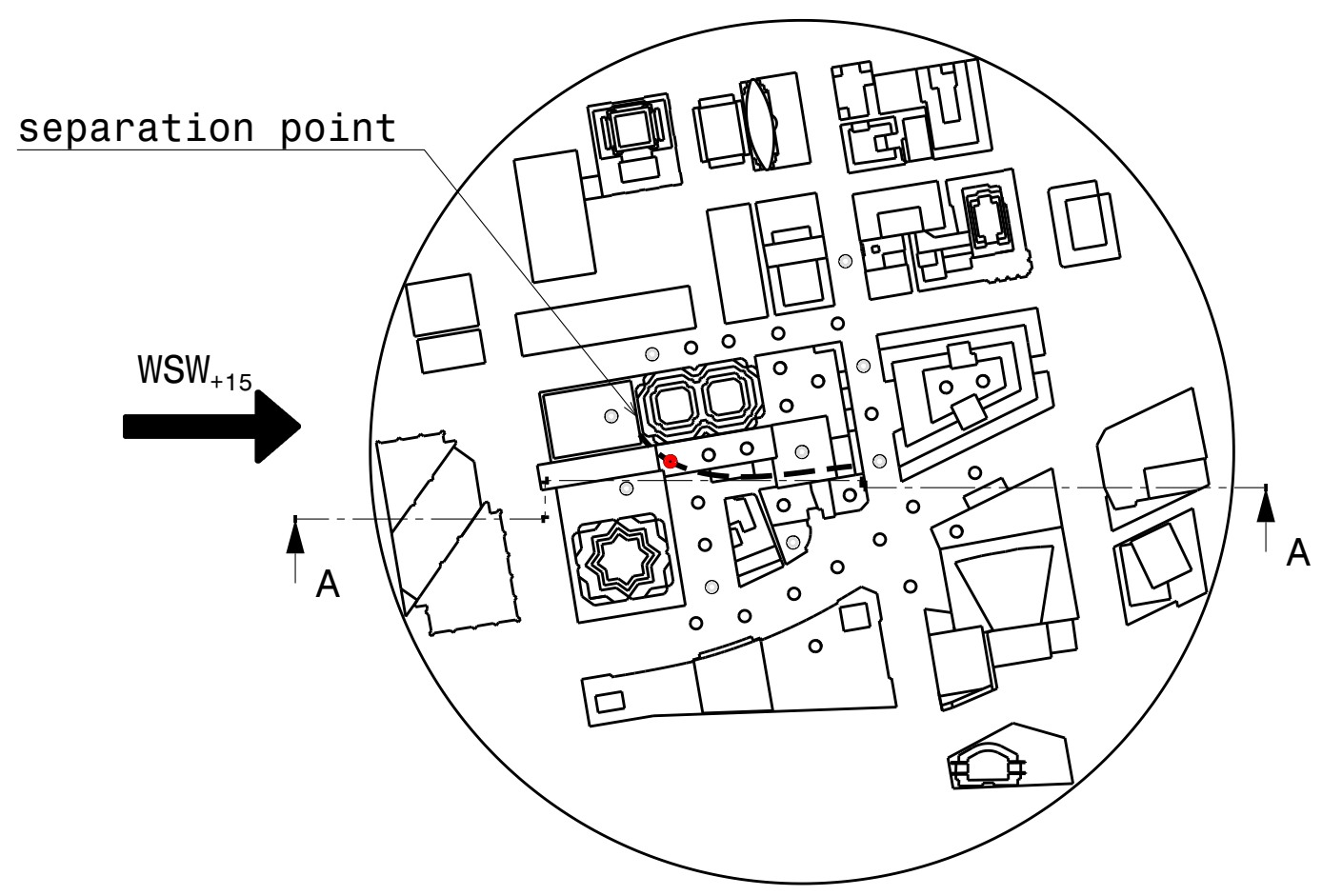

Figure 4.22: The location of Spot 21 (red markers) with a model orientation representing $\mathrm{WSW}_{+15}$ wind direction.

(Adverse case of flow acceleration) 


\section{Flow speed reduction}

Severe cases of percent decrease in the mean wind speed were found downstream of buildings, particularly in the main wake region. Increment 3 of Spot 9 in a WSW wind direction was subjected to the maximum flow reduction of $61 \%$, the location of which is shown in Figure 4.23. The reduction in the mean wind speed in the wake of buildings was highly expected since the wake region is characterized by high levels of turbulence. Figure 4.24(a) shows the mean wind speed profile of Spot 9 with and without the model and 4.24(b) is showing the mean wind speed profile against the overall turbulence intensity profile. These plots confirm the expected flow nature in the wake of buildings since the mean wind speed was in a state of reduction until Increment 13 which is the first point past the height of the upstream building to Spot 9. Along with the decrease in the mean wind speed, high levels of turbulence were observed at measurements taken below the upstream building level (below $0.52 \mathrm{~m}$ ). 

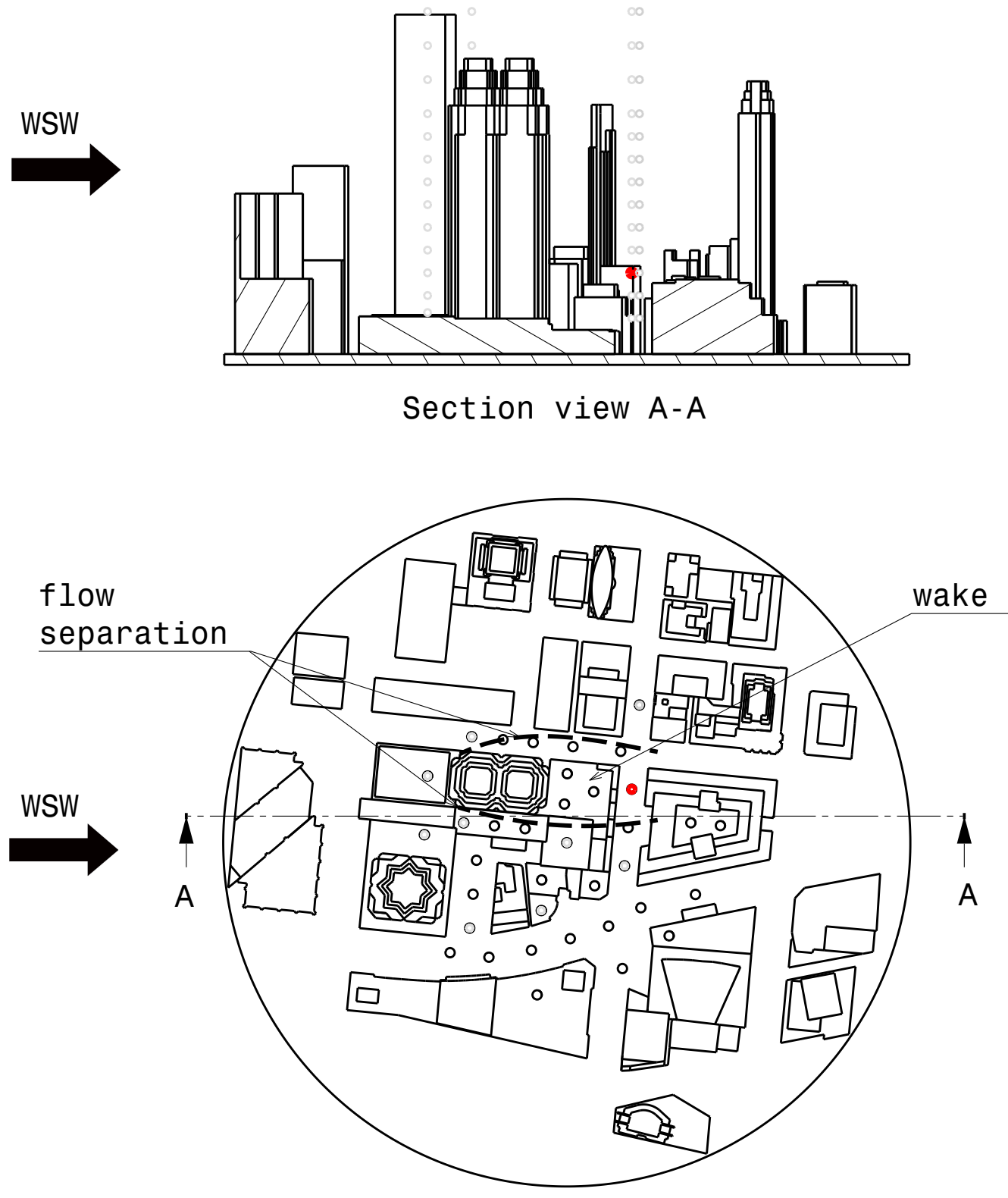

Figure 4.23: The location of Spot 9 (red markers) with a model orientation representing WSW wind direction.

(Severe case of flow deceleration) 


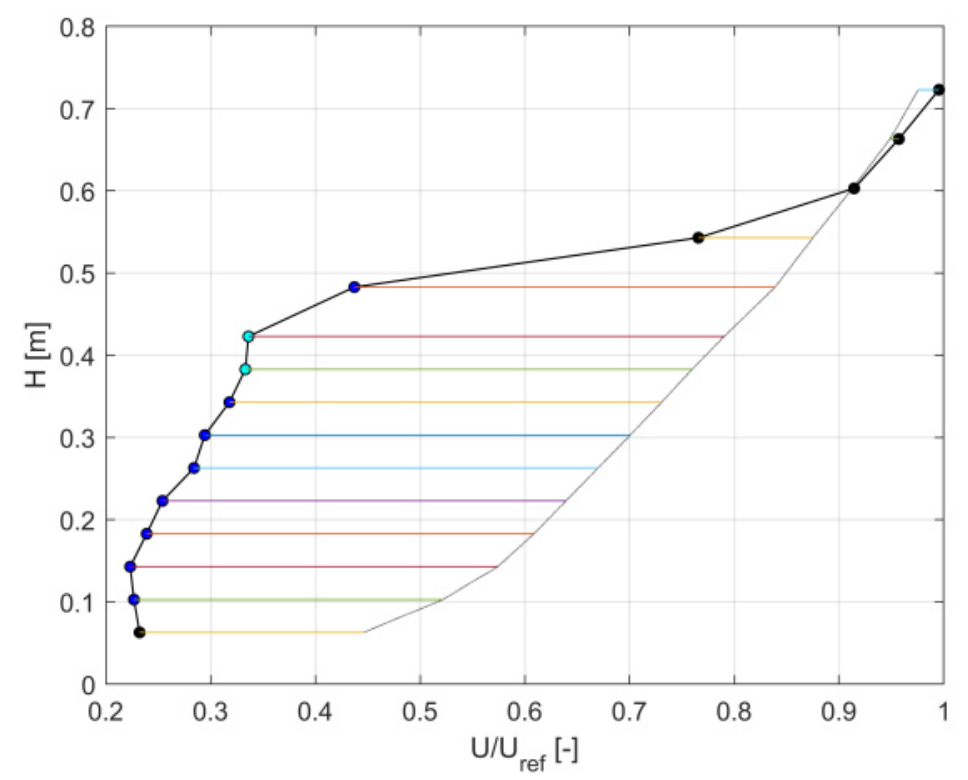

(a)

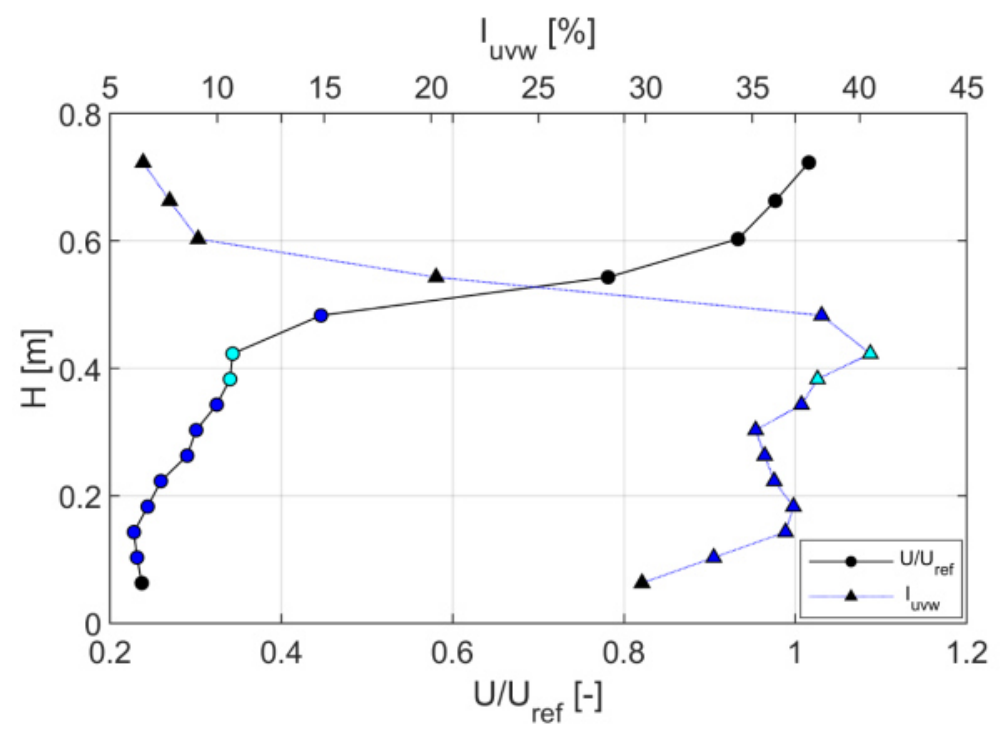

(b)

Figure 4.24: (a) Mean wind speed profiles with and without the model at Spot 9 in WSW wind direction; (b) Mean wind speed against overall turbulence intensity at Spot 9 in WSW wind direction. Total increments $=15$. 
Some adverse cases of percent decrease in the mean wind speed were also found above buildings level for measuring spots located in close-proximity to the buildings. The wake region in three dimensional flows includes the separation from the sides as well as the top surface of a building, therefore, measurements taken in close-proximity to a building would be affected by the wake and its turbulent nature for some distance past the height of the building. For example, Spot 5 is located immediately downwind of a low-rise building as seen in Figure 4.25, in which flow speed reduction was observed in Increments 1 through 5 as illustrated in Figure 4.26.

From the plot, it can be deduced according to the colour scheme defined earlier in this chapter that the flow conditions were bad below the building top surface (Increments 1-2). Immediately past the building roof (Increment 3), the flow condition was less severe but still $50 \%$ flow reduction was determined. It is suggested that the proximity to buildings plays a major role in the severity of the flow condition since Spot 9 in the previous example was also located in the wake region of a building (medium-proximity); however, the Cobra probe was able to capture at least $80 \%$ of the data at most of the increments within the height of the upstream building. On the other hand, the flow was not captured by the probe at the first Increment and was poorly captured at the second Increment of Spot 5 due to the close-proximity to the upstream building.

Flow speed reduction was also observed at spots located upstream of a tall building. Figure 4.27 shows the reduction in the mean wind speed at Spot 24 for wind direction $\mathrm{E}_{17.1}$.

The reduction in the profile was increasing gradually from Increments 3 to 8, which are represented by filled markers in Figure 4.28. Considering the perpendicular wind direction to the windward face of Building I, the stagnation zone from which the flow separates was expected to be located close to Increment 8, which is located at approximately $70 \%$ of the height of the building. This expectation was based 
on the general flow pattern around tall buildings, where flow separation from the windward side of a building occurs at approximately $70 \%-80 \%$ of the height of the building [73]. From the stagnation zone (high pressure zone), the flow deviates to areas of lower pressure, and thus, flow moves upwards, sideward and strongly downward as explained in Ref. [73]. To verify the location of the stagnation zone, the pitch profile was examined (see Figure 4.29), and the results match well with the expectation since the flow shifted from being downward (positive values) to being upward (negative values) past Increment 8. By examining Spot 24 mean wind speed profile, which was shown earlier in Figure 4.27, the maximum reduction of $34 \%$ was found at Increment 8. This suggests that the maximum flow reduction upstream of buildings would likely occur close to the zone of flow separation. 


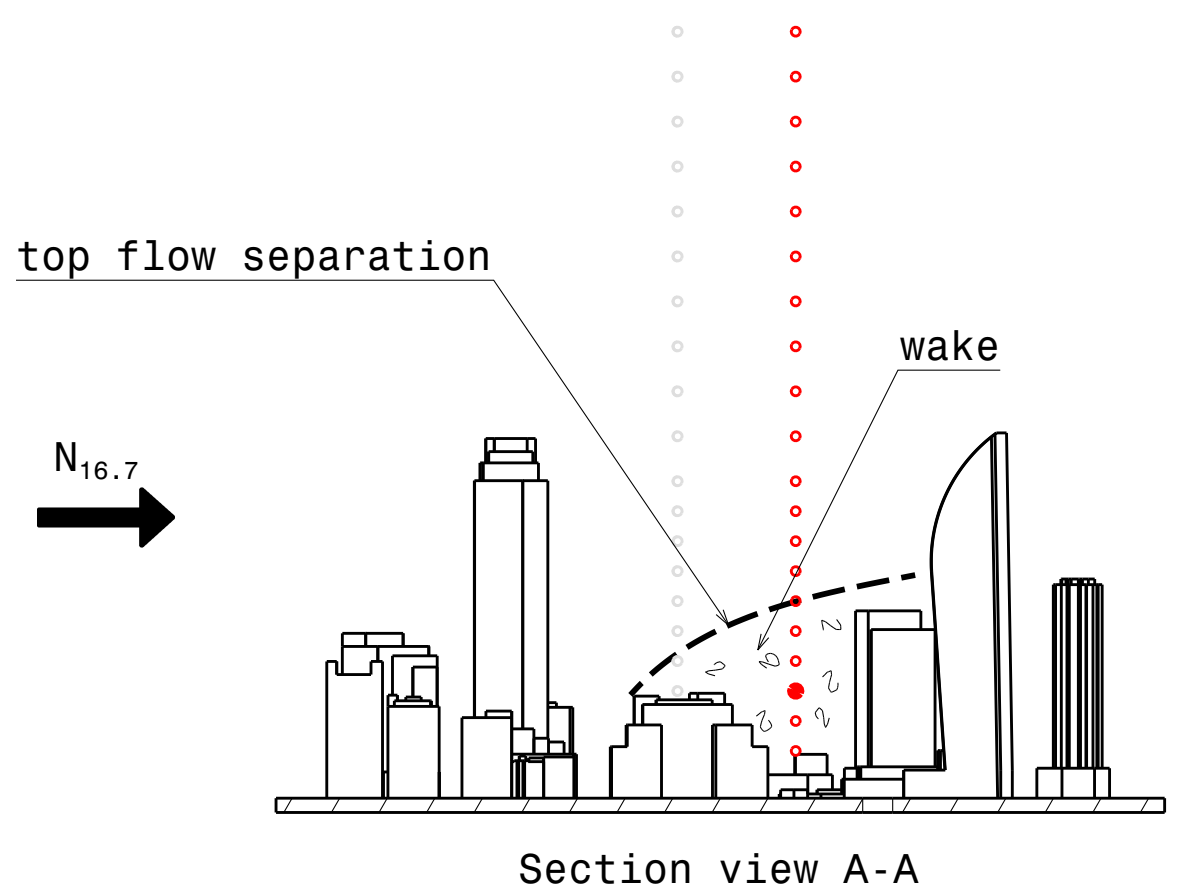

\section{side flow \\ separations}

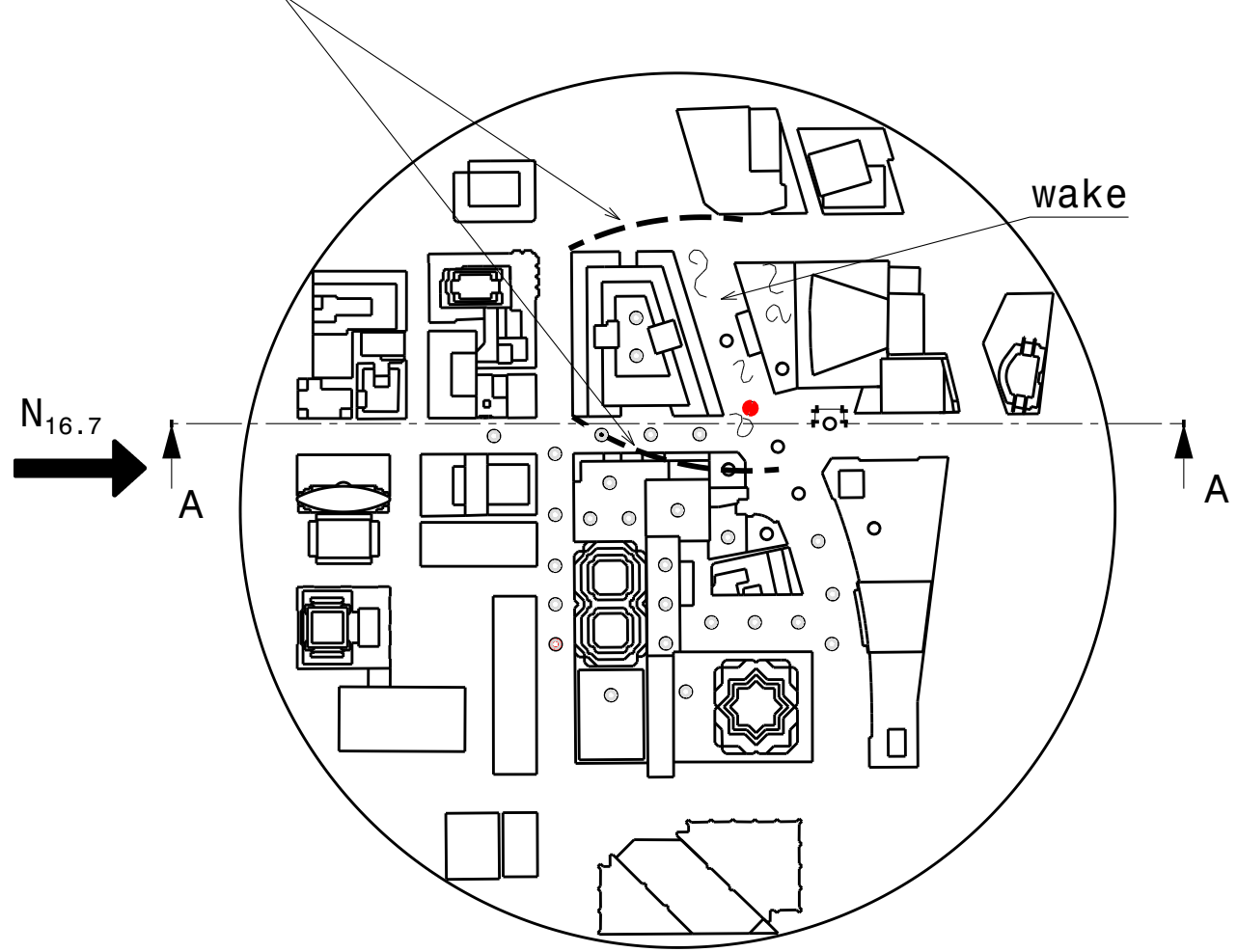

Figure 4.25: The location of Spot 5 (red markers) with a model orientation representing $\mathrm{N}_{16.7}$ wind direction.

(Adverse case of flow deceleration) 


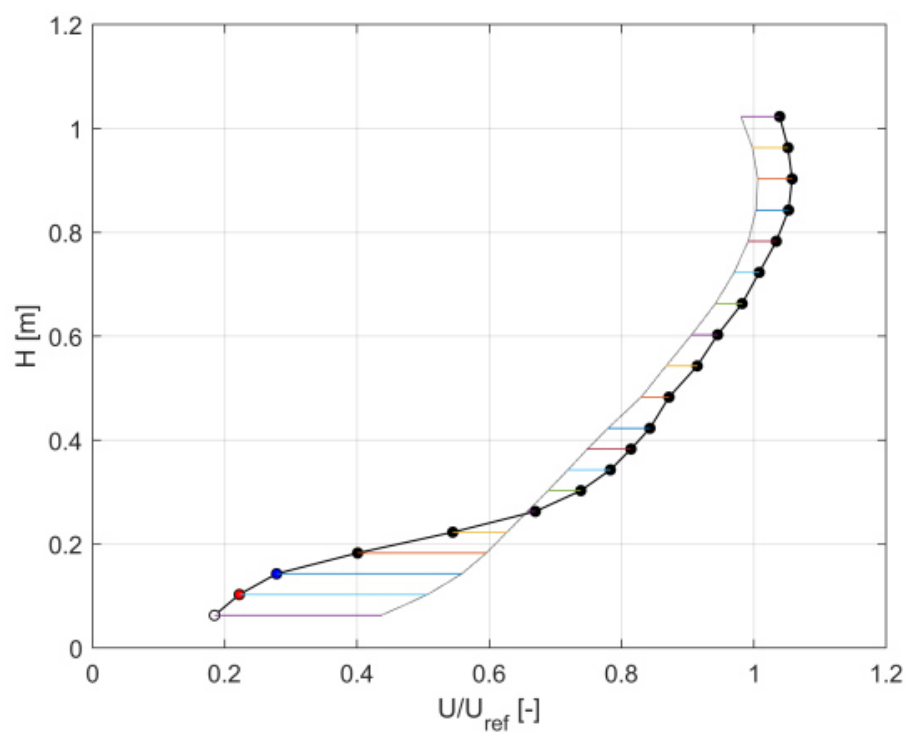

Figure 4.26: Example of the flow speed reduction at increments above upstream building level (Spot 5 in $\mathrm{N}_{16.7}$ wind direction). Total increments $=20$.

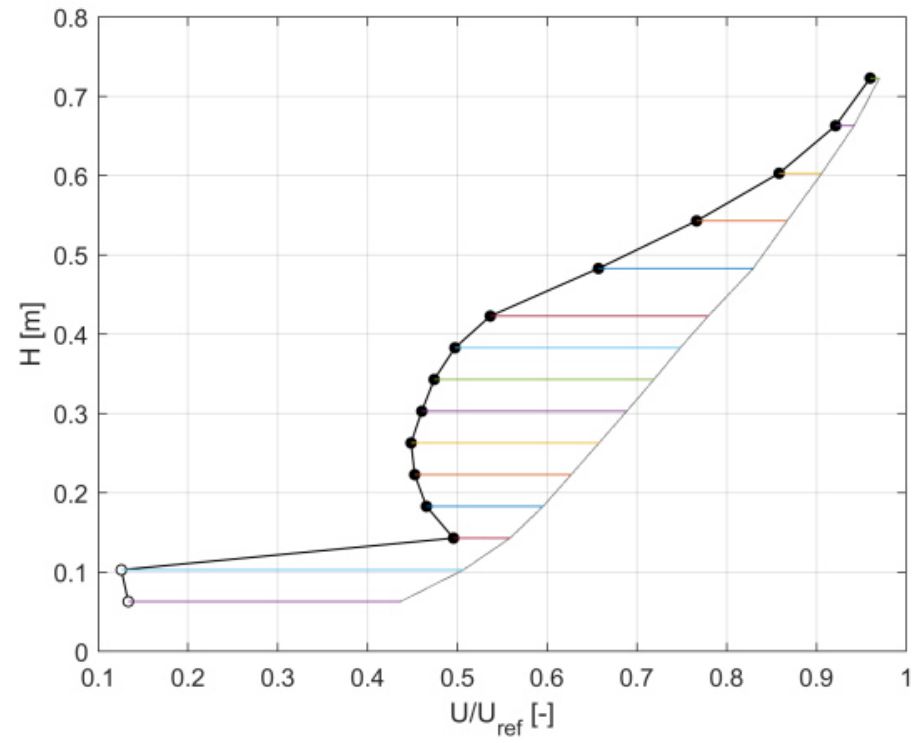

Figure 4.27: Example of the flow speed reduction at measuring spot located upstream of a tall building. Measurement at Spot 24 for $\mathrm{E}_{17.1}$ wind direction. Total increments $=15$. 

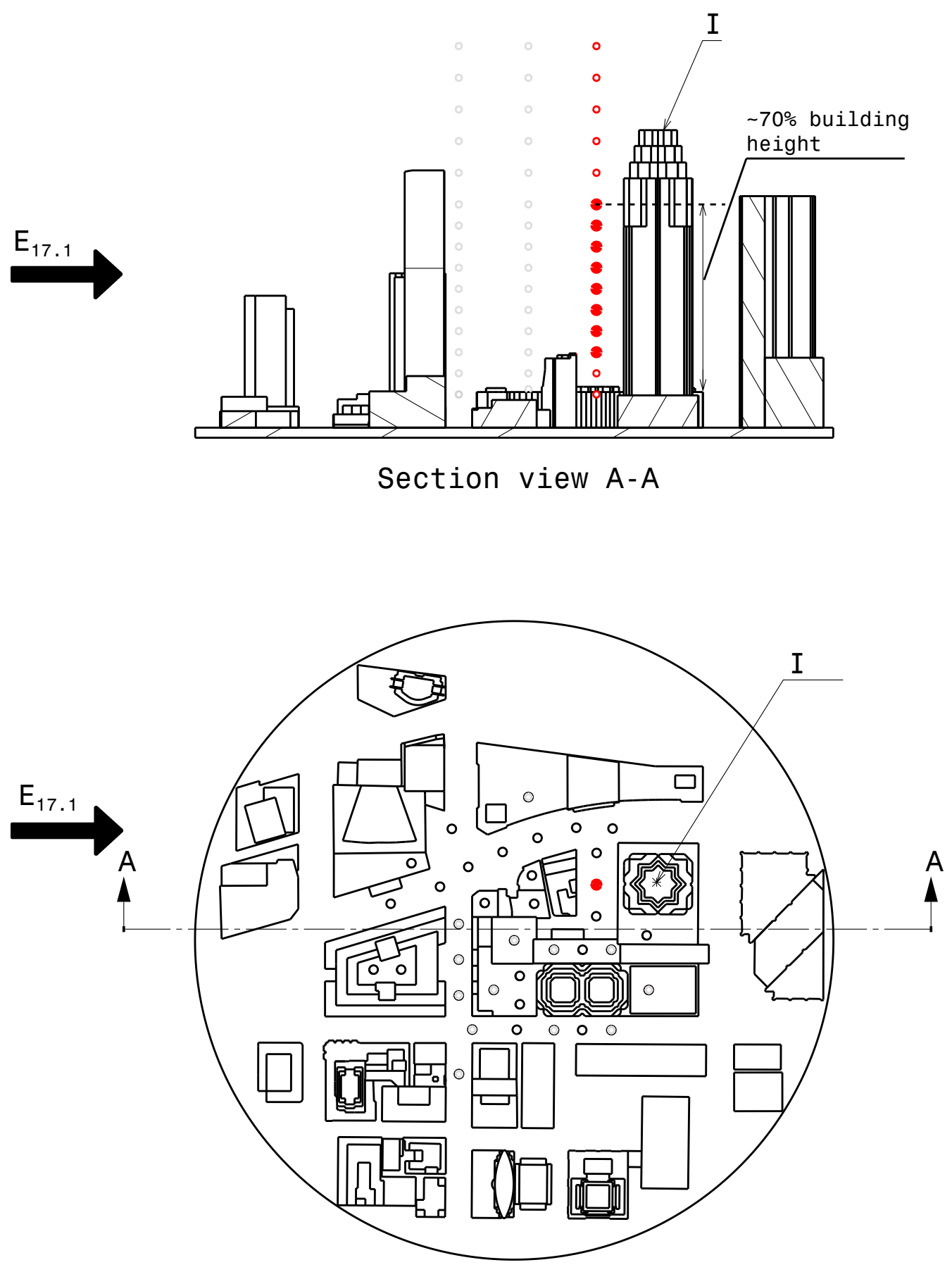

Figure 4.28: The location of Spot 24 (red markers) with a model orientation representing $\mathrm{E}_{17.1}$ wind direction.

(Moderate case of flow deceleration) 


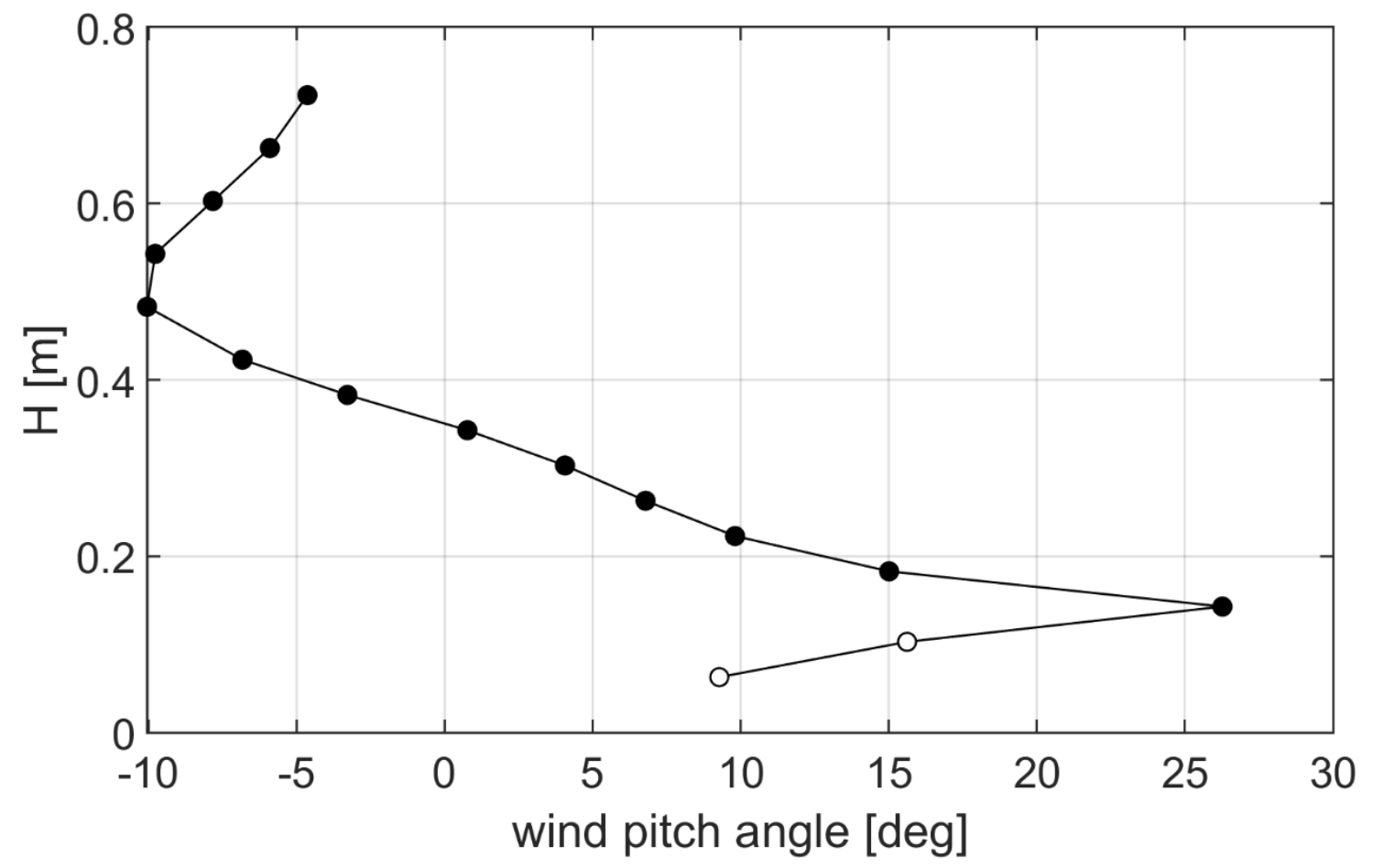

Figure 4.29: Pitch angle profile at Spot 24 for wind direction $\mathrm{E}_{17.1}$. Total increments $=15$. 


\subsubsection{Wind direction}

Wind direction is highly influenced by the complexity of the urban settings. Therefore, a flying taxi may encounter different wind directions that are not reported by the local weather stations when flying within the urban canopy. Wind direction can be divided into three categories: upward flow (updraft), downward flow (downdraft), and horizontal flow. The upward and downward flows can be determined from the pitch flow angle while the horizontal flow wind direction can be determined from the flow yaw angle. This section demonstrates how wind directions in an urban setting can be markedly different from the freestream value.

\section{Vertical airflow}

Some of the extreme maximum updrafts and downdrafts were found upstream of buildings as a result of the flow separation from the stagnation zone as discussed earlier in Section 4.3.2.1. An example of this case is shown in Figure 4.30, which demonstrates the maximum updraft and downdraft found in the WSW wind direction, particularly at Spot 19. The stagnation zone marked in Figure 4.30 was identified from Increments 2-15 of the flow pitch angle profile shown in Figure 4.31 by observing the shift in the flow direction from being downward (positive values) to being upward (negative values). The maximum updraft of $10.4^{\circ}$ and downdraft of $15.4^{\circ}$ were found at Increments 11 and 5, respectively. The maximum updraft in $\mathrm{WSW}_{+15}$ wind direction was also found at Increment 11 of Spot 19, but with higher amount of distortion, that is $12.3^{\circ}$.

In line with the previous cases of vertical flows, the maximum updraft and downdraft in the measurements taken at $\mathrm{N}_{16.7}$ wind direction were also found upstream of a building as shown in Figure 4.32, in which a maximum updraft of $18.8^{\circ}$ and downdraft of $23.3^{\circ}$ were found at Increments 11 and 8, respectively, of Spot 17. Furthermore, the most severe case of $26.3^{\circ}$ downdraft was found in measurements taken 
at $\mathrm{E}_{17.1}$ wind direction, particularly at Increment 3 of Spot 24 upstream of Building I (refer back to Figure 4.28).

Another case of adverse updrafts of $17.6^{\circ}$ was found in the measurements taken very close to the building top surface, which is Increment 1 of Spot 28 for wind direction $\mathrm{E}_{17.1}$. The location of the spot, denoted in red in Figure 4.33, was also downstream of a tall building making it more challenging to determine the major cause of the upward motion of the flow. However, it is believed that the separation from the top surface of the building where the spot is located is the major cause for the upward flow motion since the flow was deflected downward (positive values) from Increments 2 through 11, which is the last Increment within the height of the upstream building (see Figure 4.34).

For some of the extreme cases, it was challenging to make conclusions about the major cause of the vertical flow distortions owing to the complexity of the urban setting and the existence of multiple buildings. For instance, the most severe updraft case of $20.5^{\circ}$ was found at Increment 1 of Spot 5 in a $S_{15}$ wind direction (without the future buildings). As can be seen in Figure 4.35, Spot 5 is located immediately below the rooftop of Building I, likely in the wake of Building II and upstream of Building III. With the oblique wind direction to the buildings windward surfaces (more or less than $90^{\circ}$ ), it was even more challenging to determine a subtle reasoning for the upward flow motion. In fact, it was expected that the flow will be deflected downward after the separation from Building I rooftop. However, the upward flow motion observed at Spot 5 could be related to the flow passing between Buildings I and II which may deflected upwards as it reaches Building III windward face. Therefore, the fact that the flow was deflecting upward could be an evidence of the complex and unexpected airflow behaviour in urban settings that an urban air taxi may encounter when flying through different parts of the urban canopy.

There were definitely more extreme cases of updrafts and downdrafts, however, 
the analysis was restricted by Cobra probe measurements with at least $80 \%$ GD, and thus, larger flow angles recorded with less than $80 \%$ GD were not considered. Additionally, any pitch angle greater than $45^{\circ}$ was not captured by the probe, so the fact that small angles were included in this study as extremes does not imply that large angle vertical flow distortions do not exist. 

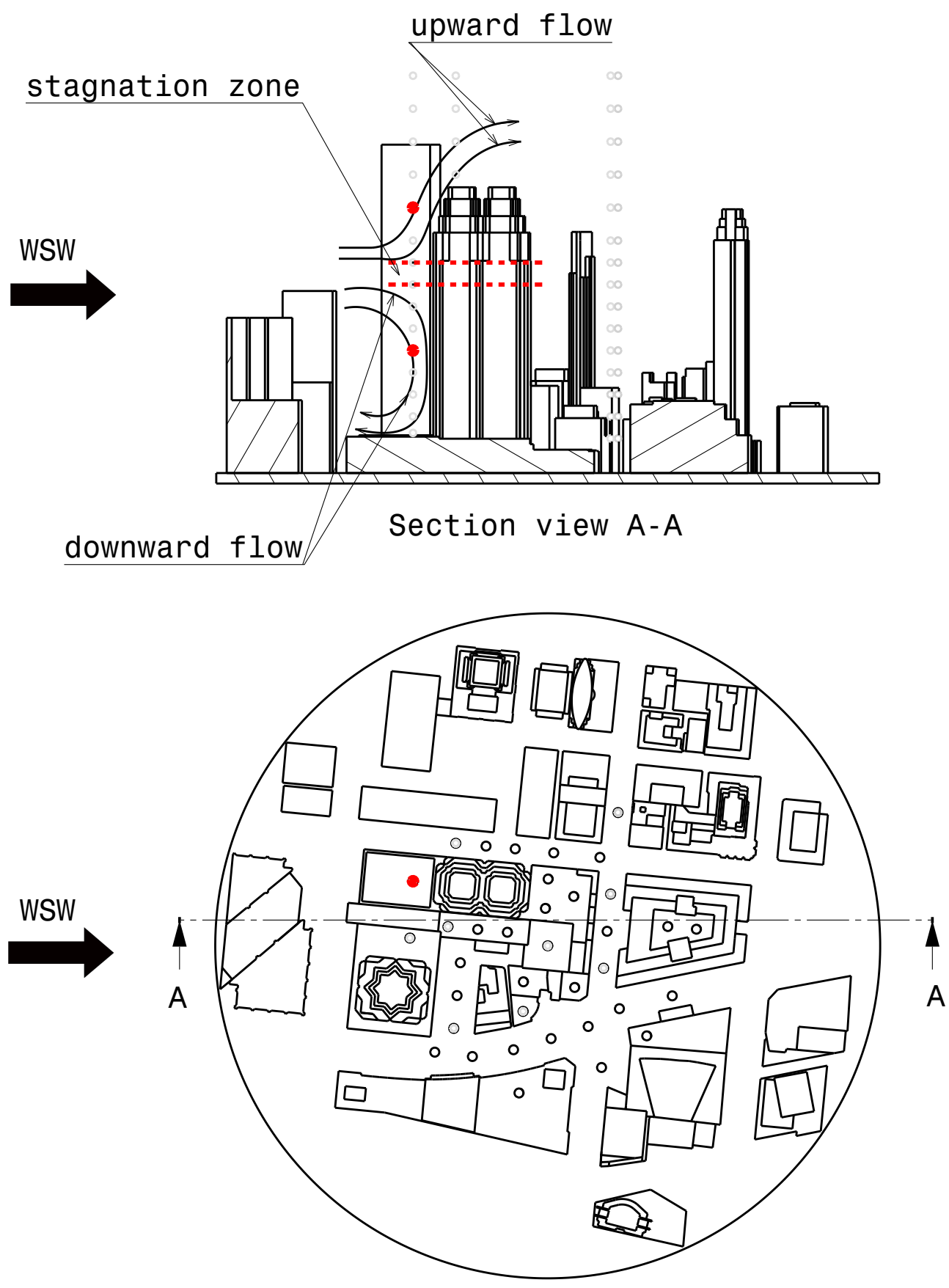

Figure 4.30: The location of Spot 19 (red markers) with a model orientation representing WSW wind direction.

(Moderate cases of updraft and downdraft) 


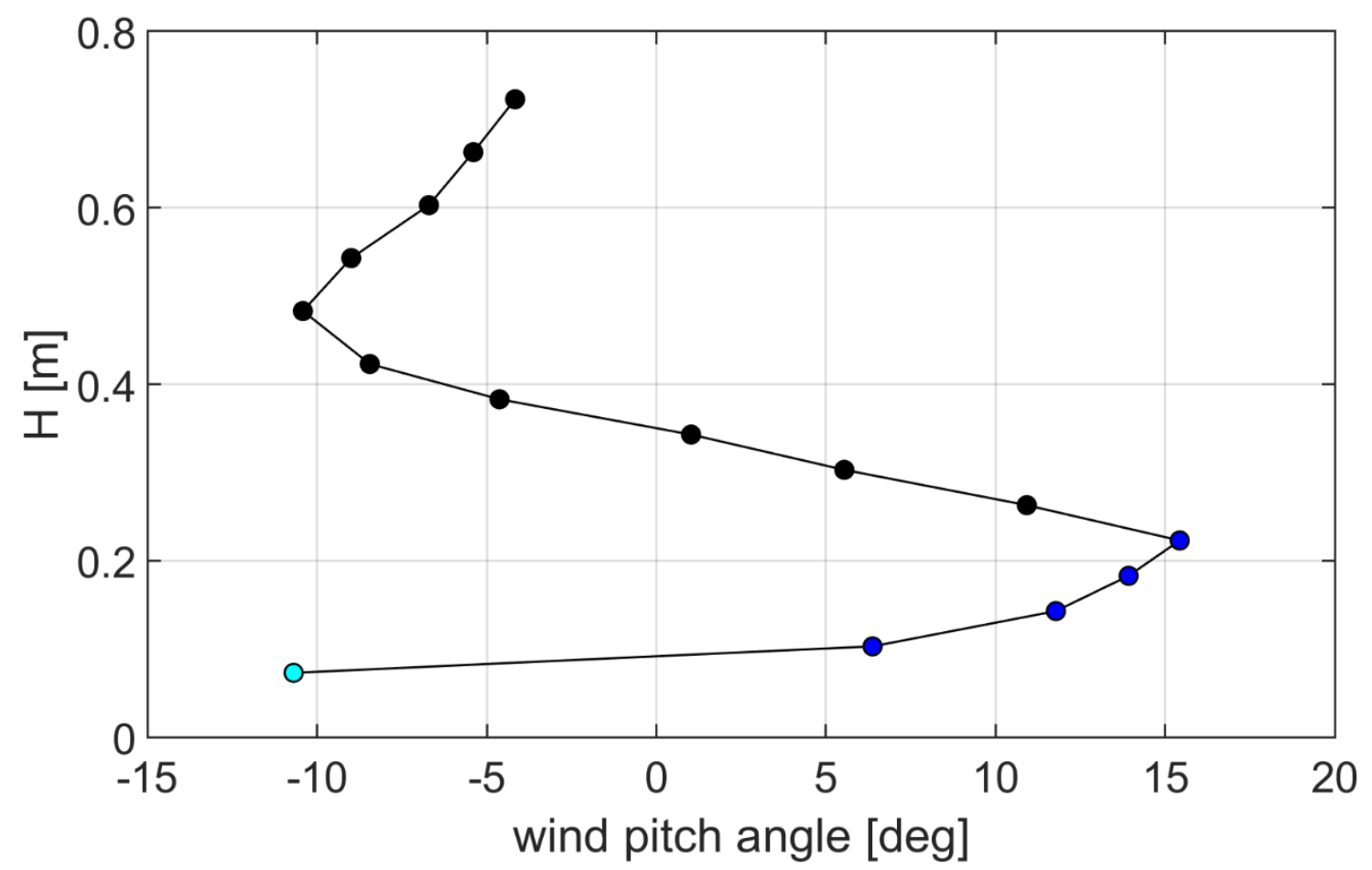

Figure 4.31: Pitch angle profile at Spot 19 for wind direction WSW.

Total increments $=15$. 

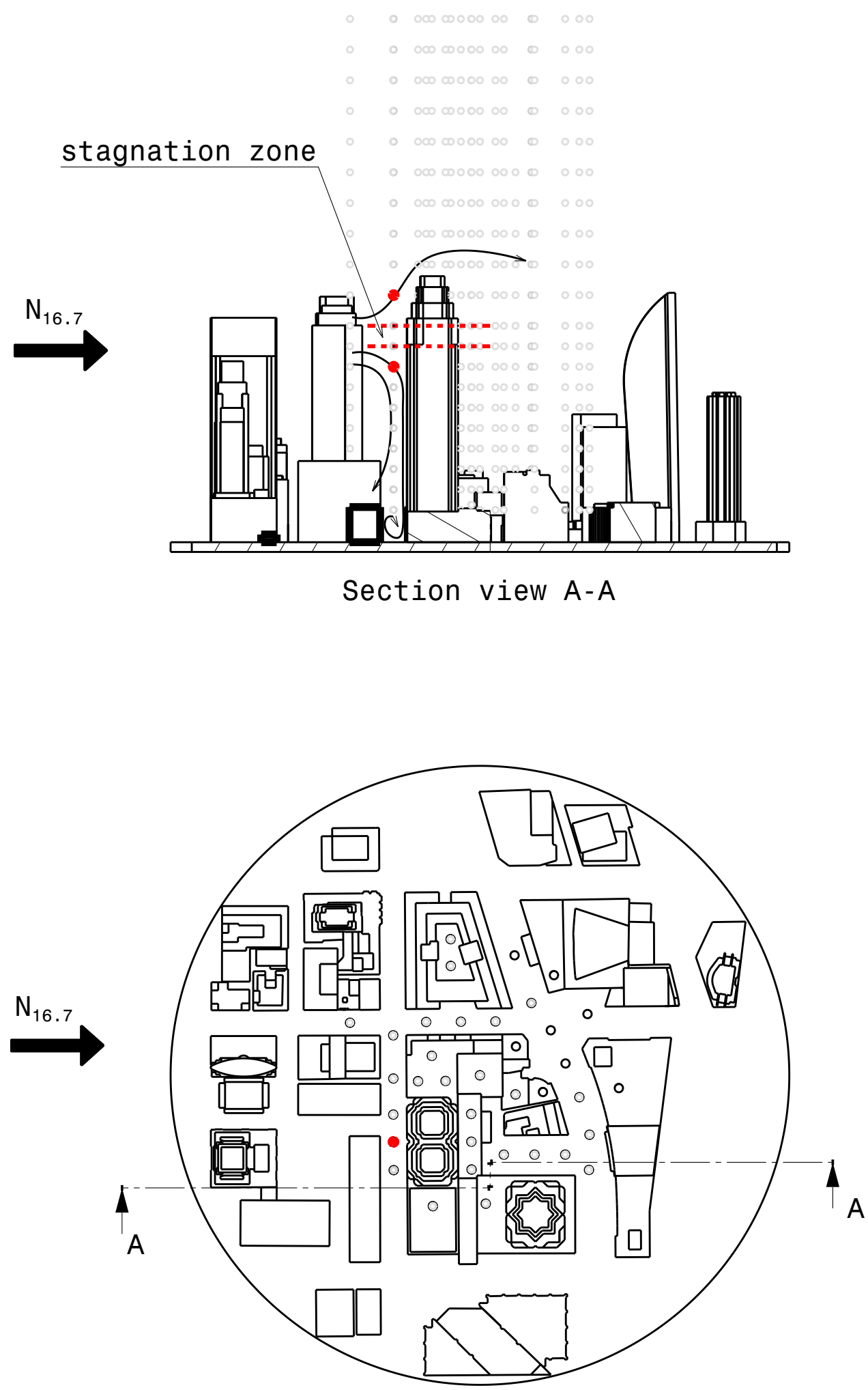

Figure 4.32: The location of Spot 17 (red markers) with a model orientation representing $\mathrm{N}_{16.7}$ wind direction.

(Adverse cases of updraft and downdraft) 

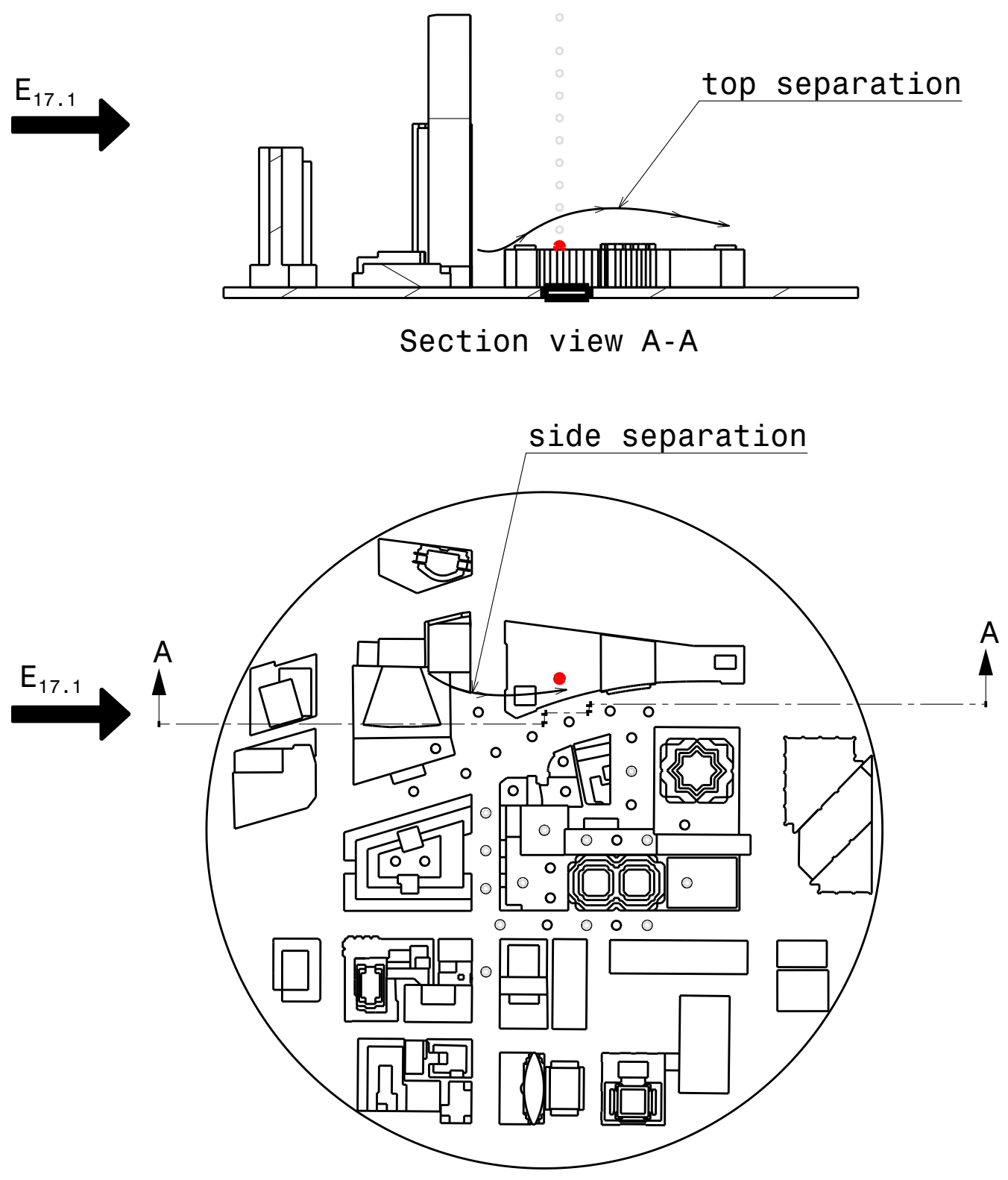

Figure 4.33: The location of Spot 28 (red markers) with a model orientation representing $\mathrm{E}_{17.1}$ wind direction.

(Adverse case of updraft) 


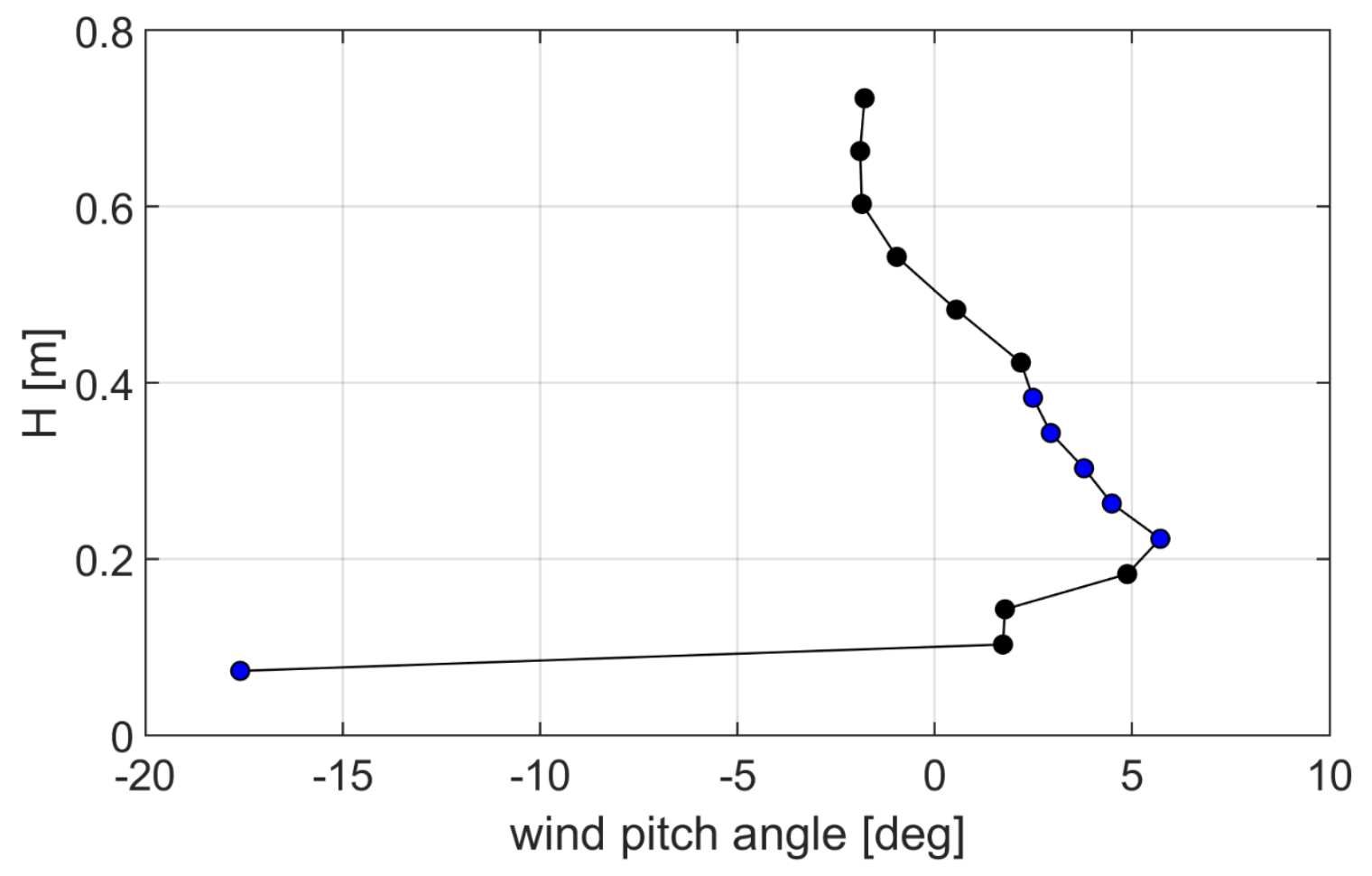

Figure 4.34: Pitch angle profile at Spot 28 for wind direction $\mathrm{E}_{17.1}$. Total increments $=15$. 


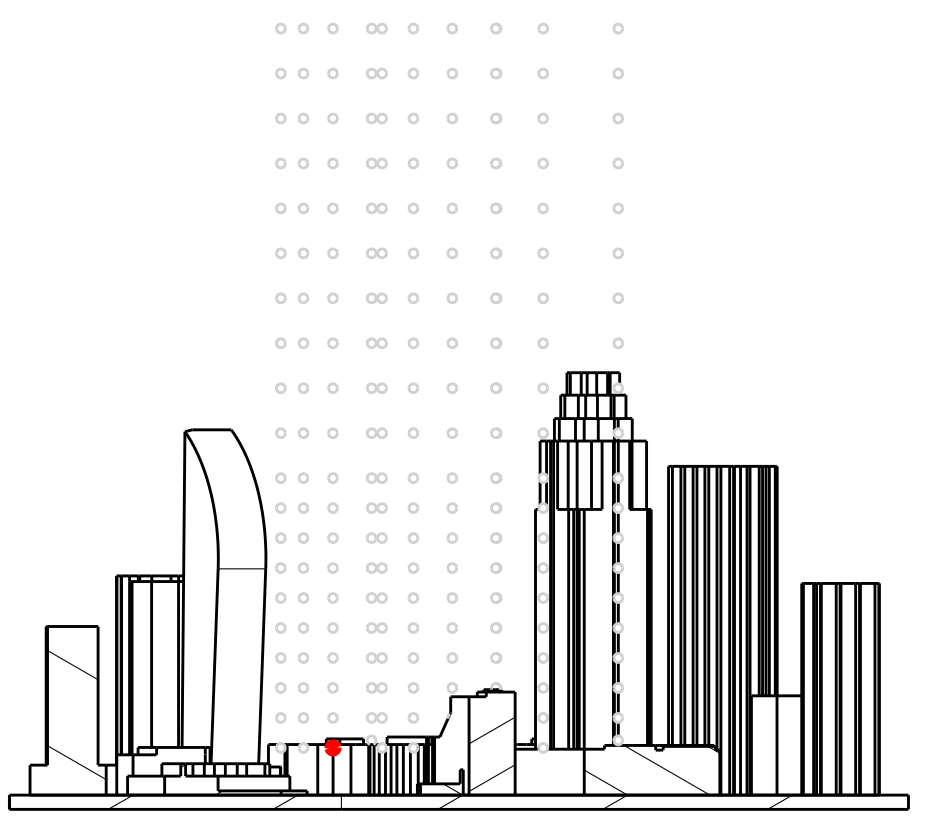

Section view A-A

$S_{15}$ is out of the page

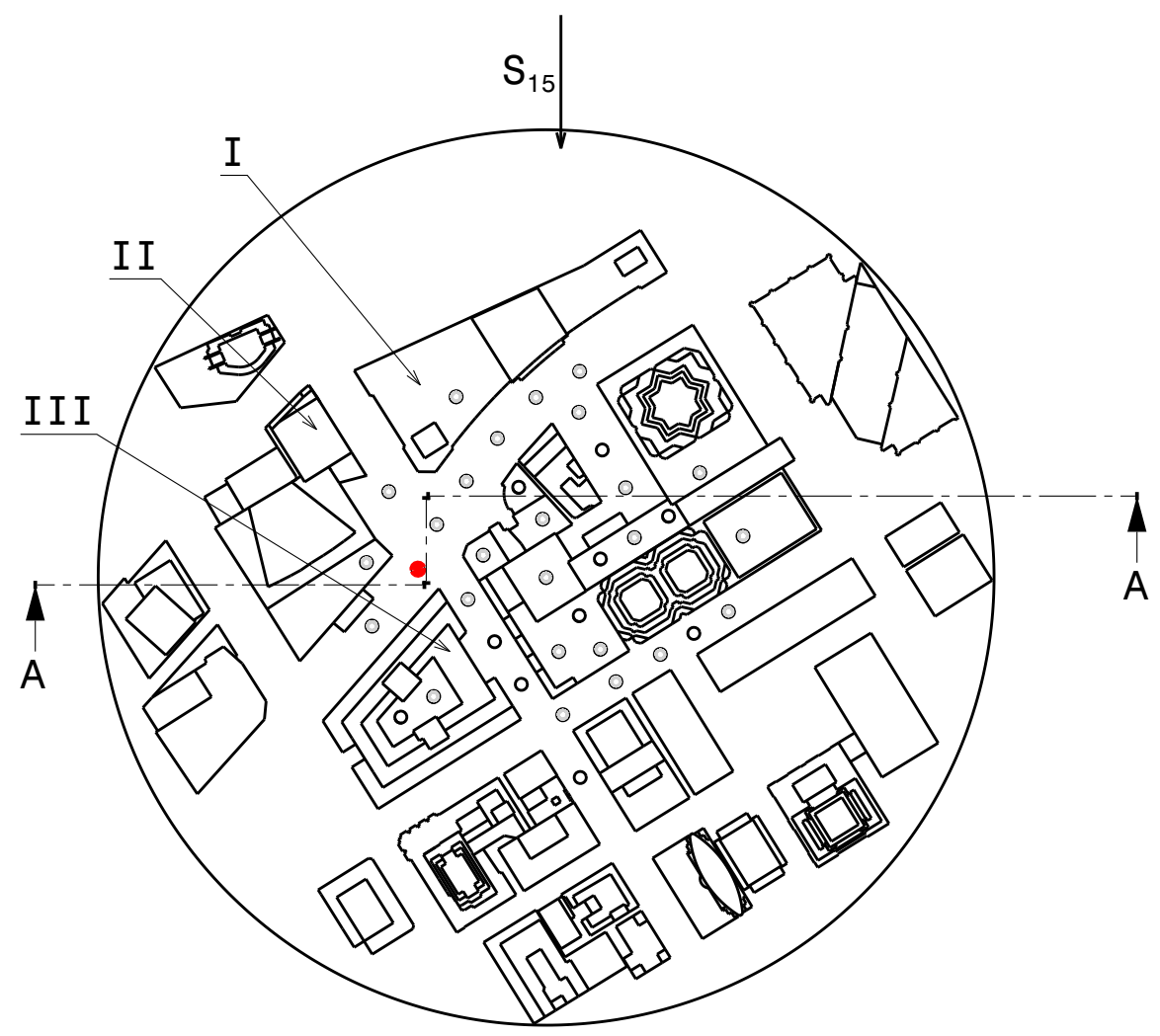

Figure 4.35: The location of Spot 5 (red markers) with a model orientation representing $S_{15}$ wind direction.

(Severe case of updraft) 


\section{Horizontal airflow}

The severe change in horizontal flow direction was observed at various increments within the height of the buildings. However, the spots in which severe flow yaw angles were measured were all located at the edge of the wake, possibly at the buildings shear layers. Figure 4.36 shows the location of Spots 18, 23, and 26 for a wind coming from $\mathrm{N}_{16.7}$, at which flow yaw angles were found to be $77^{\circ}, 58^{\circ}$, and $74^{\circ}$, respectively. As can be seen in the figure, Spots 18 and 26 were located at the edge of the separated boundary layer from Buildings I and III, respectively. Spot 23 was likely located at the edge of the separated boundary layer from Building II, which was likely distorted by the existence of Building III. The change in horizontal flow direction was predominant in each spot within the height of the nearby building as demonstrated in Figure 4.37. which shows the yaw angle profiles measured at each of the three spots.

Although flow measurements with less than $80 \%$ GD were not involved when extreme values were determined, the Cobra probe yaw angle was optimized by the indexing system of the probe holder. Therefore, despite the fact that the Cobra probe was not capturing high percentage of GD at some locations even when it was rotated, the indicated angles in the yaw profile plots are the ones in which the flow was captured the most. There were cases in which the probe was not capturing any flow data until it was rotated by $180^{\circ}$, entirely opposite to the flow direction. In such a case, it is likely that the probe was in a re-circulation zone, and therefore the probe was capturing low \% GD even after being rotated. An example of such as case is the measurement taken at Increment 2 of Spot 11 for wind direction WSW, the location of which is shown in Figure 4.38. Similar cases of change in horizontal flow direction at the edge of the separated boundary layer were found at:

- Increment 1 of Spot 28 in $\mathrm{E}_{17.1}$ wind direction;

- Increment 1 of Spot 23 in $\mathrm{S}_{15}$ wind direction without the future buildings; and 
- Increment 6 of Spot 26 in $S_{15}$ wind direction with the future buildings.

Each of the listed locations represent the extreme maximum at specific model orientation to the incoming wind, and thus, the horizontal angles of each case can be found in Table 4.2. Similarly, the spots location can be identified from the images provided earleier, especially Figure 4.33 for Spot 28 in $E_{17.1}$ wind direction, Figure 4.35 for Spot 23 in $\mathrm{S}_{15}$ wind direction (without the future buildings), and Figure 4.18 for Spot 26 in $\mathrm{S}_{15}$ wind direction (with the future buildings). The spots location, if not indicated in the image, can always be extracted from the spots designation provided in Figure 3.13 . 

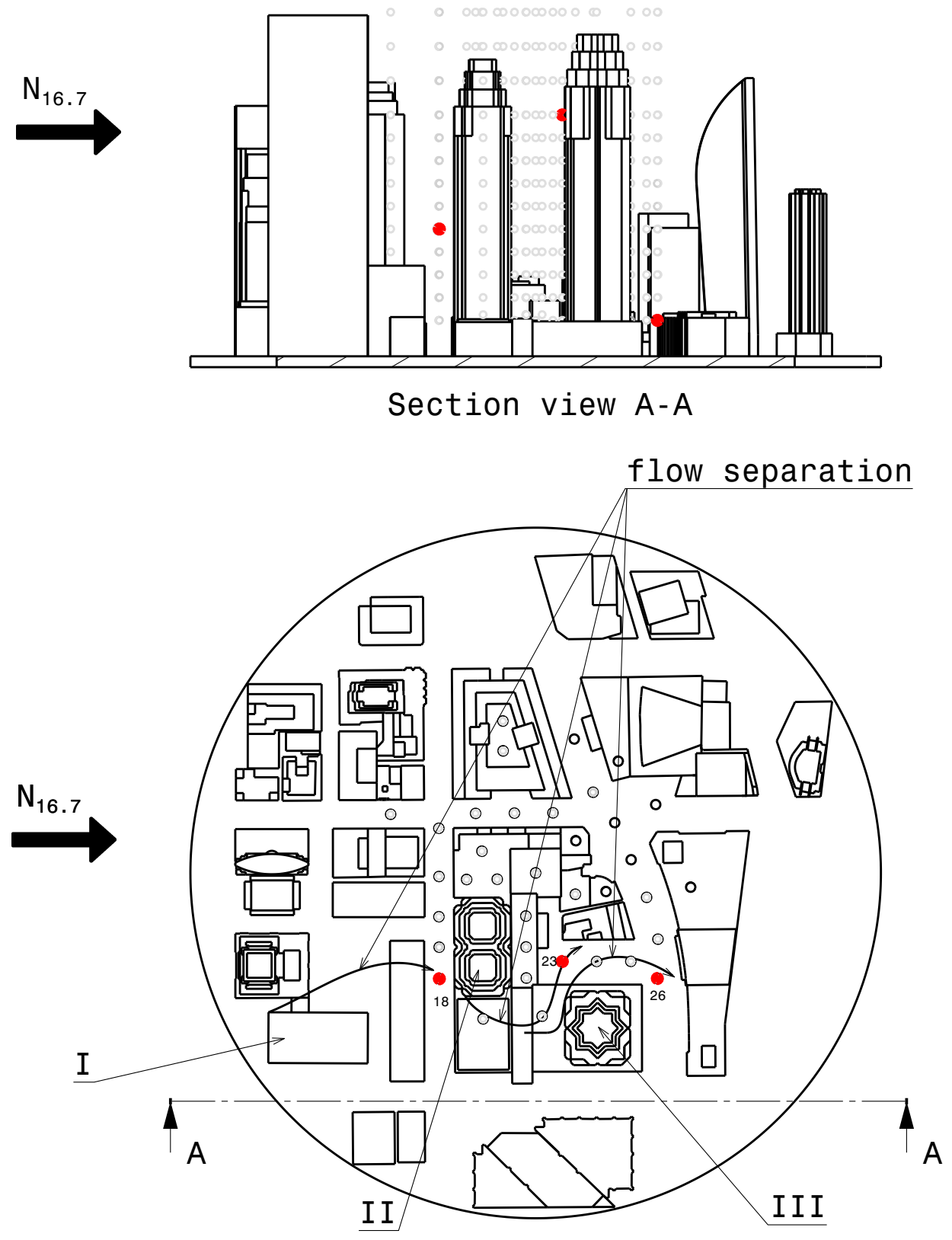

Figure 4.36: The location of Spots 18, 23 and 26 (red markers) with a model orientation representing $\mathrm{N}_{16.7}$ wind direction.

(Severe/adverse cases of change in horizontal flow direction) 


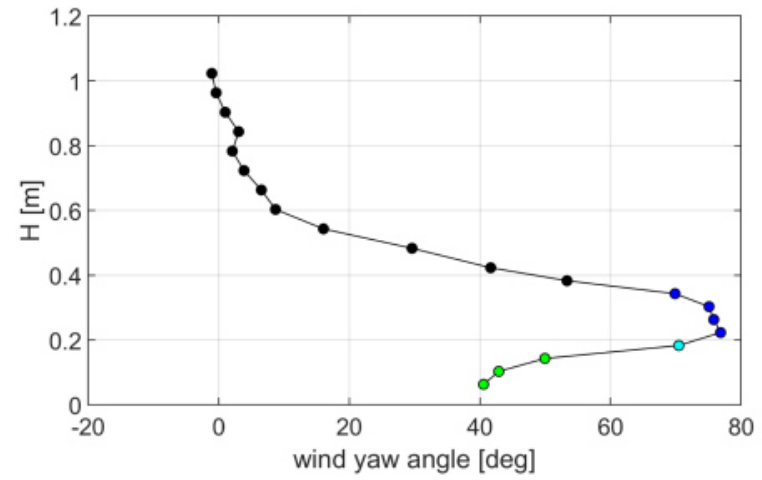

(a)

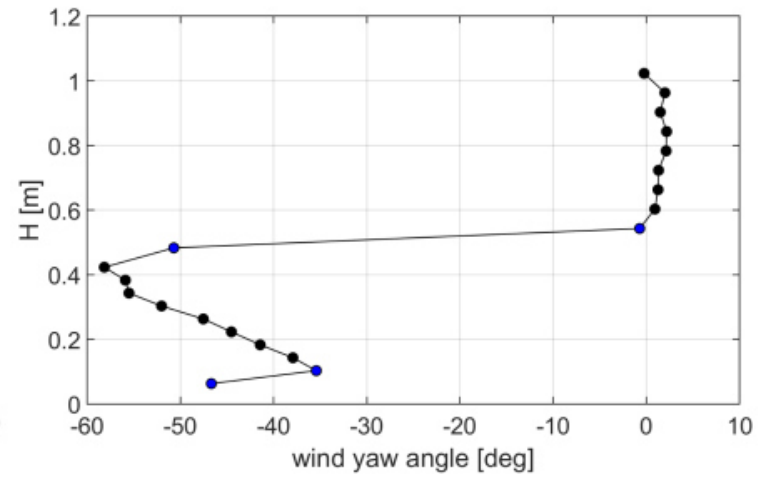

(b)

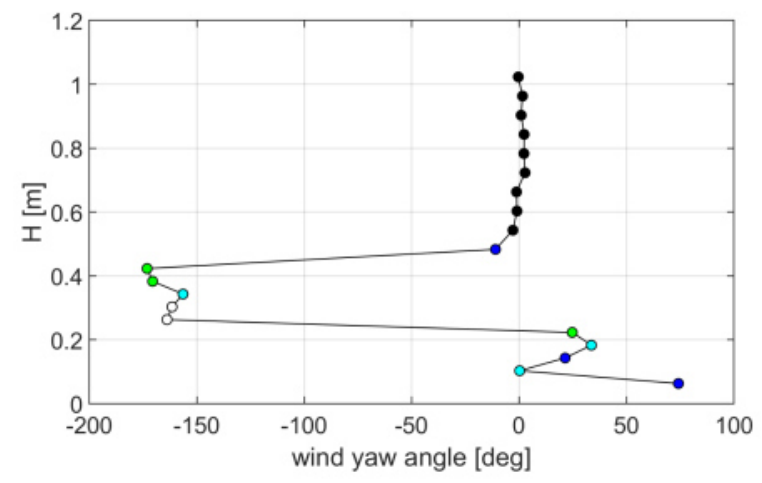

(c)

Figure 4.37: Yaw angle profile at Spots: (a) 18, (b) 23 and (c) 26 for wind direction $\mathrm{N}_{16.7}$. Total increments $=20$. 

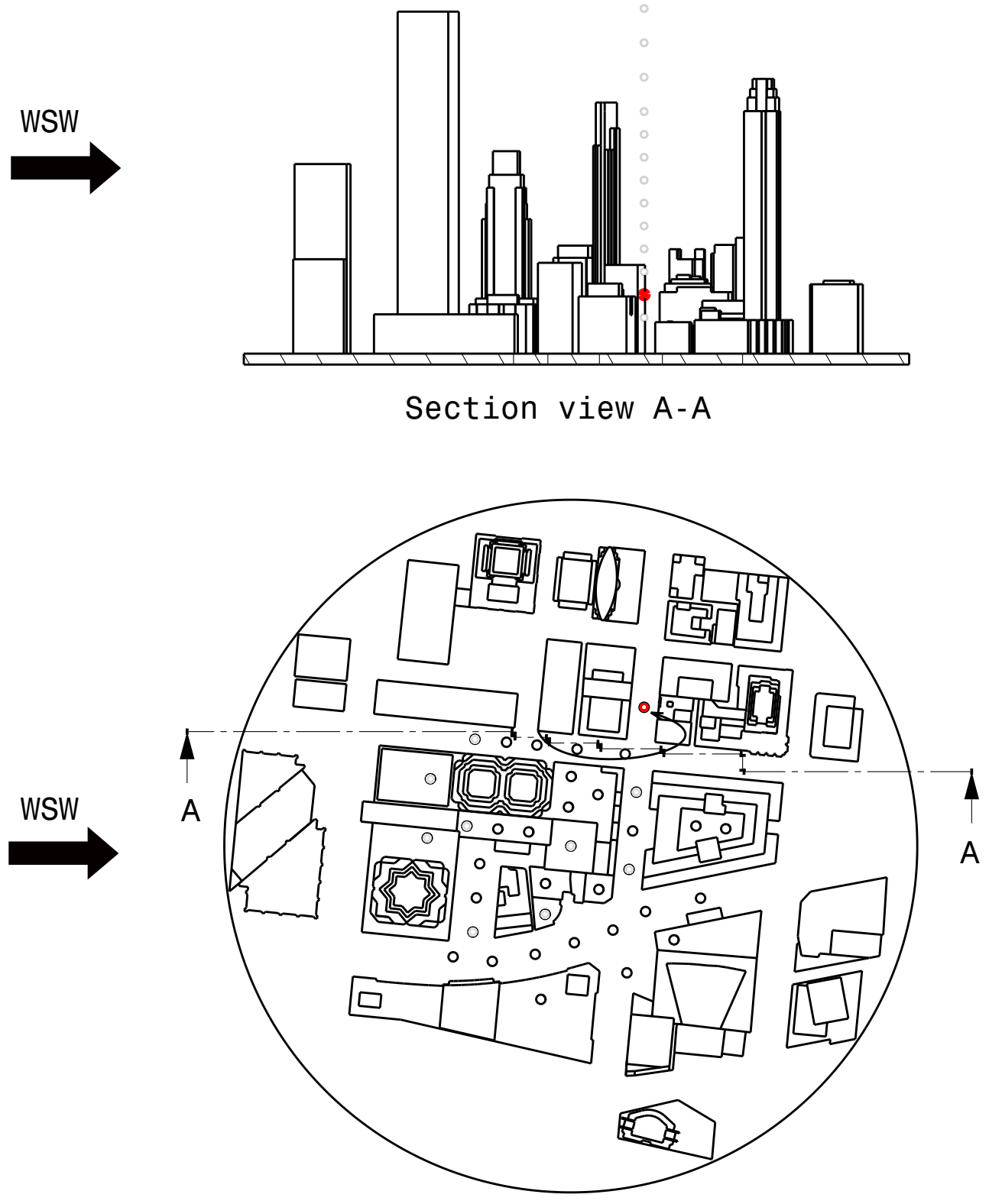

Figure 4.38: The location of Spot 11 (red markers) with a model orientation representing WSW wind direction.

(Possible case of flow re-circulation) 


\subsubsection{Wind shear}

The urban airflow features were analyzed with considerations to the UAM operations. Therefore, it was decided to examine the wind shear by determining the extent of variations in wind speed and direction between two points in space that lie between two consecutive vertical layers, which is significant when considering Vertical Take-Off and Landing (VTOL) urban air taxis such as the Bell Nexus.

\section{Vertical speed shear}

The severe cases of shear in wind speed were found between two vertically adjacent layers in which airflow was obstructed by an upstream building at one layer and open to the incoming flow at the other layer. This behaviour in shear can be strongly linked to the turbulence level found in the wake of buildings as compared to the freestream flow. Figure 4.39 shows both the normalized mean wind speed and overall turbulence intensity profiles at three spots, which are: (a) 23 in $\mathrm{N}_{16.7}$ wind direction, (b) 26 in $\mathrm{S}_{15}$ wind direction and (c) 9 in WSW wind direction. The degree of wind shear at Spots 23, 26 and 9 was found to be $6.93 / \mathrm{m}$ between Increments $12-13,5.81 / \mathrm{m}$ between Increments 13-14 and 5.59/m between Increments 11-12, respectively, of the vertical traverse.

The location of the spots and the corresponding vertical layers at which shear was found to be maximum are denoted by filled red markers and dashed lines in Figures 4.40, 4.41 and 4.42, The degree of speed shear for Spot 23 corresponded to a change in wind speed by $4.45 \mathrm{~m} / \mathrm{s}$ over a height of $0.06 \mathrm{~m}$ (24 m FS). The maximum shear in wind speed for Spot 23 was found between the two layers above the rooftop of Building II (see Figure 4.40), which suggests that the wake effect of Building I was persisted at least to the location of Spot 23; therefore, the extreme change in wind speed occurred between the layers below and above the rooftop of Building I. From the profiles shown in Figure 4.39(a), it can be seen that the level of turbulence 


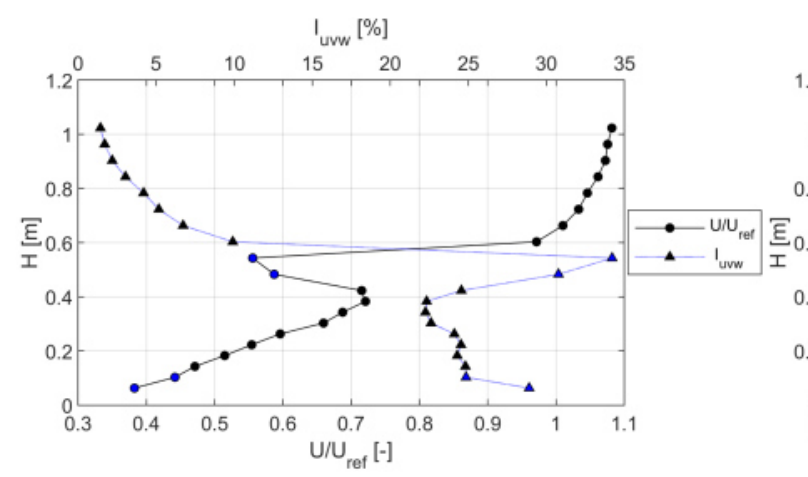

(a)

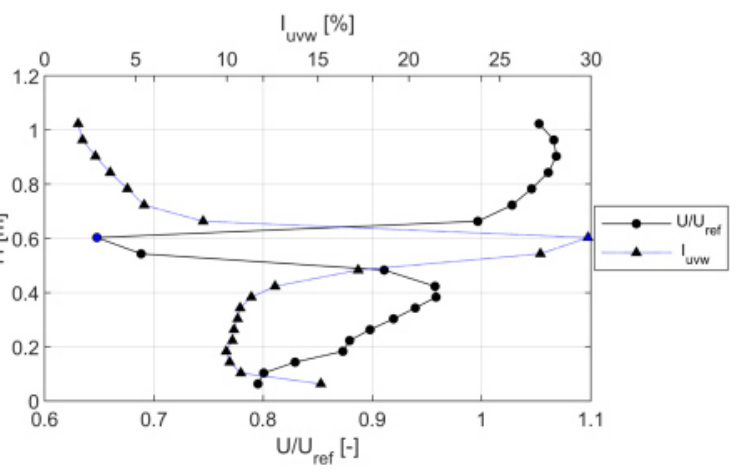

(b)

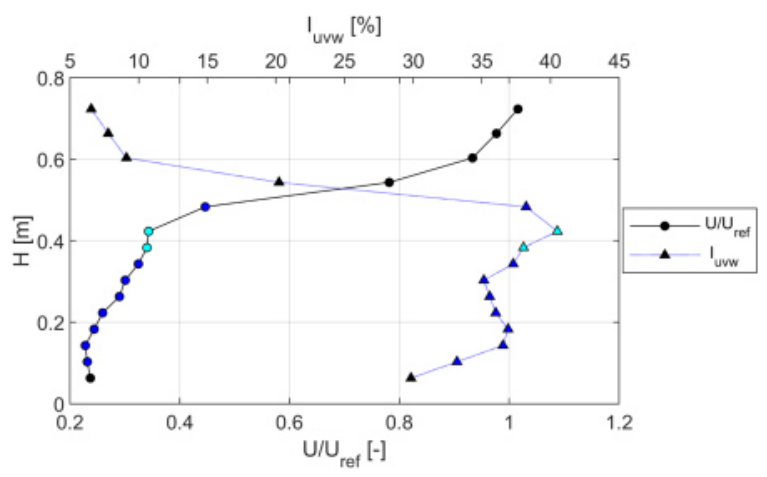

(c)

Figure 4.39: Normalized mean wind speed and overall turbulence intensity profiles at Spots: (a) 23 in $\mathrm{N}_{16.7}$ wind direction with total increments $=20$, (b) 26 in $\mathrm{S}_{15}$ wind direction with total increments $=20$ and (c) 9 in WSW wind direction with total increments $=15$.

transitioned from $34 \%$ at Increment 12 to $10 \%$ at Increment 13, which concurs with the assumption of persisted wake effect from Building I.

Similarly, the degree of speed shear at Spots 26 and 9, was corresponding to a change in the mean wind speed by $3.75 \mathrm{~m} / \mathrm{s}$ and $3.53 \mathrm{~m} / \mathrm{s}$, respectively, over a height of $0.06 \mathrm{~m}$ ( $24 \mathrm{~m} \mathrm{FS})$. The shear in both spots was found between layers below and above the corresponding upstream buildings as shown in Figure 4.41 and 4.42 . In both cases, the immediate upstream building was the tallest; therefore, the degree of speed shear was less as compared to the case of Spot 23 which was exposed to the effect of two tall buildings. Nonetheless, the level of turbulence in Spots 26 and 9 was 
much higher below the buildings rooftop (Increment 13 for Spot 26 and Increment 11 for Spot 9) as seen in Figure 4.39(b) and (c), which is consistent with the pattern found at Spot 23. Thus, the shear in wind speed can be associated with extreme changes in turbulence level, a condition that could jeopardize the safe landing of a flying vehicle. 


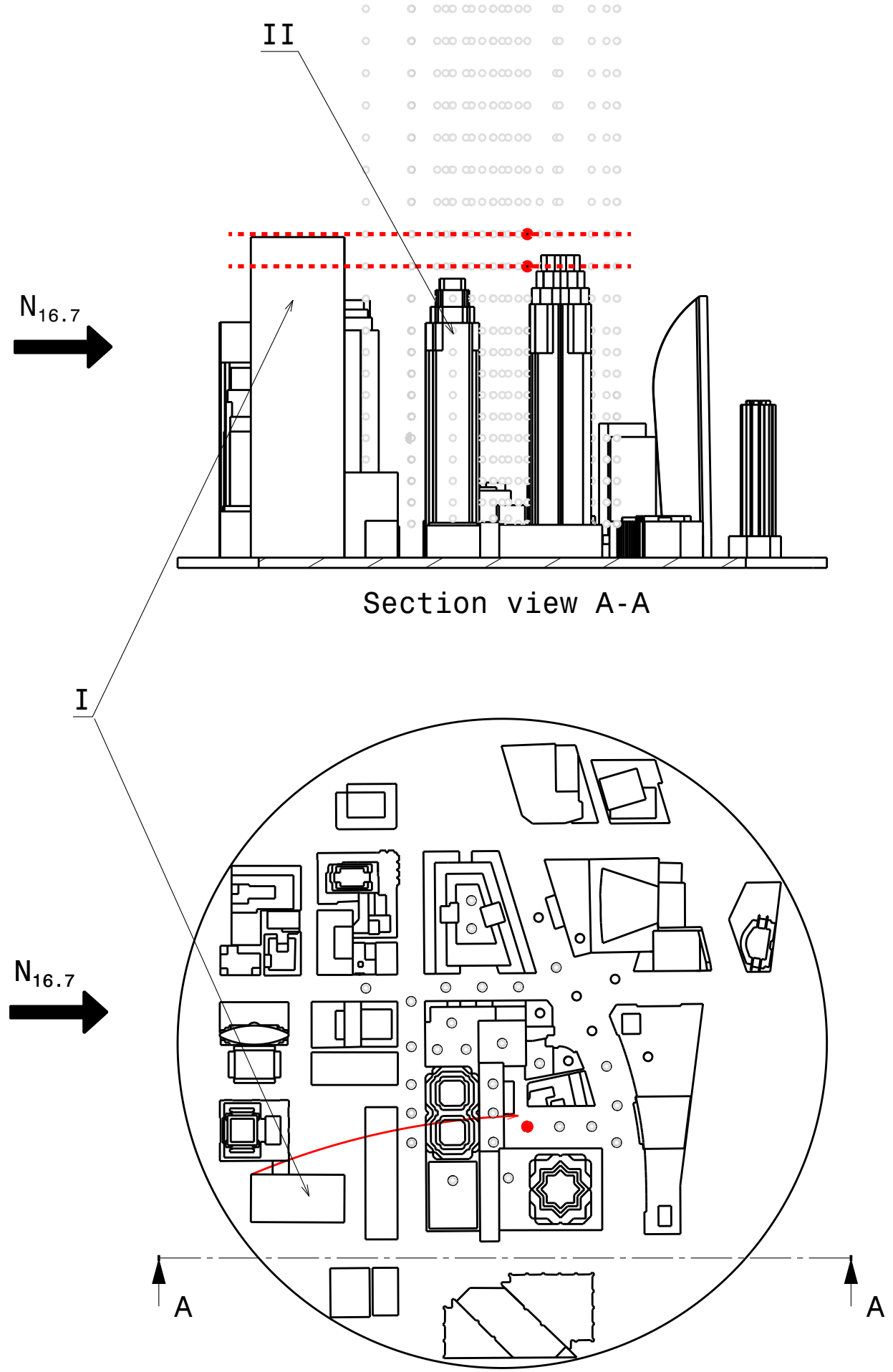

Figure 4.40: The location of Increments 12-13 at Spot 23 (red markers) with a model orientation representing $\mathrm{N}_{16.7}$ wind direction.

(Severe case of vertical speed shear) 

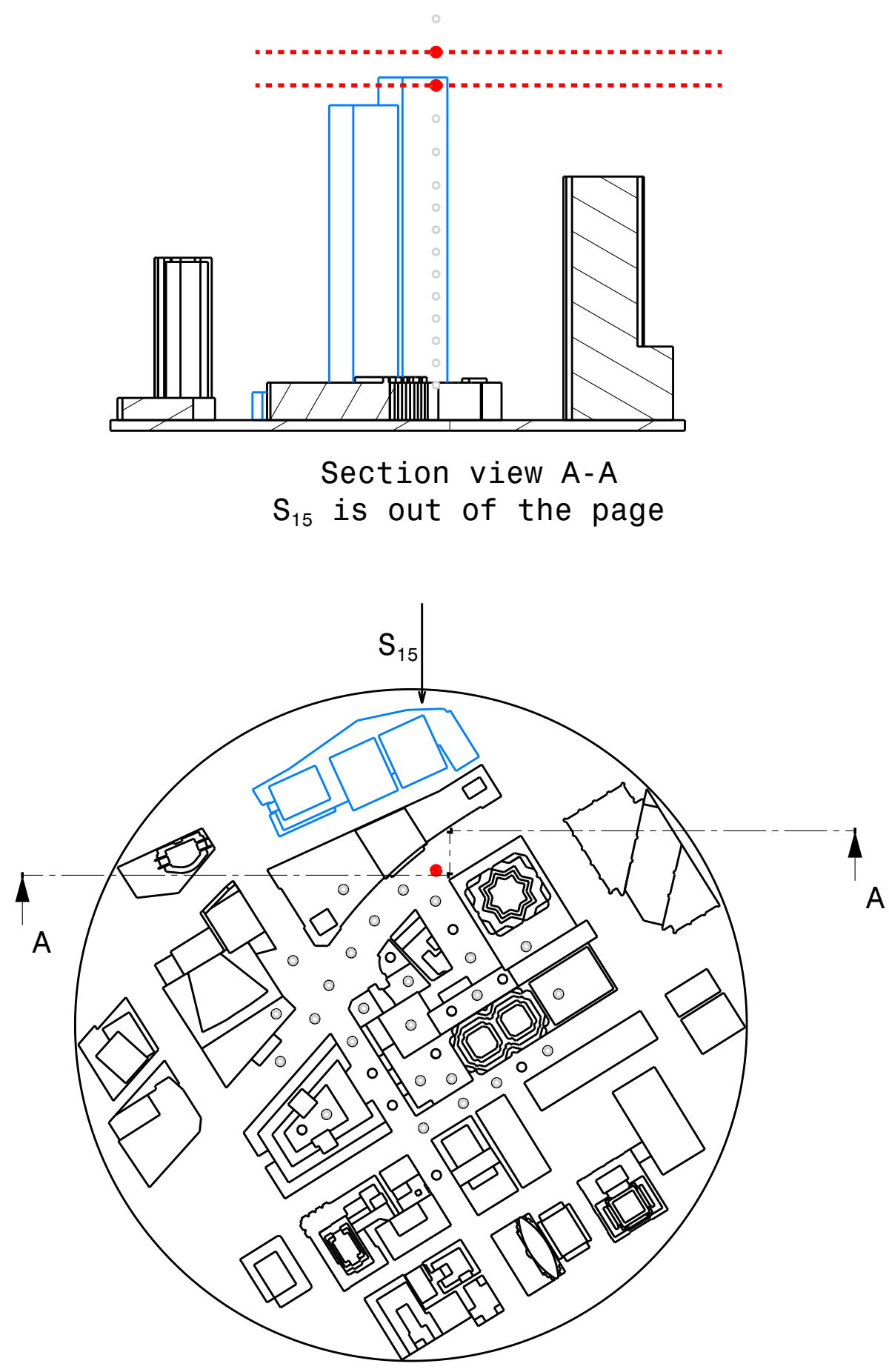

Figure 4.41: The location of Increments 13-14 at Spot 26 (red markers) with a model orientation representing $\mathrm{S}_{15}$ wind direction.

(Adverse case of vertical speed shear) 

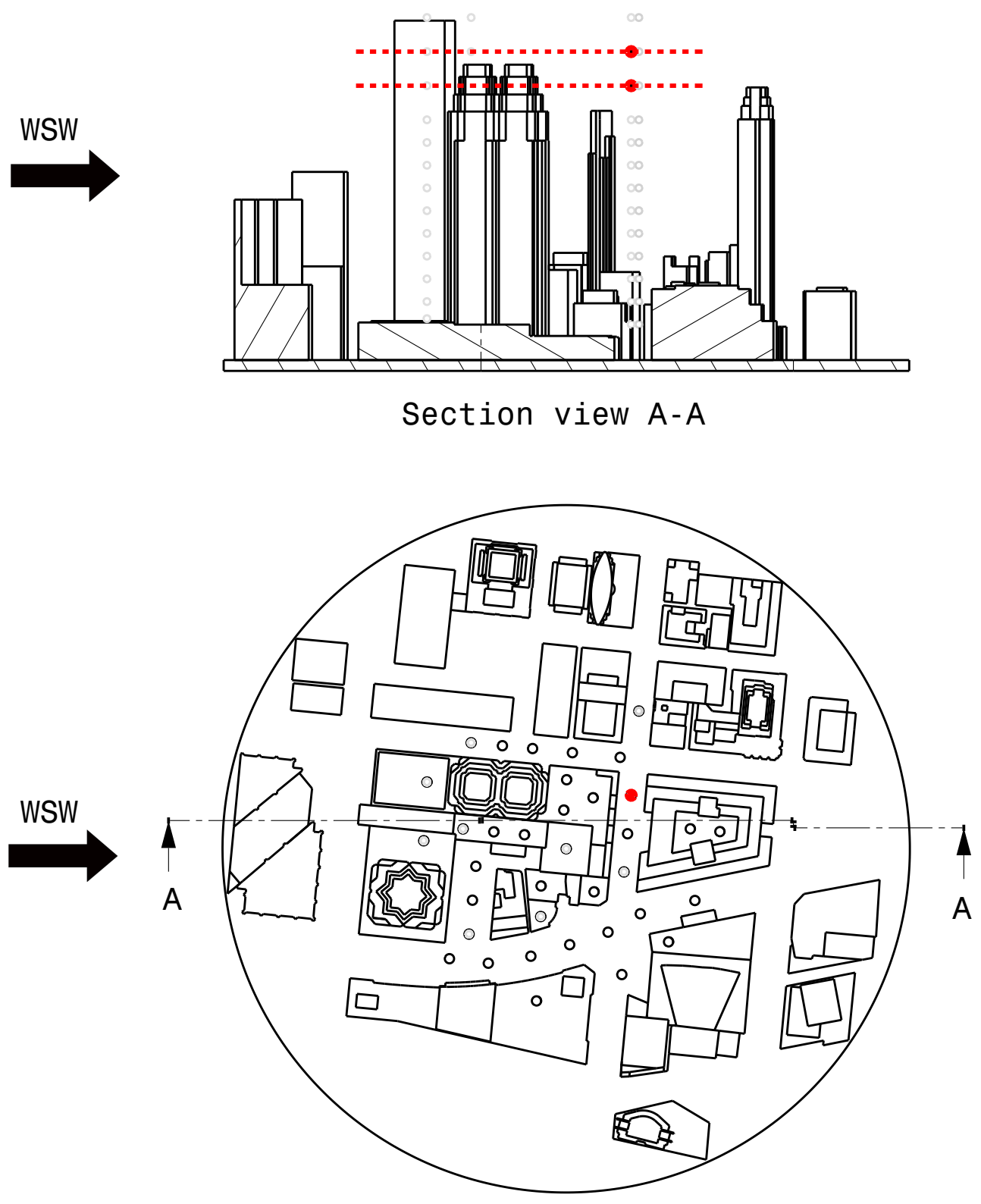

Figure 4.42: The location of Increments 11-12 at Spot 9 (red markers) with a model orientation representing WSW wind direction.

(Adverse case of vertical speed shear) 


\section{Vertical directional shear}

Vertical directional shear reflects the amount of change in vertical flow direction between two consecutive layers. The change could be from an upward flow direction to a downward flow direction (or vice versa), or simply a change in the flow angle within the same direction (upward/downward). The most severe case of vertical directional shear was found between Increments 9 and 10 of Spot 17 upstream of a building for wind coming from $\mathrm{N}_{16.7}$, the location of which was previously shown in Figure 4.32 with Increments 9-10 being denoted by dashed lines that represents the stagnation zone. The degree of vertical directional shear between Increments 9 and 10 was equal to $541 \mathrm{deg} / \mathrm{m}$, which corresponds to a change from $16.3^{\circ}$ downward flow to $5.3^{\circ}$ upward flow, a total of $22.4^{\circ}$ over a height of $0.04 \mathrm{~m}(16 \mathrm{~m}$ FS) as shown in Figure 4.43 .

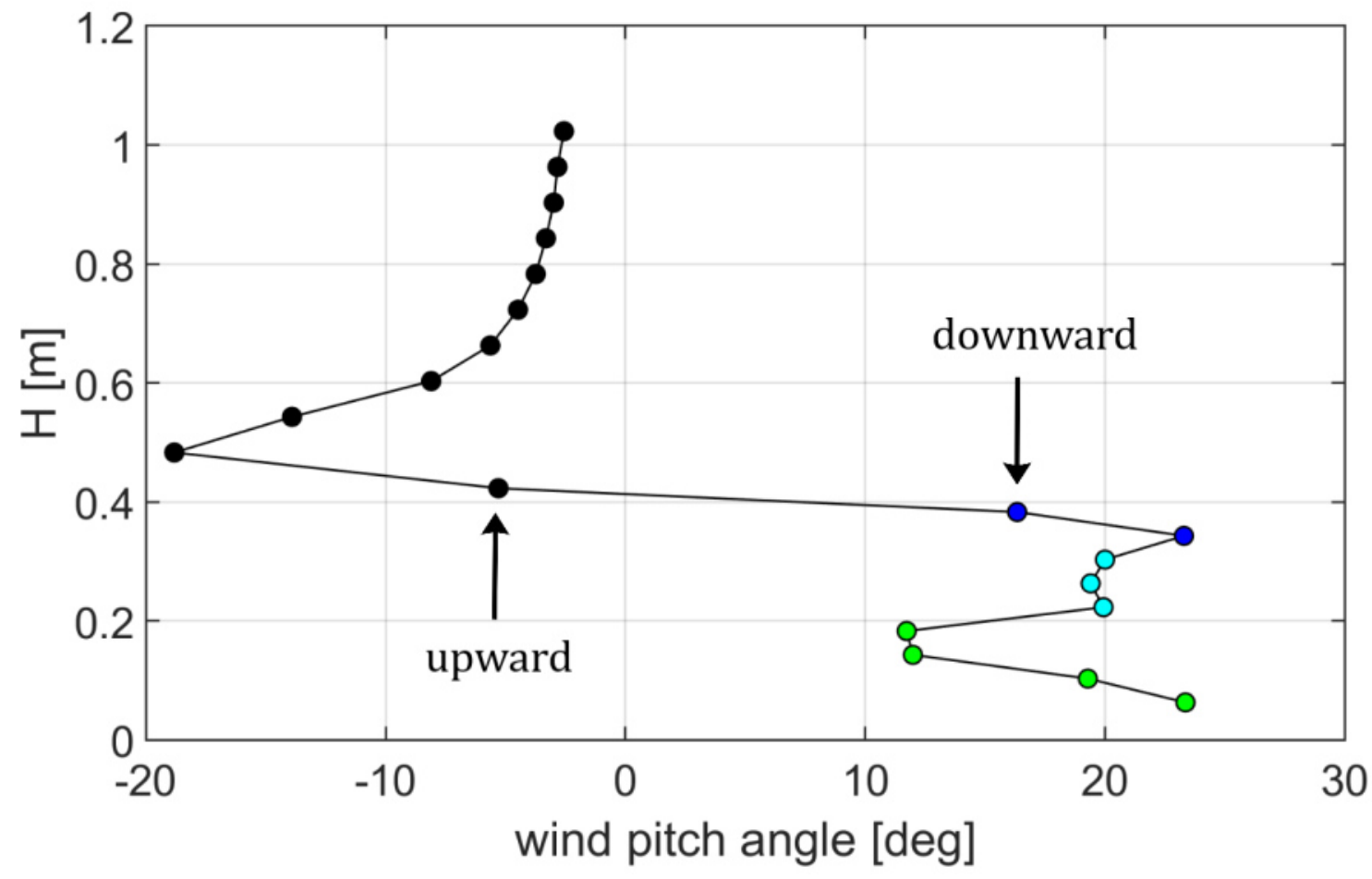

Figure 4.43: Pitch angle profile at Spot 17 for wind direction $\mathrm{N}_{16.7}$. Total increments $=20$. 
The vertical directional shear was also found on buildings rooftop, an example of which is the shear between Increments 1 and 2 of Spot 28 for a wind coming from $\mathrm{E}_{17.1}$, whose location can be identified from Figure 4.33. The degree of vertical directional shear at Spot 28 was found to be $483 \mathrm{deg} / \mathrm{m}$, which corresponds to a change by $19.3^{\circ}$ in vertical flow direction over a height of $0.04 \mathrm{~m}$ (16 m FS). The change in the vertical flow by $19.3^{\circ}$ between Increments $1-2$ was due to the shift from $17.6^{\circ}$ downward flow to $1.7^{\circ}$ upward flow as seen in Figure 4.34 .

A case of vertical directional shear within the same flow direction (upward/downward) was found in the shear between Increments 1-2 of Spot 6 for wind coming from $\mathrm{S}_{15}$, without the future buildings (see Figure 4.44). The vertical directional shear was found to be $380 \mathrm{deg} / \mathrm{m}$, which corresponds to a change in the downward flow from $19.7^{\circ}$ at Increment 1 to $4.5^{\circ}$ at Increment $2(\sim 4$ times the direction of Increment 1) as illustrated in Figure 4.45. The spot location was between two buildings at the height of Increment 1 as seen in Figure 4.44. Consequently, the large amount of downdraft at Increment 1 as compared to Increment 2 is expected to be the result of the flow motion over Building I along with the flow separation from the tall building located nearby Spot 6 . 


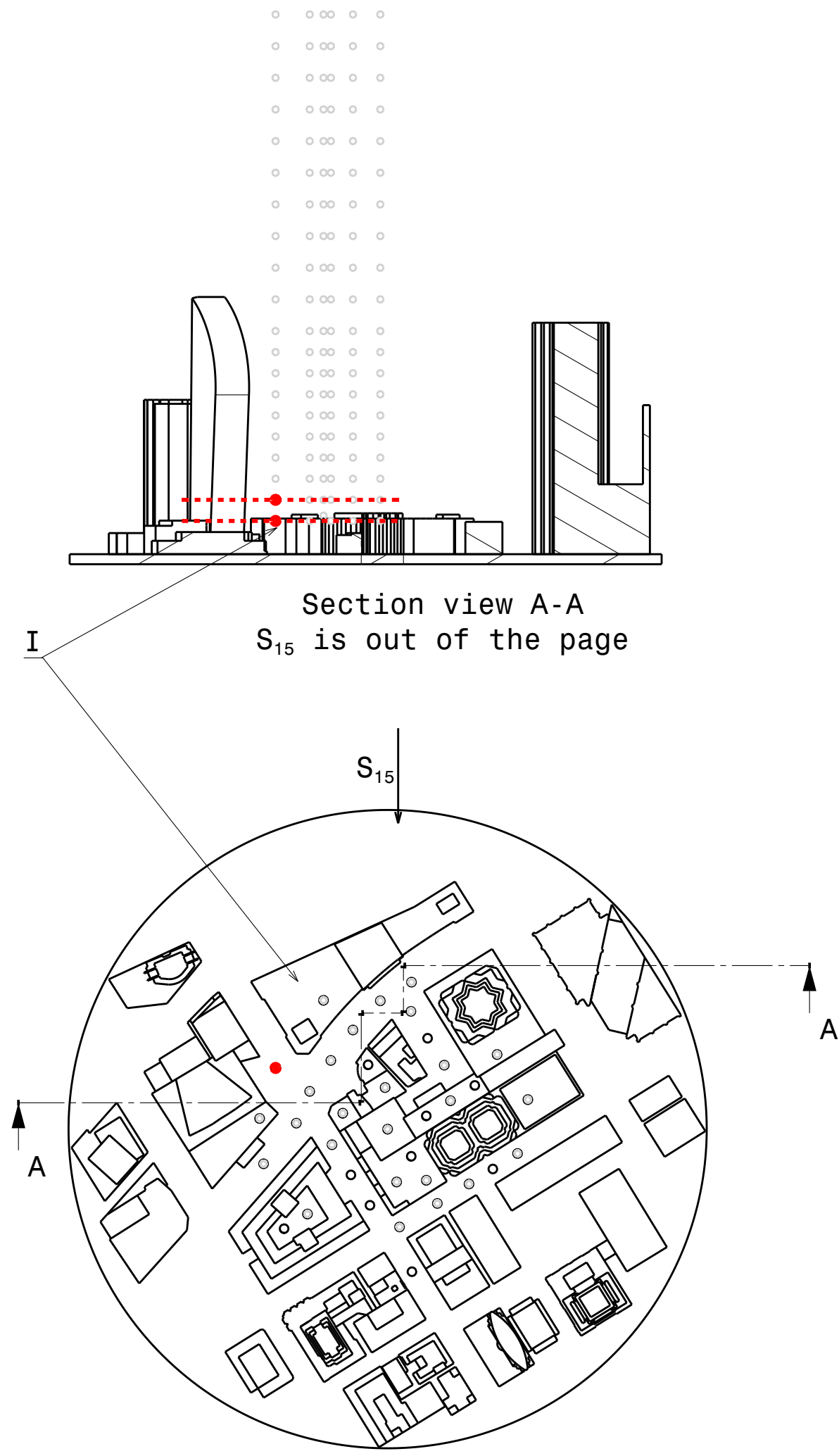

Figure 4.44: The location of Increments 1-2 at Spot 6 (red markers) with a model orientation representing $\mathrm{S}_{15}$ wind direction.

(Adverse case of virtical directional shear) 


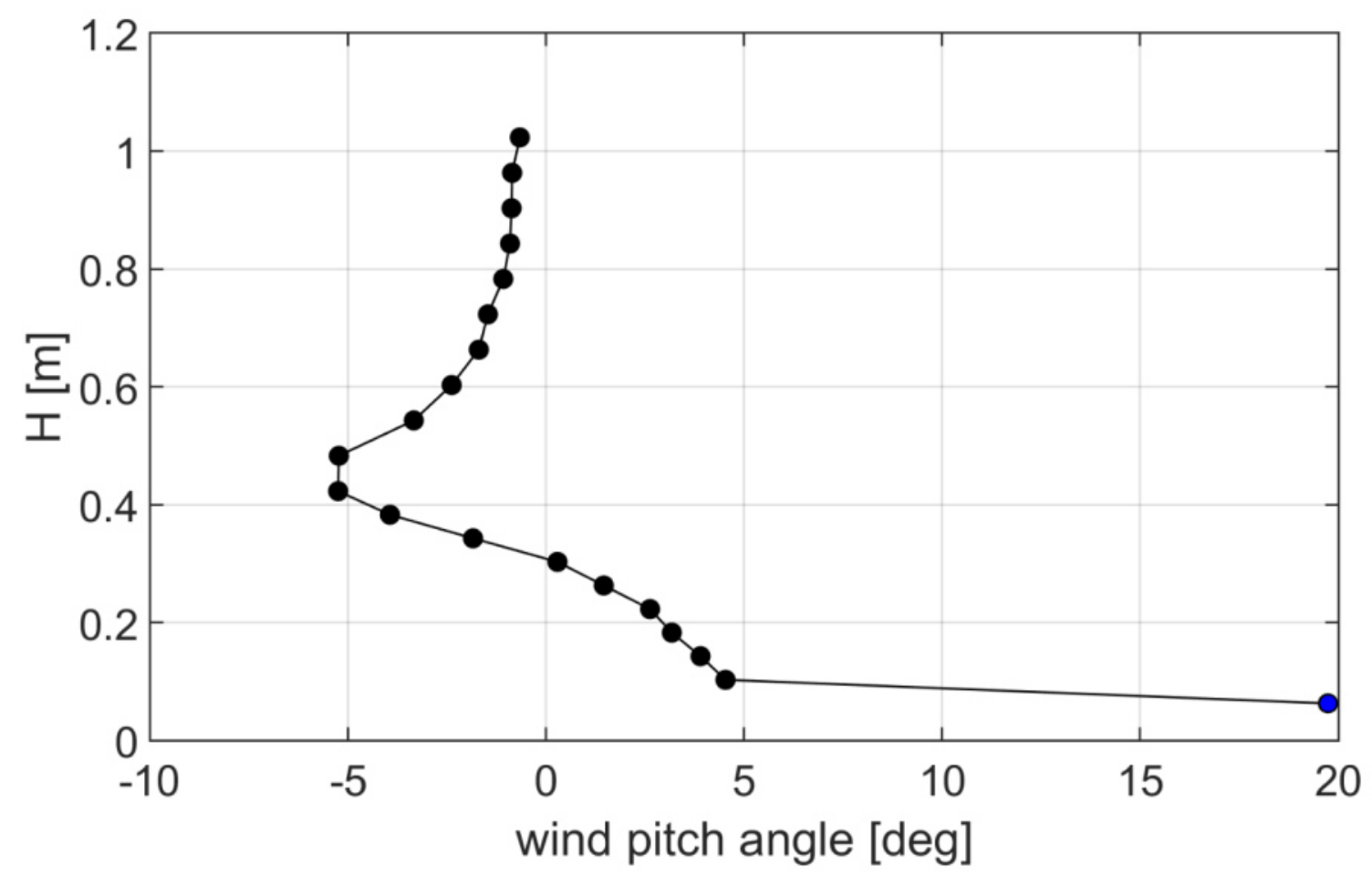

Figure 4.45: Pitch angle profile at Spot 6 for wind direction $\mathrm{S}_{15}$. Total increments $=20$. 


\section{Horizontal directional shear}

The horizontal directional shear represents the variations in wind yaw angle between two vertically adjacent layers. The most severe change in horizontal wind direction was found between Increments 11 and 12 of Spot 23 for wind coming from $\mathrm{N}_{16.7}$, the location of which is shown in Figure 4.46. The corresponding degree of horizontal directional shear between the two layers was found to be $833 \mathrm{deg} / \mathrm{m}$, which yields to a change in yaw angle by $50^{\circ}$ over a height of $0.06 \mathrm{~m}$ (24 m FS). As seen in Figure 4.46 . the wind yaw angle at Increment 12 was almost aligned with the incoming flow since the measurement was taken above the rooftop of Building I; therefore, the change in wind yaw angle from $0.7^{\circ}$ at Increment 12 to $51.7^{\circ}$ at Increment 11 (consult Figure $4.37(\mathrm{~b})$ ) can be attributed to the flow separation from Building I second top layer, causing the flow to twist by the indicated amount.

Some adverse cases of horizontal directional shear were found at low height in the wake of buildings. Figure 4.47 shows the location of Increments 2-3 of Spot 9 in WSW wind direction, where the degree of horizontal wind shear was equal to 635 $\mathrm{deg} / \mathrm{m}$, which is equivalent to a change of $25.4^{\circ}$ in wind yaw angle over a height of $0.04 \mathrm{~m}$ (16 m FS). As can be seen in Figure 4.47, both Increments 2 and 3 are located downstream of a tall building that has a uniform cross-sectional geometry within the increments height. By considering the negative yaw angles at Increments 2-3 (see Figure 4.48, which are denoted by red arrows in Figure 4.47, it is expected that the horizontal directional shear found between the two vertical layers is due to the flow passing through the opening of an upstream building at the height of Increment 3 as illustrated in Figure 4.47 .

Horizontal directional shear was also found in spots that are not located in closeproximity to buildings, an example of which is the shear between Increment 11-12 of Spot 31, that are located below the future buildings rooftops for a model orientation representing $\mathrm{S}_{15}$ wind direction (see Figure 4.49). The shear between both layers was 
equal to $442 \mathrm{deg} / \mathrm{m}$, which corresponds to a change of $25.1^{\circ}$ over a height of $0.06 \mathrm{~m}$ (24 m FS).

Although the presented results of horizontal directional shear show a maximum of $50^{\circ}$ of change between two consecutive vertical measurements, there are significantly more extreme cases that have not been included in the analysis due to the low \%GD. However, the use of the probe indexing mechanism during the process of data sampling allowed for obtaining the best estimate of the flow horizontal direction despite the fact that the probe was not capturing the entire data, which is evident of the extreme wind flapping. An example of such cases is the $162^{\circ}$ horizontal directional shear between Increments 10-11 of Spot 26 for wind coming from $\mathrm{N}_{16.7}$, the yaw profile of which was previously shown in Figure 4.37(c). 

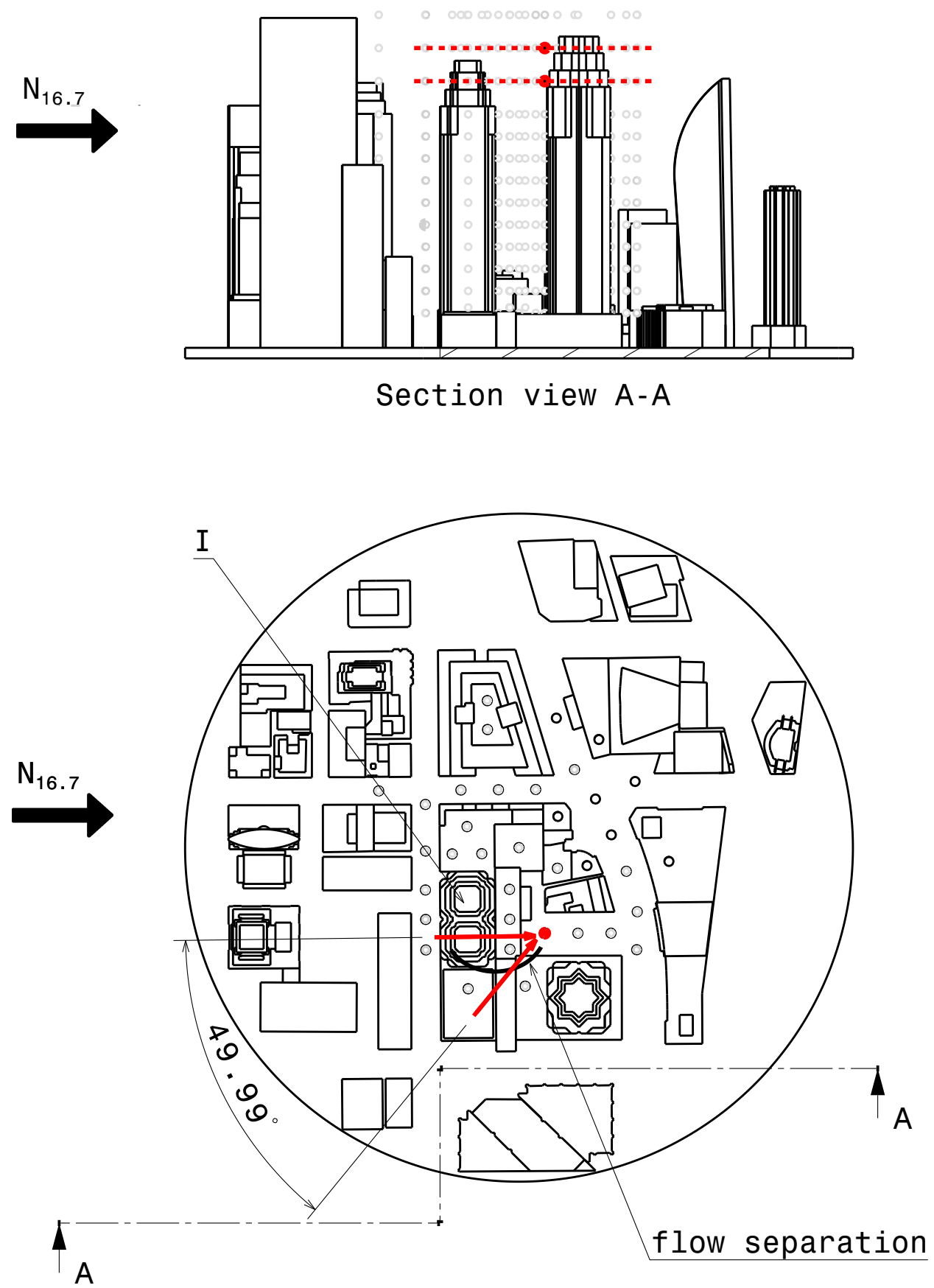

Figure 4.46: The location of Increments 11-12 at Spot 23 (red markers) with a model orientation representing $\mathrm{N}_{16.7}$ wind direction.

(Severe case of horizontal directional shear) 


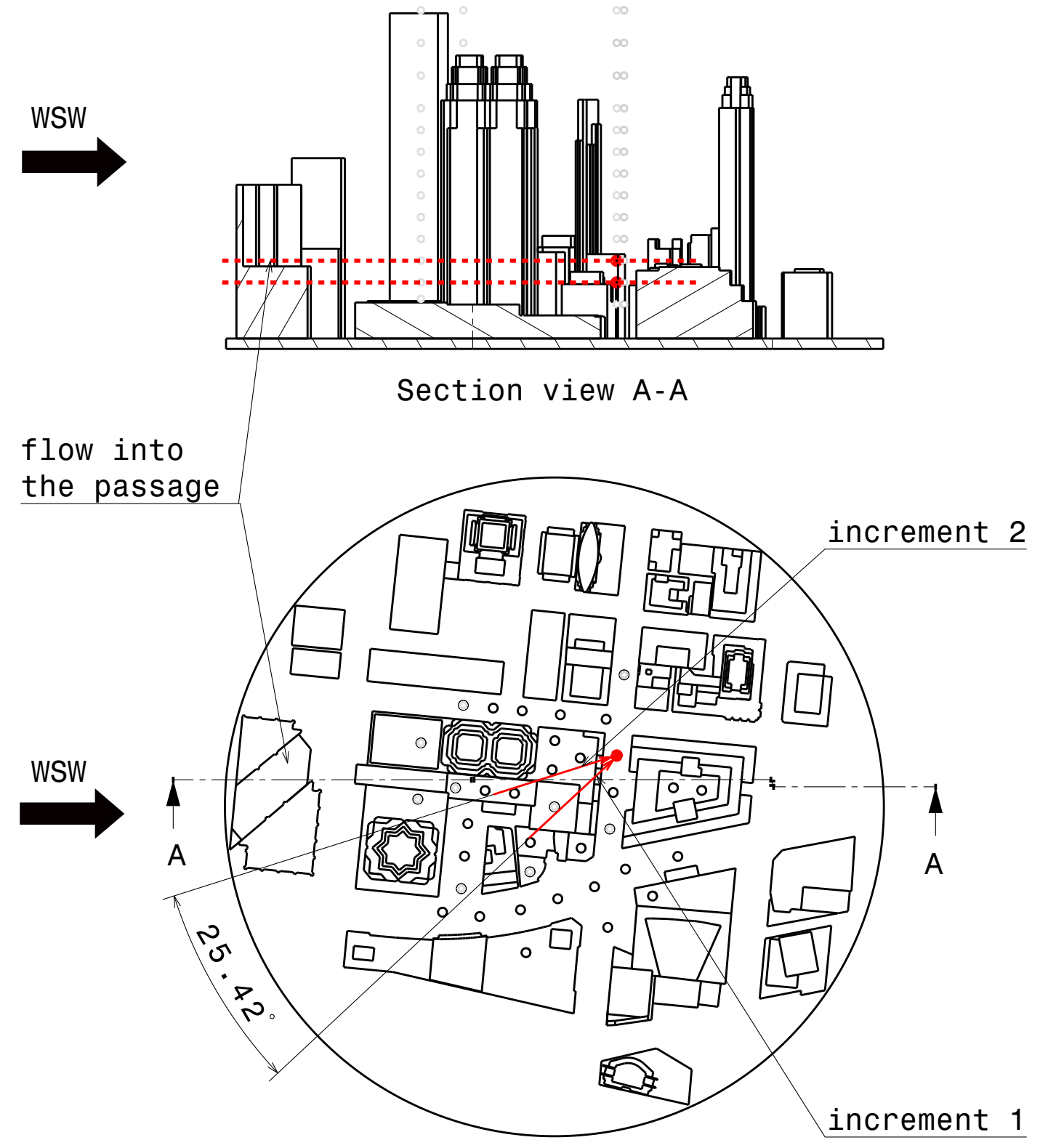

Figure 4.47: The location of Increments 2-3 at Spot 9 (red markers) with a model orientation representing WSW wind direction.

(Adverse case of horizontal directional shear) 


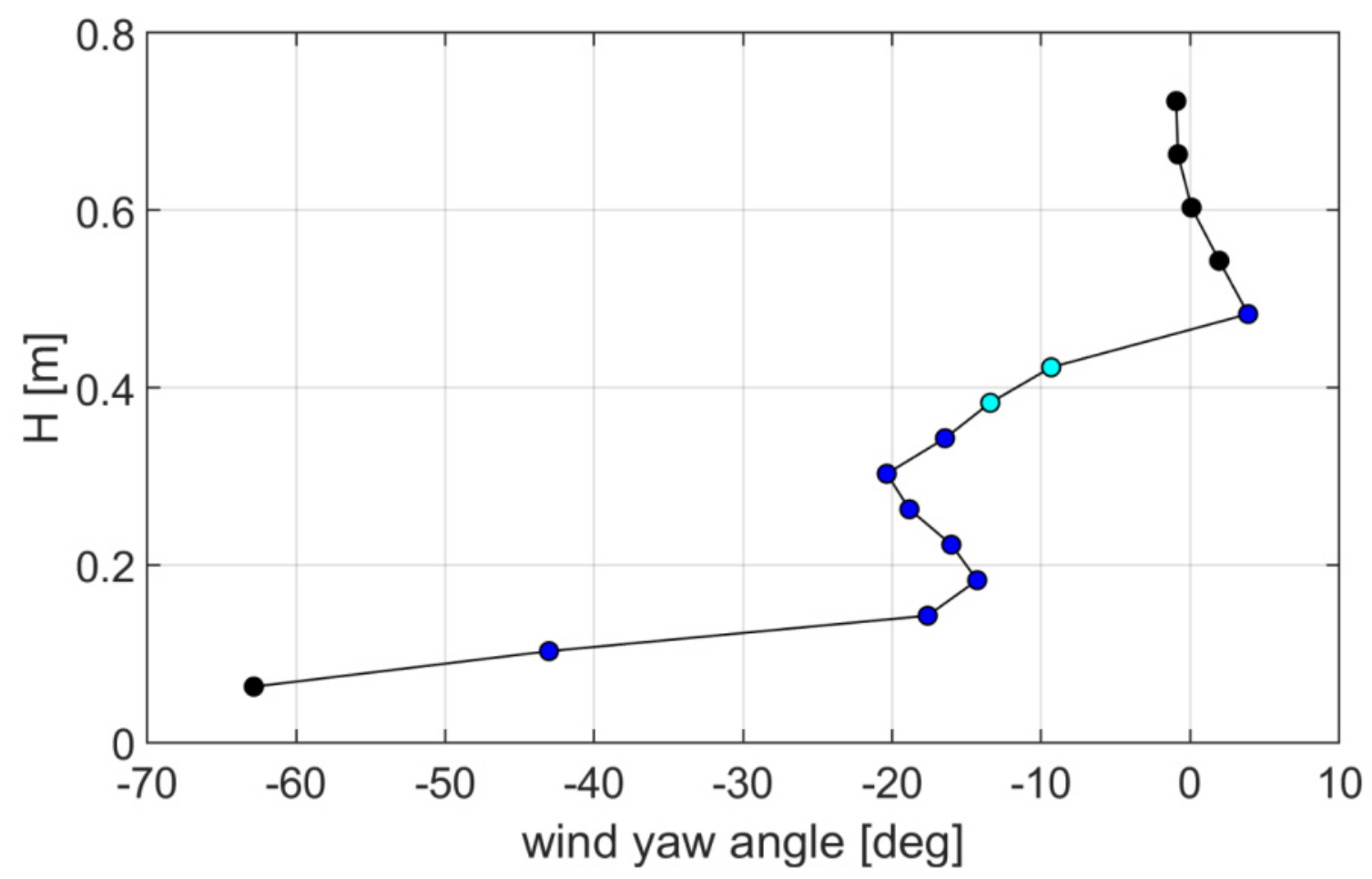

Figure 4.48: Yaw angle profile at Spot 9 for wind direction WSW. Total increments $=15$. 


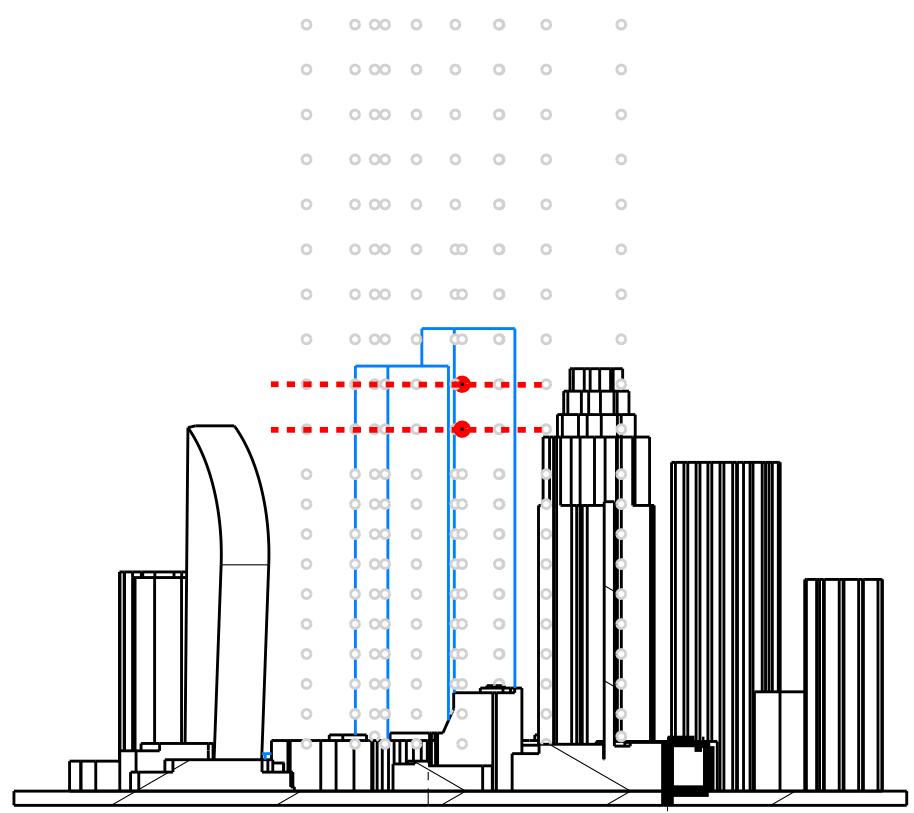

Section view A-A

$S_{15}$ is out of the page

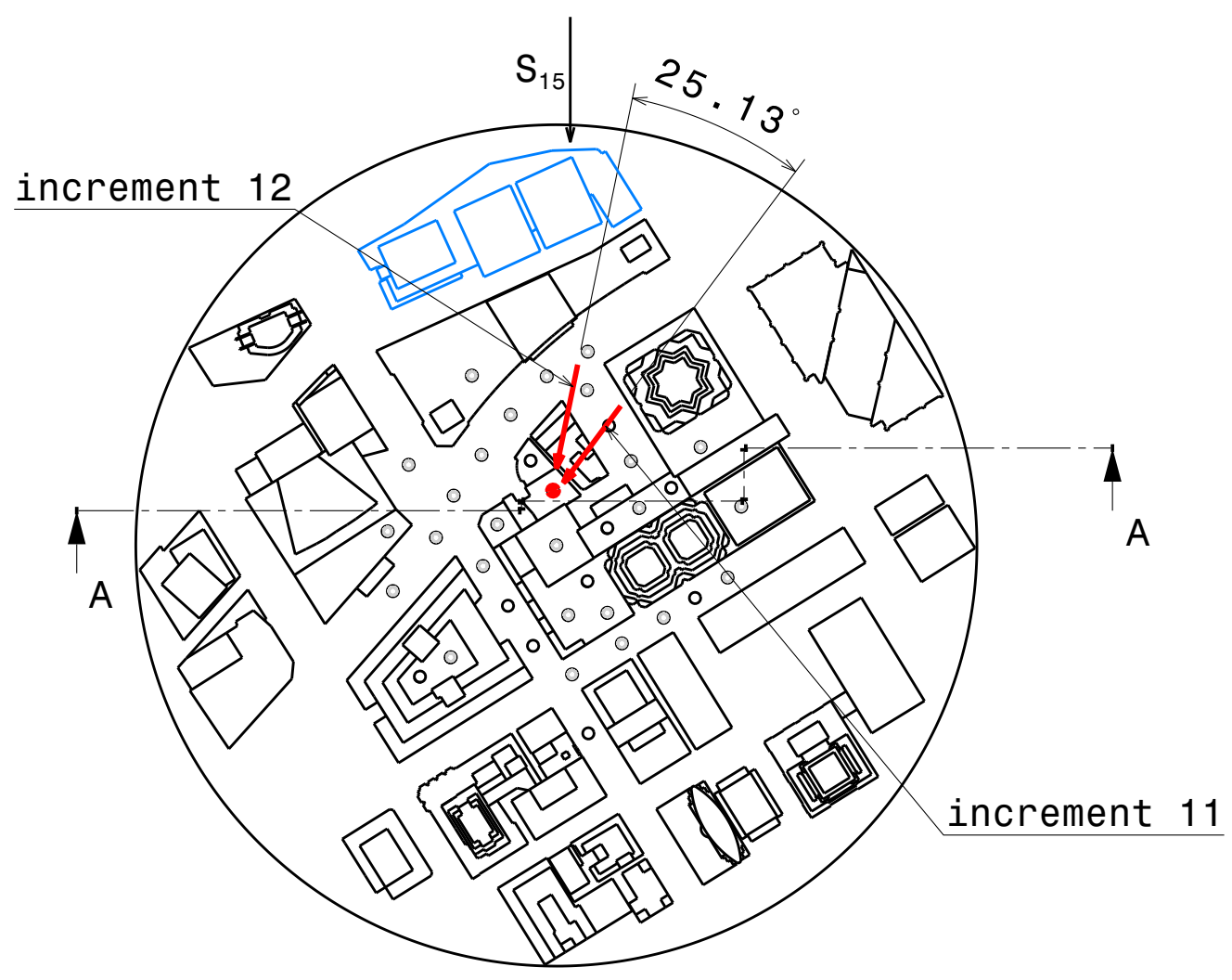

Figure 4.49: The location of Increments 11-12 at Spot 31 (red markers) with a model orientation representing $\mathrm{S}_{15}$ wind direction.

(Adverse case of horizontal directional shear) 


\subsubsection{Turbulence intensity}

The atmospheric turbulence is one of the major factors that can make flight operations hazardous. Within the urban boundary layer, the effect of 'mechanical' turbulence is highly pronounced as it is directly related to the terrain roughness and wakes of tall structures. Studies have shown that turbulence levels in urban settings can be four times [33] and two times [46] more intense than the levels found in the free atmosphere and in rural areas, respectively. This is due to the impact of urban airflow on the steadiness of mean wind speed, hence, the more the changes in the wind speed, the more severe the turbulence.

In this research, the turbulence intensity was investigated by looking into the fluctuations of each velocity component with respect to the local mean wind speed as opposed to the fluctuations in the magnitude of the velocity vector with respect to the local mean wind speed. This better reflects the amount of flow unsteadiness that an urban air taxi may experience along any of the three axes, namely, longitudinal, lateral, and vertical axes. Furthermore, since turbulence is known to be affected by the surface roughness, it was decided to divide the model into 5 vertical zones, as shown in Figure 4.50, and to find the maximum turbulence intensities from the measurements taken at each zone for each of the considered wind directions. This ensures better probing of the urban 3D space that an urban air taxis will fly through. For each of the wind directions, the maximum turbulence intensities found at each of the vertical zones are given in Table 4.4, and the corresponding locations in the form: (spot,increment) are given in Table 4.5. From Table 4.4, the maximum turbulence intensities across each zone are summarized in the following statements.

In Zone 1, the maximum longitudinal, lateral, and vertical turbulence intensities were found to be $40 \%, 45 \%$, and $38 \%$, respectively.

In Zone 2, the maximum longitudinal, lateral, and vertical turbulence intensities 
were found to be $46 \%, 45 \%$, and $36 \%$, respectively.

In Zone 3, the maximum longitudinal, lateral, and vertical turbulence intensities were found to be $42 \%, 47 \%$, and $37 \%$, respectively.

In Zone 4, the maximum longitudinal, lateral, and vertical turbulence intensities were found to be $35 \%, 39 \%$, and $36 \%$, respectively.

In Zone 5, the maximum longitudinal, lateral, and vertical turbulence intensities were found to be $6 \%, 5 \%$, and $5 \%$, respectively.

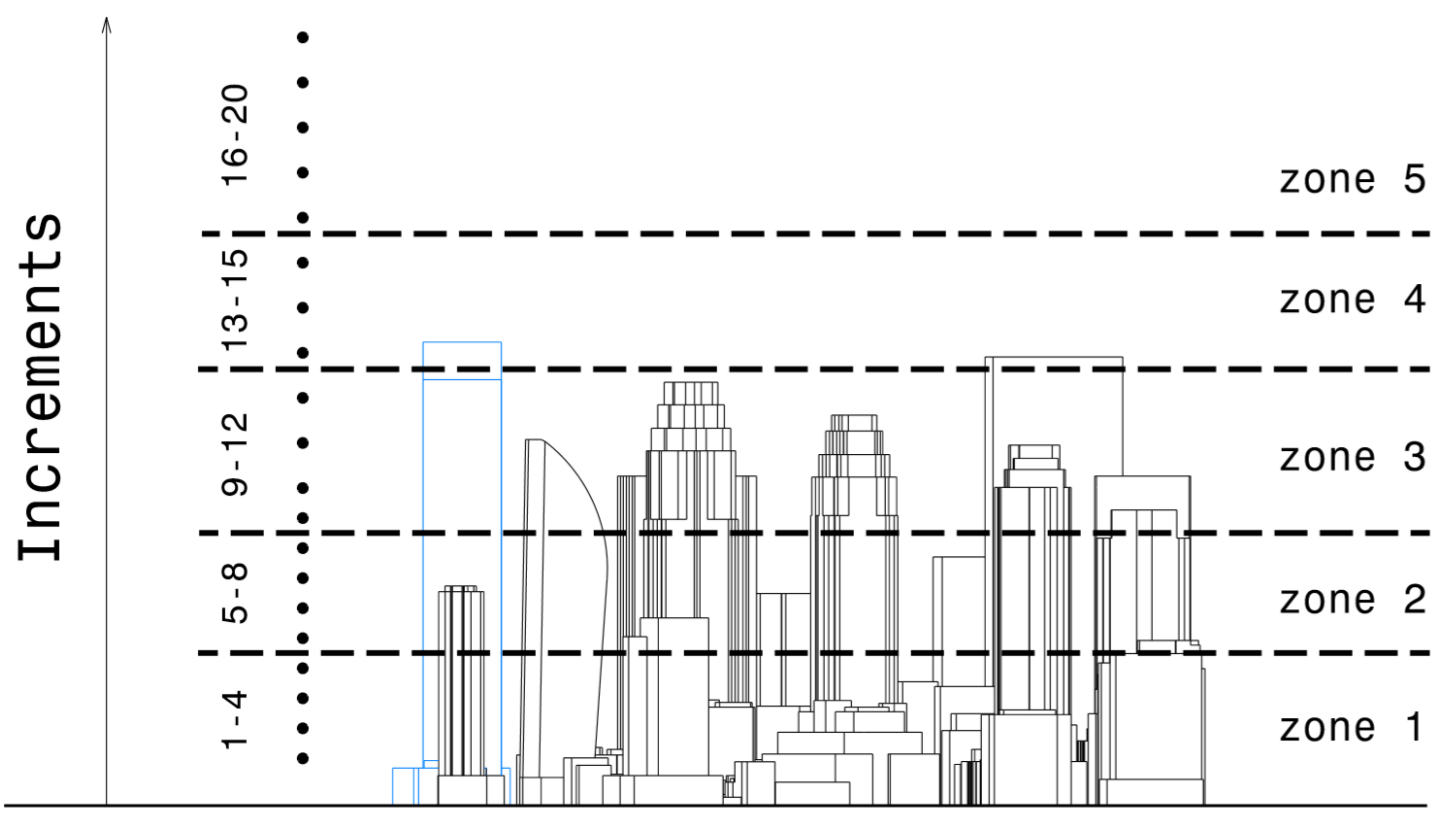

Figure 4.50: Vertical zones for the assessment of turbulence intensity within and above the city model. 
Table 4.4: Severe turbulence intensity values (in \%) at each of the vertical zones of the city model.

\begin{tabular}{|c|c|c|c|c|c|c|c|c|c|c|c|c|c|c|c|c|c|c|}
\hline \multirow{3}{*}{ Zone } & \multicolumn{18}{|c|}{ Wind directions } \\
\hline & \multicolumn{3}{|c|}{ WSW } & \multicolumn{3}{|c|}{$\mathrm{WSW}_{+15}$} & \multicolumn{3}{|c|}{$\mathrm{E}_{17.1}$} & \multicolumn{3}{|c|}{$\mathrm{N}_{16.7}$} & \multicolumn{3}{|c|}{$\mathrm{S}_{15, \text { without }}$} & \multicolumn{3}{|c|}{$\mathrm{S}_{15, w i t h}$} \\
\hline & $\mathrm{I}_{u u}$ & $\mathrm{I}_{v v}$ & $\mathrm{I}_{w w}$ & $\mathrm{I}_{u u}$ & $\mathrm{I}_{v v}$ & $\mathrm{I}_{w w}$ & $\mathrm{I}_{u u}$ & $\mathrm{I}_{v v}$ & $\mathrm{I}_{w w}$ & $\mathrm{I}_{u u}$ & $\mathrm{I}_{v v}$ & $\mathrm{I}_{w w}$ & $\mathrm{I}_{u u}$ & $\mathrm{I}_{v v}$ & $\mathrm{I}_{w w}$ & $\mathrm{I}_{u u}$ & $\mathrm{I}_{v v}$ & $\mathrm{I}_{w w}$ \\
\hline 1 & 40 & 45 & 36 & 32 & 32 & 34 & 34 & 27 & 31 & 40 & 34 & 35 & 37 & 38 & 34 & 39 & 42 & 38 \\
\hline 2 & 38 & 42 & 35 & 36 & 35 & 33 & 32 & 27 & 25 & 40 & 36 & 36 & 36 & 38 & 35 & 46 & 45 & 33 \\
\hline 3 & 38 & 42 & 36 & 38 & 42 & 31 & 30 & 27 & 25 & 42 & 39 & 34 & 36 & 38 & 33 & 42 & 47 & 37 \\
\hline 4 & 23 & 18 & 19 & 32 & 31 & 31 & 11 & 9 & 10 & 26 & 28 & 25 & 27 & 21 & 19 & 35 & 39 & 36 \\
\hline 5 & - & - & - & - & - & - & - & - & - & 6 & 4 & 5 & 6 & 5 & 5 & 6 & 4 & 5 \\
\hline
\end{tabular}

Table 4.5: Locations of severe turbulence intensity values at each of the vertical zones of the city model.

\begin{tabular}{|c|c|c|c|c|c|c|c|c|c|c|c|c|c|c|c|c|c|c|}
\hline \multirow{3}{*}{ Zone } & \multicolumn{18}{|c|}{ Wind directions } \\
\hline & \multicolumn{3}{|c|}{ WSW } & \multicolumn{3}{|c|}{$\mathrm{WSW}_{+15}$} & \multicolumn{3}{|c|}{$\mathrm{E}_{17.1}$} & \multicolumn{3}{|c|}{$\mathrm{N}_{16.7}$} & \multicolumn{3}{|c|}{$\mathrm{S}_{15, \text { without }}$} & \multicolumn{3}{|c|}{$\mathrm{S}_{15, w i t h}$} \\
\hline & $\mathrm{I}_{u u}$ & $\mathrm{I}_{v v}$ & $\mathrm{I}_{w w}$ & $\mathrm{I}_{u u}$ & $\mathrm{I}_{v v}$ & $\mathrm{I}_{w w}$ & $\mathrm{I}_{u u}$ & $\mathrm{I}_{v v}$ & $\mathrm{I}_{w w}$ & $\mathrm{I}_{u u}$ & $\mathrm{I}_{v v}$ & $\mathrm{I}_{w w}$ & $\mathrm{I}_{u u}$ & $\mathrm{I}_{v v}$ & $\mathrm{I}_{w w}$ & $\mathrm{I}_{u u}$ & $\mathrm{I}_{v v}$ & $\mathrm{I}_{w w}$ \\
\hline 1 & 30,3 & 30,3 & 9,4 & 19,2 & 18,1 & 33,4 & 10,2 & 10,1 & 10,2 & 5,3 & 26,1 & 13,1 & 10,1 & 10,1 & 10,2 & 31,4 & 32,4 & 12,2 \\
\hline 2 & 33,6 & 9,8 & 9,8 & 7,5 & 9,8 & 7,5 & 10,8 & 10,8 & 28,5 & 2,5 & 29,8 & 18,5 & 18,6 & 18,6 & 18,6 & 31,6 & 32,5 & 3,8 \\
\hline 3 & 25,11 & 9,11 & 9,11 & 30,9 & 30,9 & 25,9 & 10,9 & 10,9 & 10,9 & 26,11 & 29,11 & 26,11 & 23,12 & 16,12 & 16,12 & 31,9 & 35,10 & 5,12 \\
\hline 4 & 11,13 & 11,13 & 11,13 & 11,14 & 11,14 & 11,14 & 18,13 & 18,13 & 18,13 & 19,13 & 19,13 & 19,13 & 16,13 & 16,13 & 20,13 & 35,13 & 35,13 & 35,13 \\
\hline 5 & - & - & - & - & - & - & - & - & - & 7,16 & 7,16 & 1,16 & 10,16 & 22,16 & 18,16 & 14,16 & 15,16 & 22,16 \\
\hline
\end{tabular}




\section{Along-wind turbulence intensity}

The most severe cases of $40 \%$ longitudinal turbulence in Zone 1 were found in the near wake of low-rise building, particularly at increments located approximately at the same height as the upstream buildings rooftop. Two examples are Increment 3 of Spot 5 for wind direction $\mathrm{N}_{16.7}$, the location of which was previously shown in Figure 4.25, and Increment 3 of Spot 30 for wind direction WSW, whose location is shown in Figure 4.51. Although both spots are exposed to approximately the same flow conditions and turbulence level in the first Zone of the model, Spot 30 is located in the wake of other tall buildings, which explains the lower wind speed and higher turbulence level in the vertical profiles of Spot 30 as compared to those of Spot 5 (see Figure 4.52.

The maximum longitudinal turbulence intensities in Zones 2 and 3, were found in Increments 6 and 9 of Spot 31 in the wake of the future buildings for wind direction $\mathrm{S}_{15}$ as illustrated in Figure 4.53. Although Spot 31 is $128 \mathrm{~m}$ (FS) distant from the future buildings, the turbulence level was severe at the spot location, where longitudinal turbulence intensities were equal to $46 \%$ and $42 \%$ in Zones 2 and 3, respectively. In Zone 3, the longitudinal turbulence intensity at Increment 11 of Spot 26 for wind direction $\mathrm{N}_{16.7}$ was also equal to $42 \%$. The spot location can be extracted from Figure 4.36. Although Increment 11 of Spot 26 is located well above ground level, the turbulence was considerably severe due to the spot location being downstream of multiple tall buildings (Buildings I, II and III).

In Zone 4, the maximum turbulence of $35 \%$ was found at Increment 13 of Spot 35, that is $14.25 \mathrm{~mm}$ below the rooftop of one of the future buildings as seen in Figure 4.53. Due to the spot location and height, it is expected that the turbulence is mainly driven by the future buildings.

For Zone 5, which is above buildings level, the maximum longitudinal turbulence was found to be $6 \%$. Considering the measurements in Zone 5 for wind directions 
$\mathrm{N}_{16.7}$ and $\mathrm{S}_{15}$ with and without the future buildings, the maximum turbulence was always found in Increment 16, which is the closest to the buildings rooftops. The turbulence in this zone was almost similar for all spots (variations are less than $2 \%$ ) and no specific spot warranted additional discussion. 


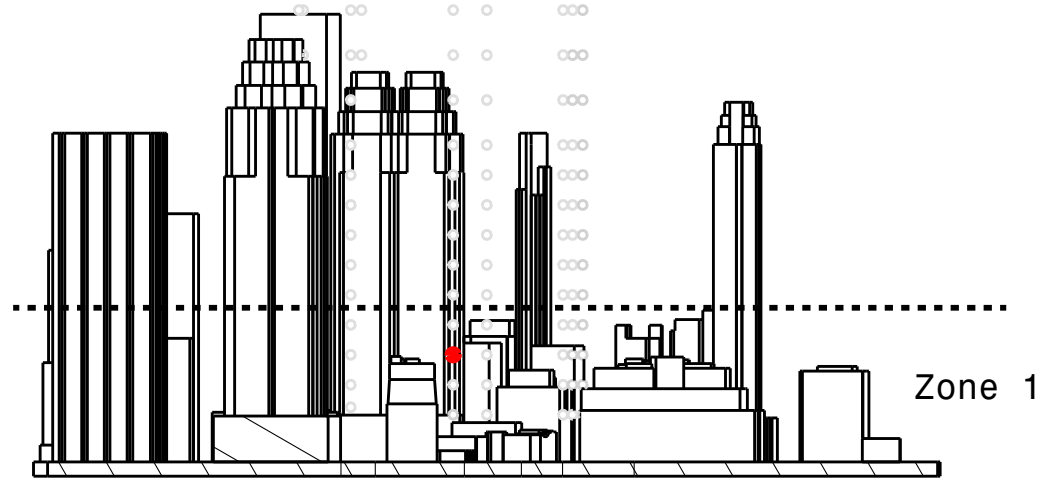

Section view $A-A$
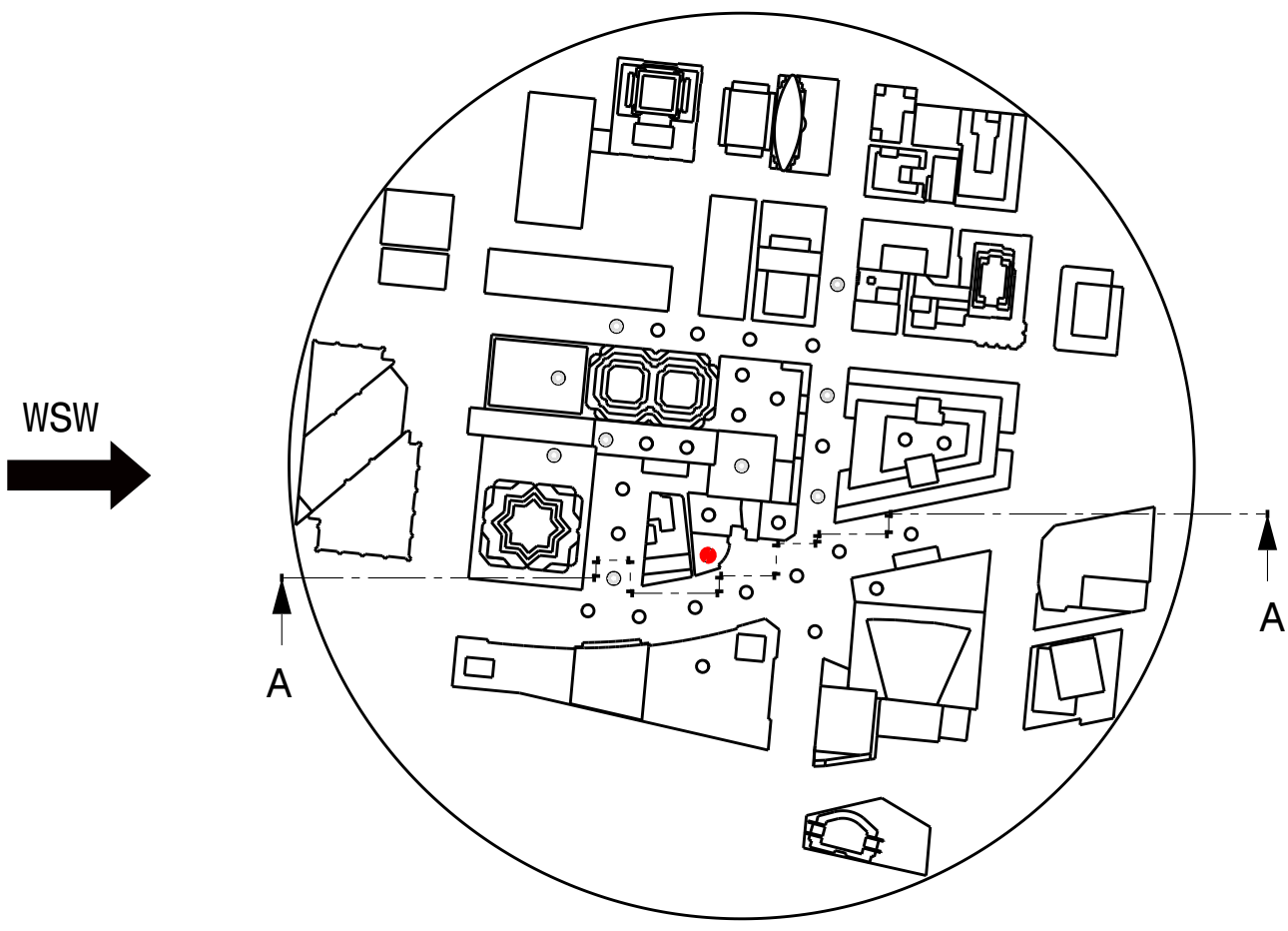

Figure 4.51: The location of Spot 30 (red markers) with a model orientation representing WSW wind direction.

(Severe case of longitudinal turbulence intensity in Zone 1) 


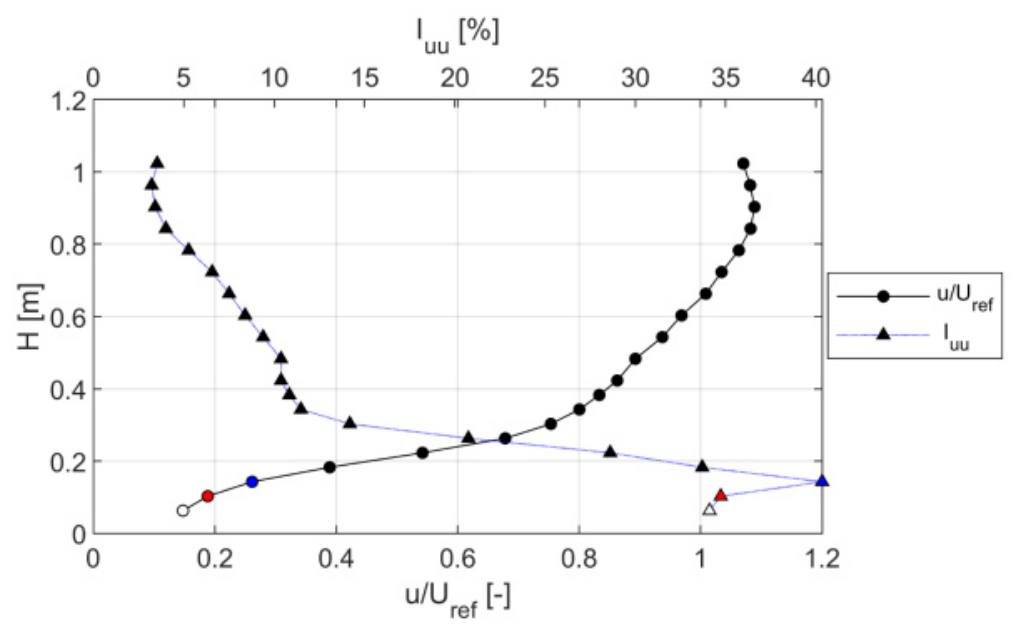

(a)

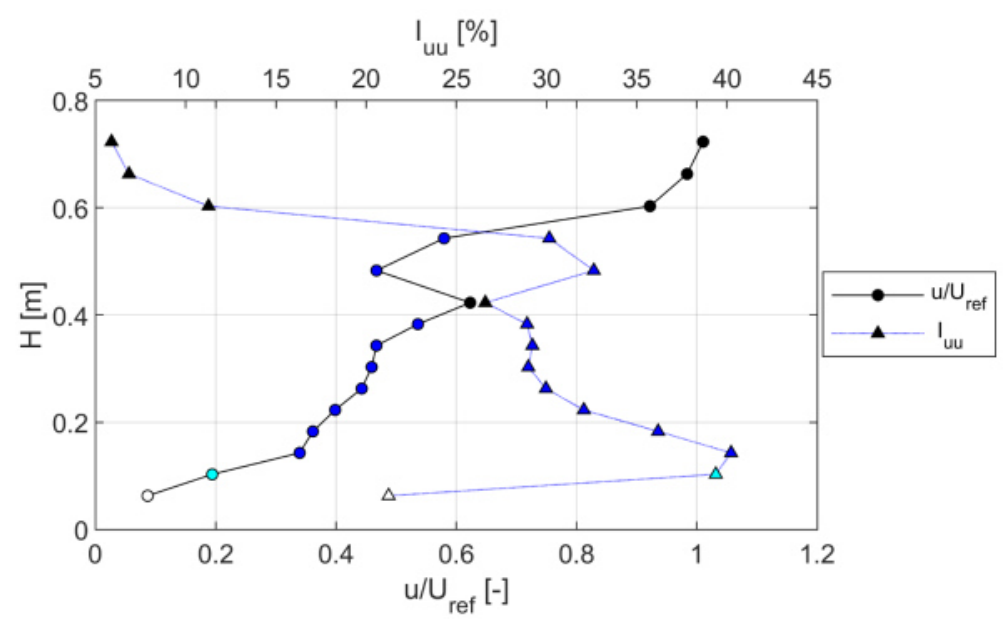

(b)

Figure 4.52: (a) Longitudinal velocity and turbulence intensity profiles at Spots 5 for wind direction $\mathrm{N}_{16.7}$ (total increments $=20$ ) and (b) 30 for wind direction WSW (total increments $=15)$. 


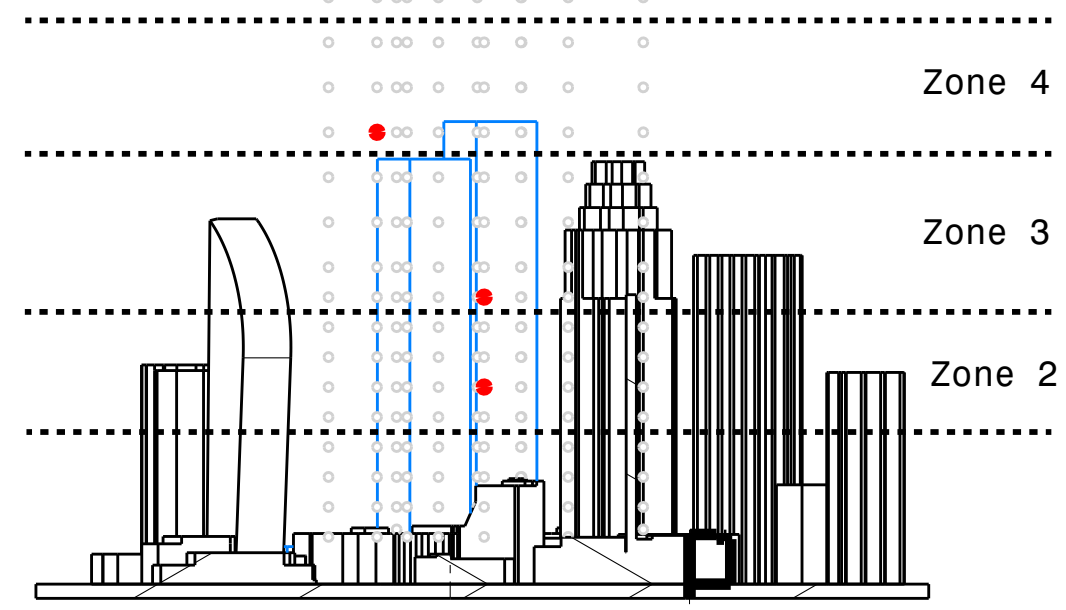

Section view A-A

$S_{15}$ is out of the page

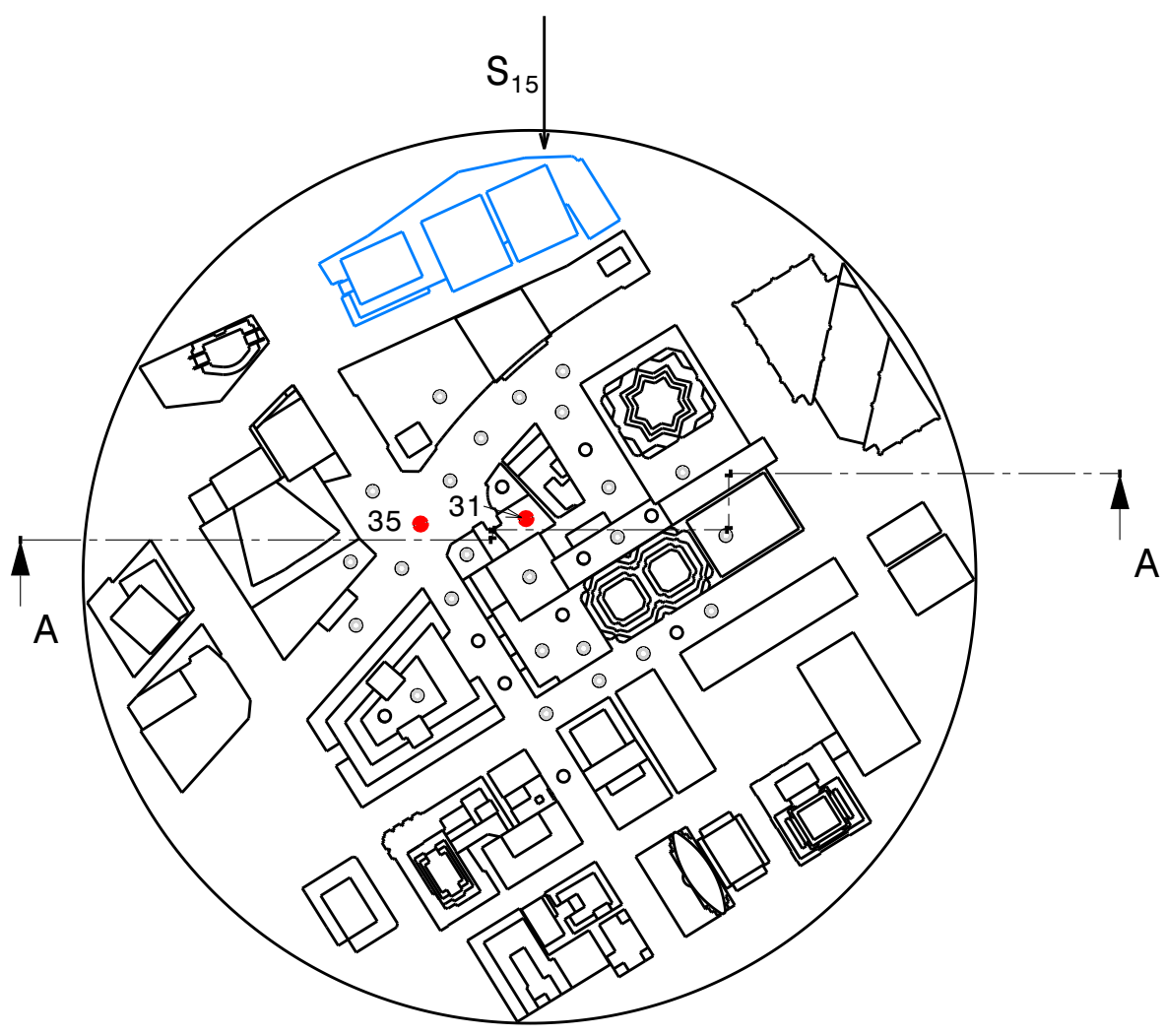

Figure 4.53: The location of Spots 31 and 35 (red markers) with a model orientation representing $\mathrm{S}_{15}$ wind direction.

(Severe cases of longitudinal turbulence intensity in Zones 2, 3 and 4) 


\section{Across-wind turbulence intensity}

The across-wind disturbances strongly impact the stability of both fixed-wing and rotary-wing aircraft, which are more susceptible to disturbances along their longitudinal axes [46]. From Table 4.4, it can be seen that in many instances, the lateral turbulence intensity exceeded both the longitudinal and the vertical ones.

For the first Zone of the model, the lateral turbulence intensity of $45 \%$ was found at the same location of maximum longitudinal turbulence, that is Increment 3 of Spot 30 for wind direction WSW (refer back to Figure 4.51).

The maximum values of lateral turbulence intensity in Zones 2, 3 and 4 were found in $\mathrm{S}_{15}$ wind direction, specifically in the wake of the future buildings. Figure 4.54 shows the location of the spots for each of the zones, where $45 \% \mathrm{I}_{v v}, 47 \% \mathrm{I}_{v v}$ and $39 \% \mathrm{I}_{v v}$ were found at Increment 5 of Spot 32, Increment 10 of Spot 35 and Increment 13 of Spot 35, respectively. The lateral velocity, lateral turbulence intensity and yaw angle profiles at Spot 35 are shown in Figure 4.55. It can be seen in the figure that changes in the across-wind velocities and yaw angles were more pronounced between Increments 1 and 14, where the latter is located $0.046 \mathrm{~m}(18.40 \mathrm{~m} \mathrm{FS})$ above the future buildings. Within the same increments (1-14), the turbulence level was also high especially within the height of the two future buildings (Increments 1-12). The reader can also observe the shift in turbulence level from approximately $40 \%$ to $20 \%$ between Increments 13 and 14, which are below and above one of the future buildings.

For Zone 5, the maximum lateral turbulence intensity was equal to 5\%, and it follows the same pattern for longitudinal turbulence intensity in Zone 5, where the maximum was found at Increment 16, the closest to the buildings rooftops. Additionally, the turbulence level was approximately the same in all spots (variations are within 1\%), and thus, no specific location will be presented. 


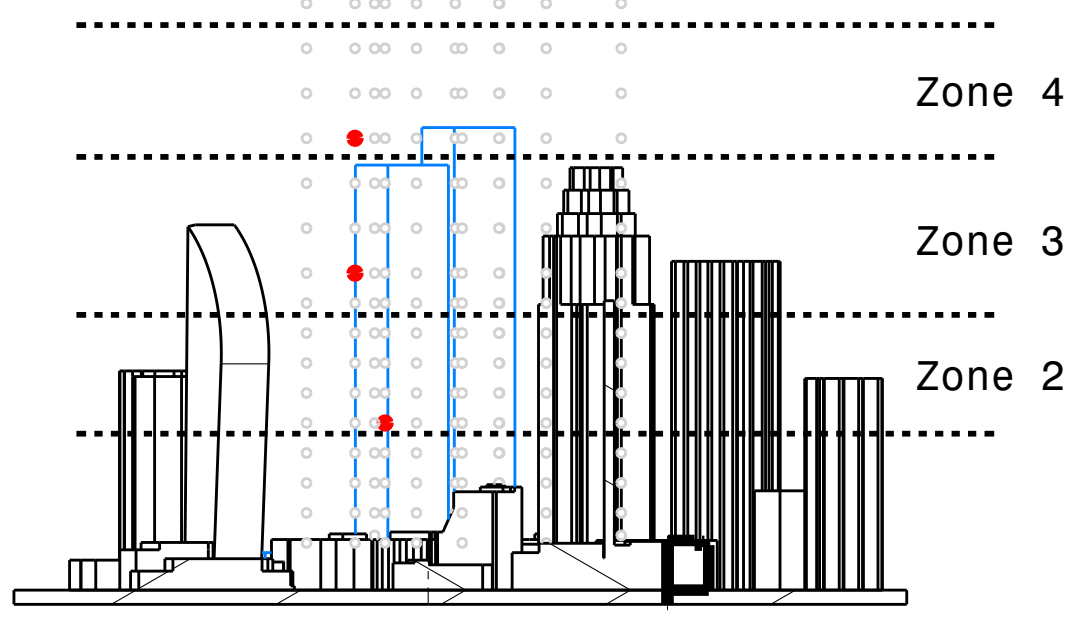

Section view A-A

$S_{15}$ is out of the page

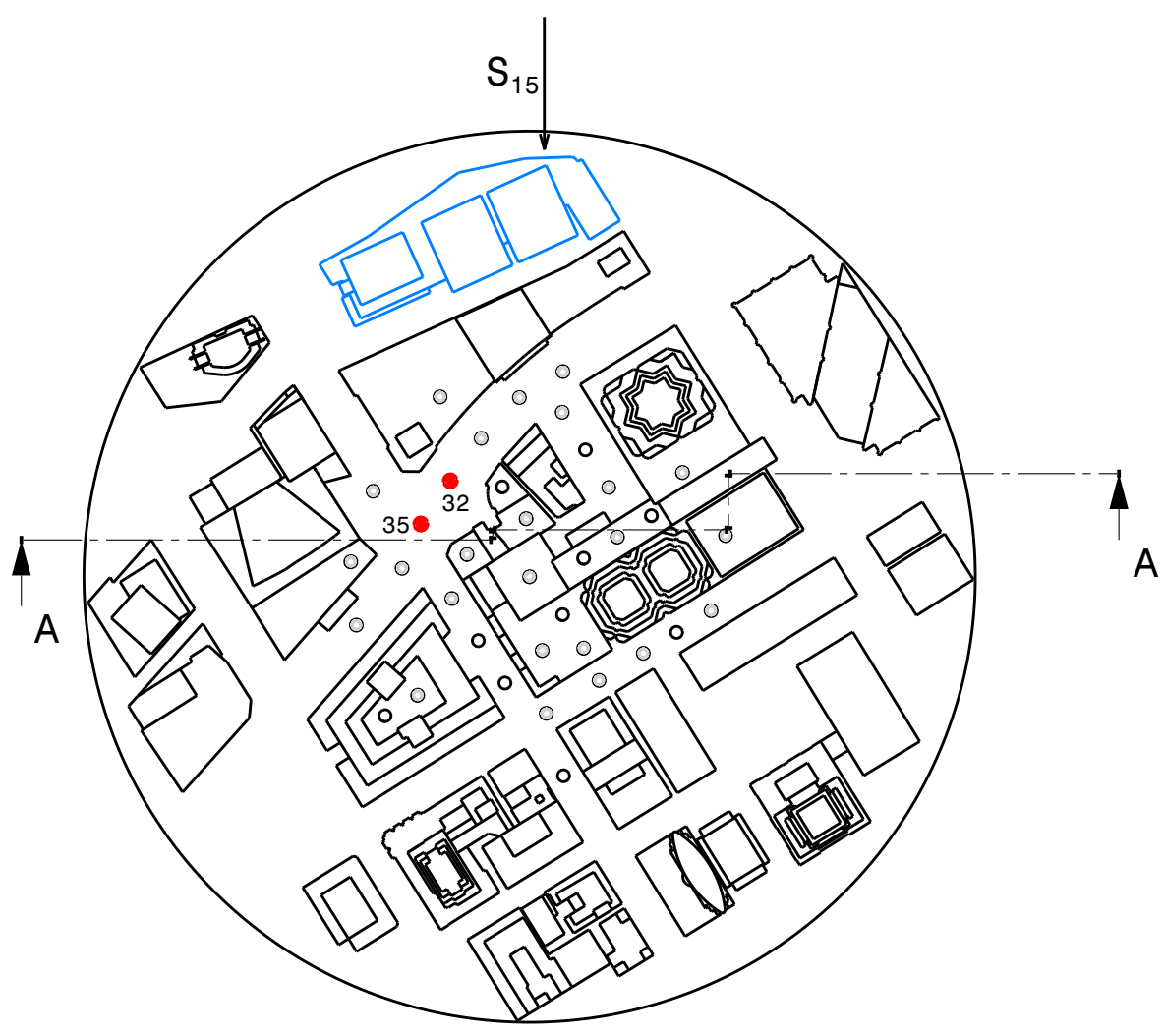

Figure 4.54: The location of Spots 32 and 35 (red markers) with a model orientation representing $\mathrm{S}_{15}$ wind direction.

(Severe cases of lateral turbulence intensity in Zones 2, 3 and 4) 


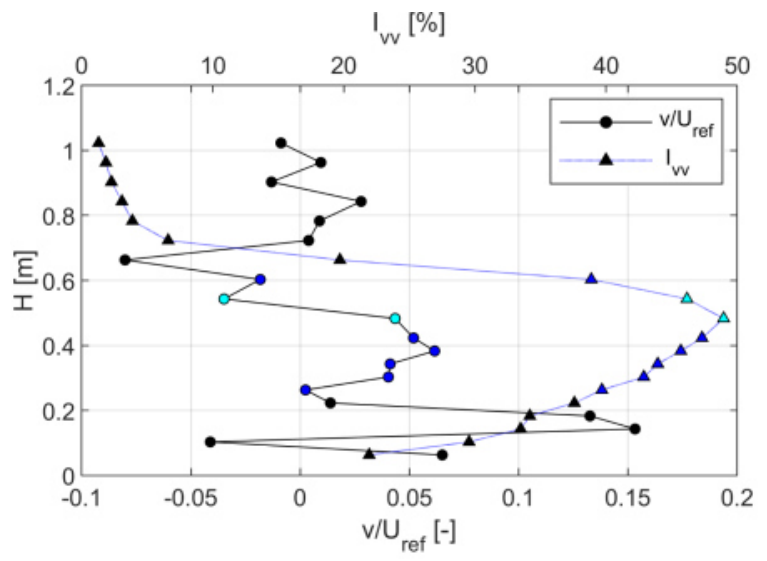

(a)

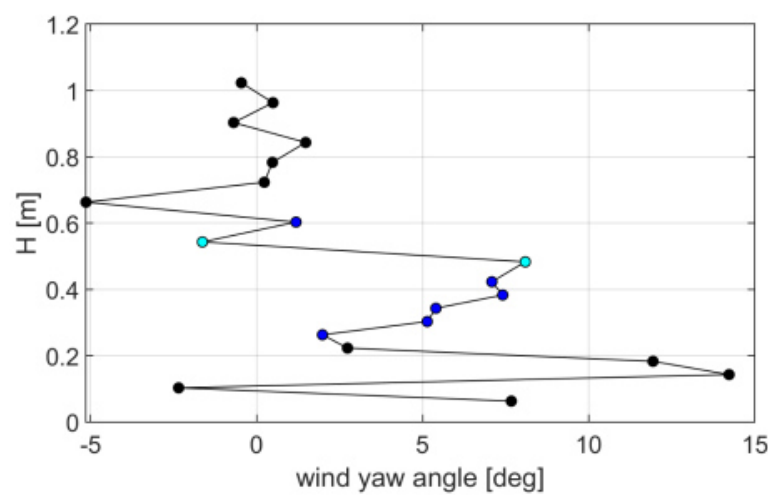

(b)

Figure 4.55: (a) Lateral velocity and lateral turbulence intensity profiles and (b) yaw angle profile at Spot 35 for wind direction $\mathrm{S}_{15}$. Total increments $=20$.

\section{Vertical turbulence intensity}

The most severe vertical turbulence intensity in Zone 1 was found to be $38 \%$ at Increment 2 of Spot 12 for wind direction $\mathrm{S}_{15}$ (see Figure 4.56). The spot is located downstream of multiple buildings, two of which are the future buildings. However, the increment location and height suggests that the vertical turbulence was mainly produced by the low-rise upstream building (labeled I in Figure 4.56). This suggestion was made based on a comparison between the vertical turbulence intensities with and without the future buildings, which revealed that vertical turbulence levels in Increments 1-2 were in fact more severe in measurements without the future buildings as seen in Figure 4.57. The maximum turbulence of $36 \%$ in Zone 2 was found at Increment 5 of Spot 18 for wind direction $\mathrm{N}_{16.7}$ as shown in Figure 4.58. The spot was located upstream of Building I and in the wake of Buildings II and III. Therefore, it is believed that all three buildings must influence the turbulence measured at the spot; however, the effect of Building I could be predominant since the vertical velocities at the spot were positive from Increments 1-9 and negative afterwards as seen in Figure 4.59, which is consistent with the flow pattern at Spot 17 in which a stagnation zone 
was found between Increments 9 and 10 as was shown previously in Figure 4.32 .

At Zones 3 and 4 of the model, the maximum vertical turbulence intensities were found to be $37 \%$ at Increment 12 of Spot 5 and $36 \%$ at Increment 13 of Spot 35, respectively. The location of Spot 5 was in the wake of one of the future buildings (the lower building), most likely close to the edge of the wake as seen in Figure 4.60. Spot 35 was above the lower future building and below the other, so it was possibly affected by the flow separation from the rooftop of the lower building and the flow separation from the sides of the other. The vertical velocity and turbulence intensity profiles at Spots 5 and 35 are shown in Figure 4.61. The vertical turbulence at both spots seems to increase with height (within the height of the buildings) which suggests that vertical turbulence is most severe in the top layers of buildings.

For Zone 5, maximum vertical turbulence intensity was found to be $5 \%$ at Increment 16, where vertical turbulence intensities were approximately the same for all spots (variations are less than $1.50 \%$ in $\mathrm{TI}$ ). The vertical rms values across all measurement locations in Increment 16 range between 0.4 and $0.5 \mathrm{~m} / \mathrm{s}$. 

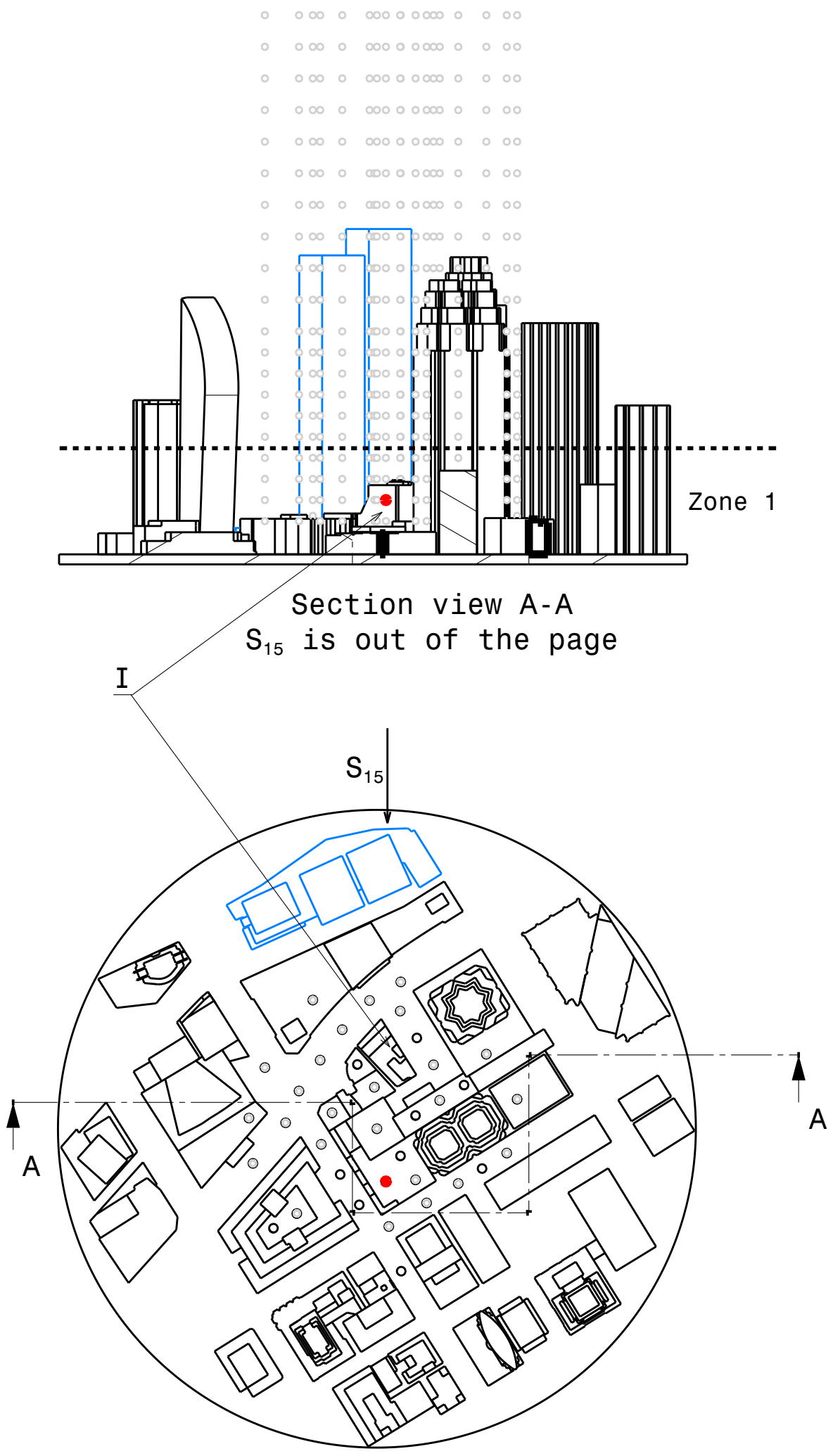

Figure 4.56: The location of Spot 12 (red markers) with a model orientation representing $\mathrm{S}_{15}$ wind direction.

(Severe case of vertical turbulence intensity in Zone 1) 


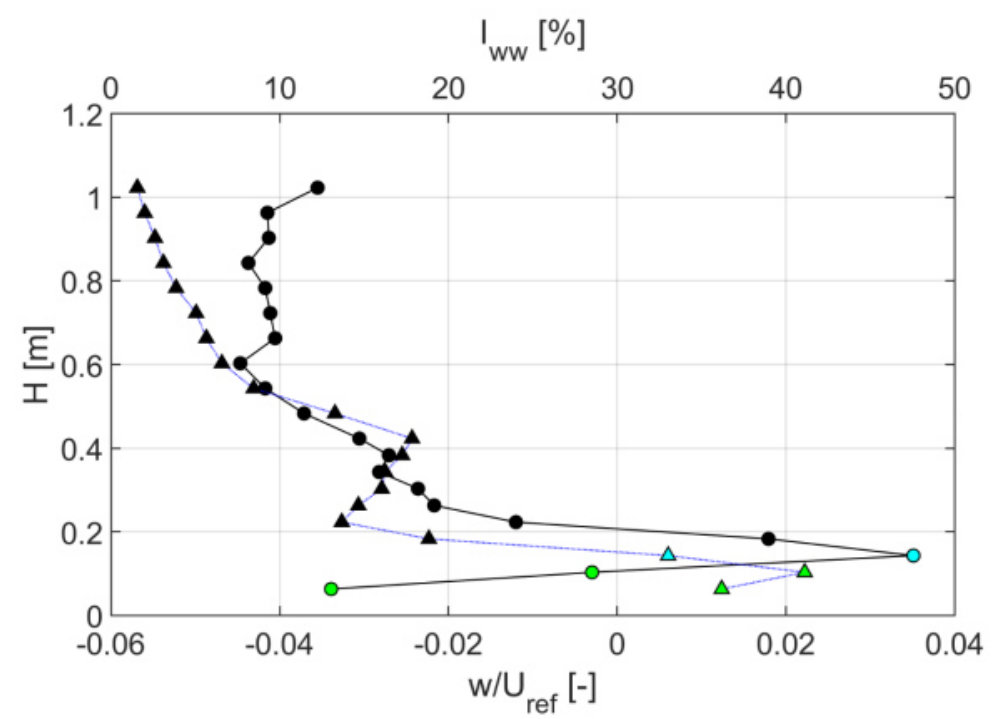

(a)

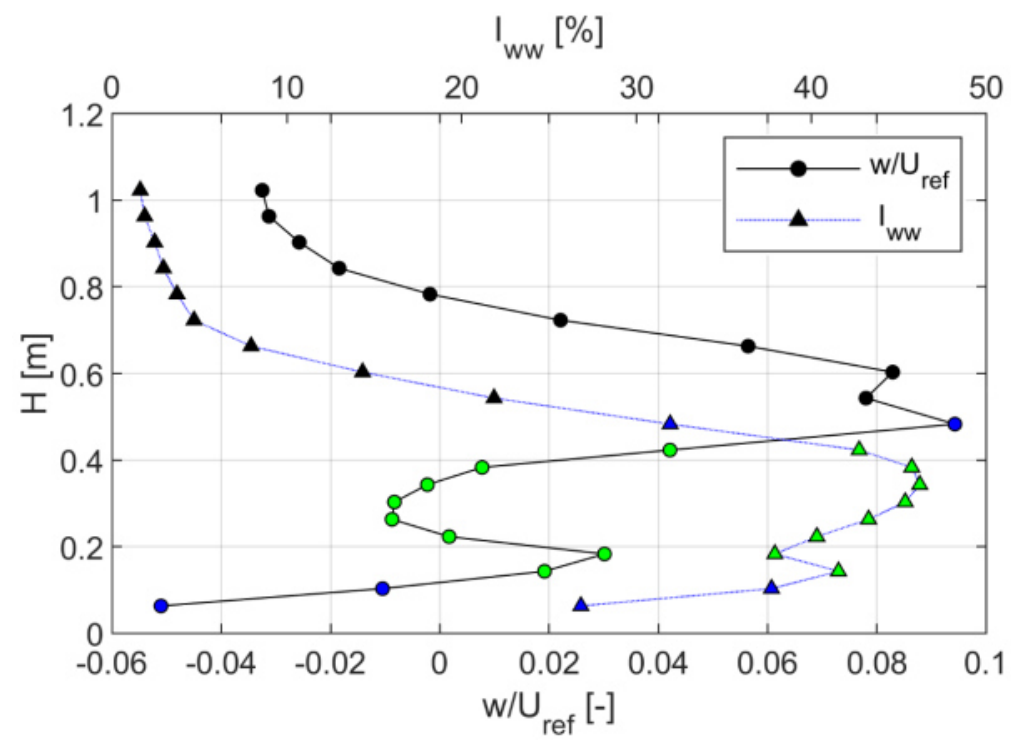

(b)

Figure 4.57: Vertical velocity and vertical turbulence intensity profiles without the future buildings (a) and with the future buildings (b) at Spot 12 for wind direction $\mathrm{S}_{15}$. Total increments $=20$. 

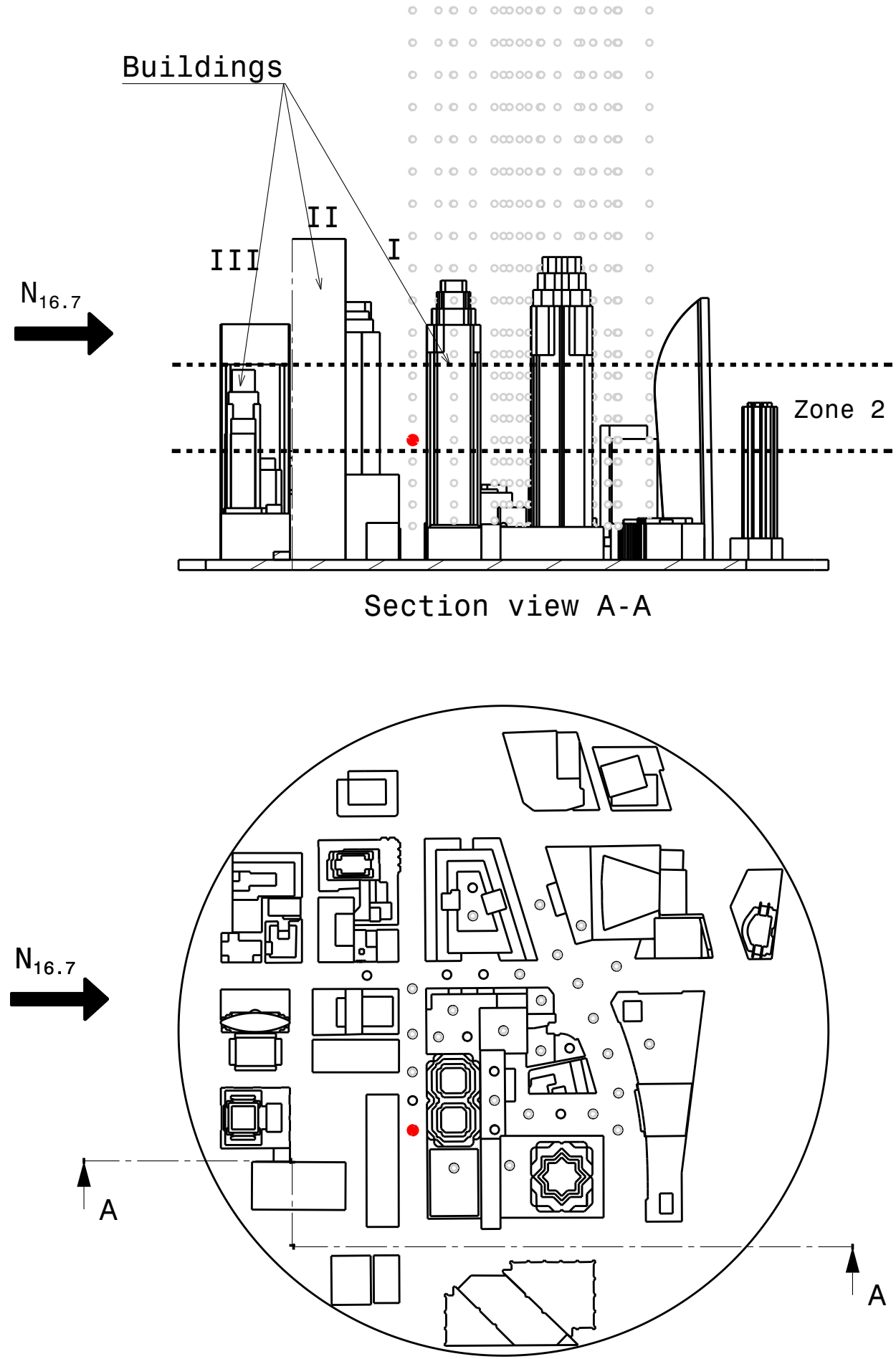

Figure 4.58: The location of Spot 18, increment 5 (red markers) with a model orientation representing $\mathrm{N}_{16.7}$ wind direction.

(Severe case of vertical turbulence intensity in Zone 2) 


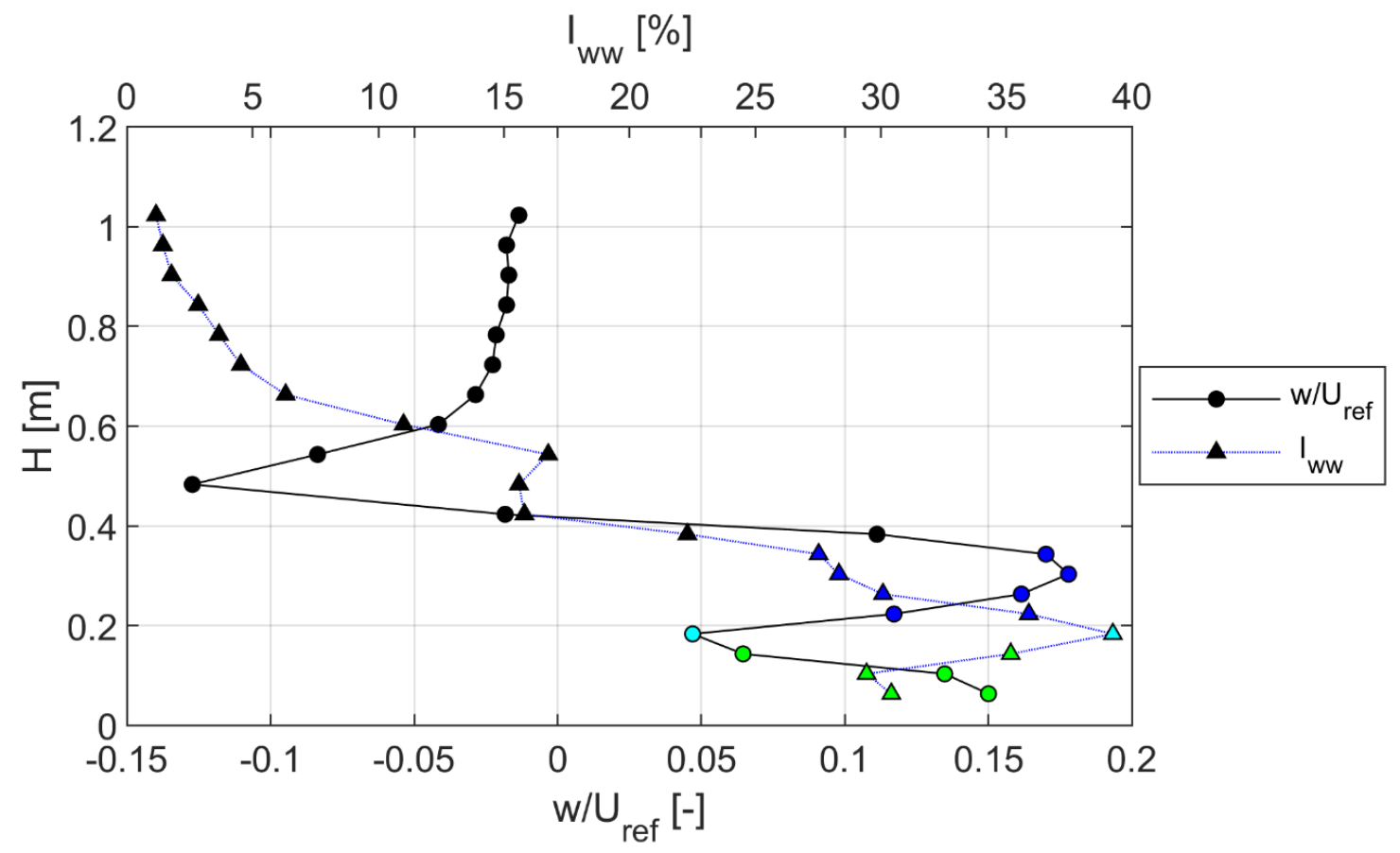

Figure 4.59: Vertical velocity and vertical turbulence intensity profiles at Spot 18 for wind direction $\mathrm{N}_{16.7}$. Total increments $=20$. 


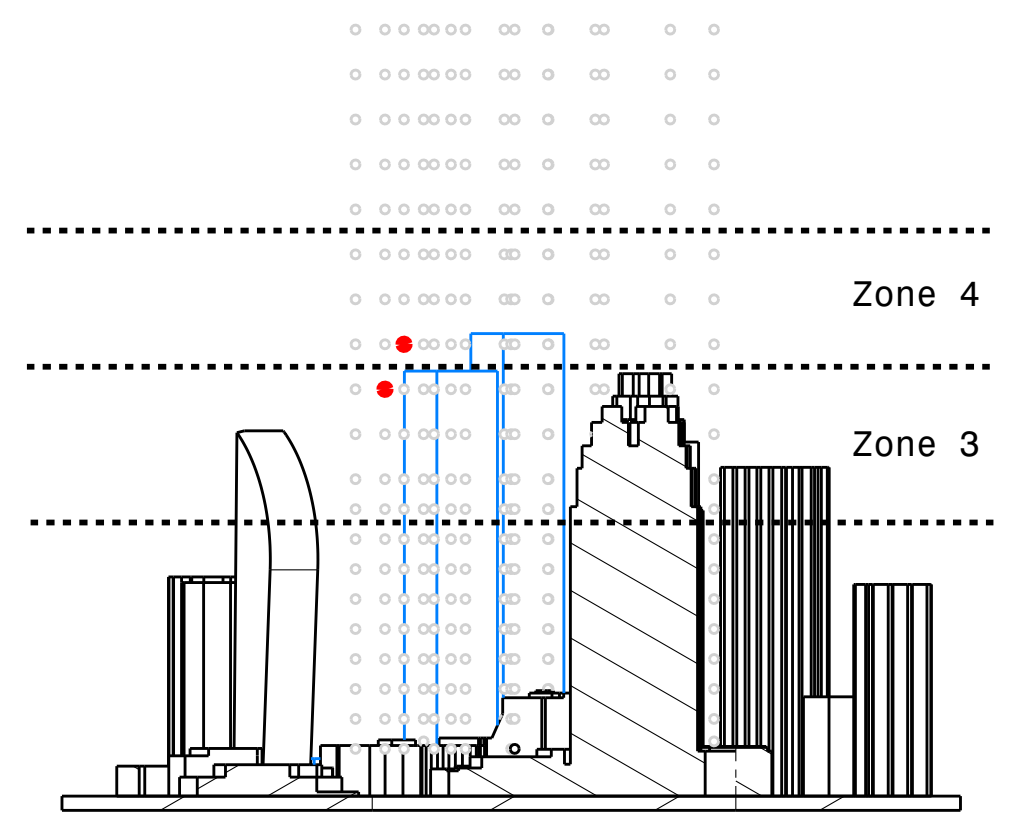

Section view A-A

$S_{15}$ is out of the page

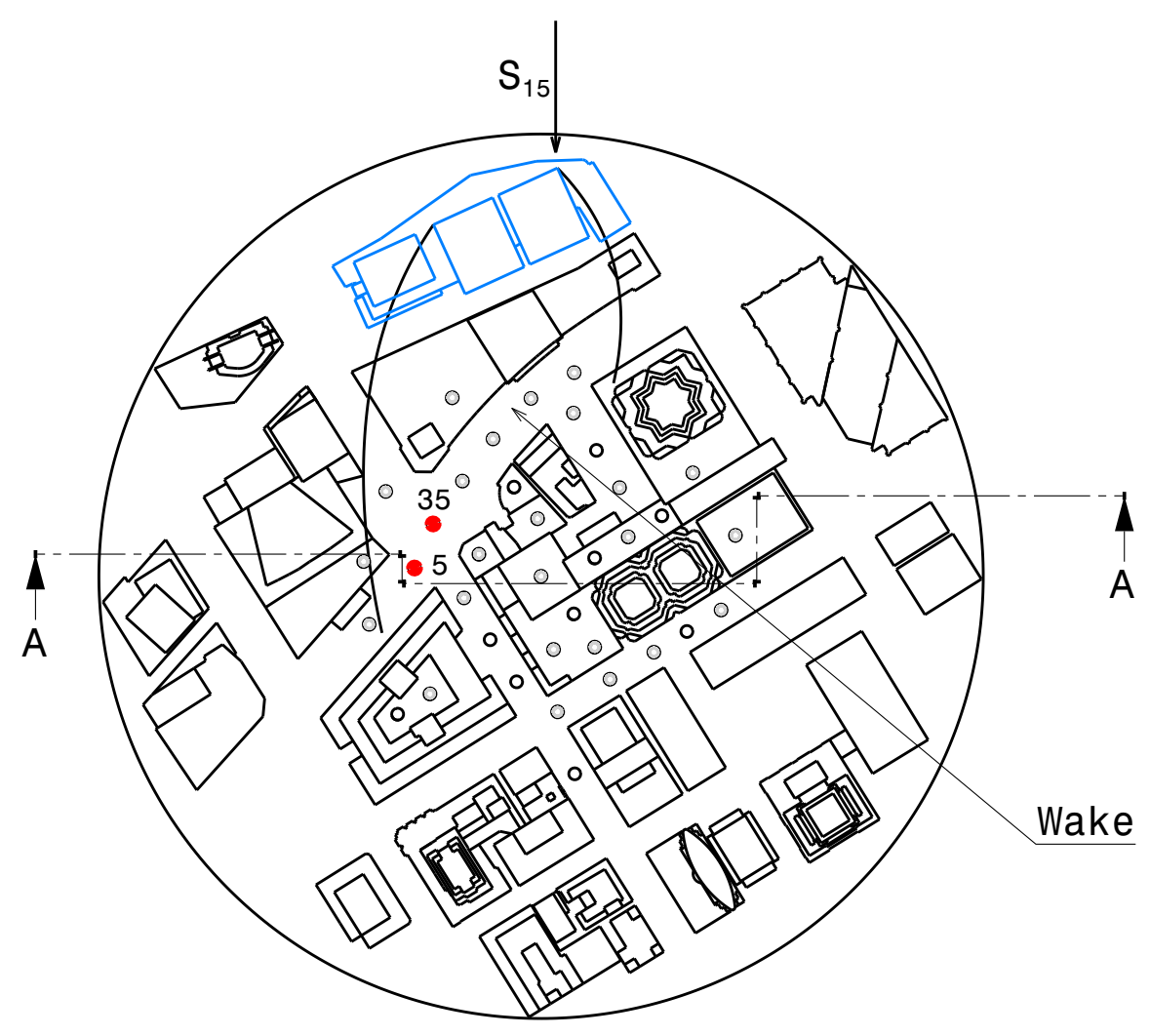

Figure 4.60: The location of Spot 5 and 35 (red markers) with a model orientation representing $\mathrm{S}_{15}$ wind direction.

(Severe cases of vertical turbulence intensity in Zones 3 and 4) 


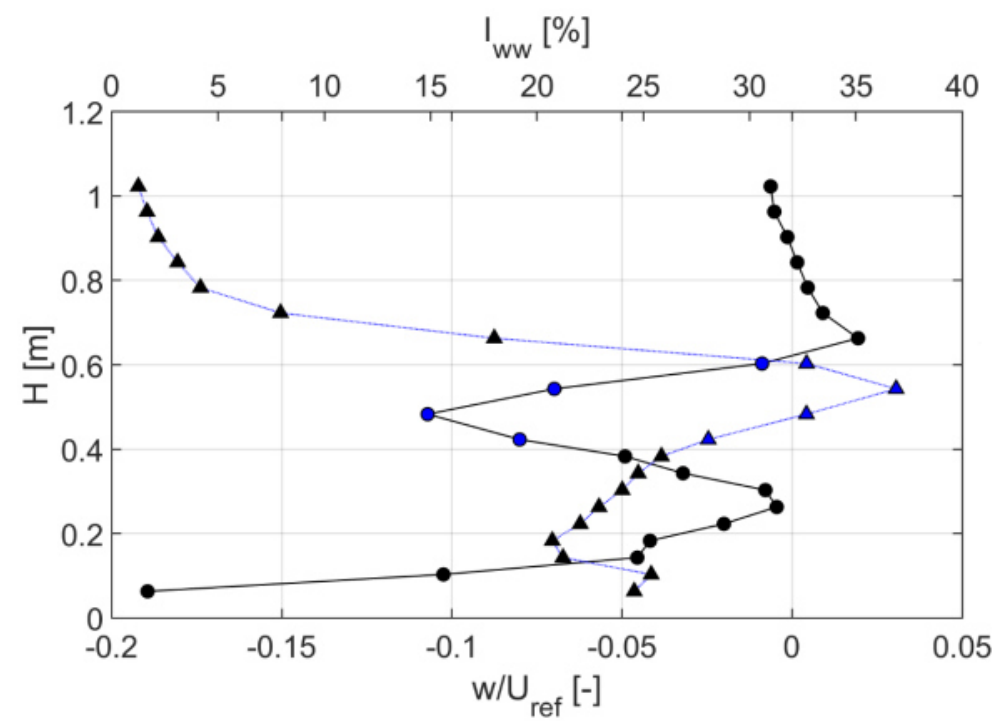

(a)

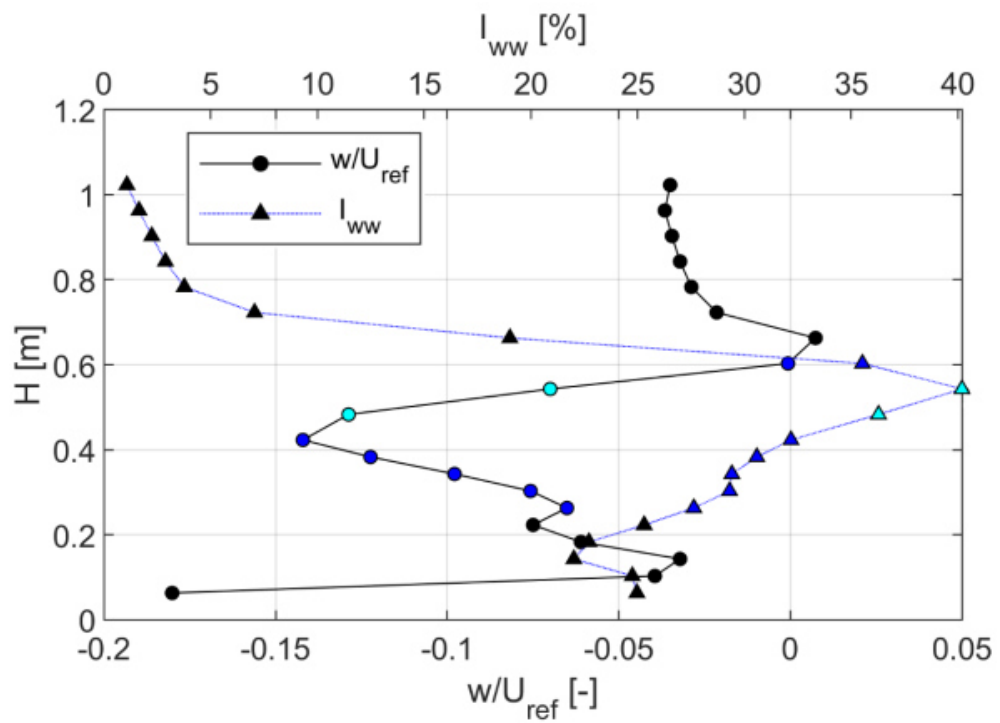

(b)

Figure 4.61: Vertical velocity and vertical turbulence intensity profiles at Spots: (a) 5 and (b) 35 for wind direction $\mathrm{S}_{15}$. Total increments $=20$. 


\section{Effect of surface roughness on turbulence intensity}

Although turbulence intensities were relatively low above buildings level, the effect of terrain roughness due to buildings was apparent in the measurements taken at the highest increment (Increment 20), which is $0.43 \mathrm{~m}$ (172 m FS) above the tallest building rooftop (future buildings are not included). Bar graphs were utilized to show the turbulence intensities at Increment 20 across all measurement spots for $\mathrm{N}_{16.7}$ and $\mathrm{S}_{15}$ wind directions. Figure 4.62 shows the along-wind, across-wind, and vertical turbulence intensities at Increment 20 for measurement locations in $\mathrm{N}_{16.7}$ wind direction, whereas Figure 4.63 shows the three components of turbulence at Increment 20 for measurement locations in $\mathrm{S}_{15}$ wind direction without the future buildings. From Figure 4.62, it can be seen that the turbulence intensities are below $2 \%$ for most of the spots; however, Spots 1 and 2 have higher turbulence intensities, especially in the longitudinal and lateral components. Both Spots 1 and 2, the location of which is shown in Figure 4.64(a), are located downstream of a tall building, where Increment 20 is $0.54 \mathrm{~m}(216 \mathrm{~m} \mathrm{FS})$ above the building rooftop. The same pattern was found in the measurements taken at $\mathrm{S}_{15}$ wind direction, in which higher turbulence intensities were found in regions where tall buildings exist (see Figures 4.63 and 4.64 (b)). The fact that the turbulence intensities across all measurement locations were not equal at the same elevation above buildings should be evident of the influence of urban buildings on atmospheric turbulence, which may extends to several hundred meters (in FS) above certain buildings rooftops. 

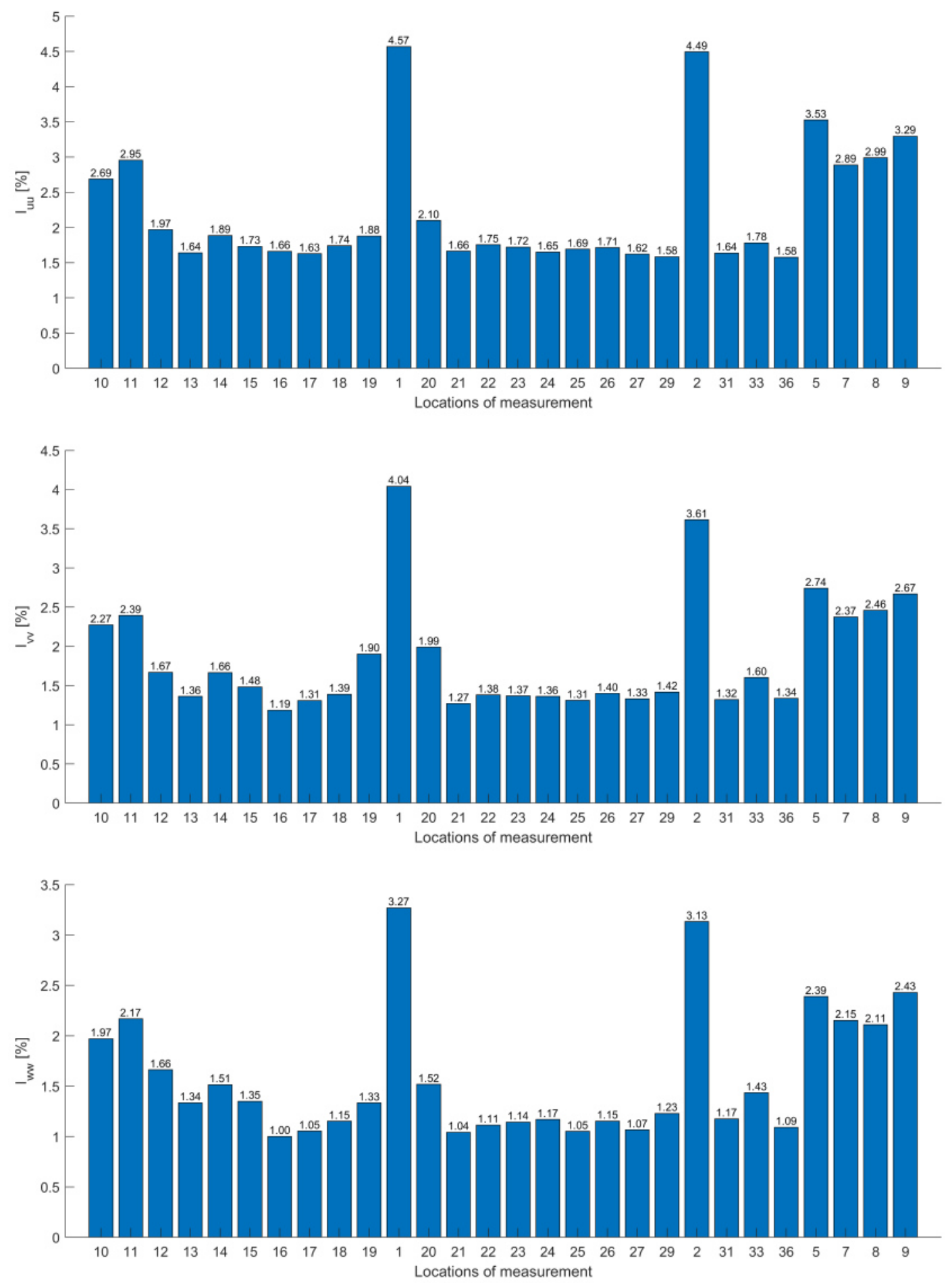

Figure 4.62: Longitudinal (top), lateral (middle), and vertical (bottom) turbulence intensities for measurements taken at Increment 20 in $\mathrm{N}_{16.7}$ wind direction. 

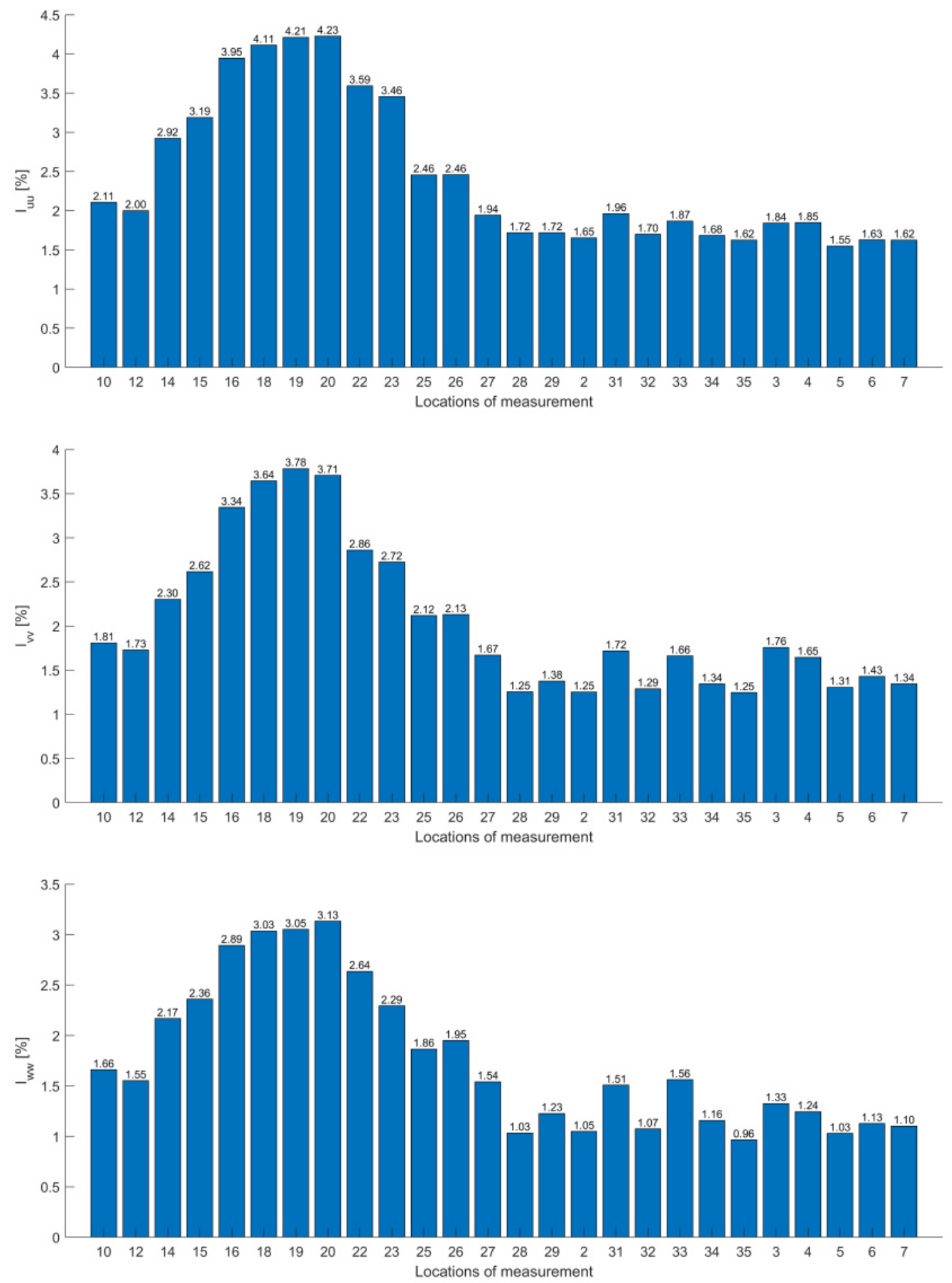

Figure 4.63: Longitudinal (top), lateral (middle), and vertical (bottom) turbulence intensities for measurements taken at Increment 20 in $\mathrm{S}_{15}$ wind direction. 

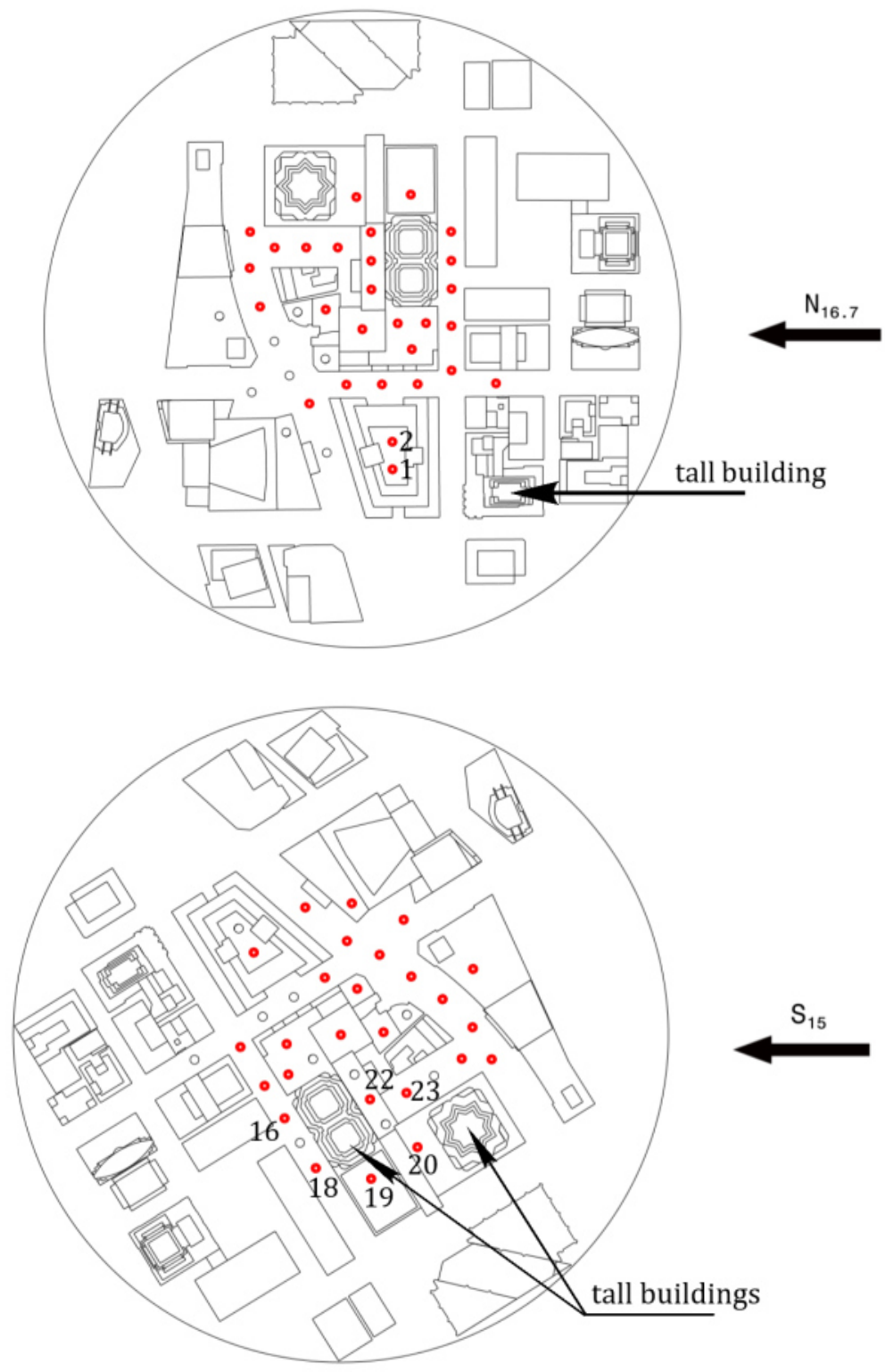

Figure 4.64: Measurement locations for: (a) $\mathrm{N}_{16.7}$ and (b) $\mathrm{S}_{15}$ wind directions. 


\subsubsection{Vortex shedding}

As mentioned earlier in this chapter, the vortex shedding phenomenon was investigated in general, and thus, the aim of the discussion included herein is not to show maximums, but rather, to determine if vortex shedding phenomenon occurs in regions where multiple buildings exist. This is worth investigating since the presence of other buildings may cause flow characteristics that disrupt the formation of shedding structures from individual buildings, and thus, the formation of the well-organized pattern of vortex shedding, known as Kármán vortex street, is not usually a concern in large urban centers where turbulence level is usually high 47].

For the analysis, the determination of vortex shedding phenomenon was accomplished through comparisons between the theoretical frequency of vortex shedding, obtained by Strouhal relation based on the across-wind building dimension, and the prominent energy peaks in the power spectral density function of a velocity signal for measurement taken at a specific location. If a clear frequency peak in the spectral density of the velocity signal was close to a predicted shedding frequency using an expected $\mathrm{S}_{t}$ range, this was taken as an evidence of vortex shedding. For the determination of the theoretical frequency of vortex shedding $\left(f_{v}\right)$, it was decided to consider $\mathrm{S}_{t}$ in the range 0.1-0.2 since most buildings in the model are of square or rectangular shapes, and Strouhal number is shape specific constant, which is typically in the range 0.1 to 0.3 for all shapes, 0.1 to 0.14 for square cross-sectional shapes and approximately 0.2 for rough circular cylindrical shapes $[71]$. The PSD functions of the measured velocity signals are presented in non-dimensionalized form, that is by considering the Von Kármán spectrum form [74]:

$$
f S_{i}(f) / \sigma^{2}
$$

where $S_{i}(f)$ is the spectrum of the velocity component at frequency $f$ and $\sigma^{2}$ is 
the variance of the corresponding velocity component. The subscript $i$ refers to the velocity components, where in this section, it is either $u$ or $v$.

It is important to note that the natural frequencies of the Cobra probe holder (stainless steal tube) were checked to ensure that the peaks found on the lower end of the spectrum, where high energy peaks are dominant, are not related to the vibrations from the tube itself.

By careful examination of the collected data, there was no evidence of the formation of coherent vortex shedding along the height of the buildings, and the results point to the likelihood of side-to-side vortices that shed at 'different frequencies' along the height of the buildings. Figure 4.65 shows the location of Increments 7, 8, and 9 of Spot 9 which is located downstream of a tall building. Considering the slightly oblique wind angle ( $85^{\circ}$ to the windward face of Building I), vortices are expected to form at and detach from the building sides. Fluctuations from vortex shedding in longitudinal $(u)$ and lateral $(v)$ velocity components are strongly related since vortices are shed from one side of the building to the other. Therefore, the PSD of $u$ and $v$ velocity signals were considered for the determination of possible evidence of vortex shedding.

By examining the PSD plots of both $u$ and $v$ velocity signals at each increment (see Figures 4.66 4.67 and 4.68, , high amplitude peaks were found at various frequencies. 

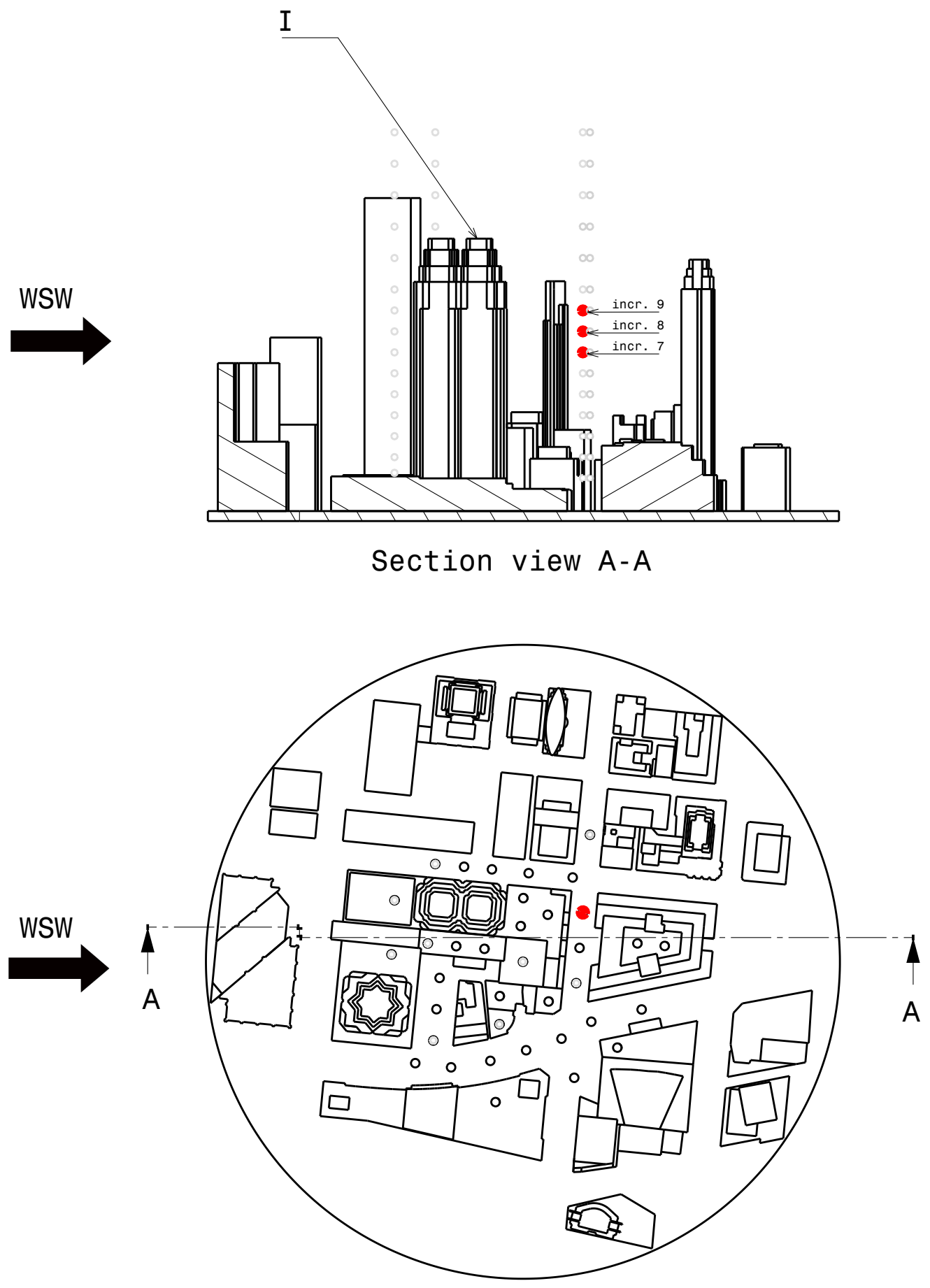

Figure 4.65: The location of Increments 7, 8 and 9 of Spot 9 (red markers) with a model orientation representing WSW wind direction.

(Vortex shedding phenomena) 

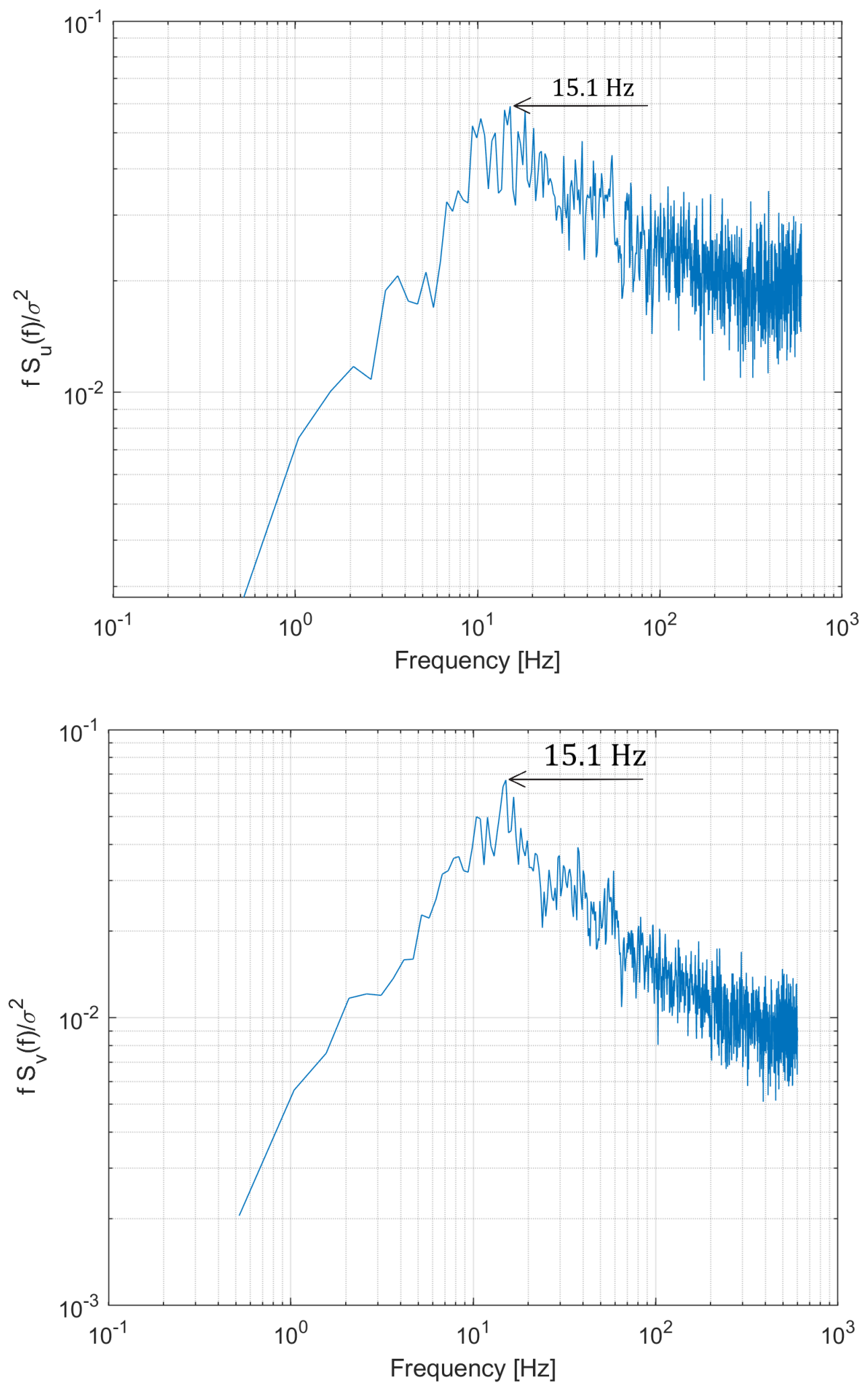

Figure 4.66: PSD plots for longitudinal (top) and lateral (bottom) velocity signals at Increment 7 of Spot 9 for wind direction WSW. 

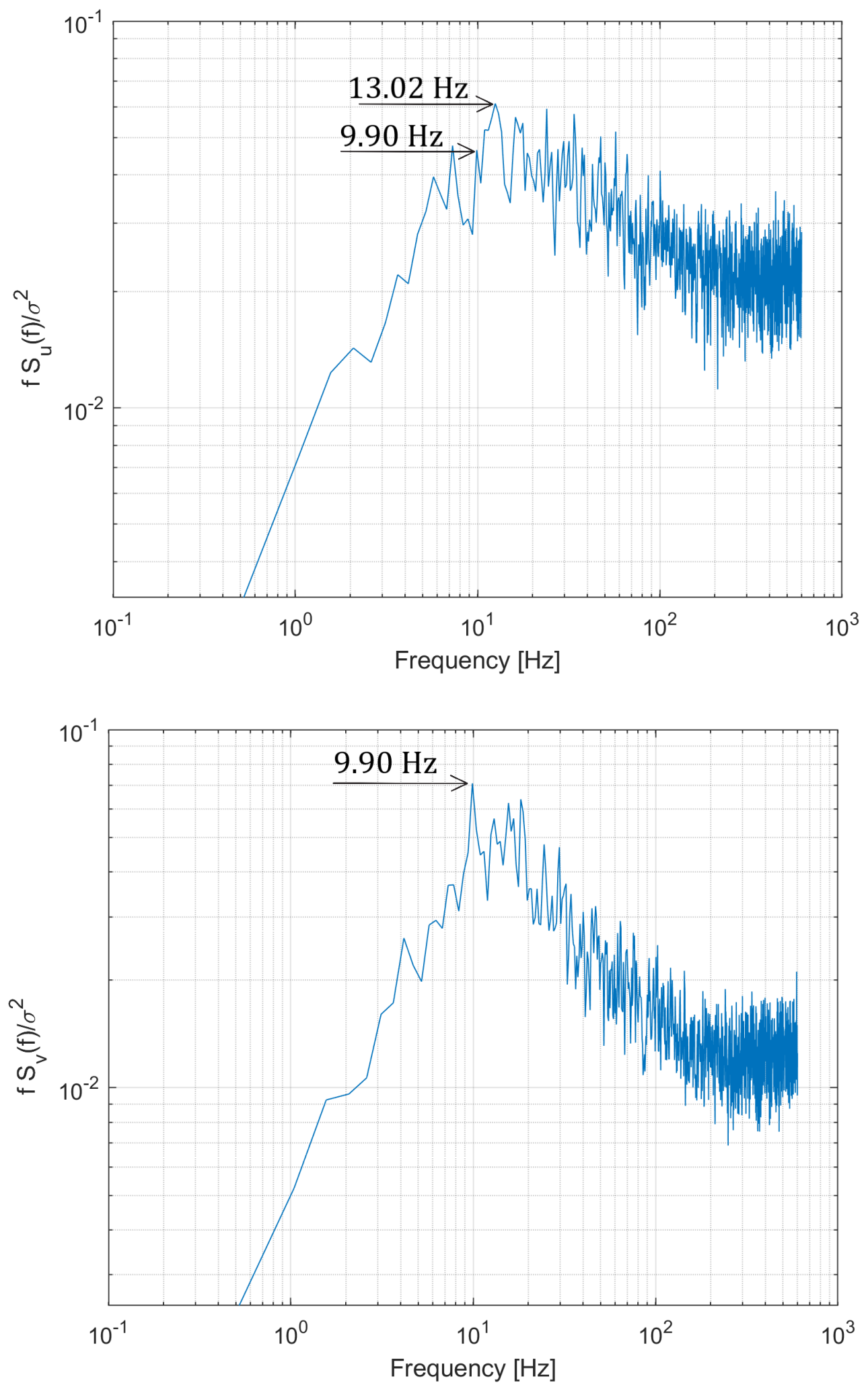

Figure 4.67: PSD plots for longitudinal (top) and lateral (bottom) velocity signals at Increment 8 of Spot 9 for wind direction WSW. 

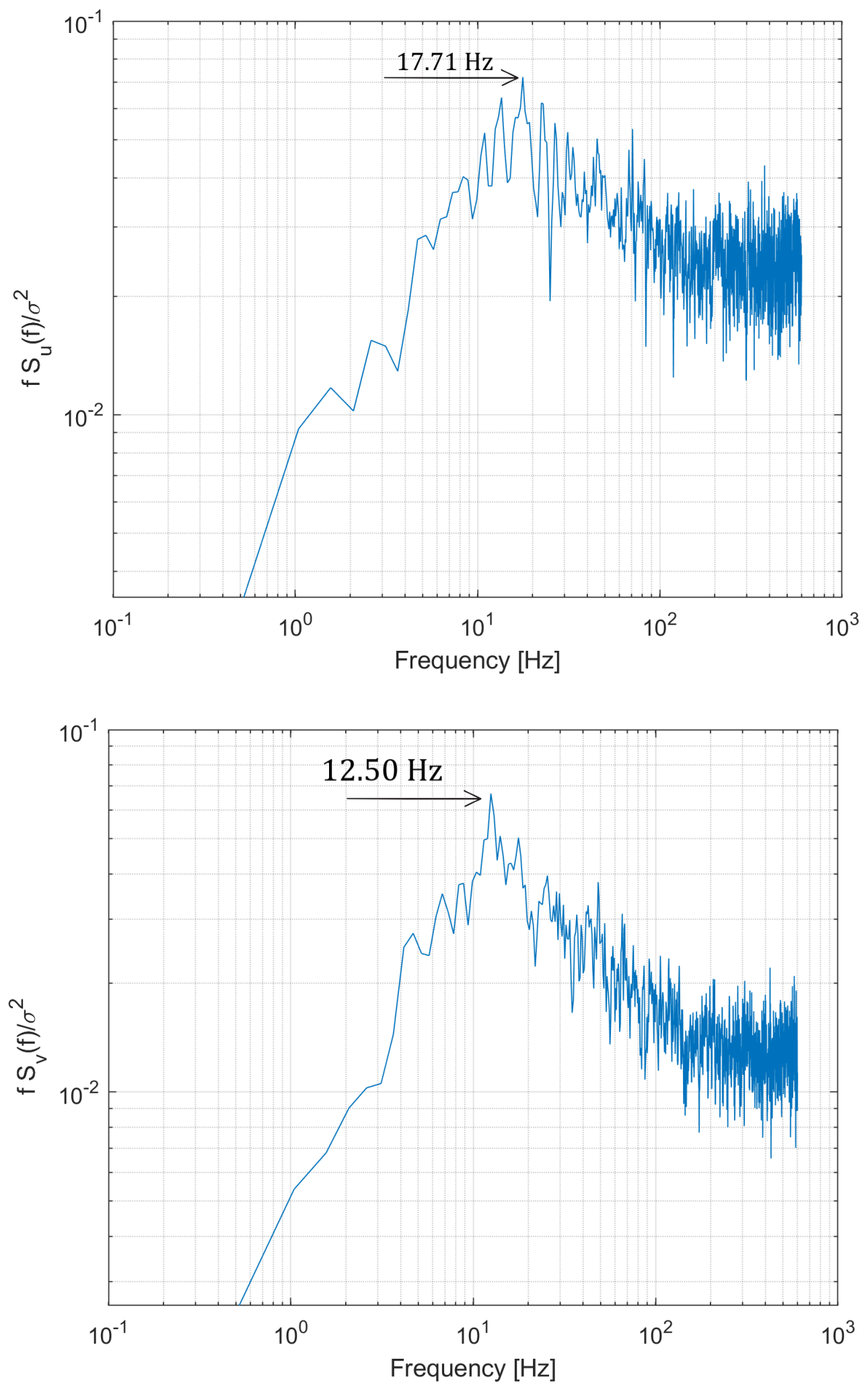

Figure 4.68: PSD plots for longitudinal (top) and lateral (bottom) velocity signals at Increment 9 of Spot 9 for wind direction WSW. 
At Increment 7 , the highest energy peak in both $u$ and $v$ spectra was found at 15.10 Hz. By checking the theoretical frequency of vortex shedding from Strouhal relation, $f_{v}$ was found to be $15.08 \mathrm{~Hz}$ considering $\mathrm{S}_{t}$ of 0.16 . At Increment 8 , the high energy peaks in $u$ and $v$ spectra were found at different frequencies, which suggests the possibility of shedding at $13.02 \mathrm{~Hz}$ in $u$ and $9.90 \mathrm{~Hz}$ in $v$ components. Based on this assumption, there are two different Strouhal numbers in which one is related to frequency of vortex shedding in $u$, and the other is related to the frequency of vortex shedding in $v$. Thus, from Strouhal relation with $\mathrm{S}_{t}=0.14, f_{v}$ was found to be $12.82 \mathrm{~Hz}$ in $u$, whereas $f_{v}$ was found to be $10.07 \mathrm{~Hz}$ in $v$ with $\mathrm{S}_{t}=0.11$. The shedding pattern at Increment 9 follows the same pattern found at Increment 8, where high energy peaks were found at different frequencies in $u$ and $v$ spectra plots. The frequencies observed from the PSD plots are close to the values obtained from Strouhal relation with $\mathrm{S}_{t}$ in the range 0.1-0.2. It can be deduced from the results of the three increments that for a building with the same cross section, different Strouhal numbers may be found for the same flow component, which could be related to the influence of surrounding buildings.

Another example, which presents a stronger likelihood of vortex shedding, is measurements taken at Spot 11 which is located downstream of a tall building of uniform shape as seen in Figure 4.69. The PSD plots of $\mathrm{u}$ and $\mathrm{v}$ components at both Increments 9 and 11 are shown in Figures 4.70 and 4.71,respectively, in which high energy peaks were found at $6.77 \mathrm{~Hz}$ (in $u$ and $v$ ) and $6.25 \mathrm{~Hz}$ (in $u$ and $v$ ) at Increments 9 and 11, respectively. For Increments 9 and 11, Strouhal relation gives $f_{v}$ values equal to $6.61 \mathrm{~Hz}$ and $6.06 \mathrm{~Hz}$ with $\mathrm{S}_{t}$ of 0.12 and 0.11 , respectively. 


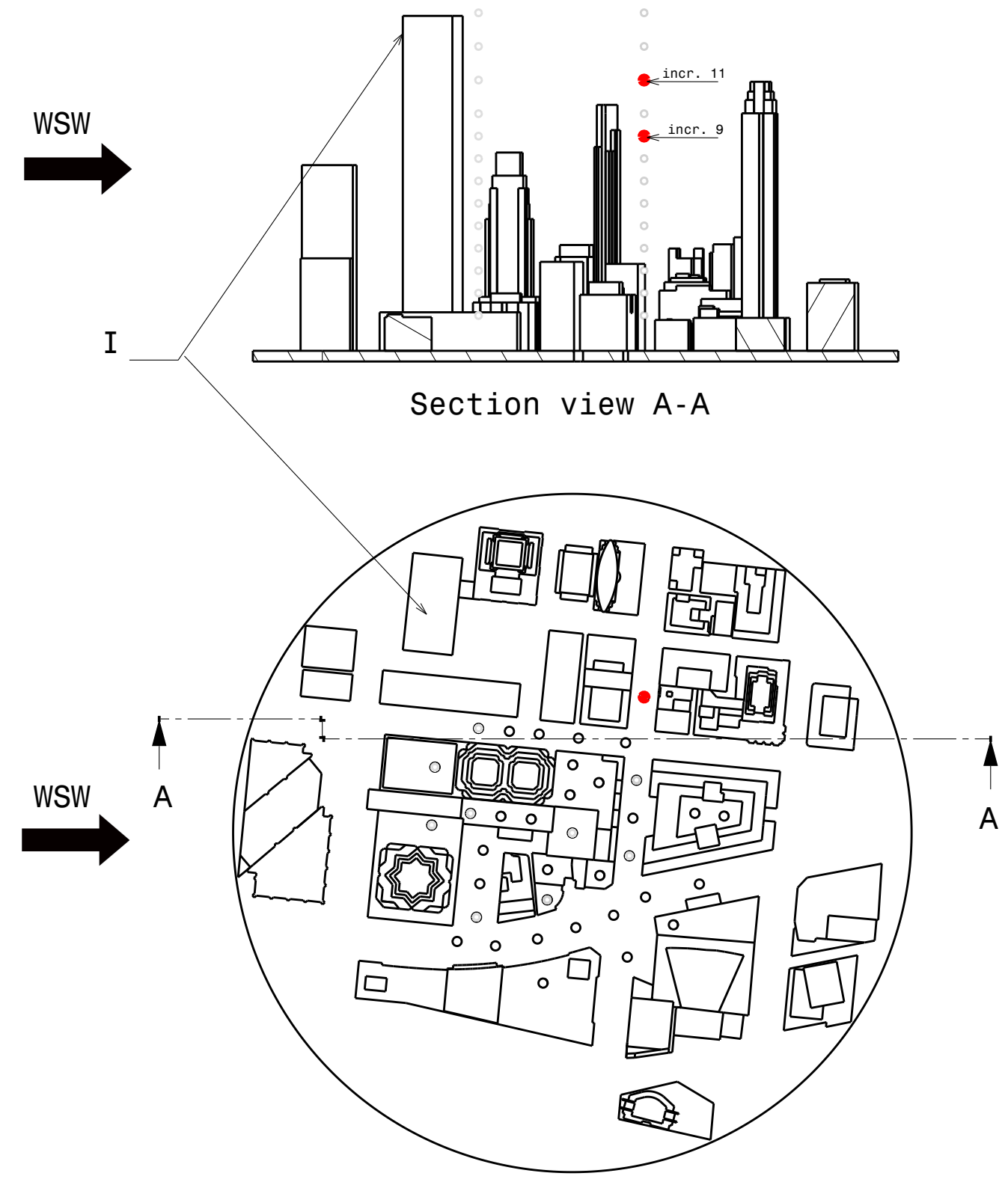

Figure 4.69: The location of Increments 9 and 11 of Spot 11 (red markers) with a model orientation representing WSW wind direction.

(Vortex shedding phenomena) 

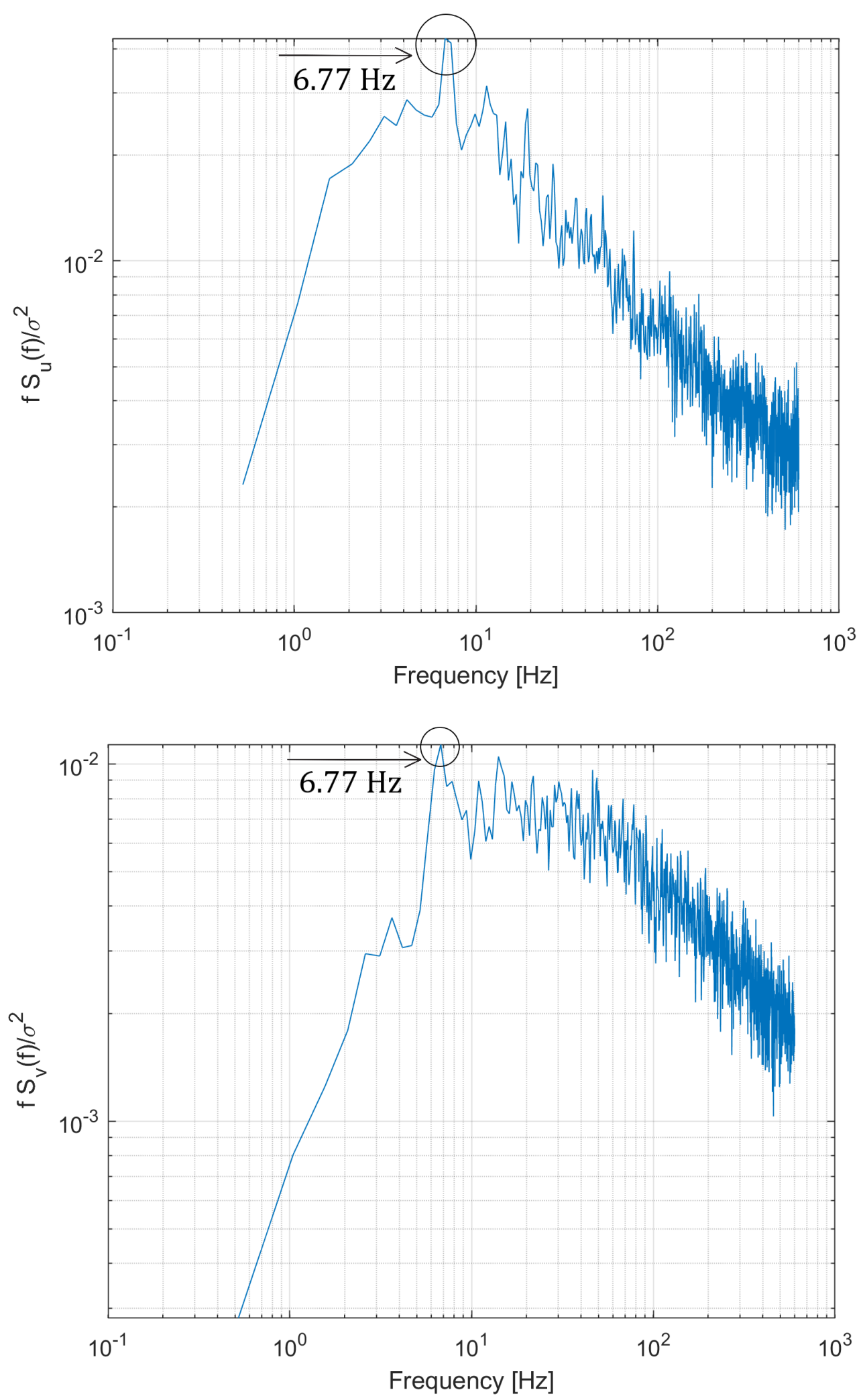

Figure 4.70: PSD plots for longitudinal (top) and lateral (bottom) velocity signals at Increment 9 of Spot 11 for wind direction WSW. 

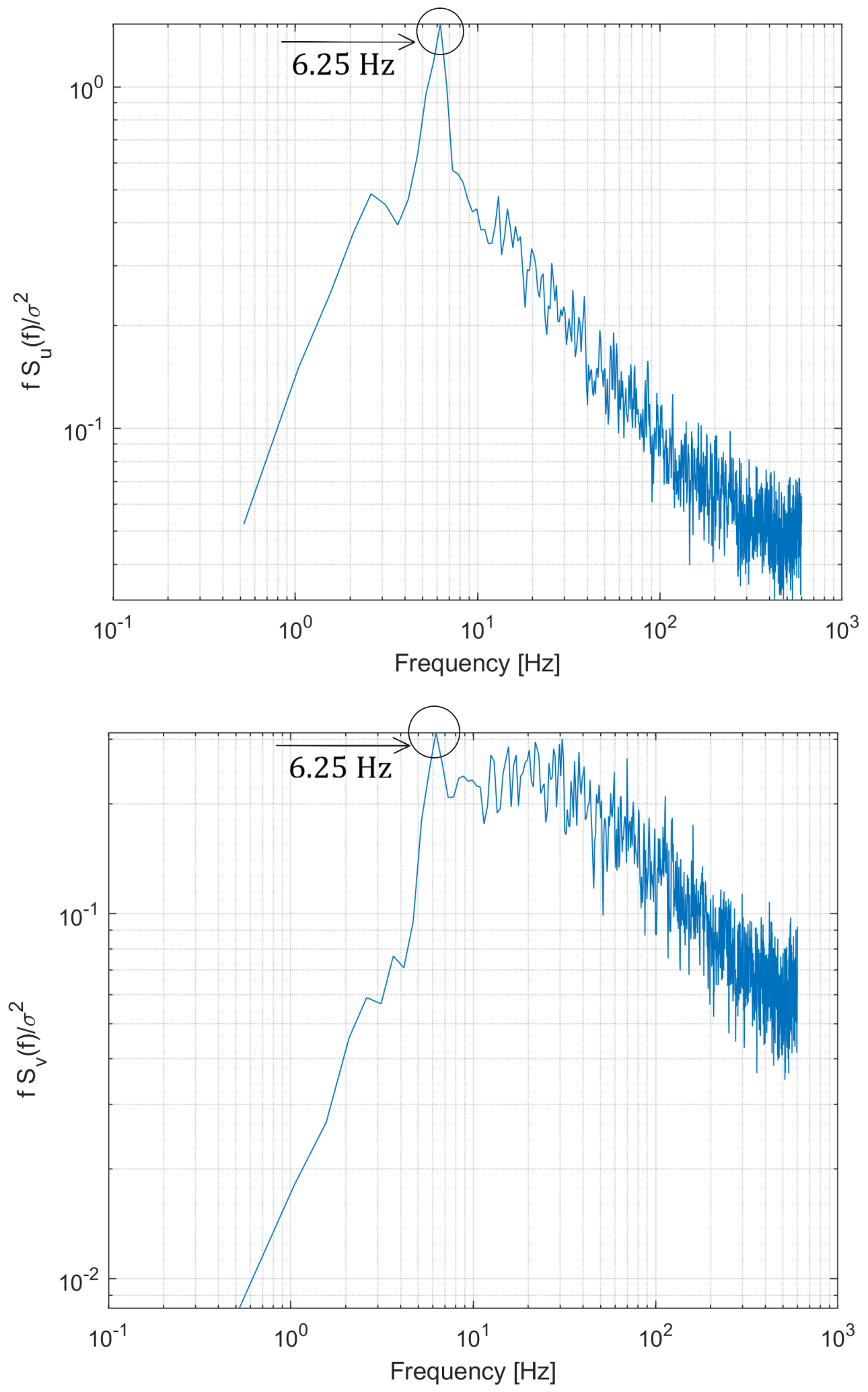

Figure 4.71: PSD plots for longitudinal (top) and lateral (bottom) velocity signals at Increments 11 of Spot 11 for wind direction WSW. 
Evidence of vortex shedding was also observed in measurements where wind angle was perpendicular to the windward face of buildings, an example of which is Spot 1 downstream of Building I for a model orientation representing wind coming from the $\mathrm{N}_{16.7}$ direction (see Figure 4.72). The lateral flow component PSD plots for flow measurement at Increments 8 and 9 show high energy peak at $25.52 \mathrm{~Hz}$ (see Figure 4.73), which is in close agreement with the theoretical frequency of vortex shedding of $25.32 \mathrm{~Hz}$ obtained from Strouhal relation with $\mathrm{S}_{t}$ of 0.14 . 


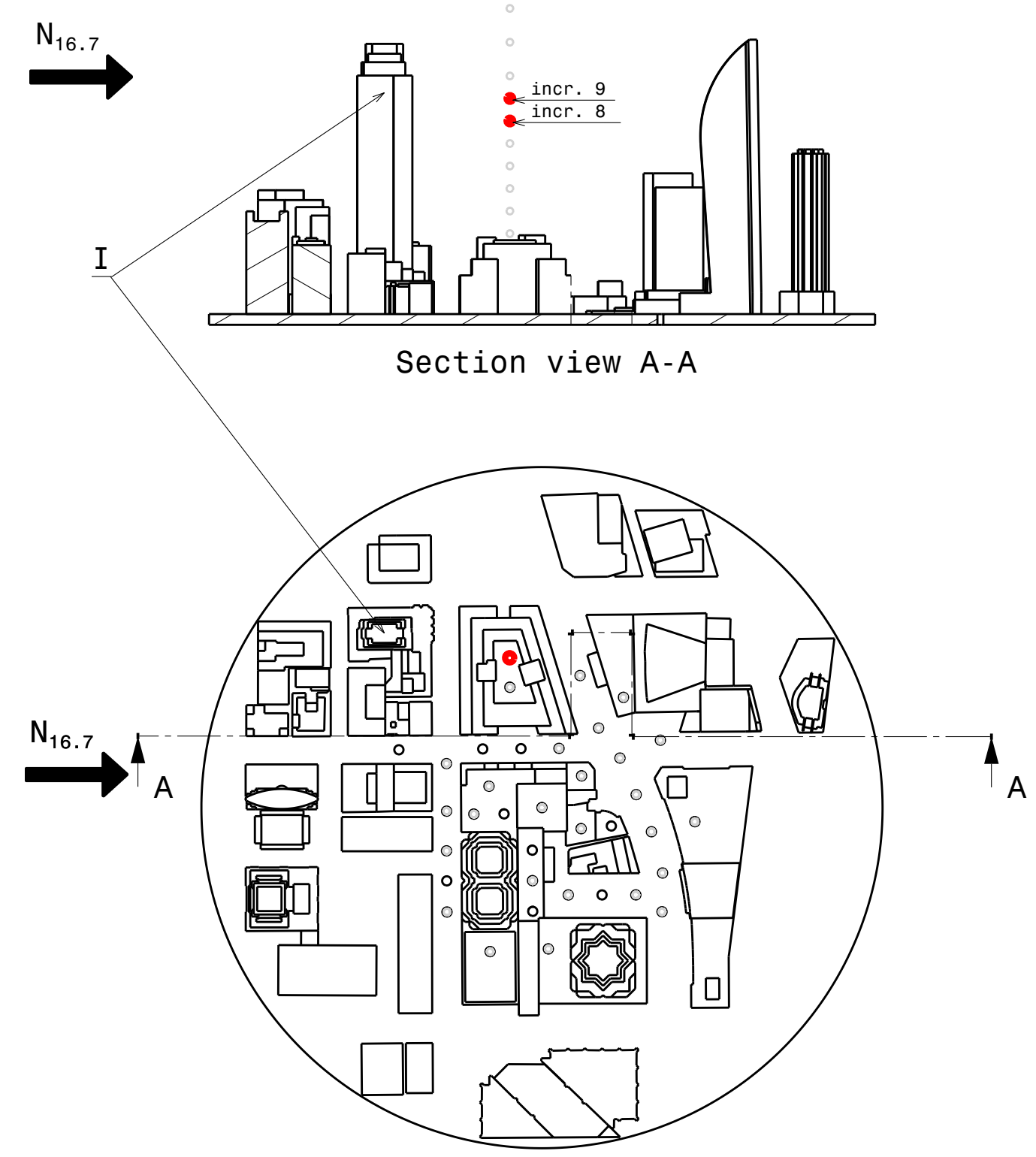

Figure 4.72: The location of Increments 8 and 9 of Spot 1 (red markers) with a model orientation representing $\mathrm{N}_{16.7}$ wind direction.

(Vortex shedding phenomena) 

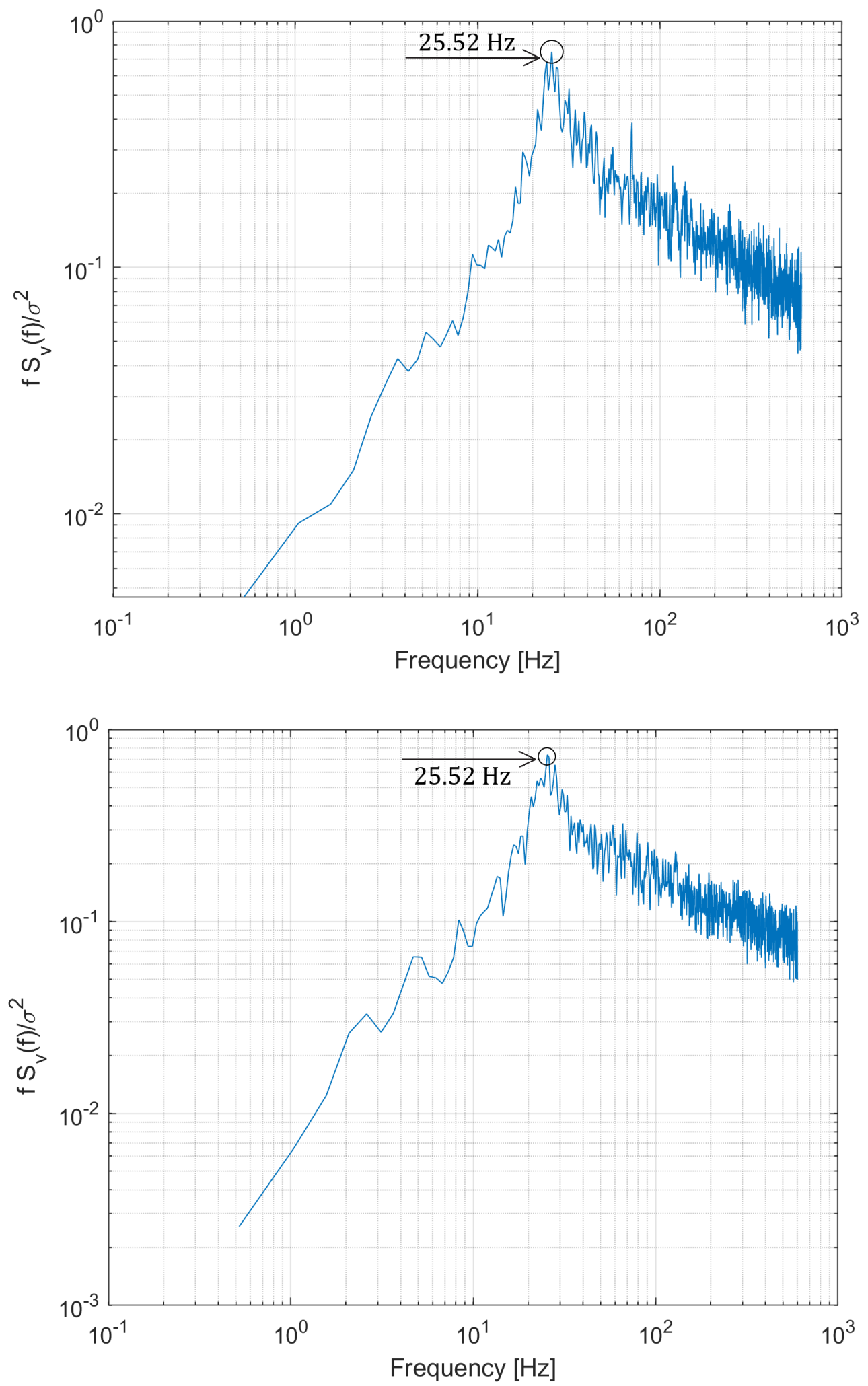

Figure 4.73: PSD plots for lateral velocity signal at Increments 8 (top) and 9 (bottom) of Spot 1 for wind direction $\mathrm{N}_{16.7}$. 


\subsubsection{Regions with complex flow conditions}

The data collection from the model measurement revealed the severity of the flow condition at certain measuring locations without having to perform further analysis to the data. This is based on the \%GD that the TFI software was providing for each data sampling, which for some measuring locations was equal to $0 \%$ despite the use of the probe indexing mechanism. Figure 4.74 shows the locations of $0 \%$ GD for data collected in $\mathrm{E}_{17.1}$ wind direction. The locations of $0 \% \mathrm{GD}$ were coloured in red, green, and blue to help the reader identify the measuring points locations as presented in different perspectives. It was observed that in all cases of $0 \% \mathrm{GD}$, the measurement locations were in close-proximity to the upstream buildings, hence, in the near-wake region. This suggests that the flow was re-circulating at the identified locations, which is consistent with the flow pattern in the near-wake region that is characterized by flow reversals $(\mathrm{U}<0)[12$.

The locations of $0 \%$ GD in $\mathrm{N}_{16.7}$ wind direction are denoted by red markers in Figure 4.75. The measuring points were located in close-proximity to the upstream buildings; therefore, it is expected that flow reversal and/or span-wise vortices are what caused the flow to be unmeasured by the probes. Some flow features were captured from the flow visualization experiment as seen in Figure 4.75. For the location of Point 1, the tuft was moving totally opposite to the wind direction; plus, it was fluctuating with high pitch angles. For the points locations denoted by 2 in Figure 4.75 , the tufts were rotating around the rod, which is likely an indication of the span-wise rotating vortices. 

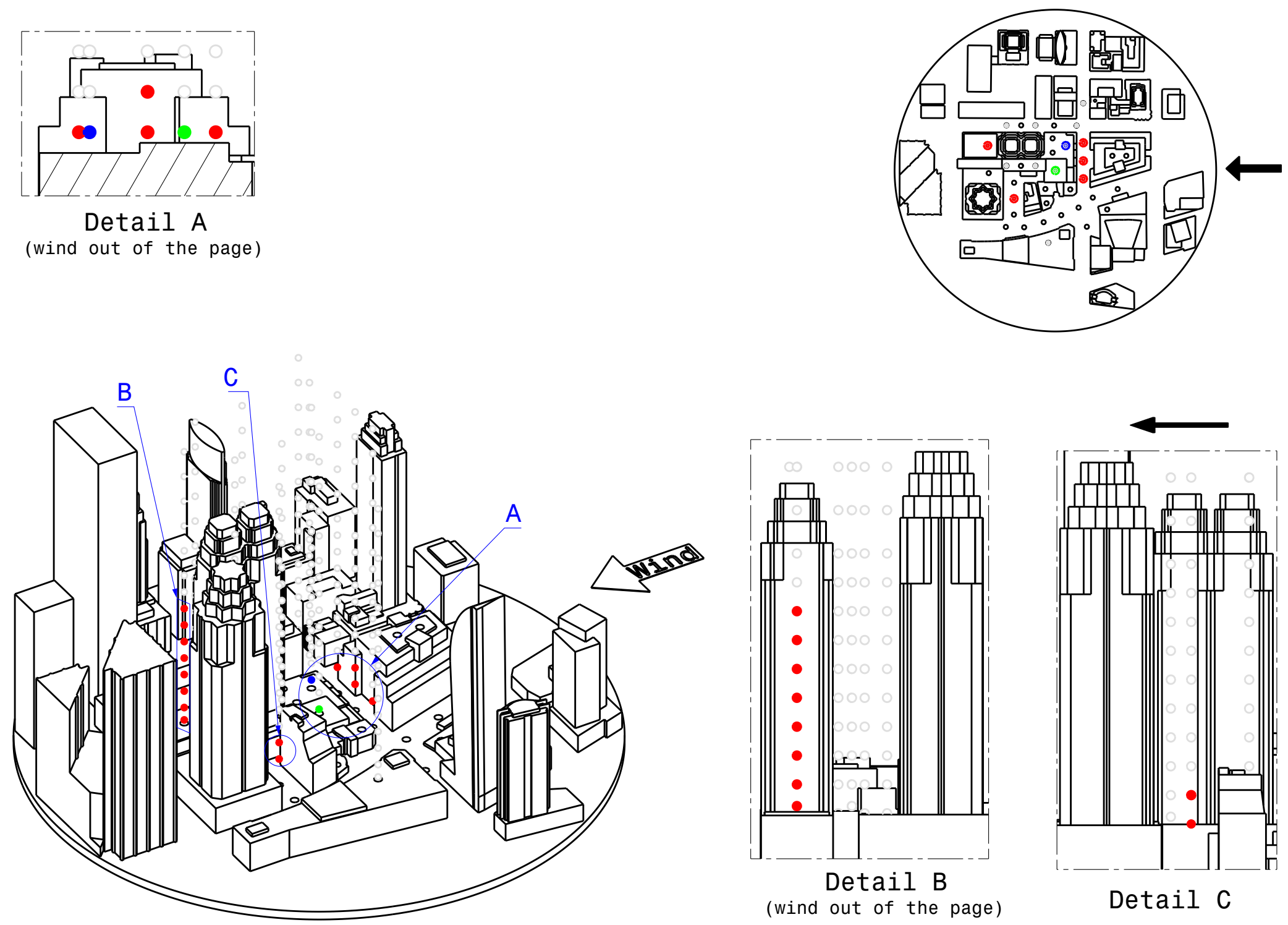

Figure 4.74: Locations of the more complex flow regions (red, green and blue markers) for wind direction $\mathrm{E}_{17.1}$. 

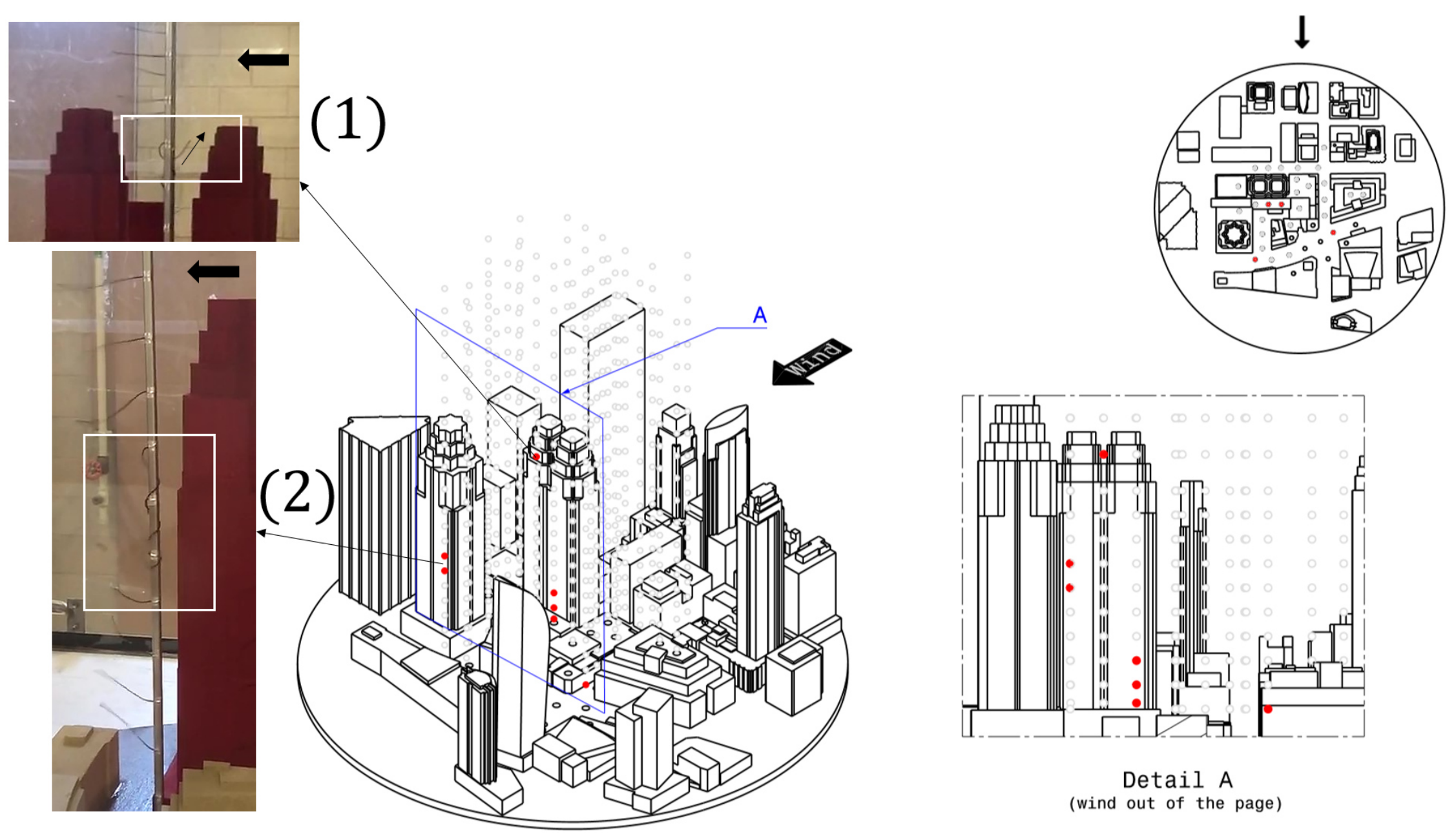

Figure 4.75: Locations of the more complex flow regions (red markers) for wind direction $\mathrm{N}_{16.7}$. 


\subsubsection{Flow conditions above the Hockey Hall of Fame}

The extreme flow features analysis shows no evidence of maximums at Spot 33, which is centered at the rooftop of the HHF (refer to Figures 3.13, 3.31 and 3.32 for the spot location and model orientations to the incoming wind). Since the spot is a potential landing zone in this research for the Bell Nexus flying taxi, the flow condition at the spot for the various wind directions considered will be briefly discussed in this section.

Figures 4.76, 4.77, 4.78, 4.79, 4.80, and 4.81 show the vertical profiles of the flow speed magnitude, three velocity components, overall turbulence intensity, three turbulence intensity components, flow yaw angle and flow pitch angle at Spot 33 for wind coming from WSW, WSW $+15, \mathrm{E}_{17.1}, \mathrm{~N}_{16.7}, \mathrm{~S}_{15}$ without and $\mathrm{S}_{15}$ with the future buildings, respectively. The term 'Not GD' in the colour bar of the plots refer to the 0\% GD. From the vertical measurements at the spot, maximums based on $80 \%$ GD were found to be:

- $38 \%$ along-wind turbulence intensity;

- $34 \%$ across-wind turbulence intensity;

- $33 \%$ vertical turbulence intensity;

- $16 \%$ flow speed increase;

- $41 \%$ flow speed decrease;

- $5^{\circ}$ updraft;

- $13^{\circ}$ downdraft;

- $31^{\circ}$ flow yaw angle;

- $1 \mathrm{~m} / \mathrm{s}$ vertical speed shear;

- $7^{\circ}$ vertical directional shear; and 
- $11^{\circ}$ horizontal directional shear.

The reason behind listing the overall maximums at Spot 33 as opposed to the maximum at the spot for each of the wind directions is to avoid giving the reader an erroneous impression that the flow conditions were more severe in wind directions where the maximums were highest, which is not always the case. This is due to the fact that maximums were determined from data collected with at least $80 \%$ GD. Therefore, it could be misleading to compare the maximums at Spot 33 from the perspective of different wind directions, hence different approach flow, since a maximum does not necessarily mean more severe, but rather, it is the maximum that was determined from the flow measurement with adequate accuracy level (\%GD > 80).

By general comparisons between the profiles, it can be seen that flow condition at Spot 33 are more severe for $\mathrm{WSW}_{+15}$ as compared to WSW wind direction. This could be related to the flow separation from the nearby building (see Figure 4.82), in which Spot 33 was likely located within the wake or the shear layer of the nearby building for wind direction $\mathrm{WSW}_{+15}$.

The flow condition at Spot 33 in $\mathrm{E}_{17.1}$ and $\mathrm{S}_{15}$ wind directions without the future buildings was relatively smooth above Increments 2 and/or 3 since the spot was open to the incoming flow with no buildings obstruction. Moderate flow conditions at the spot were observed for the case of wind coming from $\mathrm{N}_{16.7}$ direction. The high turbulence values seen in the plots of Figure 4.79 are expected to be related to the flow separation from the nearby building (see Figure 4.83 for illustration), where Spot 33 is likely located in the buildings wake or shear layer. The influence of the future buildings on the flow condition at Spot 33 is clearly seen by comparing the profiles shown in Figures 4.80 and 4.81 . 

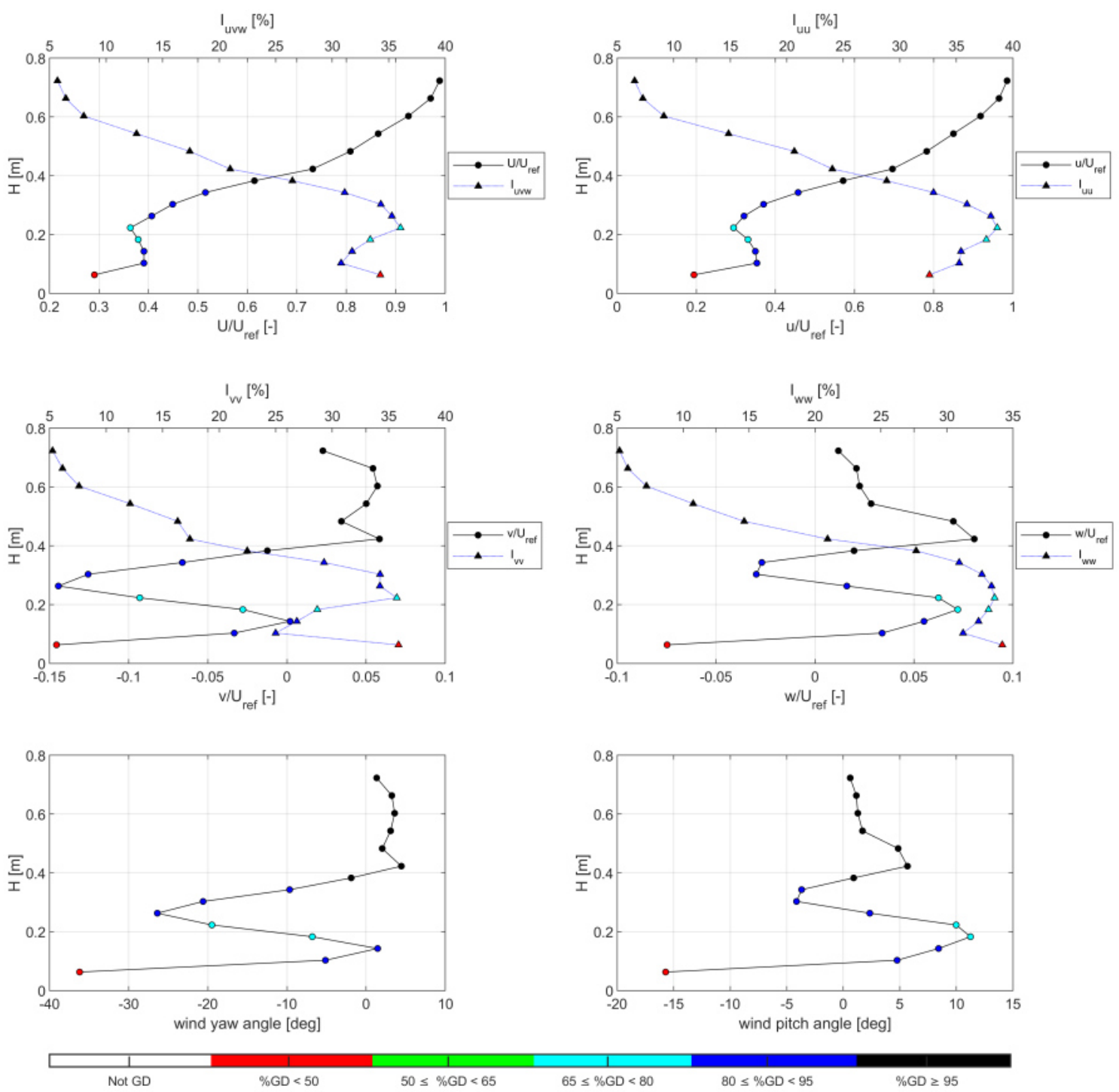

Figure 4.76: Velocities, turbulence intensities and flow angles profiles at Spot 33 for WSW wind direction. Total increments $=15$. 

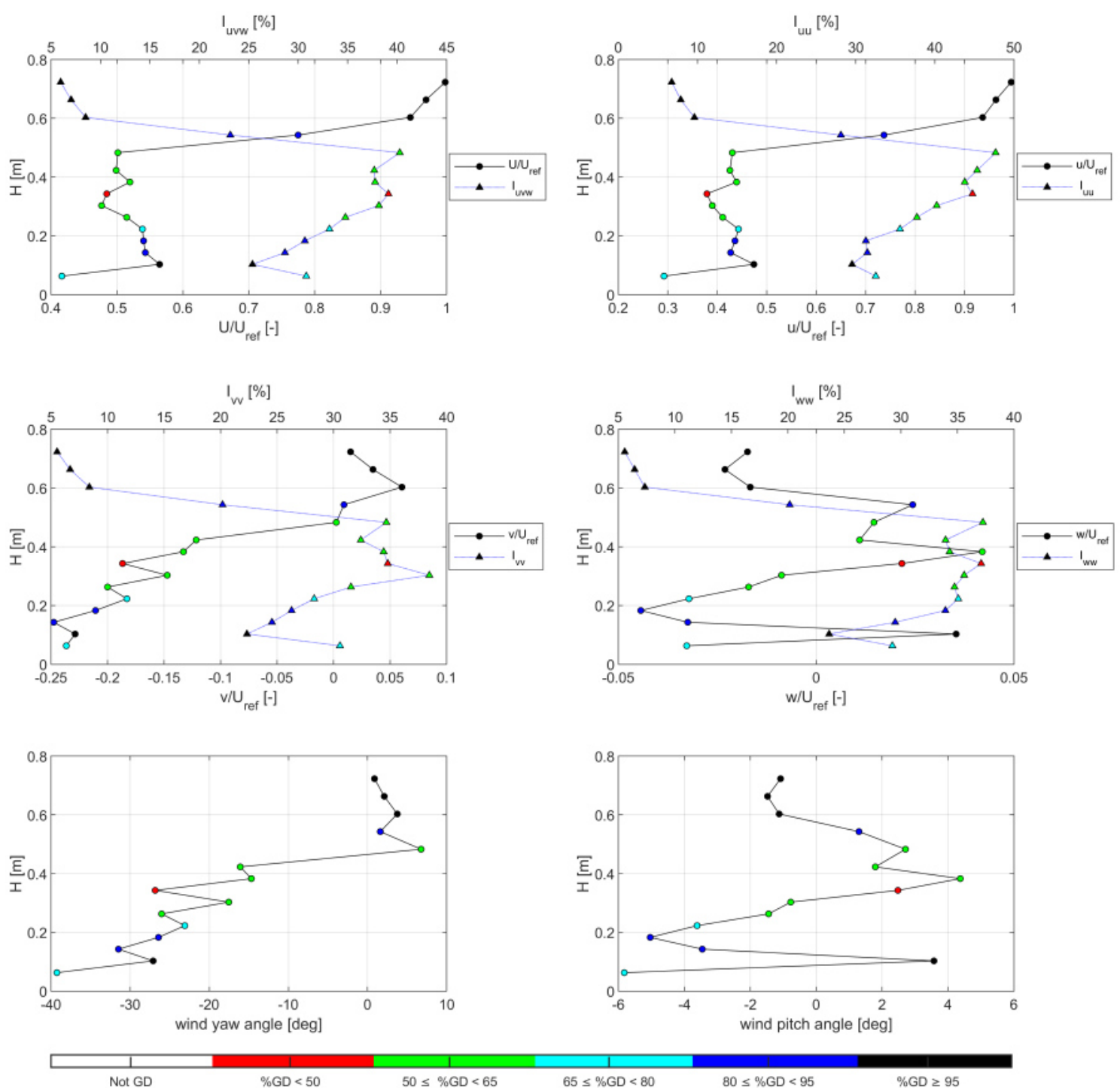

Figure 4.77: Velocities, turbulence intensities and flow angles profiles at Spot 33 for $\mathrm{WSW}_{+15}$ wind direction. Total increments $=15$. 

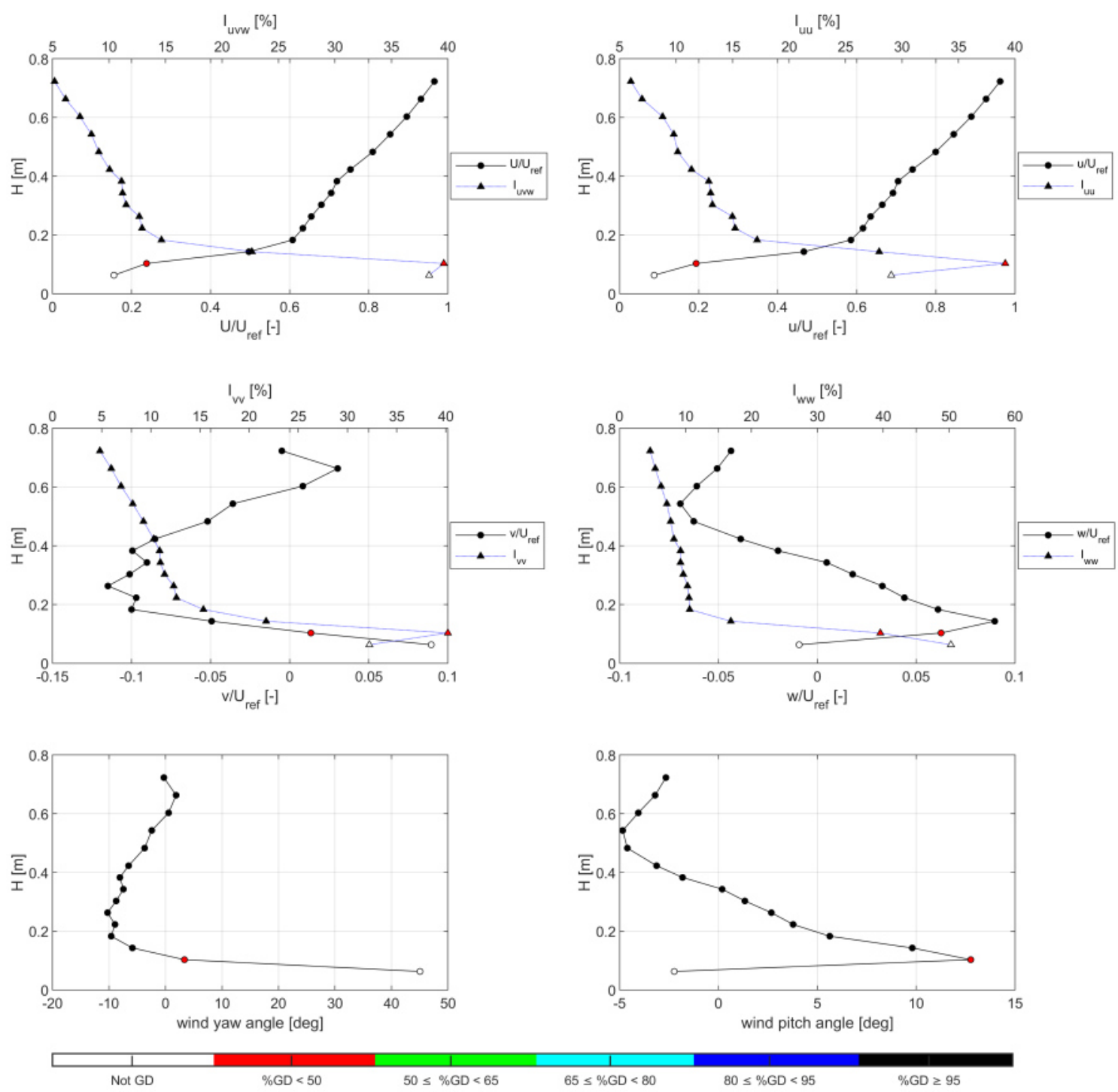

Figure 4.78: Velocities, turbulence intensities and flow angles profiles at Spot 33 for $\mathrm{E}_{17.1}$ wind direction. Total increments $=15$. 

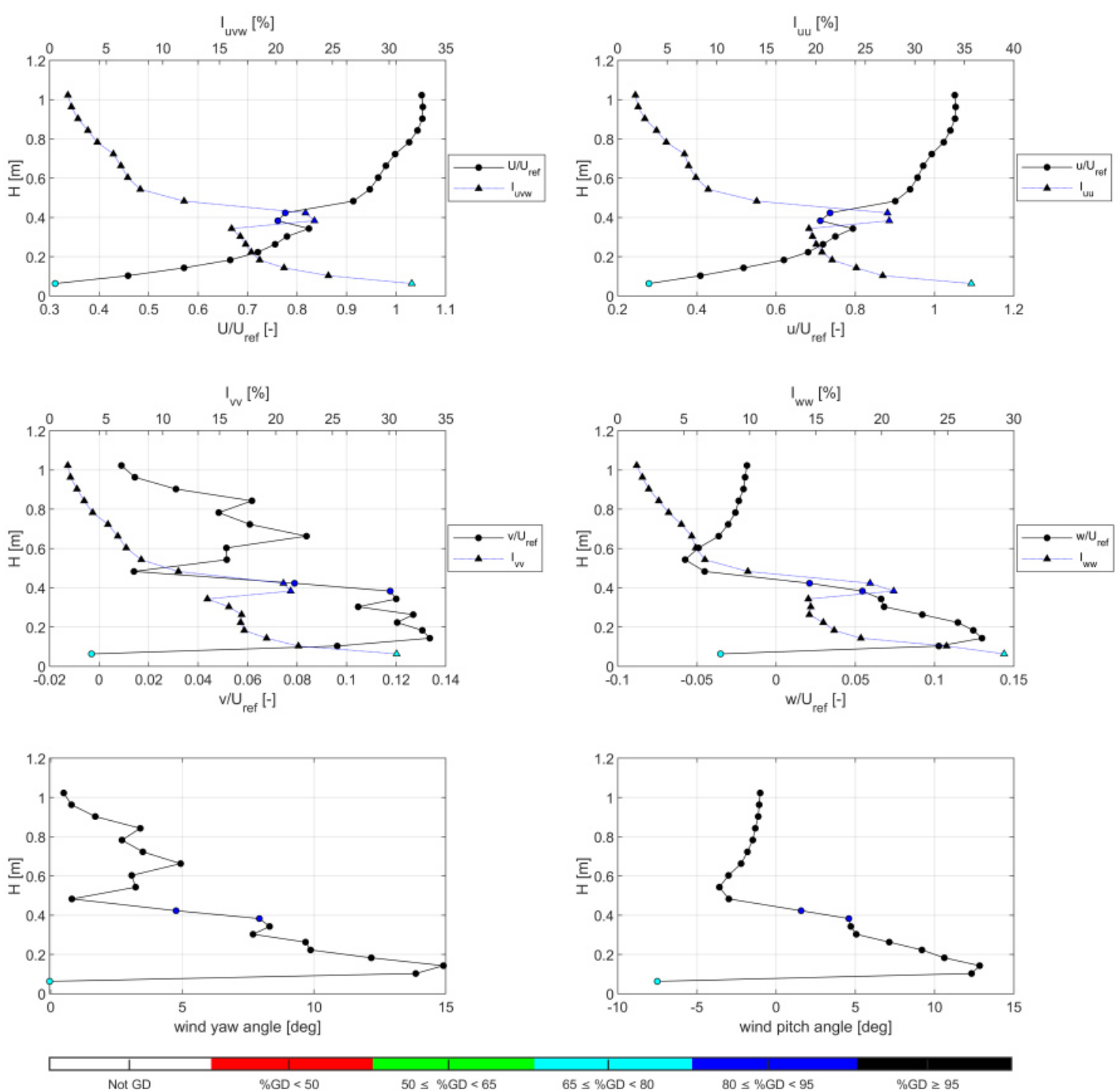

Figure 4.79: Velocities, turbulence intensities and flow angles profiles at Spot 33 for $\mathrm{N}_{16.7}$ wind direction. Total increments $=20$. 

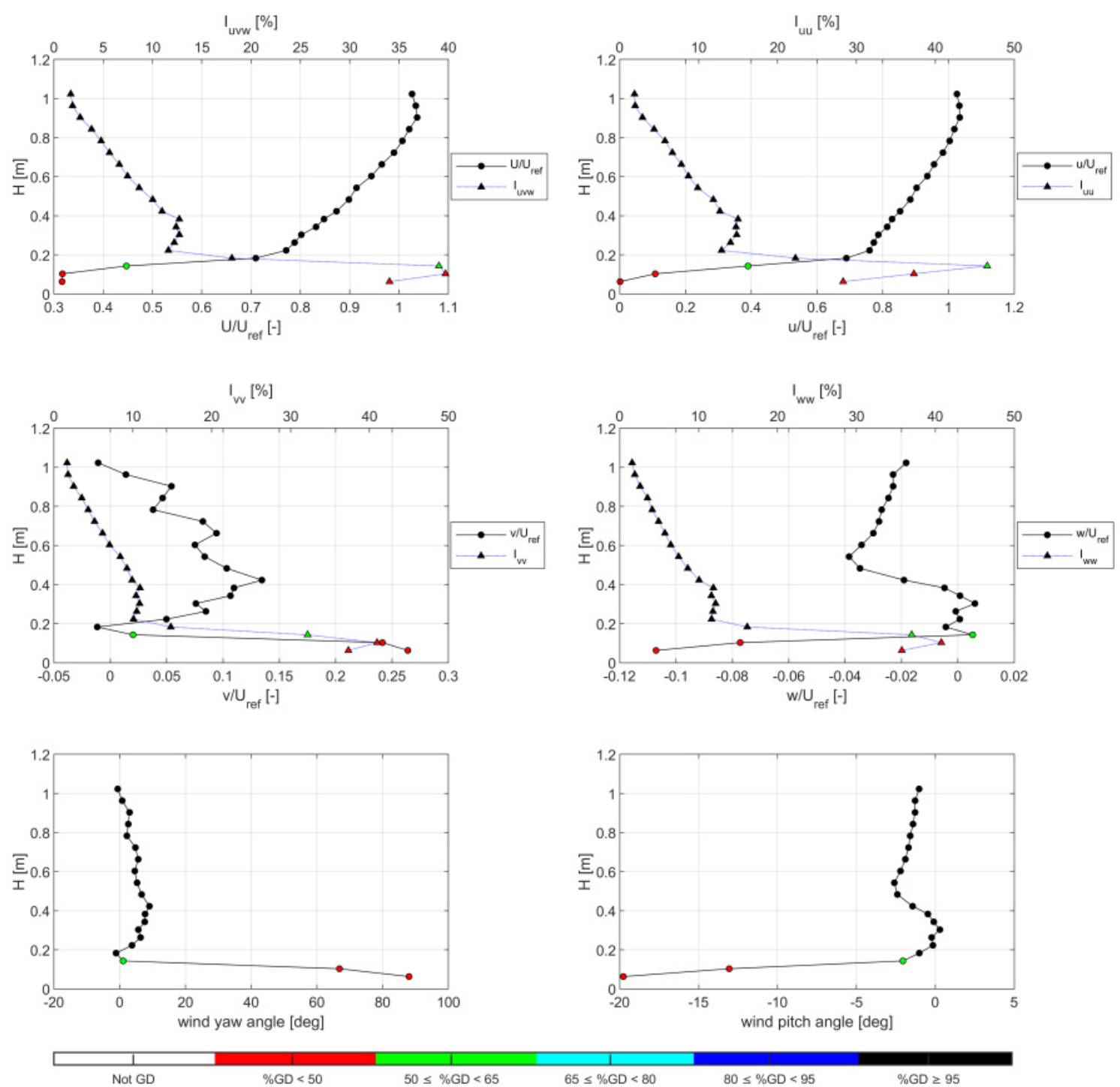

$65 \leq \% \mathrm{GD}<80$

$80 \leq \% \mathrm{GD}<95$

$\% G D \geq 95$

Figure 4.80: Velocities, turbulence intensities and flow angles profiles at Spot 33 for $\mathrm{S}_{15}$ wind direction without the future buildings. Total increments $=20$. 

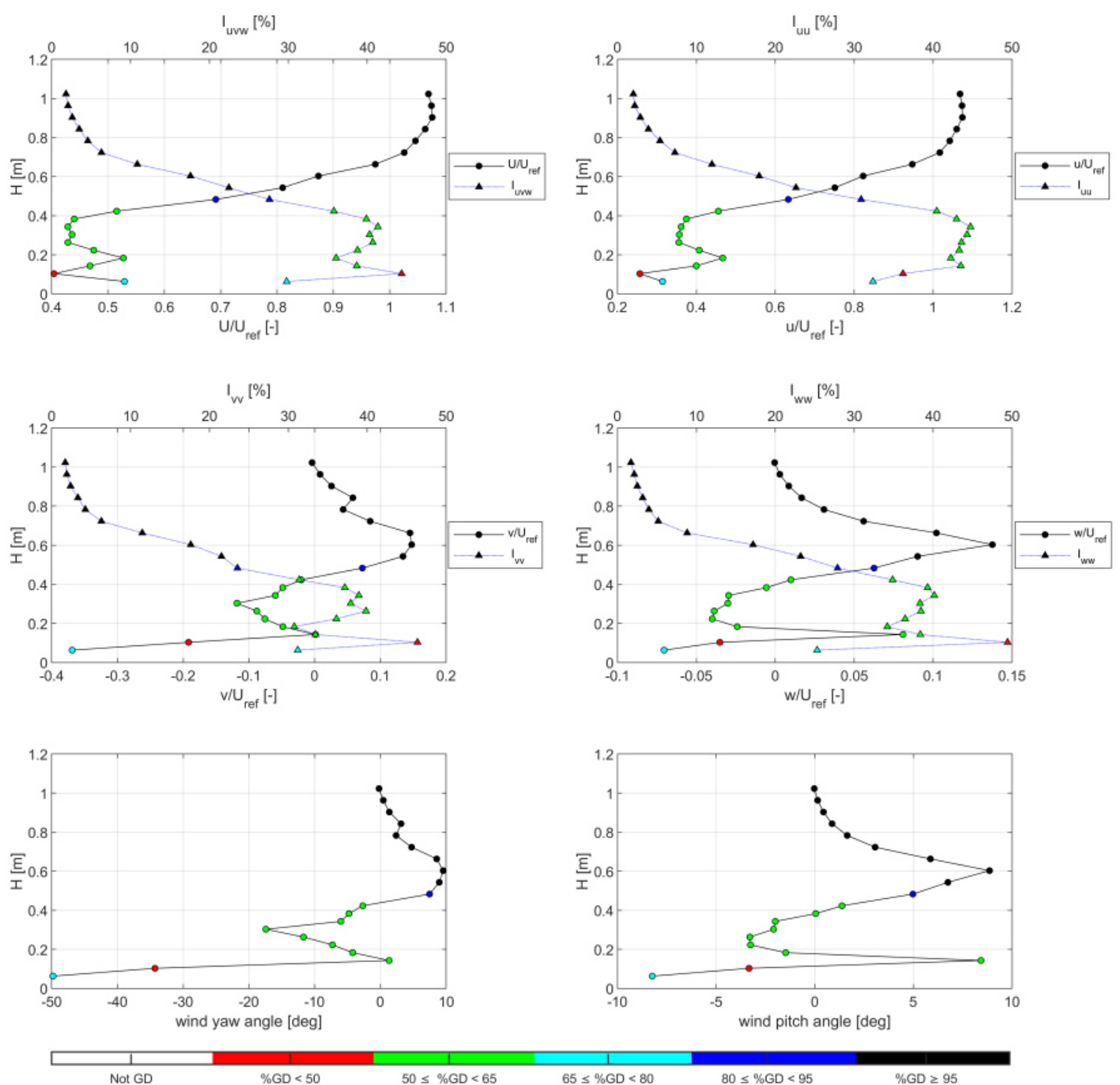

Figure 4.81: Velocities, turbulence intensities and flow angles profiles at Spot 33 for $\mathrm{S}_{15}$ wind direction with the future buildings. Total increments $=20$. 

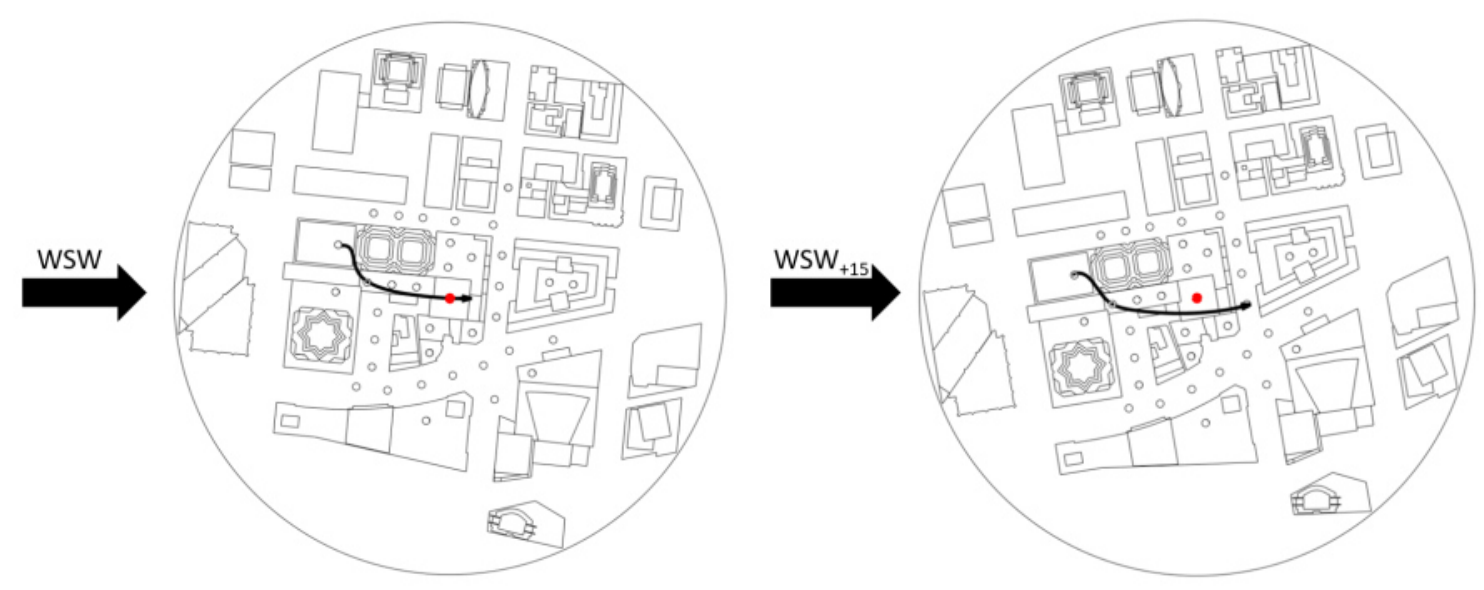

Figure 4.82: Location of Spot 33 with model orientations representing WSW (left) and $\mathrm{WSW}_{+15}$ (right) wind directions. Thin black arrows represent flow separation from the building side wall.

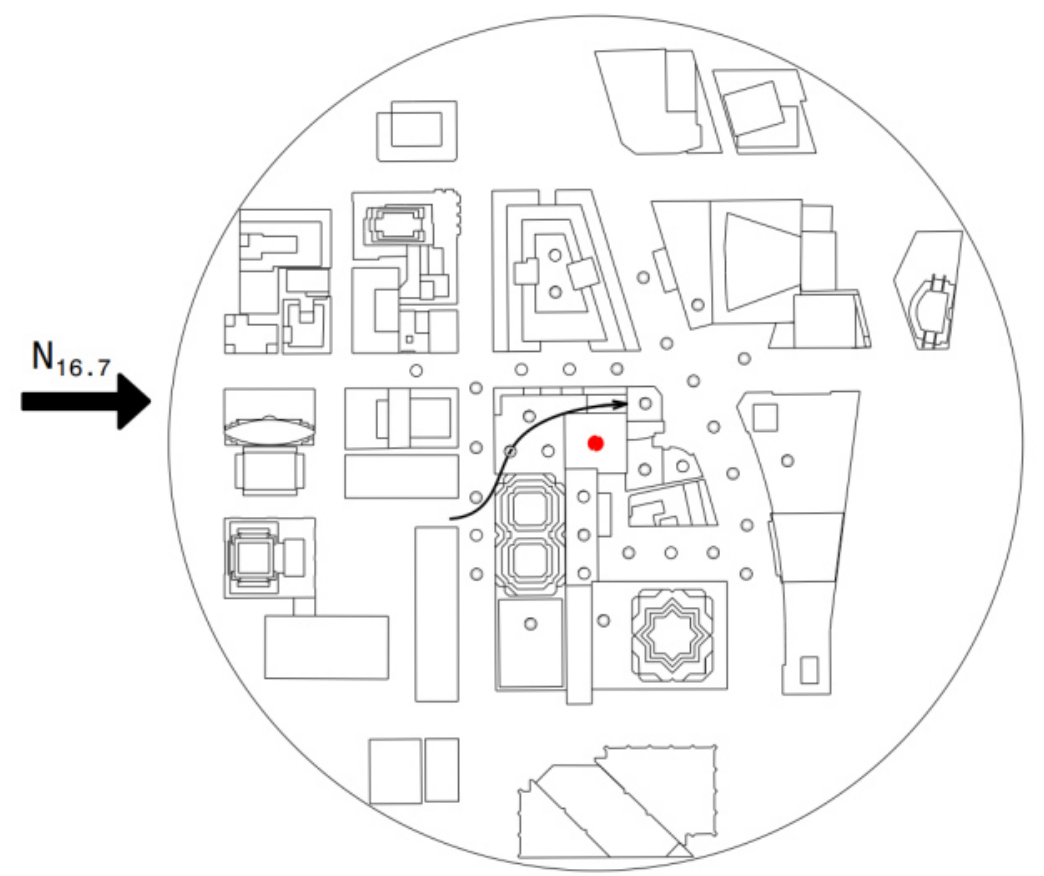

Figure 4.83: Location of Spot 33 with a model orientation representing $\mathrm{N}_{16.7}$ wind direction. Thin black arrow represents flow separation from the building side wall. 


\subsubsection{Effect of adding the future buildings to the model}

Data collection from the model in $\mathrm{S}_{15}$ wind direction was performed twice, that is before and after the installation of the future buildings. This was done to assess the impact of adding new buildings on the flow field conditions, particularly, on turbulence level.

Figures 4.84 through 4.104 show the overall turbulence intensity at various measuring spots with and without the future buildings. The figures show the turbulence level at each measuring increment, starting from Increment 1 and ending at Increment 20, in the form of coloured circles that correspond to specific turbulence intensity ranges defined in a colour bar. Since all measuring spots are involved in this assessment, measurements with less than $80 \%$ GD are additionally marked with plus symbol $(+)$ to indicate that at these spots, more than $20 \%$ of the data were not captured by the Cobra probes. The reader is referred again to Figure 3.34 in order to recall the increments locations with respect to the height of the buildings. Additionally, Increment 1 of the traverse was located $0.063 \mathrm{~m}$ above the wind tunnel floor for the majority of the spots. However, as mentioned previously in Section 3.1.6.2. Increment 1 was located $0.073 \mathrm{~m}$ above the wind tunnel floor for few spots, whose location was above low-rise buildings rooftops. For spots located above higher buildings like Spot 2, the traverse started at Increment 3 (0.143 m above the wind tunnel floor) since Increments 1 and 2 are contained within the height of the building.

By comparing the results, it was observed that the impact of the future buildings extended at least to a distance equal to the height of the buildings. This is based on examining the changes in TI level at Spot 10 (refer to Figure 4.105 for the spot location), which is approximately $247 \mathrm{~m}$ (FS) distant from the buildings leeward faces. Moreover, it can be seen in Figures 4.84 through 4.87 that the addition of the future buildings caused a reduction in overall turbulence level at the location of the 
spots that are distant from the future buildings. Interestingly, the turbulence level at Increment 3 of Spot 31 was above $40 \%$ (based on the captured data, which is less than $80 \%$ ) before adding the future buildings. However, when the future buildings were added to the model, the turbulence level were found to be lower at the spot, and more than $80 \%$ of data were captured by the probe. Nonetheless, the majority of the spots were subjected to higher turbulence level when the future buildings were added, and the fluctuations in the flow direction were more severe, causing appreciable amount of data to be lost. It was only after Increment 18, which is $114 \mathrm{~m}$ (FS) above the future buildings, that the changes in the turbulence level between both cases diminished. 

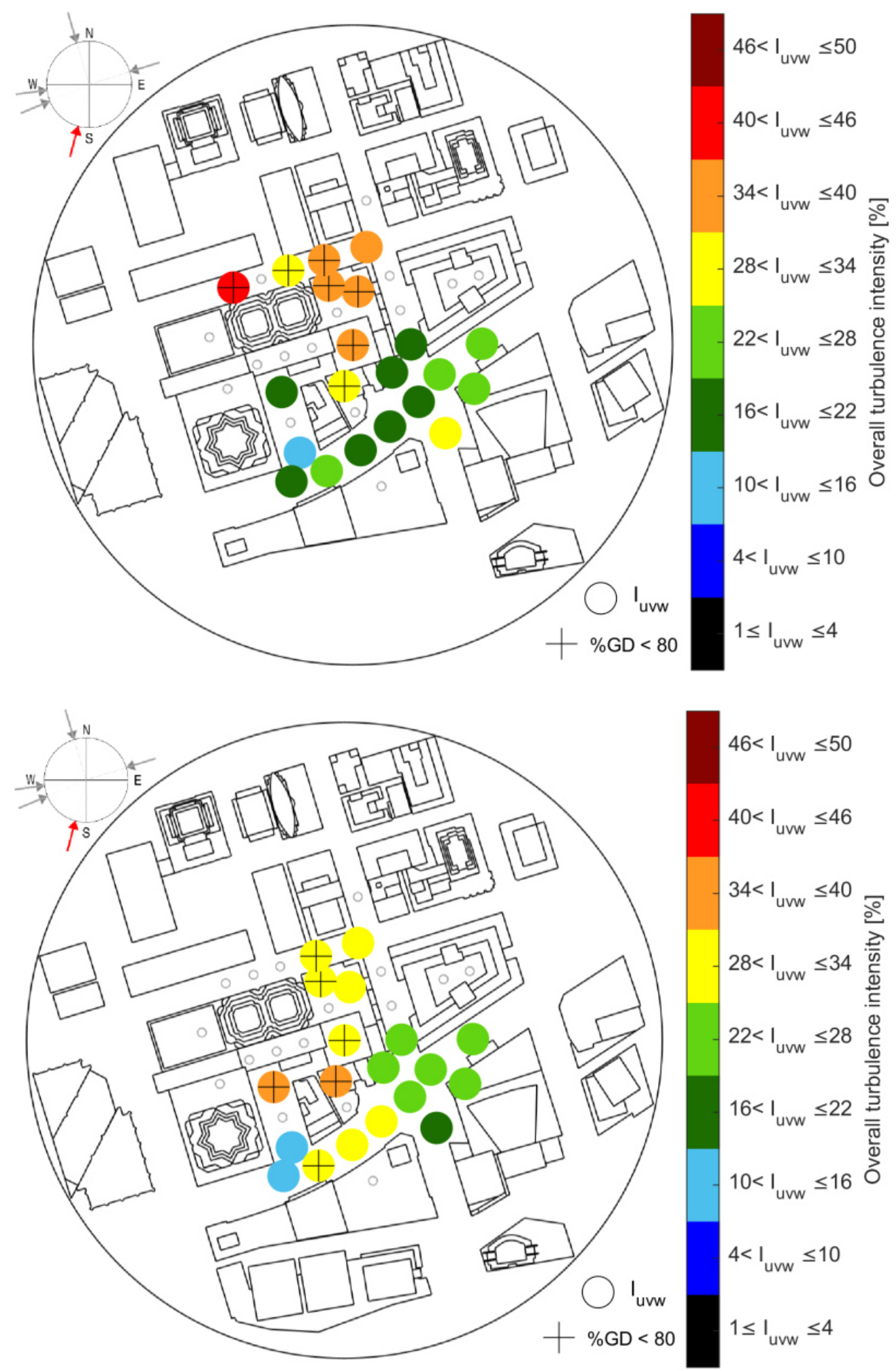

$46<\mathrm{I}_{\mathrm{urw}} \leq 50$

$40<\mathrm{I}_{\mathrm{uvw}} \leq 46$

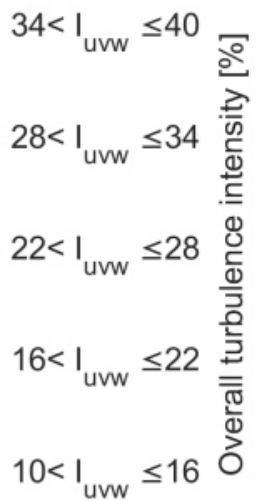

$4<\mathrm{I}_{\mathrm{uvw}} \leq 10$

$1 \leq \mathrm{I}_{\mathrm{uvw}} \leq 4$

Figure 4.84: Overall turbulence intensity at Increment 1 (0.063 m above wind tunnel floor) without (top) and with (bottom) the future buildings. 

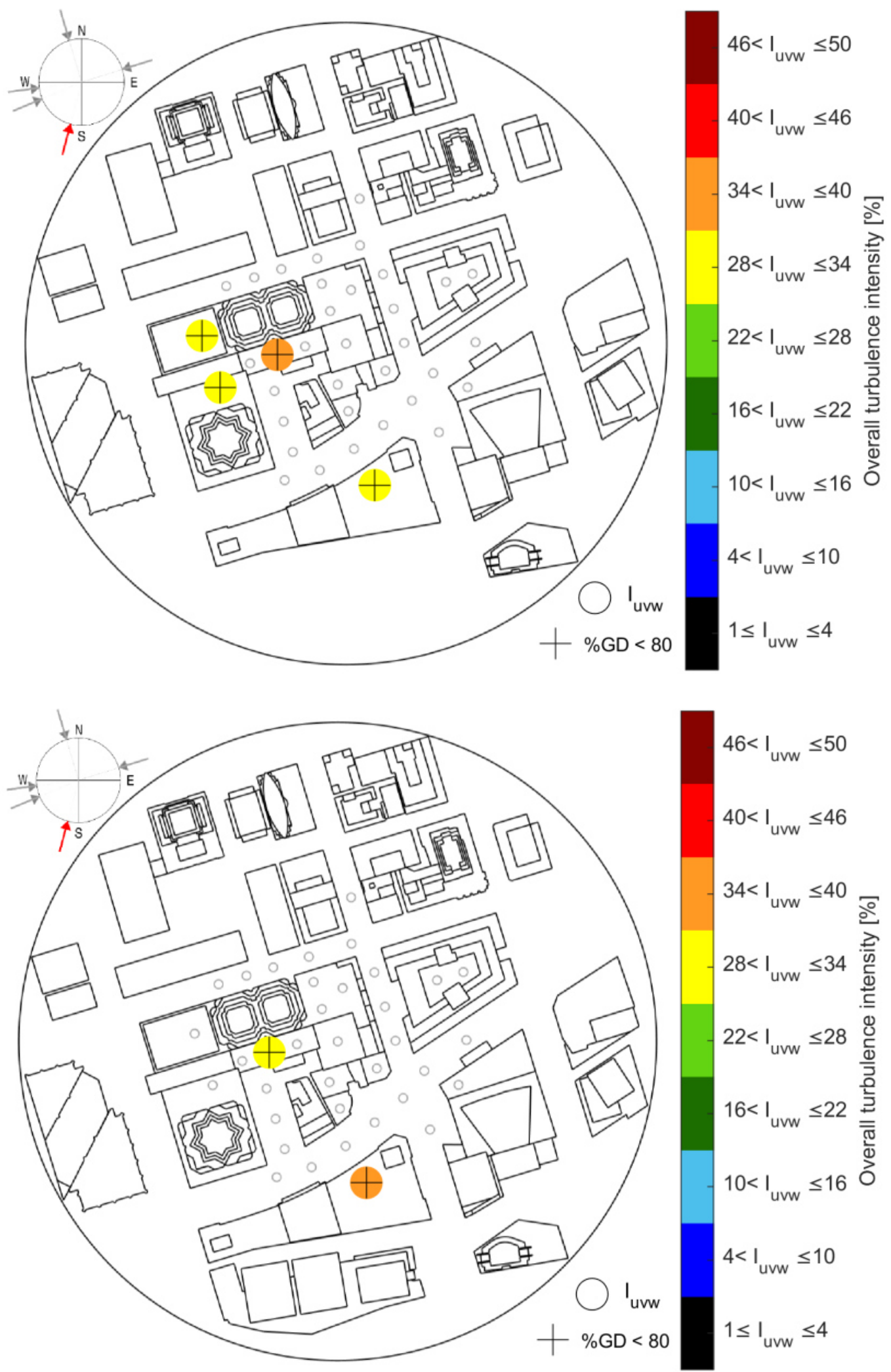

$46<$ I $_{\text {uvw }} \leq 50$

$40<I_{\text {uvw }} \leq 46$

$34<$ I urw $\leq 40$

$28<1_{\text {uvw }} \leq 34$

.

$22<I_{u v w} \leq 28$

$16<$ I $_{\text {urw }} \leq 22$

$10<\mathrm{I}_{\mathrm{uvw}} \leq 16$ ठे

$4<I_{\text {uvw }} \leq 10$

$1 \leq \mathrm{I}_{\mathrm{uvw}} \leq 4$

Figure 4.85: Overall turbulence intensity at Increment 1 (0.073 $\mathrm{m}$ above wind tunnel floor) without (top) and with (bottom) the future buildings. 

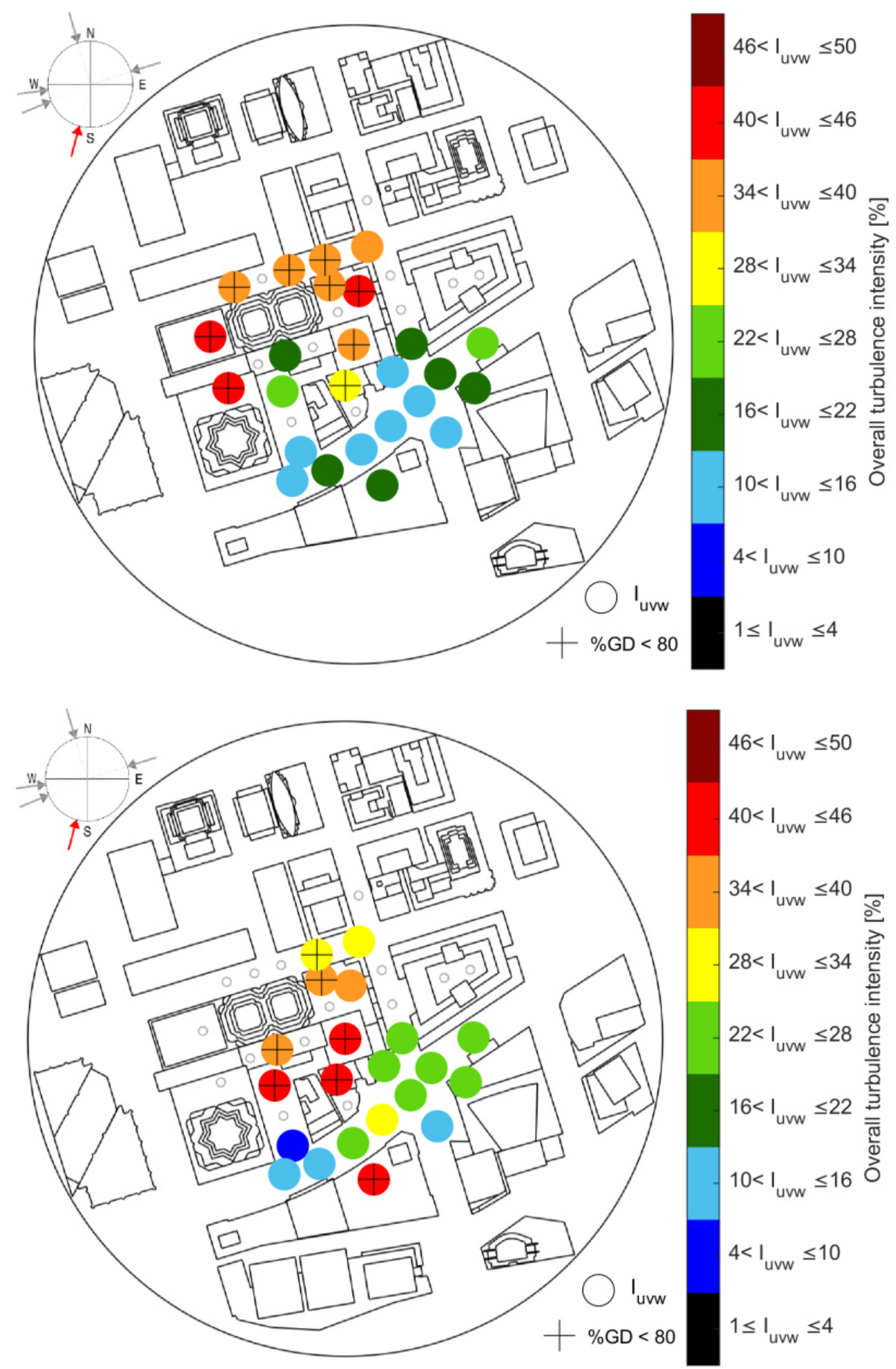

$46<\mathrm{I}_{\mathrm{urw}} \leq 50$

$40<\mathrm{I}_{\mathrm{uvw}} \leq 46$

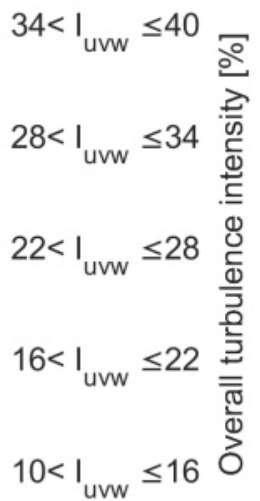

$4<\mathrm{I}_{\mathrm{uvw}} \leq 10$

$1 \leq \mathrm{I}_{\mathrm{uvw}} \leq 4$

Figure 4.86: Overall turbulence intensity at Increment 2 (0.103 $\mathrm{m}$ above wind tunnel floor) without (top) and with (bottom) the future buildings. 

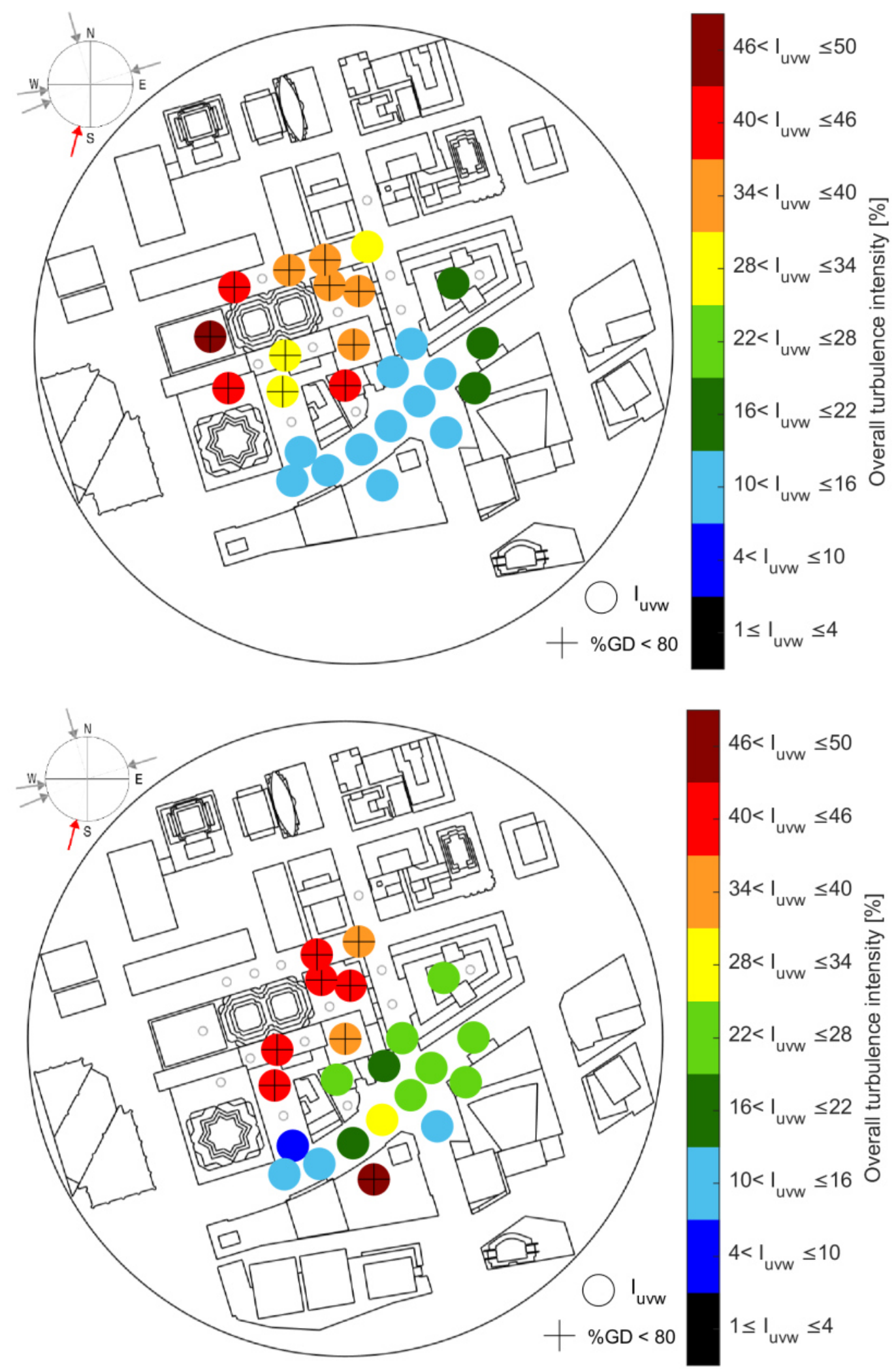

$46<\mathrm{I}_{\mathrm{urw}} \leq 50$

$40<\mathrm{I}_{\mathrm{uvw}} \leq 46$

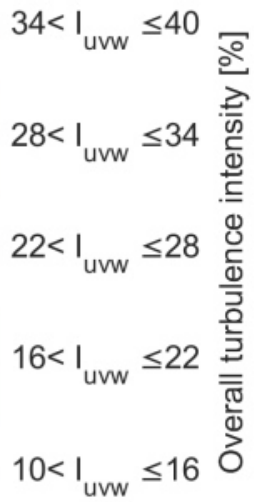

$4<\mathrm{I}_{\mathrm{uvw}} \leq 10$

$1 \leq \mathrm{I}_{\mathrm{uvw}} \leq 4$

Figure 4.87: Overall turbulence intensity at Increment 3 (0.143 m above wind tunnel floor) without (top) and with (bottom) the future buildings. 

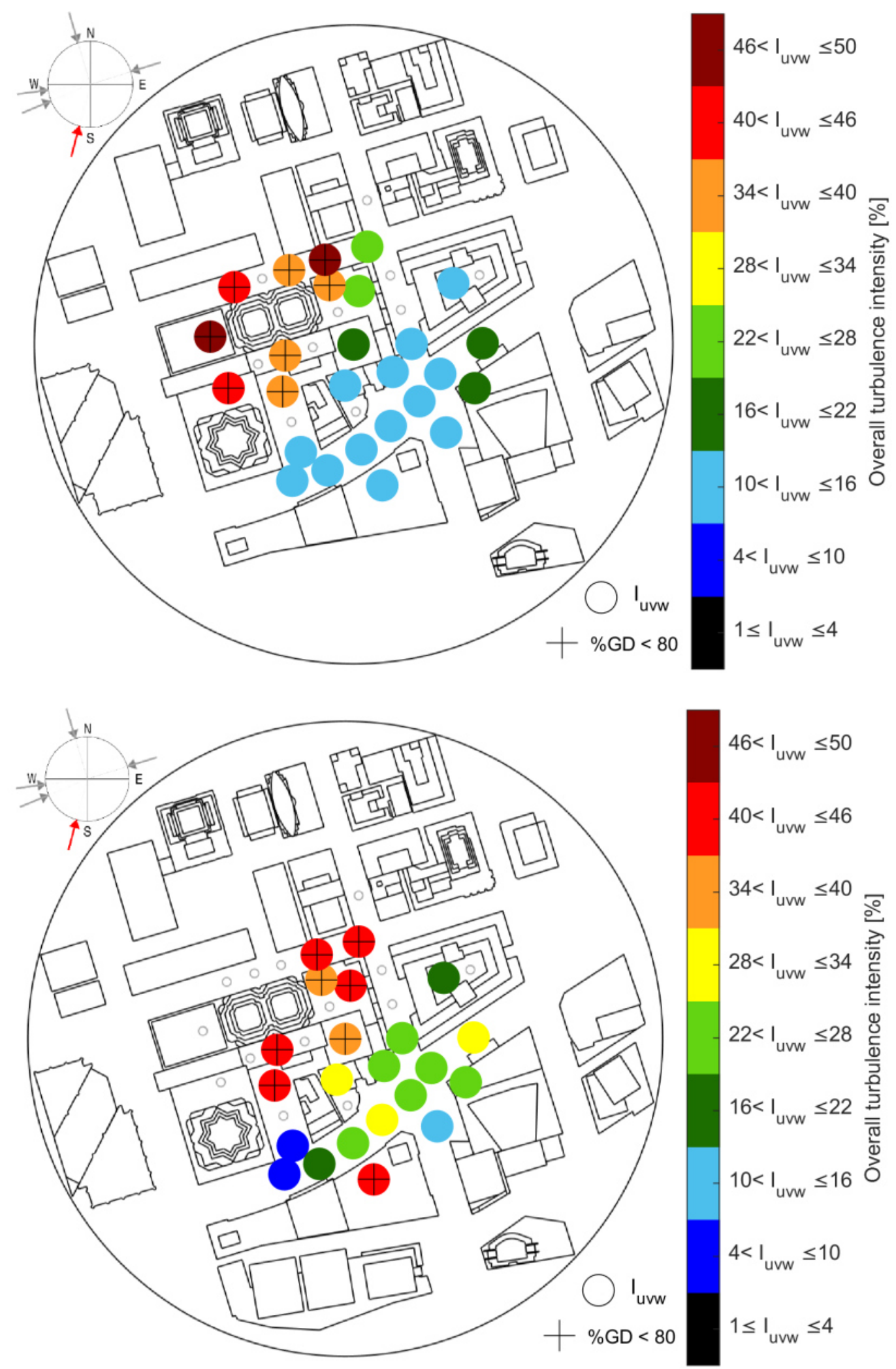

$46<\mathrm{I}_{\mathrm{uvw}} \leq 50$

$40<I_{\text {uvw }} \leq 46$

$34<I_{\text {uvw }} \leq 40$

$28<1_{\text {unw }} \leq 34$

.

$22<1_{\text {uvw }} \leq 28$

$16<$ I $_{\text {uvw }} \leq 22$

$10<$ I $_{\text {uvw }} \leq 16$ ठे

$4<I_{\text {uvw }} \leq 10$

$1 \leq \mathrm{I}_{\text {urw }} \leq 4$

Figure 4.88: Overall turbulence intensity at Increment 4 (0.183 $\mathrm{m}$ above wind tunnel floor) without (top) and with (bottom) the future buildings. 

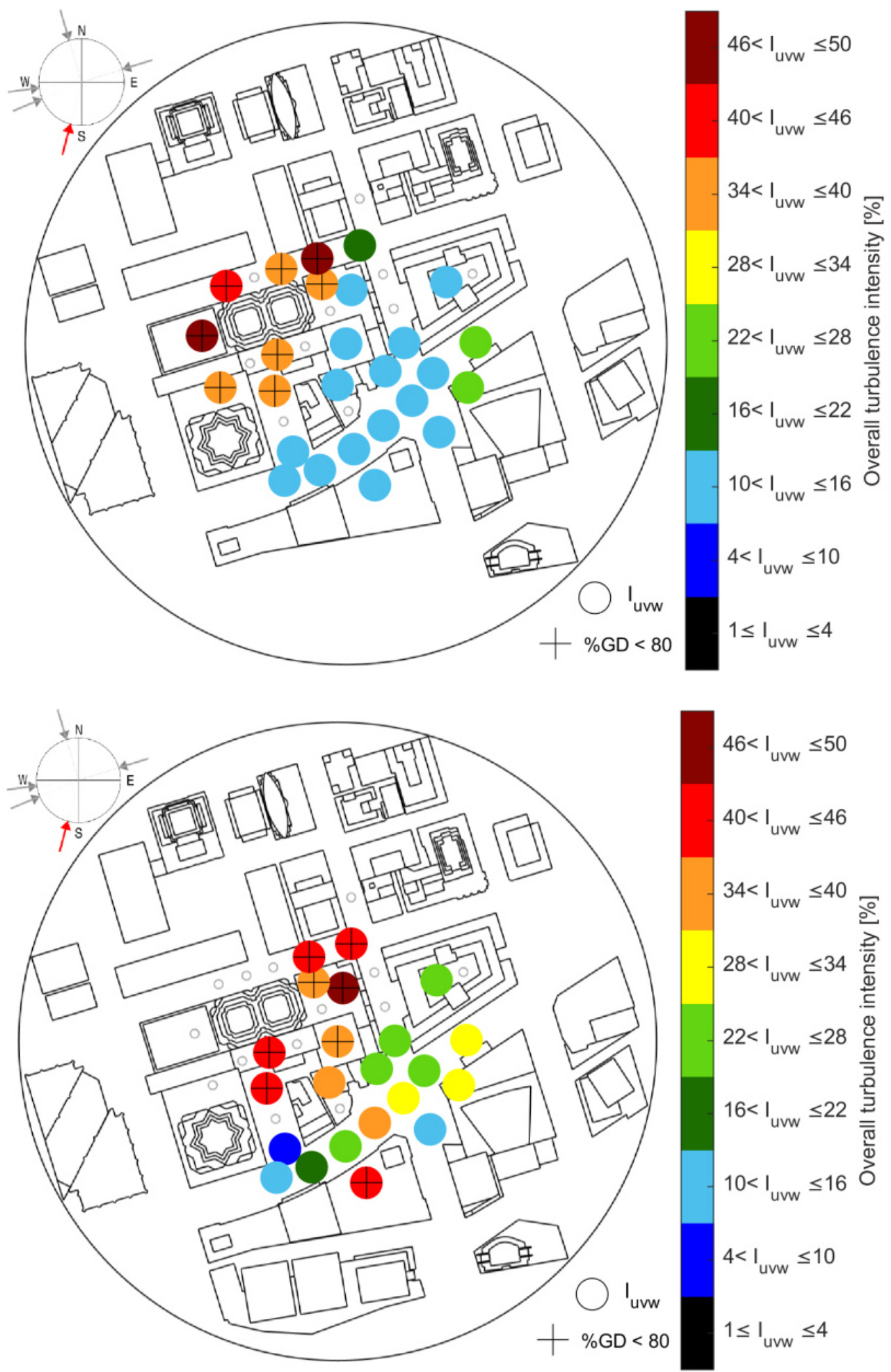

$46<\mathrm{I}_{\mathrm{uvw}} \leq 50$

$40<I_{\text {uvw }} \leq 46$

$34<I_{\text {uvw }} \leq 40$

$28<1_{\text {unw }} \leq 34$

$22<$ I $_{\text {uvw }} \leq 28$

$22<1_{\text {uvw }} \leq 28$

$16<$ I $_{\text {uvw }} \leq 22$

$10<1_{\text {uvw }} \leq 16$ ठे

$4<I_{\text {uvw }} \leq 10$

$1 \leq \mathrm{I}_{\text {urw }} \leq 4$

Figure 4.89: Overall turbulence intensity at Increment 5 (0.223 $\mathrm{m}$ above wind tunnel floor) without (top) and with (bottom) the future buildings. 

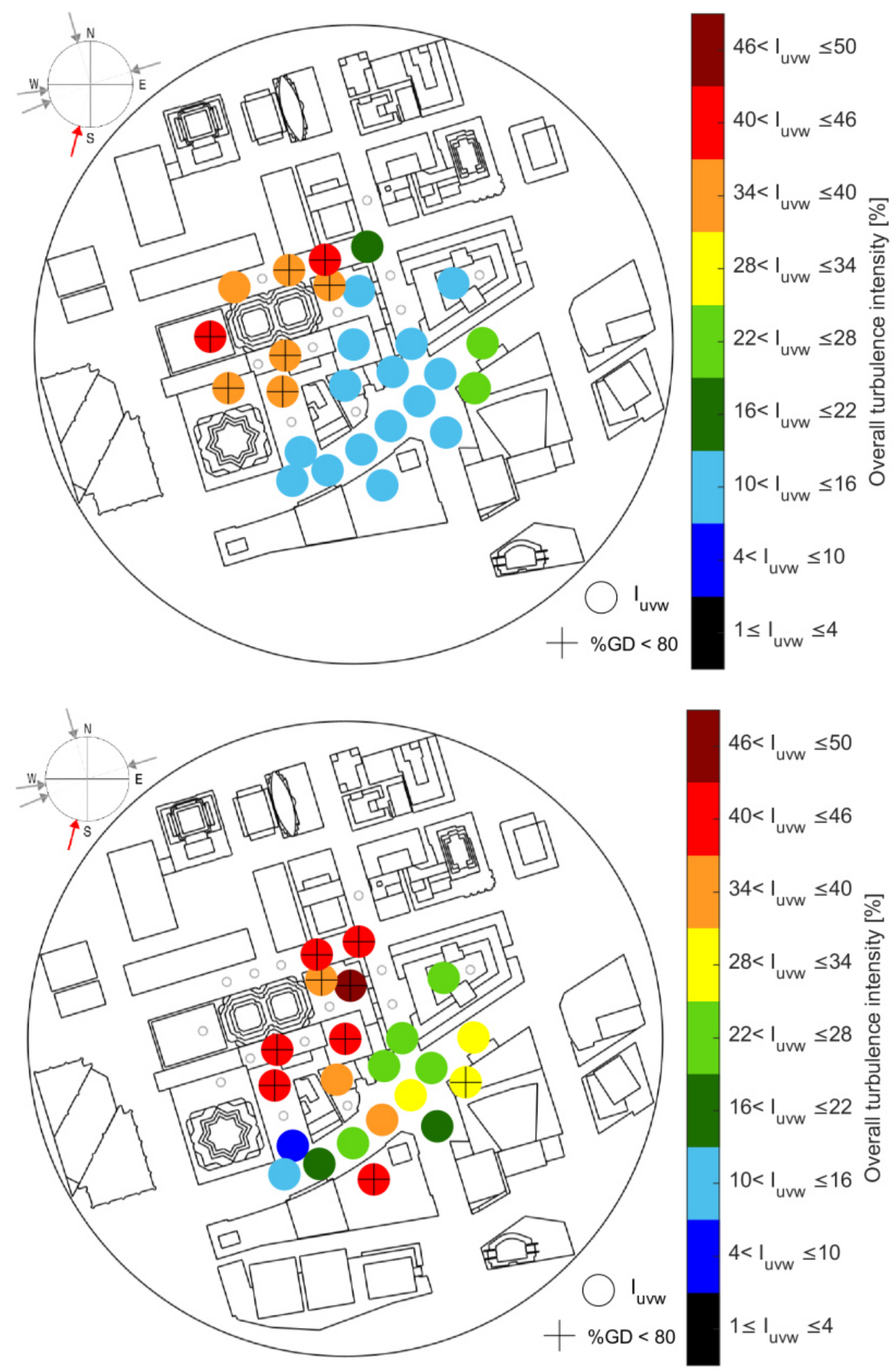

$46<\mathrm{I}_{\mathrm{urw}} \leq 50$

$40<\mathrm{I}_{\mathrm{uvw}} \leq 46$

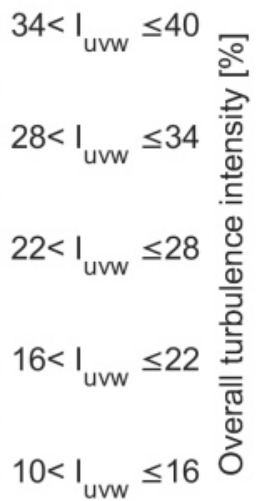

$4<\mathrm{I}_{\mathrm{uvw}} \leq 10$

$1 \leq \mathrm{I}_{\mathrm{uvw}} \leq 4$

Figure 4.90: Overall turbulence intensity at Increment 6 (0.263 $\mathrm{m}$ above wind tunnel floor) without (top) and with (bottom) the future buildings. 

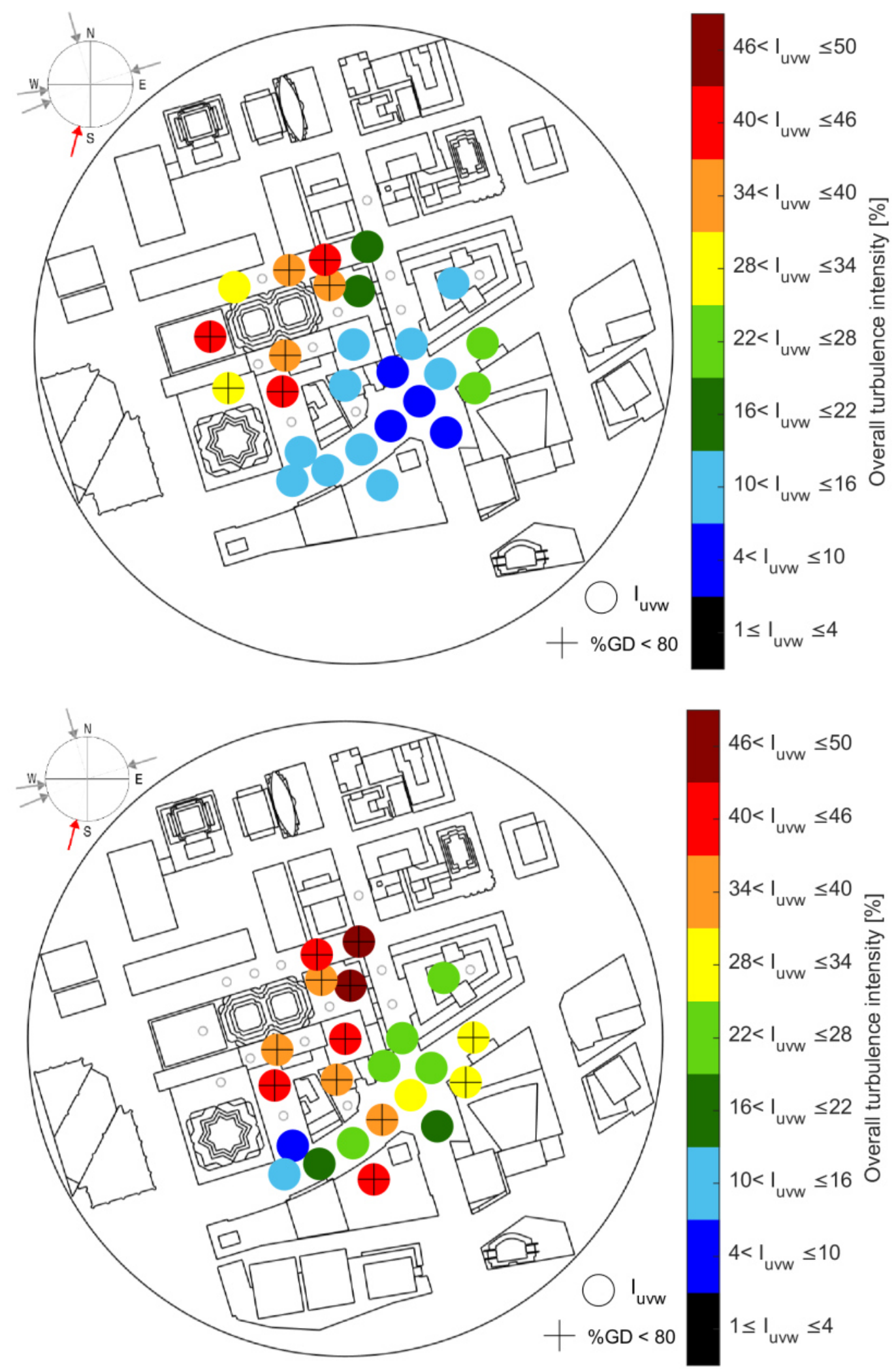

$46<\mathrm{I}_{\mathrm{uvw}} \leq 50$

$40<I_{\text {uvw }} \leq 46$

$34<$ I urw $\leq 40$

$28<1_{\text {unw }} \leq 34$

$22<1 \quad \leq 28$

$22<1_{u v w} \leq 28$

$16<$ I $_{\text {uvw }} \leq 22$

$10<1_{\text {uvw }} \leq 16$ ठे

$4<I_{\text {uvw }} \leq 10$

$1 \leq \mathrm{I}_{\text {urw }} \leq 4$

Figure 4.91: Overall turbulence intensity at Increment 7 (0.303 $\mathrm{m}$ above wind tunnel floor) without (top) and with (bottom) the future buildings. 

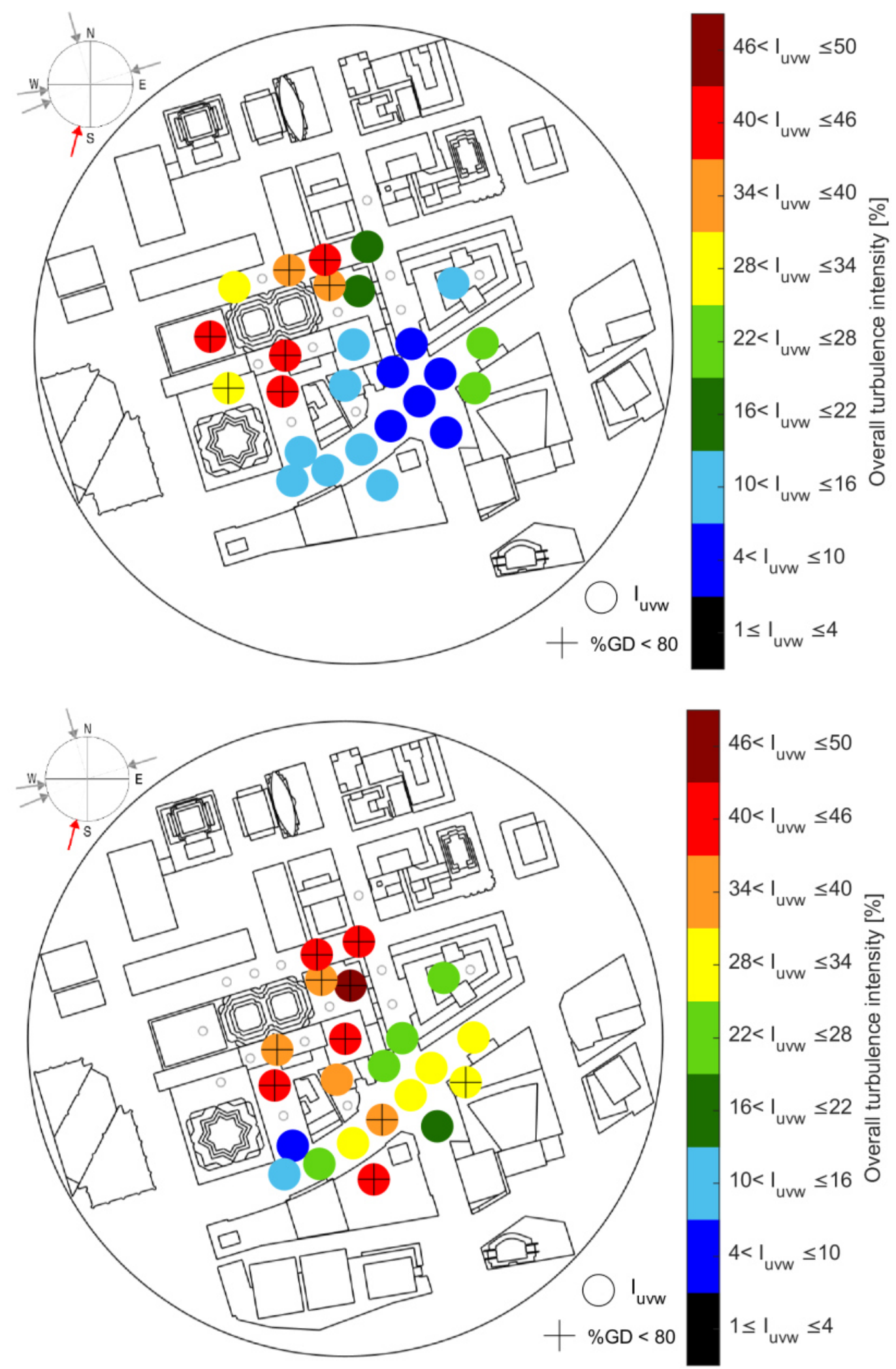

$46<\mathrm{I}_{\mathrm{urw}} \leq 50$

$40<\mathrm{I}_{\mathrm{uvw}} \leq 46$

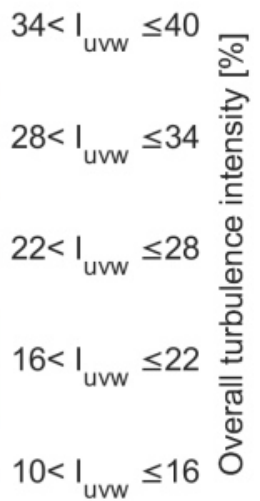

$4<\mathrm{I}_{\mathrm{uvw}} \leq 10$

$1 \leq \mathrm{I}_{\mathrm{uvw}} \leq 4$

Figure 4.92: Overall turbulence intensity at Increment 8 (0.343 $\mathrm{m}$ above wind tunnel floor) without (top) and with (bottom) the future buildings. 

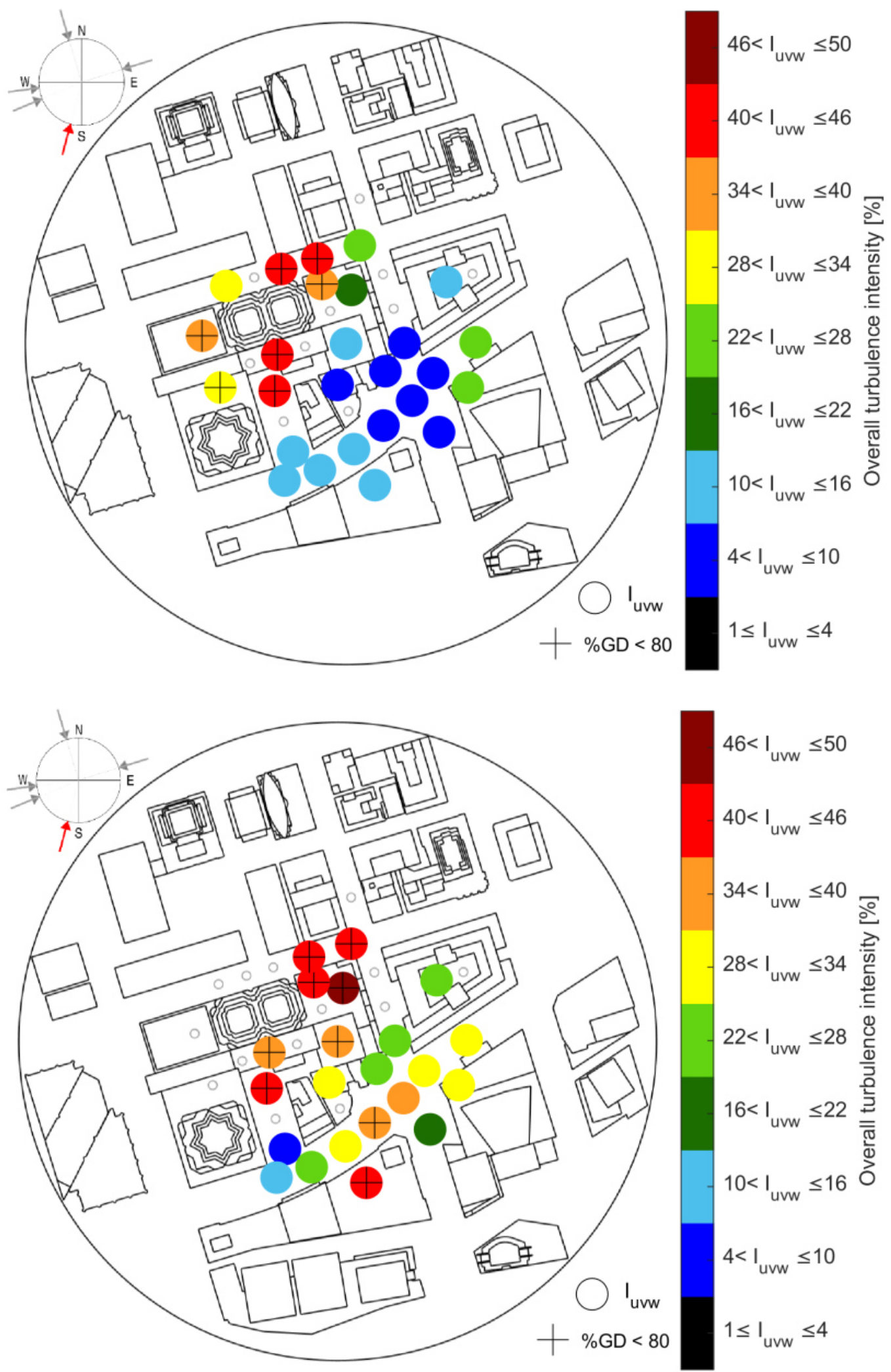

$46<\mathrm{I}_{\mathrm{urw}} \leq 50$

$40<\mathrm{I}_{\mathrm{uvw}} \leq 46$

$34<$ I $_{\text {urw }} \leq 40$

$28<1_{\text {uvw }} \leq 34$

$22<1_{\text {uvw }} \leq 28$

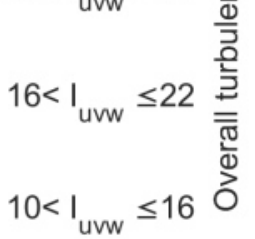

$4<\mathrm{I}_{\mathrm{urw}} \leq 10$

$1 \leq \mathrm{I}_{\text {urw }} \leq 4$

Figure 4.93: Overall turbulence intensity at Increment 9 (0.383 $\mathrm{m}$ above wind tunnel floor) without (top) and with (bottom) the future buildings. 

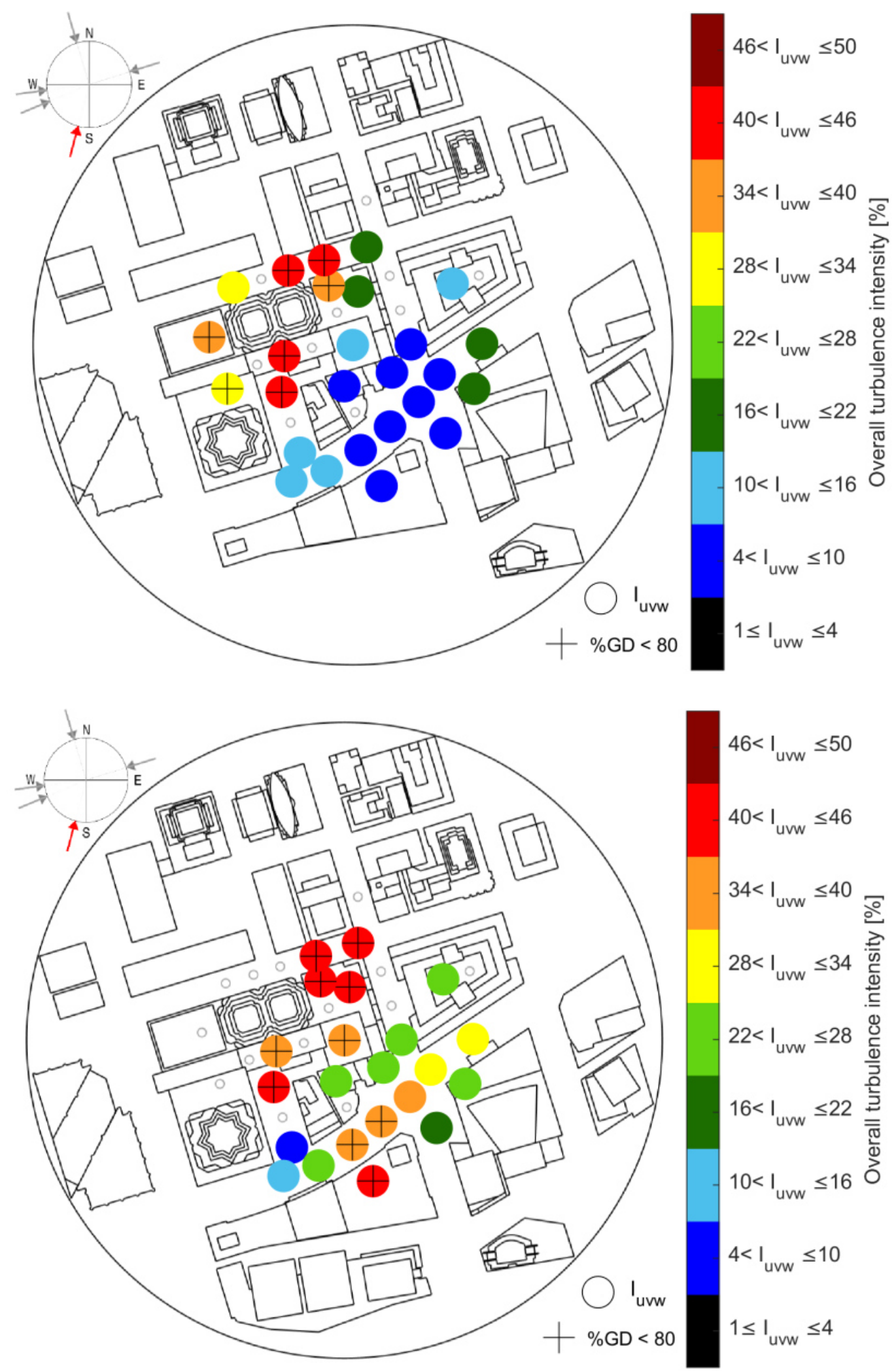

$46<\mathrm{I}_{\mathrm{uvw}} \leq 50$

$40<I_{\text {uvw }} \leq 46$

$34<$ I urw $\leq 40$

$28<1_{\text {unw }} \leq 34$

.

$22<1_{\text {uvw }} \leq 28$

$16<$ I $_{\text {uvw }} \leq 22$

$10<1_{\text {uvw }} \leq 16$ ठे

$4<I_{\text {uvw }} \leq 10$

$1 \leq \mathrm{I}_{\text {uvw }} \leq 4$

Figure 4.94: Overall turbulence intensity at Increment $10(0.4230 \mathrm{~m}$ above wind tunnel floor) without (top) and with (bottom) the future buildings. 

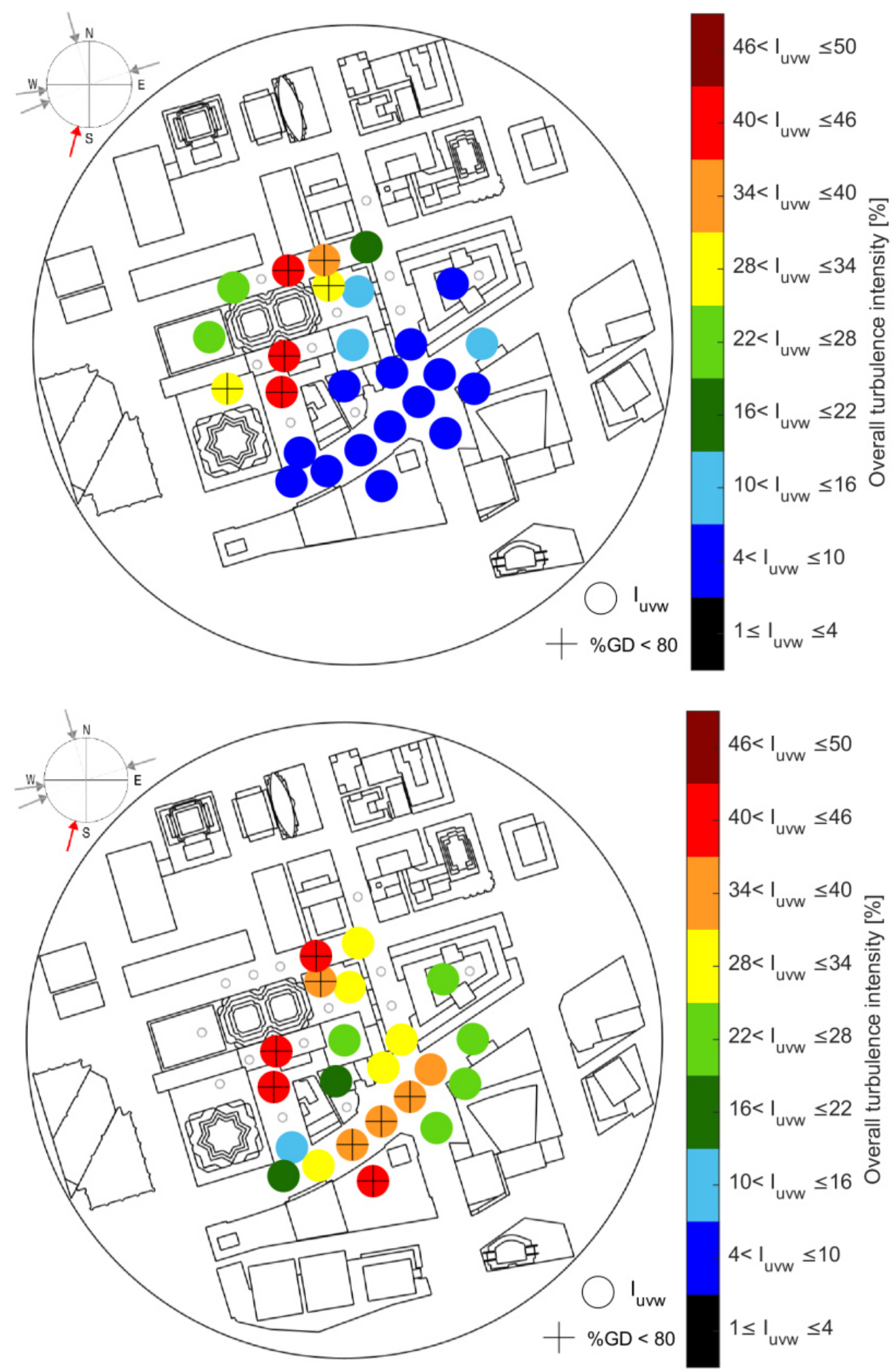

$46<I_{\text {uvw }} \leq 50$

$40<I_{\text {uvw }} \leq 46$

$34<I_{\text {uvw }} \leq 40$

$28<1_{u v w} \leq 34$

.

$22<1_{\text {uvw }} \leq 28$

$16<$ I $_{\text {uvw }} \leq 22$

$10<1_{\text {uvw }} \leq 16$ ठे

$4<I_{\text {uvw }} \leq 10$

$1 \leq \mathrm{I}_{\text {uvw }} \leq 4$

Figure 4.95: Overall turbulence intensity at Increment $11(0.483 \mathrm{~m}$ above wind tunnel floor) without (top) and with (bottom) the future buildings. 

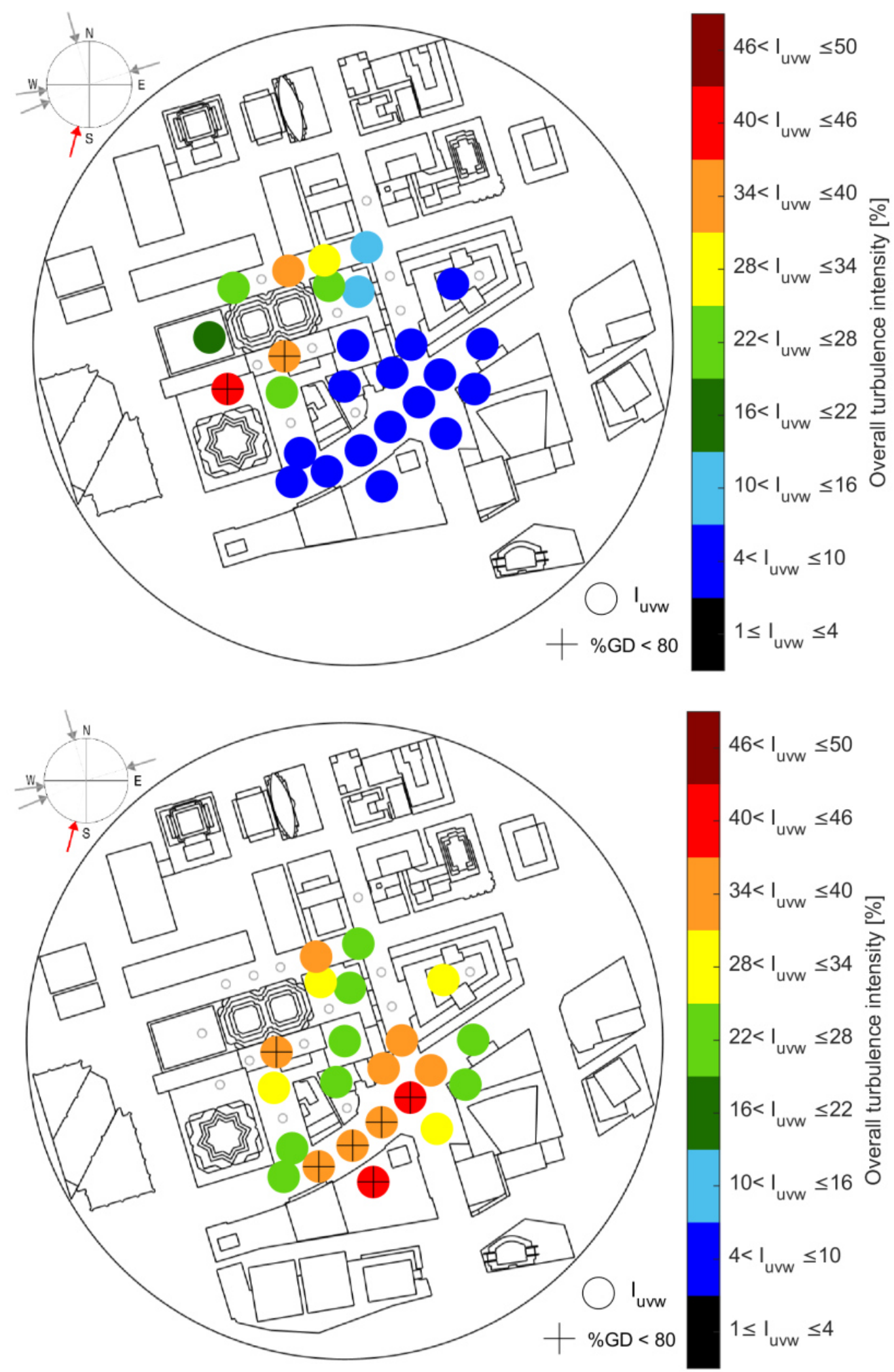

$46<I_{\text {uvw }} \leq 50$

$40<I_{\text {uvw }} \leq 46$

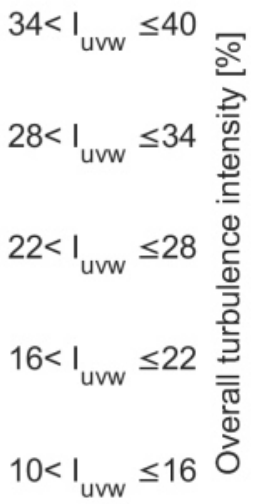

$4<\mathrm{I}_{\mathrm{uvw}} \leq 10$

$1 \leq \mathrm{I}_{\mathrm{urw}} \leq 4$

Figure 4.96: Overall turbulence intensity at Increment $12(0.543 \mathrm{~m}$ above wind tunnel floor) without (top) and with (bottom) the future buildings. 

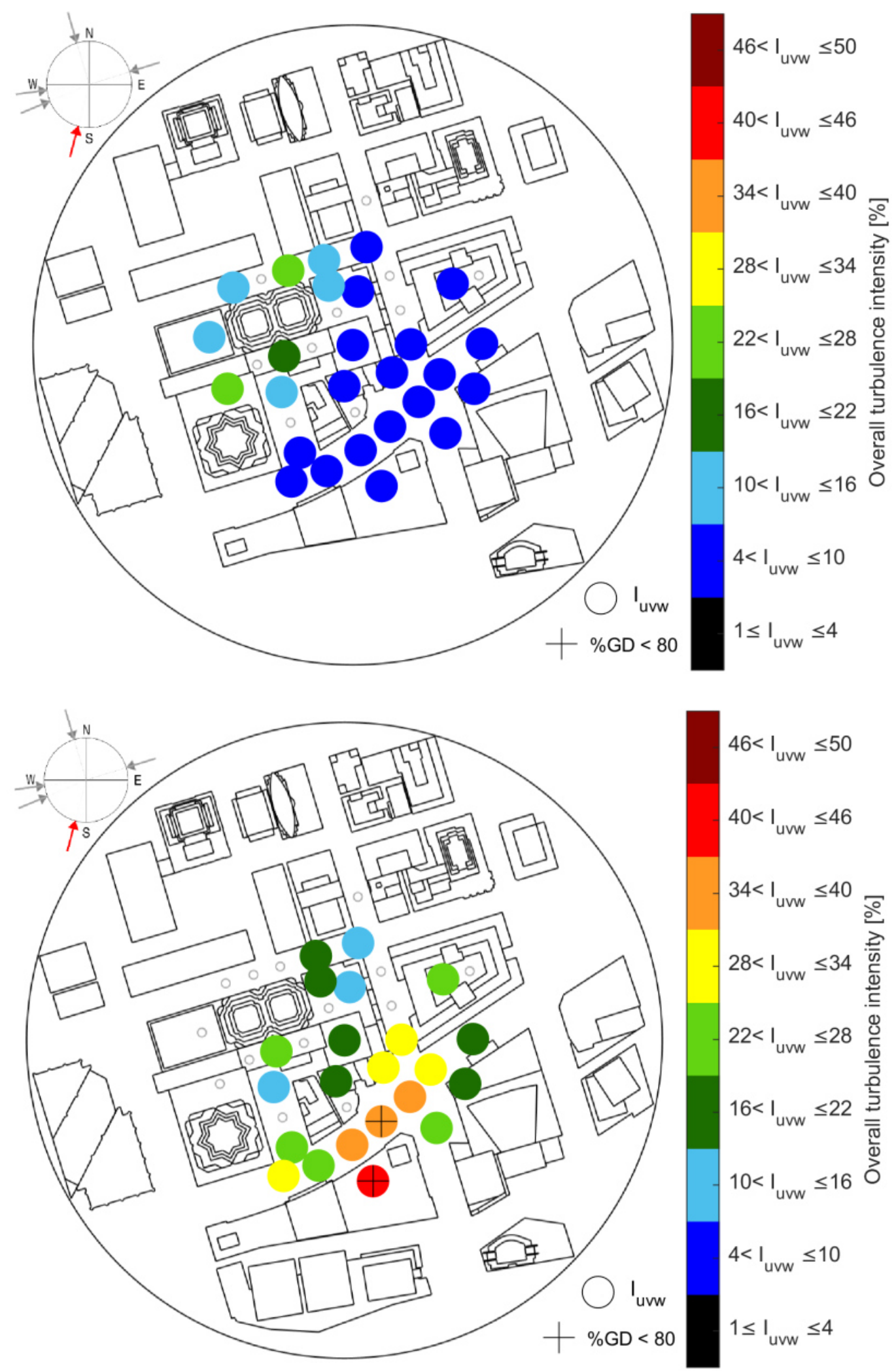

$46<I_{\text {uvw }} \leq 50$

$40<I_{\text {uvw }} \leq 46$

$34<I_{\text {uvw }} \leq 40$

$28<1_{u v w} \leq 34$

.

$22<1_{\text {uvw }} \leq 28$

$16<$ I $_{\text {uvw }} \leq 22$

$10<1_{\text {uvw }} \leq 16$ ठे

$4<I_{\text {uvw }} \leq 10$

$1 \leq \mathrm{I}_{\text {uvw }} \leq 4$

Figure 4.97: Overall turbulence intensity at Increment $13(0.603 \mathrm{~m}$ above wind tunnel floor) without (top) and with (bottom) the future buildings. 


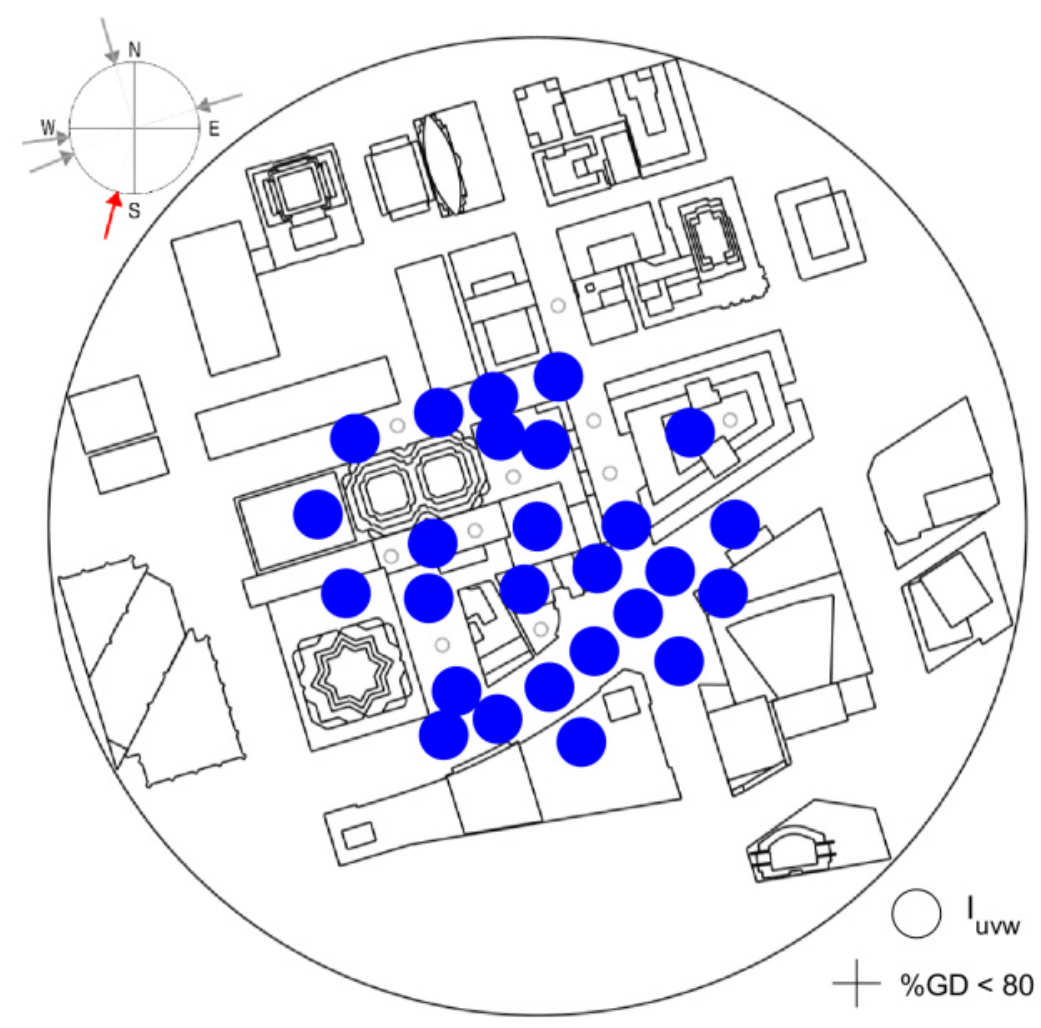

$46<\mathrm{I}_{\mathrm{uvw}} \leq 50$

$40<I_{\text {uvw }} \leq 46$

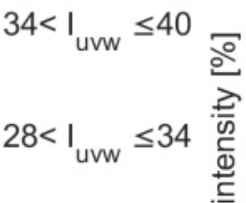

$22<$ I $_{\text {uvw }} \leq 28$

$16<$ I $_{\text {urw }} \leq 22$

$10<1_{\text {uvw }} \leq 16$ ठे

$4<I_{\text {uvw }} \leq 10$

$1 \leq \mathrm{I}_{\mathrm{uvw}} \leq 4$

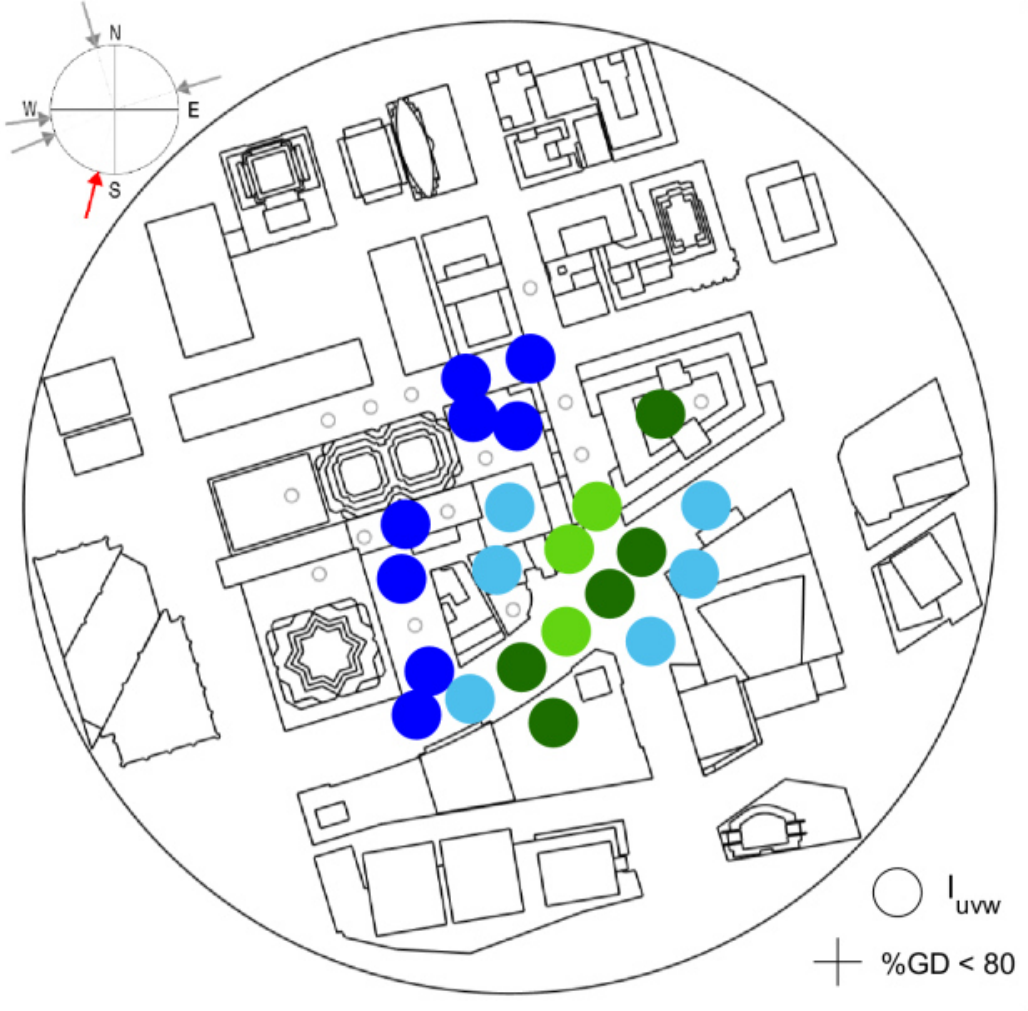

$46<I_{\text {uvw }} \leq 50$

$40<I_{\text {uvw }} \leq 46$

$34<$ I $_{\text {uvw }} \leq 40$

$28<$ I $_{\text {uvw }} \leq 34$

$22<$ I $_{\text {uvw }} \leq 28$

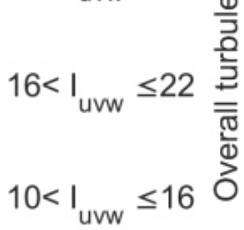

$4<I_{\text {uvw }} \leq 10$

$1 \leq \mathrm{I}_{\text {urw }} \leq 4$

Figure 4.98: Overall turbulence intensity at Increment $14(0.663 \mathrm{~m}$ above wind tunnel floor) without (top) and with (bottom) the future buildings. 


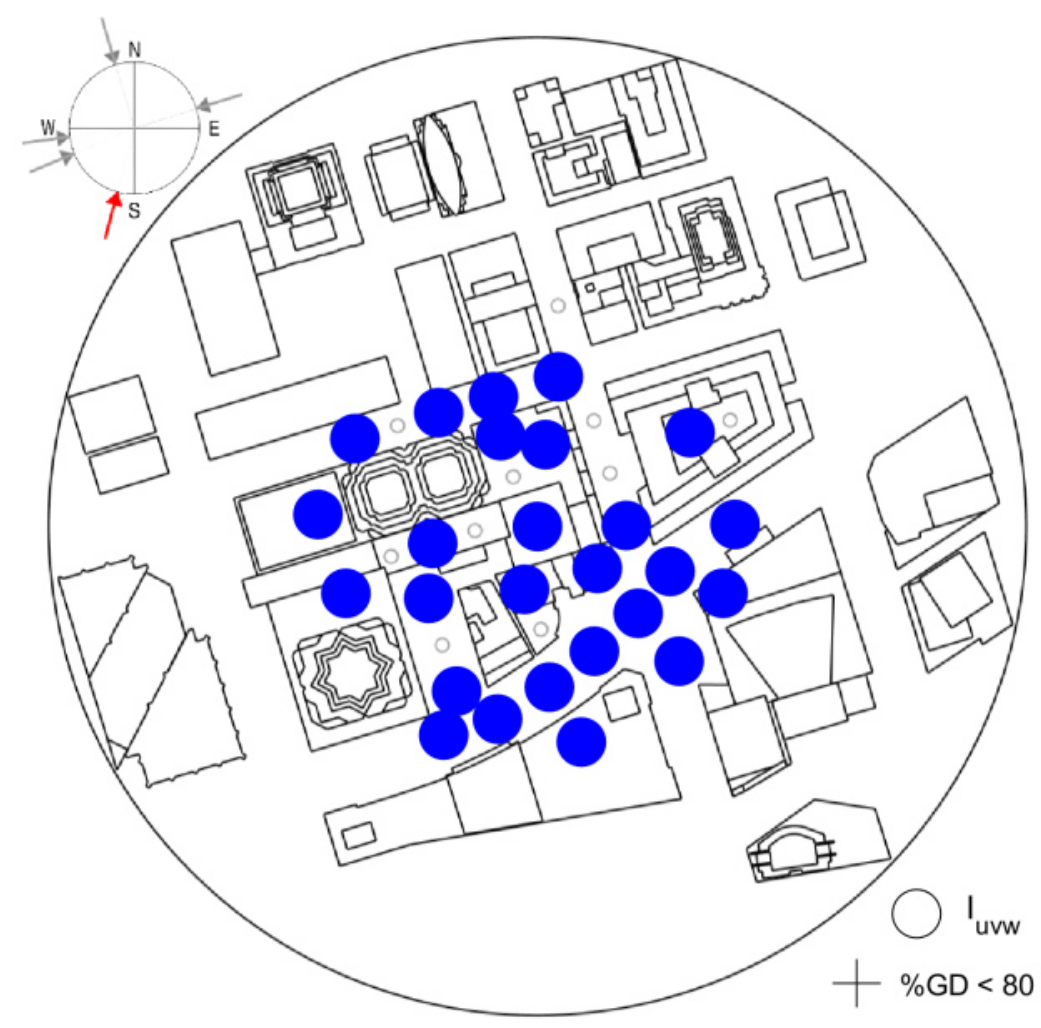

$46<\mathrm{I}_{\mathrm{uvw}} \leq 50$

$40<I_{\text {uvw }} \leq 46$

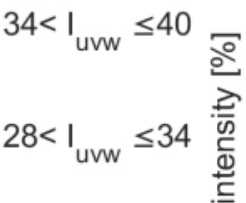

$22<$ I $_{\text {uvw }} \leq 28$

$16<$ I $_{\text {urw }} \leq 22$

$10<1_{\text {uvw }} \leq 16$ ठे

$4<I_{\text {uvw }} \leq 10$

$1 \leq \mathrm{I}_{\mathrm{uvw}} \leq 4$

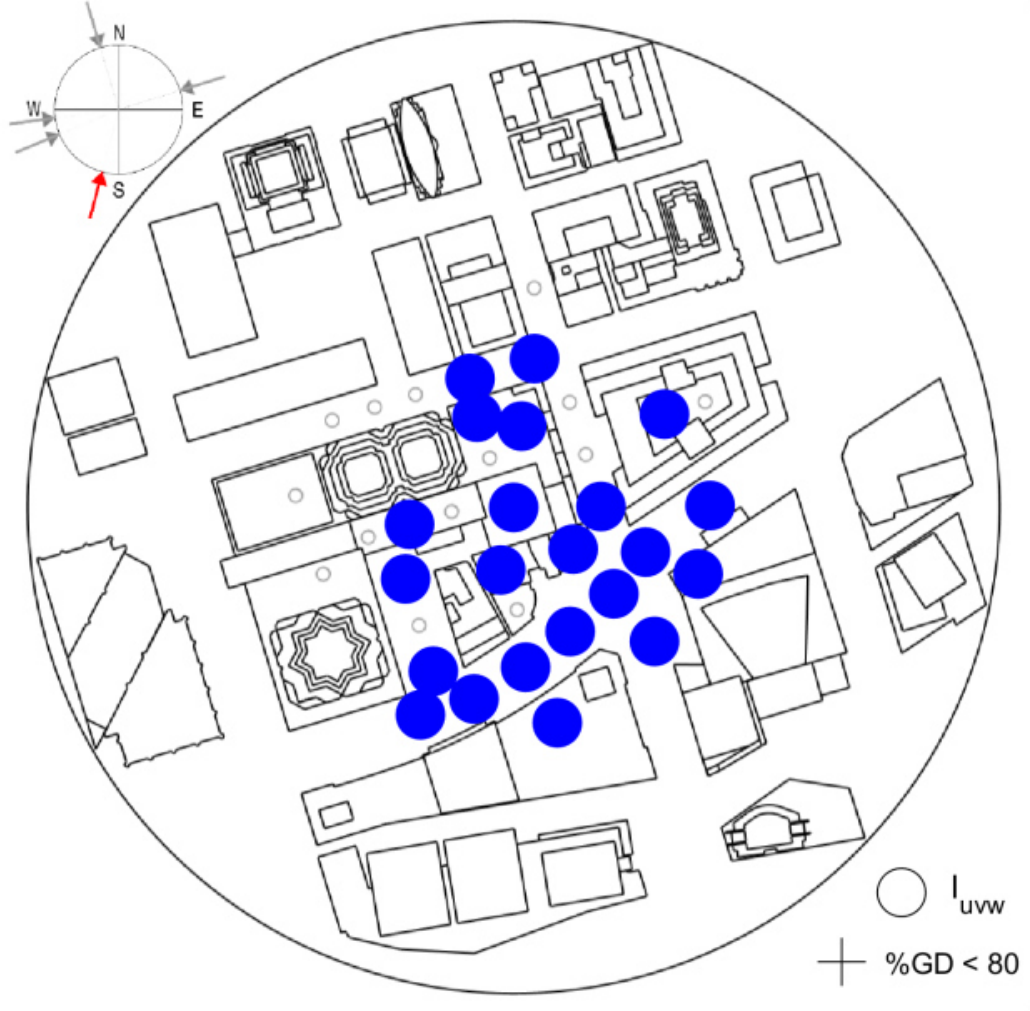

$46<I_{\text {uvw }} \leq 50$

$40<I_{\text {uvw }} \leq 46$

$34<$ I $_{\text {uvw }} \leq 40$

$28<1_{\text {uvw }} \leq 34$

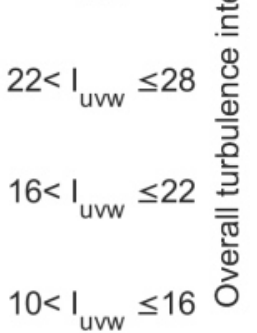

$4<I_{\text {uvw }} \leq 10$

$1 \leq \mathrm{I}_{\text {urw }} \leq 4$

Figure 4.99: Overall turbulence intensity at Increment $15(0.723 \mathrm{~m}$ above wind tunnel floor) without (top) and with (bottom) the future buildings. 


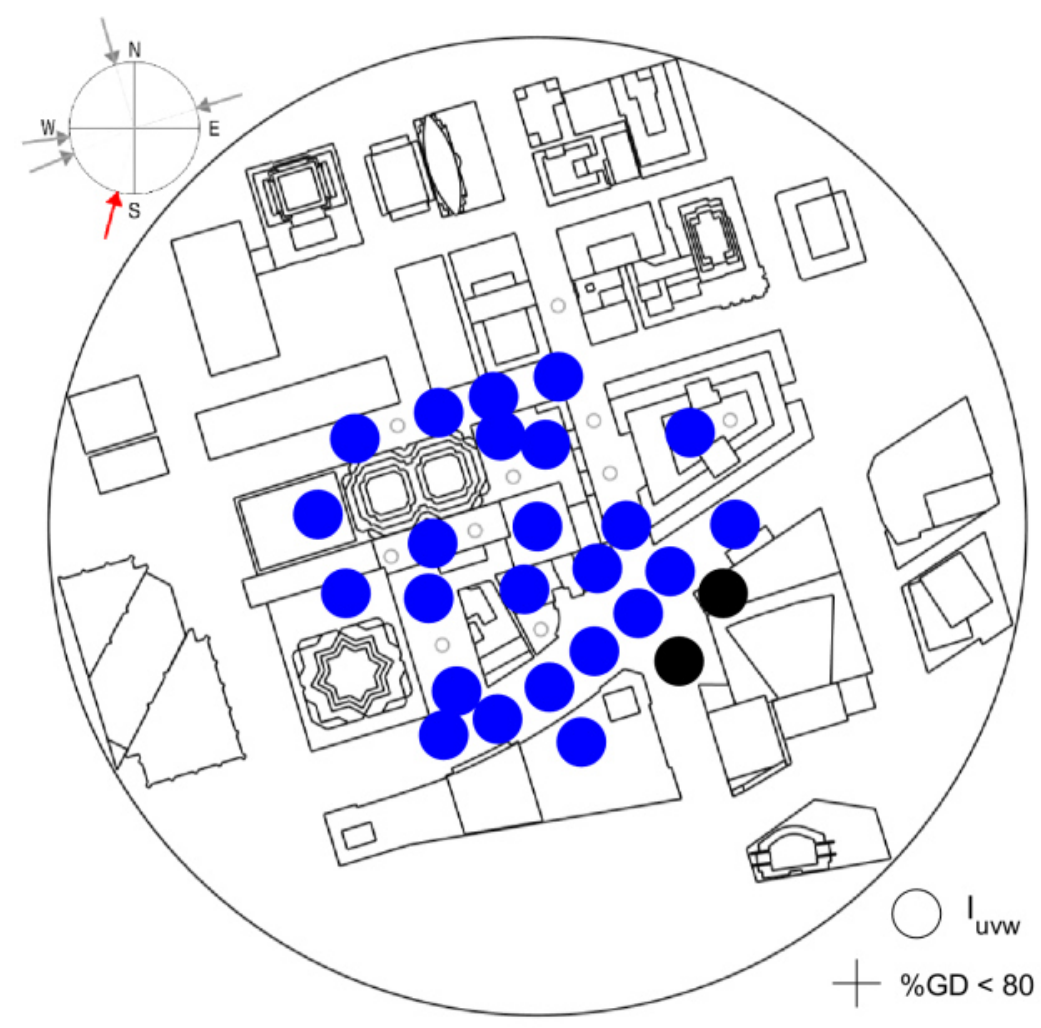

$46<\mathrm{I}_{\mathrm{uvw}} \leq 50$

$40<I_{\text {uvw }} \leq 46$

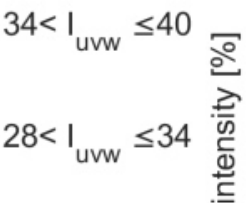

$22<$ I $_{\text {uvw }} \leq 28$

$16<$ I $_{\text {uvw }} \leq 22$

$10<I_{\text {uvw }} \leq 16$ ठे

$4<I_{\text {uvw }} \leq 10$

$1 \leq \mathrm{I}_{\mathrm{uvw}} \leq 4$

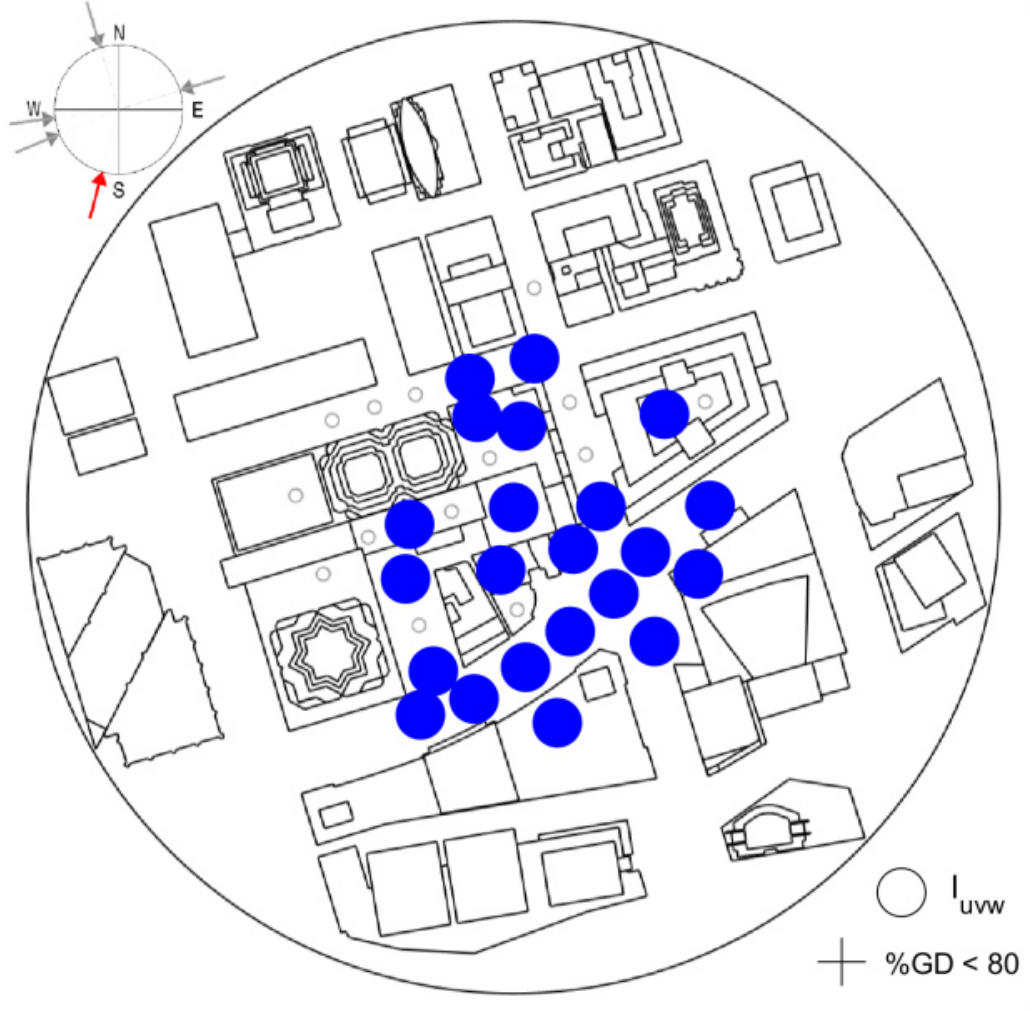

$46<I_{\text {uvw }} \leq 50$

$40<I_{\text {uvw }} \leq 46$

$34<I_{\text {urw }} \leq 40$

$28<$ I $_{\text {uvw }} \leq 34$

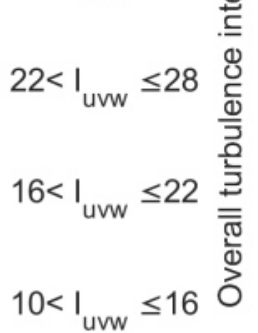

$4<I_{\text {uvw }} \leq 10$

$1 \leq \mathrm{I}_{\text {urw }} \leq 4$

Figure 4.100: Overall turbulence intensity at Increment $16(0.783 \mathrm{~m}$ above wind tunnel floor) without (top) and with (bottom) the future buildings. 


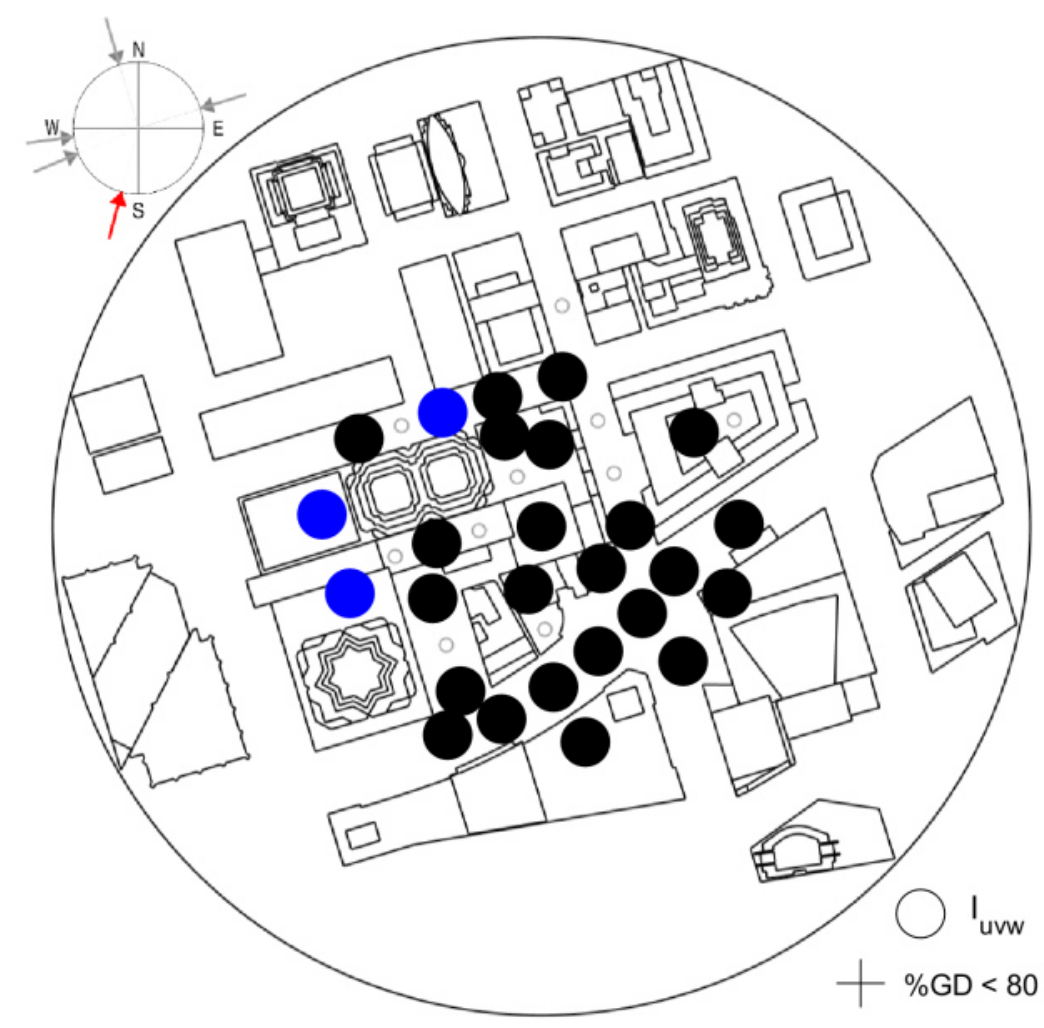

$46<\mathrm{I}_{\mathrm{uvw}} \leq 50$

$40<I_{\text {uvw }} \leq 46$

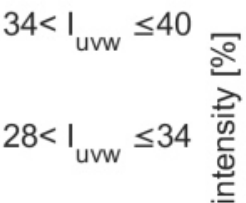

$22<$ I $_{\text {uvw }} \leq 28$

$16<$ I $_{\text {urw }} \leq 22$

$10<I_{\text {uvw }} \leq 16$ ठे

$4<I_{\text {uvw }} \leq 10$

$1 \leq \mathrm{I}_{\mathrm{uvw}} \leq 4$

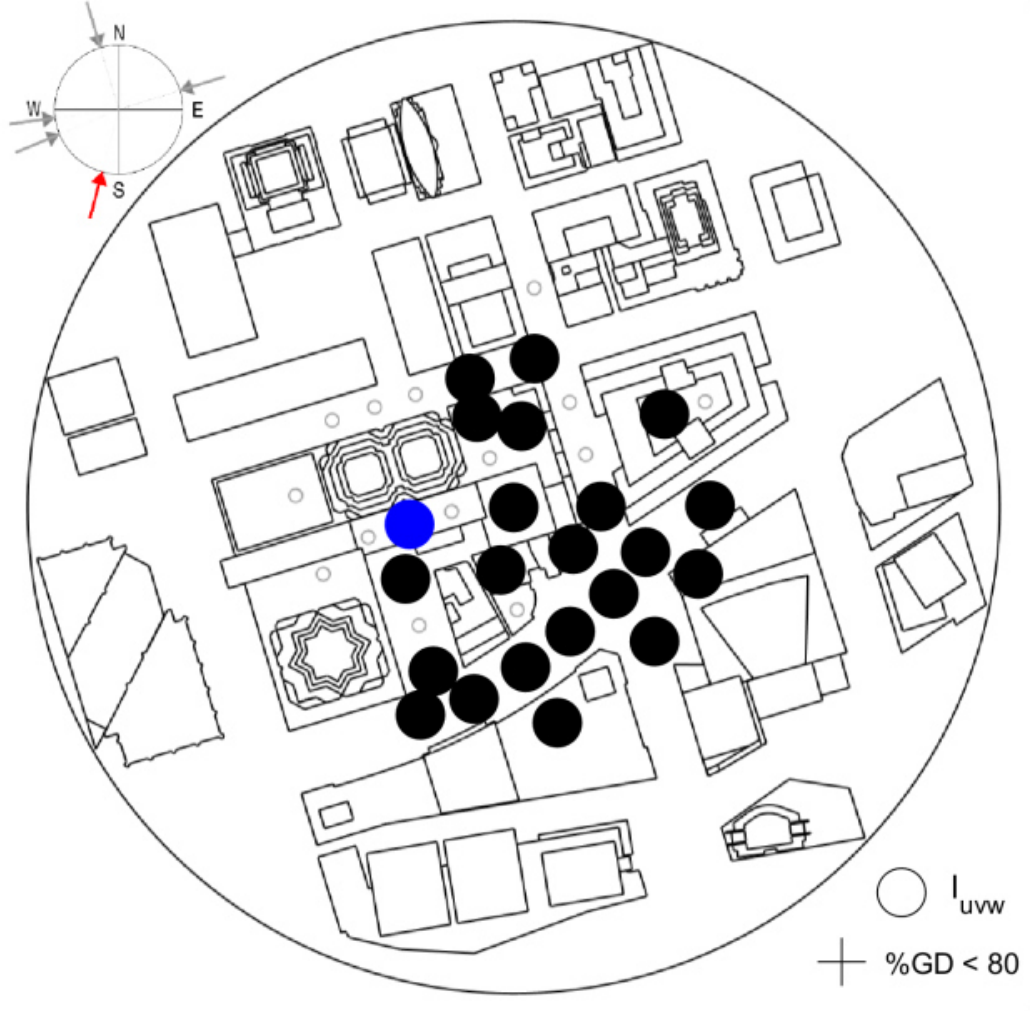

$46<I_{\text {uvw }} \leq 50$

$40<I_{\text {uvw }} \leq 46$

$34<I_{\text {urw }} \leq 40$

$28<$ I $_{\text {uvw }} \leq 34$

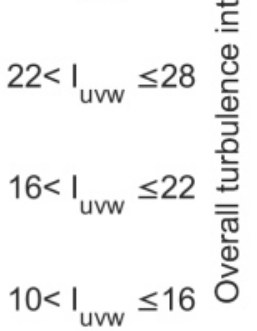

$4<I_{\text {uvw }} \leq 10$

$1 \leq \mathrm{I}_{\text {urw }} \leq 4$

Figure 4.101: Overall turbulence intensity at Increment 17 (0.843 m above wind tunnel floor) without (top) and with (bottom) the future buildings. 


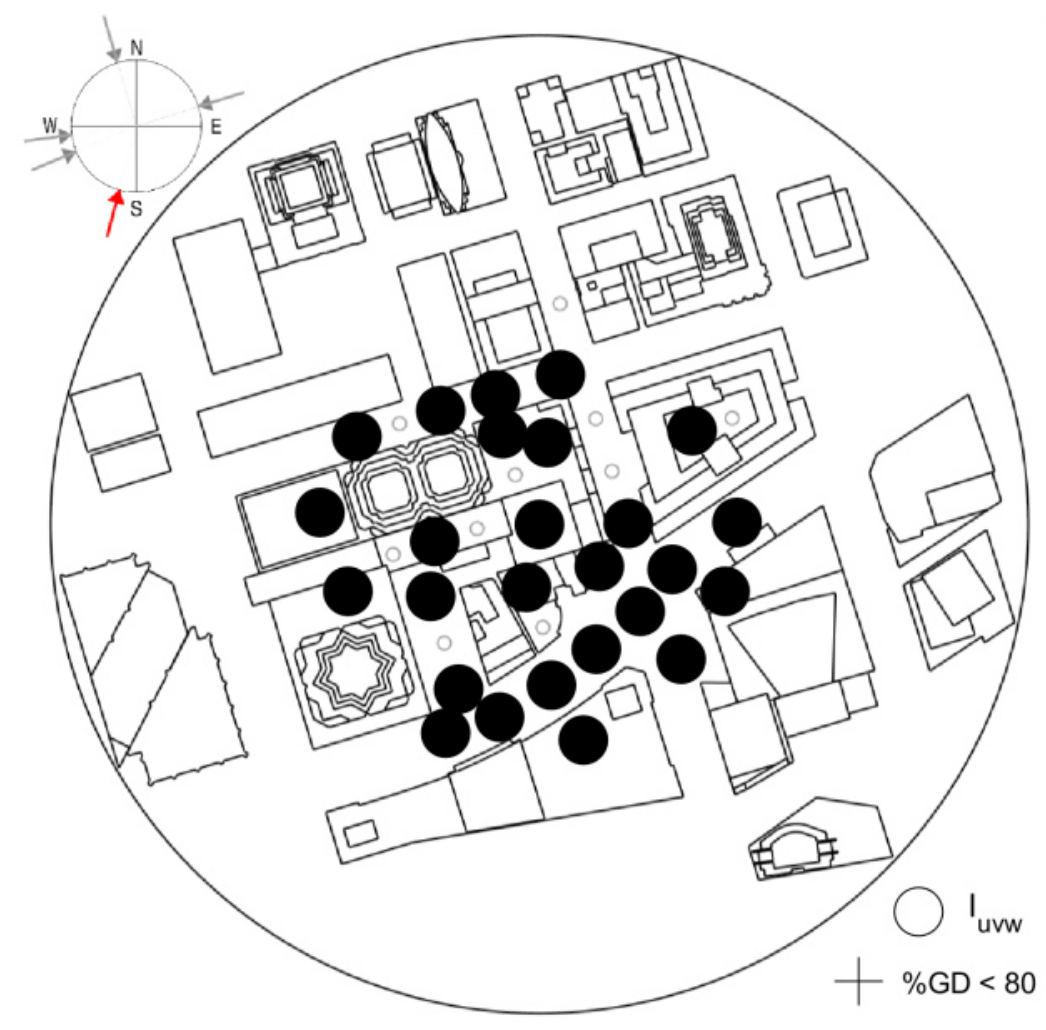

$46<\mathrm{I}_{\mathrm{uvw}} \leq 50$

$40<I_{\text {uvw }} \leq 46$

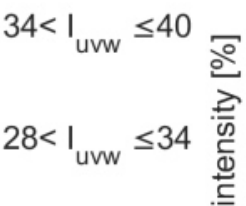

$22<$ I $_{\text {uvw }} \leq 28$

$16<$ I $_{\text {uvw }} \leq 22$

$10<I_{\text {uvw }} \leq 16$ ठे

$4<I_{\text {uvw }} \leq 10$

$1 \leq \mathrm{I}_{\mathrm{uvw}} \leq 4$

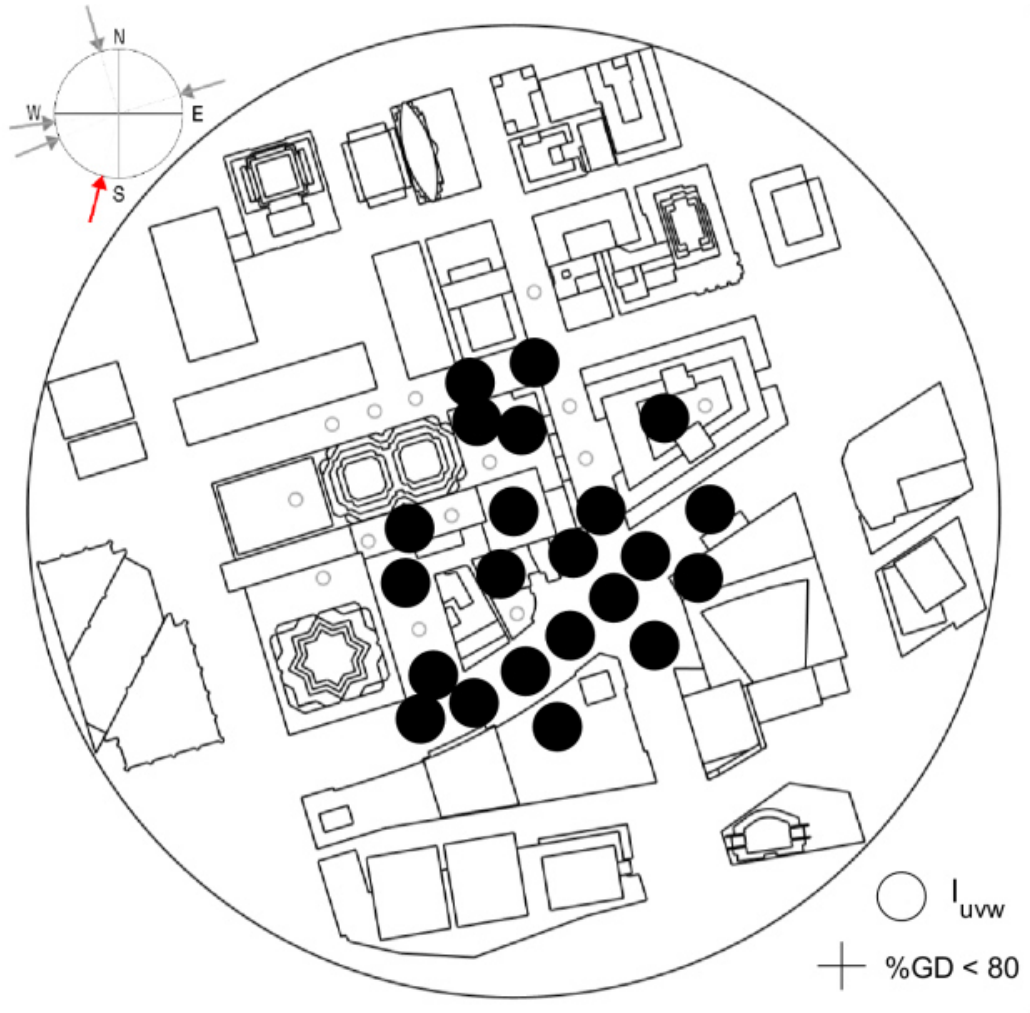

$46<I_{\text {uvw }} \leq 50$

$40<I_{\text {uvw }} \leq 46$

$34<I_{\text {urw }} \leq 40$

$28<1_{\text {uvw }} \leq 34$

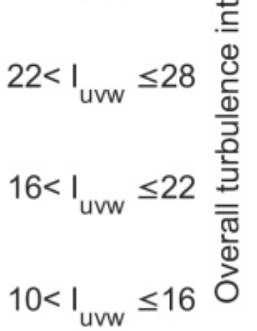

$4<I_{\text {uvw }} \leq 10$

$1 \leq \mathrm{I}_{\text {urw }} \leq 4$

Figure 4.102: Overall turbulence intensity at Increment 18 (0.903 m above wind tunnel floor) without (top) and with (bottom) the future buildings. 


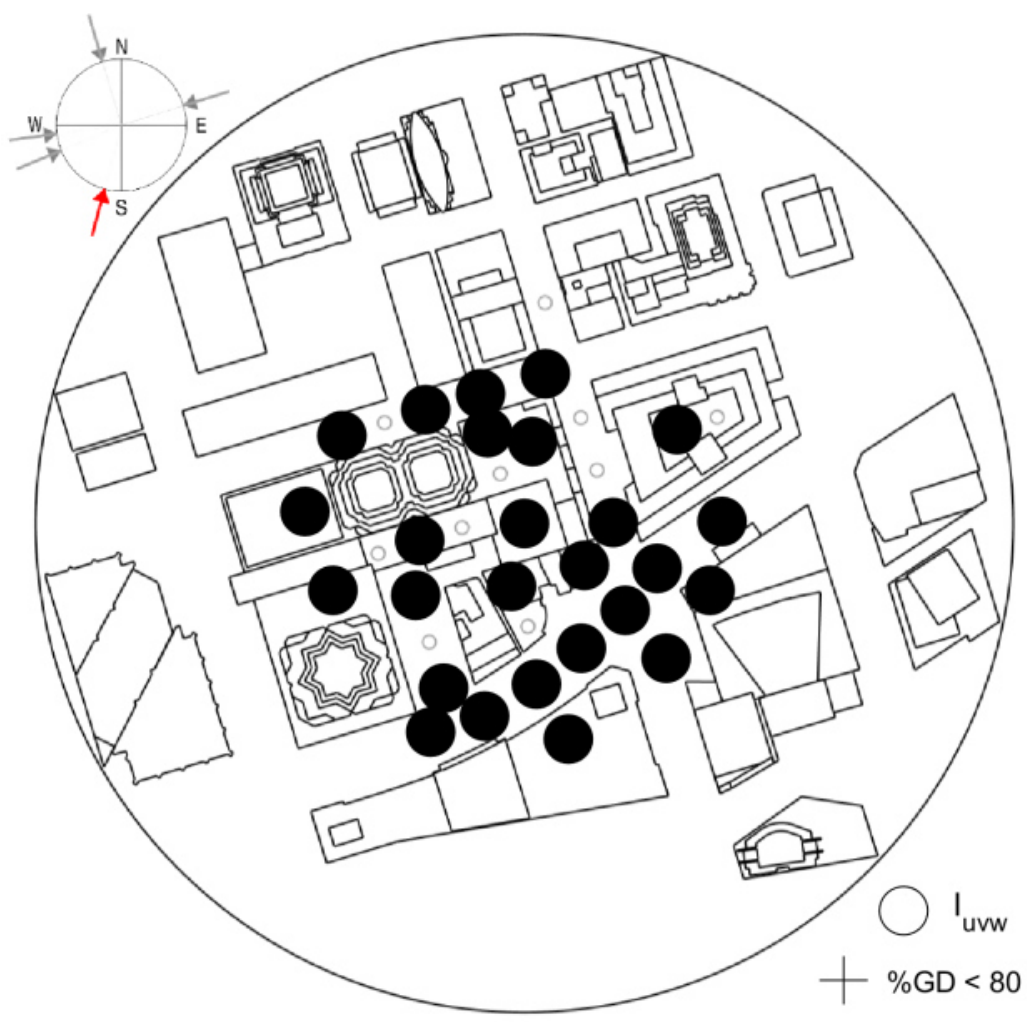

$46<\mathrm{I}_{\mathrm{uvw}} \leq 50$

$40<I_{\text {uvw }} \leq 46$

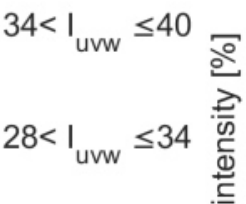

$22<$ I $_{\text {uvw }} \leq 28$

$16<$ I $_{\text {urw }} \leq 22$

$10<I_{\text {uvw }} \leq 16$ ठे

$4<I_{\text {uvw }} \leq 10$

$1 \leq \mathrm{I}_{\mathrm{uvw}} \leq 4$

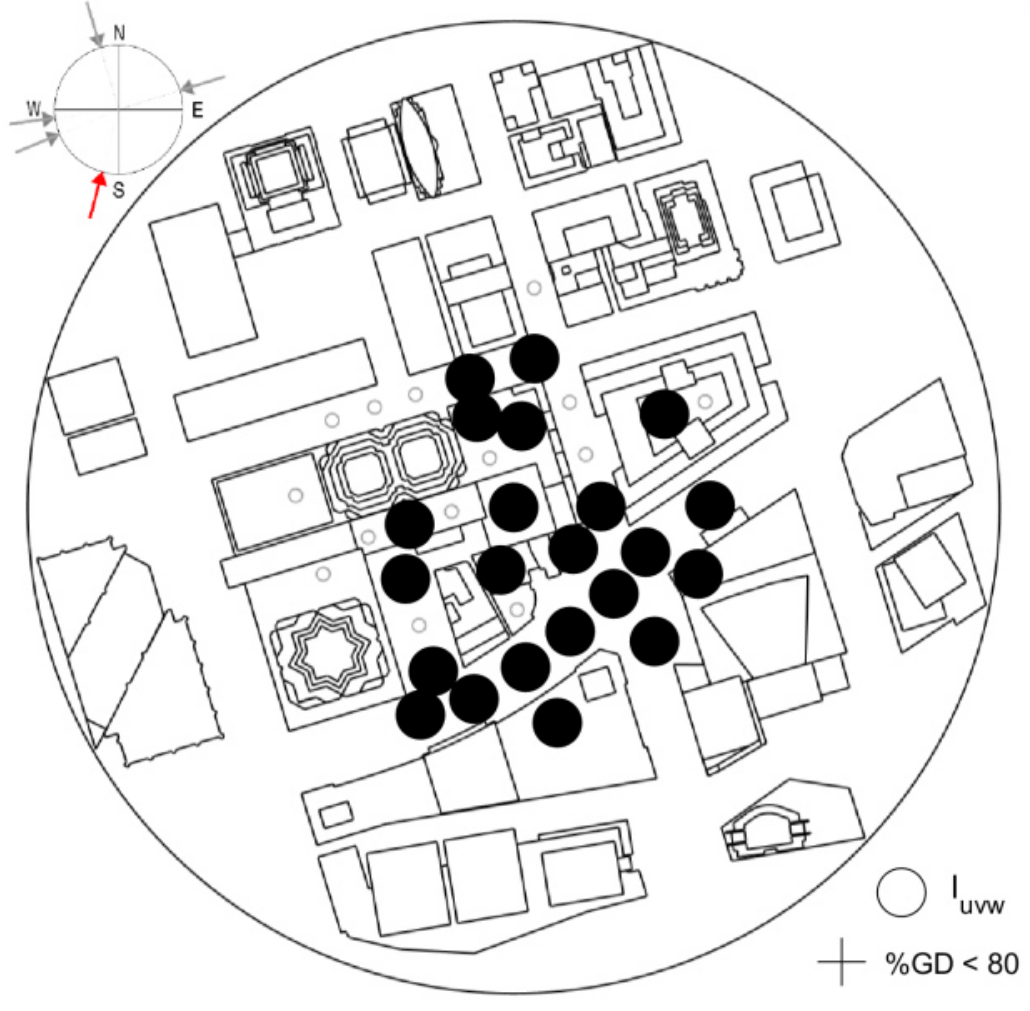

$46<I_{\text {uvw }} \leq 50$

$40<I_{\text {uvw }} \leq 46$

$34<I_{\text {urw }} \leq 40$

$28<1_{\text {uvw }} \leq 34$

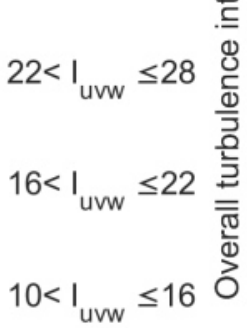

$4<I_{\text {uvw }} \leq 10$

$1 \leq \mathrm{I}_{\text {urw }} \leq 4$

Figure 4.103: Overall turbulence intensity at Increment 19 (0.963 m above wind tunnel floor) without (top) and with (bottom) the future buildings. 


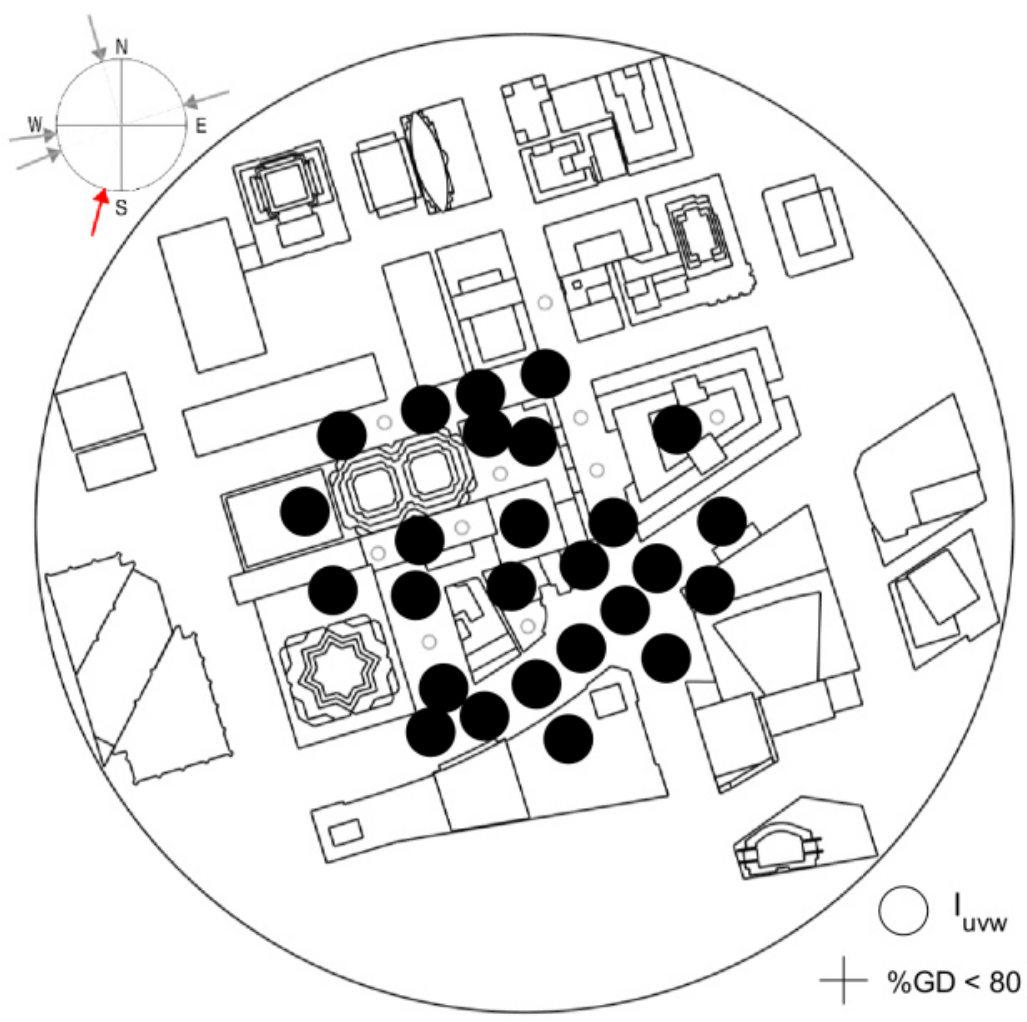

$46<\mathrm{I}_{\mathrm{uvw}} \leq 50$

$40<I_{\text {uvw }} \leq 46$

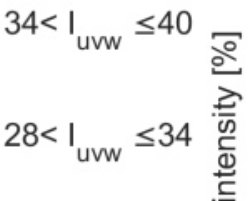

$22<$ I $_{\text {uvw }} \leq 28$

$16<\mathrm{I}_{\mathrm{uvw}} \leq 22$

$10<1_{\text {uvw }} \leq 16$ ठे

$4<I_{\text {uvw }} \leq 10$

$1 \leq \mathrm{I}_{\mathrm{uvw}} \leq 4$

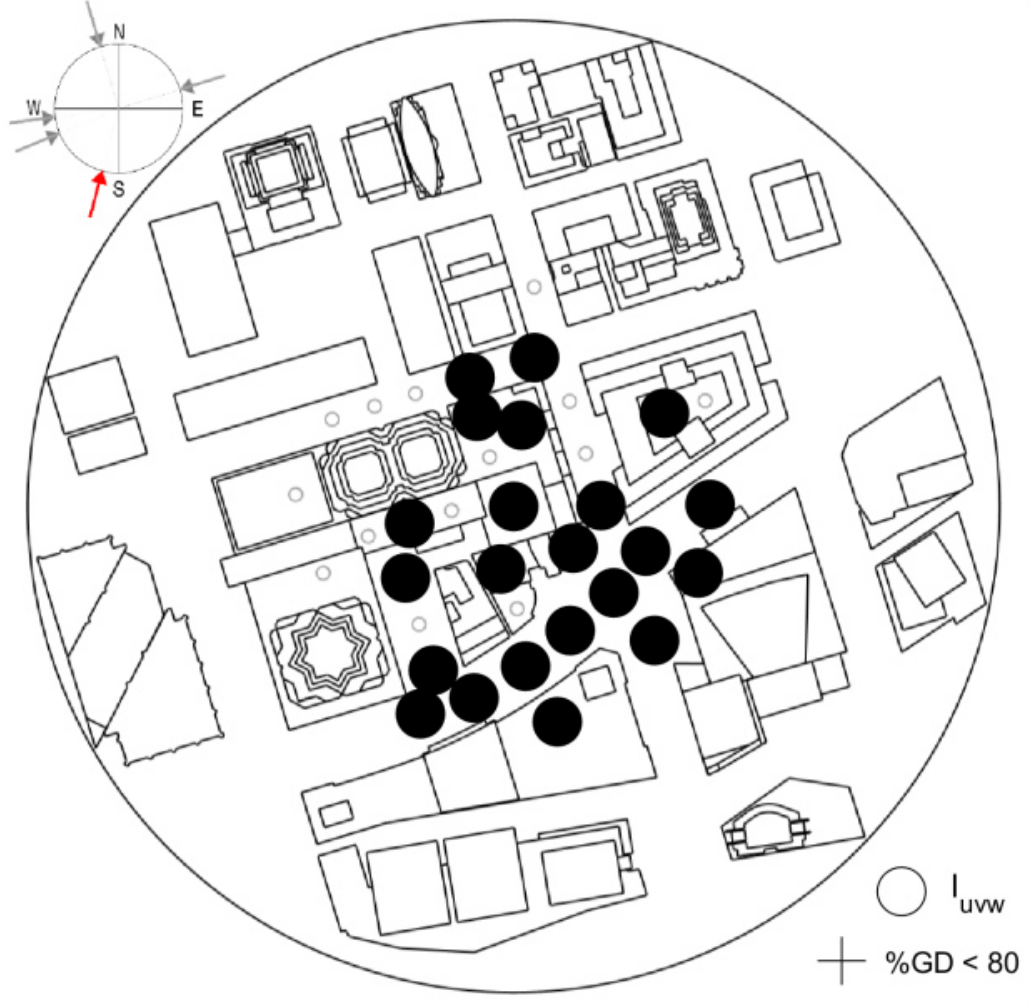

$46<I_{\text {uvw }} \leq 50$

$40<\mathrm{I}_{\text {unw }} \leq 46$

$34<$ I $_{\text {uvw }} \leq 40$

$28<1_{\text {uvw }} \leq 34$

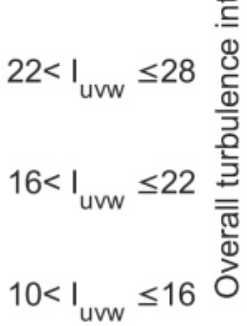

$4<I_{\text {uvw }} \leq 10$

$1 \leq \mathrm{I}_{\text {urw }} \leq 4$

Figure 4.104: Overall turbulence intensity at Increment $20(1.023 \mathrm{~m}$ above wind tunnel floor) without (top) and with (bottom) the future buildings. 
The effect of adding the future buildings on the mean wind speed was also investigated. Figure 4.105 shows the locations of 4 measuring spots that range in proximity to the future buildings from being close to being distant. Measurements of mean wind speed profiles with and without the future buildings at each of the spots are plotted in Figure 4.106. The addition of the future buildings caused considerable velocity deficit even for the distant spots. This suggests that the spots are located in the main wake region of the future buildings, which is characterized by a flow momentum deficit that decays with distance $[12$.

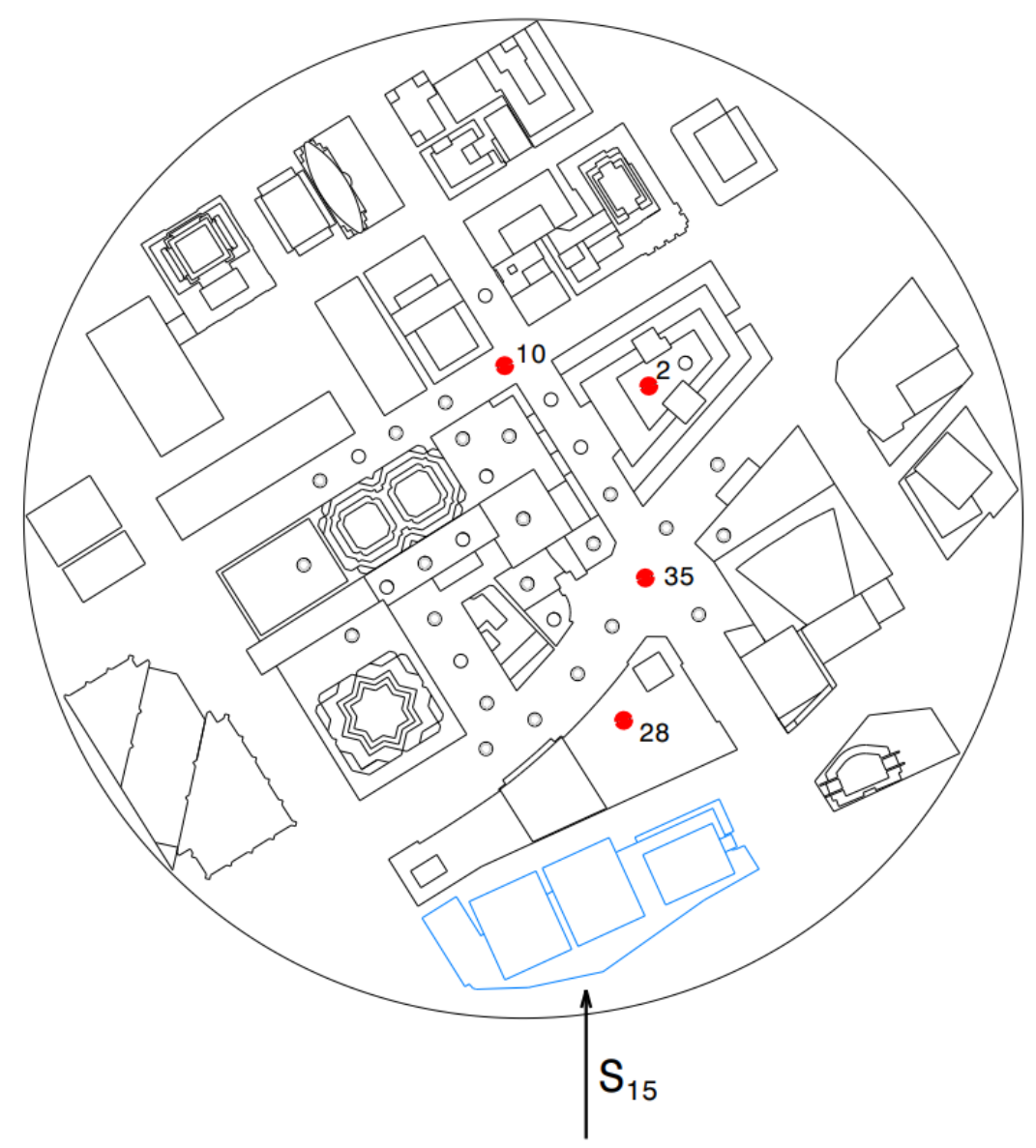

Figure 4.105: Locations of Spots 2, 10, 28 and 35 with a model orientation representing $\mathrm{S}_{15}$ wind direction. 


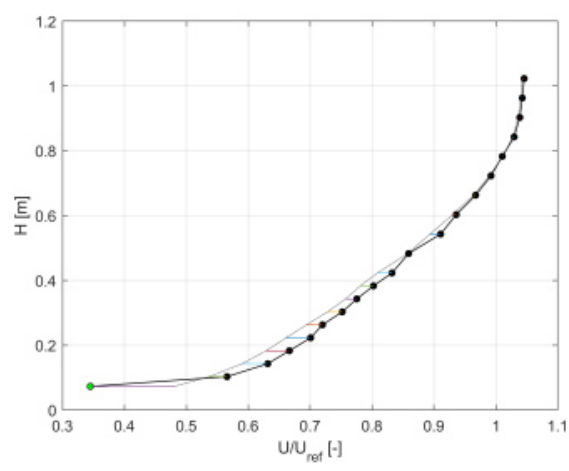

(a) Spot 28: without FB

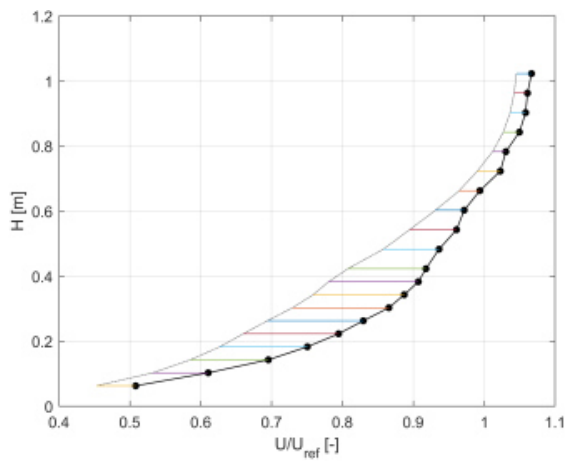

(c) Spot 35: without FB

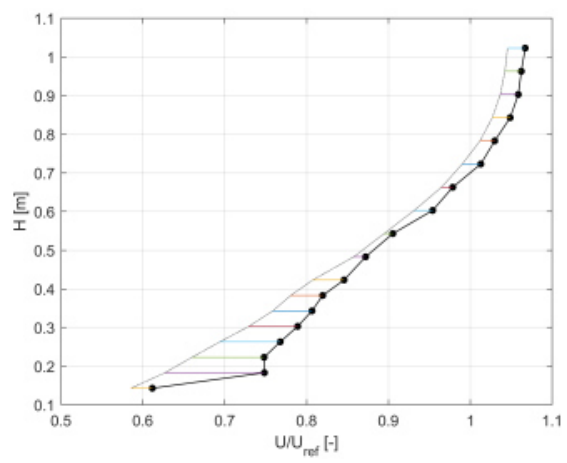

(e) Spot 2: without FB

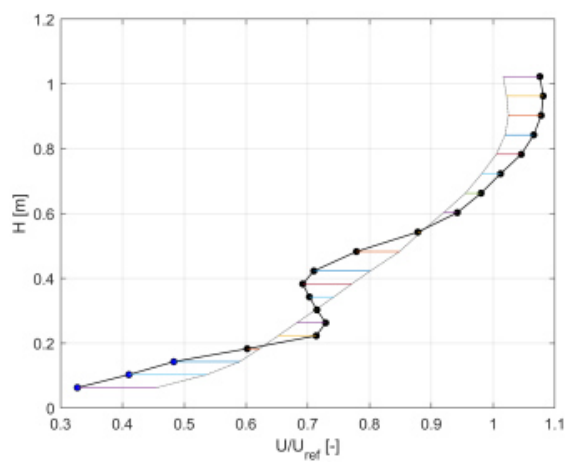

(g) Spot 10: without FB

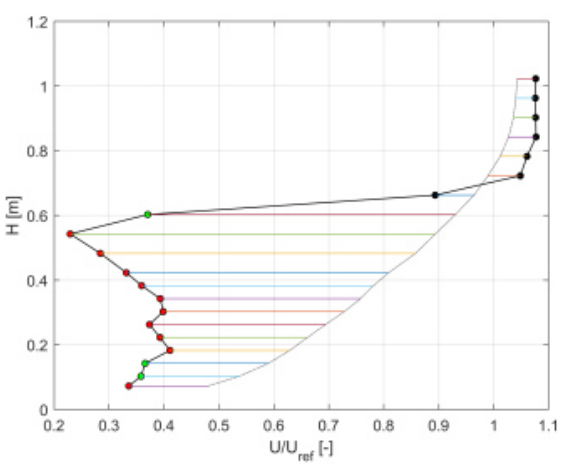

(b) Spot 28: with FB

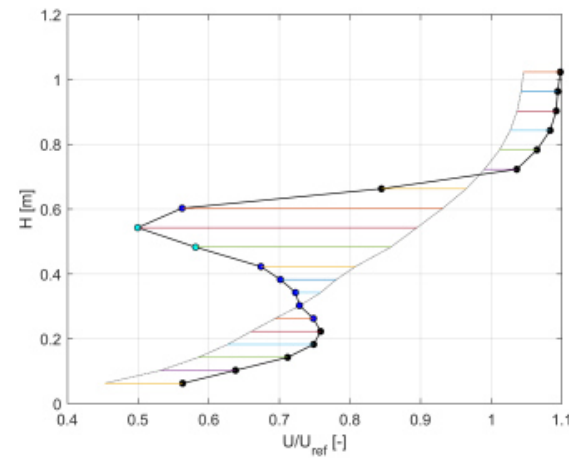

(d) Spot 35: with FB

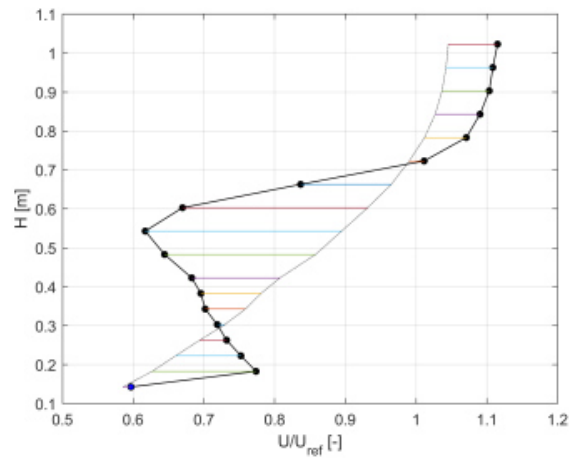

(f) Spot 2: with FB

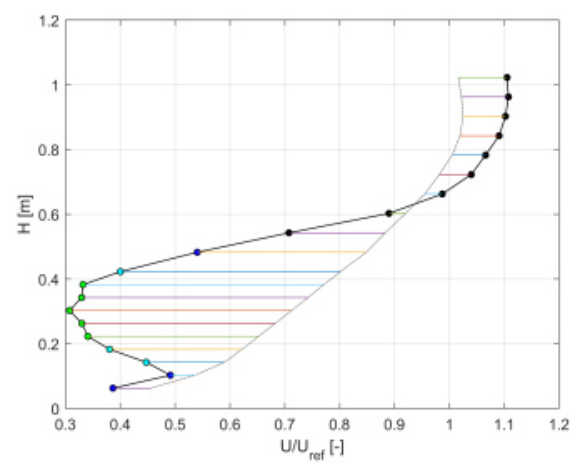

(h) Spot 10: with FB

Figure 4.106: Mean wind speed profiles for Spots 28, 35, 2 and 10 with and without the future buildings. Total increments $=20$. 
Changes on the wind direction due to the future buildings are presented visually in Figures 4.107 through 4.127. The figures follow the same general format as the preceding ones; however, each of the figures shows the changes in horizontal flow direction (yaw), updraft and downdraft collectively. Large circles were used to represent the horizontal flow direction (yaw), small circles were used to represent the updraft (up) and small squares were used to represent the downdraft (down). The colour of the symbols follows the defined ranges in the provided colour bar. Similar conclusions can be drawn for the effect of adding the future buildings on the flow angles, where changes in airflow angles were observed in multiple spots including those that are distant the most from the future buildings. Additionally, shift in the flow vertical direction from being upward to being downward (or vice versa) was also observed. Clearly, the addition of the future buildings caused notable changes in the flow field. 

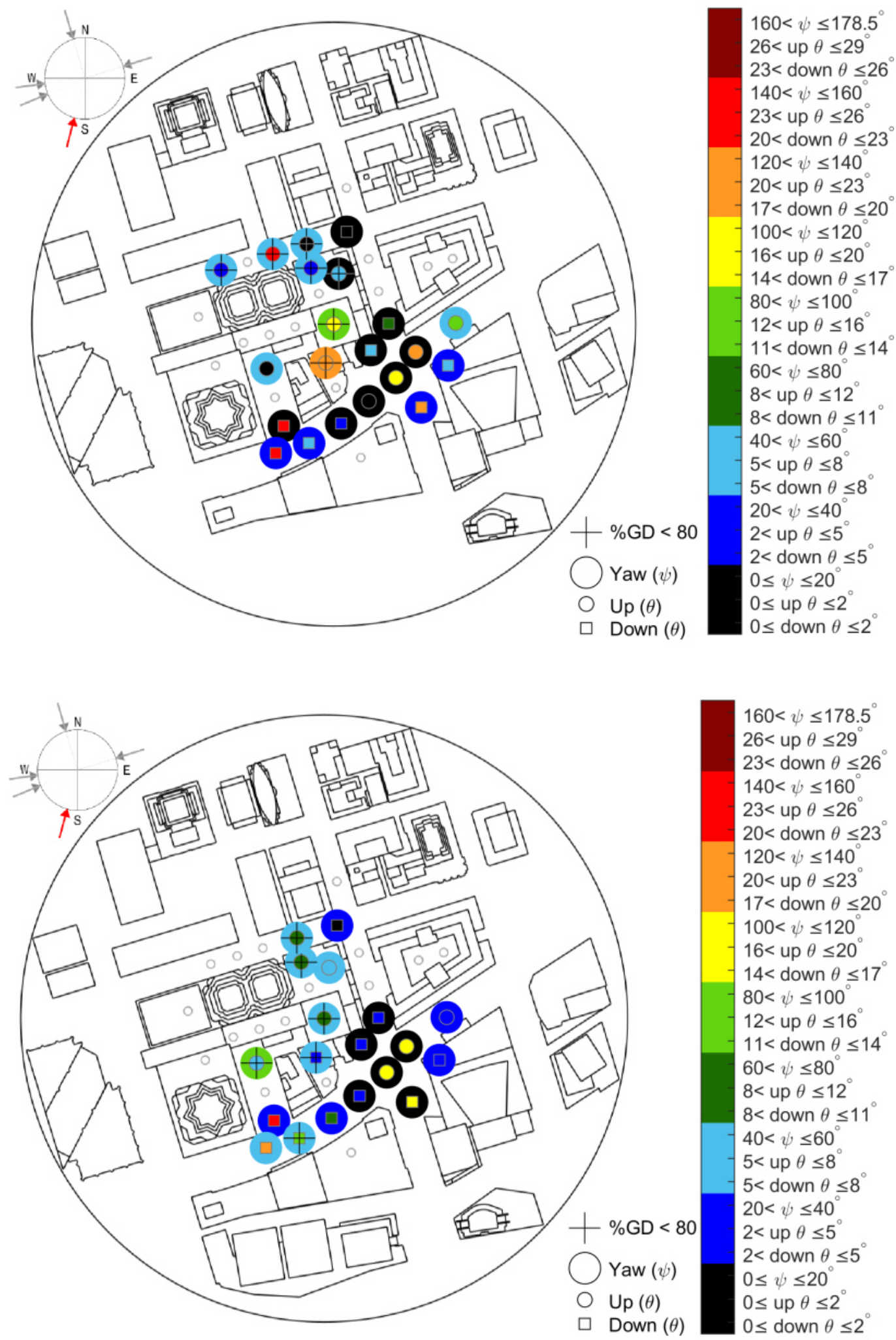

Figure 4.107: Flow angles at Increment 1 (0.063 m above wind tunnel floor) without (top) and with (bottom) the future buildings. 

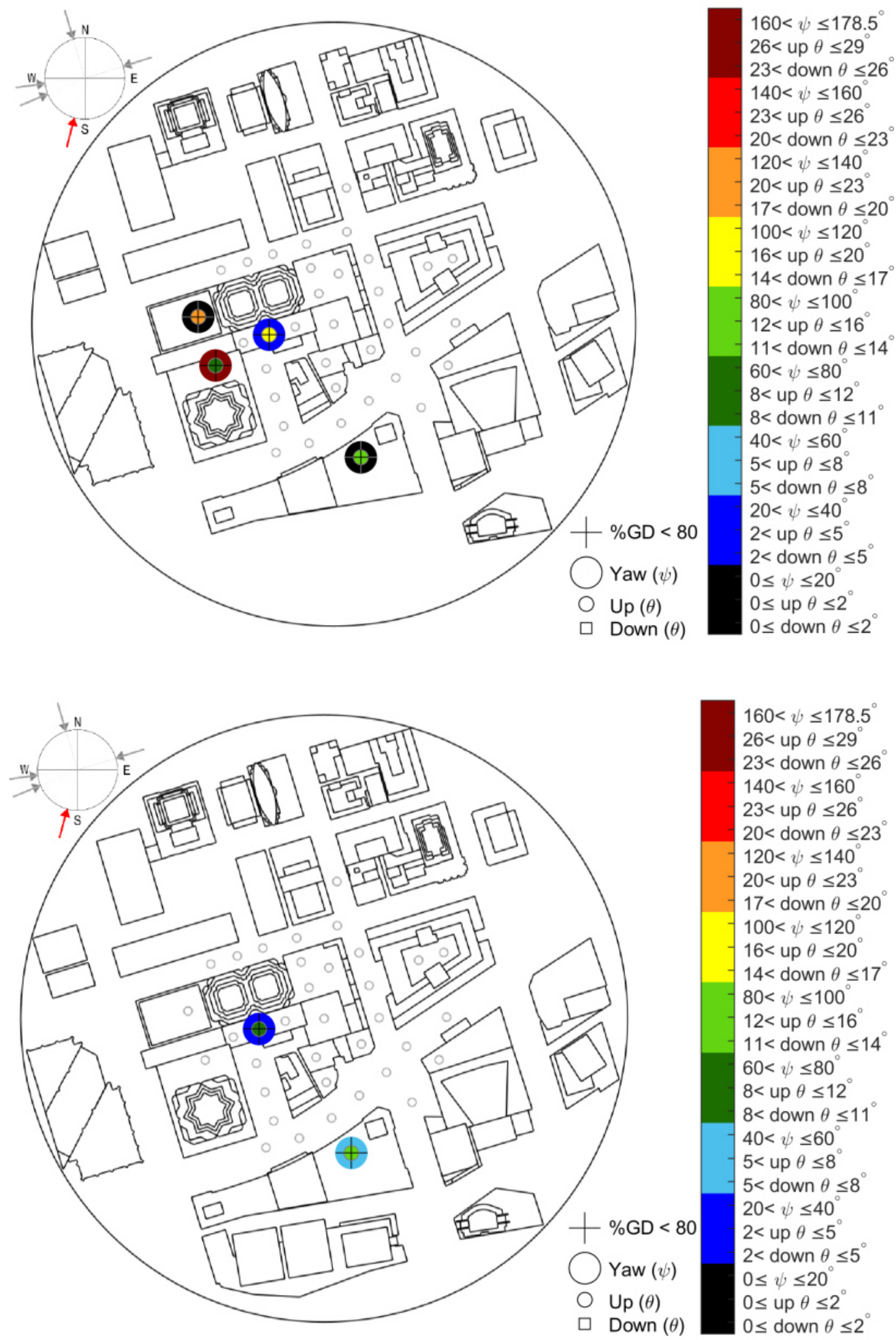

Figure 4.108: Flow angles at Increment 1 (0.073 $\mathrm{m}$ above wind tunnel floor) without (top) and with (bottom) the future buildings. 

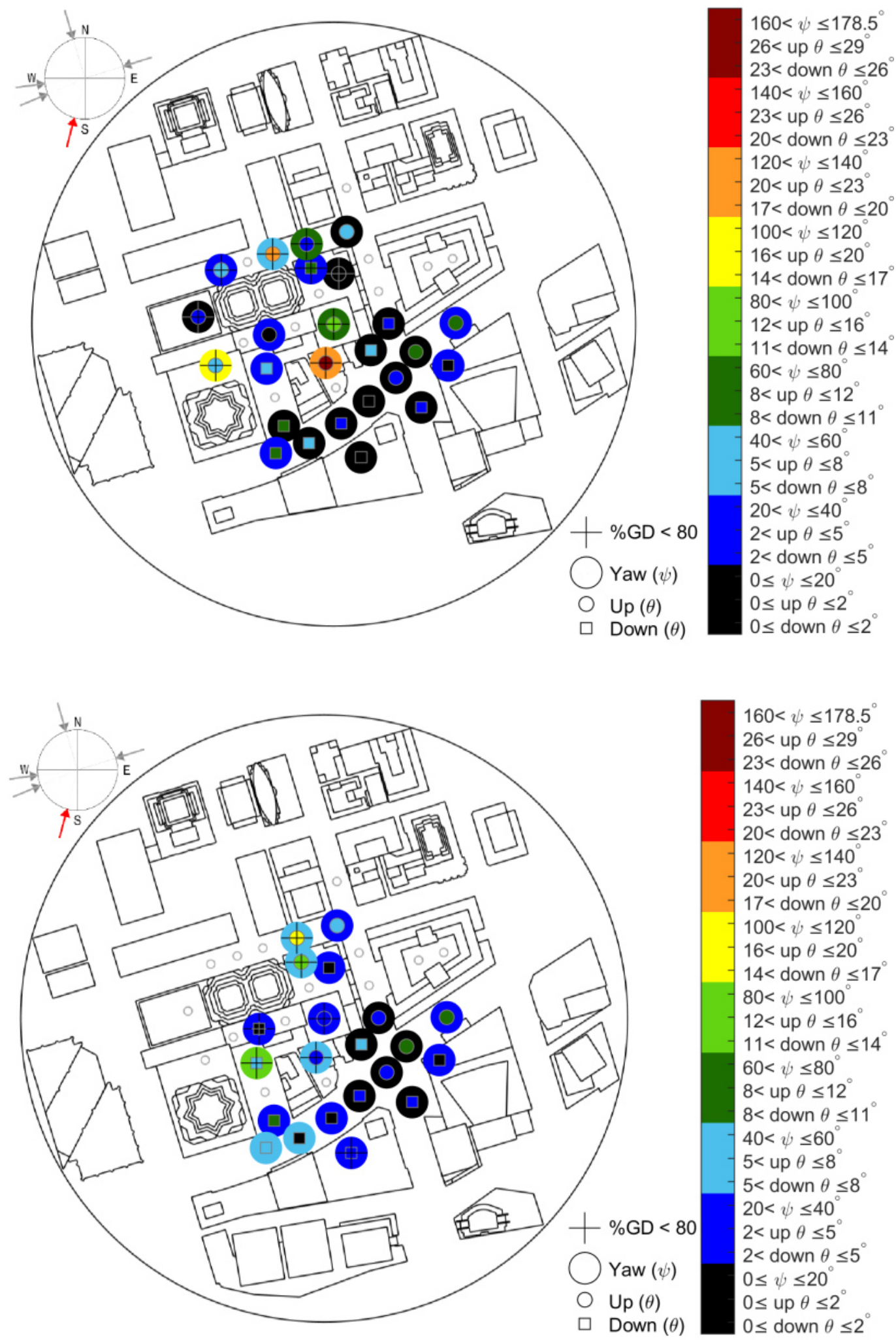

Figure 4.109: Flow angles at Increment 2 (0.103 $\mathrm{m}$ above wind tunnel floor) without (top) and with (bottom) the future buildings. 

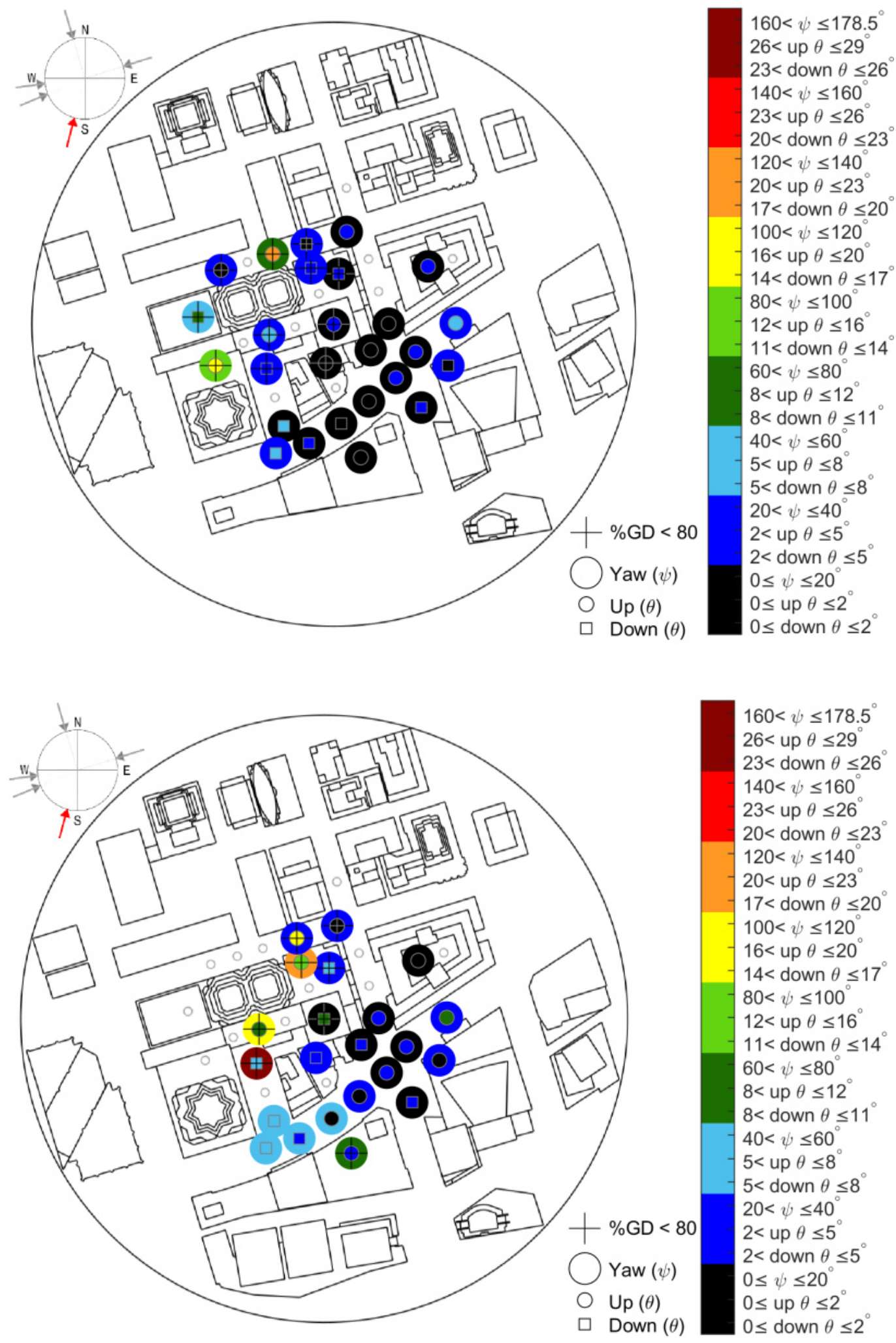

Figure 4.110: Flow angles at Increment 3 (0.143 $\mathrm{m}$ above wind tunnel floor) without (top) and with (bottom) the future buildings. 

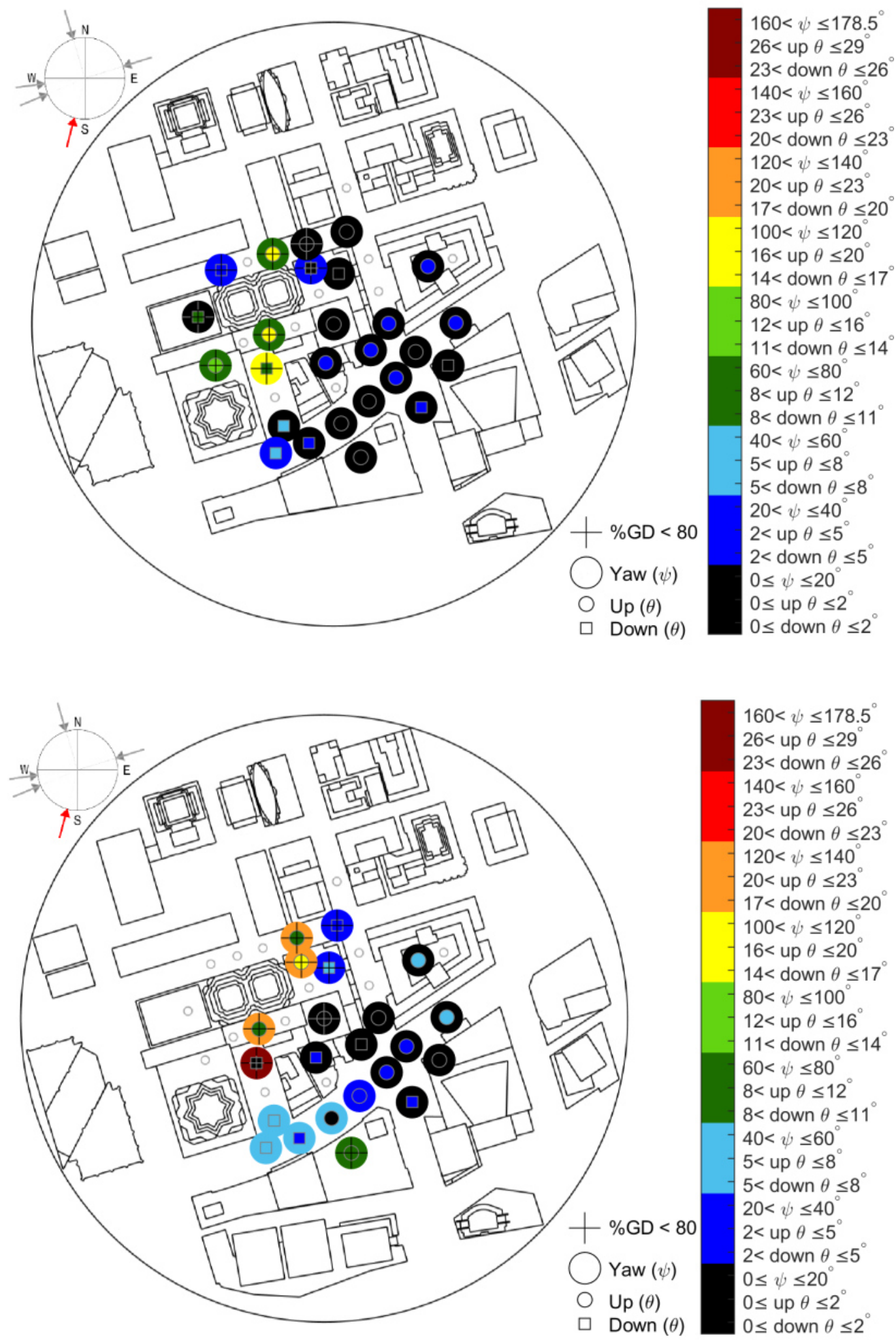

Figure 4.111: Flow angles at Increment 4 (0.183 $\mathrm{m}$ above wind tunnel floor) without (top) and with (bottom) the future buildings. 

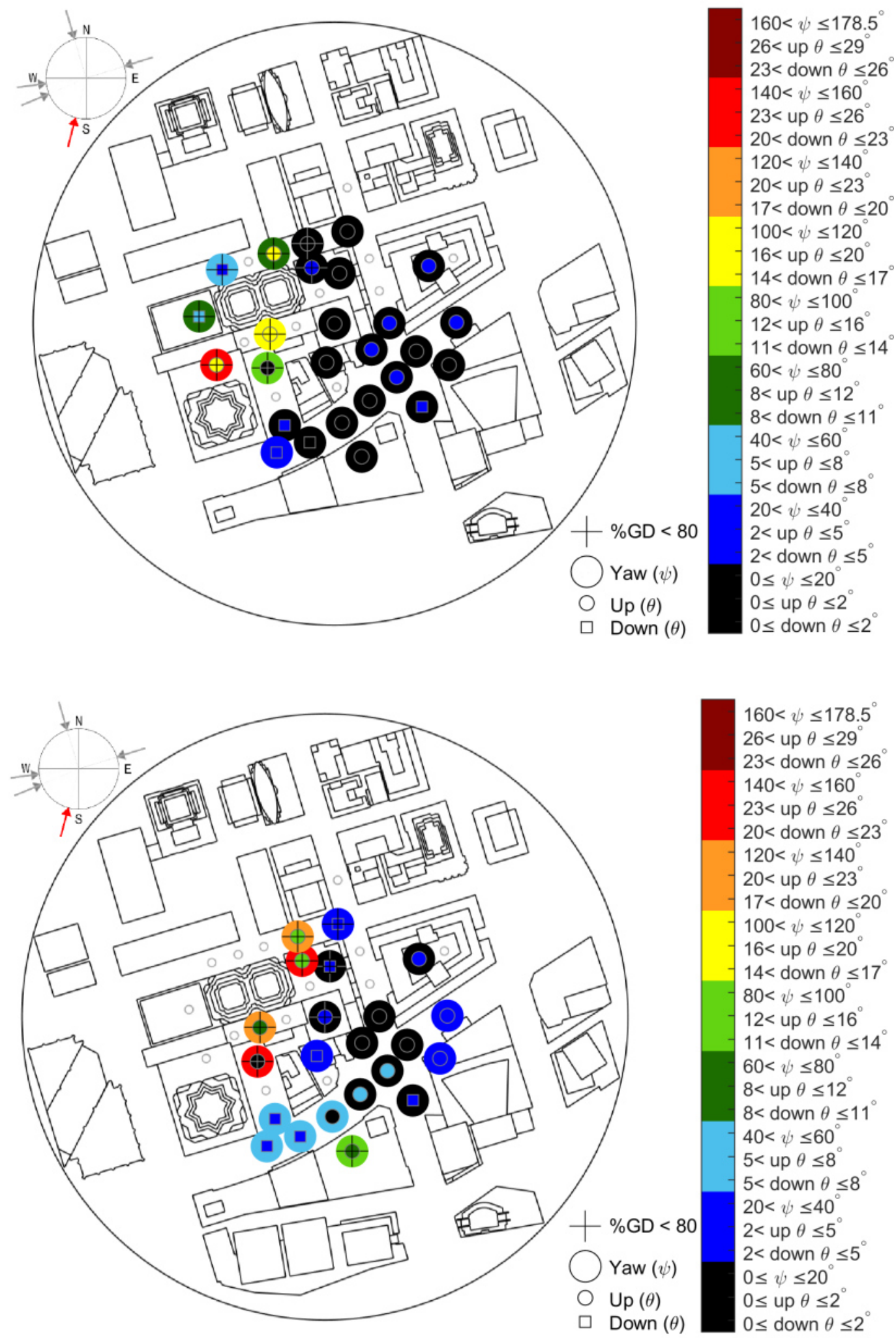

Figure 4.112: Flow angles at Increment 5 (0.223 $\mathrm{m}$ above wind tunnel floor) without (top) and with (bottom) the future buildings. 

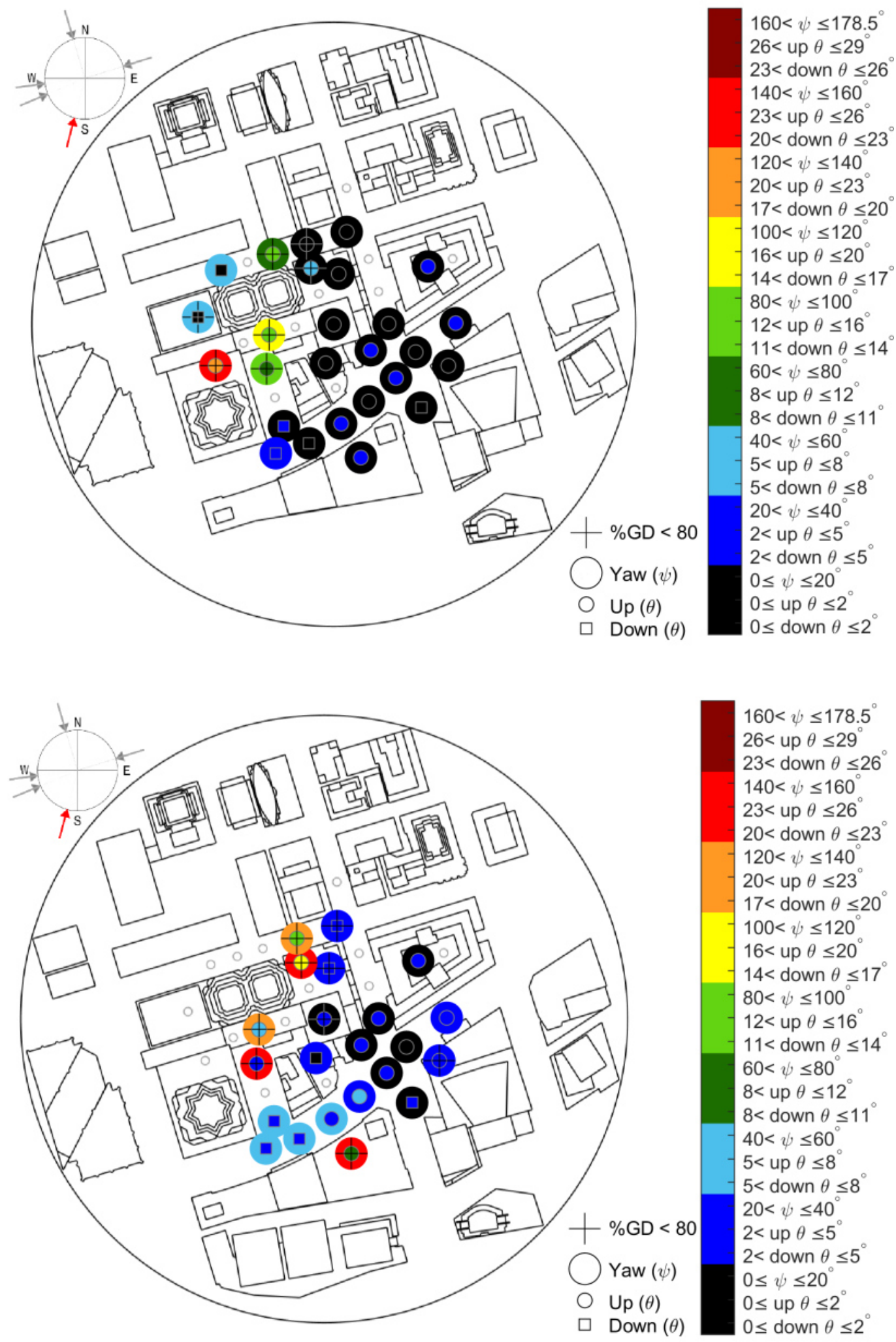

Figure 4.113: Flow angles at Increment 6 (0.263 $\mathrm{m}$ above wind tunnel floor) without (top) and with (bottom) the future buildings. 

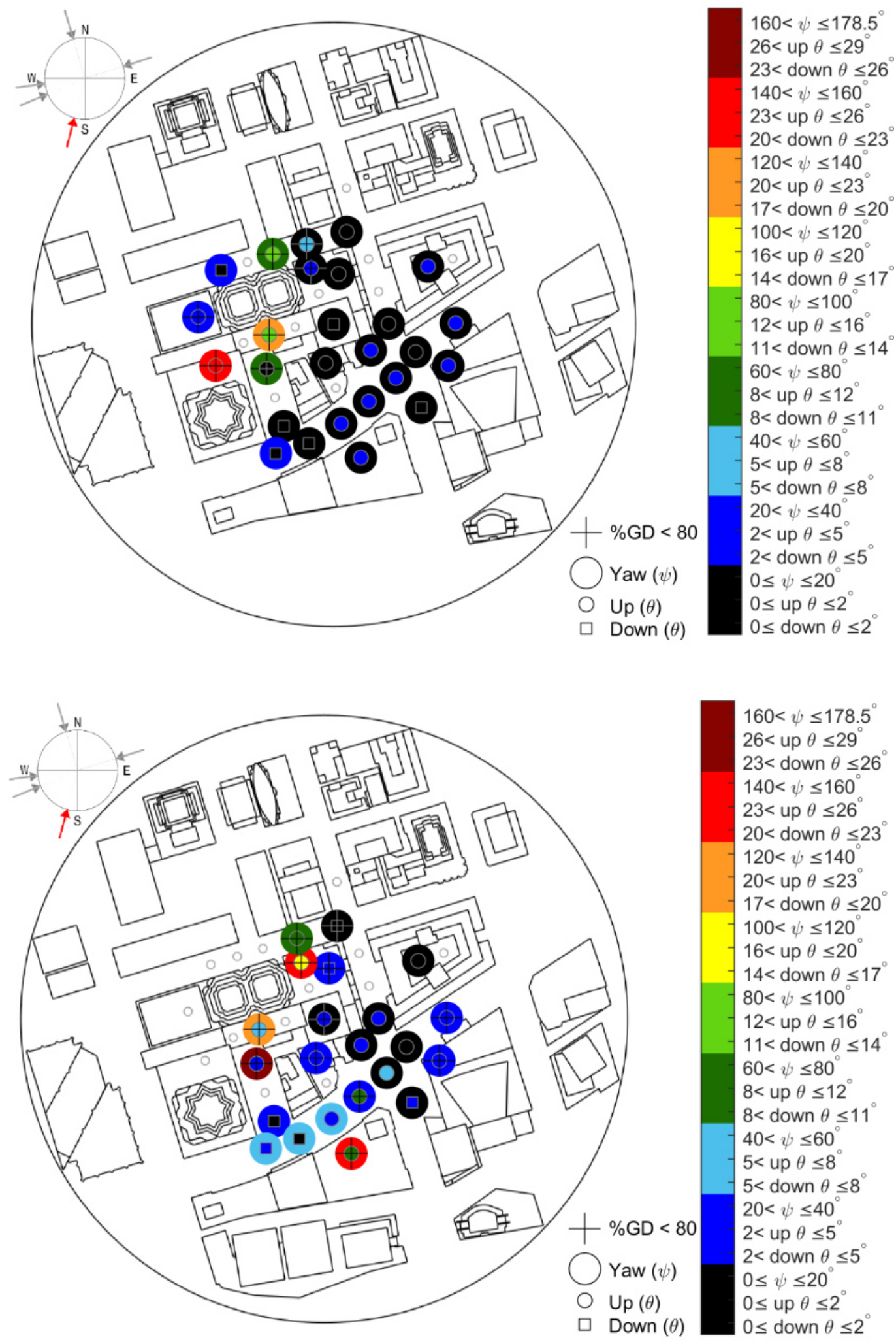

Figure 4.114: Flow angles at Increment 7 (0.303 $\mathrm{m}$ above wind tunnel floor) without (top) and with (bottom) the future buildings. 

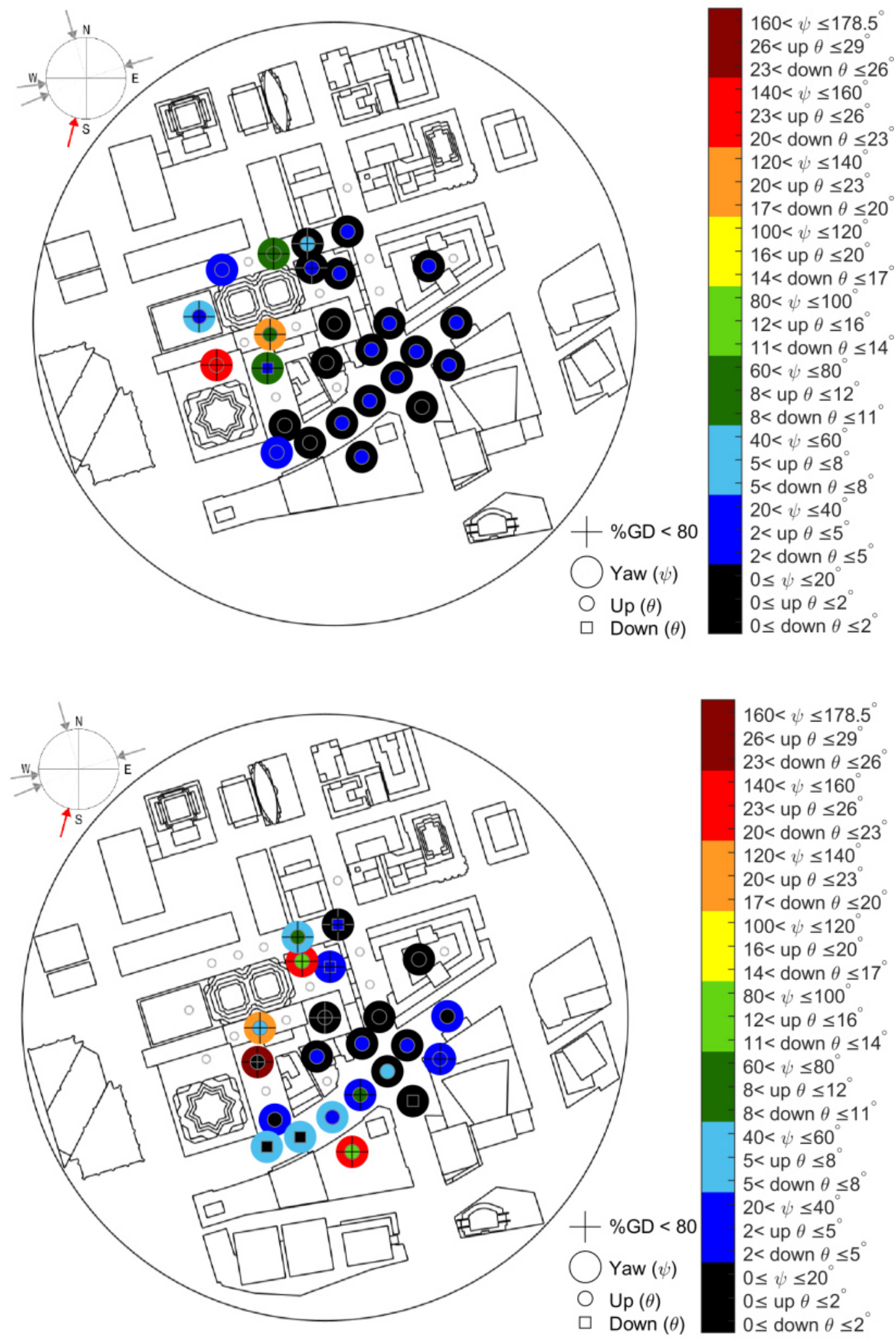

Figure 4.115: Flow angles at Increment 8 (0.343 $\mathrm{m}$ above wind tunnel floor) without (top) and with (bottom) the future buildings. 

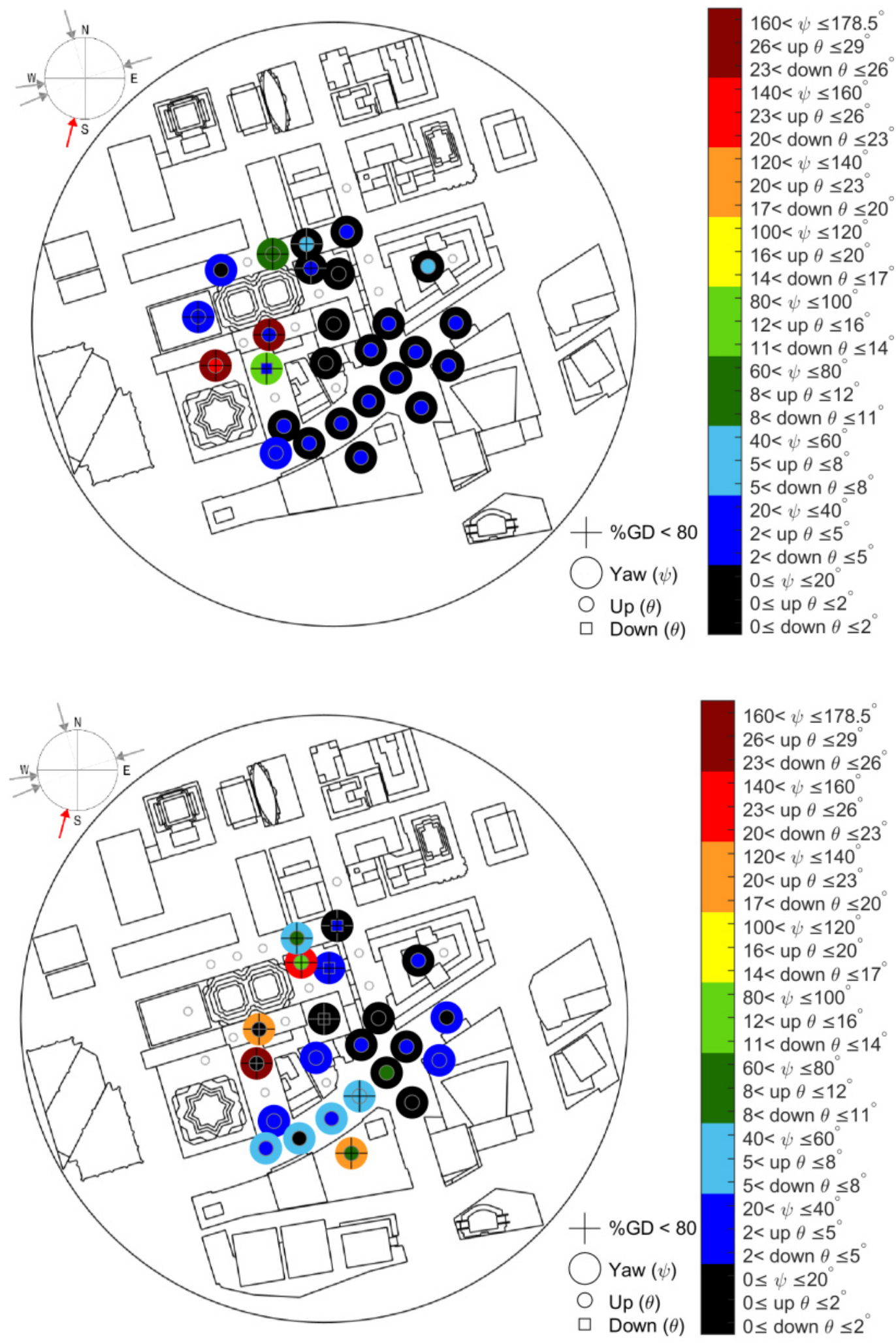

Figure 4.116: Flow angles at Increment 9 (0.383 $\mathrm{m}$ above wind tunnel floor) without (top) and with (bottom) the future buildings. 

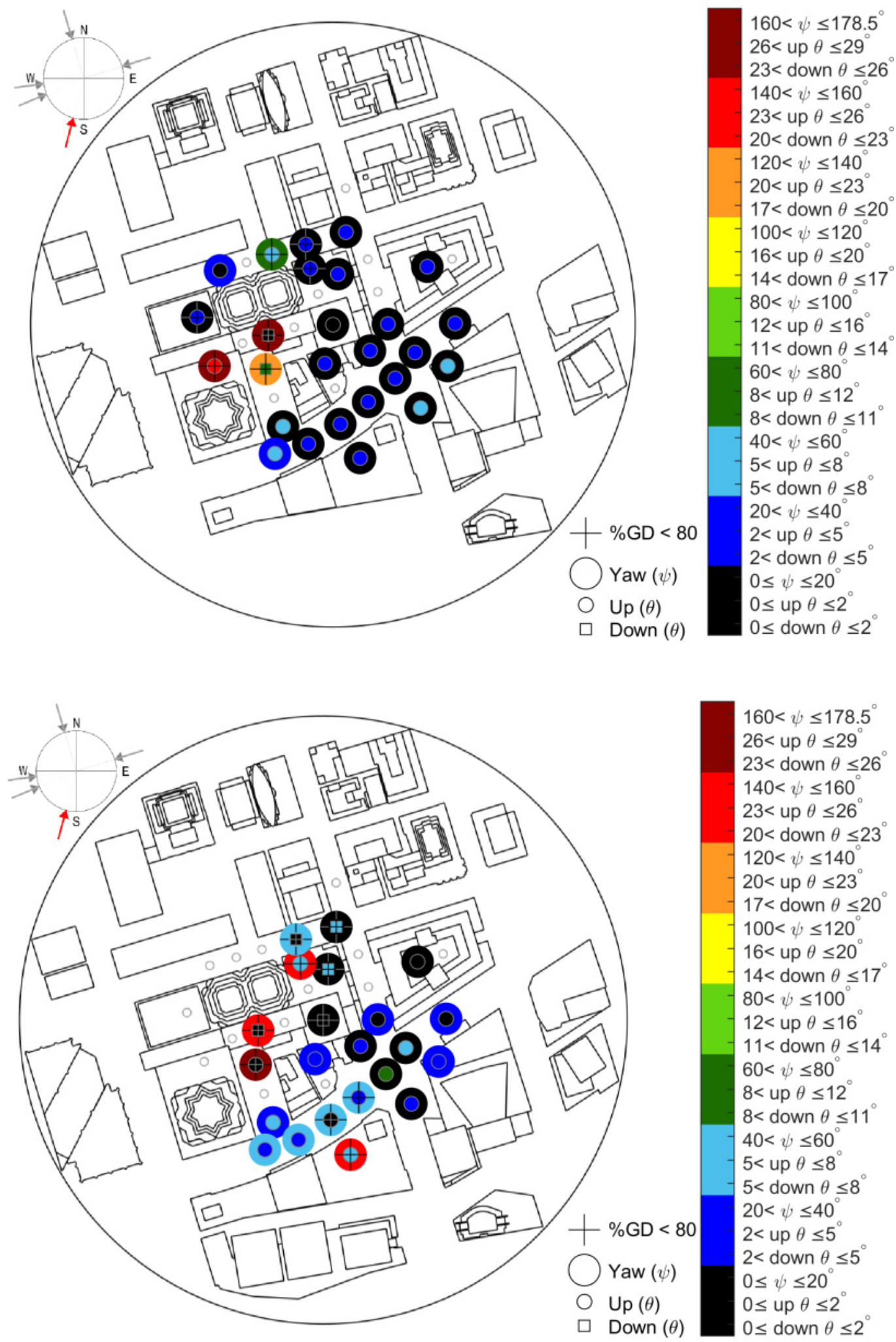

Figure 4.117: Flow angles at Increment 10 (0.4230 $\mathrm{m}$ above wind tunnel floor) without (top) and with (bottom) the future buildings. 

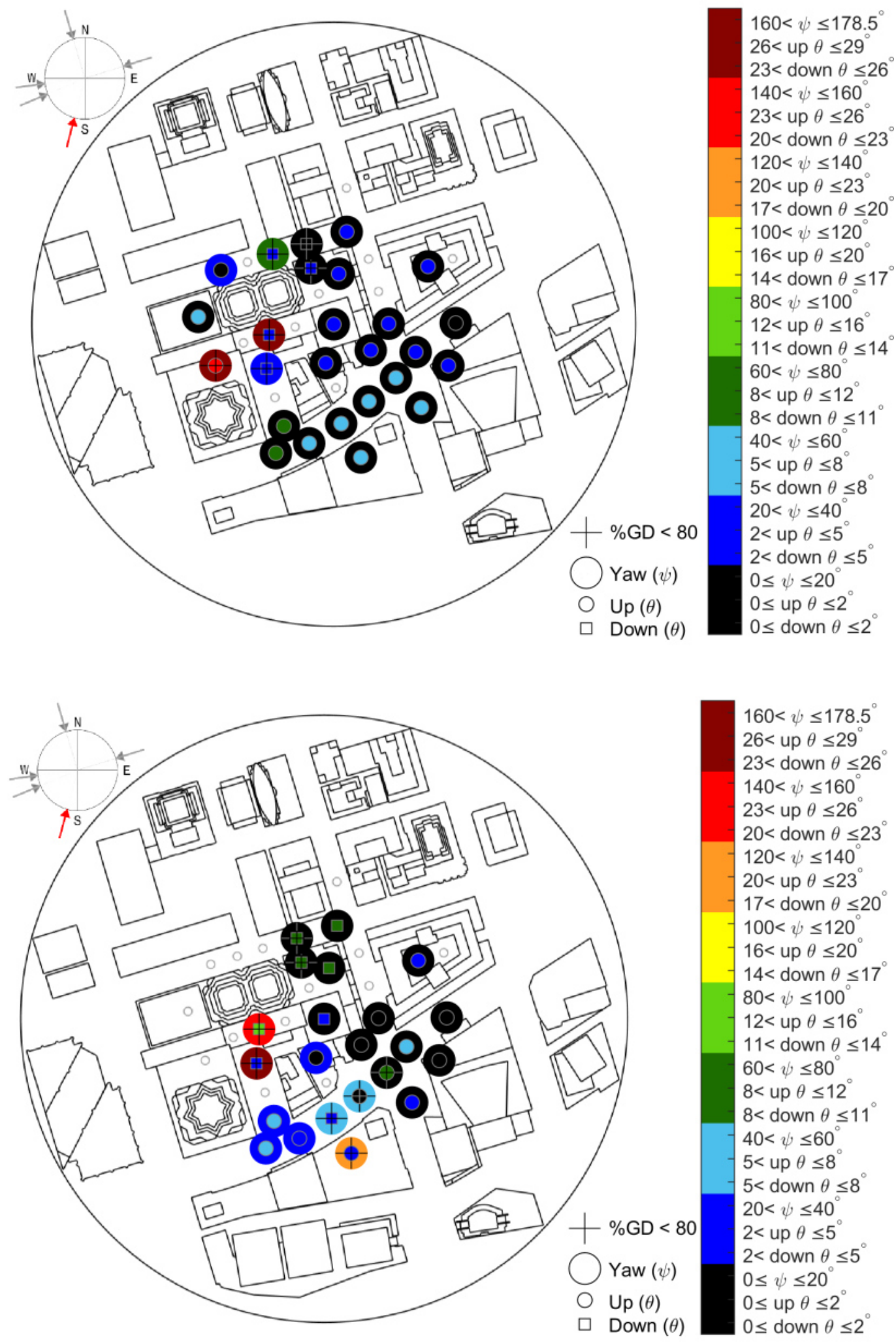

Figure 4.118: Flow angles at Increment 11 (0.483 $\mathrm{m}$ above wind tunnel floor) without (top) and with (bottom) the future buildings. 

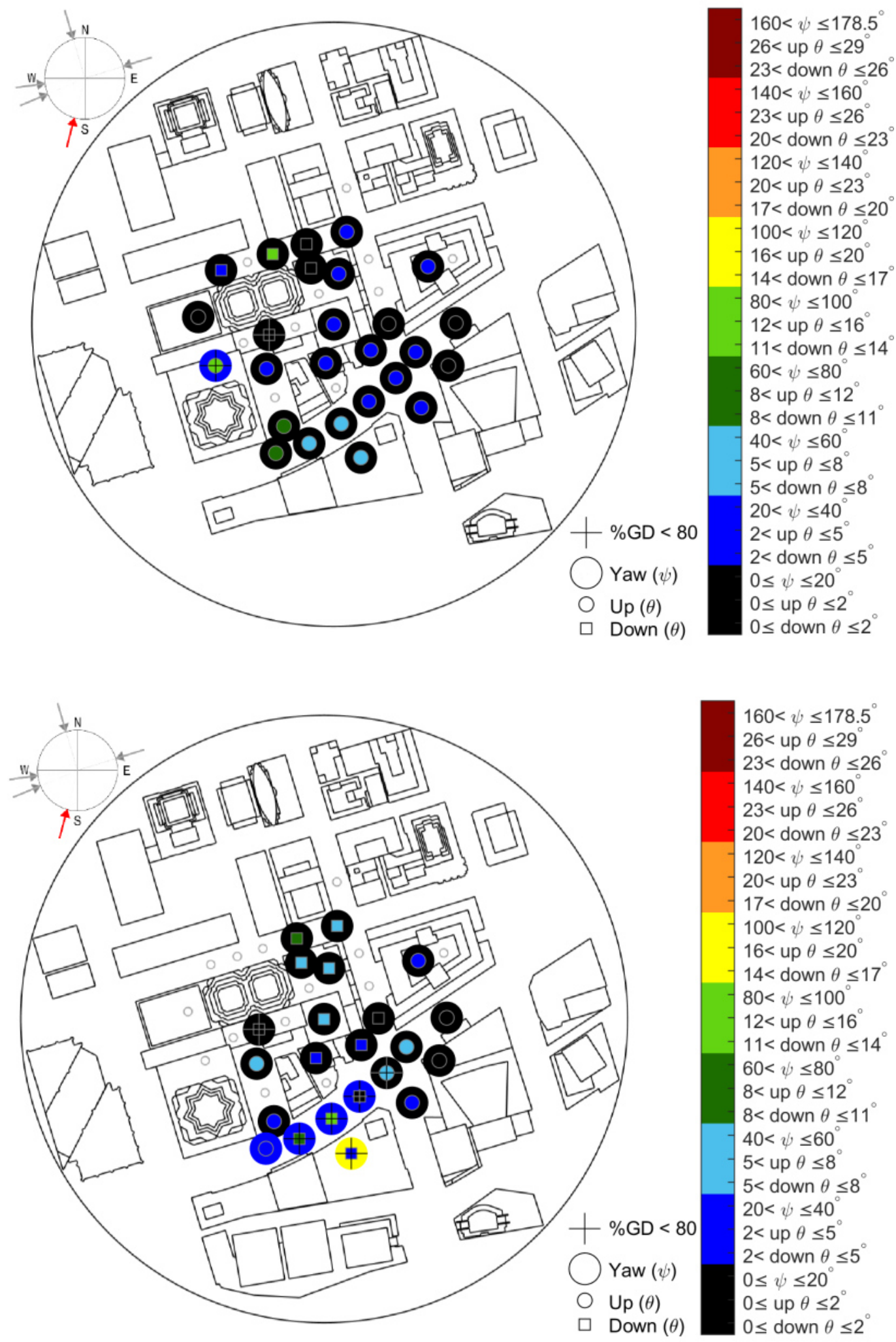

Figure 4.119: Flow angles at Increment 12 (0.543 $\mathrm{m}$ above wind tunnel floor) without (top) and with (bottom) the future buildings. 

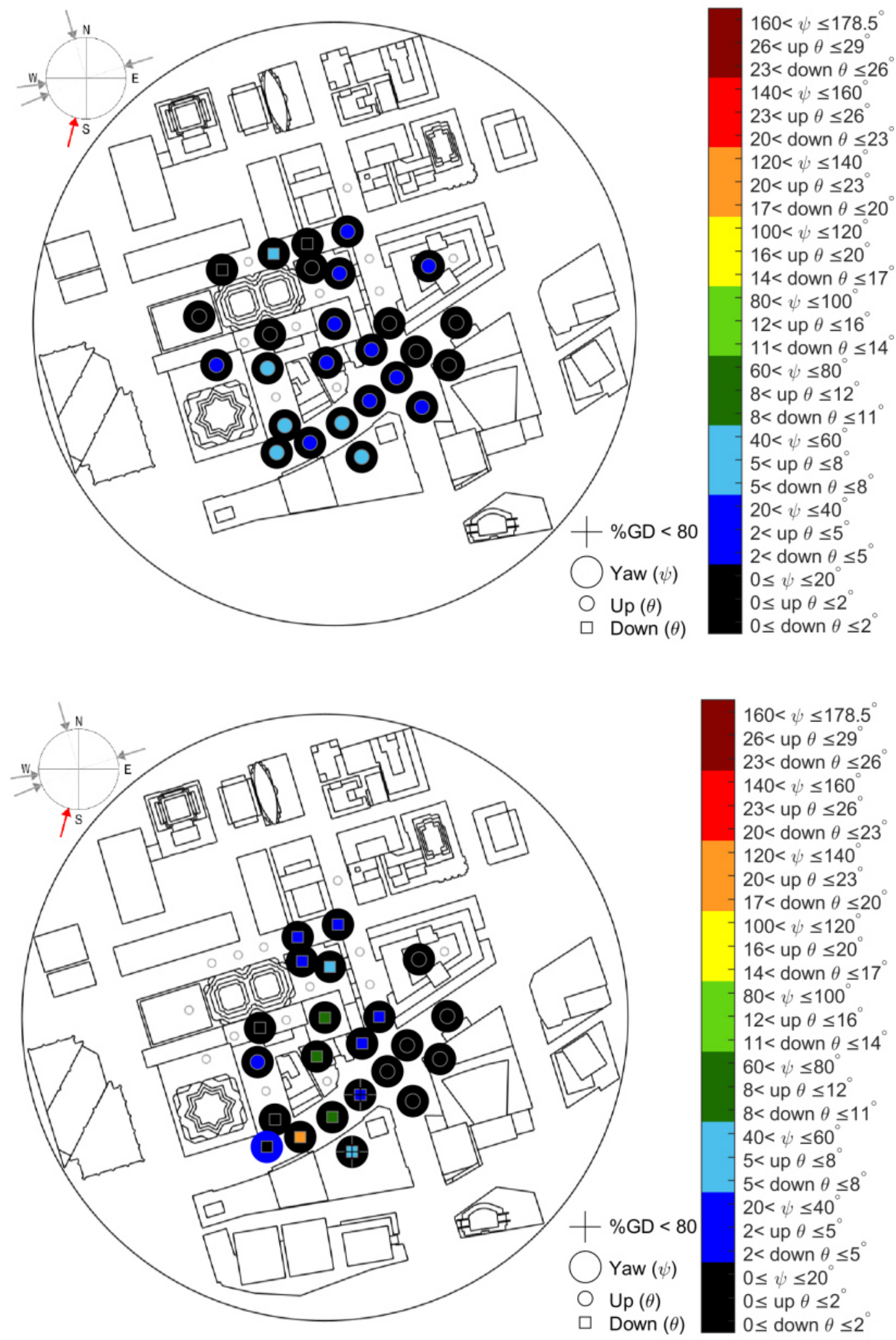

Figure 4.120: Flow angles at Increment 13 (0.603 $\mathrm{m}$ above wind tunnel floor) without (top) and with (bottom) the future buildings. 

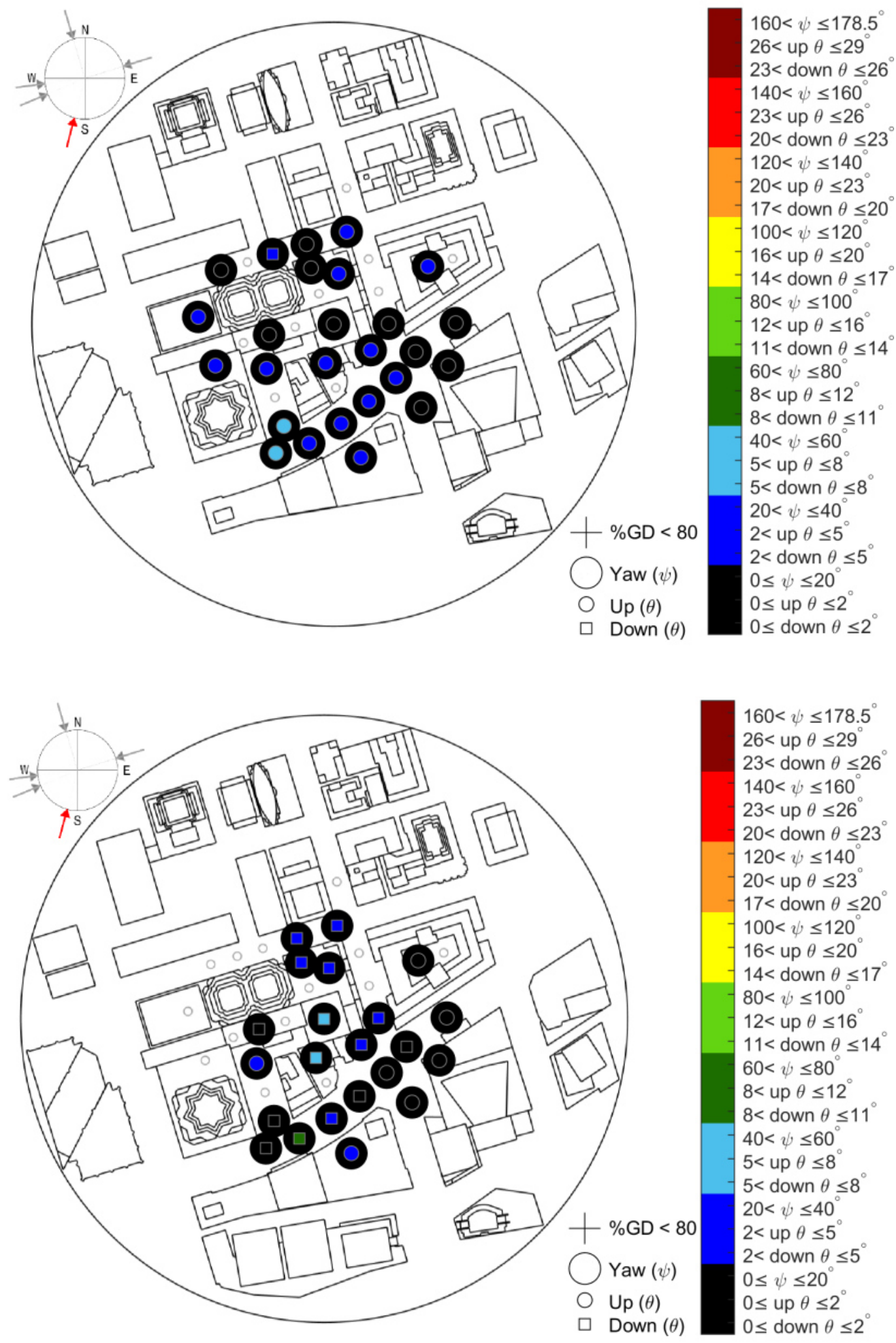

Figure 4.121: Flow angles at Increment 14 (0.663 $\mathrm{m}$ above wind tunnel floor) without (top) and with (bottom) the future buildings. 

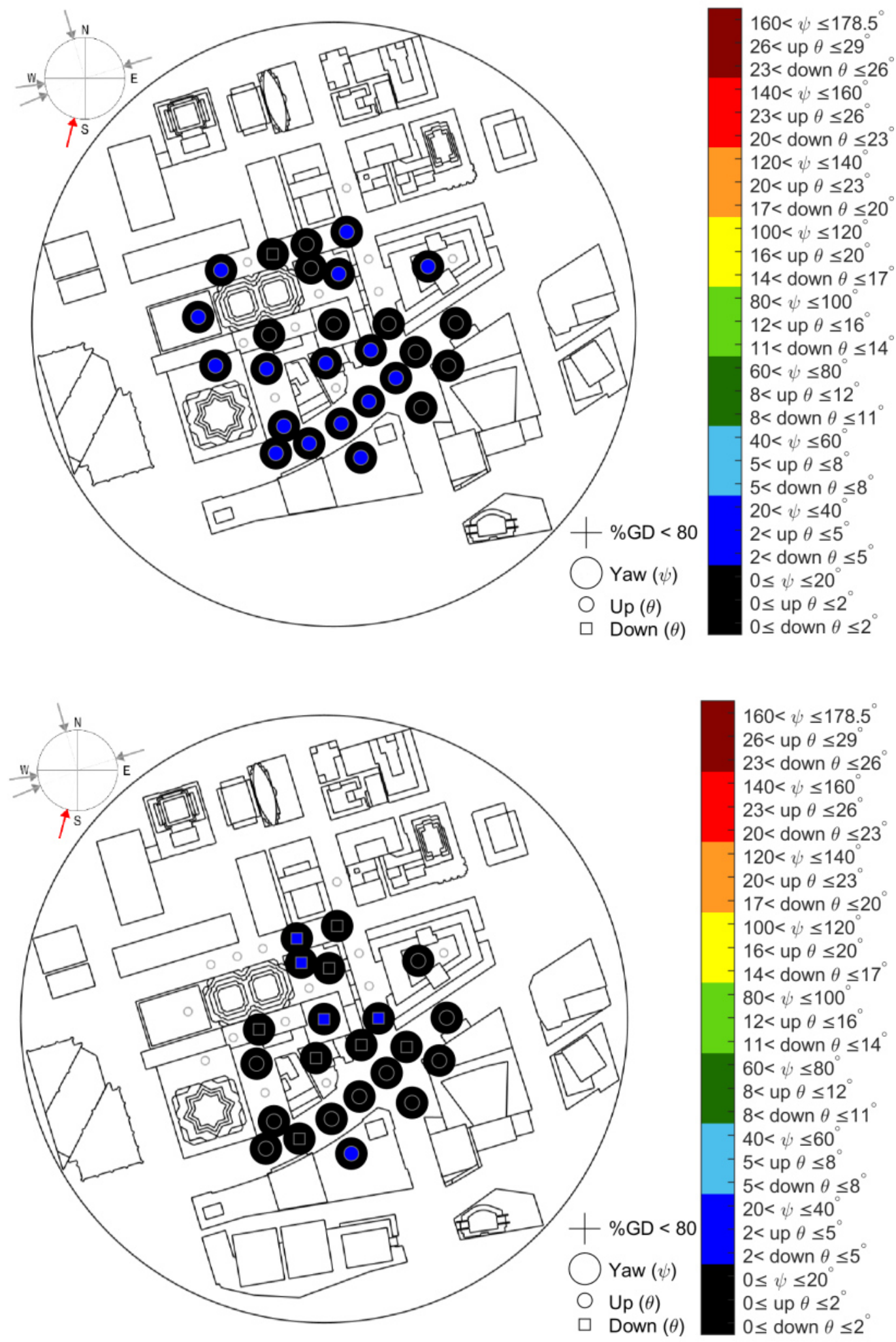

Figure 4.122: Flow angles at Increment 15 (0.723 $\mathrm{m}$ above wind tunnel floor) without (top) and with (bottom) the future buildings. 

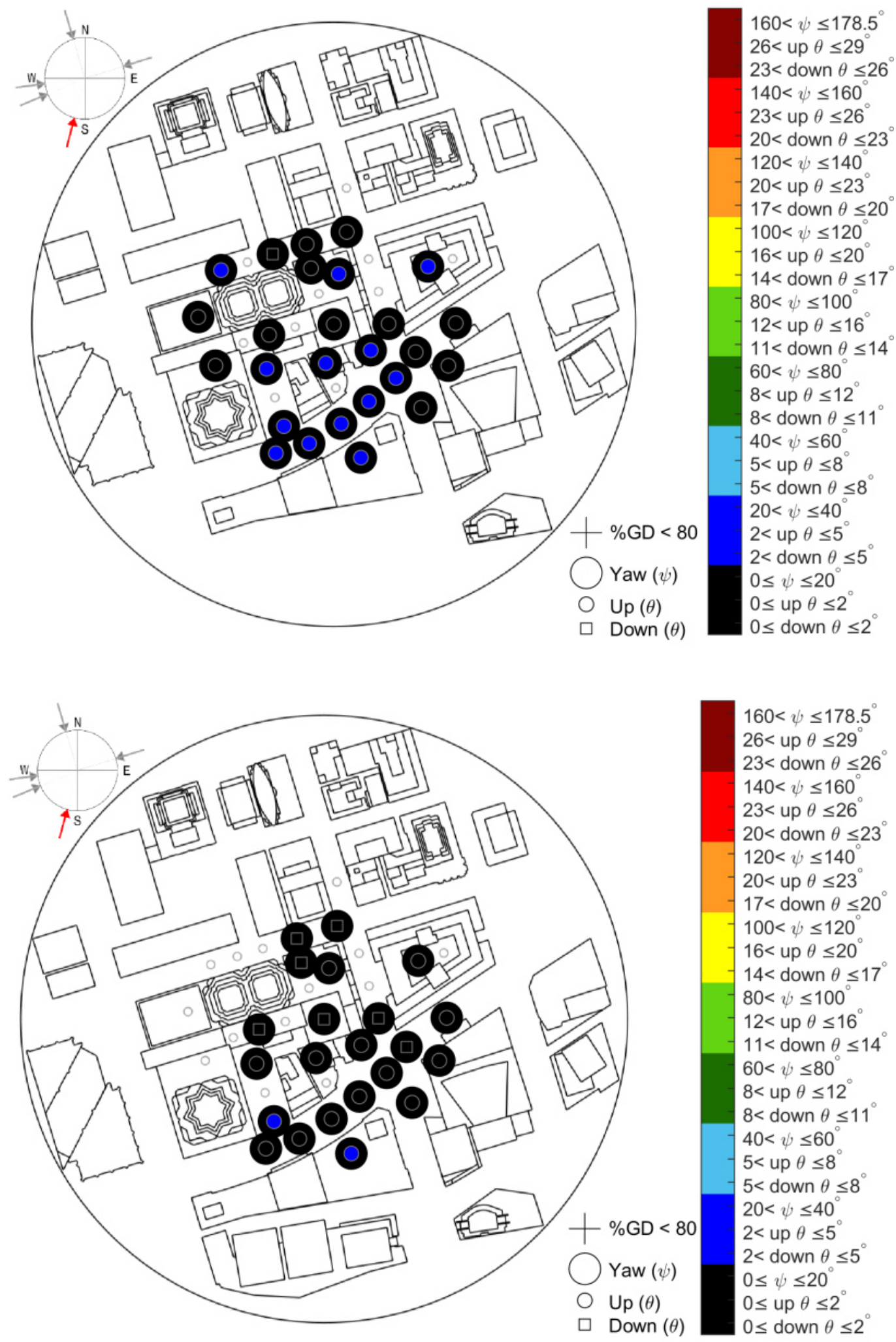

Figure 4.123: Flow angles at Increment 16 (0.783 $\mathrm{m}$ above wind tunnel floor) without (top) and with (bottom) the future buildings. 

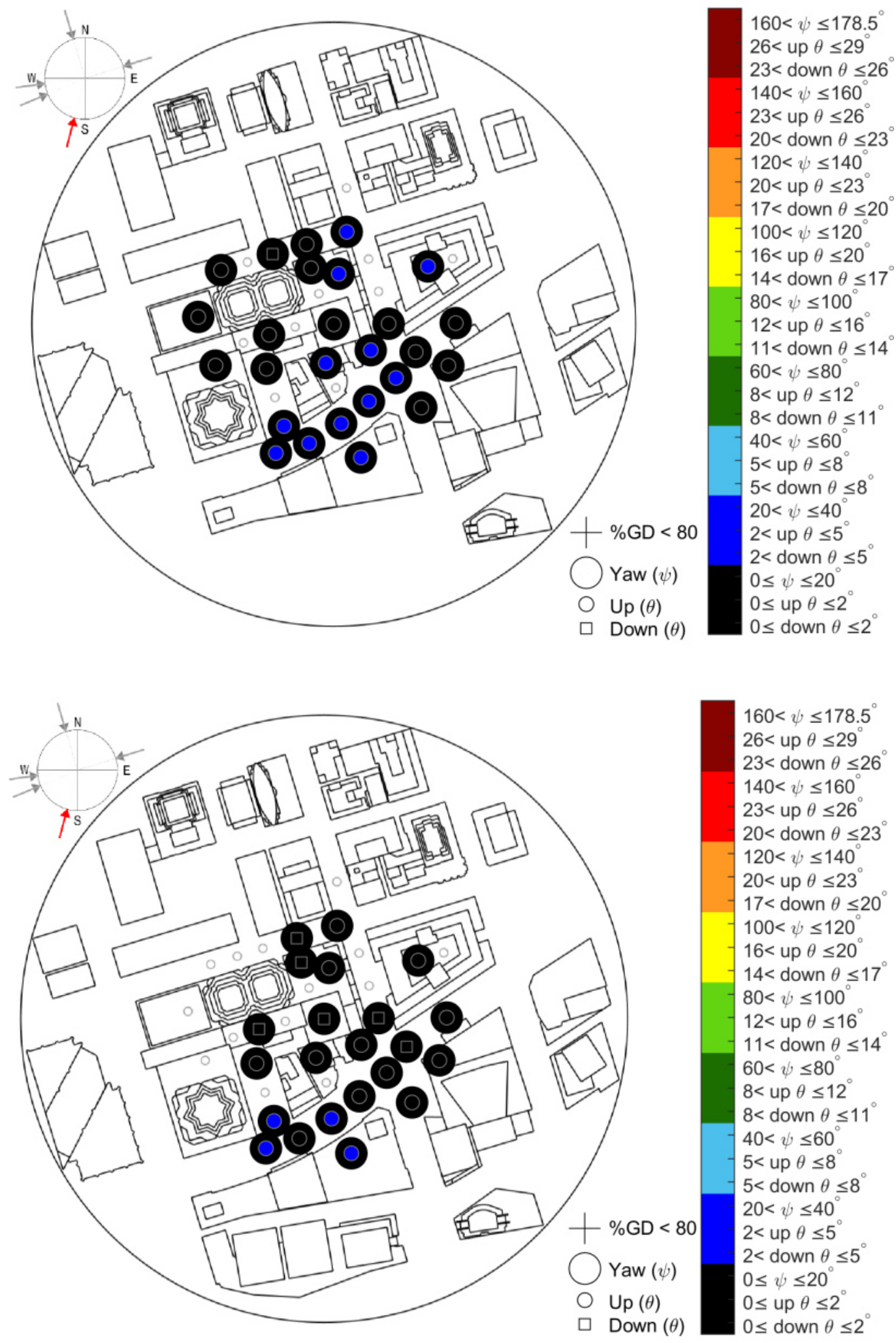

Figure 4.124: Flow angles at Increment 17 (0.843 $\mathrm{m}$ above wind tunnel floor) without (top) and with (bottom) the future buildings. 

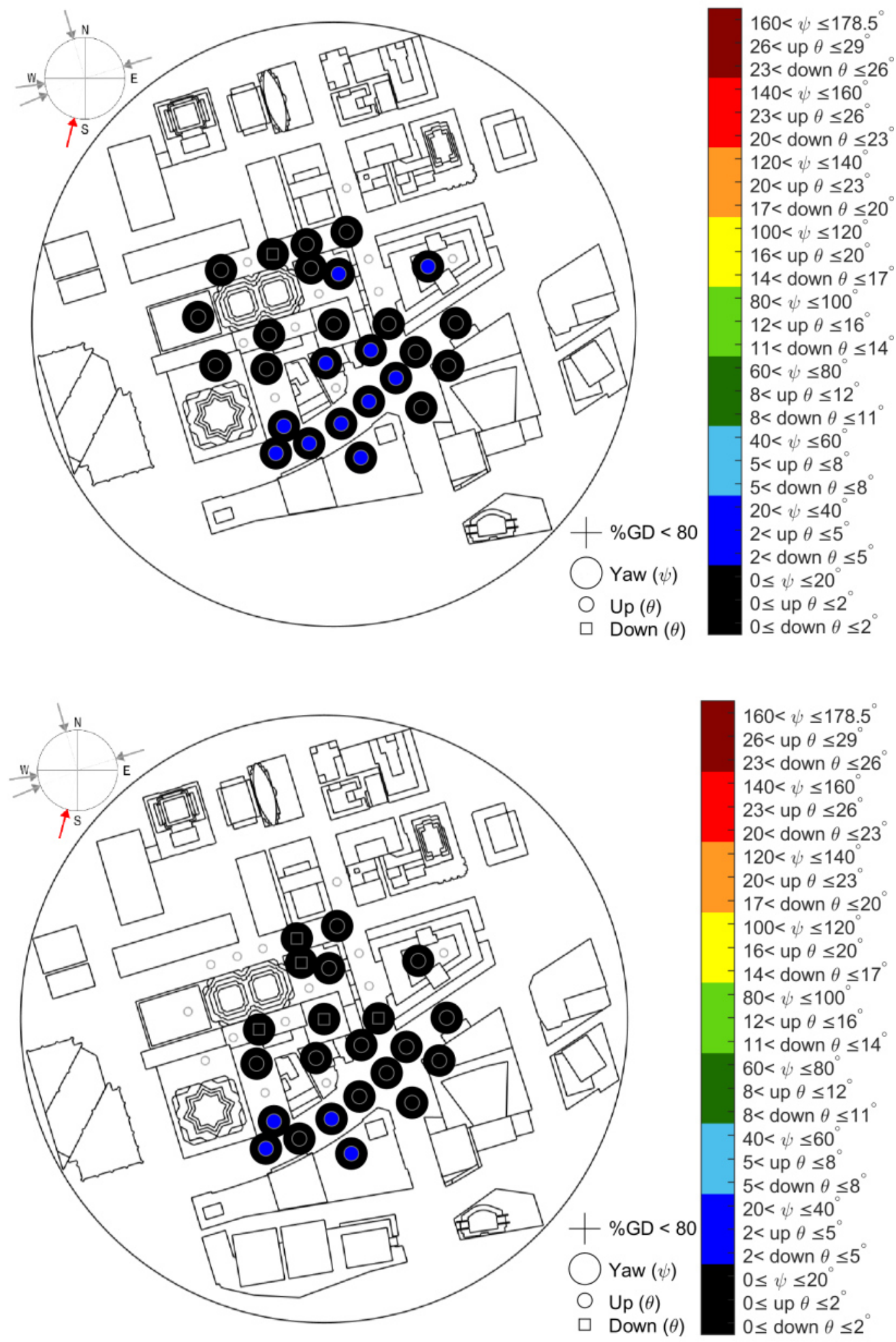

Figure 4.125: Flow angles at Increment 18 (0.903 $\mathrm{m}$ above wind tunnel floor) without (top) and with (bottom) the future buildings. 

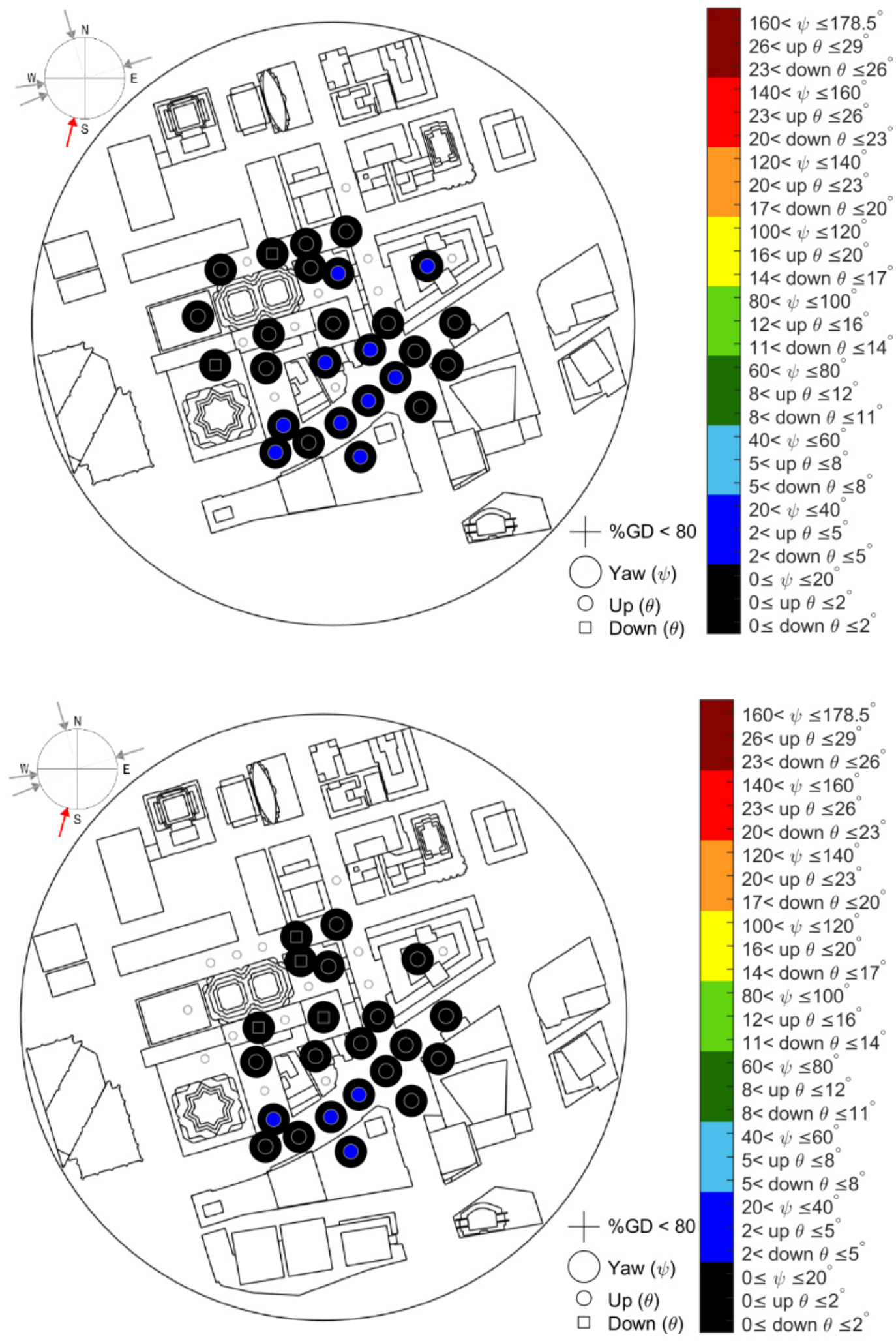

Figure 4.126: Flow angles at Increment 19 (0.963 m above wind tunnel floor) without (top) and with (bottom) the future buildings. 

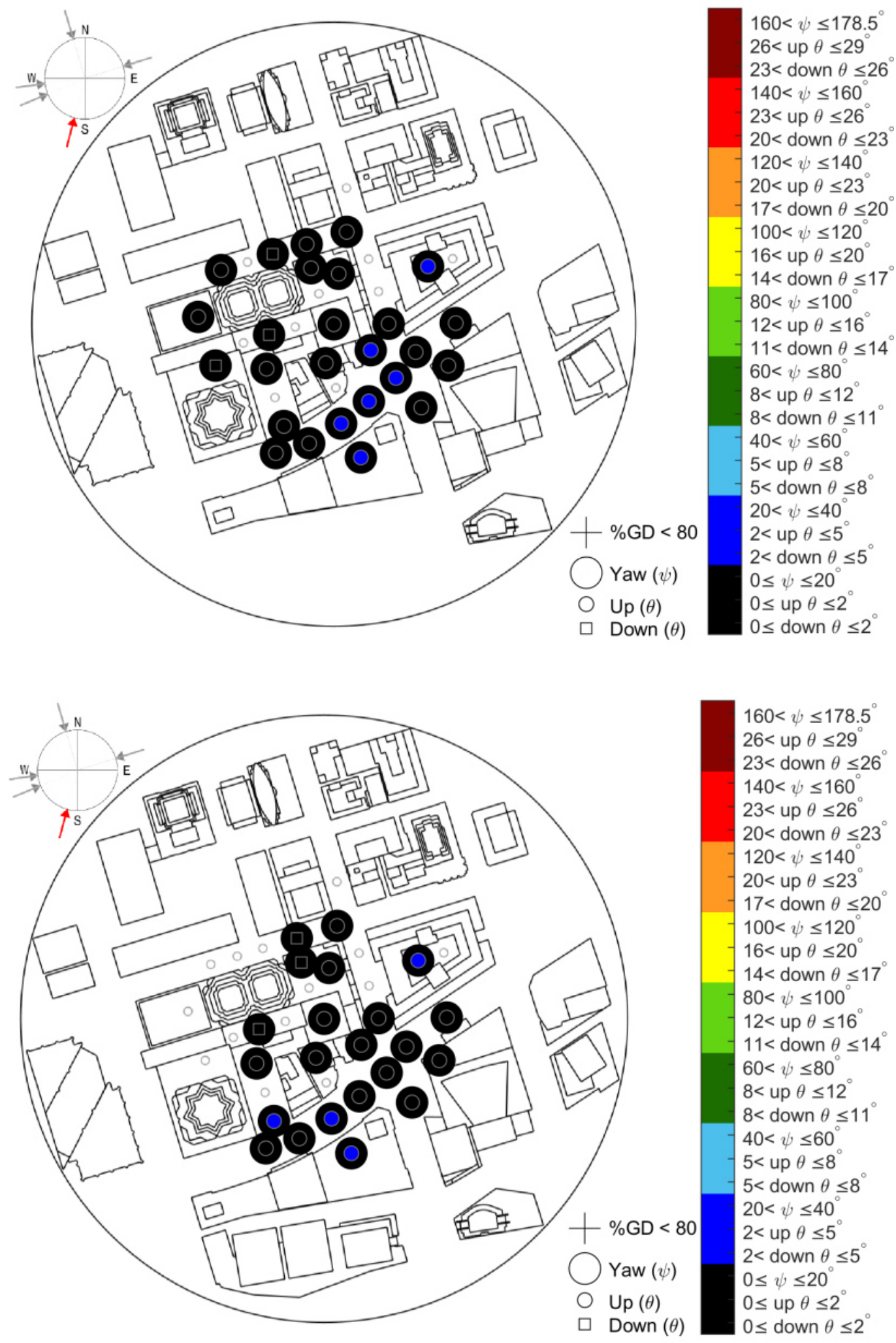

Figure 4.127: Flow angles at Increment 20 (1.023 m above wind tunnel floor) without (top) and with (bottom) the future buildings. 


\subsection{RWDI wind tunnel experiment}

The reader is encouraged to consult Figures 3.41 and 3.13 for vertical measurement spots location and wind directions, and Figure 3.42 for horizontal profiles locations

and designations. Furthermore, the \% GD was presented in the plots following the same colour scheme shown in Figure 4.7. For ease of reference, the data collected at Carleton University ABL wind tunnel will be referred to as 'CU', while the data collected at RWDI B12 wind tunnel will be referred to as 'RWDI'.

\subsubsection{Empty wind tunnel test}

As discussed previously in Section 3.2.4.1, vertical and horizontal profiles were measured at three different exposures prior to model installation in the test section of the B12 wind tunnel. From the vertical measurements, both mean wind speed and longitudinal turbulence intensity profiles were plotted for each exposure, and compared against the approach flow boundary layer profiles developed at Carleton University ABL wind tunnel facility. As can be seen in Figure 4.128, CU mean wind speed profile seems to be in good agreement with RWDI mean wind speed profile for urban exposure. However, the developed turbulence intensity profile at Carleton University ABL wind tunnel is less turbulent as compared to the RWDI turbulence profile for urban exposure. The difference in turbulence profiles for urban exposure allows the assessment of the impact of approach flow turbulence level on the measured data.

The test section lateral flow uniformity was checked by examining the flow speed and turbulence level across the width of the turntable for all three exposures. The results of each exposure show variations within $\pm 5 \%$ from the mean value. This slight deviations is deemed insignificant for wind engineering purposes; thus, the flow is considered uniform. 

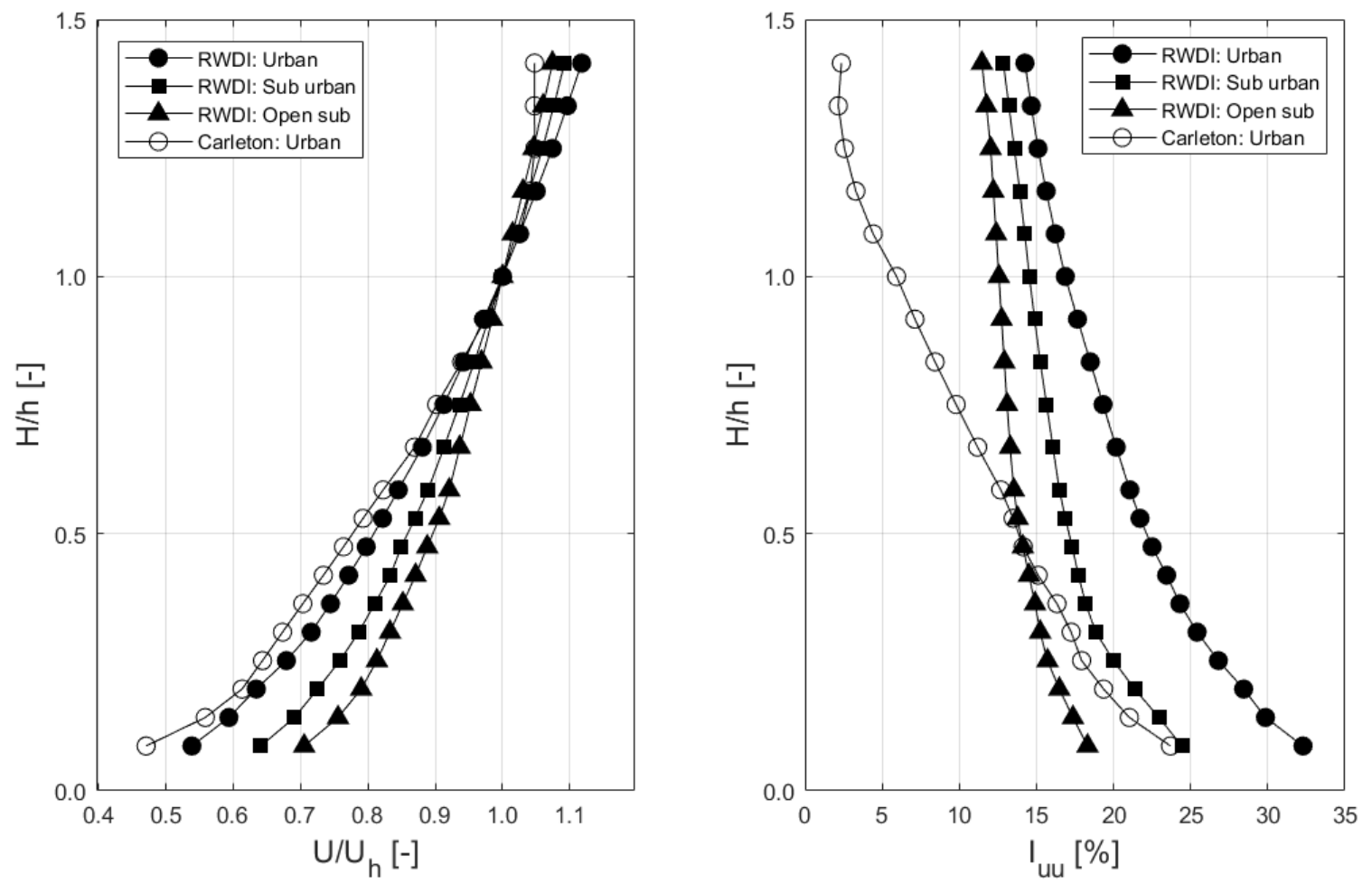

Figure 4.128: Comparisons between RWDI exposure profiles and CU urban profiles. Left: normalized mean wind speed profiles. Right: longitudinal turbulence intensity profiles. Total increments $=20$.

\subsubsection{Effect of model scale and testing facility}

One of the main objectives of performing the wind tunnel experiment at RWDI was to cross-check the measurements taken at Carleton University wind tunnel against data collected in a different facility. The physical model was constructed at a scale of 1:300, which is different from the 1:400 one tested at Carleton University ABL wind tunnel. This is due to the new model being slated to be used for another experiment while providing an opportunity to verify that buildings scale within the appropriate range (1:300-1:600) [26] should have no impact on the measured data.

To accomplish the aforementioned objectives, measurements were collected from the 1:300 scale model at two different spots which have approximately the same upstream buildings layer as the 1:400 scale model, except for one high-rise building 
located in far-proximity to the spots. Figure 4.129 shows the normalized mean wind speed and overall turbulence intensity profiles at Spot 7 for wind coming from WSW.
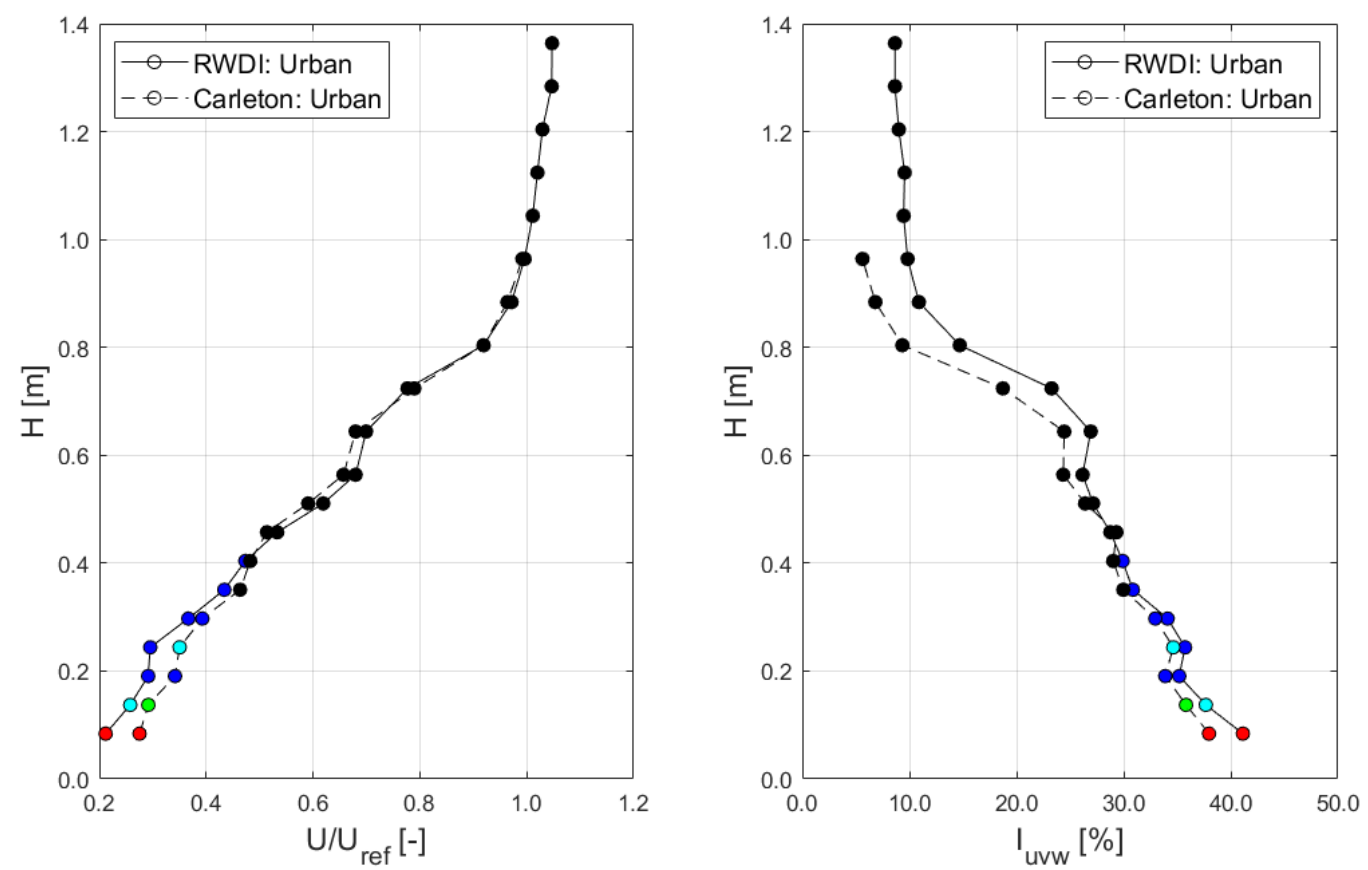

Figure 4.129: Comparisons between RWDI and CU profiles at Spot 7 in WSW wind direction for urban exposure. Left: normalized mean wind speed profiles. Right: overall turbulence intensity profiles. Total increments $=20$.

From the plots, it was observed that the profiles pattern was generally consistent between both experiments. The preserved profile pattern indicates the minimal impact of model scale on the measured data. For the mean wind speed profiles, the results of both experiments show good agreement despite the slight variations in the values of the lower increments, which is consistent with the variations found in the approach flow mean wind speed profiles that were measured without the model. The turbulence profiles shows better agreement in increments located within the height of the upstream buildings, while deviations were more significant in increments located above the height of the upstream buildings since the measurements are directly influenced by the approaching flow characteristics. 
Figure 4.130 shows the normalized mean wind speed and overall turbulence intensity profiles for measurements taken at Spot 9 in WSW wind direction.
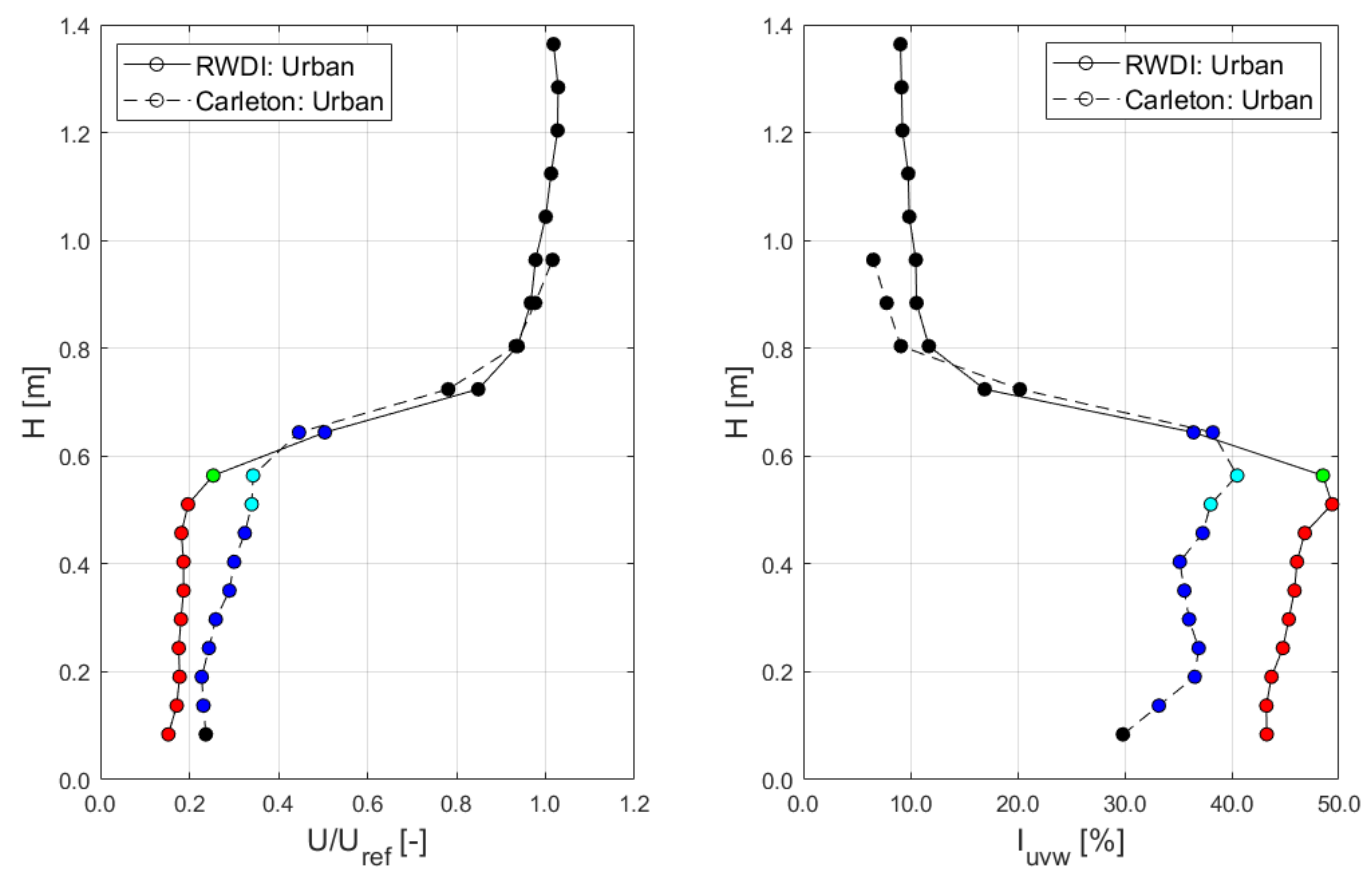

Figure 4.130: Comparisons between RWDI and CU profiles at Spot 9 in WSW wind direction for urban exposure. Left: normalized mean wind speed profiles. Right: overall turbulence intensity profiles. Total increments $=20$.

The conclusions deduced from the plots support the findings obtained from Spot 7 flow information. The profiles pattern of RWDI experiment matches very well the profiles pattern obtained from CU experiment. The mean wind speed values of both experiments were very close, except for the first 10 increments, where the \%GD was found to be very low in RWDI measurements as compared to CU data. The reason for the significant difference in the $\%$ GD between the profiles of both experiments is attributed to the indexing mechanism which was only utilized for the experiment at CU. Therefore, the data collected at RWDI were influenced by the probe acceptance cone of $\pm 45^{\circ}$, which explains the deviation of RWDI profiles from the ones obtained at CU. The deviation was only significant in the increments where appreciable amount of data was not captured. The same conclusion applies to the turbulence data, which 
were close within the height of the upstream buildings, with the exception of the region where data was not captured.

Based on the data comparison between both Spots 7 and 9 , it is believed that model scale has no impact on the measured data and the effect of testing facility is also minimal. Therefore, deviations between the profiles could be related to either the approach flow characteristics or the few extra buildings included in the 1:300 city model for WSW wind direction at RWDI. This is verified by the discussions included in the following subsections.

\subsubsection{Effect of approach flow boundary layer}

The effect of the mean wind speed and turbulence characteristics of the approach flow on the measured data was investigated by collecting data from the model using different exposure profiles, which are urban, sub-urban, and open sub-urban (opensub). The mean wind speed and turbulence characteristics of each of the exposure profiles was shown previously in Figure 4.128.

To determine the influence of the approach flow profile, the measuring spots were selected based on the following criteria.

- A spot that has light upstream building density: Spot 28 in $\mathrm{E}_{17.1}$ (see Figure 4.131 for the spot location).

- A spot that has heavy upstream building density with majority of low-rise buildings: Spot 33 in $\mathrm{N}_{16.7}$ (see Figure 4.132 for the spot location).

- A spot that has heavy upstream building density with majority of high-rise buildings: Spot 7 in WSW (see Figure 4.133 for the spot location). 


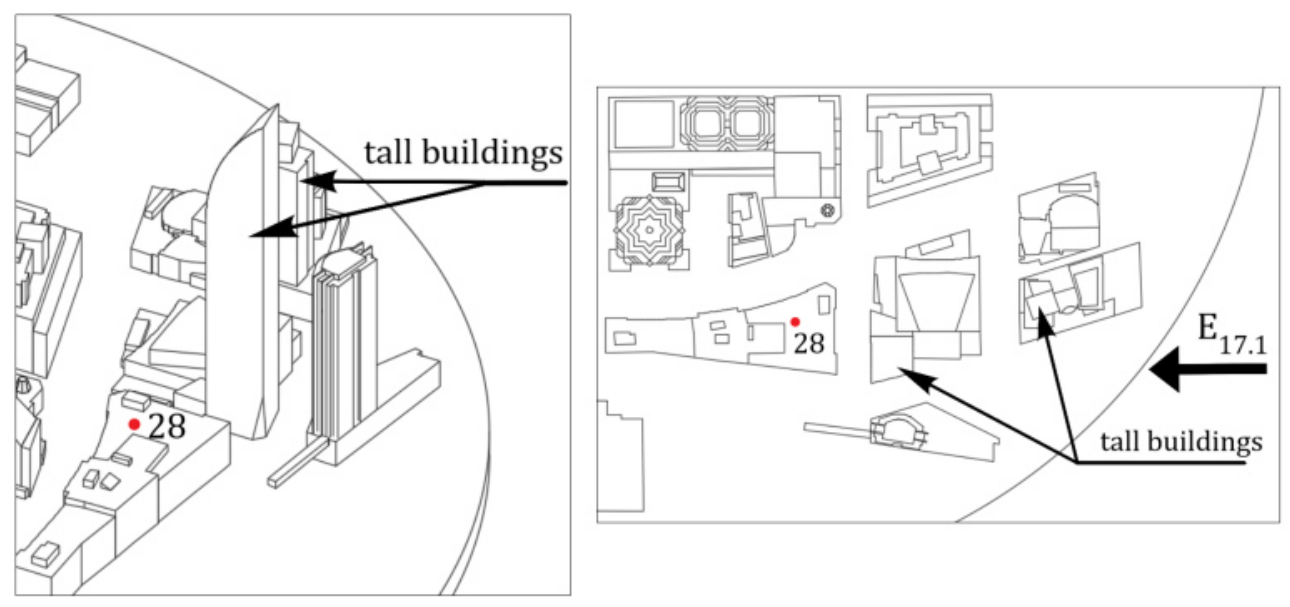

Figure 4.131: Location of Spot 28 for wind direction $\mathrm{E}_{17.1}$.
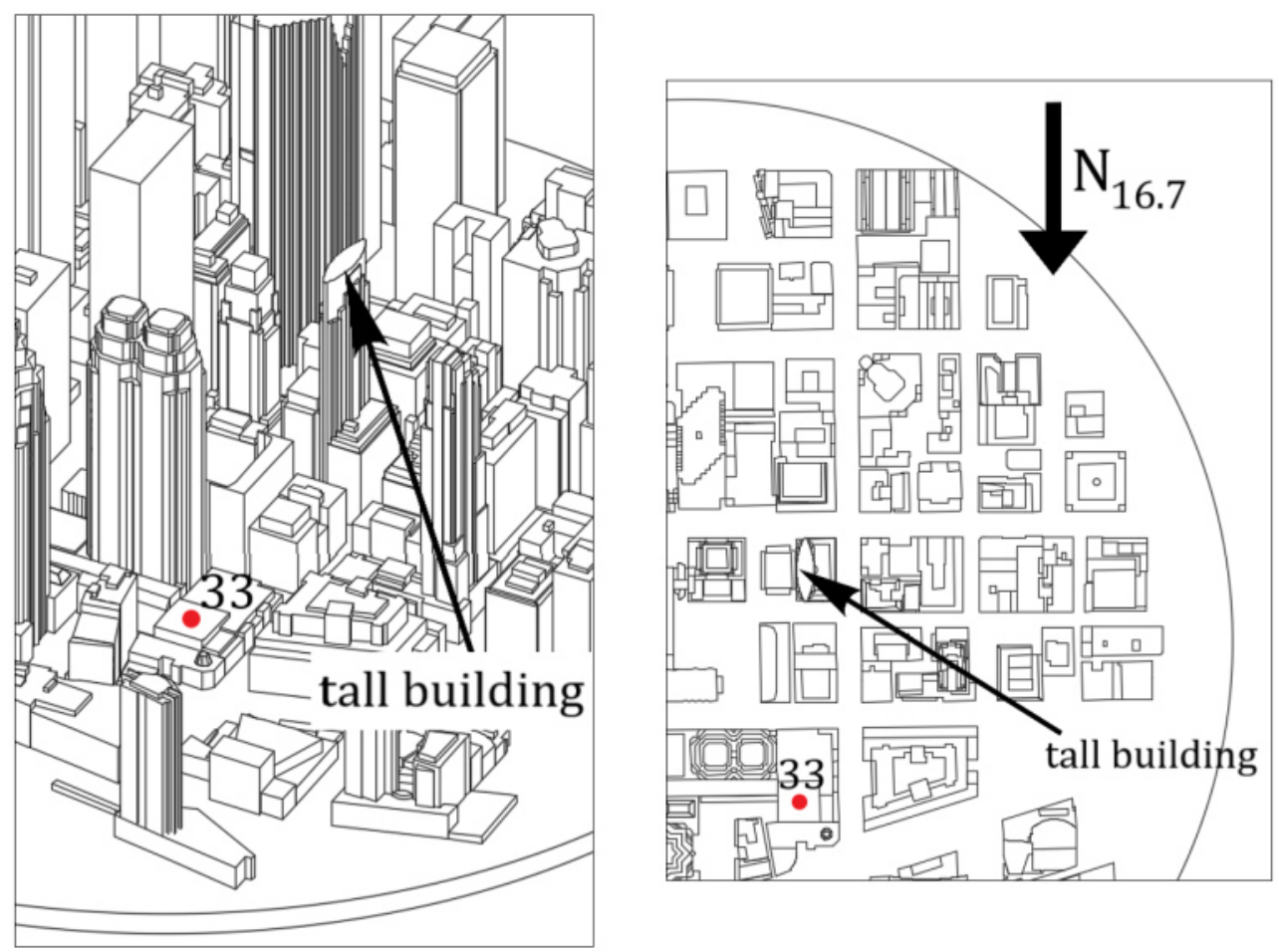

Figure 4.132: Location of Spot 33 for wind direction $\mathrm{N}_{16.7}$. 

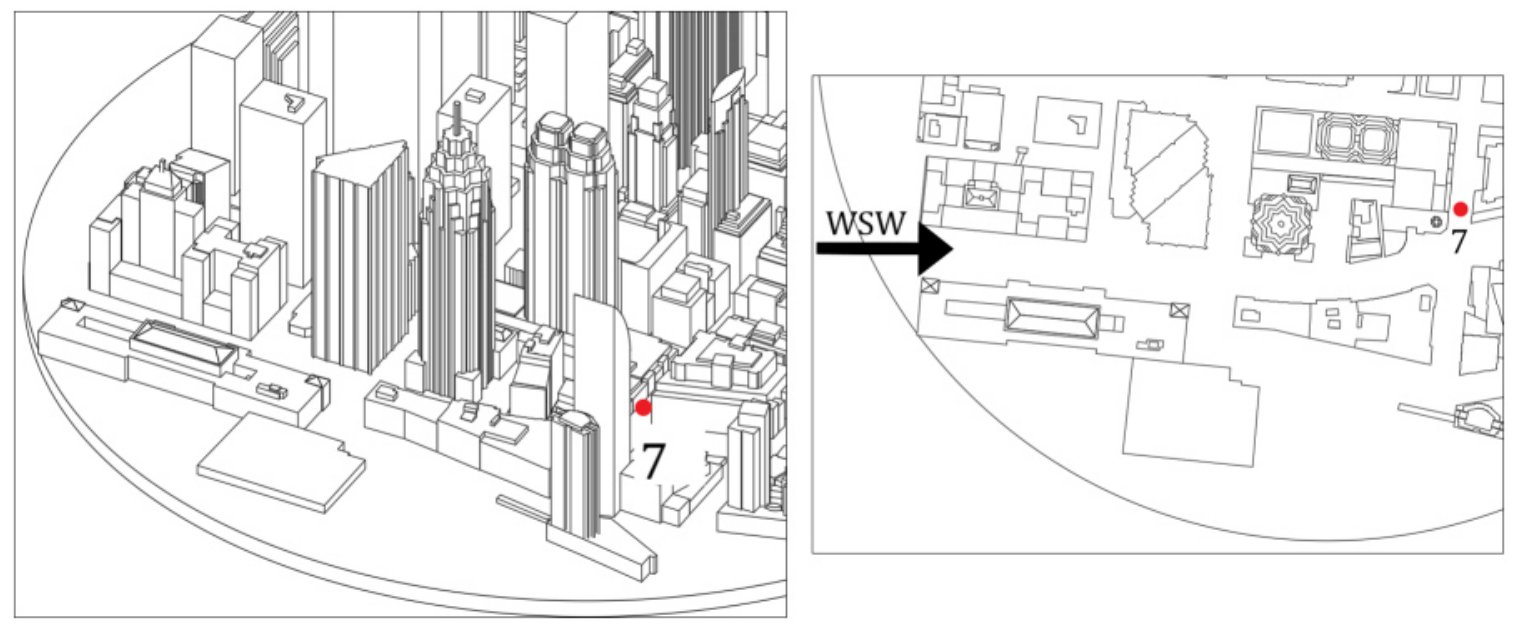

Figure 4.133: Location of Spot 7 for wind direction WSW.

The normalized mean wind speed and overall turbulence intensity profiles at Spots 28, 33 and 7 are shown in Figures 4.134, 4.135 and 4.136, respectively. By comparing the results, it was observed that the effect of the approach flow characteristics on the measured data strongly depends on the spot location. For the case of Spot 28, the spot was located close to the model leading edge with few upstream buildings, the majority of which are low-rise. Therefore, the flow measurements at the spot were almost directly exposed to the approach flow, which explains the variations between the measured profiles that was more notable as compared to variations found at the other two spots. Spot 33 was located downstream of heavy building density, but the majority were low-rise. The only high-rise building upstream of the spot has a cross-section similar to an airfoil shape, and thus, the turbulence level in its wake at the spot location should not be severe. The profiles measured at Spot 33 are in good agreement for the first 5 increments, which are within the height of the upstream low-rise buildings. However, deviations between the profiles started beyond the 5th Increment, where the measurements were almost subjected to the approaching flow. For Spot 7, multiple high-rise buildings were located upstream of the spot; therefore, 
measurements were highly influenced by the buildings turbulent wake, and the effect of the approach flow characteristics on the measured data was almost negligible. The data collected at Spot 7 from the 1:400 scale city model are also shown in Figure 4.136. where reasonable agreement was found between the profiles. However, since RWDI profiles for the three different exposures show strong agreement, it is believed that the slight discrepancy found in CU profiles could be related to the few extra buildings, which were not part of the 1:400 city model. The effect of near field modelling will be discussed in more depth in Section 4.4.4.
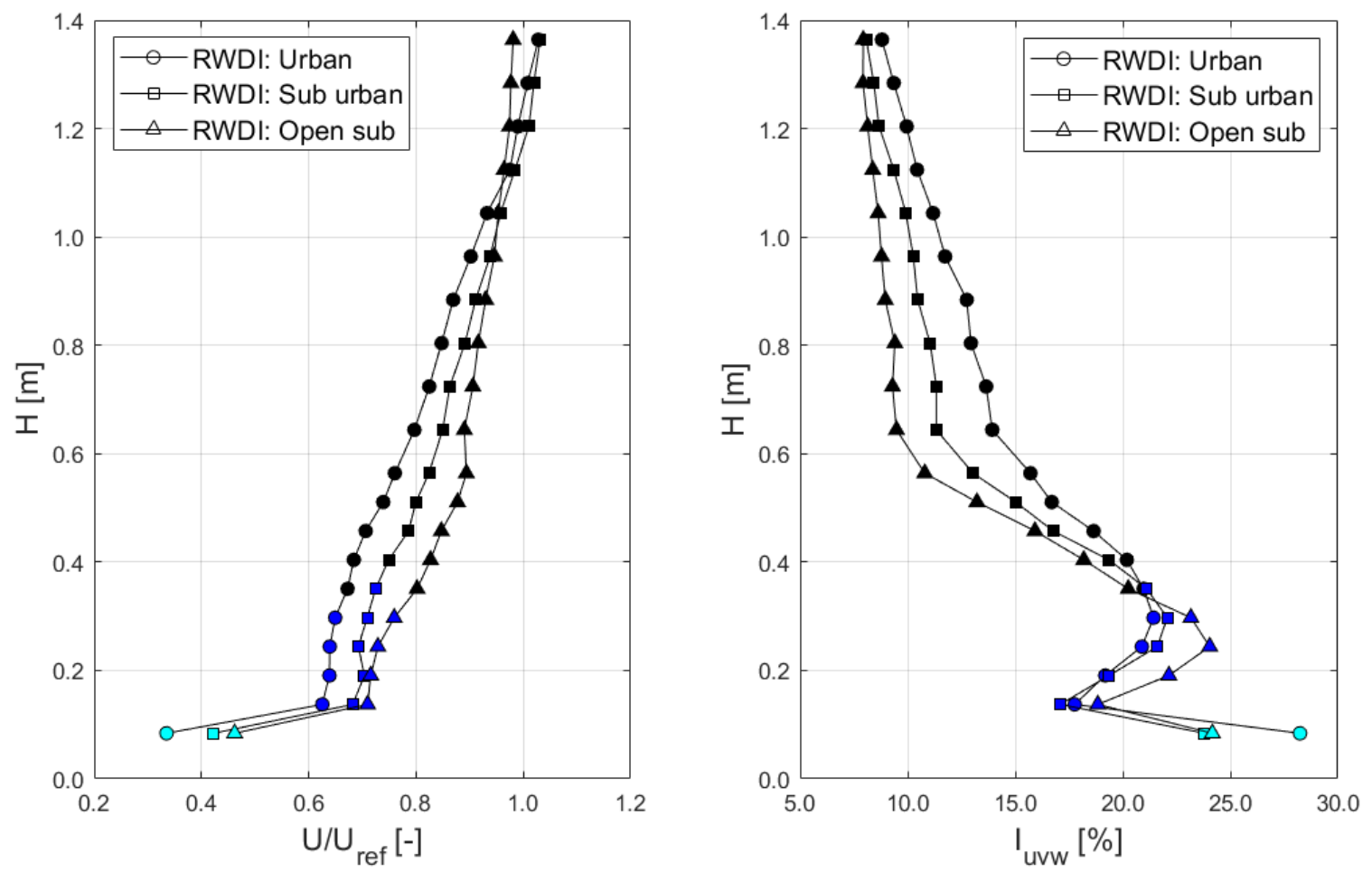

Figure 4.134: Comparisons between the profiles measured at Spot 28 in $\mathrm{E}_{17.1}$ wind direction for all three exposures. Left: normalized mean wind speed profiles. Right: overall turbulence intensity profiles. Total increments $=20$. 

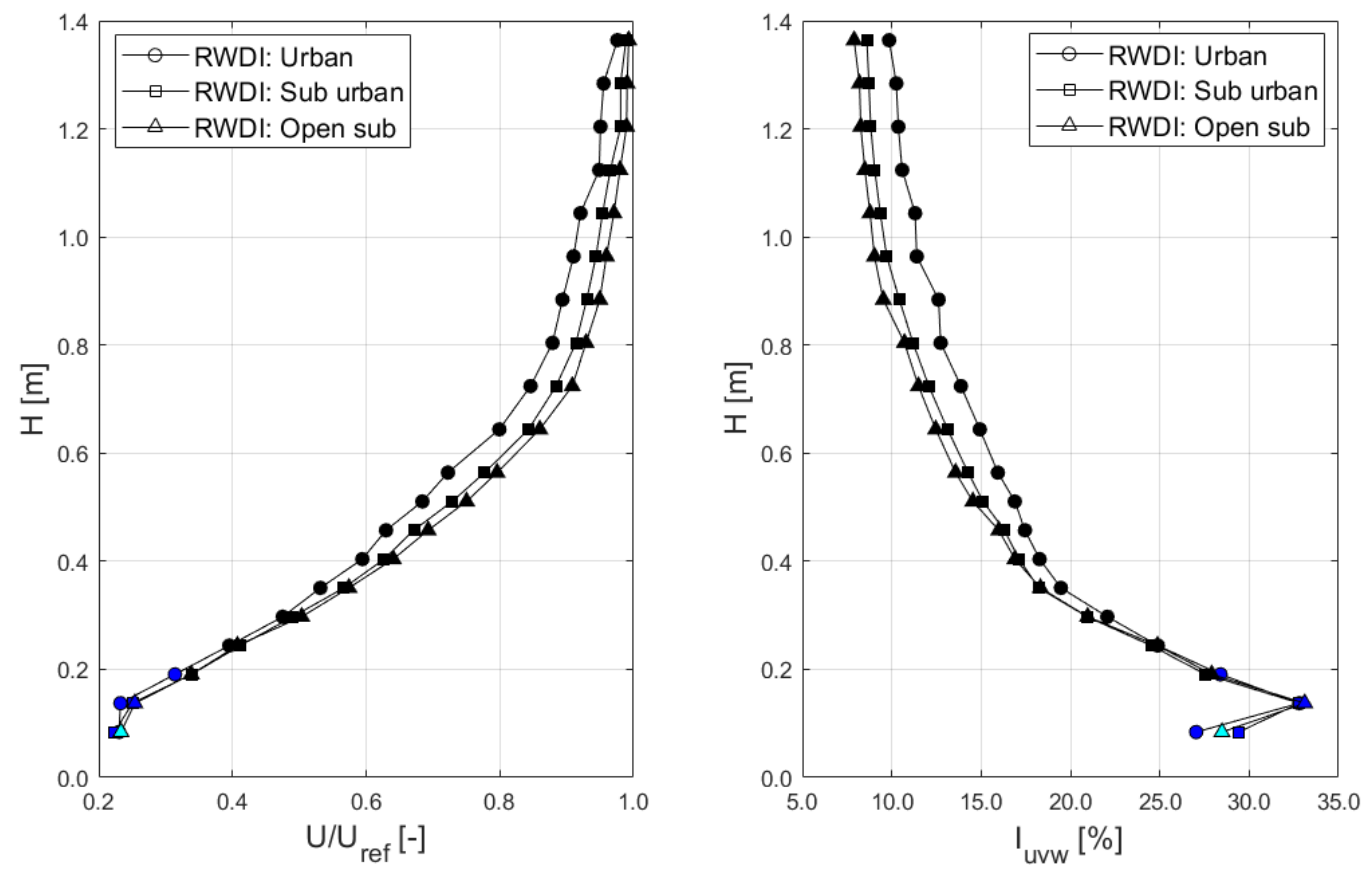

Figure 4.135: Comparisons between the profiles measured at Spot 33 in $\mathrm{N}_{16.7}$ wind direction for all three exposures. Left: normalized mean wind speed profiles. Right: overall turbulence intensity profiles. Total increments $=20$.
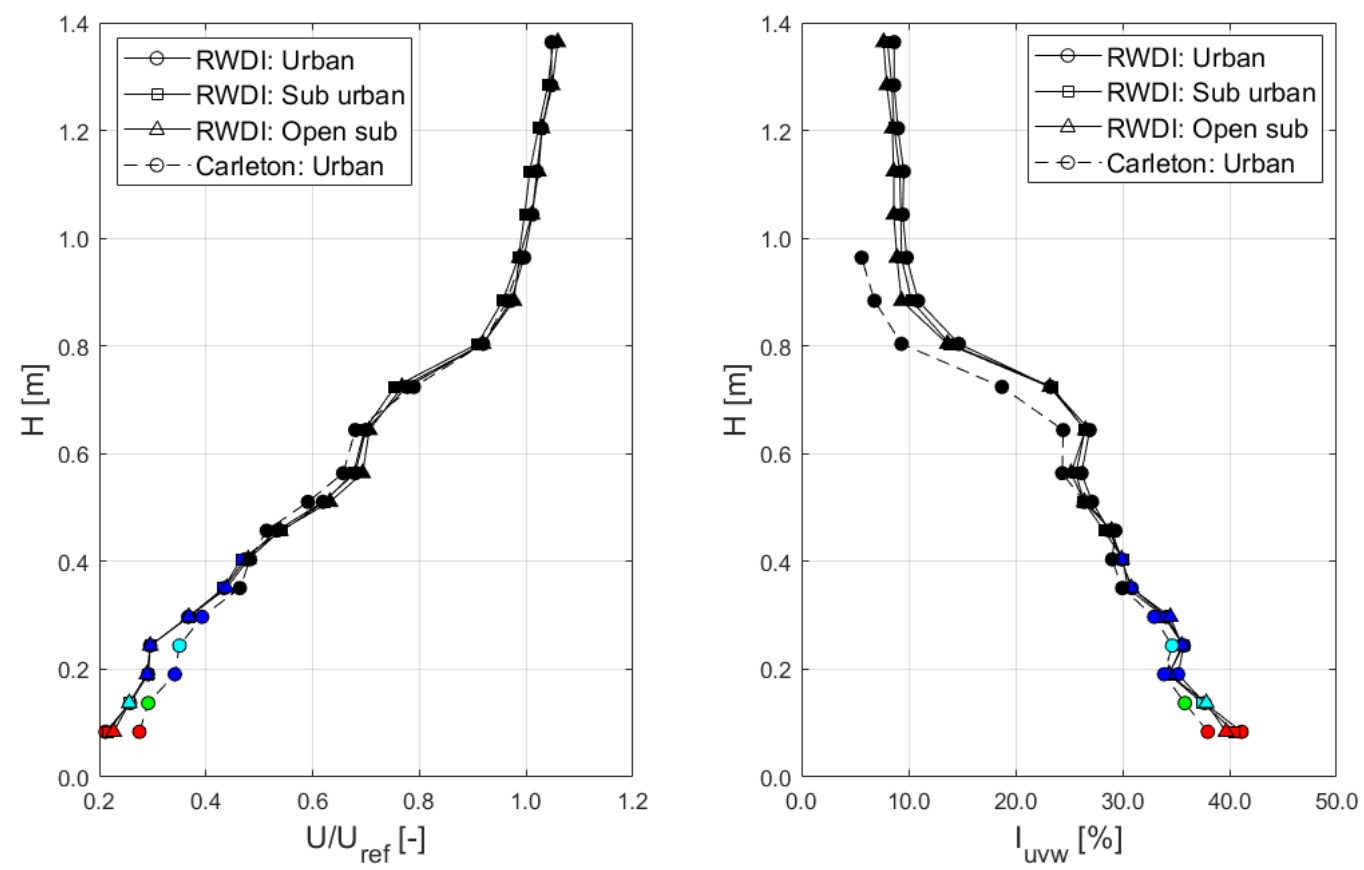

Figure 4.136: Comparisons between the profiles measured at Spot 7 in WSW wind direction for all three exposures. Left: normalized mean wind speed profiles. Right: overall turbulence intensity profiles. Total increments $=20$. 


\subsubsection{Effect of near field modelling}

The effect of near field modelling on the measured data was investigated in a model orientation representing wind coming from $\mathrm{N}_{16.7}$ direction. The reason behind choosing this specific wind angle is attributed to the addition of approximately four rows of buildings in the 1:300 scale city model. To carry out the investigation, measurements have been collected from the 1:300 scale city model at the measuring spots shown in Figure 4.137, and the results were compared against the measurements of the 1:400 city model at the same spots.

Figures 4.138 through 4.141 show the normalized mean wind speed and overall turbulence intensity profiles of both experiments for urban exposure. Both Spots 10 and 16 are located downstream of low- and mid-rise buildings. The profiles comparisons show consistency in the general trend except for increments with low \%GD, the reason of which was explained earlier in this section. However, CU data seems to have higher velocities and lower turbulence levels, which is expected owing to the more complex upstream flow in the 1:300 city model. The velocity and turbulence measurements at Spot 33 show similar behaviour to Spots 10 and 16 profiles data, where the velocities were higher and the turbulence level was lower in CU data. However, CU measurements show a specific pattern in Increments 9-10 (see Figure 4.141, where turbulence level was increased by approximately $7 \%$ from Increment 8 to Increment 9. This behaviour can be explained by the difference in the upstream buildings of both models as shown in Figure 4.142. The two dashed lines in the 1:400 city model represent the location of Increments 9-10, where the change in the profile pattern had occurred. As can be seen in Figure 4.142, Building I, which is the nearby building to Spot 33, was directly subjected to the incoming flow in the 1:400 city model, whereas Building I was obstructed by another building, labelled as II, in the 
1:300 city model. Additionally, Building II is distant from Building I; hence, the effect of flow separation from Building II should decay and diminish as it reaches Spot 33. For the 1:400 city model, the effect of flow separation from Building I on Spot 33 should be more intense since Spot 33 is directly located in the building wake or shear layer, and this could explain the increase in turbulence level found in CU data despite the less complex upstream building density.

The profiles measured at Spot 18 seem to be the least consistent between both experiments since not only the values are different, but also the profiles general trend seem to vary. This could be attributed to the tall buildings located upstream the spot.

The near field modelling seems to be crucial for wind tunnel testing of buildings and structures, and it has a dominant influence over the approach flow characteristics. Therefore, the slight variations found in CU data for Spots 7 and 9 in WSW wind direction are expected to be influenced, to a great extent, by the near field modelling as opposed to the approach flow characteristics. 


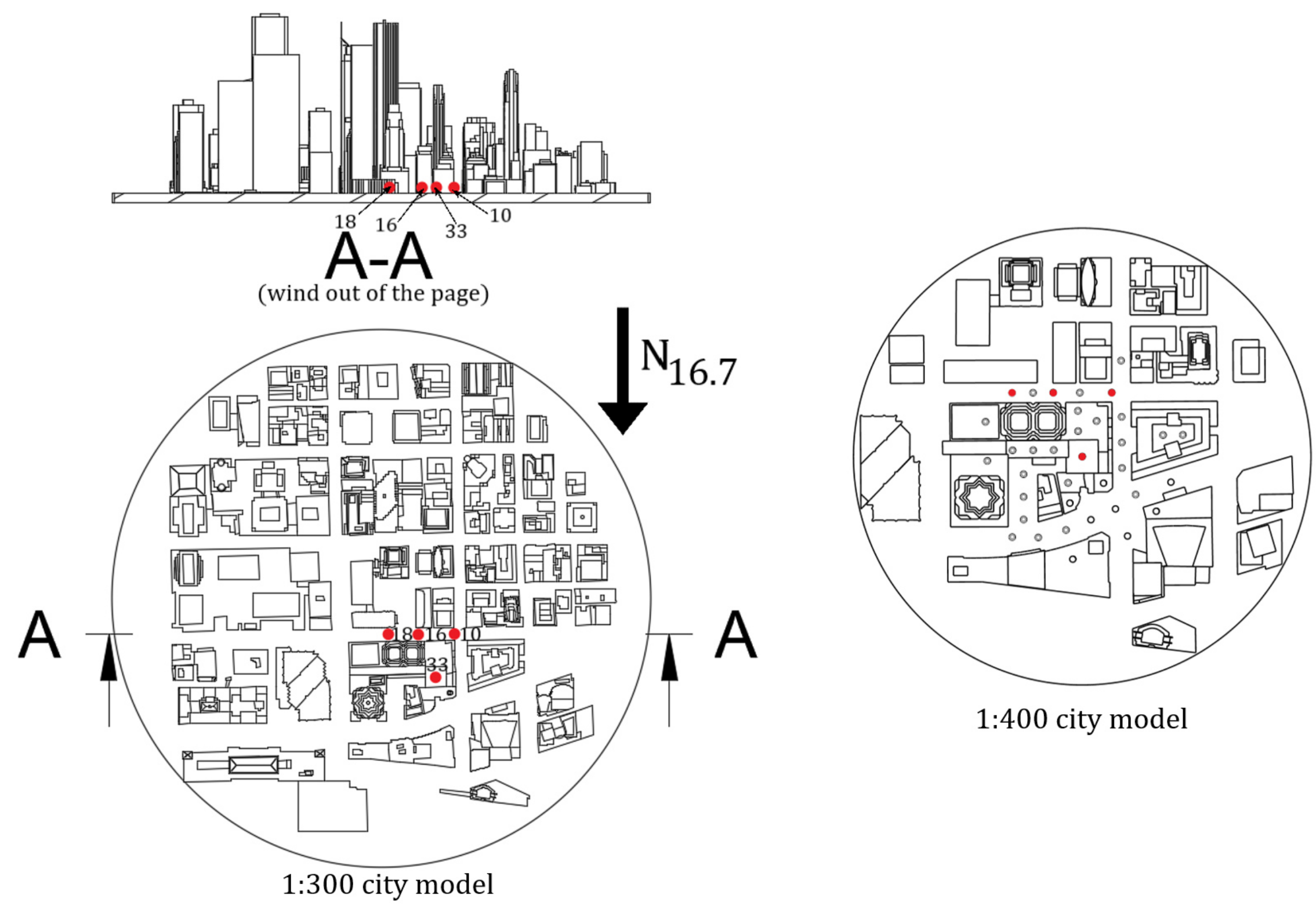

Figure 4.137: Measurement locations for the investigation of the near field modelling effect on data measurements. 

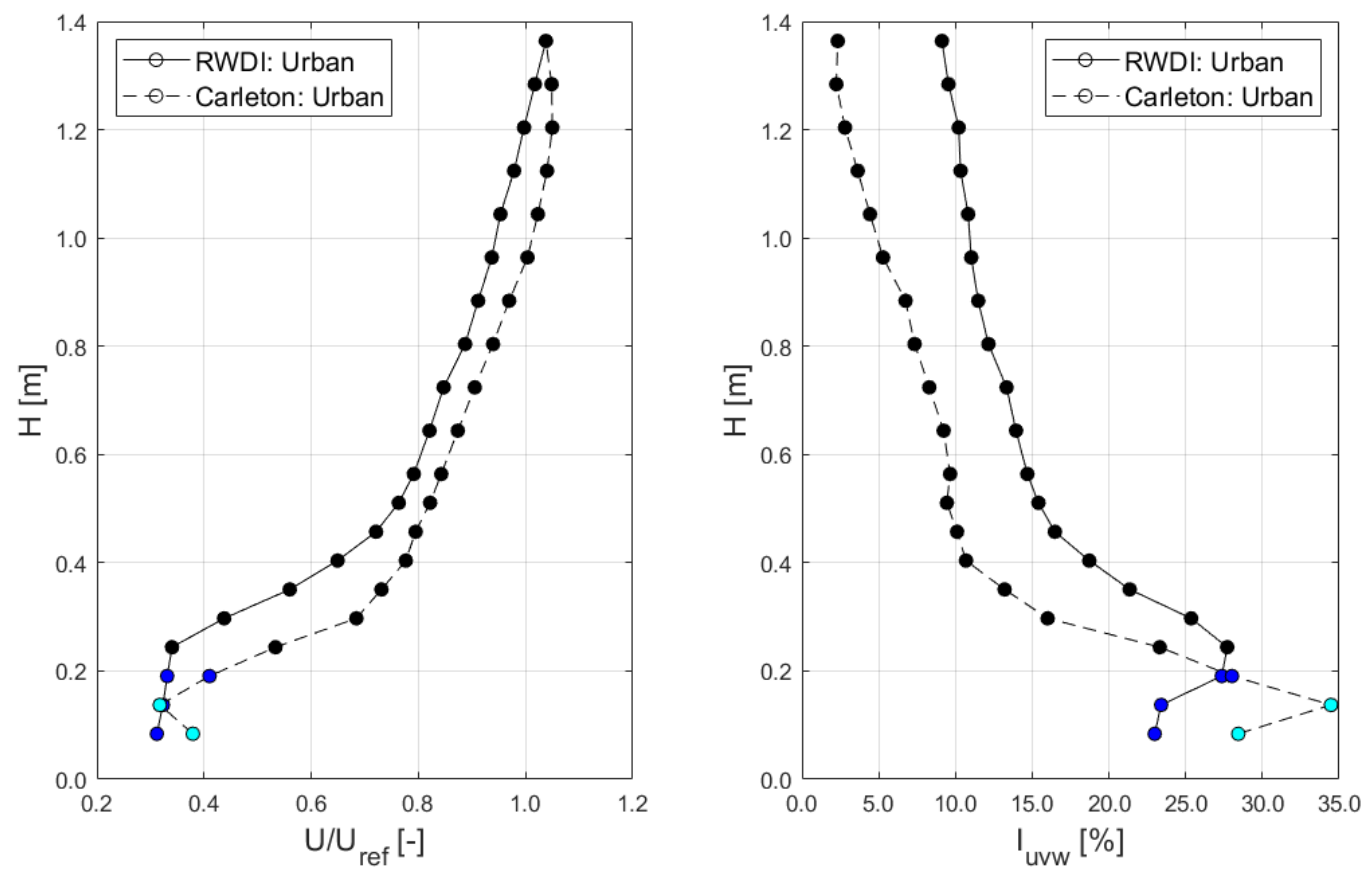

Figure 4.138: Comparisons between RWDI and CU profiles at Spot 10 in $\mathrm{N}_{16.7}$ wind direction for urban exposure. Left: normalized mean wind speed profiles. Right: overall turbulence intensity profiles. Total increments $=20$.
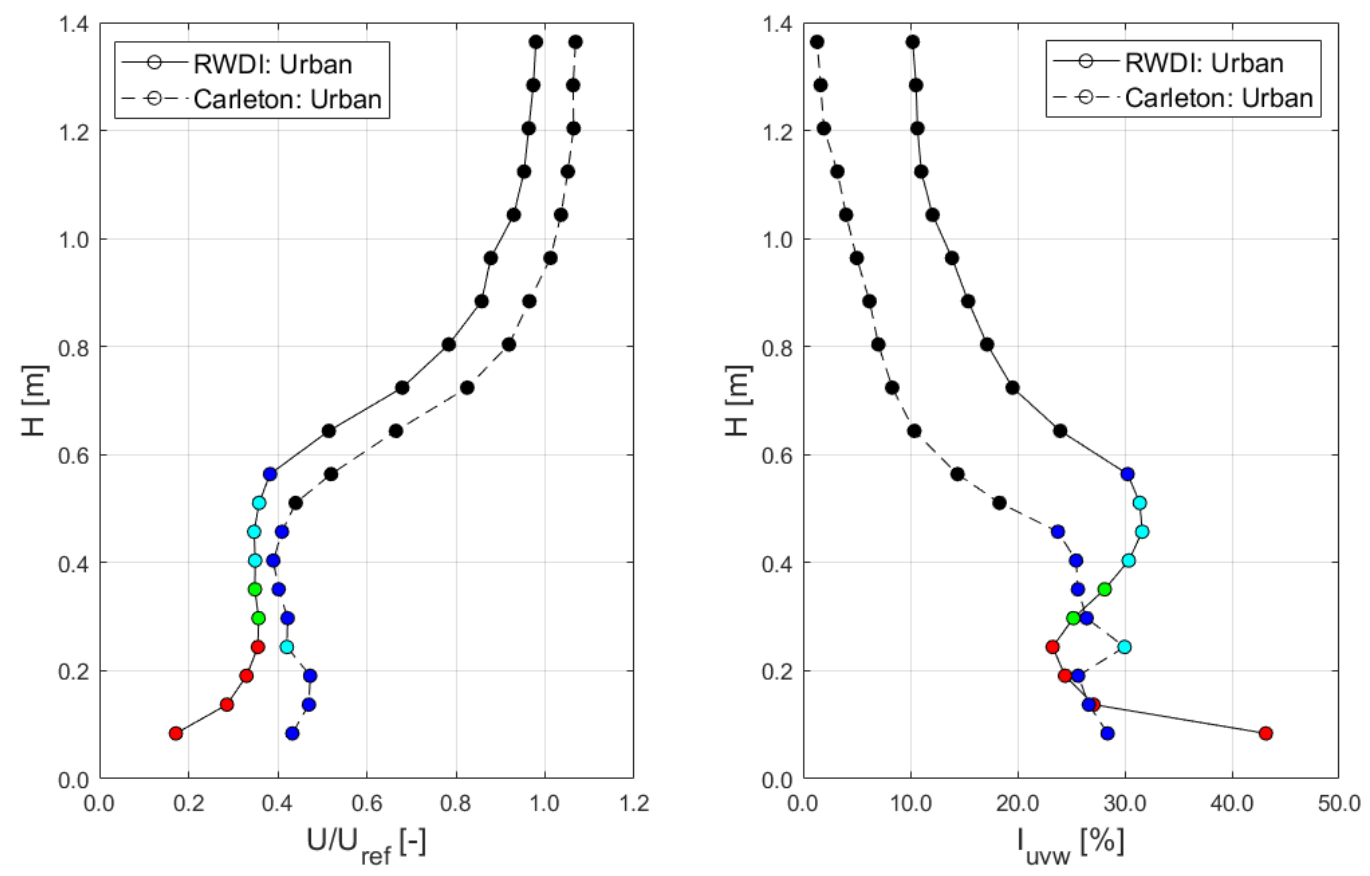

Figure 4.139: Comparisons between RWDI and CU profiles at Spot 16 in $\mathrm{N}_{16.7}$ wind direction for urban exposure. Left: normalized mean wind speed profiles. Right: overall turbulence intensity profiles. Total increments $=20$. 

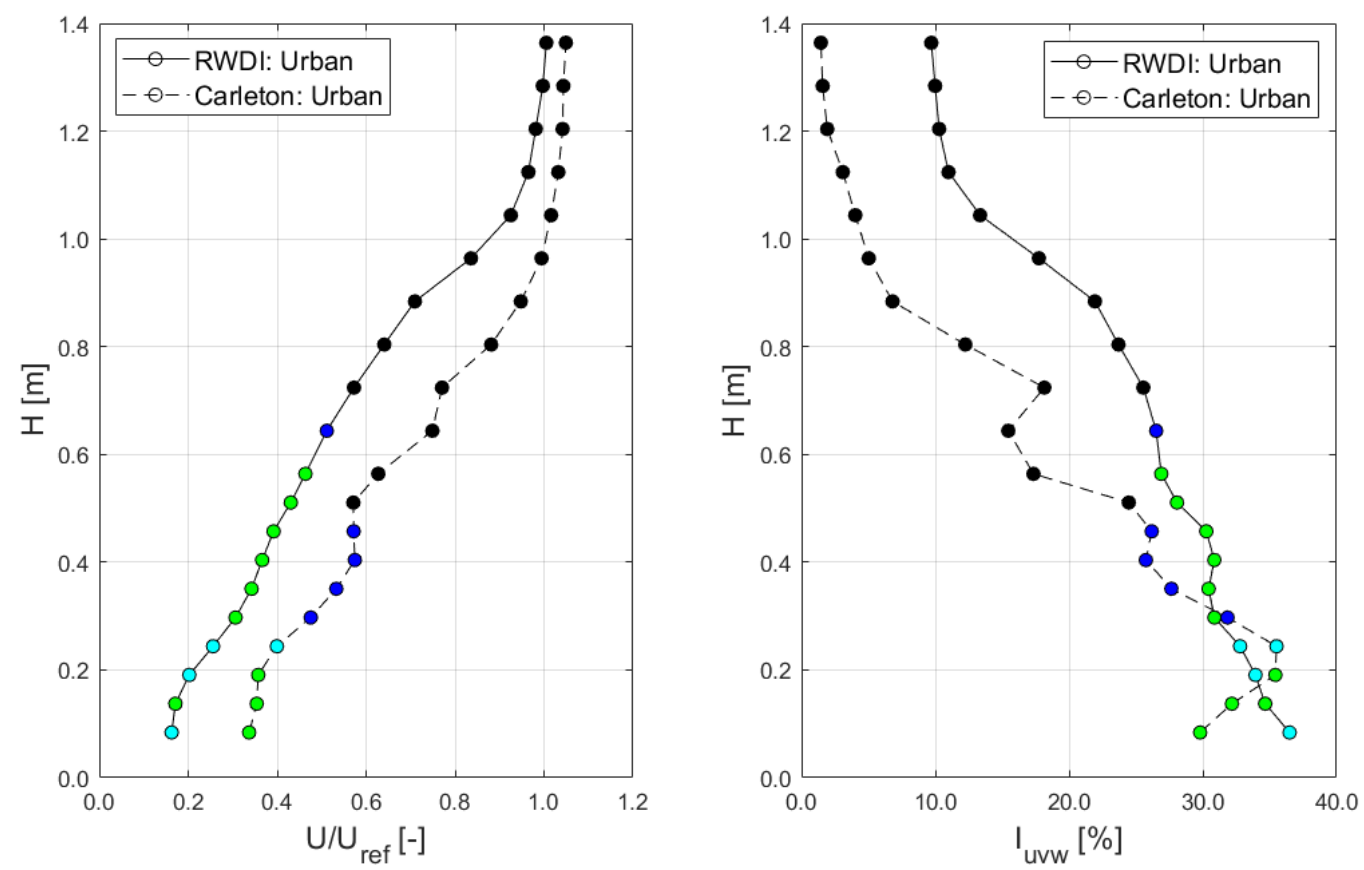

Figure 4.140: Comparisons between RWDI and CU profiles at Spot 18 in $\mathrm{N}_{16.7}$ wind direction for urban exposure. Left: normalized mean wind speed profiles. Right: overall turbulence intensity profiles. Total increments $=20$.
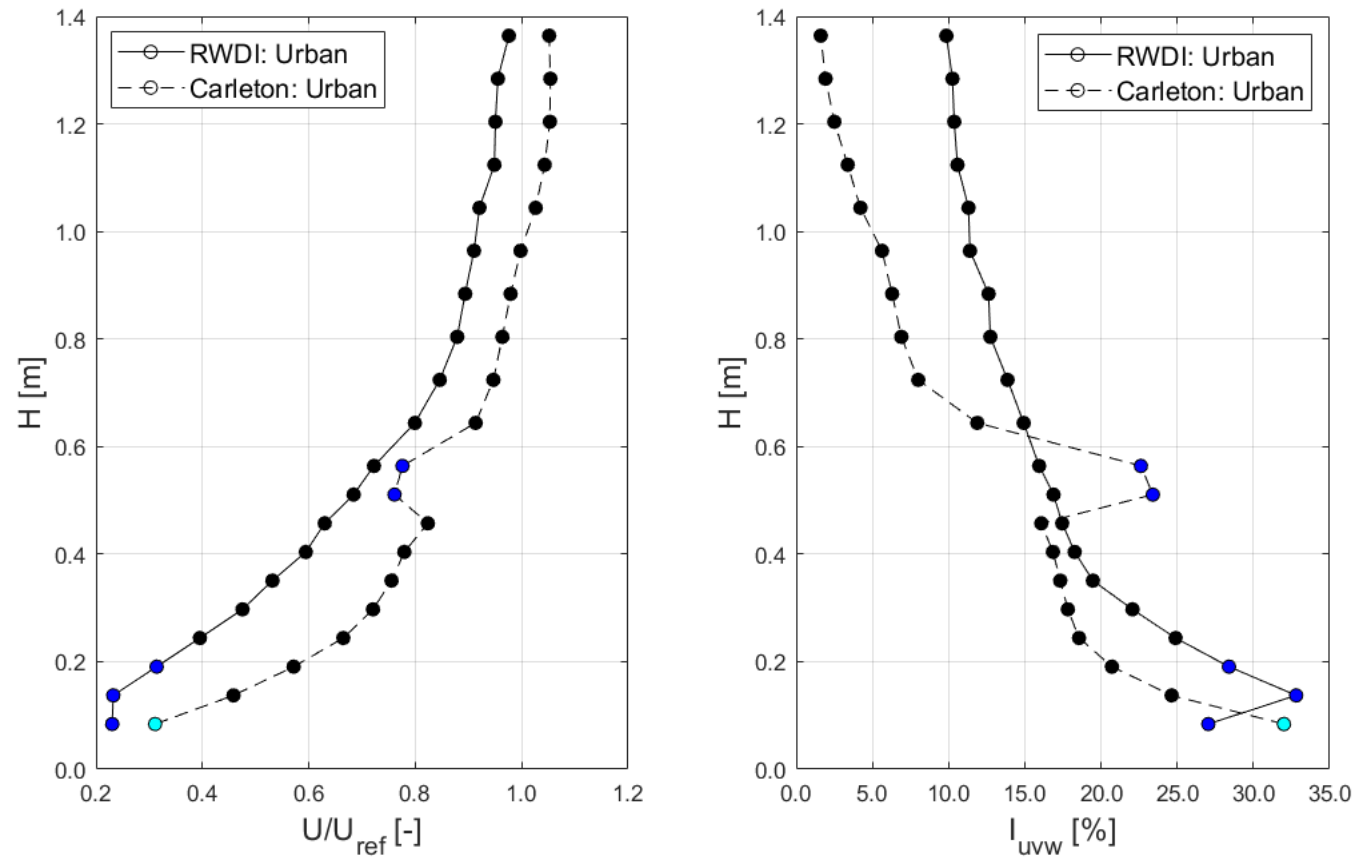

Figure 4.141: Comparisons between RWDI and CU profiles at Spot 33 in $\mathrm{N}_{16.7}$ wind direction for urban exposure. Left: normalized mean wind speed profiles. Right: overall turbulence intensity profiles. Total increments $=20$. 


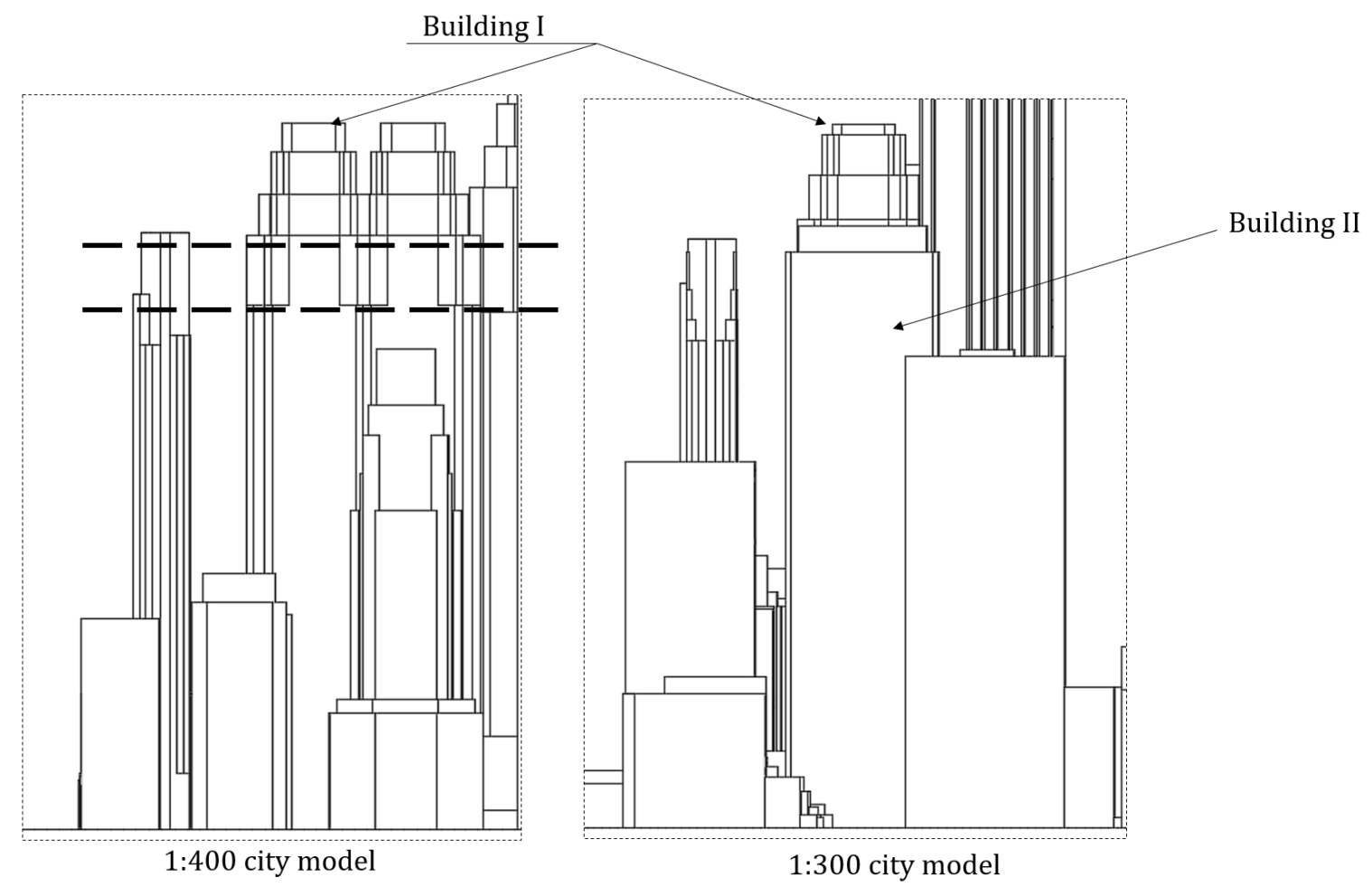

Figure 4.142: The upstream building layers of Spot 33 for both scaled models (wind direction is into the page).

\subsubsection{Lateral wake characteristics}

The reader is encouraged to consult Figure 3.42 for horizontal transects designations and locations, and Table 3.13 for the height at which the data were collected. Additionally, the 'velocity deficit' term used in this research refers to the reduction in the wind speed as opposed to the difference between the velocity values in and out of the wake.

\subsubsection{Transect 12}

Across-wind measurement were collected along $\mathrm{T}_{12}$ at $0.3507 \mathrm{~m}$ and $0.5640 \mathrm{~m}$ from the wind tunnel floor in $\mathrm{N}_{253.2}$ wind direction (see Figure 4.143). The velocity, turbulence 
intensity and flow angles data are plotted in Figures 4.144 and 4.145 for measurements at $0.3057 \mathrm{~m}$ and $0.5640 \mathrm{~m}$, respectively.

As can be seen in Figure 4.144, the normalized mean wind speed was in a state of reduction due to the upstream buildings wake with the maximum velocity deficit at $0.4 \mathrm{~m}$, which lies in the wake of the building that is closer to the transect with its width denoted by red lines in the same figure. Furthermore, the maximum velocity deficit, the location of which is denoted by red point in Figure 4.143 , was found at a region of high turbulence level, which is expected since turbulence and velocity are inversely related; in other words, regions with high turbulence are characterized by lower wind speeds. It should be noted that the flow yaw angle steeply changed from $-16^{\circ}$ to $4^{\circ}$ across the width of the nearby building, that is between Side Walls (SW) 3 and 4 . This could be related to the flow separation from the building side walls and the possible formation of counter-rotating vortices, which may justify the large amount of data that was not captured within the width of the building. Similarly, the flow pitch angle was steadily decreasing from $\mathrm{SW}_{2}$ to approximately the centerline of the building close to the transect.

The same pattern was found in measurements taken at $0.564 \mathrm{~m}$, with the maximum velocity deficit located at $0.4 \mathrm{~m}$, which is in the wake of the nearby building at the location of the highest turbulence level. However, the behaviour of the pitch flow angle was different as the flow was gradually deflecting downward until the traverse reached $\mathrm{SW}_{3}$, where a sudden reduction in the downdraft was continued across the width of the building. This is likely due to building I, which is located upstream of both tall buildings (see Figure 4.143). At a height of $0.3507 \mathrm{~m}$, building I was blocking the opening between both tall buildings; however, the opening was partially exposed to the incoming flow at $0.564 \mathrm{~m}$. 

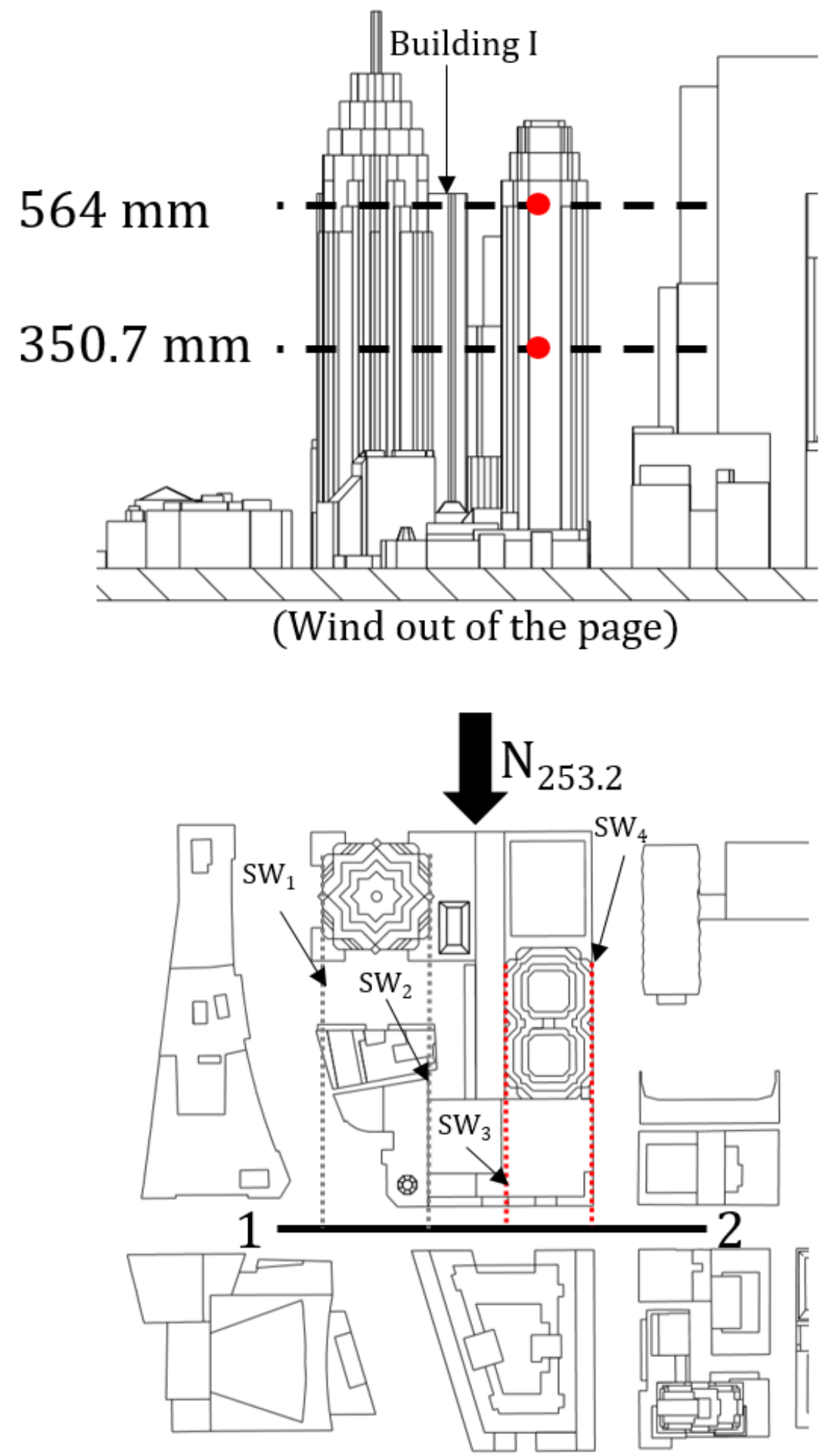

Figure 4.143: Location of $\mathrm{T}_{12}$. Top: locations of measurement height of the horizontal traverses. Bottom: starting point (1) and ending point (2) of the traverse. 

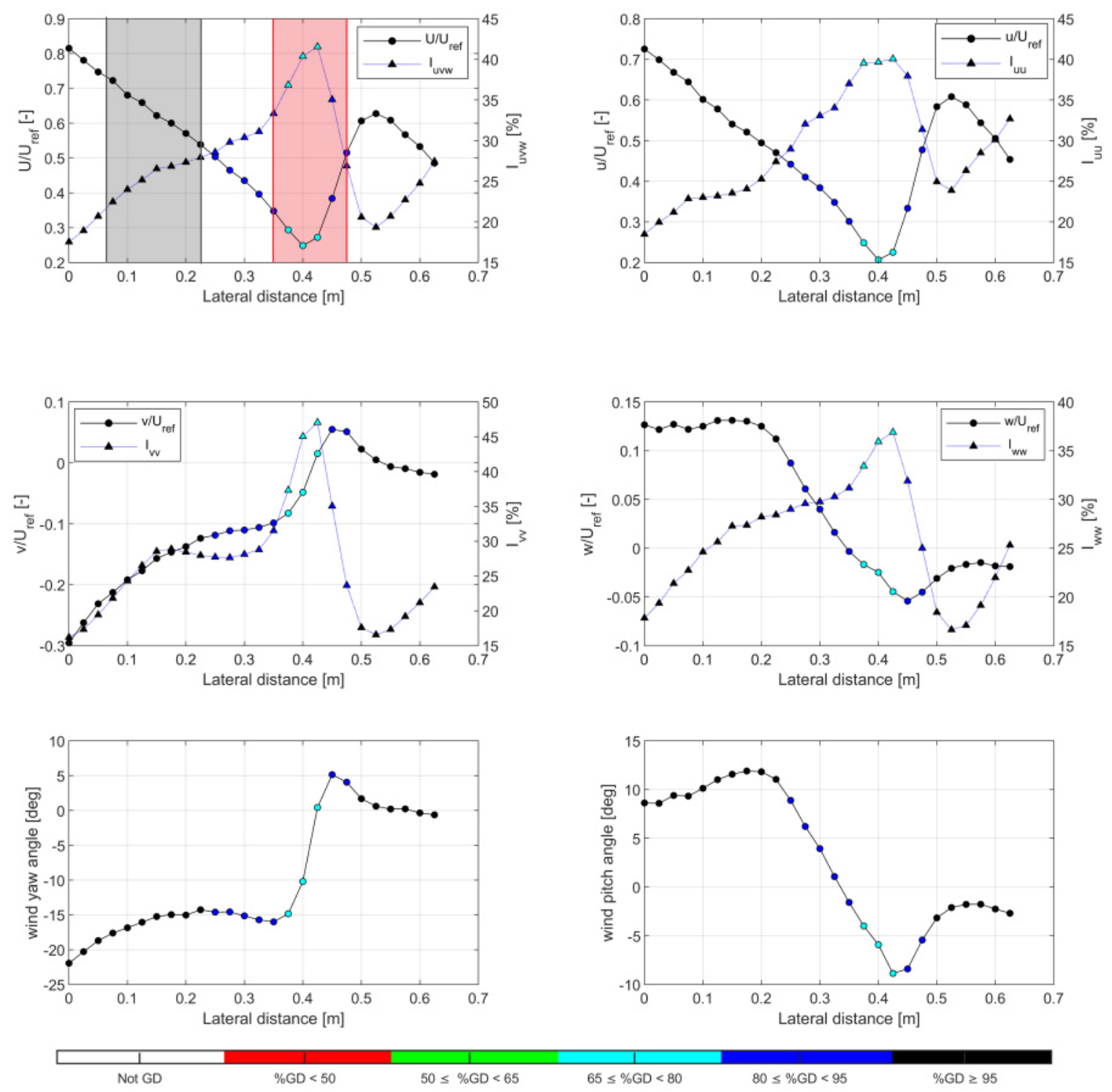

Figure 4.144: Lateral wake velocity, turbulence and flow angles data for $\mathrm{T}_{12}$ at $0.3507 \mathrm{~m}$ from the wind tunnel floor. The shaded regions in the mean wind speed plot represents the upstream buildings width. 

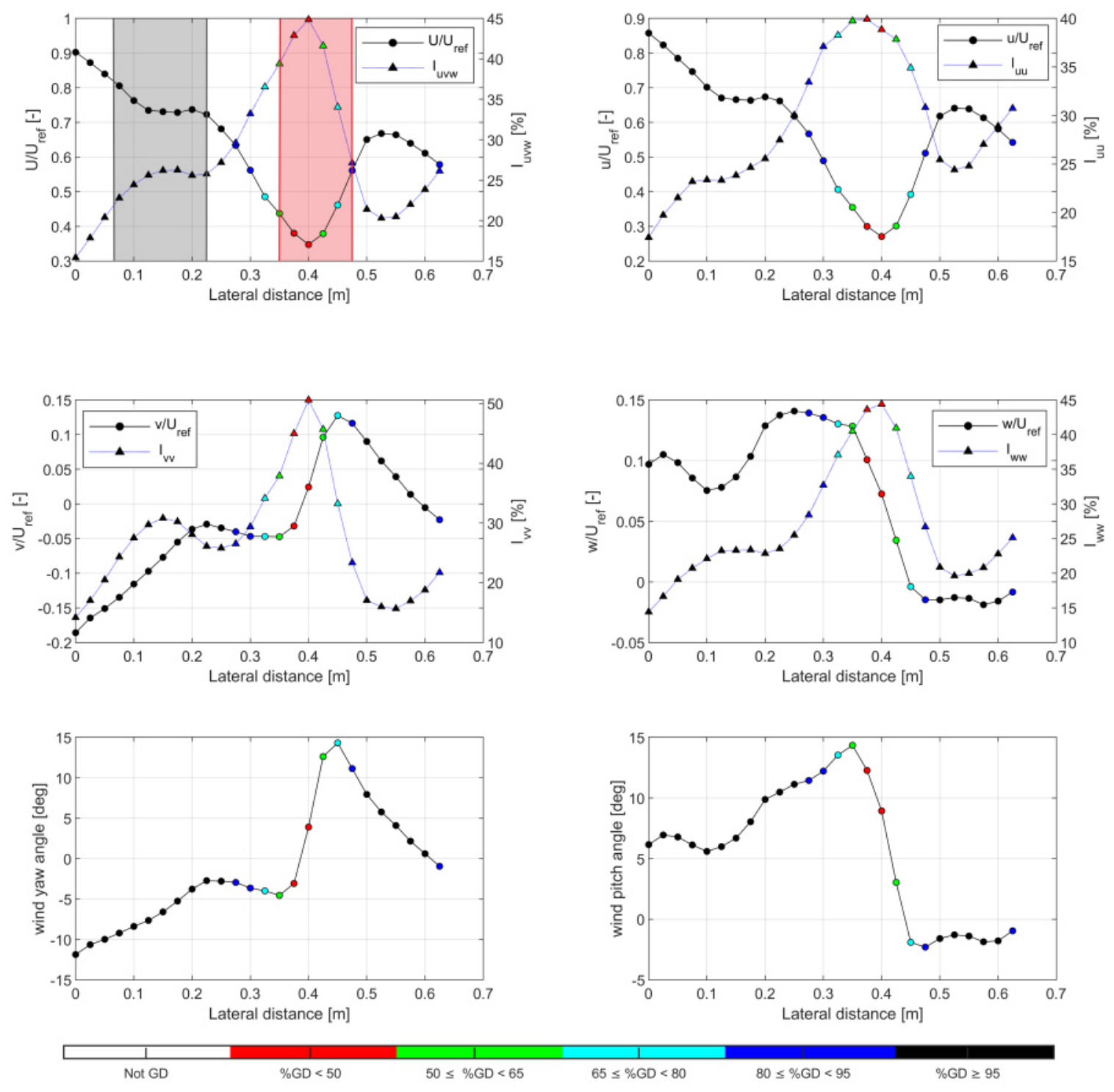

Figure 4.145: Lateral wake velocity, turbulence and flow angles data for $\mathrm{T}_{12}$ at $0.5640 \mathrm{~m}$ from the wind tunnel floor. The shaded regions in the mean wind speed plot represents the upstream buildings width. 


\subsubsection{Transect 34}

Lateral measurements were also collected along $\mathrm{T}_{34}$ at $0.564 \mathrm{~m}$ from the wind tunnel floor for wind direction $\mathrm{N}_{335}$ (see Figure 4.146). Transect 34 was subjected to the influence of the same buildings as transect 12, but from a different wind angle. The spacing between the buildings is narrow and it is obstructed by other buildings located upstream of the two buildings of interest. Additionally, the transect is located in close proximity to one of the tall buildings, whose sides are designated as $\mathrm{SW}_{1}$ and $\mathrm{SW}_{2}$. The lateral flow data of $\mathrm{T}_{34}$ are plotted in Figure 4.147 with shaded areas in the first plot to represent the upstream buildings width. From the plots, the maximum velocity deficit was found at a lateral distance of $0.175 \mathrm{~m}$ from the traverse starting point 3 $(0 \mathrm{~m})$, the location of which is denoted by red point in Figure 4.146. The location of the maximum velocity deficit indicates that the wake is not symmetrical, which is probably due to the oblique wind angle in addition to the multiple layers of buildings located upstream of the two buildings of interest. The turbulence intensity within the width of the first building $\left(\mathrm{SW}_{1}-\mathrm{SW}_{2}\right)$ shows large gradients with sharp peaks, which is related to the large fluctuations in the longitudinal velocity component in relation to the local mean wind speed. The sharp peaks in TI are more clear in the top right plot, which shows the longitudinal flow speed and turbulence data. The flow seems to recover within the wake of the second building $\left(\mathrm{SW}_{3}-\mathrm{SW}_{4}\right)$, which is consistent with the relatively steady pattern found in the wake of building $\left(\mathrm{SW}_{1}-\mathrm{SW}_{2}\right)$ in $\mathrm{T}_{12}$ at the same height, where both buildings were far from the transect according to their respective wind angles.

Some fluctuations in wind flow angles were observed, particularly within the wake of the first building $\left(\mathrm{SW}_{1}-\mathrm{SW}_{2}\right)$. The flow yaw angles were negative near to and past $\mathrm{SW}_{1}$ due to the flow separation from $\mathrm{SW}_{1}$, which was accompanied by upward flow. Within the width of the same building, the yaw angles were gradually changing 
from negative to positive values since the data measured were affected by the flow separation from the other side of the building. On the other hand, the variations in wind pitch angle could be related to the vortices and eddies that forms in the near wake of buildings since the transect was in very close-proximity to the building of $\mathrm{SW}_{1}-\mathrm{SW}_{2}$.

Some of the conclusions made in this section are based on data measured at low \%GD, which implies less accuracy in the data. However, the results are demonstrating useful qualitative information and trends, which provide insights about flow features of interest for urban air mobility operations. 

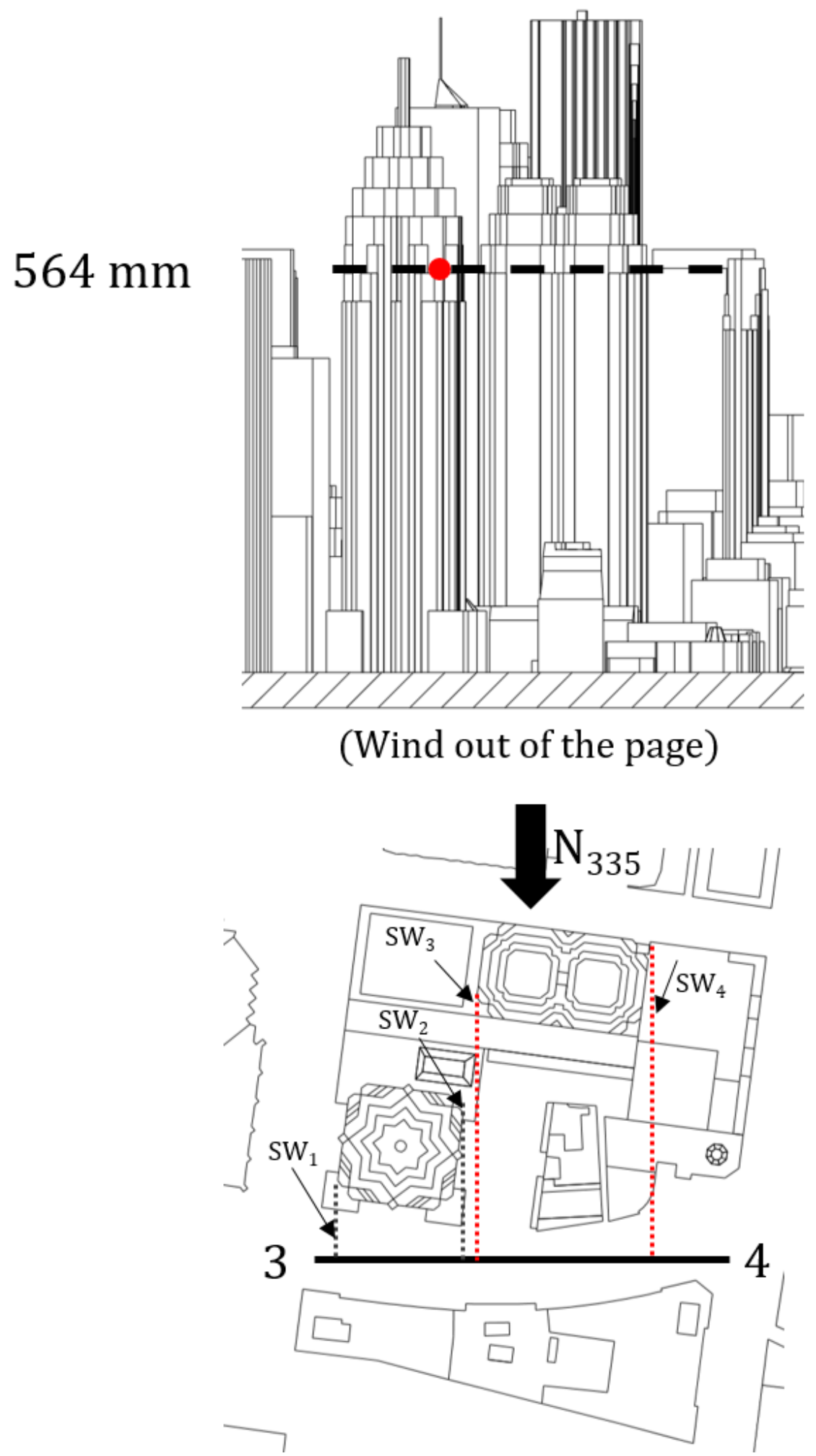

Figure 4.146: Location of $\mathrm{T}_{34}$. Top: location of measurement height of the horizontal traverse. Bottom: starting point (3) and ending point (4) of the traverse. 

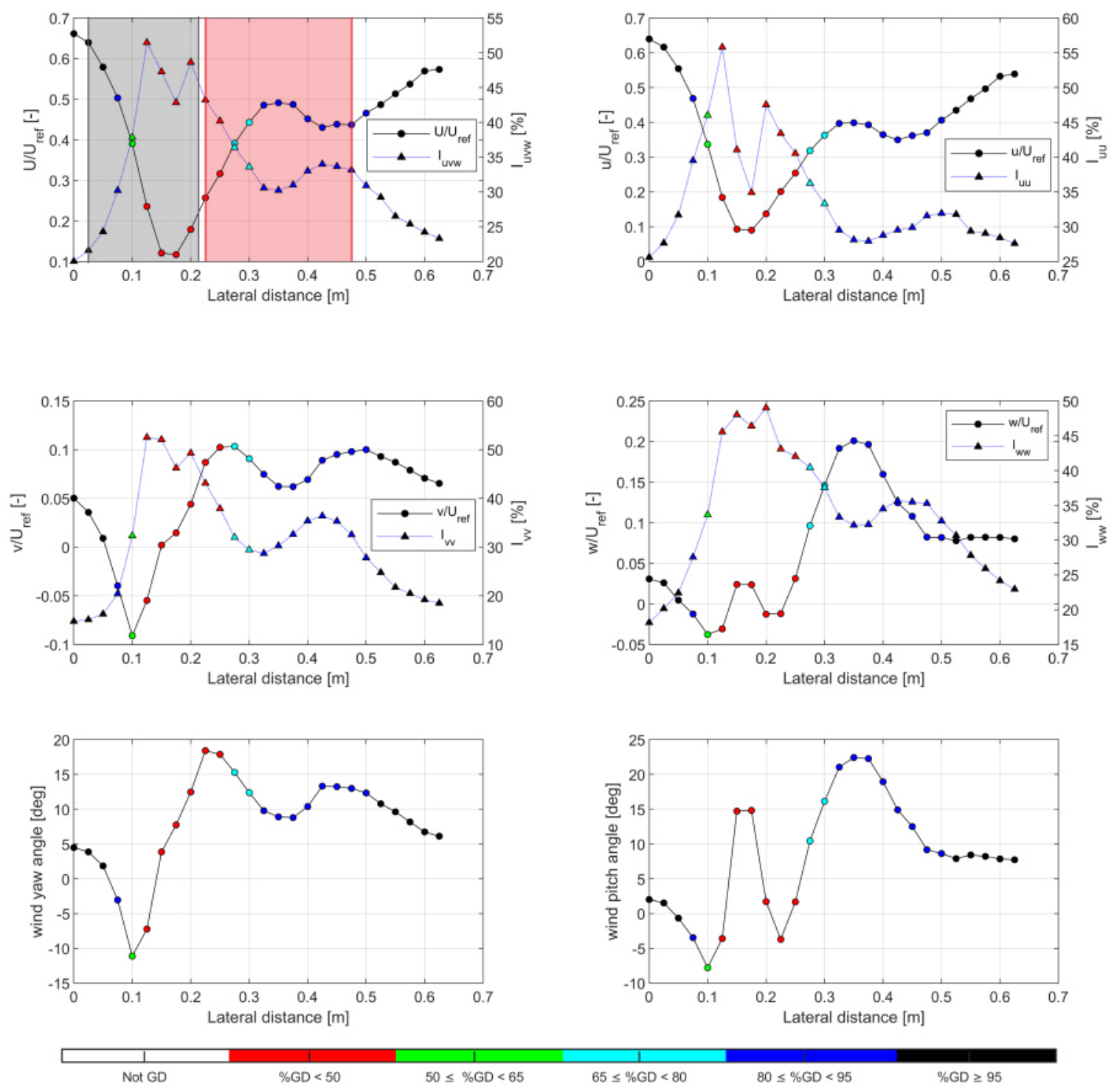

Figure 4.147: Lateral wake velocity, turbulence and flow angles data for $\mathrm{T}_{34}$ at $0.5640 \mathrm{~m}$ from the wind tunnel floor. The shaded regions in the mean wind speed plot represents the upstream buildings width. 


\subsubsection{Transect 56}

Horizontal profiles were measured along $\mathrm{T}_{56}$ at $0.4573 \mathrm{~m}$ from the wind tunnel floor to assess the impact of wind angle variations on the mean wind speed and turbulence characteristics downwind of buildings. The transect location and height with respect to the upstream building are shown in Figure 4.148. It should be noted that WSW and $\mathrm{WSW}_{+15}$ corresponds to the azimuth angles of $247.5^{\circ}$ and $262.5^{\circ}$, respectively.

The results plotted in Figure 4.149 demonstrate closer correspondence between the measurements at $\mathrm{N}_{253.2}$ and WSW $\left(247.5^{\circ}\right)$ wind directions; however, this closer agreement between the results is possibly related to the small change in wind angle between WSW and $\mathrm{N}_{253.2}$, which is equal to $5.7^{\circ}$. In contrast, the difference in wind angles between $\mathrm{N}_{253.2}$ and $\mathrm{WSW}_{+15}\left(262.5^{\circ}\right)$ is equal to $9.3^{\circ}$, which is probably a contributing factor for the distinguished offset of $\mathrm{WSW}_{+15}$ from the $\mathrm{N}_{253.2}$ profile. It is also believed that the upstream buildings have an influence on the velocity and turbulence behaviour since the model orientation either permits or obstructs the flow

movement around the building. This effect is clearly seen in $\mathrm{WSW}_{+15}$ data, which shows lower velocities and higher levels of turbulence outside the immediate upstream building boundaries since building I was obstructing the flow in the region to the right behind the tall building of interest. It should be noted that the building boundaries shown in the first plot of Figure 4.149 would slightly shift for WSW and WSW +15 wind angles.

The location of maximum velocity deficit appears to be insensitive to the model orientation giving the small angle change. However, it is believed that large angles may cause a shift in the location of the maximum velocity deficit. 


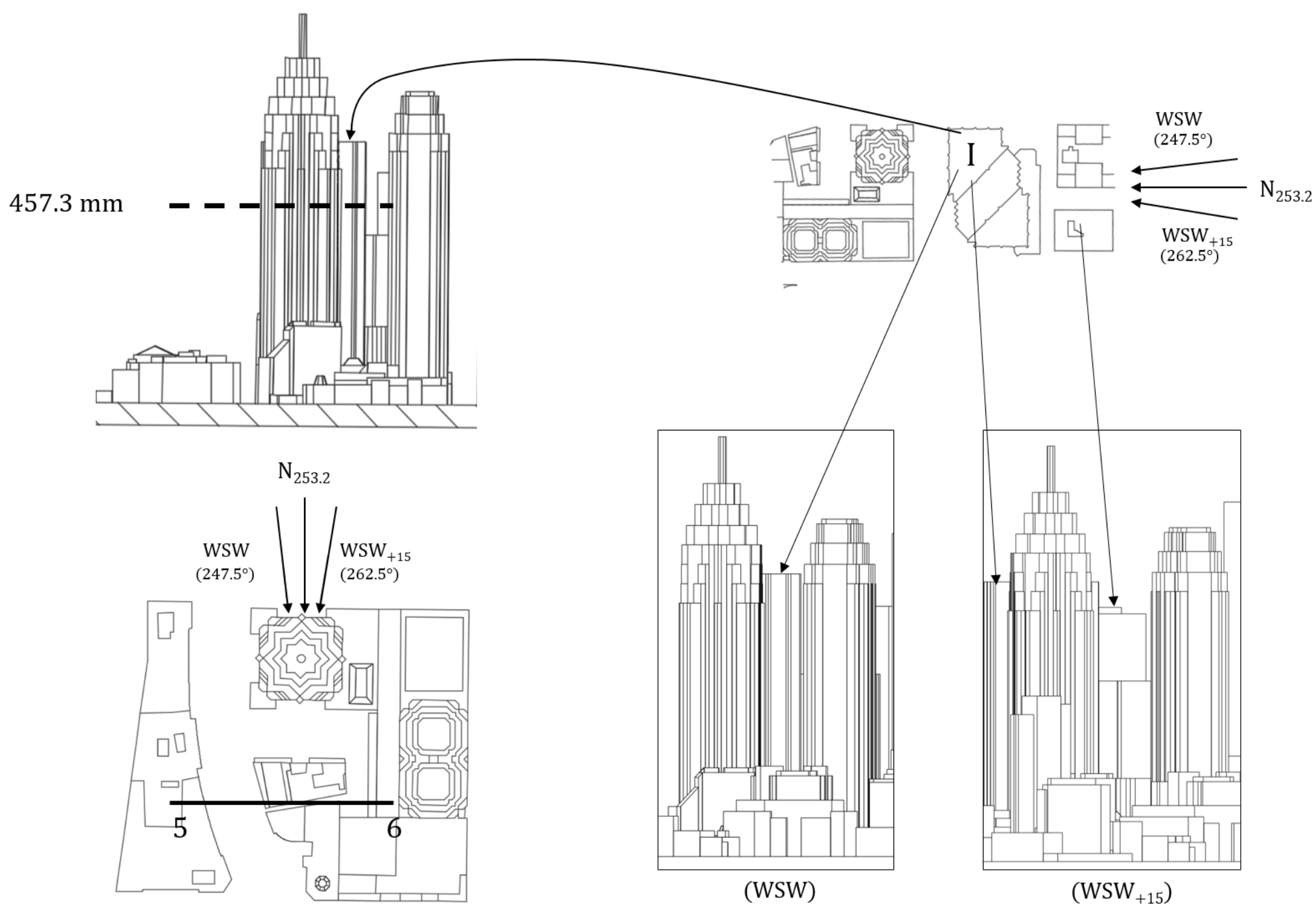

Figure 4.148: Location of $\mathrm{T}_{56}$. Top left: location of measurement height of the horizontal traverse. Bottom left: starting point (5) and ending point (6) of the traverse. Bottom right: the model profile views for WSW and $\mathrm{WSW}_{+15}$ wind directions. 

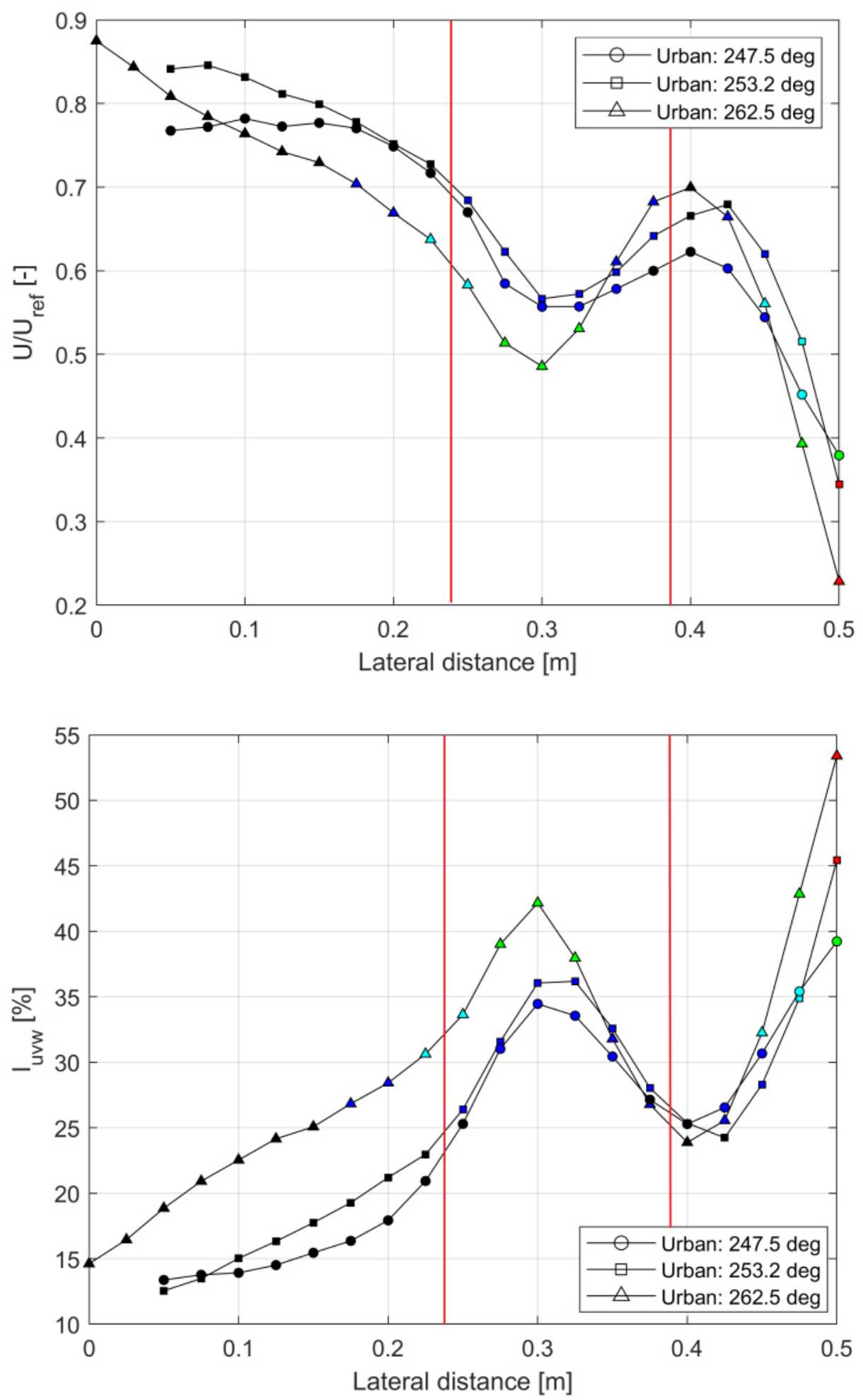

Figure 4.149: Mean wind speed and turbulence intensity profiles along $\mathrm{T}_{56}$ at 0.4573 $\mathrm{m}$ from the wind tunnel floor for three different wind directions. Vertical red lines represents the upstream building width for perpendicular wind direction $\left(\mathrm{N}_{253.2}\right)$. 


\section{Chapter 5}

\section{Conclusions, Contributions and Recommendations for Future Work}

Through wind tunnel testing on subscale city models, the research included within this thesis has successfully accomplished the stated goals, which were:

1. to analyse worst-case urban airflow characteristics that are most significant for the safe operation of VTOL air vehicles; and

2. to assess the significance of certain testing parameters on the quality of the measured data.

The main contributions of the presented work, the conclusions drawn from the complete body of work and the recommendations for future work are outlined in the following sections.

\subsection{Contributions}

The research encompassed within this thesis makes the following contributions:

- Investigation of the urban flow field characteristics of relevance to UAM applications had been conducted using an experimental approach through wind 
tunnel testing on scaled city models.

- Flow visualization experiment was performed to determine regions of high instabilities and to understand possible causes of the flow unsteadiness at certain locations.

- Investigations were done to assess the influence of certain testing parameters including model scale, approach flow exposure profile and near field modelling on the measured data.

- Ranges of certain flow features were identified from pre-determined extremes based on data measured with adequate accuracy.

- Locations of smooth and severe flow features were identified with respect to buildings geometry.

- Assessment of the lateral wake characteristics was done with primary focus on the velocity deficit, turbulence level and changes in flow angles downwind of buildings.

- Investigations were done to assess the impact on the flow field characteristics due to placing future buildings into the existing model.

- The influence of wind incidence angle on the flow speed, turbulence level, and flow angles was investigated for a selected spot that represents a potential vertipad location.

\subsection{Conclusions}

The main conclusions drawn from Carleton University wind tunnel experiment on the 1:400 scale city model are summarized below: 
- Extreme values:

- The wind speed within the urban environment may increase by $74 \%$ and decrease by $61 \%$ from the conditions found in flat areas/open lands.

- The wind direction within and above buildings level is generally different from what is reported by local weather stations. Additionally, the wind direction within the city itself varies based on the locality, where wind direction may change laterally by $77^{\circ}$, vertically upward by $20^{\circ}$ and vertically downward by $26^{\circ}$ from the direction of the free-stream flow. Larger wind angles are expected.

- The normalized wind speed in urban environments may change by 0.42 over a vertical distance of $24 \mathrm{~m}$ (FS). Horizontal and vertical wind directions may change by $50^{\circ}$ over a vertical distance of $24 \mathrm{~m}(\mathrm{FS})$ and $22^{\circ}$ over a vertical distance of $16 \mathrm{~m}(\mathrm{FS})$, respectively.

- The turbulence intensities within the height of the buildings can be severe, where $46 \%, 47 \%$ and $38 \%$ turbulence maybe found along-wind, acrosswind, and in the vertical direction, respectively.

- Flow features and model geometry:

- Smooth flow conditions were generally found well above buildings level and in spots clear of buildings obstruction.

- Flow speed increase and severe change in horizontal wind direction were found around buildings, particularly, at the edge of the separated flow.

- Severe reduction in wind speed, and hence, high levels of turbulence were heavily found in the wake of buildings.

- Moderate reduction in wind speed, high updrafts and downdrafts were found immediately upstream of a building due to the flow separation from 
the stagnation zone at the windward face of the building.

- Moderate updrafts were found on buildings rooftops, close to the surface level.

- Severe difference in wind speed (vertical speed shear) and horizontal wind direction (horizontal directional shear) can be found downwind of buildings, in layers below and above the buildings rooftop.

- Shift in vertical wind direction (vertical directional shear) can be found upstream of buildings at the stagnation zone due to the flow divergence in the upward and downward directions.

- High turbulence levels are not necessarily found at lower elevations, and can actually be found at higher elevations within the height of buildings.

- Effect of tall buildings can persist to a distance of over $200 \mathrm{~m}$ (FS) above buildings rooftops.

- The main wake of tall buildings can persist downstream for at least the same distance as the height of the buildings.

- No evidence of coherent vortex shedding was found along the entire height of a building.

- Evidences of vortex shedding were observed at different frequencies for the same flow components along the height of buildings of the same crosssection.

- Evidences of vortex shedding were observed at the same and different frequencies in $\mathrm{u}$ and $\mathrm{v}$ flow components at certain elevations downwind of buildings of uniform cross-section.

- Evidence of flow reversals and re-circulation was observed in buildings near-wake from the flow visualization experiment. 
The main conclusions drawn from the RWDI wind tunnel experiment on the 1:300 scale city model are summarized below.

- Model scale has no impact on model measurements considering that the model scale is within the recommended range for model testing of large buildings and structures.

- The effect of approach flow exposure profile is most notable in measurement locations that are above the average height of buildings or in measurement locations at the edge of the model.

- The effect of approach flow exposure profile is minimal for measurements in wellmixed flow with highly dense upwind terrain that consists of high-rise buildings.

- The effect of near field modelling is highly significant and it impacts the accuracy of the measured data.

- Velocity deficit in the wake of buildings was found to be utmost within the boundaries of the most nearby building.

Clearly, flow conditions in cities are not smooth, and severe flow features could be found in various locations and altitudes within the urban canopy. Adding to the severe flow conditions which may be expected at certain locations, some flow features could be found at unexpected locations owing to the combined effects of buildings arrangement. Such factors strongly impact the flying vehicle stability, and impose challenges in defining safe zones of operation within the height of buildings. Therefore, UAS operators need to consider those factors and account for such unexpected flow behaviours. Plus, well defined regulations need to be issued before allowing the use of UAS within cities since loss of control of a flying vehicle over a populated area would result in catastrophic events that could have been avoided with proper flight 
planning and laws, along with robust control systems that are designed to overcome the complex airflow structures.

\subsection{Future work}

Recommendations for possible future work are summarized in the following list.

1. Flight dynamics models of different air vehicle configurations with different control systems could be developed and tested by using the experimental airflow data collected in this research to investigate their dynamics response and control within the identified severe airflow features. Work related to this recommendation is already being carried out for a Bell Nexus-like configuration by a colleague within the research group 75$]$.

2. Following Task 1, critical ranges of each of the investigated flow features could be identified for different air vehicle configurations.

3. Red-flag (complex flow) regions for UAM applications can be identified in the city model by considering the combined effects of the flow features at certain locations.

4. Additional data collection techniques such as Laser Doppler Anemometry could be used to capture flow reversals.

5. Wind tunnel experiments of different urban topologies could be conducted to:

(a) determine the range of each of the flow features based on multiple models of different cities or multiple models representing different parts of a large city; and

(b) develop additional confidence in relating certain flow features to the buildings geometry. 
6. In this research, airflow measurements were conducted from a wind engineering point of view, where natural wind was approximated with quasi-steady approach by considering turbulent boundary layer flow with locally stationary mean and turbulent speed characteristics. In nature, flow data are dependent on the incoming wind characteristics, which change continuously with time, and hence are non-stationary. Therefore, flow measurements could be done with considerations to the airflow non-stationarity. 


\section{List of References}

[1] K. Davis, "The origin and growth of urbanization in the world," American Journal of Sociology, vol. 60, no. 5, pp. 429-437, 1955.

[2] E. Ng, "Policies and technical guidelines for urban planning of high-density cities - air ventilation assessment (ava) of hong kong," Building and Environment, vol. 44, no. 7, pp. 1478-1488, 2009. The 6th International Conference on Indoor Air Quality, Ventilation \& Energy Conservation in Buildings (IAQVEC 2007), Sendai, Japan, 28-31 October, 2007.

[3] I. Panagiotou, M. K.-A. Neophytou, D. Hamlyn, and R. E. Britter, "City breathability as quantified by the exchange velocity and its spatial variation in real inhomogeneous urban geometries: An example from central london urban area," Science of The Total Environment, vol. 442, pp. 466-477, 2013.

[4] J. Song, S. Fan, W. Lin, L. Mottet, H. Woodward, M. D. Wykes, R. Arcucci, D. Xiao, J.-E. Debay, H. ApSimon, E. Aristodemou, D. Birch, M. Carpentieri, F. Fang, M. Herzog, G. R. Hunt, R. L. Jones, C. Pain, D. Pavlidis, A. G. Robins, C. A. Short, and P. F. Linden, "Natural ventilation in cities: the implications of fluid mechanics," Building Research \& Information, vol. 46, no. 8, pp. 809-828, 2018.

[5] W. Janssen, B. Blocken, and T. van Hooff, "Pedestrian wind comfort around buildings: Comparison of wind comfort criteria based on whole-flow field data for a complex case study," Building and Environment, vol. 59, pp. 547-562, 2013.

[6] V. N. Varapaev and A. V. Doroshenko, "Methodology for the prediction and the assessment of pedestrian wind environment around buildings," Procedia Engineering, vol. 91, pp. 200-203, 2014. XXIII R-S-P Seminar, Theoretical Foundation of Civil Engineering (23RSP) (TFoCE 2014). 
[7] E. J. Plate and H. Kiefer, "Wind loads in urban areas," Journal of Wind Engineering and Industrial Aerodynamics, vol. 89, no. 14, pp. 1233-1256, 2001. Bluff Body Aerodynamics and Applications.

[8] A. Elshaer, A. Gairola, K. Adamek, and G. Bitsuamlak, "Variations in wind load on tall buildings due to urban development," Sustainable Cities and Society, vol. 34, pp. 264-277, 2017.

[9] J. Fackrell, "Parameters characterising dispersion in the near wake of buildings," Journal of Wind Engineering and Industrial Aerodynamics, vol. 16, pp. 97-118, 1984.

[10] Y. Tominaga and T. Stathopoulos, "Ten questions concerning modeling of nearfield pollutant dispersion in the built environment," Building and Environment, vol. 105, pp. 390-402, 2016.

[11] S. Karra, L. Malki-Epshtein, and M. K.-A. Neophytou, "Air flow and pollution in a real, heterogeneous urban street canyon: A field and laboratory study," Atmospheric Environment, vol. 165, pp. 370-384, 2017.

[12] D. Hertwig, H. L. Gough, S. Grimmond, J. F. Barlow, C. W. Kent, W. E. Lin, A. G. Robins, and P. Hayden, "Wake characteristics of tall buildings in a realistic urban canopy," Boundary-Layer Meteorology, vol. 172, no. 2, pp. 239-270, 2019.

[13] D. Galway, J. Etele, and G. Fusina, Modeling of the Urban Gust Environment with Application to Autonomous Flight.

[14] D. Galway, J. Etele, and G. Fusina, "Development and implementation of an urban wind field database for aircraft flight simulation," Journal of Wind Engineering and Industrial Aerodynamics, vol. 103, pp. 73-85, 2012.

[15] S. A. Raza and J. Etele, Autonomous Position Control Analysis of Quadrotor Flight in Urban Wind Gust Conditions.

[16] M. J. Logan, E. Bird, L. Hernandez, and M. Menard, Operational Considerations of Small UAS in Urban Canyons.

[17] B. Z. Cybyk, B. E. McGrath, T. M. Frey, D. G. Drewry, J. F. Keane, and G. Patnaik, "Unsteady airflows and their impact on small unmanned air systems in urban environments," Journal of Aerospace Information Systems, vol. 11, no. 4, pp. 178-194, 2014. 
[18] S. A. Raza, Autonomous UAV Control for Low-Altitude Flight in an Urban Gust Environment. PhD thesis, Carleton University, 2015.

[19] M. Llaguno-Munitxa, E. Bou-Zeid, and M. Hultmark, "The influence of building geometry on street canyon air flow: validation of large eddy simulations against wind tunnel experiments," Journal of Wind Engineering and Industrial Aerodynamics, vol. 165, pp. 115-130, 2017.

[20] "Considerations in vertipad design." https://rwdi.com/assets/factsheets/ RWDI-Insights-Considerations-in-Vertipad-Design.pdf. RWDI Inc.

[21] K. Jayyaratnam, R. Shilston, and D. Hackett, "The use of cfd and wind tunnel testing in wind microclimate assessments," in Proceedings of the Symposium on Simulation for Architecture and Urban Design, SIMAUD '17, (San Diego, CA, USA), Society for Modelling and Simulation International, 2017.

[22] D. C. Ompad, S. Galea, and D. Vlahov, "Urbanicity, urbanization, and the urban environment," in Macrosocial determinants of population health, pp. 5369, Springer, 2007.

[23] F. Emejeamara, A. Tomlin, and J. Millward-Hopkins, "Urban wind: Characterisation of useful gust and energy capture," Renewable Energy, vol. 81, pp. 162$172,2015$.

[24] H. Teunissen, "Characteristics of the mean wind and turbulence in the planetary boundary layer," tech. rep., Toronto Univ Downsview (Ontario) Inst For Aerospace Studies, 1970.

[25] R. D. Blevins, "Applied fluid dynamics handbook," New York, 1984.

[26] "Wind-tunnel studies of buildings and structures," Journal of Aerospace Engineering, vol. 9, no. 1, pp. 19-36, 1996.

[27] ESDU, "Characteristics of atmospheric turbulence near the ground," Part I, defnitions and general infromation, Engineering Sciences Data, no. 74030, 1974.

[28] M. Lateb, R. N. Meroney, M. Yataghene, H. Fellouah, F. Saleh, and M. Boufadel, "On the use of numerical modelling for near-field pollutant dispersion in urban environments- a review," Environmental Pollution, vol. 208, pp. 271-283, 2016.

[29] L. Wang, D. Li, Z. Gao, T. Sun, X. Guo, and E. Bou-Zeid, "Turbulent transport of momentum and scalars above an urban canopy," Boundary-layer meteorology, vol. 150, no. 3, pp. 485-511, 2014. 
[30] P. Kastner-Klein, M. Rotach, and E. Fedorovich, "Experimental study on mean flow and turbulence characteristics in an urban roughness sublayer (formerly paper p6b. 2)," in 14th Symposium on Boundary Layer and Turbulence, 2000.

[31] A. G. Davenport, "The dependence of wind loads on meteorological parameters," Proceedings International Research Seminar on Wind effects on buildings and structures, vol. Vol 1, pp. P19-82, Sept.1967.

[32] S. Recoskie, E. Lanteigne, and W. Gueaieb, "A high-fidelity energy efficient path planner for unmanned airships," Robotics, vol. 6, no. 4, p. 28, 2017.

[33] H. Barber and A. Wall, "Urban airflow: what drone pilots need to know," tech. rep., National Research Council of Canada. Aerospace Research Centre, 2021.

[34] D. Micallef and G. Van Bussel, "A review of urban wind energy research: aerodynamics and other challenges," Energies, vol. 11, no. 9, p. 2204, 2018.

[35] L. Merlier, F. Kuznik, G. Rusaouën, and S. Salat, "Derivation of generic typologies for microscale urban airflow studies," Sustainable cities and society, vol. 36, pp. 71-80, 2018.

[36] G. Buresti, "Lecture notes in bluff-body aerodynamics." https://www.mech.kth. se/courses/5C1211/BluffBodies.pdf. Department of Aerospace Engineering University of Pisa, June, 2000.

[37] W. Rodi, "Comparison of les and rans calculations of the flow around bluff bodies," Journal of Wind Engineering and Industrial Aerodynamics, vol. 69-71, pp. 55-75, 1997. Proceedings of the 3rd International Colloqium on Bluff Body Aerodynamics and Applications.

[38] W. Beranek, Wind Environment Around Single Buildings of Rectangular Shape. Stevin-Laboratory of the Department of Civil Engineering, Delft University ..., 1984.

[39] B. Blocken and J. Carmeliet, "Pedestrian wind environment around buildings: Literature review and practical examples," Journal of Thermal Envelope and Building Science, vol. 28, no. 2, pp. 107-159, 2004.

[40] J. Leene, D. Delaunay, and A. Jensen, "Handbook on obstacle wake effects related to wind turbine siting," tech. rep., TNO, 1990. 
[41] S. Dutta, K. Muralidhar, and P. Panigrahi, "Influence of the orientation of a square cylinder on the wake properties," Experiments in fluids, vol. 34, no. 1, pp. 16-23, 2003.

[42] M. M. ALAM, Y. ZHOU, and X. W. WANG, "The wake of two side-by-side square cylinders," Journal of Fluid Mechanics, vol. 669, p. 432-471, 2011.

[43] T. R. Oke, "Street design and urban canopy layer climate," Energy and buildings, vol. 11, no. 1-3, pp. 103-113, 1988.

[44] M. Jedrzejewski, M. Pocwierz, and K. Zielonko-Jung, "The problem of airflow around building clusters in different configurations," Archive of Mechanical Engineering, vol. 64, no. 3, 2017.

[45] J. Mironova, "Wind impact on low-rise buildings when placing high-rises into the existing development," in IOP Conference Series: Materials Science and Engineering, vol. 890, p. 012055, IOP Publishing, 2020.

[46] K. A. Adkins, "Urban flow and small unmanned aerial system operations in the built environment," International Journal of Aviation, Aeronautics, and Aerospace, vol. 6, no. 1, p. 10, 2019.

[47] P. Irwin, "Vortices and tall buildings: A recipe for resonance," Physics Today, vol. 63, pp. 68-69, 2010.

[48] "Flying your drone safely and legally." https://tc.canada.ca/en/aviation/ drone-safety/learn-rules-you-fly-your-drone/flying-your-drone-safely-legally Transport Canada, Sept. 2020.

[49] S. Tavoularis, Measurement in fluid mechanics. Cambridge University Press, 2005.

[50] J. B. Barlow, W. H. Rae, and A. Pope, Low-speed wind tunnel testing. John wiley \& sons, 1999.

[51] S. McTavish, Identification of wind turbine testing practices and investigation of the performance benefits of closely-spaced lateral wind farm configurations. $\mathrm{PhD}$ thesis, Carleton University, 2013.

[52] J. E. Cermak and N. Isyumov, Wind Tunnel Studies of Buildings and Structures. Reston, VA: American Society of Civil Engineers, 1999. 
[53] H. P. A. H. Irwin, "The design of spires for wind simulation," Journal of Wind Engineering and Industrial Aerodynamics, vol. 7, pp. 361-366, 1981.

[54] H. P. A. H. Irwin, "Design and use of spires for natural wind simulation," tech. rep., Ottawa, Canada, 1979.

[55] "Bell nexus." https://www.bellflight.com/products/bell-nexus. Bell Textron Inc., Accessed on June 27, 2021.

[56] "Personal communication." RWDI Inc., Feb., 2020.

[57] N. Isyumov, "Wind engineering terminology," in 14th International Conference of Wind Engneering (ICWE-14). Porto Alegre, Brazil. 18p, 2015.

[58] G. L. Larose and N. Franck, "Early wind engineering experiments in denmark," Journal of Wind Engineering and Industrial Aerodynamics, vol. 72, pp. 493499, 1997. Selected papers from the Ninth International Symposium on Wind Engineering.

[59] B. W. J, Wind Environment around Single Buildings of Rectangular Shape, vol. 29. Delft University of Technology, 1984.

[60] S. J. Lee, G. S. Mun, Y. G. Park, and M. Y. Ha, "A numerical study on fluid flow around two side-by-side rectangular cylinders with different arrangements," Journal of Mechanical Science and Technology, vol. 33, no. 7, pp. 3289-3300, 2019.

[61] J. Mao and N. Gao, "The airborne transmission of infection between flats in high-rise residential buildings: A review," Building and environment, vol. 94, pp. 516-531, 2015.

[62] X. Xie, Z. Huang, and J.-s. Wang, "Impact of building configuration on air quality in street canyon," Atmospheric Environment, vol. 39, no. 25, pp. 4519 4530, 2005.

[63] S. C. Yen and J. H. Liu, "Wake flow behind two side-by-side square cylinders," International Journal of Heat and Fluid Flow, vol. 32, no. 1, pp. 41-51, 2011.

[64] ASHRAE@ Handbook-Fundamentals (SI Edition). Ch.24 Flow Patterns around Isolated, Rectangular Block-Type Buildings. American Society of Heating, Refrigerating and Air-Conditioning Engineers, Inc. (ASHRAE), 2017. 
[65] "Getting started series 100 cobra probe." https://www.turbulentflow.com.au/ Downloads/Getting\%20Started\%20-\%20Cobra\%20Probe.pdf, 2015. Turbulent Flow Instrumentation. Accessed on June 16, 2021.

[66] "Cobra probe." https://www.turbulentflow.com.au/Downloads/Cat_ CobraProbe.pdf. Turbulent Flow Instrumentation. Accessed on June 16, 2021.

[67] S. Mallipudi, M. Selig, and K. Long, "Use of a four hole cobra pressure probe to determine the unsteady wake characteristics of rotating objects," in 24th AIAA Aerodynamic Measurement Technology and Ground Testing Conference, p. 2299, 2004.

[68] W. J. Yang, Handbook of flow visualization. Routledge, 2018.

[69] "Personal communication." RWDI Inc., June, 2021.

[70] C. K. Choi and D. K. Kwon, "Determination of the strouhal number based on the aerodynamic behavior of rectangular cylinders," Wind and Structures, vol. 3, no. 3, pp. 209-220, 2000.

[71] P. Irwin, R. Denoon, and D. Scott, Wind tunnel testing of high-rise buildings: an output of the CTBUH Wind Engineering Working Group. Routledge, 2019.

[72] L. W. Chew, A. A. Aliabadi, and L. K. Norford, "Flows across high aspect ratio street canyons: Reynolds number independence revisited," Environmental Fluid Mechanics, vol. 18, no. 5, pp. 1275-1291, 2018.

[73] J. D. Holmes and S. A. Bekele, Wind Loading of Structures. Boca Raton: CRC Press, 4th ed., 2021.

[74] J. V. Healey, "Establishing a database for flight in the wakes of structures," Journal of Aircraft, vol. 29, no. 4, pp. 559-564, 1992.

[75] R. McKercher, "Modelling, flight dynamics and inner-loop control of an urban air mobility vehicle subject to empirically-developed urban airflow disturbances," Master's thesis, Carleton University, 2021. 